SAND94-3069 - UC-721

Unlimited Release

RECENES

Printed April 1996

MY 17 ใหล

OST1

\title{
Computational Implementation of a Systems Prioritization Methodology for the Waste Isolation Pilot Plant: A Preliminary Example
}

J. C. Helton, D. R. Anderson, B. L. Baker, J. E. Bean, J. W. Berglund, W. Beyeler, R. Blaine, K. Economy, J. W. Garner, S. C. Hora, R. C. Lincoln, M. G. Marietta, F. T. Mendenhall, N. H. Prindle, D. K. Rudeen, J. D. Schrieber, A. W. Shiver, L. N. Smith, P. N. Swift, P. Vaughn

Prepared by

Sandia National Laboratories

Albuquerque, New Mexico 87185 and Livermore, California 94550 for the United States Department of Energy under Contract DE-AC04-94AL85000

Approved for public release; distribution is unlimited.

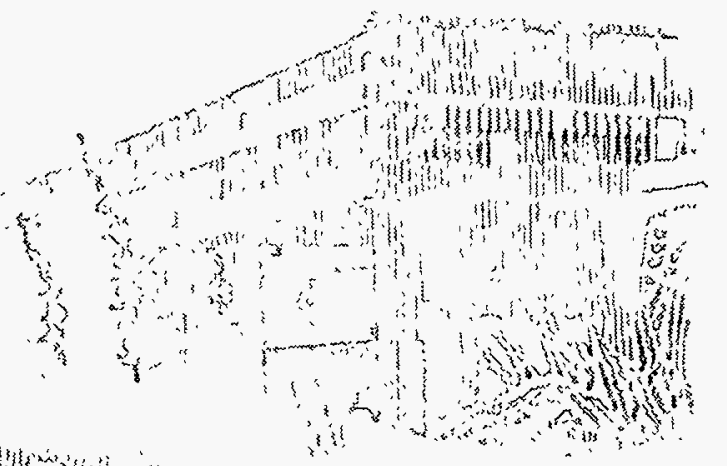


Issued by Sandia National Laboratories, operated for the United States Department of Energy by Sandia Corporation.

NOTICE: This report was prepared as an account of work sponsored by an agency of the United States Government. Neither the United States Government nor any agency thereof, nor any of their employees, nor any of their contractors, subcontractors, or their employees, makes any warranty, express or implied, or assumes any legal liability or responsibility for the accuracy, completeness, or usefulness of any information, apparatus, product, or process disclosed, or represents that its use would not infringe privately owned rights. Reference herein to any specific commercial product, process, or service by trade name, trademark, manufacturer, or otherwise, does not necessarily constitute or imply its endorsement, recommendation, or favoring by the United States Government, any agency thereof or any of their contractors or subcontractors. The views and opinions expressed herein do not necessarily state or reflect those of the United States Government, any agency thereof or any of their contractors.

Printed in the United States of America. This report has been reproduced directly from the best available copy.

Available to DOE and DOE contractors from Office of Scientific and Technical Information

PO Box 62

Oak Ridge, TN 37831

Prices available from (615) 576-8401, FTS 626-8401

Available to the public from

National Technical Information Service

US Department of Commerce

5285 Port Royal Rd

Springfield, VA 22161

NTIS price codes

Printed copy: A16

Microfiche copy: A01 


\title{
Computational Implementation of a Systems Prioritization Methodology for the Waste Isolation Pilot Plant: A Preliminary Example
}

\author{
J.C. Helton, ${ }^{1}$ D.R. Anderson, B.L. Baker, ${ }^{2}$ J.E. Bean, ${ }^{3}$ J.W. Berglund, ${ }^{3}$ W. Beyeler, ${ }^{4}$ R. Blaine, ${ }^{5}$ K. Economy, ${ }^{5}$ J.W. \\ Garner, ${ }^{6}$ S.C. Hora, ${ }^{7}$ R.C. Lincoln, M.G. Marietta, F.T. Mendenhall, N.H. Prindle, D.K. Rudeen, ${ }^{3}$ J.D. Schreiber, ${ }^{4}$ \\ A.W. Shiver, L.N. Smith, ${ }^{4}$ P.N. Swift, P. Vaughn \\ WIPP Performance Assessments Departments \\ Sandia National Laboratories \\ Albuquerque, New Mexico 87185-1328
}

\begin{abstract}
A systems prioritization methodology (SPM) is under development at Sandia National Laboratories (SNL) to provide guidance to the U.S. Department of Energy (DOE) on experimental programs and design modifications to be supported in the development of a successful licensing application to the U.S. Environmental Protection Agency (EPA) for the Waste Isolation Pilot Plant (WIPP) for the geologic disposal of transuranic (TRU) waste. The purpose of the SPM is to determine the probabilities that the implementation of different combinations of experimental programs and design modifications, referred to as activity sets, will lead to compliance with 40 CFR 191, Subparts B and C (Environmental Radiation Protection Standards for the Management and Disposal of Spent Nuclear Fuel, High-Level and Transuranic Waste) and 40 CFR 268.6 (Petitions to Allow Land Disposal of a Waste Prohibited under Subpart $C$ of Part 268, which implements the Resource Conservation and Recovery Act, i.e., RCRA). Appropriate tradeoffs between compliance probability, implementation cost and implementation time can then be made in the selection of the activity set to be supported in the development of a licensing application. Determination of compliance probabilities for individual activity sets involves probability spaces for (1) possible outcomes of the experimental programs, (2) uncertainty in analysis input given specific experimental outcomes and (3) possible future occurrences at the WIPP and also models for (1) fluid flow in the vicinity of the repository, (2) radionuclide release from the repository due to flowing groundwater, (3) groundwater flow and radionuclide transport in geologic formations overlying the repository, (4) radionuclide release to the surface environment due to cuttings and spallings removal in the event of a drilling intrusion and (5) transport of RCRA contaminants in gas and brine. Descriptions are given for the conceptual structure of the SPM and the manner in which this structure determines the computational implementation of an example SPM application. Due to the sophisticated structure of the SPM and the computational demands of many of its components, the overall computational structure must be organized carefully to provide the compliance probabilities for the large number of activity sets under consideration at an acceptable computational cost. Conceptually, the determination of each compliance probability is equivalent to a large numerical integration problem.
\end{abstract}

\footnotetext{
1 Department of Mathematics, Arizona State University, Tempe, AZ 85287

2 Technadyne Engineering Consultants, Albuquerque, NM 87112

3 New Mexico Engineering Research Institute, Albuquerque, NM 87106

4 Science Applications International Corp., Albuquerque, NM 87106

5 Ecodynamics Research Associates, Albuquerque, NM 87106

6 Applied Physics, Inc., Albuquerque, NM 87109

7 University of Hawaii at Hilo, Hilo, HI 96720
} 


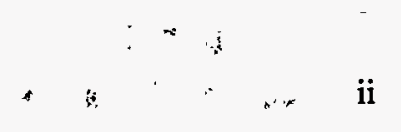




\section{Contents}

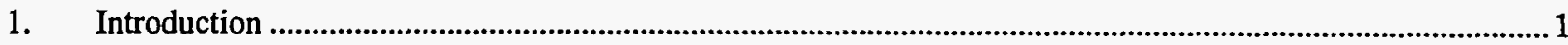

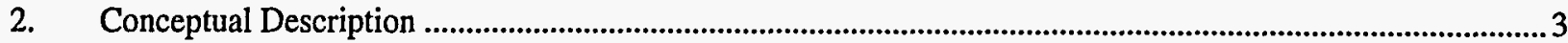

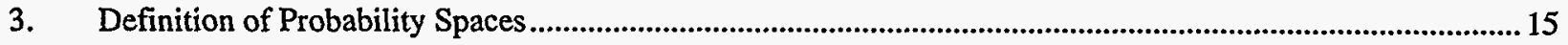

3.1 Probability Spaces $70 \mathbb{E}_{i}$ that Characterize Possible Outcomes of Individual Experimental Programs .. 15

3.2 Probability Spaces $70 \nVdash \mathcal{A} \varsigma_{j \varepsilon}$ that Characterize Input to Performance Assessment..................................22

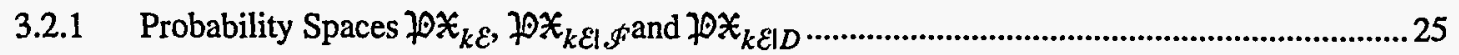

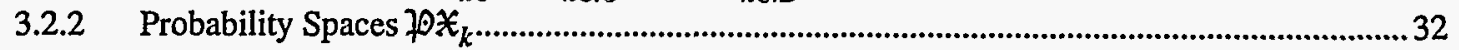

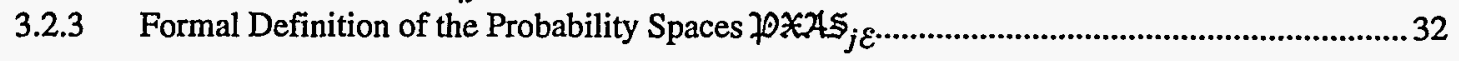

3.3 Probability Spaces $\mathcal{P}\left\{\mathcal{A} \Im_{j \mathcal{E}}\right.$ that Characterize Future Occurrences at the WIPP ..................................36

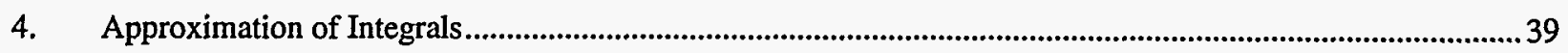

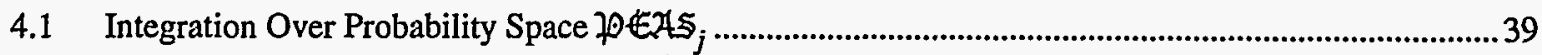

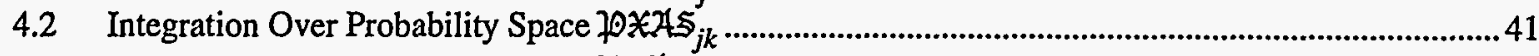

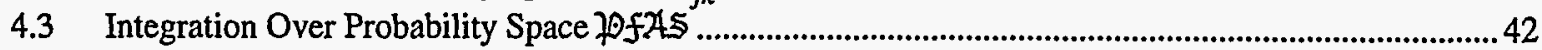

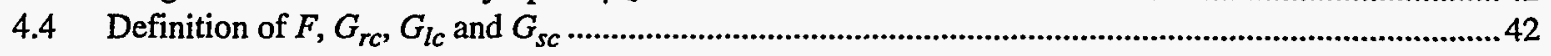

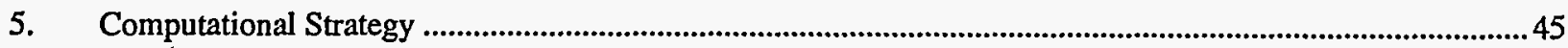

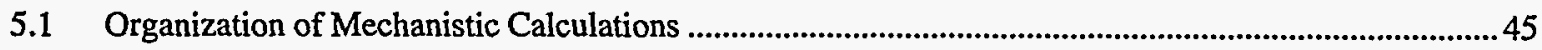

5.2 Mechanistic Calculations for $G_{r c}, G_{l c}$ and $G_{s c}$ (i.e., RCRA) ............................................................... 46

5.3 Mechanistic Calculations for $F$ (i.e., 40 CFR 191.13) .........................................................................52

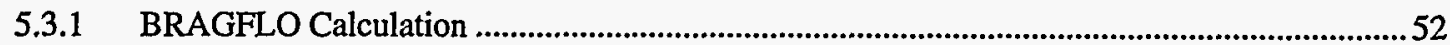

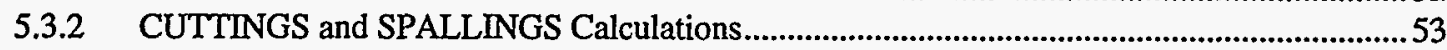

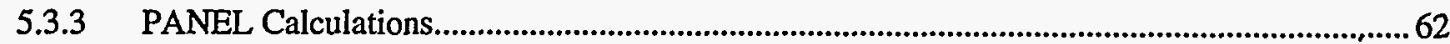

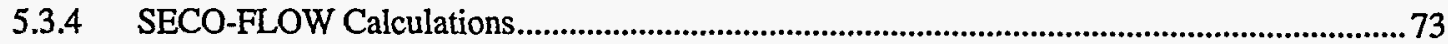

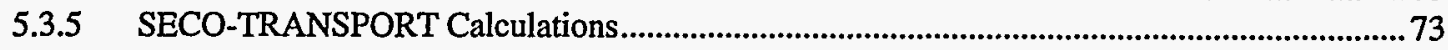

5.4 Monte Carlo Construction of CCDFs............................................................................................. 79

5.4.1 Cases Requiring Unique CCDF Constructions .........................................................................79

5.4.2 Organization of Mechanistic Results for Monte Carlo CCDF Construction................................8

5.4.3 Numerical Implementation of Monte Carlo CCDF Construction ................................................ 84

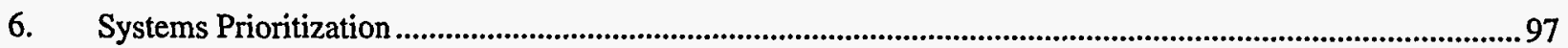

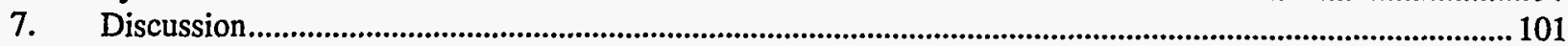

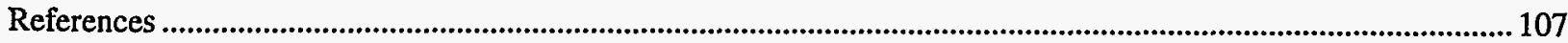




\section{Figures}

2.1 Distribution of CCDFs obtained in the 1991 WIPP PA for normalized released to the accessible environment including both cuttings removal and groundwater transport with gas generation in the repository and a dual-porosity transport model in the Culebra Dolomite...

4.1 Models used in SPM-1 to define functions $F, G_{r c}, G_{l c}$ and $G_{s c}$ in Eqs. (2.28) - (2.32) and elsewhere. The names for computer models (i.e., computer codes) are shown in capital letters

5.1 Logic diagram indicating 12 sets of BRAGFLO calculations associated with outcomes of experimental programs $E_{1}$ and $E_{2}$ and design modification $D_{2}$.

5.2 Time-dependent brine pressure in repository for 6 sets of BRAGFLO calculations with Brooks-Corey model indicated in Fig. 5.1

5.3 Time-dependent brine pressure in repository for 6 sets of BRAGFLO calculations with van Genuchten-

Parker model indicated in Fig. 5.1

5.4 Logic diagram indicating 4 sets of BRAGFLO calculations performed for outcomes of experimental program $E_{2}$

5.5 Cumulative brine flows to the Culebra calculated with BRAGFLO for E1 and E2 intrusions at $1000 \mathrm{yr}$ for subset $\varepsilon_{21}$ (i.e., for shaft permeabilities in the range $10^{-15}-10^{-13} \mathrm{~m}^{2}$ ) of $\varepsilon_{2}$

5.6 Cumulative brine flows to the Culebra calculated with BRAGFLO for E1 intrusions at $1000 \mathrm{yr}$ for subsets $\varepsilon_{22}$ (i.e., for shaft seal permeabilities in the range $10^{-18}-10^{-16} \mathrm{~m}^{2}$ ) and $\varepsilon_{23}$ (i.e., shaft seal permeabilities in the range $10^{-19}-10^{-18} \mathrm{~m}^{2}$ ) of $\varepsilon_{2}$.

5.7 Time-dependent pressures in repository calculated with BRAGFLO for intrusions at $1000 \mathrm{yrs}$

5.8 Total normalized release to the accessible environment due to cuttings removal for waste of average activity level (upper left) and to spallings for base-case spallings model (i.e., model implied by subset $\varepsilon_{81}$

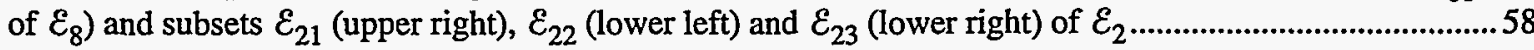

5.9 Selected time-dependent radionuclide inventories used in SPM-1

5.10 Logic diagram indicating 4 possible outcomes associated with experimental program $E_{8}$ that result in different spallings releases..

5.11 Logic diagram indicating 24 sets of PANEL calculations associated with outcomes of experimental programs $E_{3}$ and $E_{7}$ and design modification $D_{3}$ for each BRAGFLO calculation

5.12 Total release of colloidally-sorbed radionuclides to the Culebra over 10,000 yr for an E1E2 intrusion at $1000 \mathrm{yr}$, low permeability shaft seals, and different assumptions involving colloidal sorption

5.13 Total release of chemically dissolved radionuclides to the Culebra over 10,000 yr for an E1E2 intrusion at $1000 \mathrm{yr}$, low permeability shaft seals, and different assumptions involving colloidal sorption .....................68

5.14 Total release of chemically-dissolved radionuclides to the Culebra over 10,000 yr for an E1E2 intrusion at $1000 \mathrm{yr}$, low permeability shaft seals and different assumptions involving colloidal sorption and chemical solubility....

Release of individual colloidally-sorbed radionuclides to the Culebra over 10,000 yr for an E1E2 intrusion at $1000 \mathrm{yr}$, low permeability shaft seals and different assumptions involving colloidal sorption .......70

5.16 Release of individual chemically-dissolved radionuclides to the Culebra over 10,000 yr for an E1E2 intrusion at $1000 \mathrm{yr}$, low permeability shaft seals, $\mu=-9$ for colloidal solubility and different assumptions involving chemical solubility....

5.17 Example radionuclide discharges to the Culebra calculated by PANEL and used as input to radionuclide transport calculations performed with SECO-TRANSPORT for an E1E2 intrusion at $1000 \mathrm{yr}$, low permeability shaft seals, $\mu=-9$ for colloidal solubility, and different assumptions involving chemical solubility

5.18 Logic diagram indicating 20 possible sets of conditions in the Culebra associated with experimental programs $E_{4}, E_{5}$ and $E_{6}$ that could require SECO-TRANSPORT calculations 


\section{Figures (continued)}

5.19 Scatterplot for total normalized release to Culebra over 10,000 yr versus total normalized release to the accessible environment due to groundwater transport with no chemical retardation and no matrix diffusion for Scenario $S^{4-}(2,0)$, where $\mathcal{S}^{+-}(2,0)$ is used to denote an E1E2 intrusion occurring $1000 \mathrm{yr}$ after repository closure

5.20 Scatterplot for total normalized release to the accessible environment over 10,000 yr due to groundwater transport with chemical retardation, no clay lining in fractures and matrix diffusion versus CULFRSP (Culebra fracture spacing) for scenario $S^{+-}(2,0)$, where $S^{+-}(2,0)$ is used to denote an E1E2 intrusion occurring 1000 yr after repository closure.

5.21 Scatterplots for normalized releases of Am-241 and Pu-239 to the accessible environment over $10,000 \mathrm{yr}$ due to groundwater transport with no chemical retardation, no clay lining in fractures and matrix diffusion versus CULFRSP (Culebra fracture spacing) for scenario $\delta^{4-}(2,0)$, where $\delta^{4-}(2,0)$ is used to denote an E1E2 intrusion occurring $1000 \mathrm{yr}$ after repository closure

5.22 Total release of chemically-dissolved radionuclides to the accessible environment over $10,000 \mathrm{yr}$ due to groundwater transport for an E1E2 intrusion at $1000 \mathrm{yr}, \mu=-9$ for colloidal solubility, and different assumptions involving shaft seal permeability, chemical solubility and retardation.

5.23 Release of individual chemically-dissolved radionuclides to the accessible environment over $10,000 \mathrm{yr}$ due to groundwater transport for an E1E2 intrusion at $1000 \mathrm{yr}$, low permeability shaft seals, $\mu=-9$ for colloidal solubility, and different assumptions involving chemical solubility and retardation

5.24 Example of input file used in Monte Carlo CCDF construction ....................................................................8.

5.25 Excavated areas and planned seals in the WIPP repository .......................................................................... 88

5.26 Contour map of elevation to first major conductor below WIPP disposal area ................................................93

5.27 Example distributions of CCDFs for normalized release to the accessible environment constructed for low permeability shafts (ISHAFT $=3$ ), low chemical solubility $(I C H M S O L=3$ ), low colloid solubility $(J C O L S O L=8)$, complete retardation of dissolved release to the Culebra $(K C U L T R N=5)$, and four outcomes for spallings ( $L S P A L=1 \Rightarrow$ baseline, $L S P A L=2 \Rightarrow$ baseline $/ 2, L S P A L=3 \Rightarrow$ baseline $/ 10$, $L S P A L=4 \Rightarrow$ baseline $/ 100$ )

6.1 Scatterplots of cost $\left(c A S_{j}\right)$ versus compliance probability $\left(p A S_{j}\right)$ for $p A S_{j}=p A S M_{j}$ (upper frame), $p A S_{j}=$

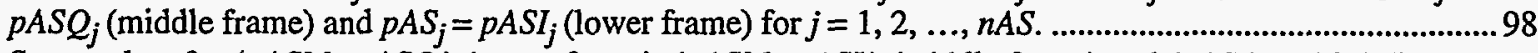

6.2 Scatterplots for $\left(p A S M_{j}, p A S Q_{j}\right)$ (upper frame), $\left(p A S M_{j}, p A S I_{j}\right)$ (middle frame) and $\left(p A S Q_{j}, p A S I_{j}\right)$ (lower frame) for $j=1,2, \ldots, n A S$.

\section{Tables}

2.1 Experimental Program $\left(E_{i}\right)$ and Design Modifications $\left(D_{i}\right)$ Considered in SPM-1 ........................................ 4

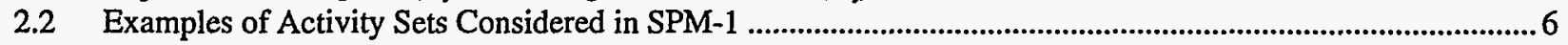

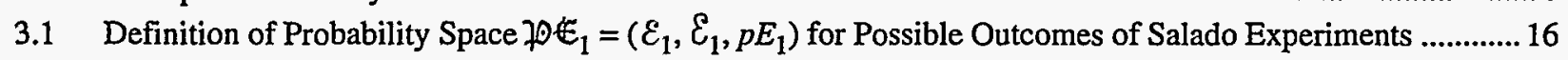

3.2 Definition of Probability Space $\mathfrak{P E _ { 2 }}=\left(\varepsilon_{2}, \varepsilon_{2}, p E_{2}\right)$ for Possible Outcomes of Seal Experiments ................17

3.3 Definition of Probability Space $\mathcal{D} E_{3}=\left(\varepsilon_{3}, \varepsilon_{3}, p E_{3}\right)$ for Possible Outcomes of Source Term Experiments to Determine Chemical Solubility...

3.4 Definition of Probability Space $\not \supset \mathbb{E}_{4}=\left(\varepsilon_{4}, \varepsilon_{4}, p E_{4}\right)$ for Possible Outcomes of Single-Well Culebra Experiments to Determine Physical Retardation.

3.5 Definition of Probability Space $\not \mathcal{B} \mathbb{E}_{5}=\left(\mathcal{E}_{5}, \mathcal{E}_{5}, p E_{5}\right)$ for Possible Outcomes of Multiwell Culebra Experimental to Determine Physical Retardation 


\section{Tables (continued)}

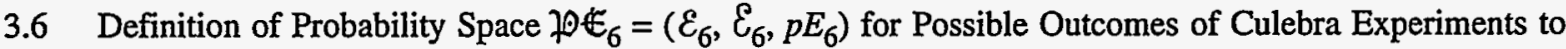
Determine Chemical Retardation

3.7 Definition of Probability Space $\not \supseteqq E_{7}=\left(\varepsilon_{7}, \varepsilon_{7}, p E_{7}\right)$ for Possible Outcomes of Source Term Experiments to Determine Actinide Attachment to Mobile Colloids Using Present and Past Waste Inventory Having Significant Amounts of High Molecular Weight Organic Materials Such As May Be Found in Soils..............21

3.8 Definition of Probability Space $7 \bigcirc E_{8}=\left(\varepsilon_{8}, \mathcal{E}_{8}, p E_{8}\right)$ for Possible Outcomes of Spallings Experiments.........22

3.9 Elements $S_{i j}$ of $\varepsilon_{i}$ for Experimental Program $E_{i}$ Used in the Definition of the Probability Spaces $70 * \mathcal{A ~}_{j} \varepsilon$ in SPM-1

3.10 Definition of Probability Spaces $\neg \bullet X_{1 \varepsilon}=\left(X_{1 \varepsilon}, X_{1 \varepsilon}, p X_{1 \varepsilon}\right)$ Associated with Possible Outcomes of Salado Experiments

3.11 Definition of Probability Spaces $\mathfrak{\mathfrak { X } _ { 2 \varepsilon }}=\left(\mathrm{c}_{2 \varepsilon}, \mathscr{X}_{2 \varepsilon}, p X_{2 \varepsilon}\right)$ Associated with Possible Outcomes of Seal Experiments

3.12 Definition of Probability Spaces $\mathfrak{P X _ { 3 \varepsilon }}=\left(X_{3 \varepsilon}, X_{3 \varepsilon}, p X_{3 \varepsilon}\right)$ Associated with Possible Outcomes of Source Term Experiments to Determine Chemical Solubility

3.13 Definition of Probability Spaces $20 \mathcal{X}_{4 \varepsilon}=\left(X_{4 \varepsilon}, X_{4 \varepsilon}, p X_{4 \varepsilon}\right)$ Associated with Possible Outcomes of Single-Well Culebra Experiments to Determine Physical Retardation

3.14 Definition of Probability Spaces $\not \cap X_{5 \varepsilon}=\left(X_{5 \varepsilon}, X_{5 \varepsilon}, p X_{5 \varepsilon}\right)$ Associated with Possible Outcomes of Multiwell Culebra Experiments to Determine Physical Retardation

3.15 Definition of Probability Spaces $\neg \otimes X_{6 \varepsilon}=\left({ }^{\circ} X_{6 \varepsilon}, X_{6 \varepsilon}, p X_{6 \varepsilon}\right)$ Associated with Possible Outcomes of Culebra Experiments to Determine Chemical Retardation

3.16 Definition of Probability Spaces $\not \cap X_{7 \varepsilon}=\left({ }^{\prime} X_{7 \varepsilon}, X_{7 \varepsilon}, p X_{7 \varepsilon}\right)$ Associated with Possible Outcomes of Source Term Experiments to Determine Actinide Attachment to Mobile Colloids Using Present and Past Waste Inventory Having Significant Amounts of High Molecular Weight Organic Materials Such as May Be Found in Soils

3.17 Definition of Probability Spaces $70 \mathcal{X}_{7 \varepsilon}=\left(\propto X_{7 \varepsilon}, X_{7 \varepsilon}, p X_{7 \varepsilon}\right), \varepsilon=\varepsilon_{7 i} \mid D_{3}$, Associated with Possible Outcomes of Source Term Experiments to Determine Actinide Attachment to Mobile Colloids Using Present and Past Waste Inventory Having Significant Amounts of High Molecular Weight Organic Materials Such As May Be Found in Soils and the Implementation of Design Modification $D_{3}$.

3.18 Definition of Probability Spaces $\not ڤ X_{8 \varepsilon}=\left({ }^{2} X_{8 \varepsilon}, X_{8 \varepsilon}, p X_{8 \varepsilon}\right)$ Associated with Possible Outcomes of Spallings Experiments

3.19 Variables Associated with the Probability Space $\mathcal{P O X}_{S}$ and Used in the Calculation of Brine Flow, Gas Generation and Gas Flow in the Vicinity of the Repository

3.20 Variables Associated with the Probability Space $10 X_{D}$ and Used in the Calculation of Cuttings and Spallings Releases

3.21 Variables Associated with the Probability Space $\mathfrak{D X}_{C}$ and Used in the Calculation of Radionuclide Transport in the Culebra Dolomite.

3.22 Definition of Probability Space $70 X \mathcal{A} S_{j \varepsilon}$ for Activity Sets $A S_{1}, A S_{2}, A S_{j}$ and $A S_{n A S}$ in Table 2.2..................37

4.1 Sets $\varepsilon A S_{j k} k=1,2, \ldots, n D=18$, Associated with Activity Set $A S_{j}$ and Used in Approximation to $p C_{j}$ in Eq. (4.3), where $A S_{j}$ is the Activity Set that Contains Experimental Programs $E_{2}, E_{3}$, and $E_{4}$ and Design Modification $D_{2}$.

4.2. Summary of Computer Models Used in SPM-1 to Define Functions $F, G_{r c}, G_{l c}$ and $G_{s c}$ Appearing in Eqs. (2.28) - (2.32) and Elsewhere . 


\section{Tables (continued)}

5.1 Definition of $G_{r c}, G_{l c}$ and $G_{s c}$ for 132 Cases Considered in SPM-1

5.2 Projected Activity Levels $\left(\mathrm{Ci}^{\mathrm{i}} / \mathrm{m}^{2}\right)$ Used in the 1992 WIPP PA Due to Waste that is Currently Stored and May be Shipped to the WIPP.

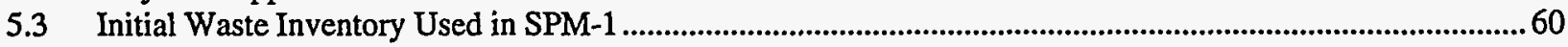

5.4 Summary of LHSs Generated for the Probability Spaces $10 \mathbb{X}_{3 \varepsilon} \times 10 \mathbb{Z}_{7 \mathscr{F}}$ Indicated in Fig. 5.11 to Define Input for PANEL Calculations.

5.5 Cumulative Brine Releases from the Repository to the Culebra over 10,000 yr for E1 and E2 Intrusions Occurring at $1000 \mathrm{yr}$ and High Permeability Shaft Seals...............................................................................66

5.6 Partial Summary of PANEL Results Calculated for a Drilling Intrusion at $1000 \mathrm{yr}$ and Saved for Use in

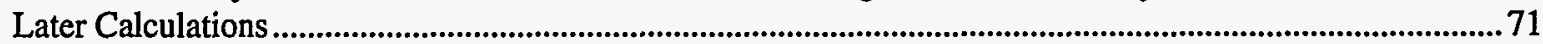

5.7 Partial Summary of SECO-TRANSPORT Results Calculated for a Drilling Intrusion at $1000 \mathrm{yr}$ and

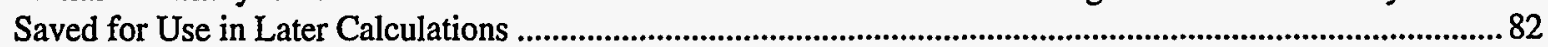

5.8 Summary of Algorithm Used to Define Normalized Releases to the Accessible Environment Due to Groundwater Transport..

5.9 Information Available for Construction of Cuttings Release, $n R C\left(\mathrm{f}_{n}\right)$, and Groundwater Release, $n R G\left(\mathrm{f}_{n}\right)$,

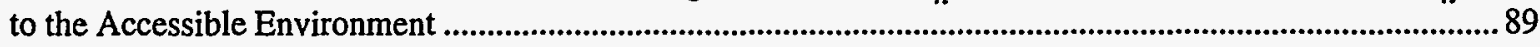

5.10 Assumptions Used in Construction of CCDFs for Groundwater Release to the Accessible Environment.........91 


$$
\text { . }
$$




\section{Introduction}

The Waste Isolation Pilot Plant (WIPP) is being developed by the U.S. Department of Energy (DOE) as a disposal facility for transuranic waste (TRU). ${ }^{1-3}$ As part of the development process, a sequence of performance assessments (PAs) for the WIPP has been carried out by Sandia National Laboratories (SNL). The initial development work for these iterative PAs was conducted in $1989^{4,5}$ and built on earlier analysis work performed at SNL for the WIPP. ${ }^{6}$ Then, PAs were performed in 1990, ${ }^{7-9} 1991^{10-15}$ and 1992.16-20 The purpose of these PAs was to organize the knowledge currently available about the WIPP and to provide guidance with respect to future research and development efforts. In particular, insights were sought on the compliance of the WIPP with applicable environmental regulations, including 40 CFR 191, Subparts B and C (Environmental Radiation Protection Standards for the Management and Disposal of Spent Nuclear Fuel, High-Level and Transuranic Radioactive Wastes) ${ }^{21,22}$ and 40 CFR 268.6 (Petitions to Allow Land Disposal of a Waste Prohibited Under Subpart C of Part 268), ${ }^{23}$ which is the regulation implementing the Resource Conservation and Recovery Act (RCRA) ${ }^{24}$ for the disposal of hazardous wastes. It is currently anticipated that the next full PA for the WIPP will be performed in 1996 and will form the basis for an application to the U.S. Environmental Protection Agency (EPA) for the licensing of the WIPP for the disposal of TRU waste.

A systems prioritization methodology (SPM) has been proposed to provide additional support in the development of a defensible application to the EPA for the licensing of the WIPP. ${ }^{25-35}$ The basic entity considered in the SPM is a set of experimental programs and design modifications referred to as an activity set. There are many possible activity sets that might be implemented at the WIPP, each with different costs, time requirements and likelihoods of leading to a favorable licensing decision. The purpose of the SPM is to provide guidance in the selection of the most appropriate experimental programs and design modifications to support in the development of a defensible and ultimately acceptable licensing application for the WIPP.

The basic outcome of the SPM is a sequence of triples of the form

$$
\left(p A S_{j}, c A S_{j}, t A S_{j}\right), j=1,2, \ldots, n A S
$$

where $p A S_{j}$ is the probability that performance of activity set $A S_{j}$ will lead to compliance with applicable regulations, $c A S_{j}$ is the cost (\$) of implementing activity set $A S_{j}, t A S_{j}$ is the time (yr) required to implement activity set $A S_{j}$ and $n A S$ is the number of activity sets under consideration. Once the information in Eq. (1.1) is available, it can be examined in various ways to determine favorable combinations of compliance probability, implementation cost and implementation time. In particular, two and three dimensional plots involving $p A S_{j}, c A S_{j}$ and $t A S_{j}$ can be examined to determine such combinations. More sophisticated search procedures are also possible, although it is not currently apparent that they will be needed. The implementation costs and times for the individual experimental programs and design modifications are inputs to the SPM. As a result, the determination of the implementation costs $c A S_{j}$ and 
times $t A S_{j}$ for the activity sets is straightforward. Further, assessment of the implications of compliance probabilities and associated implementation costs and times is also reasonably straightforward in at least a mechanical sense. The primary conceptual complexities and computational demands of the SPM are associated with the determination of the compliance probabilities. The determination of these probabilities is the primary focus of this presentation.

A central part of the SPM is the definition of a scientifically sound baseline for each experimental program under consideration. The baseline for a given experimental program is the least conservative set of assumptions about the parameters under study that can be defended with high confidence if the experimental program is not carried out. Here, conservative means having implications that tend to decrease the acceptability of the WIPP as a disposal facility for TRU waste. The designation least conservative is important because the intent is to define assumptions that are conservative but not more conservative than is implied by the information that is available if the experimental program is not carried out. If an experimental program is not included in an activity set, then the scientifically sound baseline for that program is used in the calculation of the compliance probability $p A S_{j}$ for the activity set. As a result, the compliance probabilities calculated for individual activity sets include conservative assumptions about PA parameters that are studied in experimental programs not included in these sets. The use of least conservative assumptions is critical to avoid introducing excessive pessimism into the compliance probabilities.

This presentation describes the design and implementation of a set of calculations performed to support a trial application of the SPM, designated SPM-1, in the summer of 1994. The purpose of SPM-1 and the descriptions contained in this presentation is to illustrate the ideas associated with the implementation of the SPM. Although computational assumptions and results are presented for illustration, the SPM-1 analysis is not suitable for assessing the possible suitability of the WIPP as a disposal facility for TRU waste or making selection decisions with respect to experimental programs and design modifications. Rather, SPM-1 is a vehicle for discussing the SPM and illustrating the ideas and computational requirements that underlie it. A list of detailed caveats is provided in Ref. 35. 


\section{Conceptual Description}

The purpose of the SPM is to determine subjective (i.e., degree of belief or epistemic) probabilities that the WIPP will comply with appropriate regulations after the performance of specified experimental programs and design modifications. For notational convenience, let

$$
E_{1}, E_{2}, \ldots, E_{n E}
$$

and

$$
D_{1}, D_{2}, \ldots, D_{n D}
$$

designate the experimental programs and design modifications under consideration, where $n E$ is the number of experimental programs and $n D$ is the number of design modifications. The experimental programs and design modifications considered in SPM-1 are listed in Table 2.1.

By their nature, experimental programs can have many possible outcomes. After all, if it was known a priori that an experiment could have only one possible outcome, then there would be little reason to conduct the experiment. As a result, each experimental program $E_{i}$ in Eq. (2.1) can be represented by a probability space

$$
\not \mathcal{B} E_{i}=\left(\varepsilon_{i}, \varepsilon_{i}, p E_{i}\right)
$$

where

$$
\begin{aligned}
\varepsilon_{i} & =\left\{\mathbf{e}_{i}: \mathbf{e}_{i} \text { is a possible outcome of the experiments in } E_{i}\right\}, \\
\varepsilon_{i} & =\left\{\varepsilon_{:}: \mathcal{E} \text { is a subset of } \varepsilon_{i} \text { for which a probability will be defined }\right\}, \\
p E_{i} & =\text { a function that defines probability for the elements of } \varepsilon_{i} .
\end{aligned}
$$

In general, $E_{i}$ would involve a number of individual experiments, and the $e_{i}$ would be vectors containing possible outcomes of these experiments (e.g., if $E_{i}$ involved 50 individual experiments and each of these experiments produced 6 results, then $\varepsilon_{i}$ would be a subset of $R^{300}$ ). In a careful development of the concepts of probability, $\varepsilon_{i}$ would have the properties that (1) if $\varepsilon \in \varepsilon_{i}$, then $\varepsilon^{c} \in \mathcal{E}_{i}$, where the superscript $c$ is used to denote the complement of $\mathcal{E}$, and (2) if $\mathcal{S}_{1}, \mathcal{S}_{2}, \ldots$ is a sequence of sets from $\varepsilon_{i}$, then $\cup_{j} \mathcal{S}_{j} \in \mathcal{E}_{i}$ and $\cap_{j} \mathcal{S}_{j} \in \mathcal{E}_{i}$. Further, $p E_{i}$ would have the properties that (1) if $\varepsilon \in \mathcal{E}_{i}$, then $0 \leq p E_{i}(\mathcal{E}) \leq 1$, and (2) if $\mathcal{S}_{1}, \mathcal{S}_{2}, \ldots$ is a sequence of disjoint sets from $\mathcal{E}_{i}$ (i.e., $\delta_{i} \cap$ $S_{j}=\varnothing$ if $\left.i \neq j\right)$, then $p E_{i}\left(\cup_{j} S_{j}\right)=\Sigma_{j} p E_{j}\left(S_{j}\right)$. In the terminology of probability theory, $\varepsilon_{i}$ is the sample space, the elements $\mathcal{E}$ of $\mathcal{E}$ are events, and the function $p E_{i}$ is a probability function or measure. In the SPM, the probability

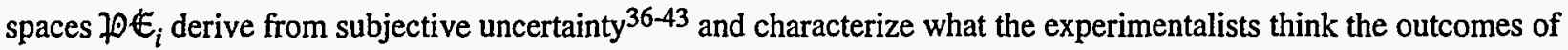


Table 2.1. Experimental Programs $\left(E_{i}\right)$ and Design Modifications $\left(D_{i}\right)$ Considered in SPM-1.

\begin{tabular}{cl}
\hline Program & \\
\hline$E_{1}$ & Salado experiments to determine physical properties of the Salado Formation. \\
$E_{2}$ & Seal experiments to determine seal permeabilities. \\
$E_{3}$ & Source term experiments to determine chemical solubility of radionuclides under repository conditions. \\
$E_{4}$ & Single-well Culebra experiments to determine physical retardation. \\
$E_{5}$ & Multiwell Culebra experiments to determine physical retardation. \\
$E_{6}$ & Culebra experiments to determine chemical retardation. \\
$E_{7}$ & $\quad \begin{array}{l}\text { Source term experiments to determine actinide attachment to colloids using present and past waste } \\
\text { inventory having no significant amounts of high molecular weight organic materials such as may be } \\
\end{array}$ \\
$E_{8}$ & found in soils. \\
$D_{1}$ & Spallings experiments. \\
$D_{2}$ & Reduction of gas generation potential by exclusion of corrodible metals. \\
$D_{3}$ & Reduction of potential for colloids by exclusion of soils. \\
\hline
\end{tabular}

their experiments might be and how likely these outcomes are. The concept of a probability space is one of the fundamental ideas of probability theory ${ }^{44-46}$ and will appear repeatedly in this description of the SPM.

As indicated in the Introduction, the basic entity considered in the SPM is designated an activity set and is a set of specific experiments and design modifications, with the goal being to determine the (subjective) probability that the WIPP will comply with the applicable regulations given that these experiments and design modifications are carried out. For notational convenience, let

$$
A S_{1}, A S_{2}, \ldots, A S_{n A S}
$$

denote the activity sets selected for consideration, where $n A S$ is the number of such sets. The properties of the individual activity sets can be further specified by

$$
\begin{aligned}
\varepsilon S_{j} & =\left\{i \text { : experimental program } E_{i} \text { is included in } A S_{j}\right\} \\
\varepsilon S_{j}^{c} & =\left\{i \text { : experimental program } E_{i} \text { is not included in } A S_{j}\right\} \\
\Phi S_{j} & =\left\{i \text { : design modification } D_{i} \text { is included in } A S_{j}\right\}
\end{aligned}
$$


for $j=1,2, \ldots, n A S$. The sets $\varepsilon S_{j}, \varepsilon S_{j}^{c}$ and $\Phi S_{j}$ will be used in the development of an exact description of the relationships between the experimental programs and design modifications associated with activity set $A S_{j}$ and the resultant input to the PA models.

The analysis for SPM-1 considered all activity sets that could be obtained from the experimental programs and design modifications in Table 2.1, which led to $n A S=1536$ activity sets. Examples of these activity sets are given in Table 2.2. The experimental activities associated with $E_{4}$ are a subset of the activities associated with $E_{5}$. Because of this relationship, an activity set $A S_{j}$ will not be indicated as containing both $E_{4}$ and $E_{5}$ as the specification of $E_{4}$ is redundant given the specification of $E_{5}$ (i.e., $\& S_{j}$ can contain 4 or 5 , but not both 4 and 5). Further, 4 is not included in $\varepsilon S_{j}^{c}$ when $A S_{j}$ includes $E_{5}$; similarly, only 5 is included in $\varepsilon S_{j}^{c}$ when $A S_{j}$ contains neither $E_{4}$ or $E_{5}$. The effects of preceding conventions appear in conjunction with each of the example activity sets in Table 2.2.

The set of all possible experimental outcomes associated with activity set $A S_{j}$ is given by

$$
\begin{aligned}
\xi I S_{j} & =\times_{i \in \varepsilon S_{j}} \varepsilon_{i} \\
& =\varepsilon_{i(1)} \times \varepsilon_{i(2)} \times \cdots \times \varepsilon_{i\left(n E S_{j}\right)} \\
& =\left\{\left[\mathbf{e}_{i(1)}, \mathbf{e}_{i(2)}, \ldots, \mathbf{e}_{i\left(n E S_{j}\right)}\right]: \quad \mathbf{e}_{i(j)} \in \varepsilon_{i(j)} \text { for } j=1,2, \ldots, n E S_{j}\right\}
\end{aligned}
$$

where

$$
\mathcal{E} S_{j}=\left\{i(1), i(2), \ldots, i\left(n E S_{j}\right)\right\}
$$

as indicated in Eq. (2.8). Associated with this set of experimental outcomes is the probability space

$$
\not 0 \in A \Im_{j}=\left(\xi \not S_{j}, E A \delta_{j}, p E A S_{j}\right)
$$

The set $\mathcal{E} \mathcal{L}_{j}$ is given by

$$
\mathcal{E} A \mathcal{S}_{j}=x_{i \in \varepsilon s_{j}} \varepsilon_{i}=\varepsilon_{i(1)} \times \varepsilon_{i(2)} \times \cdots \times \varepsilon_{i\left(n E s_{j}\right)} .
$$

Further, if the outcomes of the individual experimental programs are independent (i.e., if the probability assessments associated with individual experimental programs do not affect the probability assessments associated with other experimental programs), then

$$
\begin{aligned}
p E A S_{j}(S) & =\Pi_{i \in \varepsilon S_{j}} p E_{i}\left(S_{i}\right) \\
& =p E_{i(1)}\left(S_{i(1)}\right) p E_{i(2)}\left(S_{i(2)}\right) \cdots p E_{i\left(n E S_{j}\right)}\left(S_{i\left(n E S_{j}\right)}\right)
\end{aligned}
$$




$$
\begin{aligned}
A S_{1}= & \left\{E_{1}\right\}, \varepsilon S_{1}=\{1\}, \varepsilon S_{1}^{c}=\{2,3,5,6,7,8\}, \Phi S_{1}=\phi \\
A S_{2}= & \left\{E_{1}, E_{2}, D_{1}\right\}, \varepsilon S_{2}=\{1,2\}, \varepsilon S_{2}^{c},=\{3,5,6,7,8\}, \Phi S_{2}=\{1\} \\
& \vdots \\
A S_{j}= & \left\{E_{1}, E_{2}, E_{5}, E_{6}, D_{1}, D_{2}\right\}, \varepsilon S_{j}=\{1,2,5,6\}, \varepsilon S_{j}^{c}=\{3,7,8\}, \Phi S_{j}=\{1,2\} \\
& \vdots \\
A S_{n A S}= & \left\{E_{1}, E_{2}, E_{3}, E_{5}, E_{6}, E_{7}, E_{8}, D_{1}, D_{2}, D_{3}\right\}, \varepsilon S_{n A S}=\{1,2,3,5,6,7,8\}, \varepsilon S_{n A S}^{c}=\phi, \Phi S_{n A S}=\{1,2,3\}
\end{aligned}
$$

where

$$
\mathcal{S}=\times_{i \in E S_{j}} S_{i}=S_{i(1)} \times S_{i(2)} \times \cdots \times S_{i\left(n E S_{j}\right)}
$$

is an element of $\mathcal{E} \& \delta_{j}$. If the experimental outcomes associated with the individual experimental programs in $A S_{j}$ are not independent, then a more complex definition for $p E A S_{j}$ will be required.

As indicated earlier, the purpose of the SPM is to determine the (subjective) probabilities that the WIPP will comply with appropriate regulations given the implementation of the individual activity sets $A S_{j}$ indicated in Eq. (2.7). This determination requires the use of PA models to calculate the various quantities addressed in the regulations. In turn, this requires the determination of values for the many inputs to the PA models. For notational convenience, let

$$
\mathbf{x}=\left[x_{1}, x_{2}, \ldots, x_{n I}\right]
$$

represent the inputs to the PA models, where $n I$ is the number of inputs. The possible values for $\mathrm{x}$ will depend on the design modifications associated with an activity set $A S_{j}$ (see $\Phi S_{j}$ in Eq. (2.10)) and will also change for different experimental outcomes associated with this activity set (see $\& A S_{j}$ in Eq. (2.11)). Further, given even a single element of $\& A \mathcal{S}_{j}$ (i.e., experimental outcome $\left.\left[\mathbf{e}_{i(1)}, \mathbf{e}_{i(2)}, \ldots, \mathbf{e}_{i\left(n E S_{j}\right)}\right]\right)$, it is unlikely that $\mathbf{x}$ will be uniquely determined; rather, a number of possible values for $\mathbf{x}$ of varying degrees of plausibility will exist. Thus, given an element of $\mathcal{E A} \delta_{j}$, or more typically a subset of $\& A S_{j}$, it will be necessary to characterize where the possible values for $\mathbf{x}$ are located.

The uncertainty in $\mathbf{x}$ can be characterized with appropriately defined probability spaces. Suppose $\varepsilon \in \mathcal{E} \mathcal{A} \mathscr{S}_{j}$ (i.e., $\mathcal{E}$ is a set of experimental outcomes associated with activity set $A S_{j}$ for which a probability has been defined). The corresponding uncertainty in $\mathbf{X}$ can be represented by the probability space 


$$
\mathfrak{P * X S _ { j \varepsilon }}=\left[\propto X \not S_{j \varepsilon}, X A S_{j \varepsilon}, p X A S_{j \varepsilon}\right]
$$

where

${ } X \not A S_{j} \varepsilon=\{\mathbf{x}: \mathbf{x}$ is possibly the appropriate input to use in PA given the occurrence of $\varepsilon\}$,

$X A \delta_{j \varepsilon}=\left\{\mathrm{e} X: \mathrm{e} X\right.$ is a subset of $\mathrm{e} X \not S_{j \varepsilon}$ for which a probability will be defined $\}$,

$p X A S_{j \varepsilon}=$ a function that defines probability for elements of $2 A \ell_{j \varepsilon}$

and the subscript $\varepsilon$ indicates that the values for $\triangle X \not \mathcal{S}_{j \varepsilon}, X \mathcal{L} \mathcal{S}_{j \varepsilon}$ and $p X A S_{j \varepsilon}$ depend on the value of $\varepsilon$. In general, the uncertainty in $\mathbf{x}$ characterized by the preceding probability space will increase as the size of the set $\varepsilon$ increases; however, all uncertainty in $\mathbf{X}$ is unlikely to go away if consideration is restricted to a single element of $\varepsilon d \mathcal{S}_{j}$ (i.e., a subset of $\mathcal{E} A S_{j}$ containing only one element). The definition of $\not \nabla \nVdash \mathcal{A} S_{j \mathcal{E}}$ can also depend on the design modifications associated with the activity set under consideration as the same experimental results can have different implications for PA in the presence of different design modifications. The probabilities $p X A S_{j \varepsilon}$ are characterizing subjective uncertainty in the sense that they are indicating a degree of belief as to where a fixed but unknown quantity is located. In the unlikely situation that the vector $\mathbf{X}$ was believed to be known with complete certainty, then $\Varangle \not S_{j \varepsilon}$ would contain only this one element.

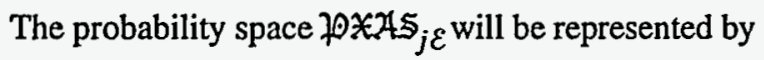

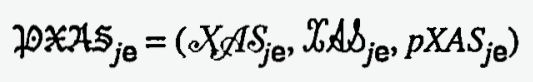

when a single element $e$ of $\varepsilon A S S_{j}$ is under consideration. Although the SPM involves the consideration of probability spaces of the form $70 * 4 \varsigma_{j \varepsilon}$, the final PA for the WIPP after the experimental programs have been completed will involve consideration of a probability space of the form $70 X \mathcal{A}_{j \mathrm{j}}$, where e corresponds to the actual experimental outcomes obtained in the experimental programs.

In practice, $70 * A \varsigma_{j \varepsilon}$ is built up by developing probability spaces

$$
\not \cap X_{j \varepsilon k}=\left(\alpha_{j \varepsilon k}, X_{j \varepsilon k}, p X_{j \varepsilon k}\right)
$$

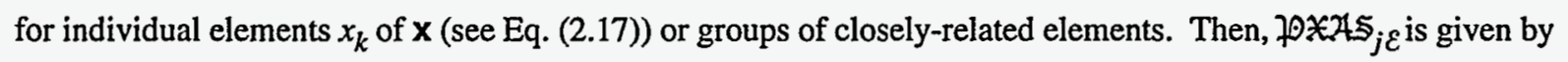

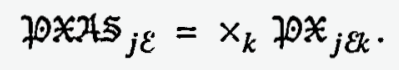

Although the notation $\not \cap \mathscr{X}_{j \xi \mathcal{K}}$ indicates a dependence on the outcomes of all experimental programs (i.e., see the definition of $\xi A S_{j}$ in Eq. (2.11), of which $\varepsilon$ is a subset) and also all design modifications, such complexity is usually not the case, with the result that a simpler notation is often possible. Further, it is possible that none of the 
experimental programs (i.e., $E_{1}, E_{2}, \ldots, E_{n E}$ ) or design modifications (i.e., $D_{1}, D_{2}, \ldots, D_{n D}$ ) under consideration will

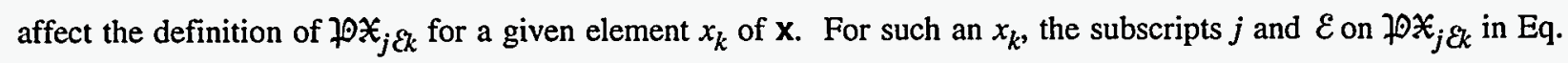
(2.21) can be dropped, and the representation for $10 \ldots \mathcal{A} \Im_{j \varepsilon}$ becomes

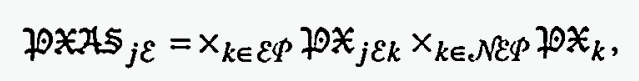

where

$$
\begin{aligned}
& \mathcal{E}=\left\{k: \text { information affecting characterization of the uncertainty in } x_{k}\right. \text { is provided by one or more of the } \\
& \text { experimental programs or design modifications under consideration }\}
\end{aligned}
$$

In the actual implementation of an analysis, $\supsetneq \bullet \mathcal{X}_{j \varepsilon k}$ and $\supsetneq \odot \Psi_{k}$ are likely to be defined for sets of related elements of $\mathbf{x}$ rather than for single elements.

An important part of the SPM is the manner in which variables are defined when experimental programs are not included in an activity set. For each variable or group of related variables investigated in an experimental program, a scientifically sound baseline distribution is defined by the relevant analysts. The purpose of this distribution is to characterize where the analysts have a high degree of confidence that the least conservative value for the variable that can be defended on the basis of all presently available information is located. Often, this distribution is degenerate in the sense that a single value is specified with a probability of 1 . The baseline distribution is used in the definition of $70 \nVdash 24 s_{j \varepsilon}$ when the relevant experimental program is not included in activity set $A S_{j}$. That is, $70 X_{j \varepsilon k}$ is the baseline distribution for $x_{k}$ if $A S_{j}$ does not contain the experimental programs intended to better characterize the possible values for $x_{k}$. In SPM-1, the baseline distribution for each variable was always associated with a possible experimental outcome. However, in general, this does not have to be the case.

The point is now being approached at which possible measures of compliance can be defined for use in the SPM. However, it is first necessary to introduce one more probability space. One of the regulations under consideration (i.e., 40 CFR 191) requires calculations for events that may occur in the future at the WIPP. Thus, a probability space that characterizes the possible future events that may take place at the WIPP is also necessary. For convenience, this space can be represented by

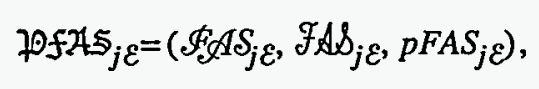

where 
$\$_{\mathscr{S}} A S_{j \varepsilon}=$ [f: $\mathbf{f}$ is a possible $10,000 \mathrm{yr}$ sequence of events at the WIPP given the occurrence of the element $\mathcal{E}$ of $\mathcal{E}\left\{\mathcal{S}_{j}\right\}$,

$\mathscr{F}_{A S_{j} \varepsilon}=\left\{\mathscr{F}: \mathscr{F}\right.$ is a subset of $\mathscr{S} \not \mathcal{A} S_{j \mathcal{E}}$ for which a probability will be defined $\}$

$p F A S_{j \varepsilon}=$ a function that defines probability for elements of $\mathcal{F} A \mathcal{S}_{j \varepsilon}$.

When a single element $e$ of $\xi A S_{j}$ is under consideration, the preceding expression becomes

$$
\not D\left\{A S_{j \mathrm{e}}=\left(S_{\mathcal{E}} \mathbb{A} S_{\mathrm{je}}, \mathcal{F} A S_{j \mathrm{e}}, p F A S_{j \mathrm{e}}\right)\right.
$$

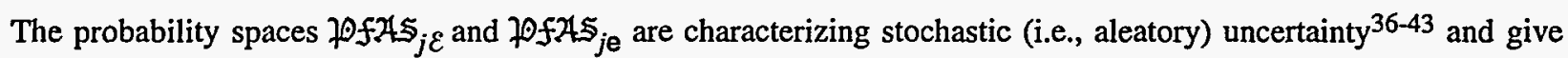
rise to the CCDF that appears in $40 \mathrm{CFR} 191$. Further, compliance with RCRA is based on the elements of $\mathcal{F} \& \&_{j \varepsilon}$ and $\mathcal{F} \delta_{j e}$ that correspond to undisturbed behavior of the WIPP. In the event that the experimental programs and design modifications associated with the activity sets $A S_{j}, j=1,2, \ldots, n A S$, do not affect the definition of $\nmid \ominus f \mathcal{H} \Im_{j} \varepsilon$ and $10\left\{\mathcal{A}_{5 \mathrm{~S}}\right.$, then the subscripts $j$, $\mathcal{E}$ and $\mathrm{e}$ can be deleted.

The SPM is based on determining the probability $p A S_{j}$ that individual activity sets $A S_{j}$ will lead to compliance with applicable regulations. If it is assumed that compliance depends on the probability spaces $70 * 245_{j \mathrm{e}}$ (i.e., on the

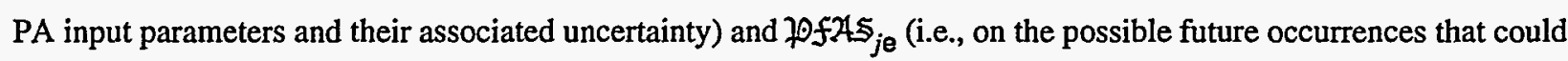
take place at the WIPP) and also that all applicable regulatory requirements must be met for compliance, then $p A S_{j}$ is given by

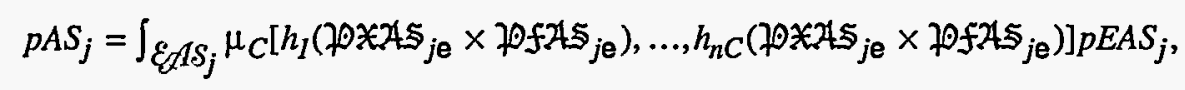

where

$$
\mu_{C}= \begin{cases}1 & \text { if } h_{1}, \ldots, h_{n C} \text { imply compliance } \\ 0 & \text { otherwise }\end{cases}
$$

and $n C$ is the number of regulatory requirements under consideration. The preceding integral can be interpreted as a Lebesgue integral where $p E A S_{j}$ is a probability measure. For readers who are more comfortable with the Riemann integral of elementary calculus, the preceding integral can be viewed as a Riemann integral with

$$
p E A S_{j}=d E A S_{j}(\mathbf{e}) d V_{\xi A S_{j}}
$$

where $d E A S_{j}$ is the density function associated with $\not 0 \notin A S_{j}$ and the differential $d V_{\varepsilon \not A S_{j}}$ denotes an increment of volume in the multidimensional space $\& \nexists S_{j}$. Each function $h_{i}$ in Eq. (2.25) defines a quantity used in assessing the compliance of the WIPP with a regulatory requirement. When each of these quantities implies compliance, the 
function $\mu_{C}$ defined in Eq. (2.26) has a value of 1, with the result that the integral in Eq. (2.25) yields the probability that activity set $A S_{j}$ will lead to compliance of the WIPP with the regulations under consideration.

In the SPM- 1 analysis, $n C=17$ and the $h_{i}$ are defined by

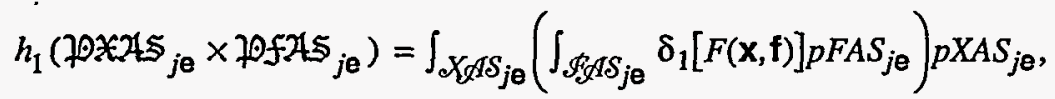

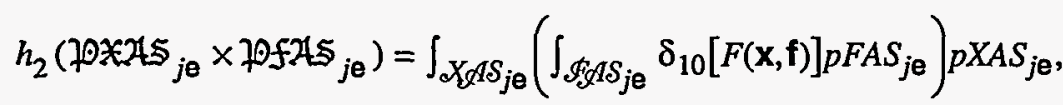

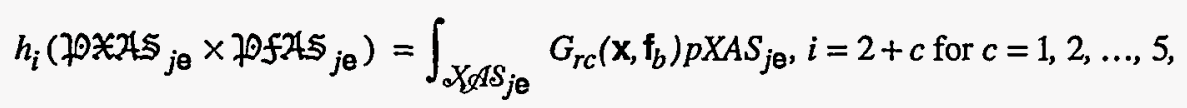

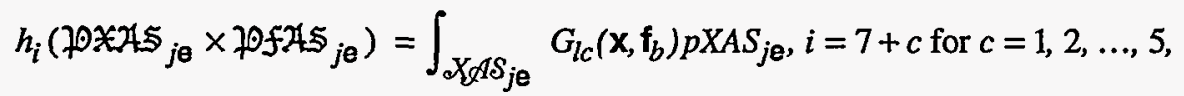

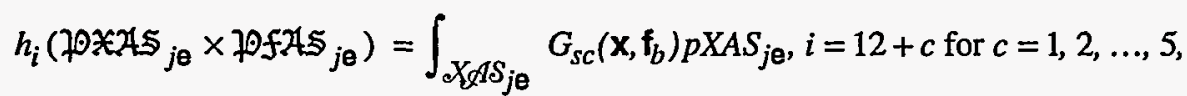

where

$F(\mathbf{x}, \mathbf{f})=$ normalized release to the accessible environment associated with the elements $\mathbf{x}$ and $\mathbf{f}$ of $\mathrm{e} \not A S_{\mathrm{je}}$ and $\nsubseteq \not I S_{j e}$, respectively,

$G_{f c}\left(\mathbf{x}, \mathbf{f}_{b}\right)=$ maximum "soil" concentration of each RCRA contaminant considered in SPM-1 ( $c=1 \Rightarrow$ carbon tetrachloride, $c=2 \Rightarrow$ methylene chloride, $c=3 \Rightarrow 1,1,1$-trichloroethane, $c=4 \Rightarrow$ trichloroethylene, $c=5 \Rightarrow$ 1,1,2-trichloro-1,2,2-trifluorothane) over 10,000 yr at right interface of BRAGFLO computational grid (see Sect. 5.3.1) and the land withdrawal boundary $(f=r)$, left interface of BRAGFLO computational grid and the land withdrawal boundary $(f=l)$, and interface between shaft and Rustler Formation in BRAGFLO computational grid $(f=s)$, respectively, associated with the

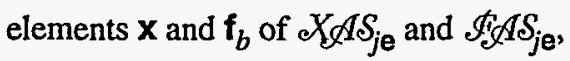

$f_{b}=$ element of $\oiint_{\mathscr{A}} A S_{j \mathrm{e}}$ corresponding to undisturbed conditions,

$$
\delta_{x}(R)= \begin{cases}1 & \text { if } R>x \\ 0 & \text { otherwise }\end{cases}
$$

The inner integrals over $\mathrm{A} \not A S_{j \varepsilon}$ in Eqs. (2.28) and (2.29) determine the probabilities of exceeding EPA normalized releases of size 1 and 10 , respectively, with these probabilities deriving from the probability space $10 \mathcal{F A}^{4} \Im_{j \mathrm{e}}$. The outer integrals in these equations determine the expected values of these two exceedance probabilities, where this expectation derives from the probability space $20 \nVdash$ F $_{j \mathrm{je}}$. The integrals in Eqs. (2.30) - (2.32) determine expected values for maximum concentrations of RCRA chemicals at three different locations, with these expectations again 
deriving from $10 \nVdash 21 s_{j e}$. Thus, the function $\mu_{C}$ in Eq. (2.25) operates on two exceedance probabilities that determine compliance with $40 \mathrm{CFR} 191$ and 15 soil concentrations that determine compliance with RCRA. In particular, $\mu_{C}$ yields a value of 1 when all 17 quantities imply compliance and a value of 0 when 1 or more of these quantities implies noncompliance.

The integrals over $\triangle \not A S_{j \varepsilon}$ in Eqs. (2.28) - (2.32) define expected values over subjective uncertainty. Thus, the probability of compliance $p A S_{j}$ in Eq. (2.25) is based on expectations over subjective uncertainty. An alternative approach is to base determinations of compliance on a specified quantile (e.g., on the 0.9 quantile) of a distribution that derives from subjective uncertainty rather than on the expected value of this distribution. In this case, the functions $h_{i}$ in Eq. (2.25) would have the form

$$
h_{i}\left(10 * 45_{j \mathrm{e}} \times 70 f \mathcal{H} \mathfrak{S}_{j \mathrm{e}}\right)=p_{i}
$$

where the $p_{i}, i=1,2, \ldots, 17$, are the solutions to the equations

$$
\begin{aligned}
& q=1-\int_{\triangle \alpha A S_{j \mathrm{e}}} \delta_{p_{1}}\left(\int_{\oiint_{\& A} S_{j \mathrm{e}}} \delta_{1}[F(\mathbf{x}, \mathbf{f})] p F A S_{j}\right) p X A S_{j},
\end{aligned}
$$

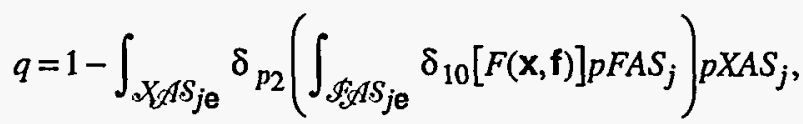

$$
\begin{aligned}
& q=1-\int_{\triangle \not A S_{j \mathrm{e}}} \delta_{p_{i}}\left[G_{r c}\left(\mathbf{x}, \mathbf{f}_{b}\right)\right] p X A S_{j \mathrm{e}}, i=2+c \text { for } c=1,2, \ldots, 5 \text {, } \\
& q=1-\int_{X_{\not A S} S_{\mathrm{je}}} \delta_{p_{i}}\left[\left(G_{l c}\left(\mathbf{x}, \mathrm{f}_{b}\right)\right] p X A S_{j \mathrm{e}}, \quad i=7+c \text { for } c=1,2, \ldots, 5\right. \text {, } \\
& q=1-\int_{X \not A S S_{j \mathrm{e}}} \delta_{p_{i}}\left[G_{s c}\left(\mathbf{x}, \mathbf{f}_{b}\right)\right] p X A S_{j \mathrm{e}}, i=12+c \text { for } c=1,2, \ldots, 5,
\end{aligned}
$$

and $q$ is the particular quantile (e.g., 0.9) under consideration. In this case, the function $\mu_{C}$ in Eq. (2.25) would determine compliance on the basis of the quantiles rather than the expected values of distributions.

The probability of compliance $p A S_{j}$ defined in Eq. (2.25) is based on measures of performance defined on the

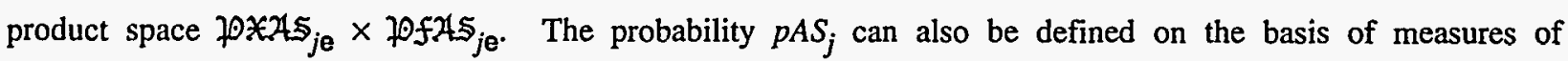
performance defined on the product space $\mathbf{x} \times \not \mathcal{F} \not \mathcal{A} \Im_{j \mathrm{j}}$ for individual elements $\mathbf{x}$ of $\mathrm{e} X \not A S_{j \mathrm{e}}$. In this case, $p A S_{j}$ would have the form

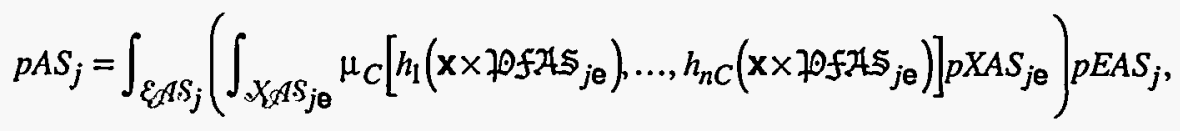

where $\mu_{C}$ and the $h_{i}$ are defined similarly to their use in Eq. (2.25) except that the $h_{i}$ are now functions of $\mathbf{x} \times$

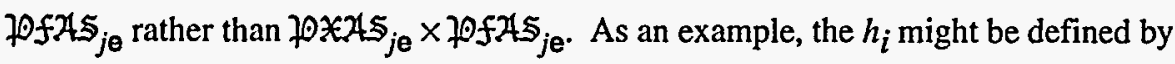




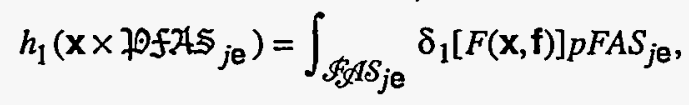

$$
\begin{aligned}
& h_{2}\left(\mathbf{x} \times \not \mathcal{f A A \Im _ { j e }}\right)=\int_{\mathscr{S} \not S_{j e}} \delta_{10}[F(\mathbf{x}, \mathbf{f})] p F A S_{j e}, \\
& h_{i}\left(\mathbf{x} \times \not \mathcal{F A} \mathcal{A} \Im_{j \mathrm{e}}\right)=G_{r c}\left(\mathrm{x}, \mathrm{f}_{b}\right), i=2+c \text { for } c=1,2, \ldots, 5, \\
& h_{i}\left(\mathbf{x} \times 70 f \mathcal{A} \Phi_{j e}\right)=G_{l c}\left(\mathbf{x}, \mathbf{f}_{b}\right), i=7+c \text { for } c=1,2, \ldots, 5
\end{aligned}
$$

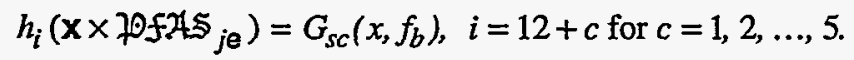

With the definition of $p A S_{j}$ in Eq. (2.40), a determination of compliance is made with the function $\mu_{C}$ before the incorporation of the effects of subjective uncertainty in the PA input parameters (i.e., before integration over dXAS $S_{\mathrm{e}}$ ). With the definition of $p A S_{j}$ in Eq. (2.25), a determination of compliance is made after the incorporation of the effects of subjective uncertainty in the PA input parameters. Even when the $h_{i}$ used in Eq. (2.25) are based on expected values as indicated in Eqs. (2.28) - (2.32) and the $h_{i}$ used in Eq. (2.40) are based on the corresponding quantities before the calculation of their expected values as indicated in Eqs. (2.41) - (2.45), the two definitions of $p A S_{j}$ will not, in general, yield the same values.

As an example, Fig. 2.1 shows a distribution of CCDFs (left frame) and the corresponding mean and percentile curves (right frame). The definition of $h_{1}$ and $h_{2}$ in Eqs. (2.28) and (2.29) corresponds to the determination of $p A S_{j}$ in Eq. (2.25) on the basis of whether or not the mean CCDF in Fig. 2.1 falls below the boundary line specified in 40 CFR 191.13. The definition of $h_{1}$ and $h_{2}$ in Eqs. (2.34) and (2.35) with $q=0.9$ corresponds to the determination of $p A S_{j}$ on the basis of whether or not the $90^{\text {th }}$ percentile curve falls below the boundary line specified in $40 \mathrm{CFR}$ 191.13. Finally, the definition of $h_{1}$ and $h_{2}$ in Eqs. (2.41) and (2.42) is equivalent to the determination of $p A S_{j}$ on the basis of the location of the individual CCDFs in Fig. 2.1 relative to the boundary line specified in 40 CFR 191.13. The corresponding definitions of the $h_{i}, i=3,4, \ldots, 17$, for RCRA contaminants have similar implications. 

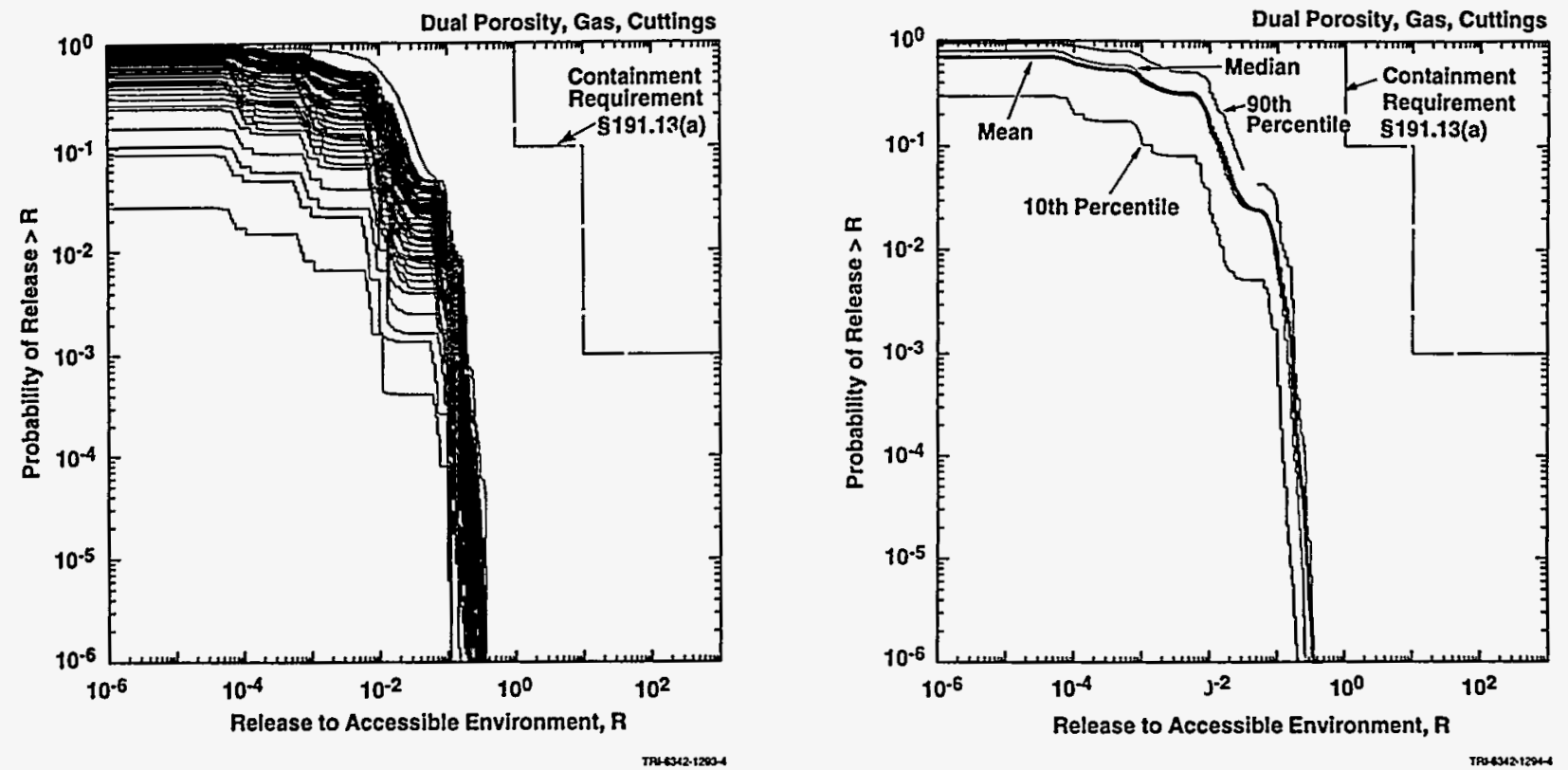

Figure 2.1. Distribution of CCDFs obtained in the 1991 WIPP PA for normalized release to the accessible environment including both cuttings removal and groundwater transport with gas generation in the repository and a dual-porosity transport model in the Culebra Dolomite (left frame: Ref. 13, Fig. 2.2-2, Ref. 47, Fig. 2; right frame: Ref. 13, Fig. 4.1-1, Ref. 47, Fig. 6). 


\section{Definition of Probability Spaces}

The preceding section provides a formal description of the manner in which compliance probabilities are determined in the SPM. This description is based on the following probability spaces: (1) probability spaces $¥ \mathbb{E}_{i}$ that characterize the possible outcomes of individual experimental programs [see Eq. (2.3)], (2) probability spaces

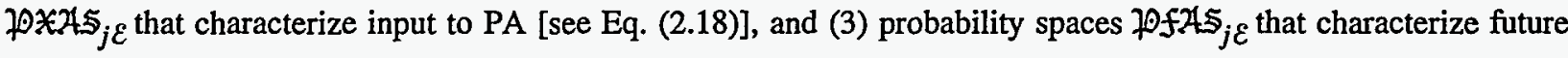
occurrences at the WIPP [see Eq. (2.23)]. These probability spaces are now discussed in the context of SPM-1.

\subsection{Probability Spaces $70 \mathbb{E}_{i}$ that Characterize Possible Outcomes of Individual Experimental Programs}

As part of SPM-1, the individuals associated with the experimental programs indicated in Table 2.1 were asked to describe the possible outcomes of the programs and to provide probabilistic characterizations of their degree of belief that these outcomes would be realized. Thus, these individuals were asked to define the probability spaces 20 $\mathbb{E}_{i}$ in Eq. (2.3), although the request was not presented in such formal terms.

The outcome of this request is documented in Ref. 34 and summarized in Table 3.1 for the Salado experimental program (i.e., experimental program $E_{1}$ ). The set $\varepsilon_{1}$ in Table 3.1 consists of all possible outcomes of the Salado experimental program. As indicated earlier, if this program involved 50 individual experiments and each experiment produced 6 numbers, then the elements of $\varepsilon_{1}$ would be vectors from $R^{300}$. In SPM-1, the nature of the Salado experimental program was not specified at a level of detail that permitted the definition of $\varepsilon_{1}$ in terms of actual experimental outcomes. Indeed, it is unlikely that the outcomes of large experimental programs in support of complex projects such as the WIPP can ever be fully-specified as vectors in high-dimensional spaces. However, this difficulty should not be taken to mean that $\varepsilon_{1}$ and other similar sets of experimental outcomes cannot or should not be defined. An adequate characterization of $\varepsilon_{1}$ and similar sets is an essential part of the SPM.

The sets $\varepsilon_{1 i}, i=1,2, \ldots, 8$, in Table 3.1 are subsets of $\varepsilon_{1}$ and elements of $\varepsilon_{1}$. These are the sets of experimental outcomes to which the experimentalists are assigning probabilities. As indicated earlier, these probabilities are subjective in the sense that they characterize the experimentalists' degree of belief that the final outcome of the Salado experimental program will produce an outcome contained in $\varepsilon_{1 i}, i=1,2, \ldots, 8$. In a complete development of $\not \emptyset \mathbb{E}_{1}$, the set $\varepsilon_{1}$ would also contain all subsets of $\mathcal{E}_{1}$ that could be obtained from forming complements, unions and intersections with the sets $\varepsilon_{1 i}$. As indicated in the assignment of probabilities in Table 3.1, the sets $\varepsilon_{1 i}$ are not necessarily disjoint.

In general, the sets $\varepsilon_{1 i}$ should be defined in terms of their properties as outcomes of the Salado experimental program. The next major step in the SPM is then to define distributions of PA input parameters conditional on the occurrence of these sets of experimental outcomes (i.e., to define the probability spaces $\not \cap \notin 245_{j} \varepsilon$ as discussed in 
Table 3.1. Definition of Probability Space $\not \supset \notin_{1}=\left(\varepsilon_{1}, \varepsilon_{1}, p E_{1}\right)$ for Possible Outcomes of Salado Experiments

Definition of Sample Space $\varepsilon_{1}$ :

$\varepsilon_{1}=\left\{e_{1}: e_{1}\right.$ a possible outcome of the Salado experiments $\}$

Definition of Set ( $\sigma$-algebra) $\varepsilon_{1}$ :

$\varepsilon_{11}=\left\{e_{1}: e_{1} \in \varepsilon_{1}\right.$ and implies baseline gas flow model $\}$

$\varepsilon_{12}=\left\{e_{1}: e_{1} \in \varepsilon_{1}\right.$ and implies altered anhydrite gas flow model based on Brooks-Corey ${ }^{48}$ characteristic curves for two-phase relative permeabilities and capillary pressures\}

$\varepsilon_{13}=\left\{e_{1}: e_{1} \in \mathcal{E}_{1}\right.$ and implies altered anhydrite gas flow model based on van Genuchten-Parker 49,50 characteristic curves for two-phase relative permeabilities and capillary pressures\}

$\varepsilon_{14}=\left\{e_{1}: e_{1} \in \varepsilon_{1}\right.$ and implies preferential flow processes increase maximum gas flow distances by factor of 10 over distances estimated with an axisymmetric flow model \}

$\varepsilon_{15}=\left\{e_{1}: e_{1} \in \mathcal{E}_{1}\right.$ and implies same outcome as $\varepsilon_{14}$ but with a factor of 6$\}$

$\varepsilon_{16}=\left\{e_{1}: e_{1} \in \mathcal{E}_{1}\right.$ and implies same outcome as $\varepsilon_{14}$ but with a factor of 4$\}$

$\varepsilon_{17}=\left\{\mathbf{e}_{1}: \mathbf{e}_{1} \in \mathcal{E}_{1}\right.$ and implies same outcome as $\varepsilon_{14}$ but with a factor of 2$\}$

$\varepsilon_{18}=\left\{\mathbf{e}_{1}: \mathbf{e}_{1} \in \mathcal{E}_{1}\right.$ and implies same outcome as $\varepsilon_{14}$ but with a factor of 1 , i.e., axisymmetric flow $\}$

All complements, unions, intersections obtainable from $\varepsilon_{1 i}, i=1,2, \ldots, 8$

Definition of Probability Function (Measure) $p E_{1}$ :

$$
\begin{aligned}
& p E_{1}\left(\varepsilon_{11}\right)=0.1, p E_{1}\left(\varepsilon_{12}\right)=0.5, p E_{1}\left(\varepsilon_{13}\right)=0.5, p E_{1}\left(\varepsilon_{14}\right)=0.2, p E_{1}\left(\varepsilon_{15}\right)=0.3, p E_{1}\left(\varepsilon_{16}\right)=0.3, p E_{1}\left(\varepsilon_{17}\right)= \\
& \left.0.1, p E_{1}\left(\varepsilon_{18}\right)=0.1, p E_{1}\left(\varepsilon_{12} \cap \varepsilon_{13}\right)=0 \text { (i.e., } \varepsilon_{12} \cap \varepsilon_{13}=\phi\right), p E_{1}\left(\varepsilon_{1 i} \cap \varepsilon_{1 j}\right)=0 \text { for } i, j=4,5,6,7,8 \text { and } i \neq j \\
& \text { (i.e., } \left.\varepsilon_{1 i} \cap \varepsilon_{1 j}=\phi\right), p E_{1}\left(\varepsilon_{11}^{c} \cap \varepsilon_{1 i} \cap \varepsilon_{1 j}\right)=p E_{1}\left(\varepsilon_{11}^{c}\right) p E_{1}\left(\varepsilon_{1 i}\right) p E_{1}\left(\varepsilon_{1 j}\right) \text { for } i=2,3, j=4,5,6,7,8
\end{aligned}
$$

Probabilities for remaining elements of $\mathcal{E}_{1}$ consistent with preceding

Section 3.2). This is a very important step because, in general, the outcomes of experimental programs do not directly define the input to the models used in PA. Rather, a considerable amount of manipulation and knowledge of both the experimental results and the PA models is required to convert from experimental results obtained on one scale to PA parameters and their associated uncertainty for use on a typically much larger scale. In SPM-1, the outcomes of experimental programs have usually been defined in terms of the resultant input to PA models rather than in terms of the experimental outcomes themselves. However, this situation is not anticipated to occur in real applications of the SPM.

In SPM-1, the first set of experimental outcomes (i.e., $\varepsilon_{i 1}$ ) is always assumed to imply the baseline assumptions associated with not conducting the corresponding experimental program. Thus, $\varepsilon_{i 1}$ contains those experimental 
outcomes that correspond to complete failure of the experiments (i.e., no more is known about the quantities under study after the experiments than was known before the experiments) as well as those outcomes that actually imply the correctness of the defensible baseline assumptions, and $p E_{i}\left(\varepsilon_{i 1}\right)$ is the probability that the experiments either fail or imply the baseline assumptions. In concept, a value of $p E_{i}\left(\varepsilon_{i 1}\right)=0$ should be assigned by an experimentalist who was very confident that the experimental program would not fail and also would not produce results that implied the baseline assumptions.

The display at the bottom of Table 3.1 presents the probabilities assigned by the experimentalists to the elements of $\varepsilon_{1}$ (i.e., to the individual sets of experimental outcomes $\varepsilon_{11}, \varepsilon_{12}, \varepsilon_{13}, \ldots$ and to certain additional sets derived from the original sets of outcomes defined by the experimentalists). The indicated assignments define the function $p E_{1}$ and complete the definition of the probability space $\not € E_{1}$.

As indicated in Tables $3.2-3.8$, the probability spaces $\longmapsto \mathbb{C}_{i}$ for experimental programs $E_{i}, i=2,3, \ldots, 8$, are defined in a similar manner.

Table 3.2. Definition of Probability Space $\not Ð E_{2}=\left(\varepsilon_{2}, \varepsilon_{2}, p E_{2}\right)$ for Possible Outcomes of Seal Experiments

\section{Definition of Sample Space $\varepsilon_{2}$ :}

$\varepsilon_{2}=\left\{\mathbf{e}_{2}: \mathbf{e}_{2}\right.$ a possible outcome of the seal experiments $\}$

Definition of Set ( $\sigma$-algebra) $\varepsilon_{2}$ :

$\varepsilon_{21}=\left\{e_{2}: e_{2} \in \varepsilon_{2}\right.$ and implies high shaft seal permeability, i.e., between $10^{-15}$ and $\left.10^{-13} \mathrm{~m}^{2}\right\}$

$\varepsilon_{22}=\left\{\mathbf{e}_{2}: \mathbf{e}_{2} \in \varepsilon_{2}\right.$ and implies intermediate shaft seal permeability, i.e., between $10^{-18}$ and $\left.10^{-16} \mathrm{~m}^{2}\right\}$

$\varepsilon_{23}=\left\{e_{2}: e_{2} \in \varepsilon_{2}\right.$ and implies low shaft seal permeability, i.e., between $10^{-19}$ and $\left.10^{-18} \mathrm{~m}^{2}\right\}$

All complements, unions, intersections obtainable from $\varepsilon_{2 i}, i=1,2,3$

Definition of Probability Function (Measure) $p E_{2}$ :

$$
\left.p E_{2}\left(\varepsilon_{21}\right)=0.01, p E_{2}\left(\varepsilon_{22}\right)=0.09, p E_{2}\left(\varepsilon_{23}\right)=0.9, p E_{2}\left(\varepsilon_{2 i} \cap \varepsilon_{2 j}\right)=0 \text { for } i \neq j \text { (i.e., } \varepsilon_{2 i} \cap \varepsilon_{2 j}=\phi\right)
$$

Probabilities for remaining elements of $\varepsilon_{2}$ consistent with preceding 
Table 3.3. Definition of Probability Space $\not \wp E_{3}=\left(\varepsilon_{3}, \varepsilon_{3}, p E_{3}\right)$ for Possible Outcomes of Source Term Experiments to Determine Chemical Solubility

Definition of Sample Space $\varepsilon_{33}$ :

$$
\varepsilon_{3}=\left\{e_{3}: e_{3} \text { a possible outcome of the source term experiments }\right\}
$$

Definition of Set ( $\sigma$-algebra) $\varepsilon_{3}$ :

$$
\begin{aligned}
& \varepsilon_{31}=\left\{e_{3}: \quad e_{3} \in \varepsilon_{3} \text { and implies infinite solubility }\right\} \\
& \varepsilon_{32}=\left\{\mathbf{e}_{3}: \mathbf{e}_{3} \in \varepsilon_{3}\right. \text { and implies intermediate solubilities, i.e., expert judgment solubility distributions } \\
& \text { developed in Refs. 51-53\} } \\
& \varepsilon_{33}=\left\{e_{3}: \quad e_{3} \in \varepsilon_{3}\right. \text { and implies low solubilities, i.e., expert judgment solubility distributions developed in }
\end{aligned}
$$

All complements, unions, intersections obtainable from $\varepsilon_{3 i}, i=1,2,3$

Definition of Probability Function (Measure) $p E_{3}$ :

$$
\left.p E_{3}\left(\varepsilon_{31}\right)=0.01, p E_{3}\left(\varepsilon_{32}\right)=0.49, p E_{3}\left(\varepsilon_{33}\right)=0.5, p E_{3}\left(\varepsilon_{3 i} \cap \varepsilon_{3 j}\right)=0 \text { for } i \neq j \text { (i.e., } \varepsilon_{3 i} \cap \varepsilon_{3 j}=\phi\right)
$$

Probabilities for remaining elements of $\varepsilon_{3}$ consistent with preceding

Table 3.4. Definition of Probability Space $\mathfrak{p} \mathbb{E}_{4}=\left(\varepsilon_{4}, \mathcal{E}_{4}, p E_{4}\right)$ for Possible Outcomes of Single-Well Culebra Experiments to Determine Physical Retardation

Definition of Sample Space $\varepsilon_{4}$ :

$$
\mathcal{E}_{4}=\left\{\mathbf{e}_{4}: \mathbf{e}_{4} \text { a possible outcome of Culebra physical retardation experiments with single well testing }\right\}
$$

Definition of Set ( $\sigma$-algebra) $\varepsilon_{4}$ :

$\varepsilon_{41}=\left\{e_{4}: e_{4} \in \mathcal{E}_{4}\right.$ and implies radionuclide transport in fractures with no diffusion into the rock matrix $\}$

$\varepsilon_{42}=\left\{e_{4}: e_{4} \in \varepsilon_{4}\right.$ and implies radionuclide transport in fractures with diffusion into the rock matrix and a fracture spacing between 0.1 and $8 \mathrm{~m}$ \}

All complements, unions, intersections obtainable from $\varepsilon_{4 i}, i=1,2$

Definition of Probability Function (Measure) $p E_{4}$ :

$$
p E_{4}\left(\varepsilon_{41}\right)=0.05, p E_{4}\left(\varepsilon_{42}\right)=0.95, p E_{4}\left(\varepsilon_{41} \cap \varepsilon_{42}\right)=0 \text { (i.e., } \varepsilon_{41} \cap \varepsilon_{42}=\phi \text { ) }
$$

Probabilities for remaining elements of $\varepsilon_{4}$ consistent with preceding 
Table 3.5. Definition of Probability Space $\not € €_{5}=\left(\varepsilon_{5}, \varepsilon_{5}, p E_{5}\right)$ for Possible Outcomes of Multiwell Culebra Experiments to Determine Physical Retardation

Definition of Sample Space $\varepsilon_{5}$ :

$\varepsilon_{5}=\left\{e_{5}: e_{5}\right.$ a possible outcome of Culebra physical retardation experiments with multiwell testing $\}$

Definition of Set ( $\sigma$-algebra) $\varepsilon_{5}$ :

$\varepsilon_{51}=\left\{\mathbf{e}_{5}: \quad \mathbf{e}_{5} \in \mathcal{E}_{5}\right.$ and implies radionuclide transport in fractures with no diffusion into the rock matrix $\}$

$\varepsilon_{52}=\left\{e_{5}: e_{5} \in \varepsilon_{5}\right.$ and implies radionuclide transport in fractures with diffusion into the rock matrix and a fracture spacing between 0.1 and $3 \mathrm{~m}$ \}

$\varepsilon_{53}=\left\{e_{5}: \mathbf{e}_{5} \in \varepsilon_{5}\right.$ and implies radionuclide transport in fractures with diffusion into the rock matrix and a fracture spacing between 0.1 and $1 \mathrm{~m}$ )

All complements, unions, intersections obtainable from $\varepsilon_{5 i}, i=1,2,3$

Definition of Probability Function (Measure) $p E_{5}$ :

$$
\left.p E_{5}\left(\varepsilon_{51}\right)=0.05, p E_{5}\left(\varepsilon_{52}\right)=0.38, p E_{5}\left(\varepsilon_{53}\right)=0.57, p E_{5}\left(\varepsilon_{5 i} \cap \varepsilon_{5 j}\right)=0 \text { for } i \neq j \text { (i.e., } \varepsilon_{5 i} \cap \varepsilon_{5 j}=\phi\right)
$$

Probabilities for remaining elements of $\varepsilon_{5}$ consistent with preceding 
Table 3.6. Definition of Probability Space $\not \mathcal{A} \mathcal{E}_{6}=\left(\varepsilon_{6}, \varepsilon_{6}, p E_{6}\right)$ for Possible Outcomes of Culebra Experiments to Determine Chemical Retardation

\section{Definition of Sample Space $\varepsilon_{6}$ :}

$\varepsilon_{6}=\left\{\mathbf{e}_{6}: \mathbf{e}_{6}\right.$ a possible outcome of Culebra experiments to determine chemical retardation $\}$

Definition of Set ( $\sigma$-algebra) $\varepsilon_{6}$ :

$\varepsilon_{61}=\left\{\mathbf{e}_{6}: \quad \mathbf{e}_{6} \in \varepsilon_{6}\right.$ and implies no chemical retardation $\}$

$\varepsilon_{62}=\left\{e_{6}: e_{6} \in \varepsilon_{6}\right.$ and implies chemical retardation with uniform distribution of clay minerals throughout Culebra\}

$\varepsilon_{63}=\left\{\mathbf{e}_{6}: \mathbf{e}_{6} \in \varepsilon_{6}\right.$ and implies chemical retardation with (1) clay minerals concentrated adjacent to subhorizontal fractures but not adjacent to high-angle fractures and (2) advective flow in subhorizontal fractures only\}

$\varepsilon_{64}=\left\{e_{6}: \quad e_{6} \in \varepsilon_{6}\right.$ and implies chemical retardation with (1) clay minerals concentrated adjacent to subhorizontal fractures but not adjacent to high-angle fractures and (2) advective flow in high-angle fractures only\}

$\varepsilon_{65}=\left\{\mathbf{e}_{6}: \mathbf{e}_{6} \in \mathcal{E}_{6}\right.$ and implies chemical retardation with (1) clay minerals concentrated adjacent to subhorizontal fractures but not adjacent to high-angle fractures and (2) advective flow alternating between subhorizontal and high-angle fractures\}

All complements, unions, intersections obtainable from $\varepsilon_{6 i}, i=1,2,3,4,5$

Definition of Probability Function (Measure) $p E_{6}$ :

$$
\begin{aligned}
& p E_{6}\left(\varepsilon_{61}\right)=0.01, p E_{6}\left(\varepsilon_{62}\right)=0.95, p E_{6}\left(\varepsilon_{6 i}\right)=0.0133 \text { for } i=3,4,5, p E_{6}\left(\varepsilon_{6 i} \cap \varepsilon_{6 j}\right)=0 \text { for } i \neq j \\
& \text { (i.e., } \varepsilon_{6 i} \cap \varepsilon_{6 j}=\phi \text { ) }
\end{aligned}
$$

Probabilities for remaining elements of $\mathcal{E}_{6}$ consistent with preceding 
Table 3.7. Definition of Probability Space $\not \supset E_{7}=\left(\varepsilon_{7}, \varepsilon_{7}, p E_{7}\right)$ for Possible Outcomes of Source Term Experiments to Determine Actinide Attachment to Mobile Colloids Using Present and Past Waste Inventory Having Significant Amounts of High Molecular Weight Organic Materials Such As May Be Found in Soils

Definition of Sample Space $\varepsilon_{7}$ :

$\varepsilon_{7}=\left\{e_{7}: \mathbf{e}_{7}\right.$ a possible outcome of colloid source term experiments with no significant inventory of high molecular weight organic materials\}

Definition of Set ( $\sigma$-algebra) $\varepsilon_{7}$ :

$\varepsilon_{71}=\left\{e_{7}: \quad e_{7} \in \varepsilon_{7}\right.$ and implies infinite colloid solubility (i.e., all actinides are attached to mobile colloids) $\}$

$\varepsilon_{7 i}=\left\{\mathbf{e}_{7}: \quad \mathbf{e}_{7} \in \varepsilon_{7}\right.$ and implies total concentration of actinides (mol/ $\ell$ ) attached to mobile colloids is approximately $\left.10^{-(i+1)} \mathrm{mol} / \ell\right\}, i=2,3,4,5,6$

$\varepsilon_{77}=\left\{e_{7}: \quad e_{7} \in \varepsilon_{7}\right.$ and implies total concentration of actinides (mol/ $\ell$ ) attached to mobile colloids is $\leq 10^{-8}$ $\mathrm{mol} / \ell\}$

All complements, unions, intersections obtainable from $\varepsilon_{7 i}, i=1,2, \ldots, 7$

Definition of Probability Function (Measure) $p E_{7}$ :

$$
\begin{aligned}
& p E_{7}\left(\varepsilon_{71}\right)=0.01, p E_{7}\left(\varepsilon_{72}\right)=0.33, p E_{7}\left(\varepsilon_{73}\right)=0.1821, p E_{7}\left(\varepsilon_{74}\right)=0.09103, p E_{7}\left(\varepsilon_{75}\right)=0.04552, p E_{7}\left(\varepsilon_{76}\right) \\
& =0.01138, p E_{7}\left(\varepsilon_{77}\right)=0.33, p E_{7}\left(\varepsilon_{77 i} \cap \varepsilon_{7 j}\right)=0 \text { for } i \neq j\left(\text { i.e., } \varepsilon_{7 i} \cap \varepsilon_{7 j}=\phi\right)
\end{aligned}
$$

Probabilities for remaining elements of $\mathcal{E}_{7}$ consistent with preceding 
Table 3.8. Definition of Probability Space $\supsetneq \bigoplus E_{8}=\left(\varepsilon_{8}, \mathcal{E}_{8}, p E_{8}\right)$ for Possible Outcomes of Spallings Experiments

Definition of Sample Space $\varepsilon_{8}$ :

$\varepsilon_{8}=\left\{e_{8}: \quad e_{8}\right.$ a possible outcome of the spallings experiments $\}$

Definition of Set ( $\sigma$-algebra) $\varepsilon_{8}$ :

$\varepsilon_{81}=\left\{\mathbf{e}_{8}: \quad \mathbf{e}_{8} \in \varepsilon_{8}\right.$ and implies baseline spallings model $\}$

$\varepsilon_{82}=\left\{e_{8}: \quad e_{8} \in \varepsilon_{8}\right.$ and implies that releases predicted with baseline spallings model are too high by a factor of 2 \}

$\varepsilon_{83}=\left\{e_{8}: \quad e_{8} \in \varepsilon_{8}\right.$ and implies same outcome as $\varepsilon_{82}$ but with a factor of 10$\}$

$\varepsilon_{84}=\left\{e_{8}: \quad e_{8} \in \varepsilon_{8}\right.$ and implies same outcome as $\varepsilon_{82}$ but with a factor of 100$\}$

All complements, unions, intersections obtainable from $\varepsilon_{8 i}, i=1,2,3,4$

Definition of Probability Function (Measure) $p E_{8}$ :

$p E_{8}\left(\varepsilon_{81}\right)=0.05, p E_{8}\left(\varepsilon_{82}\right)=0.2, p E_{8}\left(\varepsilon_{83}\right)=0.35, p E_{8}\left(\varepsilon_{84}\right)=0.4, p E_{8}\left(\varepsilon_{8 i} \cap \varepsilon_{8 j}\right)=0$ for $i \neq j$ (i.e., $\varepsilon_{8 i} \cap \varepsilon_{8 j}=\phi$ )

Probabilities for remaining elements of $\varepsilon_{8}$ consistent with preceding

\subsection{Probability Spaces $70 * 24 s_{j} \varepsilon$ that Characterize Input to Performance Assessment}

The next step in the SPM is to define the probability spaces $\not 9 X \mathcal{H} S_{j \varepsilon}$ in Eq. (2.18) that characterize the uncertainty in PA inputs conditional on a set $\varepsilon$ of experimental outcomes associated with activity set $A S_{j}$. As a reminder, an activity set involves a collection of specified experimental programs and design modifications (see Eqs. (2.8), (2.10)) and the set $\mathcal{E}$ is a subset of the set $\varepsilon A \mathcal{S}_{j}$ indicated in Eq. (2.11). Thus, $\varepsilon$ is of the form

$$
\mathcal{E}=x_{i \in \varepsilon s_{j}} S_{i k}
$$

where $\varepsilon S_{j}$ is defined in Eq. (2.12) and $S_{i k}$ is an element of the corresponding set $\varepsilon_{i}$. In practice, the $S_{i k}$ would be the sets $\varepsilon_{i j}$ defined in Tables 3.1 - 3.8 or sets constructed from the $\varepsilon_{i j}$ by forming unions or intersections, and the definition of $70 * \mathcal{H}_{j} S_{j}$ would depend on both the specific sets of experimental outcomes (i.e., $S_{i k}$ ) from which $\varepsilon$ was obtained and the design modifications associated with activity set $A S_{j}$.

The sets $S_{i j}$ used in SPM-1 are summarized in Table 3.9. With the exception of experimental program $E_{1}$, the sets $\delta_{i j}$ are the same as the sets $\varepsilon_{i j}$ used to summarize the possible outcomes of the corresponding experimental 
Table 3.9. Elements $S_{i j}$ of $\varepsilon_{i}$ for Experimental Program $E_{i}$ Used in the Definition of the Probability Spaces $70 * 15_{j}$ in SPM-1

$$
\begin{array}{ll}
E_{1}: & \varepsilon_{11}, \varepsilon_{11}^{c} \cap \varepsilon_{1 i} \cap \varepsilon_{1 j} \text { for } i=2,3, j=4,5,6,7,8\left(\text { i.e., } s_{1 j}, j=1,2, \ldots, 11\right) \\
E_{2}: & \varepsilon_{21}, \varepsilon_{22}, \varepsilon_{23}\left(\text { i.e., } \delta_{2 j}, j=1,2,3\right) \\
E_{3}: & \varepsilon_{31}, \varepsilon_{32}, \varepsilon_{33}\left(\text { i.e., } s_{3 j}, j=1,2,3\right) \\
E_{4}: & \varepsilon_{41}, \varepsilon_{42}\left(\text { i.e., } s_{4 j}, j=1,2\right) \\
E_{5}: & \varepsilon_{51}, \varepsilon_{52}, \varepsilon_{53}\left(\text { i.e., } s_{5 j}, j=1,2,3\right) \\
E_{6}: & \varepsilon_{61}, \varepsilon_{62}, \ldots \varepsilon_{65}\left(\text { i.e., } s_{6 j}, j=1,2, \ldots, 5\right) \\
E_{7}: & \varepsilon_{71}, \varepsilon_{72}, \ldots \varepsilon_{77}\left(\text { i.e., } s_{7 j}, j=1,2, \ldots, 7\right) \\
E_{8}: & \varepsilon_{81}, \varepsilon_{82}, \varepsilon_{83}, \varepsilon_{84}\left(\text { i.e., } s_{8 j}, j=1,2,3,4\right)
\end{array}
$$

program. However, this does not have to be the case. The subsets of experimental outcomes used in the definition

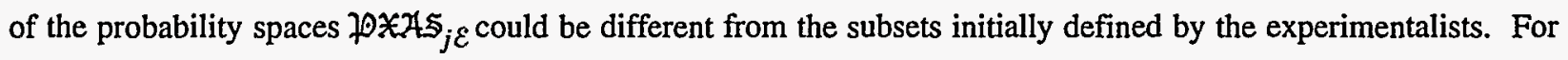
example, the sets $\varepsilon_{i j}$ defined by the experimentalists might be combined into a smaller number of sets for the definition of PA input. When the more explicit representation for the set $\varepsilon S_{j}$ in Eq. (2.12) is used, the representation for $\varepsilon$ becomes

$$
\mathcal{E}=S_{i(1), k} \times S_{i(2), l} \times \cdots \times S_{i\left(n E S_{j}\right), m}
$$

This explicit representation for $\varepsilon$ will be needed later to define probability space $10 \nVdash \mathcal{A} \varsigma_{j} \mathcal{E}$.

The probability space $10 * 2 \Im_{j} \varepsilon$ is specified as a function of $\mathcal{E}$ and $A S_{j}$ because a specific PA input may depend on the outcome of more than one experimental program and may also be influenced by one or more design modifications. As indicated in Eq. (2.22), the definition of $\not \cap * X S_{j \varepsilon}$ can also involve probability spaces that are independent of the experimental programs and design modifications under consideration. In the following discussion of $20 ※ 15_{j \varepsilon}$ for SPM-1, probability spaces that depend on experimental programs and design modifications will be considered first (i.e., the spaces $70 X_{j \varepsilon k}$ in Eq. (2.22)); then probability spaces that are not affected by the experimental programs and design modifications are considered (i.e., the spaces $70 \mathcal{X}_{k}$ in Eq. (2.22)).

Although the specification of $10 \nVdash \mathcal{A} S_{j}$ as a function of both $\mathcal{E}$ and $A S_{j}$ provides a general description of what is to be done, such inclusiveness is often not used in practice. In particular, most PA inputs are not functions of the outcomes of all the experimental programs nor functions of all the design modifications associated with activity set 
$\mathrm{AS}_{j}$, although the notation in use is sufficiently general to encompass such situations. Rather, most PA inputs are influenced by one or a relatively small number of the experimental programs and by none or a relatively small number of the design modifications.

Such is the case in SPM-1. Most PA inputs depend on the outcome of a single experimental program, with one set of PA inputs depending on the outcomes of two experimental programs. Further, most PA inputs are not influenced by any of the design modifications under consideration, with a few PA inputs influenced by a single design modification. Because of this relatively simple structure, the probability spaces $70 \nVdash 245_{j \varepsilon}$ in SPM-1 can be built from probability spaces of the form

$$
\begin{aligned}
& \not \oplus X_{k \varepsilon_{i j}}=\left(X_{k} \varepsilon_{i j}, X_{k \varepsilon_{i j}}, p X_{k \varepsilon_{i j}}\right) \\
& =\text { probability space for } k^{\text {th }} \text { set of PA input variables given the set } \varepsilon_{i j} \text { of outcomes to experimental } \\
& \text { program } E_{i} \text {, }
\end{aligned}
$$

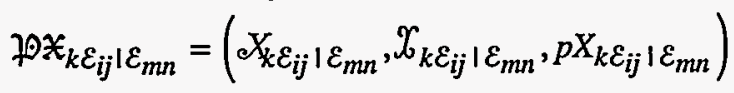

$$
\begin{aligned}
& =\text { probability space for } k^{\text {th }} \text { set of PA input variables given the sets } \varepsilon_{i j} \text { and } \varepsilon_{m n} \text { of outcomes to } \\
& \text { experimental programs } E_{i} \text { and } E_{m} \text {, } \\
& \mathfrak{P O \mathcal { X } _ { k }} \varepsilon_{i j} \mid D_{m}=\left(\mathcal{X}_{k \varepsilon_{i j} \mid D_{m}}, \mathscr{X}_{k \varepsilon_{i j} \mid D_{m}}, p X_{k \varepsilon_{i j} \mid D_{m}}\right) \\
& =\text { probability space for } k^{\text {th }} \text { set of PA input variables given the set } \varepsilon_{i j} \text { of outcomes to experimental } \\
& \text { program } E_{i} \text { and the implementation of design modification } D_{m} \text {, } \\
& \not 0 \mathcal{X}_{k}=\left(c X_{k}, \mathscr{X}_{k}, p X_{k}\right) \\
& =\text { probability space for } k^{\text {th }} \text { set of PA input variables for which the analysts' state of knowledge is }
\end{aligned}
$$

where $\varepsilon_{i j}$ and $\varepsilon_{m n}$ are sets of experimental outcomes as defined in Tables $3.1-3.8, D_{m}$ is a design modification as indicated in Table 2.1, and $k$ designates either a single PA input or a group of related PA inputs (e.g., elemental solubilities). To avoid the cumbersome use of double subscripts, the probability spaces in Eqs. (3.3) - (3.5) will often be written as

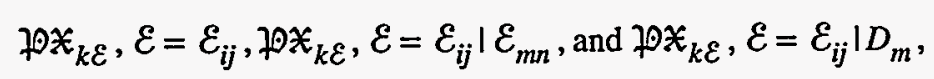

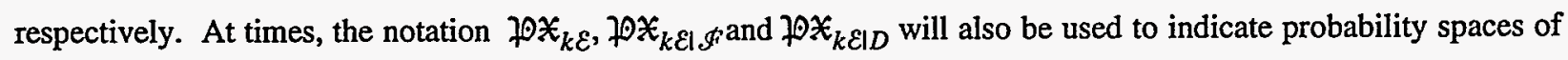
the form appearing in Eqs. (3.3) - (3.5). 
In SPM-1, a convenient labeling is to let $k$ designate the set of variables associated with experimental program

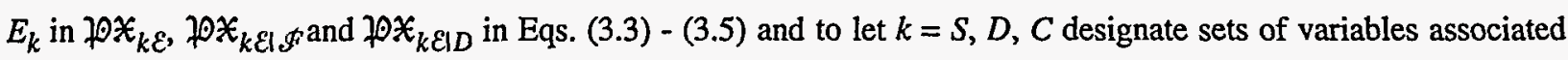
with the Salado Formation $(k=S)$, drilling intrusions $(k=D)$ and the Culebra Dolomite $(k=C)$ in $70 X_{k}$ in Eq. (3.6). Then, $10 \times A_{5} \xi_{j}$ is a product space as indicated in Eq. (2.22) obtained from probability spaces of the form appearing

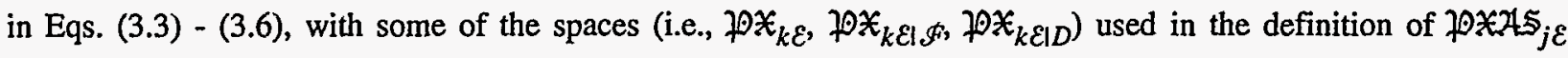
depending on the experimental programs and design modifications associated with activity set $A S_{j}$ and some of the spaces (i.e., $\not \cap X_{k}$ ) being independent of the experimental programs and design modifications under consideration.

\subsubsection{Probability Spaces $\supsetneq 0 x_{k \varepsilon}, \supsetneq 0 x_{k \varepsilon \mid g i}$ and $\not ڤ x_{k \varepsilon \mid D}$}

Probability spaces $\mathfrak{Q} \mathcal{X}_{k \mathcal{E}} \varepsilon=\varepsilon_{i j}$, that are dependent on the outcome of a single experimental program are considered first. As indicated in Table 3.9, the outcomes of experimental program $E_{1}$ considered in SPM-1 are

$$
\varepsilon_{11} \text { and } \mathcal{E}_{11}^{c} \cap \varepsilon_{1 i} \cap \varepsilon_{1 j}, i=2,3, j=4,5,6,7,8
$$

The associated probability spaces $\nmid \emptyset \mathcal{Z}_{1 \varepsilon}$ for the sets $\varepsilon$ in Eq. (3.8) are summarized in Table 3.10. These are rather uninteresting spaces because each set of experimental outcomes in Eq. (3.8) is assumed to have one, and only one, implication with respect to the PA calculations. Specifically, each set of experimental outcomes is assumed to unambiguously imply a particular model for two-phase flow. The associated probability spaces indicated in Table 3.10 are referred to as being degenerate because they imply that a single outcome occurs with a probability of 1. In general, such specificity of implication is not to be expected.

The probability spaces $f \circ x_{2 \varepsilon}, \varepsilon=\varepsilon_{21}, \varepsilon_{22}, \varepsilon_{23}$, associated with experimental program $E_{2}$ are indicated in Table 3.11. Each of these probability spaces characterizes the uncertainty in a single variable (i.e., the shaft seal permeability, SEALPRM) and describes a degree of belief as where the appropriate value of SEALPRM for use in the WIPP PA is located given that the outcome of experimental program $E_{2}$ falls in the set $\varepsilon$. Similar probability spaces $10 x_{k \varepsilon}, k=3,4,5$, associated with the experimental programs $E_{k}, k=3,4,5$, are indicated in Tables 3.12 - 3.14 .

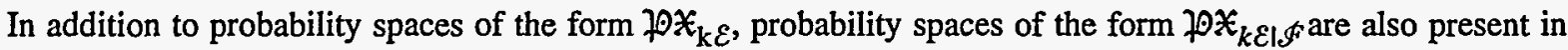
SPM-1. In particular, the implications of the possible outcomes of experimental program $E_{6}$ for PA are dependent on the possible outcomes of experimental programs $E_{4}$ and $E_{5}$. As indicated in Table 3.15, this leads to the uncertainty in PA inputs being dependent on both the outcomes of $E_{6}$ (i.e., $\varepsilon_{6 i}, i=1,2, \ldots, 5$ ) and the outcomes of $E_{4}$ (i.e., $\varepsilon_{41}, \varepsilon_{42}$ ) and $E_{5}$ (i.e., $\varepsilon_{51}, \varepsilon_{52}, \varepsilon_{53}$ ). As a reminder, $E_{4}$ and $E_{5}$ will not occur in the same activity set; thus, the probability spaces $¥ \emptyset \mathcal{X}_{6 \varepsilon \mid f_{7}}$ in Table 3.15 are conditional on outcomes associated with $E_{4}$ or $E_{5}$ but not on outcomes associated with both $E_{4}$ and $E_{5}$. 
Table 3.10. Definition of Probability Spaces $\not \cap X_{1 \varepsilon}=\left(\mathrm{C}_{1 \varepsilon}, X_{1 \varepsilon}, p X_{1 \varepsilon}\right)$ Associated with Possible Outcomes of Salado Experiments

Values for $\varepsilon: \mathcal{E}_{11}, \mathcal{E}_{11}^{c} \cap \mathcal{E}_{1 i} \cap \mathcal{E}_{1 j}, i=2,3, j=4,5,6,7,8$

Definition of $\not \supset \Psi_{1}$ for $\mathcal{\varepsilon}=\varepsilon_{11}$ :

$\not 0 X_{1 \varepsilon}$ is degenerate (i.e., there is only one possibility implied for PA input). With a probability of 1 , the baseline gas flow model is used in the Salado Formation.

Definition of $\nmid \cap \mathcal{X}_{1 \varepsilon}$ for $\varepsilon=\varepsilon_{11}^{c} \cap \varepsilon_{1 i} \cap \varepsilon_{1 j}, i=2,3, j=4,5,6,7,8$

$20 \nVdash_{1 \varepsilon}$ is degenerate. With a probability of 1 , a specific model for two-phase relative permeabilities and capillary pressures is used (i.e., $i=2 \Rightarrow$ Brooks-Corey model and $i=3 \Rightarrow$ van Genuchten-Parker model) and a specific model for preferential flow processes is used (i.e., $j=4,5,6,7,8 \Rightarrow$ preferential flow processes increase maximum gas flow distances by factors of $10,6,4,2$ and 1 , respectively).

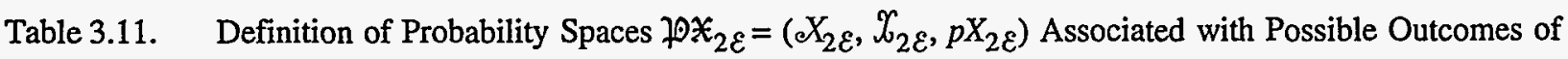
Seal Experiments .

Values for $\mathcal{E}: \varepsilon_{21}, \varepsilon_{22}, \varepsilon_{23}$

Definition of $\not D \mathbb{Z}_{2 \varepsilon}$ for $\varepsilon=\varepsilon_{21}$ :

Shaft seal permeability, SEALPRM, assigned loguniform distribution between $10^{-15}$ and $10^{-13} \mathrm{~m}^{2}$. Defines variable $k$ in Eqs. (38) and (39) of Ref. 54 for part of model domain corresponding to shaft seals.

Definition of $\not \cap \chi_{2 \varepsilon}$ for $\varepsilon=\varepsilon_{22}$ :

Shaft seal permeability, SEALPRM, assigned loguniform distribution between $10^{-18}$ and $10^{-16} \mathrm{~m}^{2}$.

Definition of $70 \mathcal{X}_{2 \varepsilon}$ for $\varepsilon=\varepsilon_{23}$ :

Shaft seal permeability, SEALPRM, assigned loguniform distribution between $10^{-19}$ and $10^{-18} \mathrm{~m}^{2}$. 


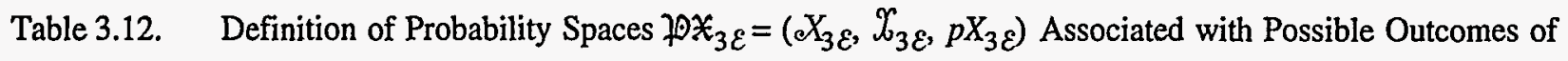
Source Term Experiments to Determine Chemical Solubility

Values for $\varepsilon: \varepsilon_{31}, \varepsilon_{32}, \varepsilon_{33}$

Definition of $70 \Psi_{3 \varepsilon}$ for $\varepsilon=\varepsilon_{31}$ :

$\mathrm{ZOX}_{3 \varepsilon}$ is degenerate. With a probability of 1 , all elements under consideration (i.e., americium, neptunium, plutonium, radium, thorium, uranium) are infinitely soluble.

Definition of $\mathcal{Q} ¥_{3 \varepsilon}$ for $\varepsilon=\varepsilon_{32}$ :

$\mathfrak{P X}_{3 \mathcal{E}}$ is defined by the distributions indicated for the solubilities of americium, neptunium, plutonium, radium, thorium and uranium in Table 3-1 of Ref. 19 (i.e., for the variables SOLAM, SOLNP, SOLPU, SOLRA, SOLTH, $S O L U$ ) and shown explicitly in Fig. 3-1 of Ref. 19. The individual solubilities are assumed to have a 0.999 rank correlation. See variable $S_{i}$ in Eq. (80) of Ref. 54 for usage of SOLAM, SOLNP, SOLPU, SOLRA, SOLTH and SOLU.

Definition of $70 \Psi_{3 \varepsilon}$ for $\varepsilon=\varepsilon_{33}$ :

$\not 0 \mathcal{F}_{3 \mathcal{E}}$ for $\mathcal{E}=\varepsilon_{33}$ is defined the same as for $\mathcal{E}=\varepsilon_{32}$ except that the solubility distributions for the individual elements are bounded above at $10^{-4} \mathrm{~mol} / \ell$ and then renormalized.

Table 3.13. Definition of Probability Spaces $\mathfrak{O Z}_{4 \varepsilon}=\left(X_{4 \varepsilon}, \mathscr{X}_{4 \varepsilon}, p X_{4 \varepsilon}\right)$ Associated with Possible Outcomes of Single-Well Culebra Experiments to Determine Physical Retardation

Values for $\mathcal{E}: \varepsilon_{41}, \mathcal{E}_{42}$

Definition of $\supsetneq \otimes x_{4 \varepsilon}$ for $\varepsilon=\varepsilon_{41}$ :

$\mathfrak{Z O X}_{4 \varepsilon}$ is degenerate. With a probability of 1 , radionuclide transport takes place in fractures with no diffusion into the rock matrix.

Definition of $\not \partial \mathcal{X}_{4 \varepsilon}$ for $\varepsilon=\varepsilon_{42}$ :

Radionuclide transport takes place in fractures with diffusion into the rock matrix. Fracture spacing, CULFRSP, is assigned a uniform distribution between 0.1 and $8 \mathrm{~m}$. The quotient CULFRSP/2 defines the sum $b_{s}+b_{f}+B$ used in Eqs. (88) and (102) and illustrated in Fig. 12 of Ref. 54. 
Table 3.14. Definition of Probability Spaces $20 \mathcal{X}_{5 \varepsilon}=\left({ }^{2} X_{5 \varepsilon}, X_{5 \varepsilon}, p X_{5 \varepsilon}\right)$ Associated with Possible Outcomes of Multiwell Culebra Experiments to Determine Physical Retardation

Values for $\varepsilon: \varepsilon_{51}, \varepsilon_{52}, \varepsilon_{53}$

Definition of $\not \circ \varkappa_{5 \varepsilon}$ for $\varepsilon=\varepsilon_{51}$ :

20* $x_{5 \varepsilon}$ is degenerate. With a probability of 1 , radionuclide transport takes place in fractures with no diffusion into the rock matrix.

Definition of $\not \mathcal{O} \mathcal{F}_{5 \varepsilon}$ for $\varepsilon=\varepsilon_{52}$ :

Radionuclide transport takes place in fractures with diffusion into the rock matrix. Fracture spacing, CULFRSP, is assigned a uniform distribution between 0.1 and $3 \mathrm{~m}$.

Definition of $10 \varkappa_{5 \varepsilon}$ for $\varepsilon=\varepsilon_{53}$ :

Radionuclide transport takes place in fractures with diffusion into the rock matrix. Fracture spacing, CULFRSP, is assigned a uniform distribution between 0.1 and $1 \mathrm{~m}$. 
Table 3.15. Definition of Probability Spaces $\not \cap X_{6 \varepsilon}=\left({ }^{\circ} X_{6 \varepsilon}, X_{6 \varepsilon}, p X_{6 \varepsilon}\right)$ Associated with Possible Outcomes of Culebra Experiments to Determine Chemical Retardation

Values for $\varepsilon: \varepsilon_{61}, \varepsilon_{6 i}\left|\varepsilon_{41}, \varepsilon_{6 i}\right| \varepsilon_{42}, \varepsilon_{6 i}\left|\varepsilon_{51}, \varepsilon_{6 i}\right| \varepsilon_{52}, \varepsilon_{6 i} \mid \varepsilon_{53}, i=2,3,4,5$

Definition of $\supsetneq \mathcal{*}_{6 \varepsilon}$ for $\varepsilon=\varepsilon_{61}$ :

$70 \mathcal{X}_{6 \varepsilon}$ is degenerate. With a probability of 1 , no chemical retardation takes place.

Definition of $\supsetneq 0 \mathcal{X}_{6 \varepsilon}$ for $\varepsilon=\varepsilon_{62}\left|\varepsilon_{41}, \varepsilon_{62}\right| \varepsilon_{42}, \varepsilon_{62}\left|\varepsilon_{51}, \varepsilon_{62}\right| \varepsilon_{52}, \varepsilon_{62}\left|\varepsilon_{53}, \varepsilon_{64}\right| \varepsilon_{41}, \varepsilon_{64} \mid \varepsilon_{51}$ :

$10 X_{6 \varepsilon}$ is derived from the distributions indicated for the fracture and matrix distribution coefficients for americium, neptunium, plutonium and uranium in Table 3-1 of Ref. 19 (i.e., for FKDAM, FKDNP, FKDPU, FKDTH, FKDU, MKDAM, MKDNP, MKDPU, MKDTH, MKDU) and shown explicitly in Fig. 3-1 of Ref. 19. In particular, the distribution coefficient $K D A M$ for americium is defined by

$$
K D A M=0.05 F K D A M+0.95 M K D A M
$$

with the distribution for $K D A M$ following from the distributions for $F K D A M$ and $M K D A M$. The distribution coefficients for neptunium, plutonium, thorium and uranium (i.e., $K D N P, K D P U, K D T H, K D U$ ) are defined in a similar manner. Further, a 0.999 rank correlation is assumed to exist between FKDAM, FKDNP, FKDPU, $F K D T H, F K D U, M K D A M, M K D N P, M K D P U, M K D T H$ and $M K D U$. $70 \mathcal{X}_{6 \varepsilon}$ is defined by the distributions for $K D A M, K D N P, D K P U, K D T H$ and $K D U$. Variables $K D A M, K D N P, K D P U, K D T H$ and $K D U$ apply in a small region adjacent to a fracture for the $\mathcal{E}$ indicated above and correspond to the variable $K_{d l}$ in Eqs. (93) and (95) of Ref. 54. In addition, $K D A M, K D N P, K D P U, K D T H$ and $K D U$ also apply in the bulk matrix of the formation for $\mathcal{E}$ $=\varepsilon_{62}\left|\varepsilon_{42}, \varepsilon_{62}\right| \varepsilon_{52}, \varepsilon_{62} \mid \varepsilon_{53}$ and correspond to the variable $K_{d l}^{\prime}$ in Eq. (98) of Ref. 54.

Definition of $7 \cap x_{6 \varepsilon}$ for $\varepsilon=\varepsilon_{63}\left|\varepsilon_{41}, \varepsilon_{63}\right| \varepsilon_{42}, \quad \varepsilon_{63}\left|\varepsilon_{51}, \quad \varepsilon_{63}\right| \varepsilon_{52}, \quad \varepsilon_{63}\left|\varepsilon_{53}, \quad \varepsilon_{65}\right| \varepsilon_{41}, \quad \varepsilon_{65}\left|\varepsilon_{42}, \quad \varepsilon_{65}\right| \varepsilon_{51}$, $\varepsilon_{65}\left|\varepsilon_{52}, \varepsilon_{65}\right| \varepsilon_{53}$

$20 \mathcal{X}_{6 \varepsilon} \varepsilon$ is defined by the distributions indicated for FKDAM, FKDNP, FKDPU, FKDTH, FKDU, MKDAM, $M K D N P, M K D P U, M K D T H$ and $M K D U$ in Table 3-1 of Ref. 19 and shown explicitly in Fig. 3-1 of Ref. 19. A 0.999 rank correlation is assumed between these variables. The fracture distribution coefficients (i.e., FKDAM, FKDNP, FKDPU, FKDTH, FKDU) are assumed to apply in a clay-dominated region adjacent to a fracture and correspond to the variable $K_{d l}$ in Eqs. (93) and (95) of Ref. 54. The matrix distribution coefficients (i.e., $M K D A M, M K D N P, M K D P U, M K D T H, M K D U$ ) are assumed to apply in the bulk matrix of the formation and correspond to the variable $K_{d l}^{\prime}$ in Eq. (98) of Ref. 54.

Definition of $7 \odot *_{6 \varepsilon}$ for $\mathcal{E}=\varepsilon_{65}\left|\varepsilon_{42}, \varepsilon_{65}\right| \varepsilon_{52}, \varepsilon_{65} \mid \varepsilon_{53}$ :

POF $6 \mathcal{E}$ defined by distributions previously indicated for $K D A M, K D N P, K D P U, K D T H, K D U, M K D A M$, MKDNP, $M K D P U, M K D T H$ and $M K D U$. Variables $K D A M, K D N P, K D P U, K D T H$ and $K D U$ apply in a small region adjacent to fracture and correspond to the variable $K_{d l}$ in Eqs. (93) and (95) of Ref. 54. Variables $M K D A M$, $M K D N P, M K D P U, M K D T H$ and $M K D U$ apply in the bulk matrix of the formation and correspond to the variable $K_{d l}^{\prime}$ in Eq. (98) of Ref. 54. 
The implications of the possible outcomes of experimental program $E_{7}$ for uncertainty in PA input are summarized in Tables 3.16 and 3.17. If design modification $D_{3}$ is not implemented, the resultant uncertainty in PA input (i.e., actinide attachment to mobile colloids) depends only on the outcome of experimental program $E_{7}$. For this case, the resultant probability spaces $\not \cap X_{7 \varepsilon}, \varepsilon=\varepsilon_{7 i}$, are summarized in Table 3.16 and are of the form in

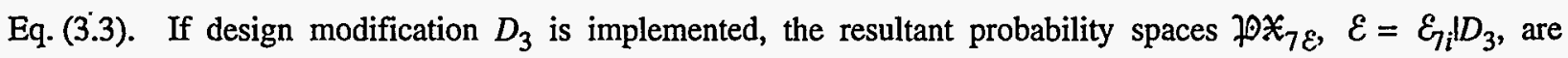
summarized in Table 3.17 and are of the form in Eq. (3.5).

The implications of the possible outcomes of experimental program $E_{8}$ for the uncertainty in PA input are summarized in Table 3.18. As the implications of these outcomes with respect to PA input are unaffected by the outcomes of other experimental programs and the implementation of the design modifications under consideration, the resultant probability spaces $10 \mathcal{X}_{8 \varepsilon}$ are of the form in Eq. (3.3).

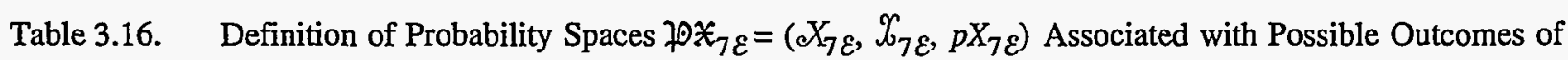
Source Term Experiments to Determine Actinide Attachment to Mobile Colloids Using Present and Past Waste Inventory Having Significant Amounts of High Molecular Weight Organic Materials Such as May Be Found in Soils

Values for $\varepsilon: \varepsilon_{7 i}, i=1,2, \ldots, 7$

Definition of $\not \circ \varkappa_{7 \varepsilon}$ for $\mathcal{\varepsilon}=\varepsilon_{71}$ :

$\supsetneq \ominus \mathcal{X}_{7 \varepsilon}$ is degenerate. With a probability of 1 , actinides have an infinite colloid solubility (i.e., all actinides are attached to mobile colloids).

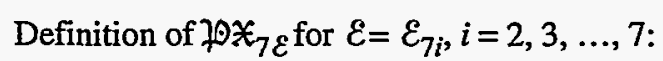

Total concentration of actinides (mol/ $\ell$ ) attached to mobile colloids assigned lognormal distribution with $\mu=$ $-(i+1)$ and $\sigma=1.03$. 
Table 3.17. Definition of Probability Spaces $70 \mathcal{*}_{7 \varepsilon}=\left(d X_{7 \varepsilon}, X_{7 \varepsilon}, p X_{7 \varepsilon}\right), \varepsilon=\varepsilon_{7 i} D_{3}$, Associated with Possible Outcomes of Source Term Experiments to Determine Actinide Attachment to Mobile Colloids Using Present and Past Waste Inventory Having Significant Amounts of High Molecular Weight Organic Materials Such As May Be Found in Soils and the Implementation of Design Modification $D_{3}$

Values for $\varepsilon: \varepsilon_{7 i} \mid D_{3}, i=1,2, \ldots, 7$

Definition of $\supsetneq \bigoplus \mathcal{X}_{7 \varepsilon}$ for $\mathcal{E}=\varepsilon_{71} \mid D_{3}$ :

$\not 0 X_{7} \varepsilon$ is degenerate. With a probability of 1 , actinides have an infinite colloid solubility (i.e., all actinides are attached to mobile colloids).

Definition of $\nmid \circ \mathcal{X}_{7 \varepsilon}$ for $\varepsilon=\varepsilon_{7 i} \mid D_{3}, i=2,3, \ldots, 7$ :

Total concentration of actinides (mol/ $\ell$ ) attached to mobile colloids assigned lognormal distribution with $\mu=-(i+2)$ and $\sigma=1.03$.

Table 3.18. Definition of Probability Spaces $70 X_{8 \varepsilon}=\left(X_{8 \varepsilon}, X_{8 \varepsilon}, p X_{8 \varepsilon}\right)$ Associated with Possible Outcomes of Spallings Experiments

Values for $\varepsilon: \varepsilon_{8 i}, i=1,2,3,4$

Definition of $70 \chi_{8 \varepsilon}$ for $\varepsilon=\varepsilon_{81}$

$\not ० \mathcal{X}_{8 \varepsilon}$ is degenerate. With a probability of 1 , the baseline spallings model is used to estimate spallings releases.

Definition of $\supsetneq \otimes \mathcal{X}_{8 \varepsilon}$ for $\varepsilon=\varepsilon_{82}, \varepsilon_{83}, \varepsilon_{84}$

$\mathfrak{P O}_{8 \varepsilon}$ is degenerate. With a probability of 1 , the baseline spallings model multiplied by a scale factor is used to estimate spallings releases, with $\varepsilon_{82}, \varepsilon_{83}$ and $\varepsilon_{84}$ implying scale factors of $1 / 2,1 / 10$ and $1 / 100$, respectively. 


\subsubsection{Probability Spaces $\not \circ x_{k}$}

Probability spaces $\not O \mathcal{X}_{k}$ of the form indicated in Eq. (3.5) for variables for which the analysts' state of knowledge is not affected by any of the experimental programs or design modifications under consideration are now described. Thirteen variables that affect the calculation of brine flow, gas generation and gas flow in the vicinity of the repository are listed in Table 3.19. These variables, together with their indicated distributions, constitute the probability space $70 \mathcal{X}_{S}$, where the subscript $k=S$ derives from the influence of these variables on processes taking place within the Salado Formation.

Four variables that affect the calculation of cuttings and spallings releases are listed in Table 3.20. These variables, together with their indicated distributions, constitute the probability space $2 \otimes \mathcal{X}_{D}$, where the subscript $k=D$ derives from the influence of these variables on processes related to drilling intrusions. In addition, the variable $D B D I A M$ associated with the probability space $\mathrm{PO}_{S}$ is also used in the calculation of cuttings and spallings releases.

Five variables that affect the calculation of radionuclide transport in the Culebra Dolomite are listed in Table 3.21. These variables, together with their indicated distributions, constitute the probability space $\mathfrak{D O}_{C}$, where the subscript $k=C$ derives from the influence of these variables on processes taking place within the Culebra Dolomite.

\subsubsection{Formal Definition of the Probability Spaces $10 \nVdash \mathcal{A} S_{j} \varepsilon$}

The SPM- 1 analysis involves $n A S=1536$ activity sets. Further, each activity set $A S_{j}$ involves from 2 to 41,580 different values for $\mathcal{E}$ in the specification of the probability spaces $70 \nVdash 24 S_{j} \varepsilon$. Thus, a complete listing of the spaces POXA $S_{j \varepsilon}$ considered in SPM-1 is not possible. However, as these spaces are constructed from the spaces $70 X_{k \varepsilon}$,

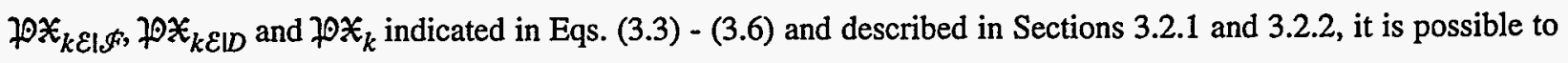
give a formal description of how the spaces $\not \otimes * X \Im_{j \varepsilon}$ are defined in SPM-1.

The probability spaces $\not 0 * A \Im_{j} \varepsilon$ can be formally defined by introducing a set $\Phi_{\&} A \mathcal{S}_{j} \varepsilon$ of integer pairs $(m, n)$ and associated sets $\mathcal{E}(m, n)$. Specifically,

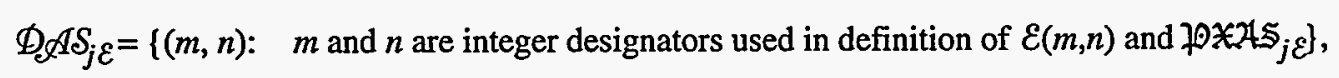

where

$$
\begin{array}{ll}
m=1,2,3,4,6,7,8 & \text { if } E_{4} \text { is in } A S_{j} \\
m=1,2,3,5,6,7,8 & \text { if } E_{4} \text { is not in } A S_{j}
\end{array}
$$


Table 3.19. Variables Associated with the Probability Space $\not \cap \gtrless_{S}$ and Used in the Calculation of Brine Flow, Gas Generation and Gas Flow in the Vicinity of the Repository

Variable Definition

BCBRSAT Residual brine saturation for Salado Formation (dimensionless). Used in BRAGFLO. Defines variable $S_{w r}$ in Eq. (33) of Ref. 54. Range: 0.0 to 0.4. Median: 0.2. Distribution: Uniform. Additional information: Sect. 2.3.1, Ref. 18; Sects. 4.2.4 and 5.1.2.4, Ref. 19.

BCEXP Brooks-Corey pore-size distribution parameter for Salado Formation (dimensionless). Used in BRAGFLO. Defines variable $\lambda$ in Eq. (33) of Ref. 54. Range: 0.2 to 10 . Median: 0.7 . Distribution: Piecewise uniform. Additional information: Same as BCBRSAT.

BCGSSAT Brooks-Corey residual gas saturation for Salado Formation (dimensionless). Used in BRAGFLO. Defines variable $S_{n r}$ in Eq. (33) of Ref. 54. Range: 0.0 to 0.4. Median: 0.2 . Distribution: Uniform. Additional information: Same as BCBRSAT.

BHPERM Borehole permeability. Used in BRAGFLO. Defines variable $k$ in Eqs. (38) and (39) of Ref. 54 for subset of model domain that corresponds to a borehole; see computational grid in Fig. 5.1-2 of Ref. 19. Range: $1 \times 10^{-14}$ to $1 \times 10^{-11} \mathrm{~m}^{2}$. Median: $3.16 \times 10^{-12} \mathrm{~m}^{2}$. Distribution: Lognormal. Additional information: Ref. 55, Table 2-2 (silty sand); Sect. 4.2.1, Ref. 18; Sect. 5.1.2.1, Ref. 19 .

BRSAT Initial fluid (brine) saturation of waste (dimensionless). Used in BRAGFLO. Defines variable $S_{n}(r, z, 0)$ in Table 4 of Ref. 24 and variable $S_{p a n}$ in Eq. (74) of Ref. 54. Range: 0 to 0.052. Median: 0.004. Distribution: Piecewise uniform. Additional information: Sect. 3.4.3, Ref. 18; Sects. 4.3 and 5.1.3, Ref. 19.

DBDIAM Drill bit diameter. Used in CUTTINGS, SPALLINGS and BRAGFLO. The quantity $D B D I A M / 2$ defines $R_{0}$ in Step 1 of algorithm given in Sect. 3.5 of Ref. 54 for calculation of borehole diameter by CUTTINGS. Similarly, DBDIAM/2 also defines borehole radius for the modeling of drilling intrusions with BRAGFLO (see Fig. 5.1-2, Ref. 19). Range: 0.267 to $0.444 \mathrm{~m}$. Median: $0.355 \mathrm{~m}$. Distribution: Uniform. Additional information: Sect. 4.2.2, Ref. 18; Chapt. 7, Ref. 19; Ref. 56.

GRCORHF Scale factor used in definition of gas generation rate for corrosion of steel under humid conditions (dimensionless). Used in BRAGFLO. Actual gas generation rate is $G R C O R H=$ GRCORHF $\bullet$ GRCORI, with GRCORH equal to the variable $r_{c h}$ in Eq. (47) of Ref. 54. Range: 0 to 0.5. Median: 0.1. Distribution: Piecewise uniform. Additional information: Sect. 3.3.5, Ref. 18; Ref. 57.

GRCORI Gas generation rate for corrosion of steel under inundated conditions. Used in BRAGFLO. Defines variable $r_{c i}$ in Eq. (47) of Ref. 54. Range: 0 to $6.4 \times 10^{-7} \mathrm{~mol} / \mathrm{m}^{2}$ surface area steel $\bullet$ s. Median: $6.3 \times 10^{-9} \mathrm{~mol} / \mathrm{m}^{2}$ surface area steel $\bullet \mathrm{s}$. Distribution: Piecewise uniform. Additional information: Same as GRCORHF.

GRMICHF Scale factor used in definition of gas generation rate due to microbial degradation of cellulosics under humid conditions (dimensionless). Used in BRAGFLO. Actual gas generation rate is $G R M I C H=G R M I C H F \bullet G R M I C I$, with $G R M I C H$ equal to the variable $r_{m h}$ in Eq. (48) of Ref. 54. Range: 0 to 0.2. Median: 0.1. Distribution: Uniform. Additional information: Same as GRCORHF. 
Table 3.19. Variables Associated with the Probability Space $20 X_{S}$ and Used in the Calculation of Brine Flow, Gas Generation and Gas Flow in the Vicinity of the Repository (continued)

Variable Definition

GRMICI Gas generation rate due to microbial degradation of cellulosics under inundated conditions. Used in BRAGFLO. Defines variable $r_{m i}$ in Eq. (48) of Ref. 54. Range: 0 to $1.6 \times 10^{-8}$ $\mathrm{mol} / \mathrm{kg}$ cellulosics $\bullet \mathrm{s}$. Median: $3.2 \times 10^{-9} \mathrm{~mol} / \mathrm{kg}$ cellulosics $\bullet \mathrm{s}$. Distribution: Piecewise uniform. Additional information: Same as GRCORHF.

MBPERM Permeability in intact anhydrite marker beds in Salado Formation. Used in BRAGFLO. Defines variable $k$ in Eqs. (38) and (39) of Ref. 54 for subset of model domain that corresponds to undisturbed anhydrite marker beds (i.e., the variable $k_{A}$ in Table 3 of Ref. 54); see Fig. 5.1-3 of Ref. 19. Range: $1 \times 10^{-21}$ to $1 \times 10^{-18} \mathrm{~m}^{2}$. Median: $1.0 \times 10^{-19.5} \mathrm{~m}^{2}$. Distribution: Loguniform. Additional information: Sect. 2.4.2, Ref. 18; Sect. 5.1.2.1, Ref. 19.

PSPERM Panel seal permeability. Used in BRAGFLO. Defines variable $k$ in Eqs. (38) and (39) of Ref. 54 for subset of model domain that corresponds to panel seals. Range: $1 \times 10^{-14}$ to $1 \times$ $10^{-12} \mathrm{~m}^{2}$. Median: $1 \times 10^{-13} \mathrm{~m}^{2}$. Distribution: Loguniform. Additional information: Sect. 32, Ref. 18.

SALPERM Permeability in intact halite component of Salado Formation. Used in BRAGFLO. Defines variable $k$ in Eqs. (38) and (39) of Ref. 54 for subset of model domain that corresponds to undisturbed halite (i.e., the variable $k_{H}$ in Table 3 of Ref. 54; see Fig. 5.1-3 of Ref. 19.). Range: $1 \times 10^{-22.4}$ to $1 \times 10^{-20.2} \mathrm{~m}^{2}$. Median: $1 \times 10^{-21.7} \mathrm{~m}^{2}$. Distribution: Piecewise loguniform. Additional information: Sect. 2.3.5, Ref. 18; Sect. 5.1.2.1, Ref. 19; Refs. 58-60.

Table 3.20. Variables Associated with the Probability Space $\not \circ X_{D}$ and Used in the Calculation of Cuttings and Spallings Releases

Variable

BLWEF

SPPERM

Waste permeability for spallings response. Used in SPALLINGS. Range: $1 \times 10^{-17}$ to $1 \times$ $10^{-12} \mathrm{~m}^{2}$. Median: $1 \times 10^{-14.5} \mathrm{~m}^{2}$. Distribution: Loguniform. Additional information: Table 3.4-1, Ref. 18; pp. 29, 37, Ref. 55 .

TAUFAIL Effective shear resistance to erosion. Used in CUTTINGS. Range: 0.048 to $9.6 \mathrm{~Pa}$. Median: 4.8 Pa. Distribution: Uniform. Additional Information: Ref. 62.

TCLOUT Driller cleanout time. Used in SPALLINGS. Range: $4.32 \times 10^{4}$ to $8.64 \times 10^{4} \mathrm{~s}$. Median: 6.48 $\times 10^{4}$ s. Distribution: Uniform. Additional information: pp. 183-184, Ref. 63 . 
Table 3.21. Variables Associated with the Probability Space $20 X_{C}$ and Used in the Calculation of Radionuclide Transport in the Culebra Dolomite

Variable Description

CULCLYF Clay filling fraction $\left(b_{c} / b\right)$ in Culebra (dimensionless), where $2 b$ is the fracture aperture and $2 b_{c}$ is the total thickness of the clay lining in the fracture. Used in SECO-TRANSPORT. Equal to ratio $b_{s} / b_{f}$ in Fig. 12 of Ref. 54. Range: 0 to 0.5. Median: 0.25 . Distribution: Uniform. Additional information: Sect. 2.6.1, Ref. 18.

CULCLYP Porosity of clay lining fractures in Culebra (dimensionless). Used in SECO-TRANSPORT. Defines porosity in region labeled "fracture skin" in Fig. 12 of Ref. 54. Range: 0.05 to 0.5 . Median: 0.275. Distribution: Uniform. Additional information: Sect. 2.6.2, Ref. 18.

CULFRPOR Fracture porosity in Culebra (dimensionless). Used in SECO-TRANSPORT. Defines variable $\phi_{f}$ in Eqs. (87) and (88) of Ref. 54. Range: $1 \times 10^{-4}$ to $1 \times 10^{-2}$. Median: $1 \times 10^{-3}$. Distribution: Lognormal. Additional information: Tables 1-2 and E-6, Ref. 6; Sect. 2.6.2, Ref. 18.

CULPOR Matrix porosity in Culebra (dimensionless). Used in BRAGFLO and SECO-TRANSPORT. Defines variable $\phi_{c}$ in definition of $\beta_{s}$ in Table 3 of Ref. 54 and variable $\phi_{m}^{\prime}$ in Eqs. (96) and (98) of Ref. 54. Range: 0.058 to 0.25. Median: $1.39 \times 10^{-1}$. Distribution: Piecewise uniform. Additional information: Table 4.4, Ref. 64; Table E-8, Ref. 6; Sect. 2.6.2, Ref. 18.

CULTRFLD Transmissivity field for Culebra. Twenty-five transmissivity fields consistent with available field data were constructed and ranked with respect to travel time to the accessible environment. CULTRFLD is a pointer variable used to select from these 25 fields, with travel time increasing monotonically with CULTRFLD. Used in SECO-FLOW. The transmissivity field identified by CULTRFLD defines the function $t(x, y)$ in Eq. (85), and thus the tensor $\mathrm{K}$ in Eqs. (83) and (84), of Ref. 54. Range: 0 to 1 . Median: 0.5. Distribution: Uniform. Additional information: Sect. 7.5, Ref. 17; Sect. 2.6.3, Ref. 18; Sect. 6-8, Ref. 19; Ref. 65.

$n=\left\{\begin{array}{l}1 \\ n\end{array}\right.$

if $E_{m}$ is not in $A S_{j}$ (i.e., $m \in E S_{j}^{c}$ in Eq. (2.9))

if $E_{m}$ is in $A S_{j}$ (i.e., $m \in E S_{j}$ in Eq. (2.8)) and the set $S_{m n}$ (see Table 3.9) is used in the definition of $\varepsilon$ (note: except for $m=1, \delta_{m n}=\varepsilon_{m n}$ )

and the sets $\mathcal{E}(m, n)$ are defined by

$$
\begin{array}{rlrl}
\mathcal{E}(m, n) & =S_{m n} & & \text { for } m=1,2,3,4,5 \text { or } 8 \\
& =\varepsilon_{61} & & \text { for } m=6, n=1 \\
& =\varepsilon_{6 n} 1 \varepsilon_{p q} & & \text { for } m=6, n=2,3,4, \text { or } 5 \text { and }(p, q)=(4,1),(4,2),(5,1),(5,2) \text { or }(5,3) \text { in } \Phi_{d} A S_{j \varepsilon} \\
& \text { (note: one, and only one, of the indicated values for }(p, q) \text { will be in } \Phi_{\varnothing} I S_{j} \delta
\end{array}
$$




$$
\begin{array}{ll}
=\mathcal{E}_{7 n} & \text { for } m=7 \text { and } D_{3} \text { not in } A S_{j} \\
=\mathcal{E}_{7 n} \mid D_{3} & \text { for } m=7 \text { and } D_{3} \text { in } A S_{j j}
\end{array}
$$

The representation

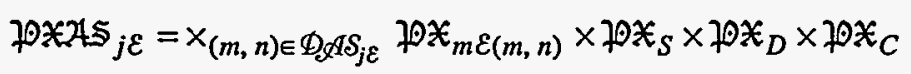

then provides a formal definition for each probability space $70 * 24 S_{j \varepsilon}$ in SPM-1. The specification of $n=1$ when experimental program $E_{m}$ is not in activity set $A S_{j}$ results in the incorporation of the defensible baseline assumptions for $E_{m}$ (i.e., $\not D X_{m \varepsilon}, \varepsilon=\varepsilon_{m 1}$ ) into the preceding definition of $\not D \nVdash \mathcal{H}_{j} \varepsilon$. As a reminder, the outcome $\varepsilon_{i 1}$ for experimental program $E_{i}$ always implies the defensible baseline assumption for $E_{i}$ in SPM-1. Examples of $70 ¥ 245_{j}$ are given in Table 3.22 .

\subsection{Probability Spaces $70 f \mathcal{A} \mathrm{s}_{j \varepsilon}$ that Characterize Future Occurrences at the WIPP}

As indicated by the subscripts $j$ and $\mathcal{E}$, the probability space $\not \mathcal{D F} 1 S_{j \varepsilon}$ can depend on both the activity set $A S_{j}$ and the particular experimental outcomes that could be obtained for $A S_{j}$. For example, an experimental program investigating the efficacy of various marker systems might be included in the portfolio of experimental programs under consideration. In this situation, $\not \mathcal{P A H} \mathrm{S}_{j \varepsilon}$ could be affected by whether or not this experimental program was contained in $A S_{j}$ and, given such containment, by the possible experimental outcomes of the program associated with $\mathcal{E}$. In SPM-1, no experimental programs or design modifications were considered that affected the definition of $20 \mathcal{F} \mathcal{H} \Im_{j} \mathcal{E}$, with the result that the subscripts $j$ and $\mathcal{E}$ can be dropped.

The SPM-1 analysis employs the same definition for $10 f 245$ as used in the 1991 and 1992 WIPP PAs. In particular, $10 \mathcal{H A} 5$ derives from the following assumptions: (1) exploratory drilling for natural resources constitutes the only disruption at the WIPP with sufficient likelihood to be retained in the analysis (Ref. 19, Chapt. 3), (2) drilling intrusions follow a Poisson process ${ }^{66-69}$ with a constant rate term $\lambda$, (3) waste is partitioned into five activity levels $\left(\mathrm{Ci} / \mathrm{m}^{2}\right)$ with a probability of $p L_{i}$ that a randomly placed borehole will intersect waste of activity level $i$, and (4) part of the repository is underlain by pressurized brine. The value for $\lambda$ is based on the default drilling rate of 30 drilling intrusions per $\mathrm{km}^{2}$ per $10,000 \mathrm{yr}$ suggested for use by the EPA in sedimentary basins. ${ }^{21,22}$

A mathematically complete definition for $10 \mathcal{F A S}$ is deferred to Sect. 5.4.3, where a full definition is needed for use in the construction of the CCDF specified in 40 CFR 191.13. 
Table 3.22 Definition of Probability Space $20 \nVdash A \Im_{j \varepsilon}$ for Activity Sets $A S_{1}, A S_{2}, A S_{j}$ and $A S_{n A S}$ in Table 2.2

Definition of $7 @ X \mathcal{A} S_{1 \varepsilon}$ for $A S_{1}=\left\{E_{1}\right\}$

$$
\begin{aligned}
& \varepsilon=S_{1 p}(\text { see Table 3.9) } \\
& \Phi_{\mathcal{L}} \mathcal{I} S_{1 \varepsilon}=\{(1, p),(2,1),(3,1),(5,1),(6,1),(7,1),(8,1)\} \\
& \varepsilon(1, p)=s_{1 p}, \varepsilon(2,1)=\varepsilon_{21}, \varepsilon(3,1)=\varepsilon_{31}, \varepsilon(5,1)=\varepsilon_{51}, \varepsilon(6,1)=\varepsilon_{61}, \varepsilon(7,1)=\varepsilon_{71}, \varepsilon(8,1)=\varepsilon_{81}
\end{aligned}
$$

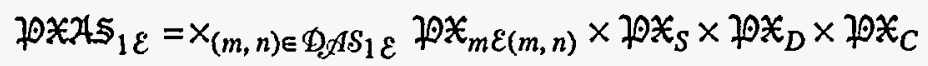

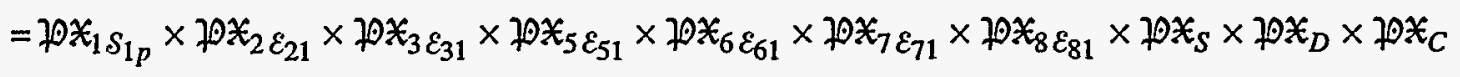

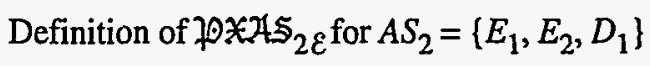

$$
\begin{aligned}
& \varepsilon=S_{1 p} \times S_{2 q}=S_{1 p} \times \varepsilon_{2 q} \text { (see Table 3.9) } \\
& \Phi_{\ell} \not S_{2 \mathcal{E}}=\{(1, p),(2, q),(3,1),(5,1),(6,1),(7,1),(8,1)\} \\
& \varepsilon(1, p)=s_{1 p}, \varepsilon(2, q)=\varepsilon_{2 q}, \mathcal{E}(3,1)=\varepsilon_{31}, \varepsilon(5,1)=\varepsilon_{51}, \varepsilon(6,1)=\varepsilon_{61}, \varepsilon(7,1)=\varepsilon_{71}, \varepsilon(8,1)=\varepsilon_{81}
\end{aligned}
$$

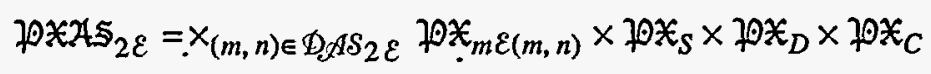

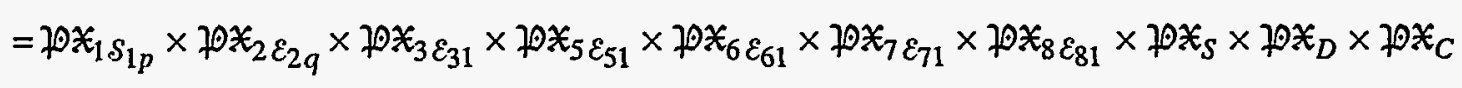$$
\text { Definition of } 10 \nVdash \mathcal{A} \Im_{j \varepsilon} \text { for } A S_{j}=\left\{E_{1}, E_{2}, E_{5}, E_{6}, D_{1}, D_{2}\right\}
$$

$\mathcal{E}=S_{1 p} \times S_{2 q} \times S_{5 s} \times S_{6 t}=S_{1 p} \times \varepsilon_{2 q} \times \varepsilon_{5 s} \times \varepsilon_{6 t}($ see Table 3.9)

$\Phi_{2} I S_{j \varepsilon}=\{(1, p),(2, q),(3,1),(5, s),(6, t),(7,1),(8,1)\}$

$\mathcal{E}(1, p)=\mathcal{S}_{1 p}, \mathcal{E}(2, q)=\varepsilon_{2 q}, \mathcal{E}(3,1)=\varepsilon_{31}, \mathcal{E}(5, s)=\varepsilon_{5 s}, \mathcal{E}(6, t)=\varepsilon_{61}$ if $t=1, \varepsilon(6, t)=\varepsilon_{6 t} \mid \varepsilon_{5 s}$ if $t=2,3,4$ or 5 , $\varepsilon(7,1)=\varepsilon_{71}, \varepsilon(8,1)=\varepsilon_{81}$

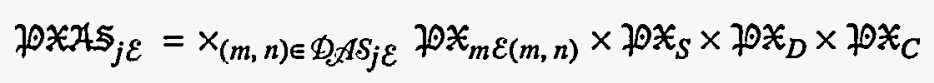

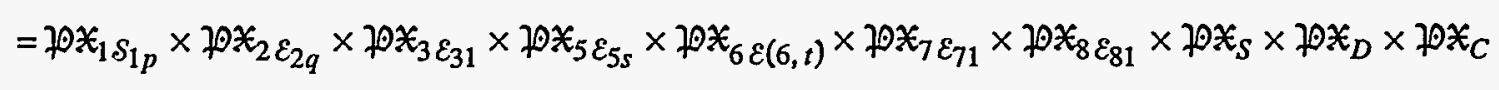



(continued)

$$
\text { Definition of } 20 * \mathcal{A} \Im_{n A S \varepsilon} \text { for } A S_{n A S} \doteq\left\{E_{1}, E_{2}, E_{3}, E_{5}, E_{6}, E_{7}, E_{8}, D_{1}, D_{2}, D_{3}\right\}
$$

$$
\begin{aligned}
& \mathcal{E}=\mathcal{S}_{1 p} \times \mathcal{S}_{2 q} \times S_{3 r} \times \mathcal{S}_{5 s} \times S_{6 t} \times \mathcal{S}_{7 u} \times \mathcal{S}_{8 v}=\mathcal{S}_{1 p} \times \varepsilon_{2 q} \times \varepsilon_{3 r} \times \mathcal{E}_{5 s} \times \varepsilon_{6 t} \times \mathcal{E}_{7 u} \times \mathcal{E}_{8 v} \text { (see Table 3.9) } \\
& \Phi_{2} \not S_{n A S \varepsilon}=\{(1, p),(2, q),(3, r),(5, s),(6, t),(7, u),(8, v)\} \\
& \varepsilon(1, p)=s_{1 p}, \varepsilon(2, q)=\varepsilon_{2 q}, \varepsilon(3, r)=\varepsilon_{3 r}, \varepsilon(5, s)=\varepsilon_{5 s}, \varepsilon(6, t)=\varepsilon_{61} \text { if } t=1, \varepsilon(6, t)=\varepsilon_{6 t} \mid \varepsilon_{5 s} \text { if } t=2,3,4 \text { or } 5 \text {, } \\
& \varepsilon(7, u)=\varepsilon_{7 u} \mid D_{3}, \varepsilon(8, v)=\varepsilon_{8 \mathrm{~V}}
\end{aligned}
$$

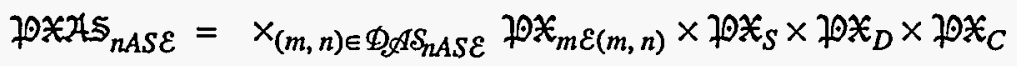

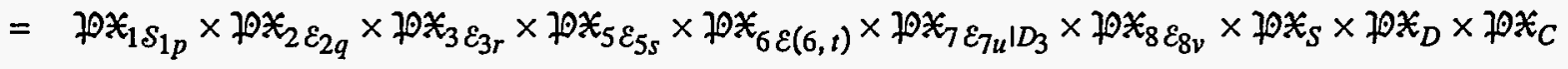




\section{Approximation of Integrals}

\subsection{Integration Over Probability Space $10 \notin 245_{j}$}

In practice, integrals of the form appearing in Eq. (2.25) do not have closed-form evaluations. Rather, they must be approximated. The first approximation is to the outer integral and produces

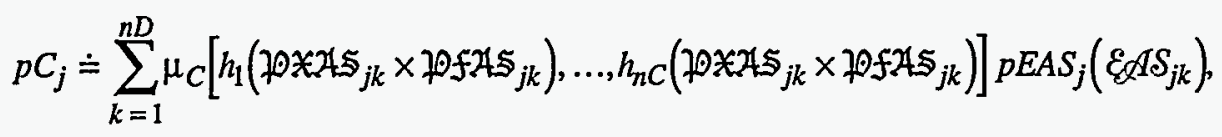

where $\xi I S_{j k}, k=1,2, \ldots, n D$, is a sequence of disjoint elements of $\mathcal{E} \& \mathcal{S}_{j}$ such that

$$
\mathscr{A} S_{j}=\bigcup_{k=1}^{n D} \xi A S_{j k}
$$

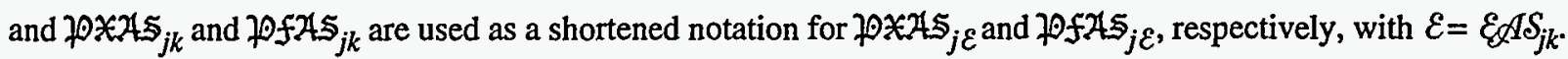

The functions $h_{1}, h_{2}, \ldots, h_{n C}$ in Eqs. (2.25) and (4.1) operate on the product of two probability spaces. However, there is a change in the definition of these spaces when the integral in Eq. (2.25) is replaced by the approximation in Eq. (4.1). In the original integral, these spaces are dependent on the occurrence of individual

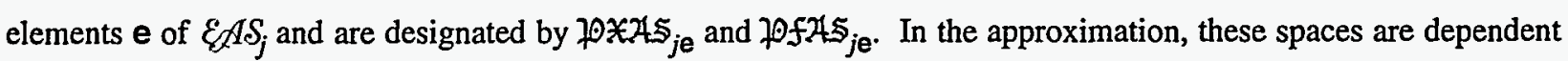

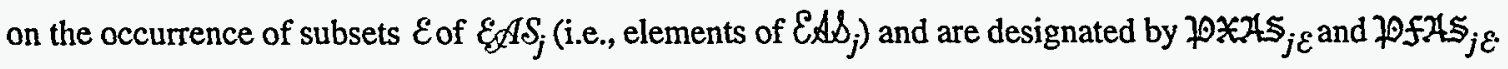

In SPM-1, the approximation in Eq. (4.1) simplifies to

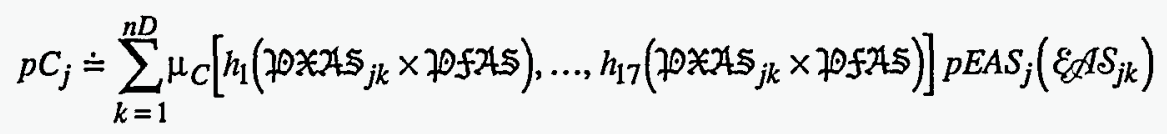

as $n C=17$ and the probability space $70 f \mathcal{A} 5$ for stochastic uncertainty does not depend on properties of the individual activity sets. Further, the sets $\varepsilon \mathbb{I} S_{j k}$ are defined by

$$
\xi A S_{j k}=\varepsilon=x_{i \in \varepsilon S_{j}} S_{i l}
$$

as indicated in Eqs. (3.1) and (3.2). As an example, the sets $\& A \delta_{j k}$ associated with activity set $A S_{j}$ that contains experimental programs $E_{2}, E_{3}$ and $E_{4}$ and design modification $D_{2}$ are listed in Table 4.1. 
Table 4.1. Sets $\& d I S_{j k}, k=1,2, \ldots, n D=18$, Associated with Activity Set $A S_{j}$ and Used in Approximation to $p C_{j}$ in Eq. (4.3), where $A S_{j}$ is the Activity Set that Contains Experimental Programs $E_{2}, E_{3}$, and $E_{4}$ and Design Modification $D_{2}$

\begin{tabular}{|c|c|c|}
\hline$\xi A S_{j 1}=\varepsilon_{21} \times \varepsilon_{31} \times \varepsilon_{41}$ & $\varepsilon \not I S_{j 7}=\varepsilon_{22} \times \varepsilon_{31} \times \varepsilon_{41}$ & $\varepsilon \not S_{j 13}=\varepsilon_{23} \times \varepsilon_{31} \times \varepsilon_{41}$ \\
\hline$\xi A I S_{j 2}=\varepsilon_{21} \times \varepsilon_{31} \times \varepsilon_{42}$ & $\varepsilon A S_{j 8}=\varepsilon_{22} \times \varepsilon_{31} \times \mathcal{E}_{42}$ & $\varepsilon \not S_{j 14}=\varepsilon_{23} \times \varepsilon_{31} \times \varepsilon_{42}$ \\
\hline$\varepsilon A S_{j 3}=\varepsilon_{21} \times \varepsilon_{32} \times \varepsilon_{41}$ & $\xi A I S_{j 9}=\varepsilon_{22} \times \varepsilon_{32} \times \varepsilon_{41}$ & $\varepsilon A S_{j 15}=\varepsilon_{23} \times \varepsilon_{32} \times \varepsilon_{41}$ \\
\hline$\varepsilon A S_{j 4}=\varepsilon_{21} \times \varepsilon_{32} \times \varepsilon_{42}$ & $\varepsilon A I S_{j 10}=\varepsilon_{22} \times \varepsilon_{32} \times \varepsilon_{42}$ & $\xi A \delta_{j 16}=\varepsilon_{23} \times \varepsilon_{32} \times \varepsilon_{42}$ \\
\hline$\varepsilon A S_{j 5}=\varepsilon_{21} \times \varepsilon_{33} \times \varepsilon_{41}$ & $\xi A S_{j 11}=\varepsilon_{22} \times \varepsilon_{33} \times \varepsilon_{41}$ & $\varepsilon d I S_{j 17}=\varepsilon_{23} \times \varepsilon_{33} \times \varepsilon_{41}$ \\
\hline$\varepsilon A S_{j 6}=\varepsilon_{21} \times \varepsilon_{33} \times \varepsilon_{42}$ & $\varepsilon d S_{j 12}=\varepsilon_{22} \times \varepsilon_{33} \times \varepsilon_{42}$ & $\xi A S_{j 18}=\varepsilon_{23} \times \varepsilon_{33} \times \varepsilon_{42}$ \\
\hline
\end{tabular}

As shown in Eqs. (2.28) - (2.32), $h_{1}$ and $h_{2}$ are defined by double integrals over $\mathrm{e} X A \mathcal{S}_{\mathrm{je}}$ and $\mathscr{I} \mathcal{A} \mathcal{S}_{\mathrm{je}}$, and $h_{3}, h_{4}$, $\ldots, h_{17}$ are defined by single integrals over $\propto \not A \delta_{j e}$. Due to the approximation of the integral in Eq. (2.25) with the

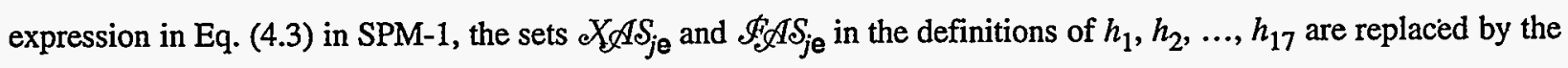
sets $\mathrm{x} \not \mathbb{A} \delta_{j k}$ and $\mathscr{F}_{\&} \mathbb{S} S$ as discussed in the two preceding paragraphs, with the result that the $h_{i}$ are defined by

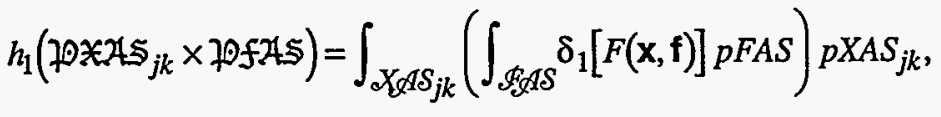

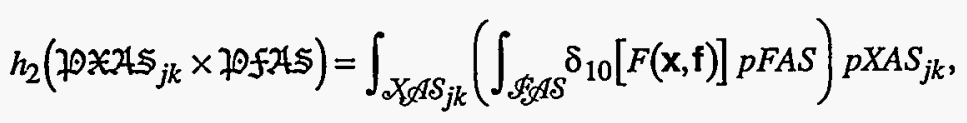

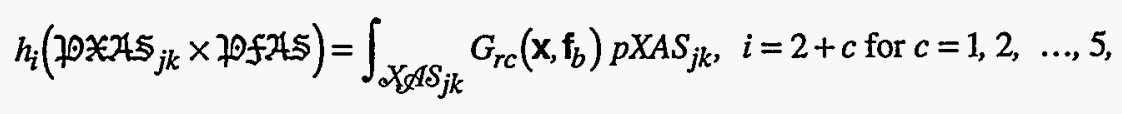

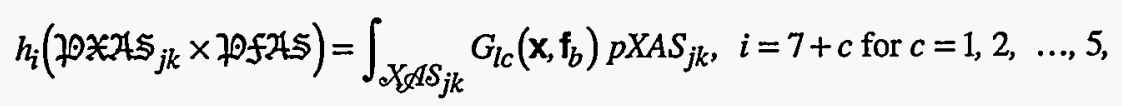

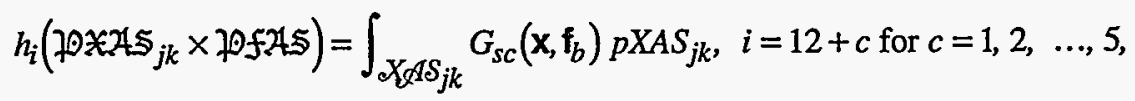

in Eq. (4.3).

The next step in evaluating the approximation to $p C_{j}$ in Eq. (4.3) is to evaluate the integrals that define the $h_{i}$. As discussed in Sect. 4.2, the integrals over $\triangle \not A S_{j k}$ in Eqs. (4.5) - (4.9) will be evaluated with a Monte Carlo technique based on Latin hypercube sampling. ${ }^{70}$ Further, as discussed in Sect. 4.3 , the integrals over $\oiint_{\mathscr{A}} \mathbb{S}$ in Eqs. (4.5) and (4.6) will be evaluated with a Monte Carlo technique based on simple random sampling. 


\subsection{Integration Over Probability Space $20 * 45_{j k}$}

In concept, evaluation of the integrals over ${ }^{X} \not I S_{j k}$ in Eqs. (4.5) - (4.9) is simple. A Latin hypercube sample (LHS)

$$
\mathbf{x}_{m}=\left[x_{m 1}, x_{m 2}, \ldots, x_{m, n I}\right], m=1,2, \ldots, n L H S
$$

is generated from $a X \not A S_{j k}$ in consistency with the definition of $20 X \mathcal{A} \varsigma_{j k}$, where $n I$ is the dimension of $\mathrm{X} \not A S_{j k}$ and $n L H S$ is the size of the sample. The approximations

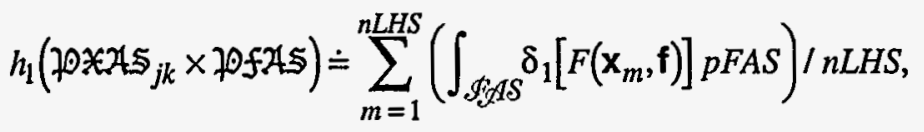

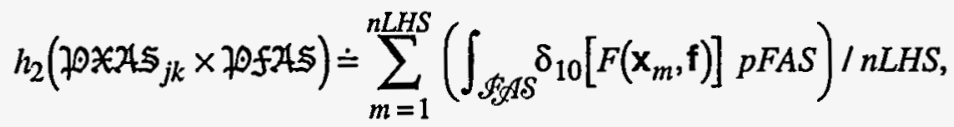

$$
\begin{aligned}
& h_{i}\left(\not \cap * \mathcal{A} \Im_{j k} \times \not \cap\{\mathcal{A} 5) \doteq \sum_{m=1}^{n L H S} G_{r c}\left(\mathbf{x}_{m}, \mathrm{f}_{b}\right) / n L H S, i=2+c \text { for } c=1,2, \ldots, 5\right. \text {, }
\end{aligned}
$$

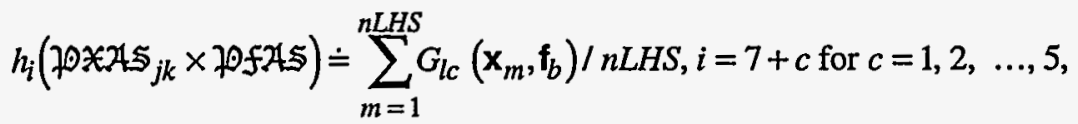

$$
\begin{aligned}
& h_{i}\left(\not 0 * 275_{j k} \times 10 f \mathcal{A} 5\right) \doteq \sum_{m=1}^{n L H S} G_{s c}\left(\mathbf{x}_{m}, \mathbf{f}_{b}\right) / n L H S, i=12+c \text { for } c=1,2, \ldots, 5 \text {, }
\end{aligned}
$$

then result for the $h_{i}$ and their defining integrals in Eqs. (4.5) - (4.9). Latin hypercube sampling was selected for use in SPM-1 because of its efficient stratification properties. ${ }^{70-73}$ In the overall design of the analysis, it is the indicated LHS that controls the total number of mechanistic (i.e., BRAGFLO, SECO-FLOW, SECO-TRANSPORT) calculations that must be performed.

Although the approximation procedure indicated in Eqs. (4.11) - (4.15) is simple in concept, its implementation must be planned very carefully to prevent total computational cost from becoming unreasonable. The indicated integrals must be evaluated for each set $c X \not A S_{j k}$ associated with each activity set $A S_{j}$. For example, the relatively simple activity set associated with Table 4.1 results in 18 different definitions for $\int X A S_{j k}$ (i.e., $\left.k=1,2, \ldots, 18\right)$ and thus evaluation $p C_{j}$ with the approximation in Eq. (4.3) requires 18 evaluations of the integrals in Eqs. (4.11) (4.15). Most of the approximately 1500 activity sets in SPM-1 involve considerably more sets $\triangle X \mathbb{I} S_{j k}, k=1,2, \ldots$, $n D$, where $n D$ equals several hundred to several thousand is typical. The evaluation of the resultant large number of integrals requires careful planning and must take into account the particular details of the analysis and the properties of the models (i.e., computer programs) that actually define the functions $F, G_{r c}, G_{l c}$ and $G_{s c}$ in Eqs. (4.11) - (4.15). The computational strategy used in SPM-1 is described in Sect. 5. 


\subsection{Integration Over Probability Space 20 f215}

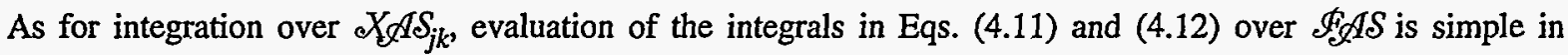
concept. A random sample

$$
\mathbf{f}_{n}, n=1,2, \ldots, n R \text {, }
$$

is generated from $\mathscr{H} \not S$ in consistency with the definition of $70 f \not A 5$, where $n R$ is the size of the sample. The integrals over $\mathscr{\&} \not \mathcal{S}$ in Eqs. (4.11) and (4.12) are then approximated by

$$
\int_{\mathscr{S A S}} \delta_{i}\left[F\left(\mathbf{x}_{m}, \mathbf{f}\right)\right] p F A S \doteq \sum_{n=1}^{n R} \delta_{i}\left[F\left(\mathbf{x}_{m}, \mathbf{f}_{n}\right)\right] / n R
$$

for $i=1,10$, with the result that the approximations to $h_{1}$ and $h_{2}$ in Eqs. (4.11) and (4.12) become

$$
h_{1}\left(20 * 24 \Im_{j k} \times \not 0 \mathcal{F} 25\right) \doteq \sum_{m=1}^{n L H S}\left(\sum_{n=1}^{n R} \delta_{1}\left[F\left(\mathbf{x}_{m}, \mathbf{f}_{n}\right)\right] / n R\right) / n L H S
$$

and

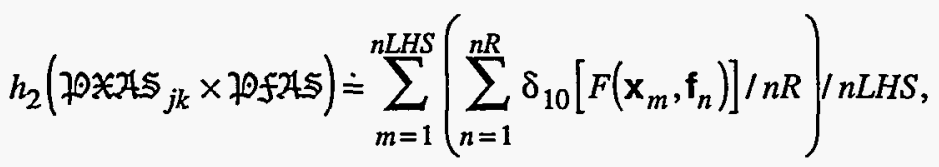

respectively.

When computationally-intensive models are in use, Monte Carlo simulation with simple random sampling can be prohibitively expensive. This procedure would not have been possible in SPM-1 if mechanistic calculations were required for every sample element $f_{n}$ in the evaluation of the integrals in Eq. (4.17). In the implementation procedure used and described in detail later, a relatively small number of mechanistic calculations were performed and then used to construct the large number of normalized releases $F\left(\mathbf{x}_{m}, \mathbf{f}_{n}\right)$ in Eq. (4.17).

\subsection{Definition of $F, G_{r c}, G_{l c}$ and $G_{s c}$}

The functions $F, G_{r c}, G_{l c}$ and $G_{s c}$ appearing in Eqs. (2.28) - (2.32) and elsewhere are evaluated through the operation of the computer programs BRAGFLO, CUTTINGS, GRASP-INV, PANEL, SANCHO, SECO-FLOW, SECO-TRANSPORT, SPALLINGS and VAST. A representation of these programs in the context of the calculations performed for SPM-1 is shown in Fig. 4.1. Further, brief summary descriptions of the individual programs are given in Table 4.2. 
The function $F$ defines normalized releases to the accessible environment. These releases have two components: a cuttings and spallings release that is based on calculations performed with BRAGFLO, CUTTINGS and SPALLINGS, and a groundwater release that is based on calculations performed with BRAGFLO, PANEL, SECO-FLOW and SECO-TRANSPORT. The functions $G_{r c}, G_{l c}$ and $G_{s c}$ are based on calculations performed with BRAGFLO and VAST.

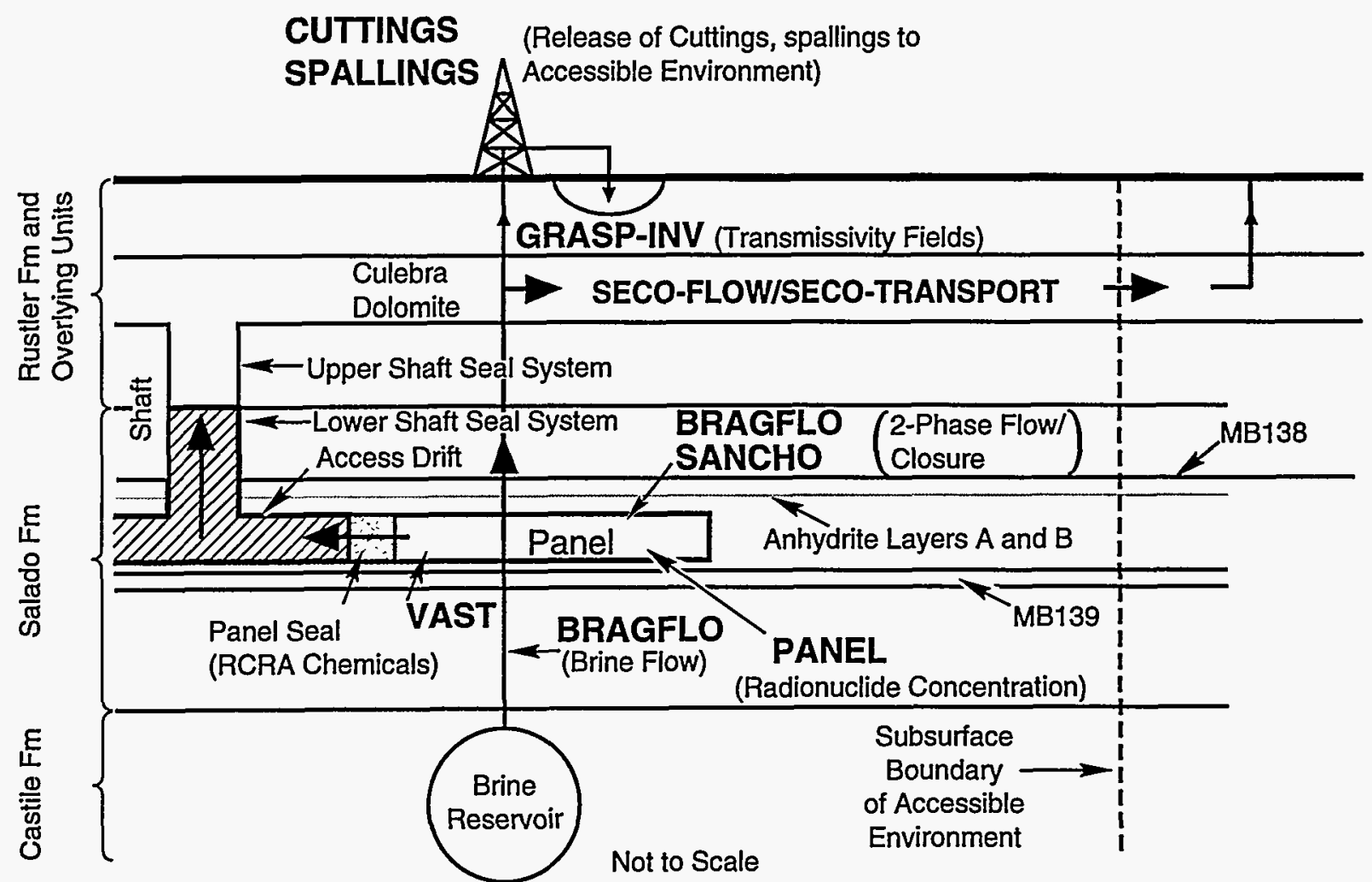

TRI-6342-3401-7

Figure 4.1. Models used in SPM-1 to define functions $F, G_{r c}, G_{l c}$ and $G_{s c}$ in Eqs. (2.28) - (2.32) and elsewhere. The names for computer models (i.e., computer codes) are shown in capital letters. 
Table 4.2. Summary of Computer Models Used in SPM-1 to Define Functions $F, G_{r c}, G_{l c}$ and $G_{s c}$ Appearing in Eqs. (2.28) - (2.32) and Elsewhere

\begin{tabular}{ll}
\hline \multicolumn{1}{c}{ Model } & \multicolumn{1}{c}{ Description } \\
\hline BRAGFLO & Describes multiphase flow of gas and brine through a porous, heterogeneous reservoir. \\
& Uses finite difference procedures to solve system of nonlinear partial differential \\
& equations that describes the mass conservation of gas and brine along with appropriate \\
& constraint equations, initial conditions and boundary conditions. Additional information: \\
& Ref. 17, App. A; Ref. 19, Chapts. 4, 5; Ref. 54, Sect. 3.1.
\end{tabular}

CUTTINGS Calculates the quantity of radioactive material brought to the surface in cuttings and cavings generated by an exploratory borehole that penetrates a waste panel. Uses brine flows calculated by BRAGFLO. Additional information: Ref. 19, Chapt. 7; Ref. 54, Sect. 3.5; Ref. 74.

GRASP-INV

Generates transmissivity fields (estimates of transmissivity values) conditioned on measured transmissivity values and calibrated to steady-state and transient pressure data at well locations using an adjoint sensitivity and pilot-point technique. Additional information: Ref. 17, App. D; Ref. 65.

PANEL

Calculates rate of discharge and cumulative discharge of radionuclides from a waste panel through an intruding borehole. Discharge is a function of fluid flow rate, elemental solubility and radionuclide inventory. Based on solution of linear ordinary differential equation. Additional information: Ref. 18, Sect. 1.4.4; Ref. 54, Sect. 3.2.

SECO-FLOW

Calculates single-phase Darcy flow for groundwater-flow problems in two dimensions. The formulation is based on a single partial differential equation for hydraulic head using fully implicit time differencing. Additional information: Ref. 17, App. C; Ref. 19, Chapt. 6; Ref. 54, Sect. 3.3; Ref. 75.

SECO-TRANSPORT Simulates fluid flow and transport of radionuclides in fractured porous media. Solves two partial differential equations: one provides two dimensional representation for convective and diffusive radionuclide transport in fractures and the other provides one dimensional representation for diffusion of radionuclides into rock matrix surrounding the fractures. Additional information: Ref. 17, App. C; Ref. 19, Chapt. 6.

SANCHO

Solves quasistatic, large deformation, inelastic response of two-dimensional solids with finite element techniques. Used in the 1992 WIPP PA and SPM-1 to determine porosity of waste as a function of time and cumulative gas generation. Additional information: Ref. 17, App. B; Ref. 76.

SPALLINGS

Calculates the quantity of radioactive material brought to the surface due to gas-driven spallings into a borehole. Uses repository pressures calculated by BRAGFLO. Additional information: Ref. 77.

VAST

Uses temporally and spatially variable gas flow fields calculated by BRAGFLO to estimate the movement of VOCs in the vicinity of the repository. Implements advectiondispersion relationship defined by Eq. (6.3.2), Ref. 78 . 


\section{Computational Strategy}

\subsection{Organization of Mechanistic Calculations}

The mechanistic calculations performed in SPM-1 had to be selected very carefully. Otherwise, the total computational cost would have been prohibitive as approximately 1500 activity sets were under consideration and individual activity sets typically required several hundred to several thousand evaluations of the integrals in Eqs. (2.28) - (2.32).

The first step in controlling computational cost was the recognition that experimental programs $E_{i}, i=2,3, \ldots$, 8, and design modification $D_{3}$ affected the function $F$ appearing in Eqs. (2.28) and (2.29) (i.e., normalized radionuclide releases to the accessible environment used in assessing compliance with $40 \mathrm{CFR} 191.13$ ), while only experimental programs $E_{i}, i=1,2$, and design modifications $D_{i}, i=1,2$, affected the functions $G_{r c}$, $G_{l c}$ and $G_{s c}$ appearing in Eqs. (2.30) - (2.32) (i.e., "soil" concentrations used in assessing compliance with RCRA). The possible outcomes of experimental program $E_{1}$ involving the Brooks-Corey and van Genuchten-Parker characteristic curves were found to have little effect on assessing compliance with 40 CFR 191.13 in the 1992 WIPP PA. Further, the remaining outcomes associated with $E_{1}$ and also the modifications associated with $D_{1}$ and $D_{2}$ are not relevant to 40 CFR 191.13. Similarly, experimental programs $E_{i}, i=2,3, \ldots, 8$, and design modification $D_{3}$ are not relevant to RCRA. Because of the preceding effects, it is possible to divide the calculations into those involved in the determination of $G_{r c}, G_{l c}$ and $G_{s c}$ (Sect. 5.2) and those involved in the determination of $F$ (Sect. 5.3). This division prevents an unnecessary increase in mechanistic calculations on the basis of factors that are not important to the outcome under consideration.

As a further part of the computational strategy, opportunities were sought to identify results calculated with computationally-demanding models that could be used as input to less computationally-demanding models. In particular, gas flow results calculated with BRAGFLO were used as input to VAST to estimate VOC transport under a number of different assumptions (Sect. 5.2), and brine flow results also calculated with BRAGFLO were used as input to PANEL to estimate radionuclide release under different solubility assumptions (Sect. 5.3.3). This usage avoided the performance of repeated BRAGFLO calculations for situations that did not involve changes in the assumptions that affected fluid flow in the vicinity of the repository. Similarly, it was possible to save pressure histories in the repository calculated with BRAGFLO and then use these histories in repeated calculations with SPALLINGS (Sect. 5.3.2). Also, only one set of SECO-FLOW calculations was required to support a large number of SECO-TRANSPORT calculations (Sect. 5.3.4).

Additional computational savings were sought by identifying situations where results could be constructed from other calculations. For example, interpolation procedures were used to extend transport results obtained with SECOTRANSPORT for large releases to transport results for smaller releases (Sect. 5.3.5). Similarly, guidance was sought from past analyses as to when the outcome of calculations could be reasonably anticipated. For example, past 
calculations have shown that little radionuclide transport takes place in the Culebra in the presence of dual porosity flow and chemical retardation. As a result, it was not necessary to perform SECO-TRANSPORT calculations for this case (Sect. 5.3.5).

Further savings were obtained by identifying the unique situations that required CCDF construction (Sect. 5.4), which significantly reduced the number of CCDF constructions (i.e., evaluations of the integrals in Eq. (4.17)) required in the analysis. In addition, the computational efficiency of CCDF construction was increased by using a Monte Carlo construction procedure rather than the importance sampling procedure used in previous WIPP PAs. ${ }^{79,80}$

The preceding devices and the overall structure of the SPM-1 calculations are described in Sects. 5.2 - 5.4.

\subsection{Mechanistic Calculations for $G_{r c}, G_{l c}$ and $G_{s c}$ (i.e., RCRA)}

The determination of $G_{r c}, G_{l c}$ and $G_{s c}$ involves mechanistic calculations performed with BRAGFLO. As already indicated, determination of $G_{r c}, G_{l c}$ and $G_{s c}$ depends on experimental programs $E_{1}$ and $E_{2}$ and on design modifications $D_{1}$ and $D_{2}$. Experimental program $E_{1}$ has 11 possible outcomes (i.e., $\mathcal{S}_{1 i}, i=1,2, \ldots, 11$ as indicated in Table 3.9) that are considered in the determination of compliance probability. However, BRAGFLO calculations are performed for experimental program $E_{1}$ only on the basis of the distinction between the Brooks-Corey and van Genuchten-Parker characteristic curves, with the remaining possibilities associated with $E_{1}$ implemented through relatively simple side calculations. Experimental program $E_{2}$ has 3 possible outcomes (i.e., $\varepsilon_{2 i}, i=1,2,3$ as indicated in Table 3.9). Each of these outcomes also requires a separate BRAGFLO calculation. As VOCs are not expressly tracked in BRAGFLO, the presence or absence of design modification $D_{1}$ does not affect the number of required BRAGFLO calculations. Rather, VOCs are simply not present when design modification $D_{1}$ is implemented. In contrast, the presence and absence of design modification $D_{2}$ does require separate BRAGFLO calculations. Thus, as indicated in Fig. 5.1, the evaluation of $G_{r c}, G_{l c}$ and $G_{s c}$ requires 12 sets of BRAGFLO calculations.

Each of the 12 sets of BRAGFLO calculations uses input defined by the probability space $\not \cap \mathcal{X}_{2 \mathcal{E}} \times \not \cap \mathcal{X}_{S}$ as indicated in Fig. 5.1. This input was obtained by generating an LHS ${ }^{70,81}$

$$
\mathbf{r}_{m}=\left[r_{m 1}, r_{m 2}, \ldots, r_{m, n I}\right], m=1,2, \ldots, n L H S=25,
$$

from the $n I=13$ variables associated with $\not \cap \mathcal{X}_{S}$ and three LHSs

$$
\mathbf{s}_{i m}=\left[s_{i m}\right], m=1,2, \ldots, n L H S=25,
$$




\begin{tabular}{|c|c|c|c|}
\hline $\begin{array}{l}\text { Outcomes: } E_{1} \\
\text { (Salado) }\end{array}$ & $\begin{array}{l}\text { Outcomes: } E_{2} \\
\text { (Seal Perm) }\end{array}$ & Design Mod: $D_{2}$ & $\begin{array}{c}\text { BRAGFLO } \\
\text { Input }\end{array}$ \\
\hline \multirow[b]{5}{*}{$\varepsilon_{12}:$ Brooks-Corey } & & metals & 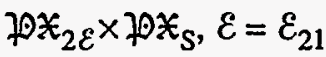 \\
\hline & $\varepsilon_{21}: 10^{-15}-10^{-13} \mathrm{~m}^{2}$ & \multirow[b]{2}{*}{ no metals } & \multirow{4}{*}{ 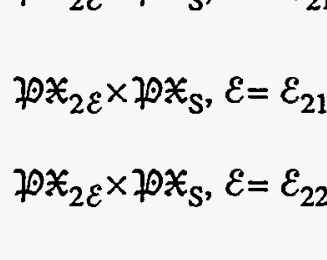 } \\
\hline & \multirow[b]{3}{*}{$\varepsilon_{22}: 10^{-18}-10^{-16} \mathrm{~m}^{2}$} & & \\
\hline & & metals & \\
\hline & & \multirow[b]{2}{*}{ no metals } & \\
\hline \multirow{13}{*}{$\begin{array}{c}\mathcal{E}_{13}: \text { van Genuchten- } \\
\text { Parker }\end{array}$} & \multirow[b]{3}{*}{$\varepsilon_{23}: 10^{-19}-10^{-18} \mathrm{~m}^{2}$} & & \multirow{3}{*}{$\begin{array}{l}\not \cap X_{2 \varepsilon} \times \not O X_{S}, \varepsilon=\varepsilon_{22} \\
\not O X_{2 \varepsilon} \times \not \cap X_{S}, \varepsilon=\varepsilon_{31}\end{array}$} \\
\hline & & metals & \\
\hline & & \multirow[b]{2}{*}{ no metals } & \\
\hline & \multirow[b]{3}{*}{$\varepsilon_{21}: 10^{-15}-10^{-13} \mathrm{~m}^{2}$} & & \multirow{3}{*}{ 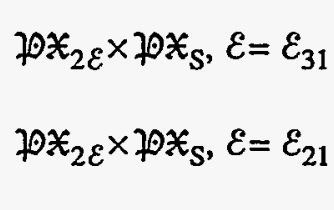 } \\
\hline & & \multirow[t]{2}{*}{ metals } & \\
\hline & & & \\
\hline & \multirow[b]{3}{*}{$\varepsilon_{22}: 10^{-18}-10^{-16} \mathrm{~m}^{2}$} & no metals & \multirow{3}{*}{ 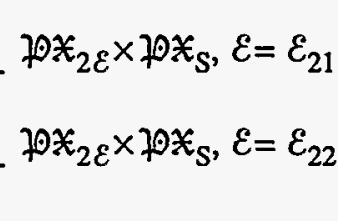 } \\
\hline & & metals & \\
\hline & & \multirow[b]{2}{*}{ no metals } & \\
\hline & \multirow{4}{*}{$\varepsilon_{23}: 10^{-19}-10^{-18} \mathrm{~m}^{2}$} & & \multirow{3}{*}{ 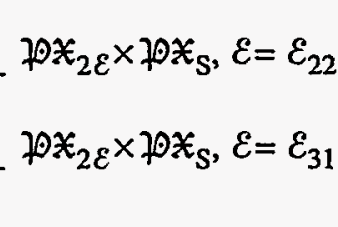 } \\
\hline & & metals & \\
\hline & & & \\
\hline & & no metals & $\not 0 \mathbb{*}_{2 \varepsilon} \times \not 0 \mathbb{X}_{S}, \varepsilon=\varepsilon_{31}$ \\
\hline
\end{tabular}

Figure 5.1.Logic diagram indicating 12 sets of BRAGFLO calculations associated with outcomes of experimental programs $E_{1}$ and $E_{2}$ and design modification $D_{2}$.

from $\not Q \mathcal{X}_{2 \varepsilon}$ for $\varepsilon=\varepsilon_{2 i}, i=1,2,3$, where $s$ corresponds to the single variable $S E A L P R M$ associated with $70 \mathcal{X}_{2 \varepsilon}$. Samples from the three probability spaces $\not \circ \mathcal{X}_{2 \varepsilon} \times \not \cap \mathcal{X}_{S}$ were then obtained by concatenating the sample elements in Eqs. (5.1) and (5.2) to produce the three new samples

$$
\mathbf{x}_{m}=\left[\mathbf{r}_{m}, \mathbf{s}_{i m}\right], m=1,2, \ldots, n L H S=25,
$$

for $i=1,2,3$, each of which correspond to a sample $\mathrm{x}_{m}, m=1,2, \ldots, n L H S$, of the form indicated in Eq. (4.10). The preceding produces three different samples with the same values from $\not 0 X_{S}$ but different values from $\not O X_{2 \varepsilon}$ for $\varepsilon$ $=\varepsilon_{21}, \varepsilon_{22}, \varepsilon_{23}$. Although the mechanics of sample generation within the SPM-1 analysis was somewhat different from the concatenation procedure described in conjunction with Eq. (5.3), both procedures produce equivalent 
outcomes. As described in conjunction with Eq. (4.10), the $\mathbf{x}_{m}$ are actually sampled from $\not \bigcirc \nVdash 24 \Im_{j k}$ rather than a

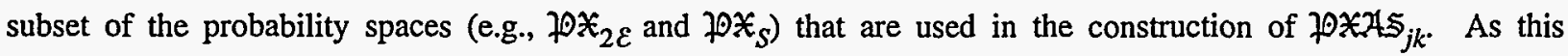
presentation progresses, sample elements $\mathbf{x}_{m}$ of this generality will be built up by additional concatenations of the form indicated in Eq. (5.3).

The BRAGFLO calculations indicated in Fig. 5.1 were performed for each sample element $\mathbf{x}_{m}$ in Eq. (5.3) to produce results that can be symbolically represented by

$$
\operatorname{BRAGFLO}\left(\varepsilon_{1 i}, \varepsilon_{2 j}, D_{2 l}, \mathbf{x}_{m}\right)
$$

where $\varepsilon_{1 i}$ implies use of the Brooks-Corey (i.e., $i=2$ ) or the van Genuchten-Parker ( $i=3$ ) characteristic curves, $\varepsilon_{2 j}$ implies high (i.e., $j=1$ ), intermediate (i.e., $j=2$ ) or low (i.e., $j=3$ ) shaft seal permeability, $D_{2 l}$ implies the absence (i.e., $l=0$ ) or presence (i.e., $l=1$ ) of design modification $D_{2}, \mathbf{x}_{m}$ designates the sample element in use, and the bold type used with BRAGFLO indicates that the associated BRAGFLO output is multidimensional (i.e., a vector). The totality of the BRAGFLO results associated with each calculation indicated in Eq. (5.4) is quite large; as a single example, time-dependent pressure histories within the repository for the 12 sets of BRAGFLO calculations under consideration are shown in Figs. 5.2 and 5.3. The plots in Figs. 5.2 and 5.3 are for brine pressure; the corresponding plots for gas pressure are visually identical. As an LHS of size 25 is in use and 12 cases are under consideration, the results presented in Figs. 5.2 and 5.3 are based on 300 (i.e., $12 \times 25$ ) BRAGFLO calculations.

The purpose of performing the BRAGFLO calculations indicated in Eq. (5.4) and illustrated in Figs. 5.2 and 5.3 is to evaluate the integrals in Eqs. (4.7) - (4.9) with the approximations in Eqs. (4.13) - (4.15). In SPM-1, only the outcomes associated with experimental programs $E_{1}$ and $E_{2}$ and design modifications $D_{1}$ and $D_{2}$ are relevant to the evaluation of $G_{r c}\left(\mathbf{x}_{m}, \mathbf{f}_{b}\right), G_{l c}\left(\mathbf{x}_{m}, \mathbf{f}_{b}\right)$ and $G_{s c}\left(\mathbf{x}_{m}, \mathbf{f}_{b}\right)$ in Eqs. (4.13) - (4.15). Thus, although the definition of $\not 0 \nVdash \mathcal{H} S_{j k}$ in Eqs. (4.13) - (4.15) derives from all experimental programs and design modifications associated with activity set $A S_{j}$, it is only the part associated with $E_{1}, E_{2}, D_{1}$ and $D_{2}$ that affects the evaluation of $G_{r c}\left(\mathbf{x}_{m}, \mathbf{f}_{b}\right)$, $G_{l c}\left(\mathbf{x}_{m}, \mathbf{f}_{b}\right)$ and $G_{s c}\left(\mathbf{x}_{m}, \mathbf{f}_{b}\right)$. As $E_{1}$ has 11 possible outcomes, $E_{2}$ has 3 possible outcomes, and $D_{1}$ and $D_{2}$ each give rise to two possible actions, 132 evaluations (i.e., $11 \times 3 \times 2 \times 2$ ) for the approximations in Eqs. (4.13) - (4.15) are required. Although not explicitly indicated by the notation in use, the functions $G_{r c}, G_{l c}$ and $G_{s c}$ actually change for each of the 132 cases under consideration. As indicated in Table 5.1, $G_{r c}, G_{l c}$ and $G_{s c}$ are not calculated directly by BRAGFLO but rather are calculated externally from results calculated with BRAGFLO. This calculation is primarily performed with the VAST program. Once $G_{r c}, G_{l c}$ and $G_{s c}$ are determined, the integrals in Eqs. (4.7) (4.9) can be approximated as shown in Eqs. (4.13) - (4.15). 

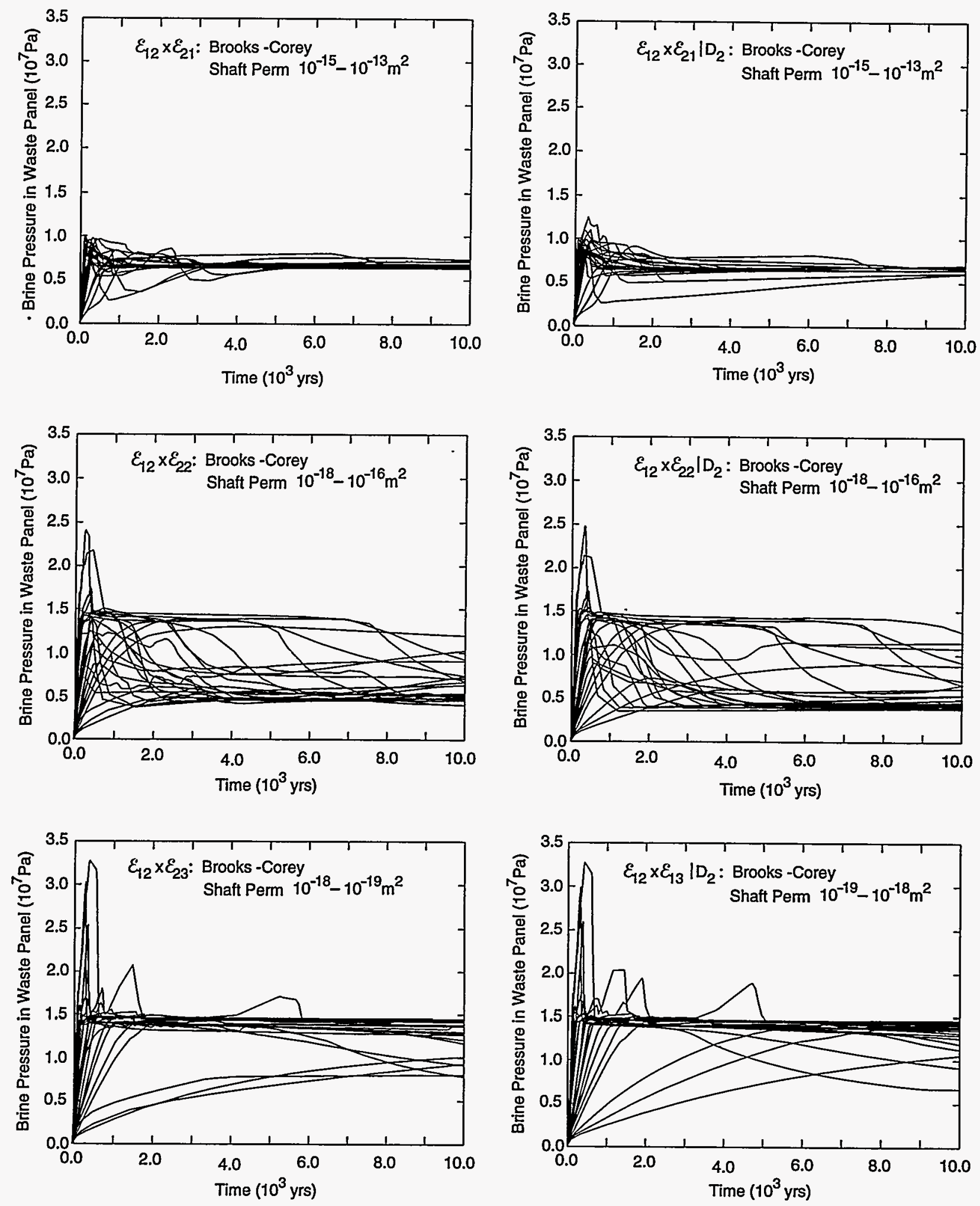

TRI-6342-4601-0

Figure 5.2. Time-dependent brine pressure in repository for 6 sets of BRAGFLO calculations with Brooks-Corey model indicated in Fig. 5.1. Each plot frame contains 25 curves due to the use of an LHS of size 25 as discussed in conjunction with Eq. (5.3). 

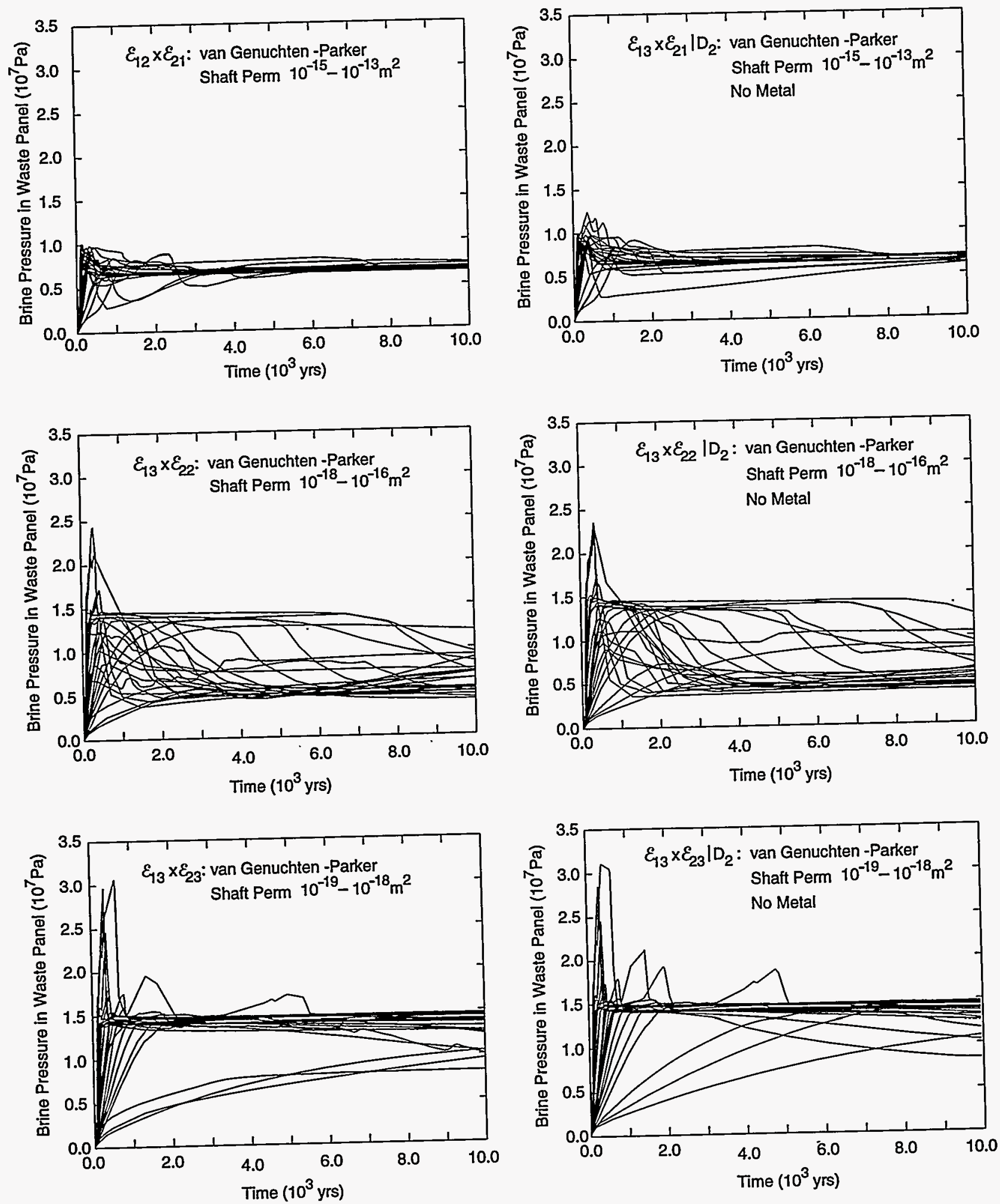

TRI-6342-4602-0

Figure 5.3. Time-dependent brine pressure in repository for 6 sets of BRAGFLO calculations with van GenuchtenParker model indicated in Fig. 5.1. Each plot frame contains 25 curves due to the use of an LHS of size 25 as discussed in conjunction with Eq. (5.3). 
Table 5.1. Definition of $G_{r c}, G_{l c}$ and $G_{s c}$ for 132 Cases Considered in SPM-1 (i.e., for all unique sets of conditions implied by the probability spaces $10 * 245_{j k}$ for the evaluation of the integrals in Eqs. (4.7) (4.9) with the approximations in Eqs. (4.13) - (4.15))

BRAGFLO Calculations with Brooks-Corey Model and without Design Modification $D_{2}: \varepsilon_{12} \times \varepsilon_{2 q}, q=1,2,3$

\begin{tabular}{|c|c|}
\hline Case & Evaluation of $G_{r c}, G_{l c}, G_{s c}$ \\
\hline$s_{11} \times s_{2 q}=\varepsilon_{11} \times \mathcal{E}_{2 q}, q=1,2,3$ & $\begin{array}{l}G_{r c} \text { and } G_{l c} \text { determined in side calculation based on } \\
\text { assumption that a fracture system extending to the } \\
\text { accessible environment develops when the gas pressure } \\
\text { in the repository reaches } 12.5 \mathrm{MPa} \text {. } G_{s c} \text { determined } \\
\text { with VAST using spatially and temporally dependent } \\
\text { gas flow fields calculated with BRAGFLO. }\end{array}$ \\
\hline $\begin{array}{l}S_{1 p} \times S_{2 q}=\left(\varepsilon_{11}^{c} \cap \varepsilon_{12} \cap \varepsilon_{1, p+2}\right) \times \varepsilon_{2 q} \\
p=2,3,4,5,6, q=1,2,3\end{array}$ & $\begin{array}{l}G_{r c}, G_{l c} \text { and } G_{s c} \text { determined with VAST using spatially } \\
\text { and temporally dependent gas flow fields calculated } \\
\text { with BRAGFLO, with distance to accessible } \\
\text { environment for } G_{r c} \text { and } G_{s c} \text { defined to be } 2.75 \mathrm{~km} / 10 \text {, } \\
2.75 \mathrm{~km} / 6,2.75 \mathrm{~km} / 4,2.75 \mathrm{~km} / 2 \text { and } 2.75 \mathrm{~km} / 1 \text { for } p= \\
2,3,4,5 \text { and } 6 \text {, respectively. }\end{array}$ \\
\hline $\begin{array}{l}\delta_{1 p} \times \delta_{2 q}=\delta_{1 p} \times \varepsilon_{2 q} \text { with } D_{1}, \\
p=1,2, \ldots, 6, q=1,2,3\end{array}$ & $G_{r c}=G_{l c}=G_{s c}=0$ due to absence of VOCs. \\
\hline
\end{tabular}

BRAGFLO Calculations with van Genuchten-Parker Model and without

Design Modification $D_{2}: \varepsilon_{13} \times \varepsilon_{2 q}, q=1,2,3$

\begin{tabular}{ll}
\hline \multicolumn{1}{c|}{ Case } & \multicolumn{1}{c}{ Evaluation of $G_{r c}, G_{l c}, G_{s c}$} \\
\hline$S_{11} \times S_{2 q}=\varepsilon_{11} \times \varepsilon_{2 q}, q=1,2,3$ & Same as above. \\
$S_{1 p} \times s_{2 q}=\left(\varepsilon_{11}^{c} \cap \varepsilon_{13} \cap \varepsilon_{1, p-3}\right) \times \varepsilon_{2 q}$, & Same as above. \\
$p=7,8,9,10,11, q=1,2,3$ & \\
$S_{1 p} \times S_{2 q}=S_{1 p} \times \varepsilon_{2 q}$ with $D_{1}$, & Same as above. \\
$p=1,2, \ldots, 6, q=1,2,3$ & \\
\hline
\end{tabular}

BRAGFLO Calculations with Brooks-Corey Model and Design Modification $D_{2}: \varepsilon_{12} \times \varepsilon_{2 q}, q=1,2,3$

Same assignments as made for $\varepsilon_{12} \times \varepsilon_{2 q}$ without design modification $D_{2}$

BRAGFLO Calculations with van Genuchten-Parker Model and Design Modification $D_{2}: \varepsilon_{13} \times \varepsilon_{2 q}, q=1,2,3$

Same assignments as made for $\varepsilon_{13} \times \varepsilon_{2 q}$ without design modification $D_{2}$ 


\subsection{Mechanistic Calculations for $F$ (i.e., 40 CFR 191.13)}

The determination of $F$ involves the full sequence of model calculations indicated in Fig. 4.1. These calculations must be organized very carefully to keep total computational cost at a reasonable level. As indicated previously, the determination of $F$ involves the outcomes of experimental programs $E_{i}, i=2,3, \ldots, 8$, and design modification $D_{3}$. Specifically, experimental program $E_{2}$ affects calculations performed with BRAGFLO; experimental programs $E_{3}$ and $E_{7}$ and design modification $D_{3}$ affect calculations performed with PANEL; experimental programs $E_{4}, E_{5}$ and $E_{6}$ affect calculations performed with SECO-TRANSPORT; and experimental program $E_{8}$ affects the spallings releases calculated with SPALLINGS. In addition, BRAGFLO provides input to the spallings calculations with SPALLINGS and the radionuclide release calculations with PANEL; the radionuclide releases determined with PANEL are input to the SECO-TRANSPORT calculations; and calculations performed with SECO-FLOW generate the Culebra flow field, which is also used as input to SECO-TRANSPORT.

The calculations indicated in Sect. 5.2 to produce values for $G_{r c}, G_{l c}$ and $G_{s c}$ were performed for undisturbed conditions (i.e., for the element $\mathbf{f}_{b}$ of $\mathscr{S}_{\mathscr{A}} A \mathcal{S}$ ). In contrast, $F$ must be evaluated for many different elements of $\mathscr{I} \not \mathcal{S}$ (i.e., patterns of drilling intrusions in SPM-1) to produce the approximation indicated in Eq. (4.17). This will be accomplished by performing a relatively small number of mechanistic calculations with the models indicated in Fig. 4.1 and then extending these results to estimate $F$ for a large number of different elements of $\mathscr{F} A \mathcal{A}$ (i.e., the $f_{n}$ indicated in Eq. (4.17)). The actual calculations performed with BRAGFLO, CUTTINGS, PANEL, SECO-FLOW, SECO-TRANSPORT and SPALLINGS are summarized in this section. The use of these calculations in the evaluation of $F\left(\mathbf{x}_{m}, \mathbf{f}_{n}\right)$ in Eq. (4.17) is discussed in Sect. 5.4.

\subsubsection{BRAGFLO Calculations}

In the 1991 and 1992 WIPP PAs, BRAGFLO calculations were performed for E1 and E2 scenarios. As a reminder, an E1 scenario corresponds to the occurrence of a drilling intrusion that penetrates both the repository and a pressurized brine pocket in the Castile Formation; an E2 scenario corresponds to the occurrence of a drilling intrusion that penetrates the repository but does not penetrate a pressurized brine pocket; and an E1E2 scenario corresponds to the occurrence of two or more drilling intrusions into a single waste panel, of which at least one penetrates a pressurized brine pocket and at least one does not penetrate a pressurized brine pocket. No BRAGFLO calculations were performed specifically for E1E2 scenarios. Rather, the results of the BRAGFLO calculations for E1 scenarios were used in conjunction with PANEL to estimate the radionuclide releases that would take place for an E1E2 scenario. Specifically, the PANEL calculations to estimate E1E2 releases were performed with the assumption that the entire radionuclide inventory of a waste panel could be accessed by the brine flows associated with an E1 scenario. In contrast, it was assumed that the brine flows associated with an E1 scenario would spread out very little through a waste panel once the panel filled with brine, with the result that E1 scenarios were assigned the same 
releases as the corresponding E2 scenarios. A similar procedure was used in SPM-1, with BRAGFLO calculations performed for E1 and E2 scenarios and then extended to produce results for E1E2 scenarios.

The actual BRAGFLO calculations performed to support the determination of $F$ are indicated in Fig. 5.4. Specifically, BRAGFLO calculations were performed for E1 and E2 scenarios with the intrusion occurring at 1000 yr for the subset $\varepsilon_{21}$ of $\varepsilon_{2}$ (i.e., for shaft permeabilities in the range $10^{-15}-10^{-13} \mathrm{~m}^{2}$ as indicated in Table 3.11 ) and for E1 scenarios with the intrusion occurring at $1000 \mathrm{yr}$ for the subsets $\varepsilon_{22}$ and $\varepsilon_{23}$ of $\mathcal{E}_{2}$ (i.e., for shaft permeabilities in the ranges $10^{-18}-10^{-16} \mathrm{~m}^{2}$ and $10^{-19}-10^{-18} \mathrm{~m}^{2}$, respectively). These calculations were performed for the three LHSs (i.e., for $\varepsilon_{21}, \varepsilon_{22}, \varepsilon_{23}$ ) discussed in conjunction with Eq. (5.3). As LHSs of size 25 are in use, the calculations indicated in Fig. 5.4 involved 100 (i.e., $4 \times 25$ ) individual BRAGFLO runs.

The BRAGFLO results that are actually used as input to PANEL are volume of brine in a waste panel and cumulative brine flow from the repository to the Culebra. The analysis also included brine flow to the surface but all such flows were predicted to be zero due to the diversion of flow into the Culebra as a result of its high permeability. For illustration, Fig. 5.5 shows the cumulative brine flows to the Culebra calculated for E1 and E2 intrusions at 1000 yr for the subset $\varepsilon_{21}$ of $\varepsilon_{2}$, and Fig. 5.6 shows similar results for E1 intrusions and the subsets $\varepsilon_{22}$ and $\varepsilon_{23}$ of $\varepsilon_{2}$.

The spallings release is dependent on the pressure in the repository, with the spallings release tending to increase as the pressure increases. Due to the assumption of high permeability boreholes as directed in the EPA guidance associated with 40 CFR 191.13, the repository was assumed to depressurize with the occurrence of the first drilling intrusion. Thus, in the evaluation of the approximation in Eq. (4.17) as discussed in Sect. 5.4.3, a spallings release can only occur for the first drilling intrusion associated with an element $f$ of $\mathscr{A} \mathcal{A} S$; later intrusions will not cause a spallings release because the repository will have been depressurized by the first intrusion. For perspective, Fig. 5.7 shows the pressure histories calculated with BRAGFLO for intrusions at $1000 \mathrm{yr}$.

\subsubsection{CUTTINGS and SPALLINGS Calculations}

The cuttings component of the direct release to the surface, which is calculated by CUTTINGS, is independent of results obtained with any other model and thus can be calculated at any point in the computational sequence. However, as just indicated, the spallings component of this release, which is calculated by SPALLINGS, is dependent on pressure in the repository and thus cannot be determined until after the pressure histories in Figs. $\mathbf{5 . 2}$ and 5.3 are calculated with BRAGFLO. To support the approximation indicated in Eq. (4.17) and discussed in more detail in Sect. 5.4.3, cuttings and spallings releases are required at a sequence of times between 100 and 10,000 yr. In SPM-1, these times are

$100 \mathrm{yr}, 125 \mathrm{yr}, 175 \mathrm{yr}, 350 \mathrm{yr}, 1000 \mathrm{yr}, 3000 \mathrm{yr}, 7250 \mathrm{yr}, 10000 \mathrm{yr}$,

which are the same times used in the 1992 WIPP PA for the calculation of cuttings releases. 


\begin{tabular}{|c|c|c|c|}
\hline $\begin{array}{c}\text { Outcomes: } E_{2} \\
\text { (Seal Perm) }\end{array}$ & Type of Intrusion & $\begin{array}{l}\text { BRAGFLO } \\
\text { Input }\end{array}$ & $\begin{array}{l}\text { BRAGFLO } \\
\text { Calculation }\end{array}$ \\
\hline \multirow[b]{2}{*}{$\varepsilon_{21}: 10^{-15}-10^{-13} \mathrm{~m}^{2}$} & E1 & \multirow{2}{*}{ 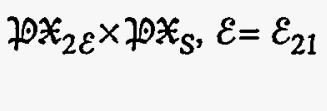 } & \multirow[t]{2}{*}{ Performed } \\
\hline & \multirow[b]{2}{*}{ E2 } & & \\
\hline & & 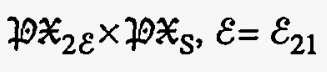 & Performed \\
\hline & E1 & \multirow[t]{2}{*}{$\not O X_{2 \varepsilon} \times \not O X_{S}, \varepsilon=\varepsilon_{22}$} & \multirow[t]{2}{*}{ Performed } \\
\hline$\varepsilon_{22}: 10^{-18}-10^{-16} \mathrm{~m}^{2}$ & \multirow[b]{2}{*}{$\mathrm{E} 2$} & & \\
\hline \multirow{4}{*}{$\varepsilon_{23}: 10^{-19}-10^{-18} \mathrm{~m}^{2}$} & & $\not \ominus \varkappa_{2 \varepsilon} \times \not \bullet \varkappa_{S}, \varepsilon=\varepsilon_{22}$ & Not Performe \\
\hline & E1 & \multirow{2}{*}{$\not \oplus \mathcal{X}_{2 \varepsilon} \times \not \ominus \mathcal{X}_{S}, \varepsilon=\varepsilon_{23}$} & \multirow[t]{2}{*}{ Performed } \\
\hline & \multirow[b]{2}{*}{ E2 } & & \\
\hline & & $\not O \mathcal{X}_{2 \varepsilon} \times \mathfrak{O O \mathcal { X } _ { S }}, \mathcal{E}=\varepsilon_{23}$ & Not Performed \\
\hline
\end{tabular}

Figure 5.4. Logic diagram indicating 4 sets of BRAGFLO calculations performed for outcomes of experimental program $E_{2}$.

Determination of the cuttings and spallings releases requires use of the variable DBDIAM associated with the probability space $7 O \mathcal{X}_{S}$ and also the variables BLWEF, SPPERM, TAUFAIL and TCLOUT associated with the probability space $\not \circ X_{D}$. As indicated in conjunction with Eq. (5.3), 3 LHSs from $\not D X_{2 \varepsilon} \times \not \cap X_{S}$ already exist, with the corresponding elements of each sample differing only in the value for shaft seal permeability (i.e., SEALPRM) due to sampling from $\supsetneq \cap \mathfrak{X}_{2 \varepsilon}$ for $\varepsilon=\varepsilon_{21}, \varepsilon_{22}, \varepsilon_{23}$. For the cuttings and spallings calculations, an additional LHS

$$
\mathbf{t}_{m}=\left[t_{m 1}, t_{m 2}, \ldots, t_{m 4}\right], m=1,2, \ldots, n L H S=25,
$$

was generated from the probability space $20 \mathcal{X}_{D}$. The cuttings and spallings calculations were then performed for the elements $\mathbf{t}_{m}$ of this sample. As these calculations also depend on the elements $\mathbf{x}_{m}$ of the samples indicated in Eq. (5.3), the analysis can be viewed as being performed for a new sample

$$
\mathbf{x}_{m}, m=1,2, \ldots, n L H S=25,
$$

where each $\mathbf{x}_{m}$ in this new sample is given by

$$
\mathbf{x}_{m}=\left[\mathbf{r}_{m}, \mathbf{s}_{i m}, \mathbf{t}_{m}\right]
$$

and is thus obtained by concatenating the elements of the samples in Eqs. (5.3) and (5.6).

At each time indicated in Eq. (5.5), a cuttings release is calculated with CUTTINGS for waste of average activity level. This produces the cuttings results shown in the box plots in the upper left frame of Fig. 5.8. As a reminder, the endpoints of the boxes in Fig. 5.8 are formed by the lower and upper quartiles of the data, that is $x_{0.25}$ 


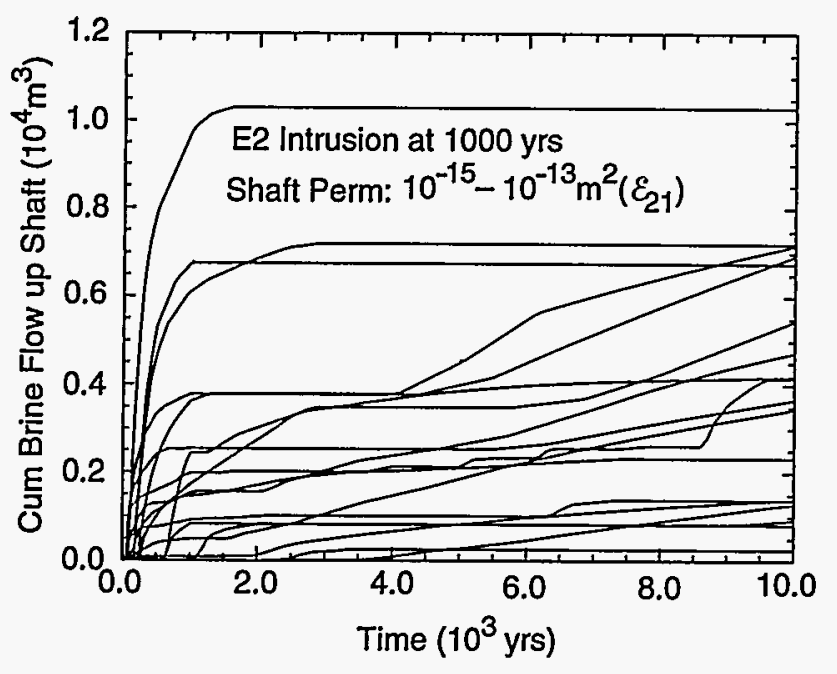

TRI-6342-4626-0

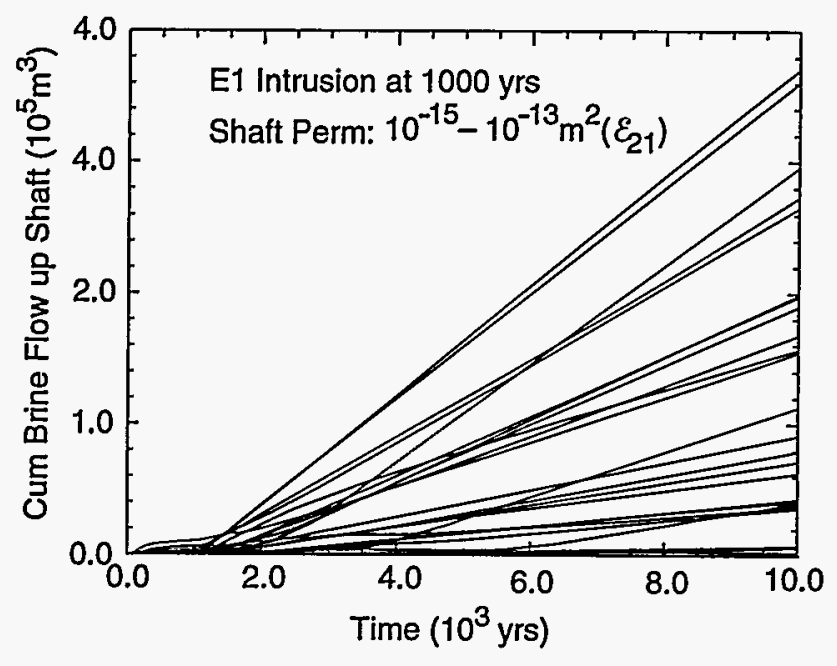

TRI-6342-4627-0

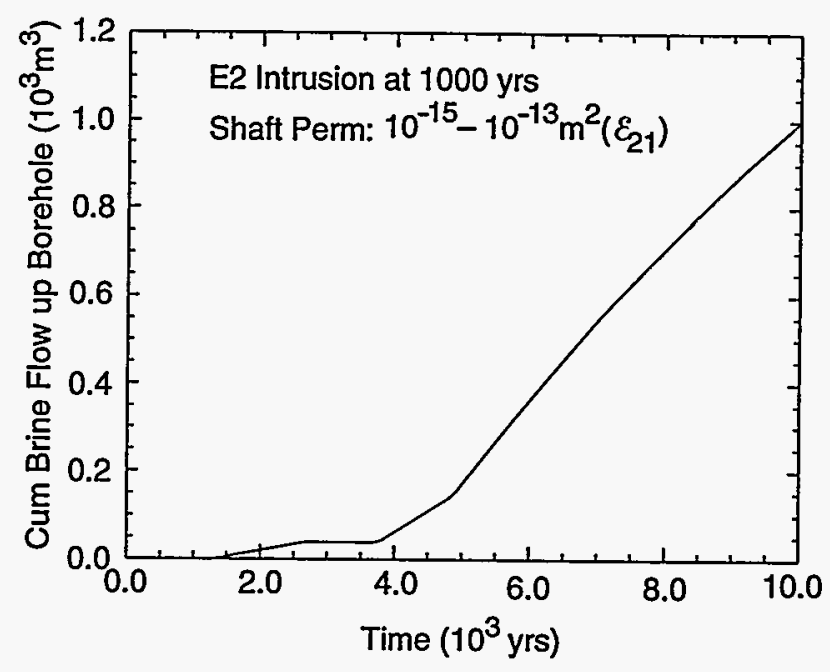

TRI-6342-4625-0

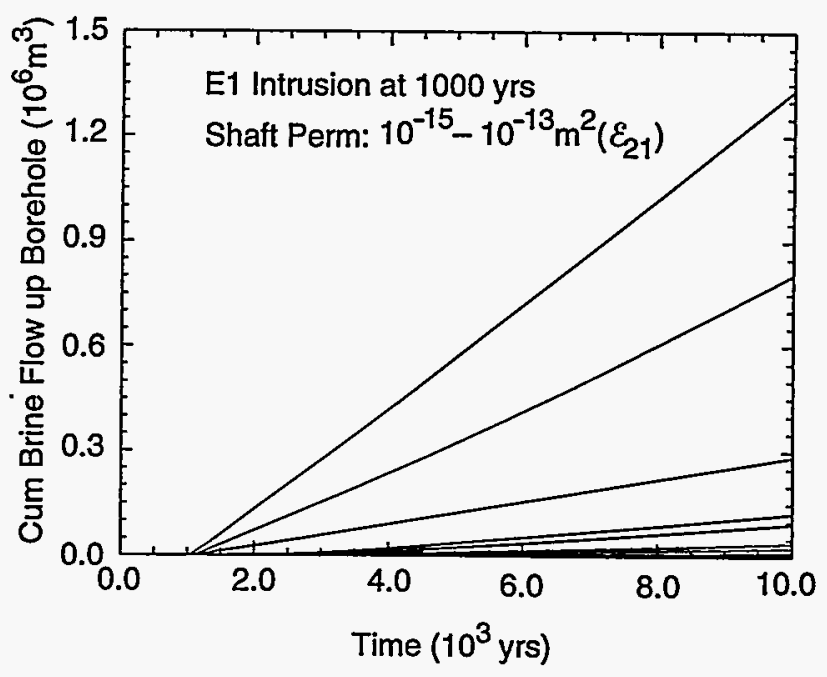

TRI-6342-4603-0

Figure 5.5. Cumulative brine flows to the Culebra calculated with BRAGFLO for E1 and E2 intrusions at $1000 \mathrm{yr}$ for subset $\varepsilon_{21}$ (i.e., for shaft permeabilities in the range $10^{-15}-10^{-13} \mathrm{~m}^{2}$ ) of $\varepsilon_{2}$. 

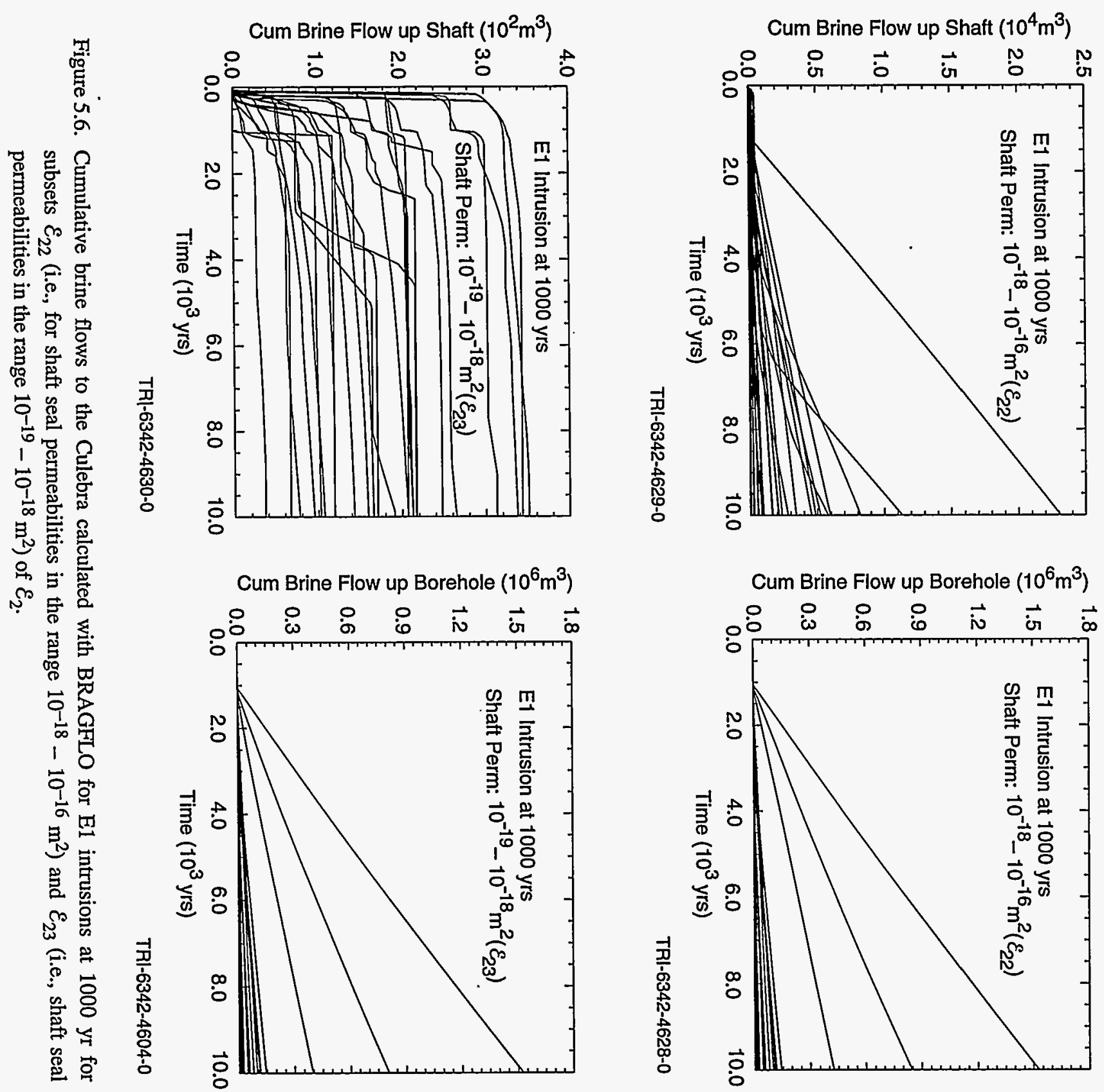


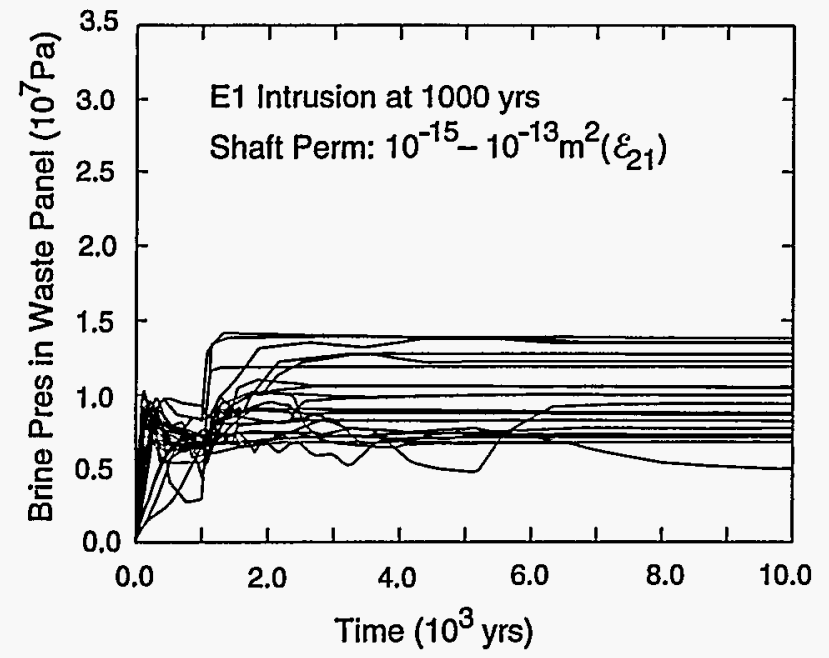

TRI-6342-4632-0

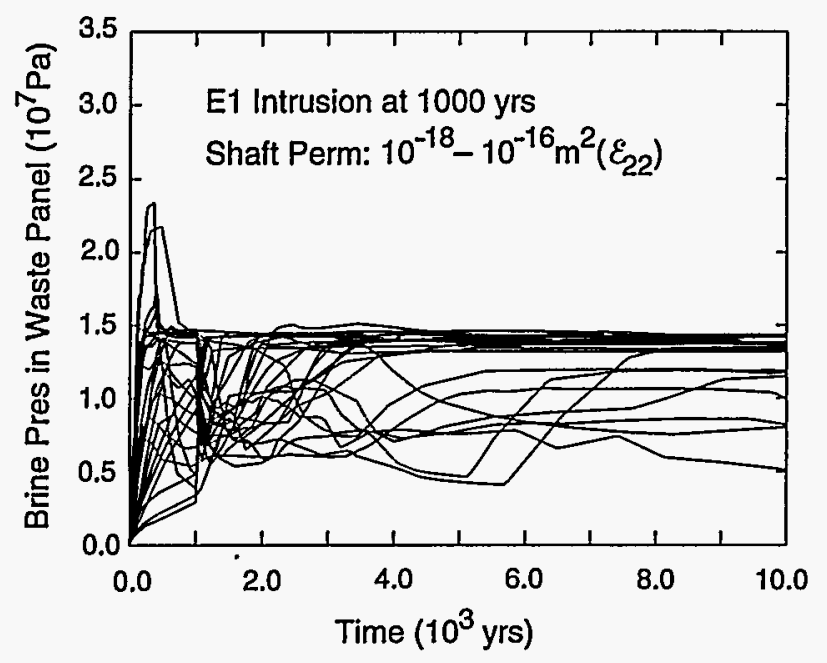

TRI-6342-4633-0

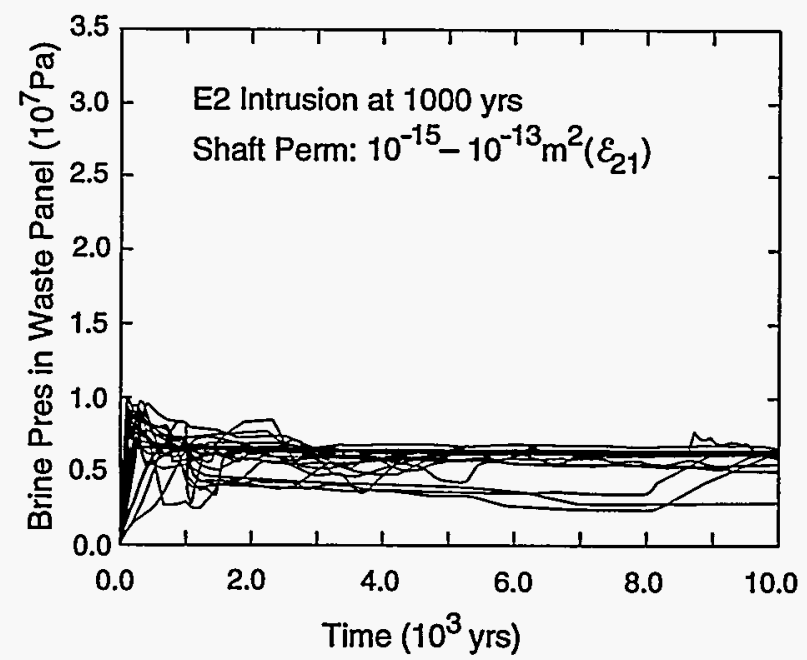

TRI-6342-4631-0

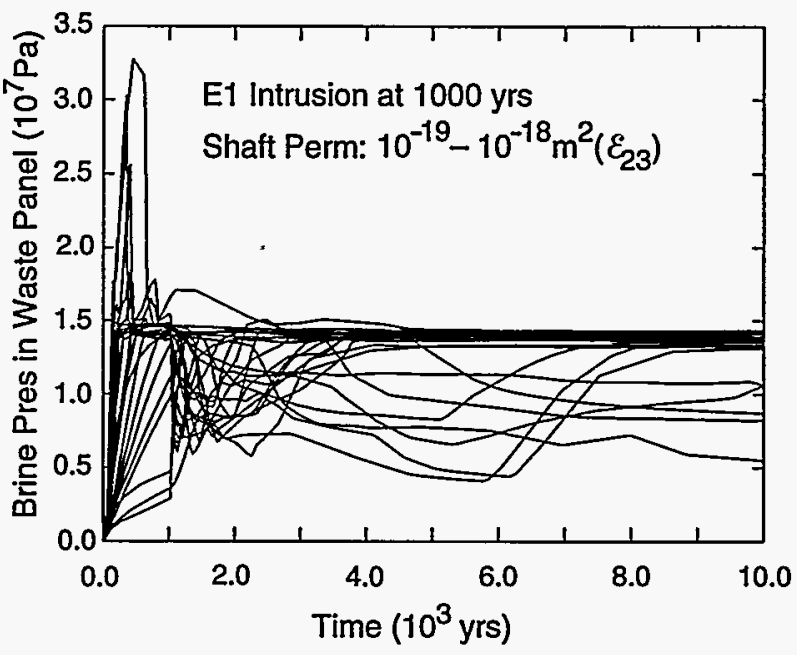

TRI-6342-4605-0

Figure 5.7. Time-dependent pressures in repository calculated with BRAGFLO for intrusions at $1000 \mathrm{yrs}$. 


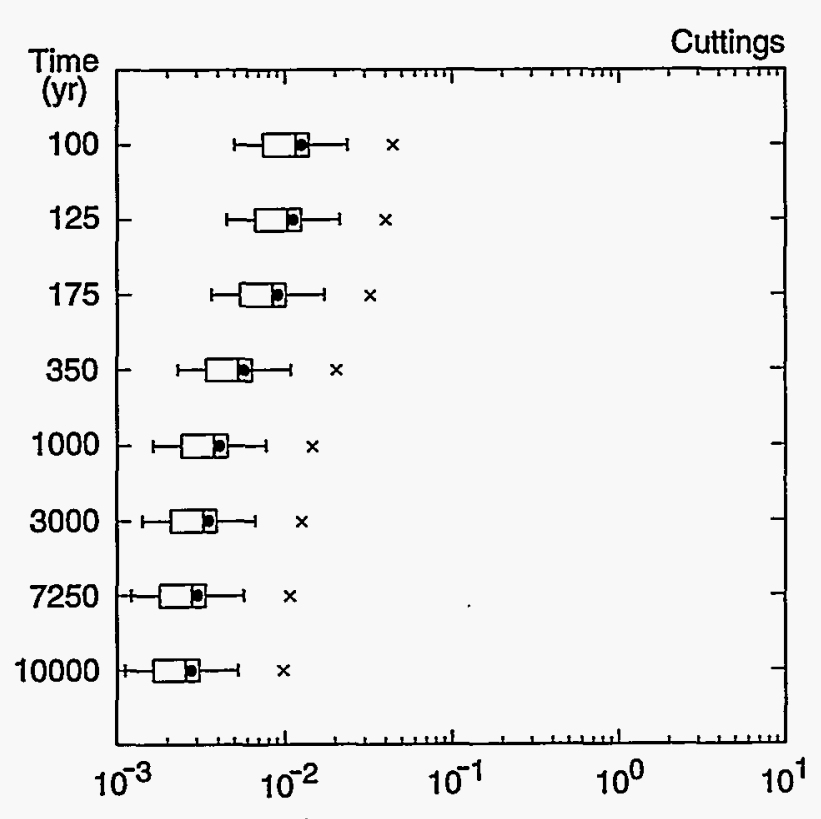

Release to Accessible Environment

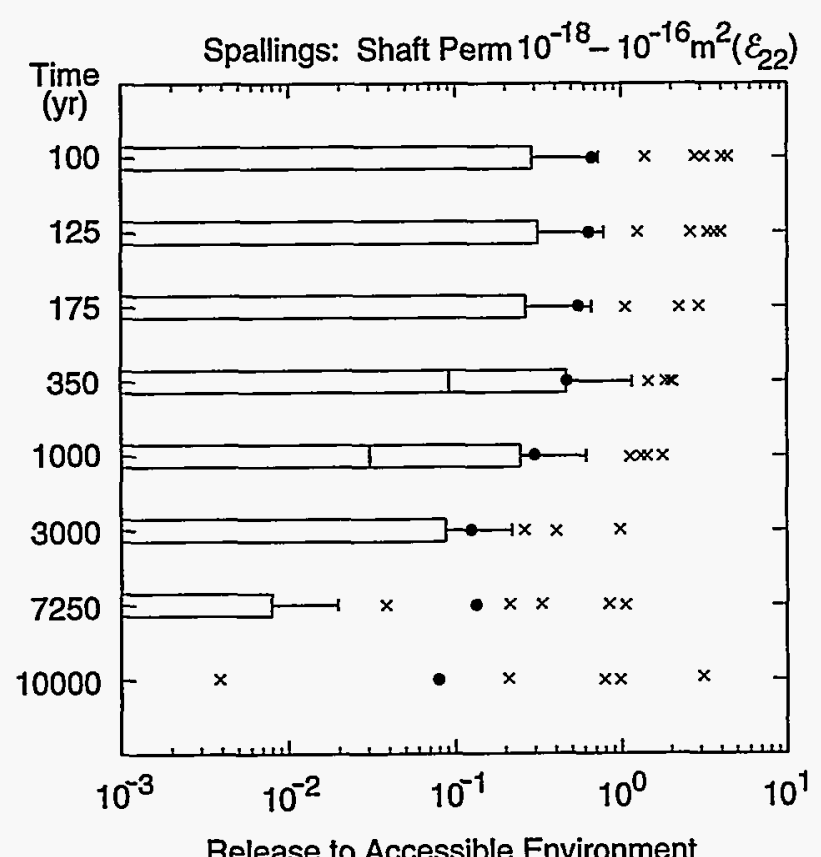

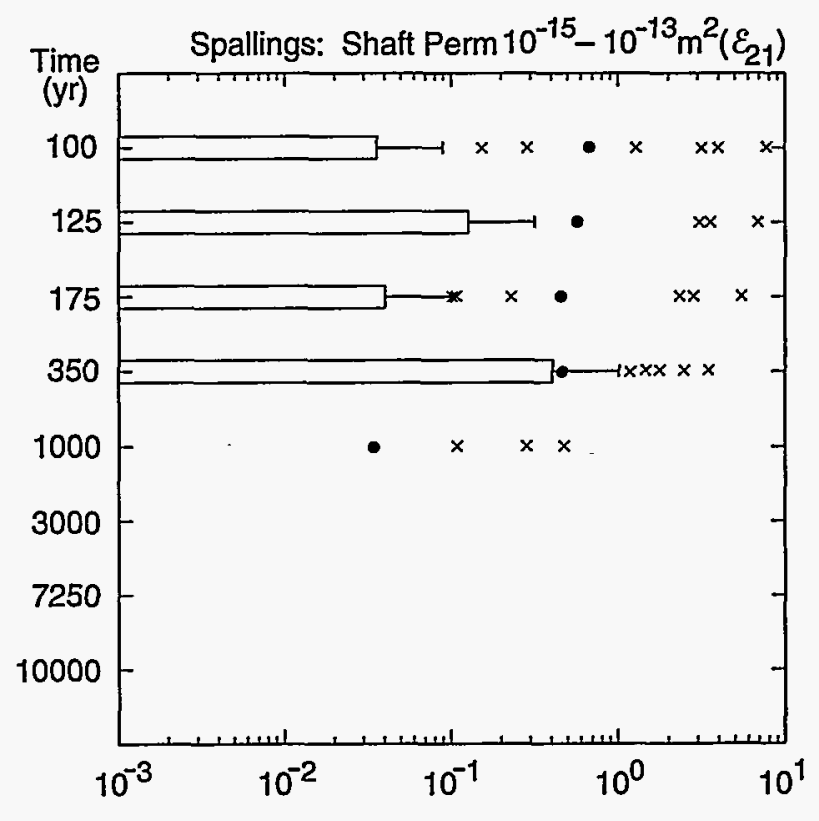

Release to Accessible Environment

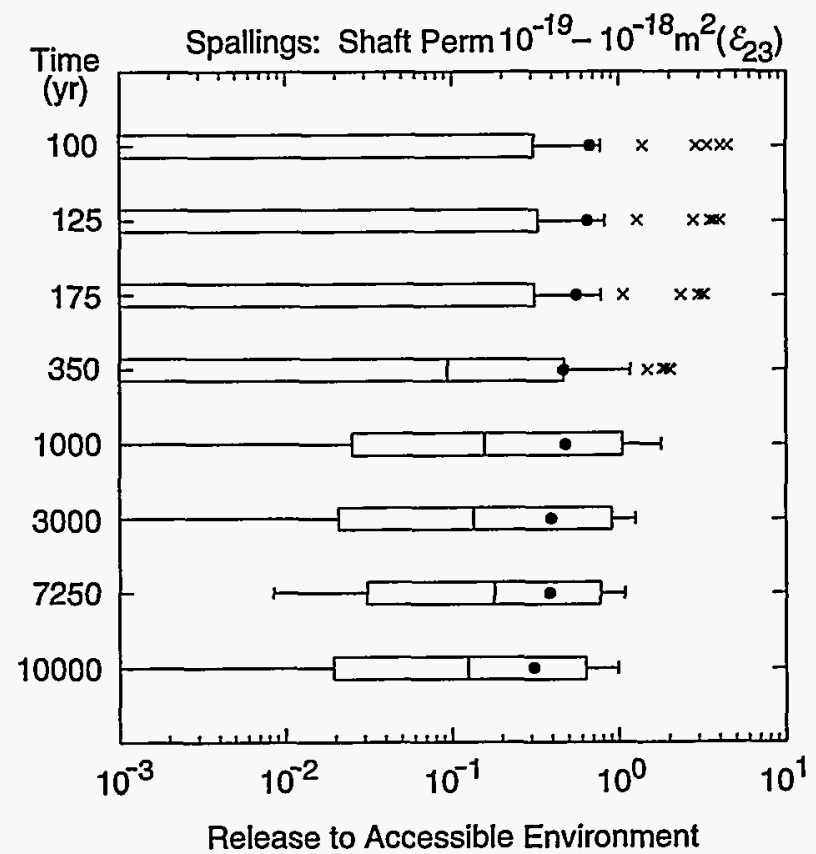

Min $\{1.5 \times$ Box, Largest Obs\}

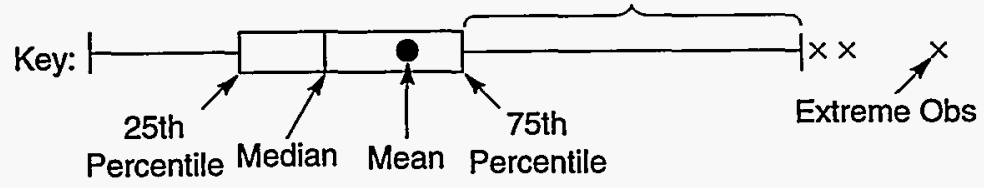

TRl-6342-4606-0

Figure 5.8. Total normalized release to the accessible environment due to cuttings removal for waste of average activity level (upper left) and to spallings for base-case spallings model (i.e., model implied by subset $\varepsilon_{81}$ of $\varepsilon_{8}$ ) and subsets $\varepsilon_{21}$ (upper right), $\varepsilon_{22}$ (lower left) and $\varepsilon_{23}$ (lower right) of $\varepsilon_{2}$. 
and $x_{0.75}$. The vertical line within the box represents the median, $x_{0.50}$. The sample mean is identified by the large dot. The bar on the right of the box extends to the minimum of $x_{0.75}+1.5\left(x_{0.75}-x_{0.25}\right)$ and the maximum observation. In a similar manner, the bar on the left of the box extends to the maximum of $x_{0.25}-1.5\left(x_{0.75}-x_{0.25}\right)$ and the minimum observation. The observations falling outside of these bars are shown with crosses. As discussed in Sect. II.D of Ref. 47, these releases for waste of average activity level are scaled with the results in Table 5.2 to produce the releases for waste of different activity levels that are used in constructing $F\left(\mathbf{x}_{m}, \mathbf{f}_{n}\right)$ in Eq. (4.17). For perspective, the initial waste inventory used in SPM-1 is shown in Table 5.3, and selected time-dependent inventories are shown in Fig. 5.9.

A spallings release is also required at each time in Eq. (5.5) for the pressure histories in Figs. 5.2 and 5.3 for $\varepsilon_{21}, \varepsilon_{22}$ and $\varepsilon_{23}$. Due to the similarity of the results in Figs. 5.2 and 5.3 for $\varepsilon_{21}, \varepsilon_{22}$ and $\varepsilon_{23}$ with the BrooksCorey and van Genuchten-Parker relationships and also design modification $D_{2}$, spallings calculations were only performed for the pressure histories in the left column of Fig. 5.2 (i.e., Brooks-Corey without design modification $D_{2}$ ). The outcome of these calculations for the base-case spallings model (i.e., the model associated with the subset $\varepsilon_{81}$ of $\varepsilon_{8}$ as indicated in Table 3.8) and the sample indicated in Eq. (5.8) is shown in Fig. 5.8. The spallings releases associated with other possible outcomes of the experimental program $E_{8}$ for spallings (i.e., $\varepsilon_{82}, \varepsilon_{83}, \varepsilon_{84}$ ) are obtained by dividing the spallings results in Fig. 5.8 by 2, 10 and 100, respectively, as indicated in Fig. 5.10. The diagram in Fig. 5.10 shows input to SPALLINGS defined by $\not 0 \mathfrak{X}_{S} \times \not 0 \mathfrak{X}_{D}$. Indirectly, the outcome of the spallings calculation also depends on $\not \circ \mathcal{X}_{2 \varepsilon}$ for $\mathcal{E}=\varepsilon_{21}, \varepsilon_{22}, \varepsilon_{23}$ due to the dependence of the pressure histories calculated by BRAGFLO on $\mathrm{PX}_{2 \varepsilon}$, with the result that the spallings calculations are actually being performed for sample elements of the form indicated in Eq. (5.8).

Table 5.2. Projected Activity Levels ( $\left.\mathrm{Ci} / \mathrm{m}^{2}\right)$ Used in the 1992 WIPP PA Due to Waste that is Currently Stored and May be Shipped to the WIPP (based on Memorandum by Peterson in App. A of Ref. 18)

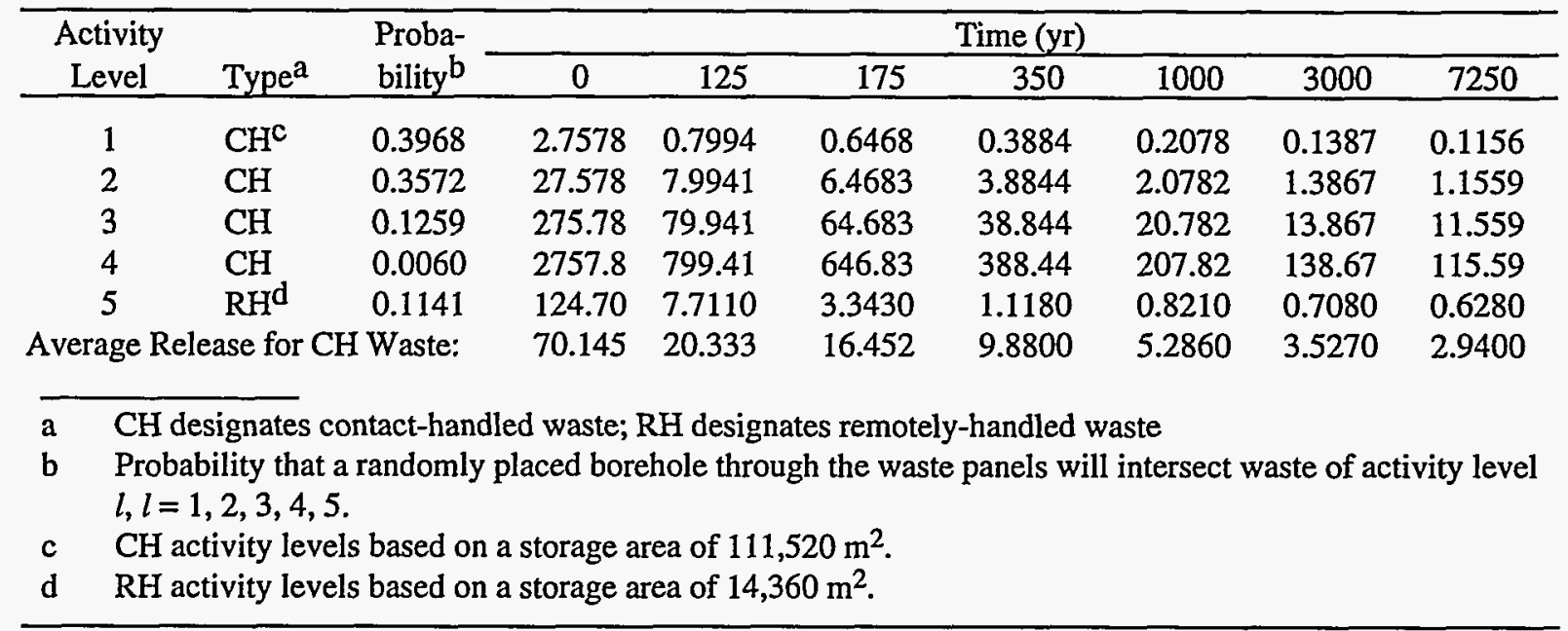


Table 5.3. Initial Waste Inventory Used in SPM-1 (from Refs. 83, 84)

\begin{tabular}{|c|c|c|c|c|c|c|}
\hline Isotope & $\begin{array}{c}\text { Half-Life } \\
(\mathrm{yr})\end{array}$ & Daughter & $\mathrm{Ci} / \mathrm{mol}$ & $\mathrm{Ci} / \mathrm{kg}$ & $\begin{array}{c}\text { Inventory } \\
\text { (Ci) }\end{array}$ & $\begin{array}{c}\text { Inventory } \\
\text { (gm) }\end{array}$ \\
\hline Sr-90 & $2.91 \times 10^{+01}$ & & $1.23 \times 10^{+04}$ & $1.36 \times 10^{+05}$ & $5.95 \times 10^{+04}$ & $4.36 \times 10^{+02}$ \\
\hline Cs-137 & $3.00 \times 10^{+01}$ & & $1.19 \times 10^{+04}$ & $8.70 \times 10^{+04}$ & $3.22 \times 10^{+04}$ & $3.70 \times 10^{+02}$ \\
\hline $\mathrm{Pb}-210$ & $2.23 \times 10^{+01}$ & & $1.60 \times 10^{+04}$ & $7.63 \times 10^{+04}$ & $0.00 \times 10^{+00}$ & $0.00 \times 10^{+00}$ \\
\hline $\mathrm{Ra}-226$ & $1.60 \times 10^{+03}$ & $\mathrm{~Pb}-210$ & $2.23 \times 10^{+02}$ & $9.89 \times 10^{+02}$ & $0.00 \times 10^{+00}$ & $0.00 \times 10^{+00}$ \\
\hline $\mathrm{Ra}-228$ & $5.75 \times 10^{+00}$ & & $6.22 \times 10^{+04}$ & $2.73 \times 10^{+05}$ & $0.00 \times 10^{+00}$ & $0.00 \times 10^{+00}$ \\
\hline Th-229 & $7.34 \times 10^{+03}$ & & $4.87 \times 10^{+01}$ & $2.13 \times 10^{+02}$ & $0.00 \times 10^{+00}$ & $0.00 \times 10^{+00}$ \\
\hline Th-230 & $7.70 \times 10^{+04}$ & $\mathrm{Ra}-226$ & $4.64 \times 10^{+00}$ & $2.02 \times 10^{+01}$ & $0.00 \times 10^{+00}$ & $0.00 \times 10^{+00}$ \\
\hline Th-232 & $1.40 \times 10^{+10}$ & $\mathrm{Ra}-228$ & $2.54 \times 10^{-05}$ & $1.10 \times 10^{-04}$ & $4.74 \times 10^{-01}$ & $4.32 \times 10^{+06}$ \\
\hline $\mathrm{Pa}-231$ & $3.28 \times 10^{+04}$ & & $1.09 \times 10^{+01}$ & $4.72 \times 10^{+01}$ & $0.00 \times 10^{+00}$ & $0.00 \times 10^{+00}$ \\
\hline U-233 & $1.58 \times 10^{+05}$ & Th-229 & $2.26 \times 10^{+00}$ & $9.68 \times 10^{+00}$ & $1.34 \times 10^{+03}$ & $1.38 \times 10^{+05}$ \\
\hline U-234 & $2.44 \times 10^{+05}$ & Th-230 & $1.46 \times 10^{+00}$ & $6.25 \times 10^{+00}$ & $0.00 \times 10^{+00}$ & $0.00 \times 10^{+00}$ \\
\hline U-235 & $7.04 \times 10^{+08}$ & $\mathrm{~Pa}-231$ & $5.08 \times 10^{-04}$ & $2.16 \times 10^{-03}$ & $3.68 \times 10^{+02}$ & $1.70 \times 10^{+08}$ \\
\hline U-236 & $2.34 \times 10^{+07}$ & Th-232 & $1.53 \times 10^{-02}$ & $6.47 \times 10^{-02}$ & $0.00 \times 10^{+00}$ & $0.00 \times 10^{+00}$ \\
\hline U-238 & $4.47 \times 10^{+09}$ & U-234 & $8.00 \times 10^{-05}$ & $3.36 \times 10^{-04}$ & $1.08 \times 10^{+01}$ & $3.22 \times 10^{+07}$ \\
\hline $\mathrm{Np}-237$ & $2.14 \times 10^{+06}$ & $\mathrm{U}-233$ & $1.67 \times 10^{-01}$ & $7.05 \times 10^{-01}$ & $2.43 \times 10^{+01}$ & $3.44 \times 10^{+04}$ \\
\hline $\mathrm{Pu}-238$ & $8.77 \times 10^{+01}$ & U-234 & $4.07 \times 10^{+03}$ & $1.71 \times 10^{+04}$ & $8.74 \times 10^{+05}$ & $5.10 \times 10^{+04}$ \\
\hline $\mathrm{Pu}-239$ & $2.41 \times 10^{+04}$ & $U-235$ & $1.49 \times 10^{+01}$ & $6.21 \times 10^{+01}$ & $2.12 \times 10^{+05}$ & $3.42 \times 10^{+06}$ \\
\hline $\mathrm{Pu}-240$ & $6.54 \times 10^{+03}$ & U-236 & $5.47 \times 10^{+01}$ & $2.28 \times 10^{+02}$ & $3.28 \times 10^{+04}$ & $1.44 \times 10^{+05}$ \\
\hline $\mathrm{Pu}-241$ & $1.44 \times 10^{+01}$ & Am-241 & $2.48 \times 10^{+04}$ & $1.03 \times 10^{+05}$ & $6.32 \times 10^{+05}$ & $6.14 \times 10^{+03}$ \\
\hline $\mathrm{Pu}-242$ & $3.76 \times 10^{+05}$ & U-238 & $9.50 \times 10^{-01}$ & $3.93 \times 10^{+00}$ & $6.90 \times 10^{+02}$ & $1.76 \times 10^{+05}$ \\
\hline $\mathrm{Pu}-244$ & $8.26 \times 10^{+07}$ & $\mathrm{Pu}-240$ & $4.33 \times 10^{-03}$ & $1.77 \times 10^{-02}$ & $0.00 \times 10^{+00}$ & $0.00 \times 10^{+\infty}$ \\
\hline Am-241 & $4.32 \times 10^{+02}$ & Np-237 & $8.27 \times 10^{+02}$ & $3.43 \times 10^{+03}$ & $1.48 \times 10^{+05}$ & $4.30 \times 10^{+04}$ \\
\hline $\mathrm{Cm}-244$ & $1.81 \times 10^{+01}$ & Pu-240 & $1.97 \times 10^{+04}$ & $8.09 \times 10^{+04}$ & $0.00 \times 10^{+00}$ & $0.00 \times 10^{+00}$ \\
\hline $\mathrm{Cm}-248$ & $3.39 \times 10^{+05}$ & $\mathrm{Pu}-244$ & $1.05 \times 10^{+00}$ & $4.25 \times 10^{+00}$ & $0.00 \times 10^{+00}$ & $0.00 \times 10^{+00}$ \\
\hline Cf- 252 & $2.64 \times 10^{+00}$ & $\mathrm{Cm}-248$ & $1.36 \times 10^{+05}$ & $5.38 \times 10^{+05}$ & $1.64 \times 10^{+02}$ & $3.05 \times 10^{-01}$ \\
\hline Pm-147 & $2.62 \times 10^{+00}$ & $\mathrm{Sm}-147$ & $1.36 \times 10^{+05}$ & $9.27 \times 10^{+05}$ & $1.86 \times 10^{+03}$ & $2.01 \times 10^{+00}$ \\
\hline Sm-147 & $1.06 \times 10^{+06}$ & & $3.37 \times 10^{-01}$ & $2.29 \times 10^{+00}$ & $0.00 \times 10^{+00}$ & $0.00 \times 10^{+00}$ \\
\hline
\end{tabular}




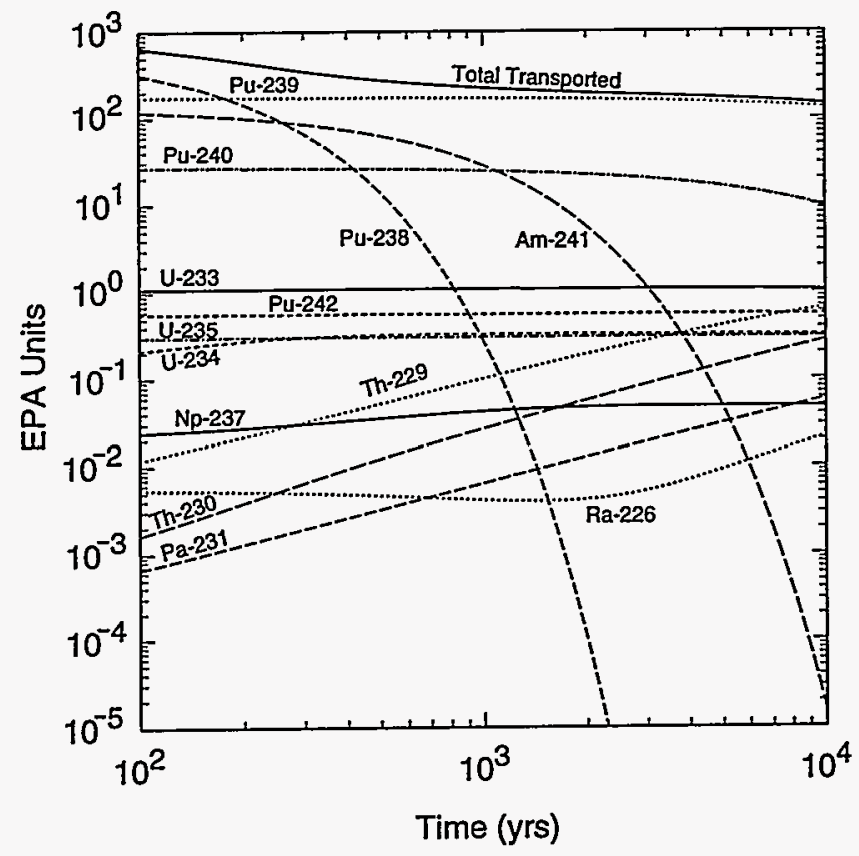

TRI-6342-4607-0

Figure 5.9. Selected time-dependent radionuclide inventories used in SPM-1 (based on initial inventories in Ref. 84).

\begin{tabular}{|c|c|}
\hline $\begin{array}{l}\text { Outcomes: } E_{8} \\
\text { (Spallings) }\end{array}$ & $\begin{array}{l}\text { SPALLINGS } \\
\text { Input }\end{array}$ \\
\hline$\varepsilon_{81}:$ Basecase & $\not O X_{S} \times \not 0 \mathcal{X}_{D}$ \\
\hline$\varepsilon_{82}:$ Basecase $/ 2$ & $10 x_{S} \times 10 x_{D}$ \\
\hline$\varepsilon_{83}:$ Basecase/10 & $10 X_{S} \times 10 X_{D}$ \\
\hline$\varepsilon_{84}:$ Basecase $/ 100$ & $\not 0 *_{S} \times \not 0 *_{D}$ \\
\hline
\end{tabular}

Figure 5.10. Logic diagram indicating 4 possible outcomes associated with experimental program $E_{8}$ that result in different spallings releases. 


\subsubsection{PANEL Calculations}

Radionuclide transport calculations from the repository to the Culebra are performed with the PANEL program after the four sets of BRAGFLO calculations indicated in Figs. 5.4, 5.5 and 5.6 are completed. Experimental programs $E_{3}$ and $E_{7}$ and design modification $D_{3}$ affect the calculations with PANEL. As indicted in Fig. 5.11, this leads to a requirement for 24 sets of PANEL calculations for each set of BRAGFLO calculations. The inputs to the

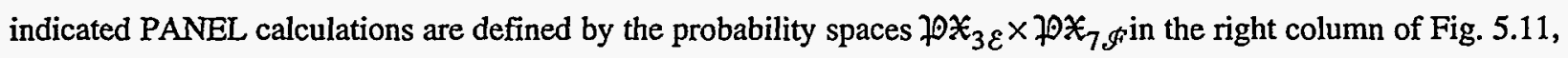
with the value for $\mathcal{E}$ changing for the different outcomes associated with experimental program $E_{3}$ (i.e., $\varepsilon_{31}, \varepsilon_{32}$, $\varepsilon_{33}$ ) and the value for $\&_{f}$ changing for the different outcomes associated with experimental program $E_{7}$ (i.e., $\varepsilon_{71}$, $\varepsilon_{72}, \ldots, \varepsilon_{77}$ ) and the effects that design modification $D_{3}$ has on the implications of these outcomes for calculations with PANEL (see Table 3.17).

In concept, each path in Fig. 5.11 defines an integration problem involving PANEL and the probability space

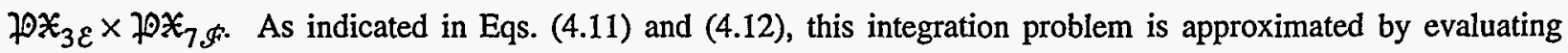
PANEL for the elements

$$
u_{m}=\left[u_{m 1}, u_{m 2}, \ldots, u_{m 7}\right], m=1,2, \ldots, n L H S=25,
$$

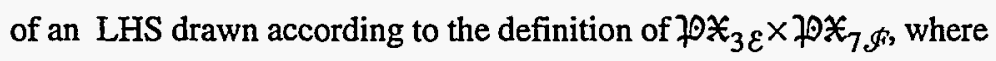

$$
\begin{aligned}
& \left.u_{1}=\text { logarithm (base } 10\right) \text { of solubility }(\mathrm{mol} / \ell) \text { for americium, } \\
& \left.u_{2}=\text { logarithm (base } 10\right) \text { of solubility }(\mathrm{mol} / \ell) \text { for neptunium, } \\
& \left.u_{3}=\text { logarithm (base } 10\right) \text { of solubility }(\mathrm{mol} / \ell) \text { for plutonium, } \\
& \left.u_{4}=\text { logarithm (base } 10\right) \text { of solubility }(\mathrm{mol} / \ell) \text { for radium, } \\
& \left.u_{5}=\text { logarithm (base } 10\right) \text { of solubility }(\mathrm{mol} / \ell) \text { for thorium, } \\
& \left.u_{6}=\text { logarithm (base } 10\right) \text { of solubility }(\mathrm{mol} / \ell) \text { for uranium, } \\
& u_{7}=\text { lognormal variable }(0.01 \text { quantile }=0.01,0.99 \text { quantile }=100) \text { used to select concentration of colloidally- } \\
& \quad \text { sorbed radionuclides }
\end{aligned}
$$

for an element

$$
\mathbf{u}=\left[u_{1}, u_{2}, \ldots, u_{7}\right]
$$




\begin{tabular}{|c|c|c|c|}
\hline & $\begin{array}{l}\text { Outcomes: } E_{3} \\
\text { (Chemical Sol) }\end{array}$ & $\begin{array}{l}\text { Outcomes: } E_{7}, D_{3} \\
\text { (Colloid Sol) }\end{array}$ & PANEL Input \\
\hline \multirow{24}{*}{ BRAGFLO } & \multirow[b]{4}{*}{$\varepsilon_{31}:$ Infinite Sol } & $\varepsilon_{71} \mid D_{3}, \varepsilon_{71}:$ Infinite Sol & \multirow{24}{*}{ 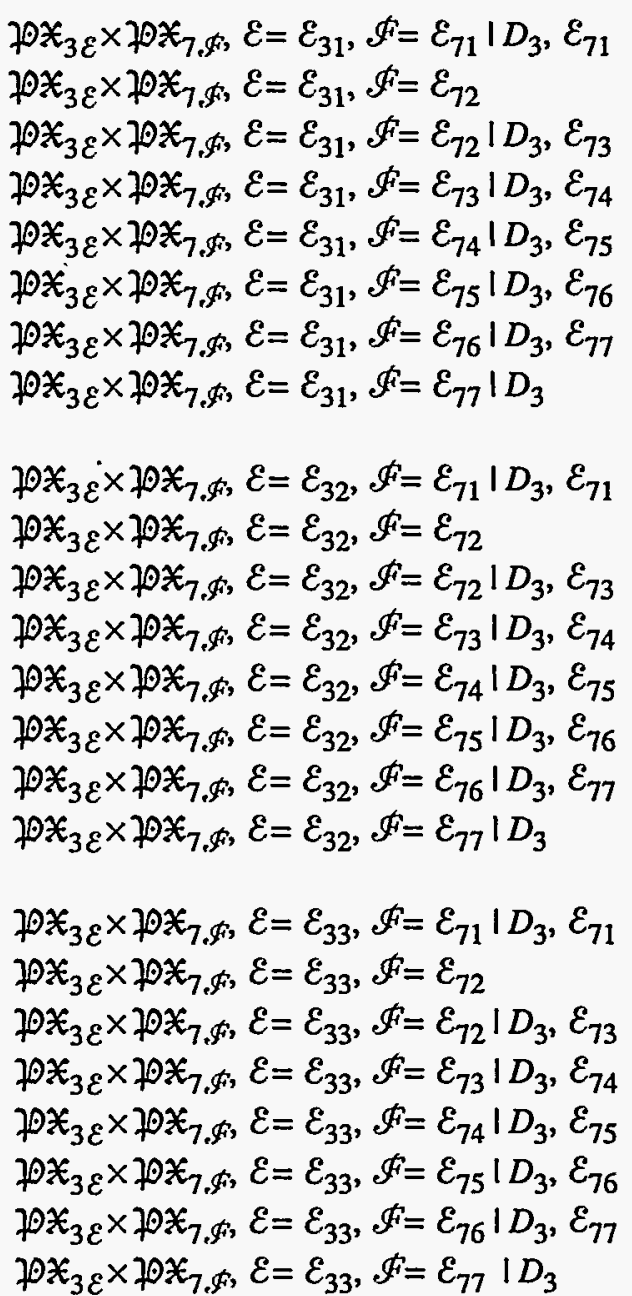 } \\
\hline & & $\varepsilon_{72}: \mu=-3$ & \\
\hline & & $\varepsilon_{72} \mid D_{3}, \varepsilon_{73}: \mu=-4$ & \\
\hline & & $\varepsilon_{73} \mid D_{3}, \varepsilon_{74}: \mu=-5$ & \\
\hline & \multirow[b]{8}{*}{$\varepsilon_{32}:$ Intermediate Sol } & $\varepsilon_{74} \mid D_{3}, \varepsilon_{75}: \mu=-6$ & \\
\hline & & $\varepsilon_{75} \mid D_{3}, \varepsilon_{76}: \mu=-7$ & \\
\hline & & $\varepsilon_{76} \mid D_{3}, \varepsilon_{77}: \mu=-8$ & \\
\hline & & $\varepsilon_{77} \mid D_{3}: \mu=-9$ & \\
\hline & & $\varepsilon_{71} \mid D_{3}, \varepsilon_{71}:$ Infinite Sol & \\
\hline & & $\varepsilon_{72}: \mu=-3$ & \\
\hline & & $\mathcal{E}_{72} \mid D_{3}, \mathcal{E}_{73}: \mu=-4$ & \\
\hline & & $\varepsilon_{73} \mid D_{3}, \varepsilon_{74}: \mu=-5$ & \\
\hline & \multirow{12}{*}{$\varepsilon_{33}:$ Low Sol } & $\varepsilon_{74} \mid D_{3}, \varepsilon_{75}: \mu=-6$ & \\
\hline & & $\varepsilon_{75} \mid D_{3}, \varepsilon_{76}: \mu=-7$ & \\
\hline & & $\varepsilon_{76} \mid D_{3}, \varepsilon_{77}: \mu=-8$ & \\
\hline & & $\mathcal{E}_{77} \mid D_{3}: \mu=-9$ & \\
\hline & & $\varepsilon_{71} \mid D_{3}, \varepsilon_{71}:$ Infinite Sol & \\
\hline & & $\varepsilon_{72}: \mu=-3$ & \\
\hline & & $\varepsilon_{72} \mid D_{3}, \varepsilon_{73}: \mu=-4$ & \\
\hline & & $\varepsilon_{73} \mid D_{3}, \varepsilon_{74}: \mu=-5$ & \\
\hline & & $\varepsilon_{74} \mid D_{3}, \varepsilon_{75}: \mu=-6$ & \\
\hline & & $\varepsilon_{75} \mid D_{3}, \varepsilon_{76}: \mu=-7$ & \\
\hline & & $\varepsilon_{76} \mid D_{3}, \varepsilon_{77}: \mu=-8$ & \\
\hline & & $\varepsilon_{77} \mid D_{3}: \mu=-9$ & \\
\hline
\end{tabular}

Figure 5.11 Logic diagram indicating 24 sets of PANEL calculations associated with outcomes of experimental programs $E_{3}$ and $E_{7}$ and design modification $D_{3}$ for each BRAGFLO calculation.

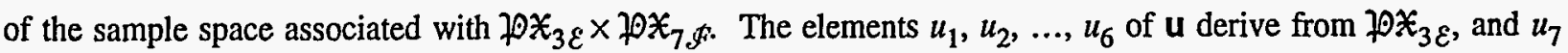

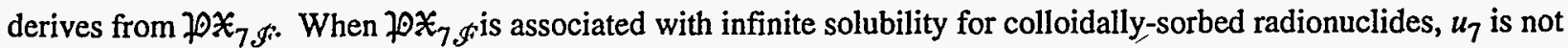
used; otherwise, the concentration for colloidally-sorbed radionuclides is assigned a value of $u_{7} 10^{\mu}$, where $\mu$ is the expected value (over subjective uncertainty) for the logarithm of the concentration of colloidally-sorbed radionuclides (see values for $\mathscr{F}$ in Fig. 5.11). The correlations between solubilities associated with $\not \vartheta \mathcal{F}_{3 \varepsilon}$ were implemented with the Iman/Conover restricted pairing technique. ${ }^{82}$

Use of a different LHS for each path in Fig. 5.11 (i.e., for each probability space $70 \mathcal{X}_{3 \varepsilon} \times 70 \mathcal{X}_{7}$ ) would require a total of 24 samples. However, as there are three unique values for $\not \supset \mathcal{X}_{3 \varepsilon}$ (i.e., for $\varepsilon_{31}, \varepsilon_{32}$ and $\varepsilon_{33}$ ) and a single 
pointer variable (i.e., $u_{7}$ ) is used to select from $70 \mathbb{X}_{7 \not 3}$, only three LHSs as indicated in Table 5.4 were used. The use of $u_{7}$ as a pointer variable induces a rank correlation of 1 between the concentrations of colloidally-sorbed radionuclides used for the different values of $\mathfrak{P X}_{7} \$$. However, as the underlying integration problems are treated separately, this should not affect the analysis outcomes. In addition, the chemical solubilities for the individual elements (i.e., $u_{1}, u_{2}, \ldots, u_{6}$ ) were assumed to have a rank-correlation of 0.999 as indicated in Table 3.12. The procedure used to generate the samples in Table 5.4 is equivalent to generating three samples from $\not \cap \mathcal{X}_{3 \mathcal{E}}$ for $\varepsilon=$ $\varepsilon_{31}, \varepsilon_{32}, \varepsilon_{33}$, generating a single sample for $u_{7}$ (i.e., from $\not \ominus \mathcal{X}_{7 \oiint^{\prime}}$ ), and then concatenating the samples from $\not \ominus \mathcal{X}_{3} \varepsilon$ and $\operatorname{lox}_{7} \xi$

Thus far, the calculations involving PANEL have been discussed in terms of integrals (i.e., samples) involving

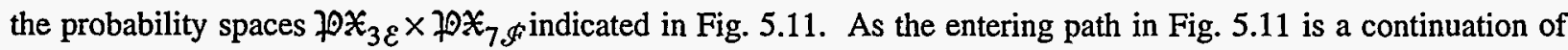
one of the paths in Fig. 5.4, these calculations are actually part of an integration over probability spaces of the form

$$
\not 0 \varkappa_{2 \varepsilon} \times 70 \varkappa_{3 \varepsilon} \times \not 0 \varkappa_{79} \times \not 0 *_{S}
$$

The BRAGFLO calculations indicated in Fig. 5.4 are performed for LHSs $\mathbf{x}_{m}, m=1,2, \ldots, 25$, of the form indicated in Eq. (5.3). Each of these calculations produces results (i.e., brine flows and brine volumes) that are inputs to PANEL. Thus, each set of PANEL calculations indicated in Fig. 5.11 is actually being performed for a sample

$$
\mathbf{x}_{m}=\left[\mathbf{r}_{m}, \mathbf{s}_{i m}, \mathbf{t}_{m}, \mathbf{u}_{j m}\right], m=1,2, \ldots, n L H S=25,
$$

drawn from the probability space indicated in Eq. (5.11), where the subscript $j$ has been added to $\mathbf{u}_{m}$.to distinguish between the three samples for $\varepsilon_{3 j}, j=1,2,3$. The elements $\mathrm{x}_{m}$ of this sample are obtained by concatenating the elements $\mathbf{r}_{m}, \mathbf{s}_{i m}, \mathbf{t}_{m}$ and $\mathbf{u}_{j m}$ of the samples indicated in Eqs. (5.1), (5.2), (5.6) and (5.9).

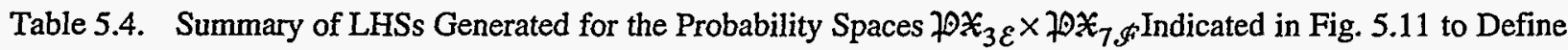
Input for PANEL Calculations

Sample 1: Used for $\varepsilon_{31}$. Variables $u_{1}, u_{2}, \ldots, u_{6}$ assigned a uniform distribution from 9 to 10 as a computationally-convenient means of implementing the assumption of infinite chemical solubility. Variability $u_{7}$ assigned lognormal distribution with 0.01 quantile $=0.01$ and 0.99 quantile $=100$.

Sample 2: Used for $\varepsilon_{32}$. Variables $u_{1}, u_{2}, \ldots, u_{6}$ assigned distributions indicated in Table 3.12 for $\varepsilon_{32}$ and shown in Fig. 3.1 of Ref. 19. Variable $u_{7}$ same as in Sample 1.

Sample 3: Used for $\varepsilon_{33}$. Variables $u_{1}, u_{2}, \ldots, u_{6}$ assigned distributions indicated in Table 3.12 for $\varepsilon_{33}$, which are the distributions shown in Fig. 3.1 of Ref. 19 truncated at $10^{-4} \mathrm{~mol} / \ell$ and then renormalized. Variable $u_{7}$ same as in Sample 1. 
As E1E2 intrusions are the dominant contributors in this analysis (i.e., in SPM-1) to the groundwater-release component in the CCDFs used for comparison with the boundary line specified in 40 CFR 191.13, the decision was made to perform PANEL calculations only for E1E2 intrusions and then to scale the results of these calculations to obtain estimates for E1 and E2 intrusions. As three different conditions for E1E2 intrusions are under consideration (i.e., for outcomes $\varepsilon_{21}, \varepsilon_{22}$ and $\varepsilon_{23}$ of experimental program $E_{2}$ ), this results in 72 (i.e., $3 \times 24$ ) sets of PANEL calculations. Each PANEL calculation for an E1E2 intrusion accessed the inventory of one waste panel; however, as discussed in Sect. 5.4.3, the CCDF construction process incorporated an estimate of the inventory that would be accessed by the drilling events that gave rise to the individual E1E2 intrusions under consideration.

The release for an E1 intrusion was assigned a value equal to $1 / 14$ of the release estimated by PANEL for an E1E2 intrusion occurring at the same time, with this scale factor corresponding to the fraction of the inventory in a waste panel that would be accessed by two closely-placed drilling intrusions that gave rise to an E1E2 intrusion (see Sect. 5.4.3). The release for an E2 intrusion was obtained by multiplying the release for an E1E2 intrusion occurring at the same time by the scale factor

$$
S F=b E 2 / b E 1,
$$

where $b E 1$ is the total brine flow $\left(\mathrm{m}^{3}\right)$ over $10,000 \mathrm{yr}$ from the repository to the Culebra for an $E 1$ intrusion at 1000 $\mathrm{yr}, b E 2$ is the total brine flow $\left(\mathrm{m}^{3}\right)$ over $10,000 \mathrm{yr}$ from the repository to the Culebra for an E2 intrusion at $1000 \mathrm{yr}$ and the brine flows for the high permeability shaft seals (i.e., outcome $\varepsilon_{21}$ for experimental program $E_{2}$ ) were used as this was the only case for which BRAGFLO calculations were performed for an E2 intrusion. The resultant values for $b E 1, b E 2$ and $S F$ are listed in Table 5.5.

The construction of CCDFs for comparison with the boundary line specified in 40 CFR 191.13 requires the consideration of drilling intrusions at many different times. However, due to computational cost, BRAGFLO calculations were only performed for drilling intrusions occurring at $1000 \mathrm{yr}$. As shown in Figs. 5.5 and 5.6, total brine flow out of the repository takes place at a constant rate once an E1 intrusion occurs, with the result that the brine flows from the repository to the Culebra that take place for an E1 intrusion at $1000 \mathrm{yr}$ can also be used to estimate the brine flows that take place for $\mathrm{E} 1$ intrusions at other times. A similar, but less pronounced, pattern can also be seen in Fig 5.5 for the brine flows associated with an E2 intrusion. The SPM-1 analysis takes advantage of this pattern by recording the cumulative radionuclide releases calculated by PANEL at a sequence of times between 1000 and 10,000 yr. After an appropriate correction for radioactive decay is made, these releases can be used to estimate the releases that would occur due to drilling intrusions at times other than $1000 \mathrm{yr}$.

Several examples of results calculated with PANEL are now presented. The colloidally-sorbed releases to the Culebra that result for an E1E2 intrusion at $1000 \mathrm{yr}$ for different assumptions with respect to colloidal solubility are illustrated in Fig. 5.12. As examination of this figure shows, the size of the colloidally-sorbed release decreases as $\mu$ 
Table 5.5. Cumulative Brine Releases from the Repository to the Culebra over 10,000 yr for E1 and E2 Intrusions Occurring at $1000 \mathrm{yr}$ and High Permeability Shaft Seals (i.e., Outcome $\varepsilon_{21}$ for Experimental Program $E_{2}$ )

\begin{tabular}{c|c|c|c|c|c|c|c}
\hline $\begin{array}{c}\text { Sample } \\
\text { Element }\end{array}$ & $b E 2$ & $b E 1$ & $\begin{array}{c}S F= \\
b E 2 / b E 1\end{array}$ & $\begin{array}{c}\text { Sample } \\
\text { Element }\end{array}$ & $b E 2$ & $b E 1$ & $\begin{array}{c}S F= \\
b E 2 / b E 1\end{array}$ \\
\hline 1 & $4.16 \times 10^{3}$ & $3.59 \times 10^{4}$ & $1.16 \times 10^{-1}$ & 14 & $5.19 \times 10^{3}$ & $1.60 \times 10^{6}$ & $3.24 \times 10^{-3}$ \\
2 & $3.46 \times 10^{3}$ & $6.45 \times 10^{5}$ & $5.36 \times 10^{-3}$ & 15 & $1.28 \times 10^{3}$ & $7.31 \times 10^{4}$ & $1.75 \times 10^{-1}$ \\
3 & 0 & $5.92 \times 10^{3}$ & 0 & 16 & $1.03 \times 10^{4}$ & $1.55 \times 10^{5}$ & $6.65 \times 10^{-2}$ \\
4 & 0 & $8.14 \times 10^{4}$ & 0 & 17 & 0 & $2.37 \times 10^{5}$ & 0 \\
5 & $1.39 \times 10^{3}$ & $7.40 \times 10^{3}$ & $1.88 \times 10^{-1}$ & 18 & $2.33 \times 10^{3}$ & $3.84 \times 10^{5}$ & $6.06 \times 10^{-3}$ \\
6 & $2.30 \times 10^{1}$ & $2.46 \times 10^{3}$ & $9.35 \times 10^{-3}$ & 19 & $9.25 \times 10^{2}$ & $2.84 \times 10^{5}$ & $3.26 \times 10^{-3}$ \\
7 & $5.46 \times 10^{3}$ & $9.90 \times 10^{4}$ & $5.52 \times 10^{-2}$ & 20 & 0 & $1.58 \times 10^{5}$ & 0 \\
8 & $6.95 \times 10^{3}$ & $3.22 \times 10^{5}$ & $2.16 \times 10^{-2}$ & 21 & $1.38 \times 10^{3}$ & $7.94 \times 10^{4}$ & $1.74 \times 10^{-2}$ \\
9 & $6.77 \times 10^{3}$ & $2.72 \times 10^{5}$ & $2.48 \times 10^{-2}$ & 22 & $3.67 \times 10^{3}$ & $1.15 \times 10^{5}$ & $3.20 \times 10^{-2}$ \\
10 & $3.56 \times 10^{-1}$ & $1.64 \times 10^{5}$ & $2.16 \times 10^{-6}$ & 23 & 0 & $9.62 \times 10^{5}$ & 0 \\
11 & $8.25 \times 10^{2}$ & $4.46 \times 10^{4}$ & $1.85 \times 10^{-2}$ & 24 & $7.19 \times 10^{3}$ & $2.13 \times 10^{5}$ & $3.38 \times 10^{-2}$ \\
12 & $4.73 \times 10^{3}$ & $1.78 \times 10^{5}$ & $2.66 \times 10^{-2}$ & 25 & $2.67 \times 10^{2}$ & $4.71 \times 10^{4}$ & $5.67 \times 10^{-3}$ \\
13 & $7.21 \times 10^{3}$ & $3.79 \times 10^{5}$ & $1.90 \times 10^{-1}$ & & & & \\
\hline
\end{tabular}

decreases. However, changes in assumptions with respect to solubility for dissolved radionuclides have little effect on the colloidal release. As the colloidally-sorbed radionuclides are assumed to transport through the Culebra to the accessible environment without retardation, the releases to the Culebra shown in Fig. 5.12 are also the releases to the accessible environment for the analysis cases under consideration, with an adjustment as discussed in Sect. 5.4.3 for the radionuclide inventory accessed by specific drilling intrusions. The corresponding releases to the Culebra for chemically-dissolved radionuclides are shown in Fig. 5.13. As examination of this figure shows, the dissolved release in conjunction with infinite colloidal solubility is zero, which results from the assumption that radionuclides attach to colloids to the maximum extent possible. For colloidal solubilities below $\mu=-5$, colloidal solubility has little effect on the dissolved radionuclide release. However, as comparison of the three individual frames in Fig. 5.13 shows, changing the assumptions for chemical solubility has a large effect on the dissolved release. An alternative display of the results in Figs. 5.12 and 5.13 that facilitates comparison of both the colloidally-dissolved and chemically-dissolved releases is given in Fig. 5.14.

The individual radionuclides in the releases to the Culebra (see Table 5.3 and Fig. 5.9) were combined into releases of the radionuclides Am-241, Pu-239 and U-233 for radionuclide transport calculations in the Culebra. ${ }^{83}$ In 


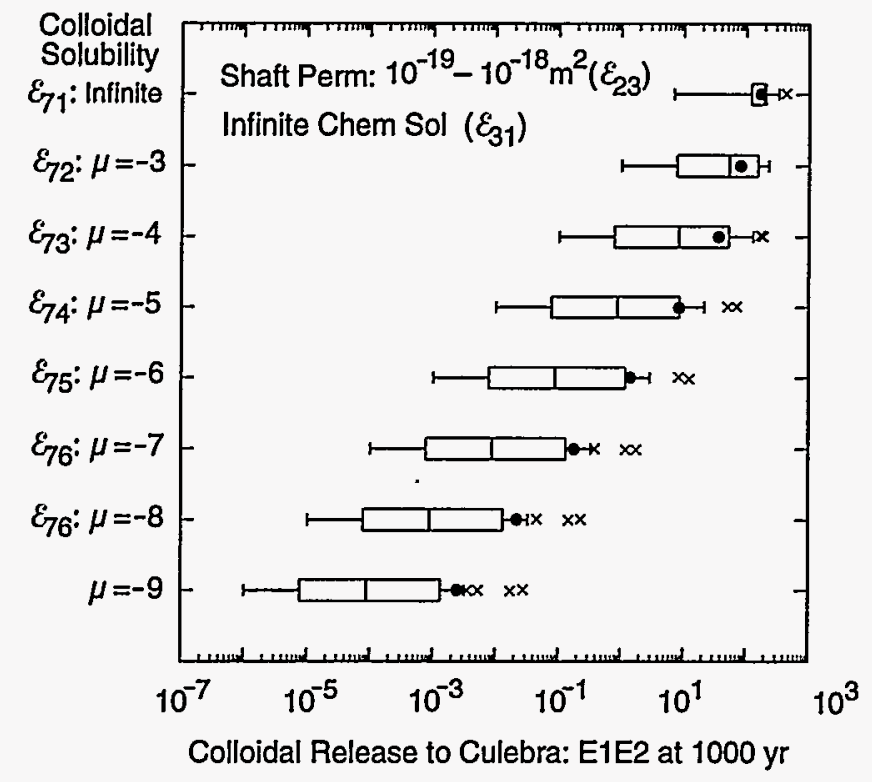

TRI-6342-4635-0

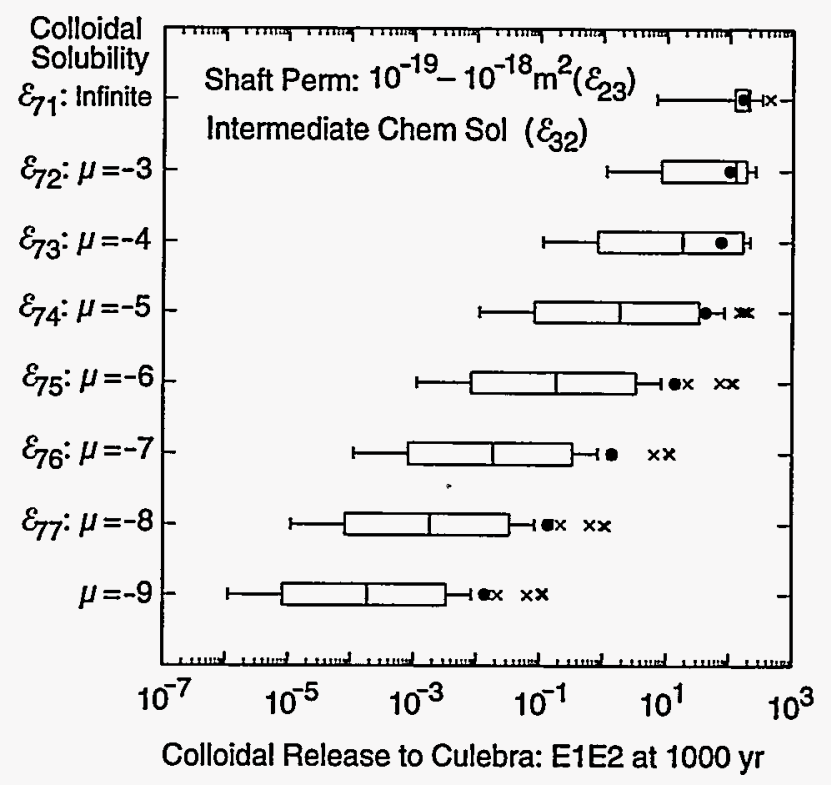

TRl-6342-4634-0

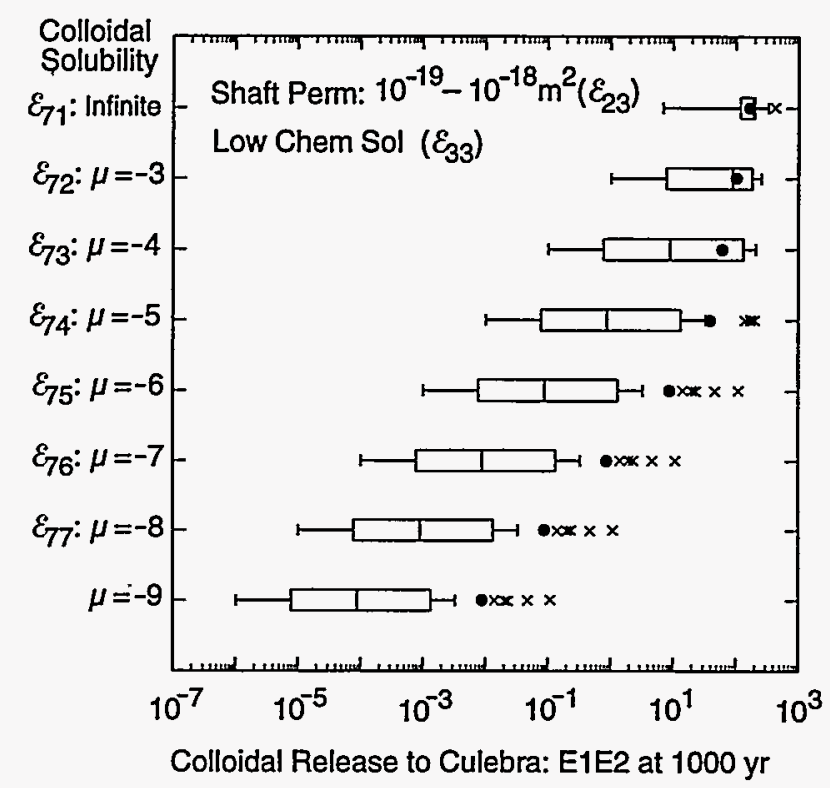

TRI-6342-4608-0

Figure 5.12. Total release of colloidally-sorbed radionuclides to the Culebra over $10,000 \mathrm{yr}$ for an E1E2 intrusion at $1000 \mathrm{yr}$, low permeability shaft seals, and different assumptions involving colloidal sorption. 


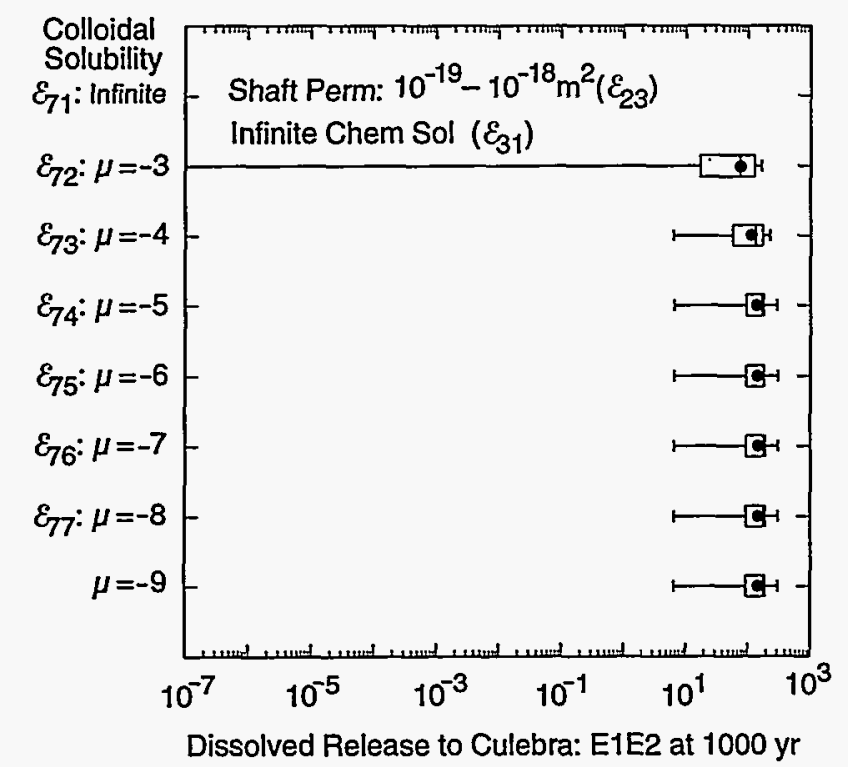

TRI-6342-4637-0

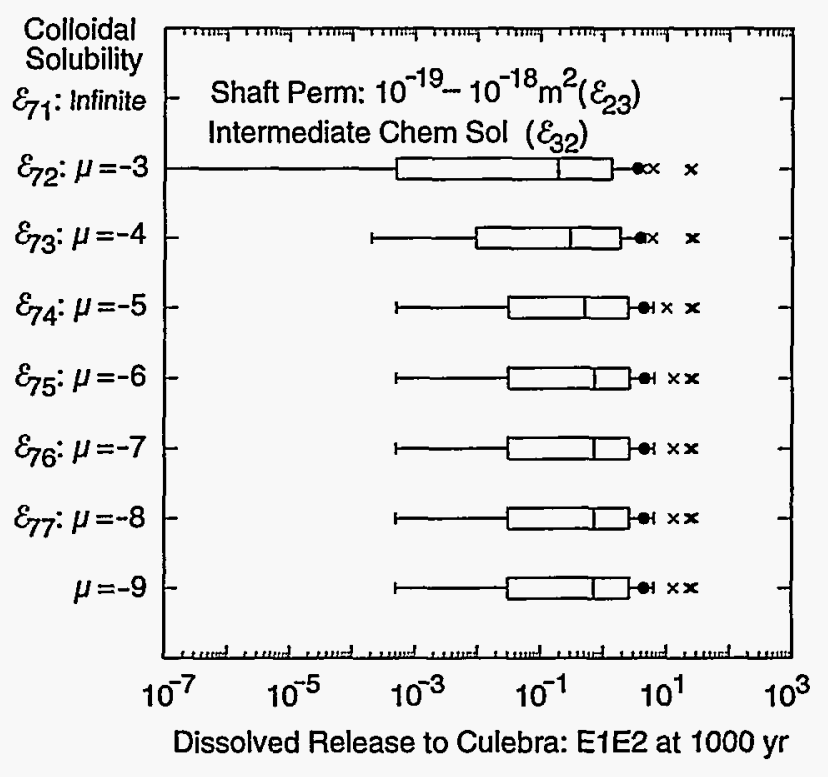

TRI-6342-4636-0

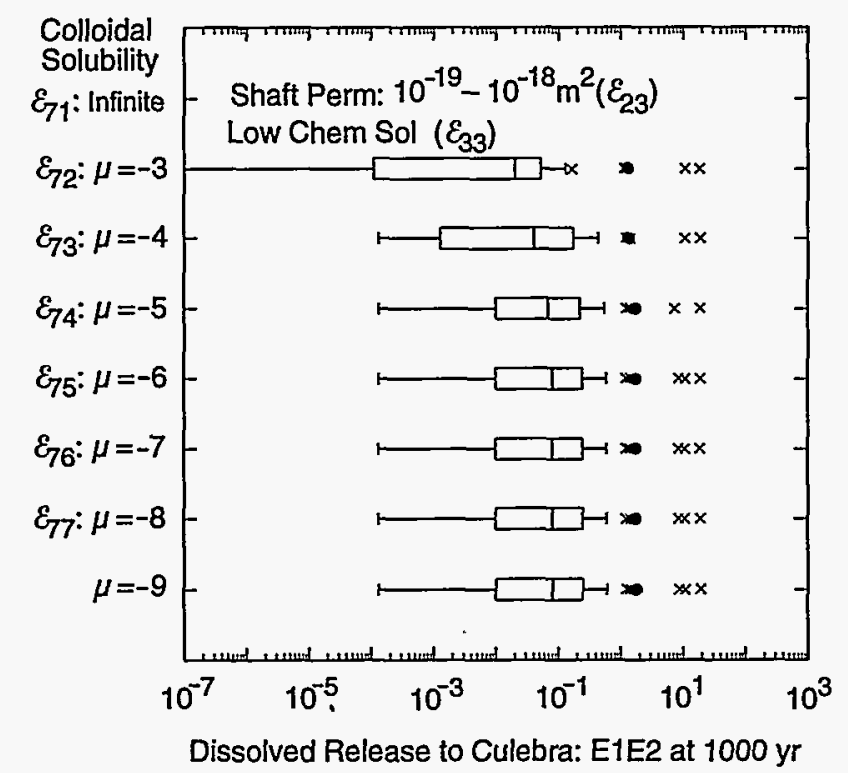

TRI-6342-4609-0

Figure 5.13. Total release of chemically dissolved radionuclides to the Culebra over $10,000 \mathrm{yr}$ for an E1E2 intrusion at $1000 \mathrm{yr}$, low permeability shaft seals, and different assumptions involving colloidal sorption. 


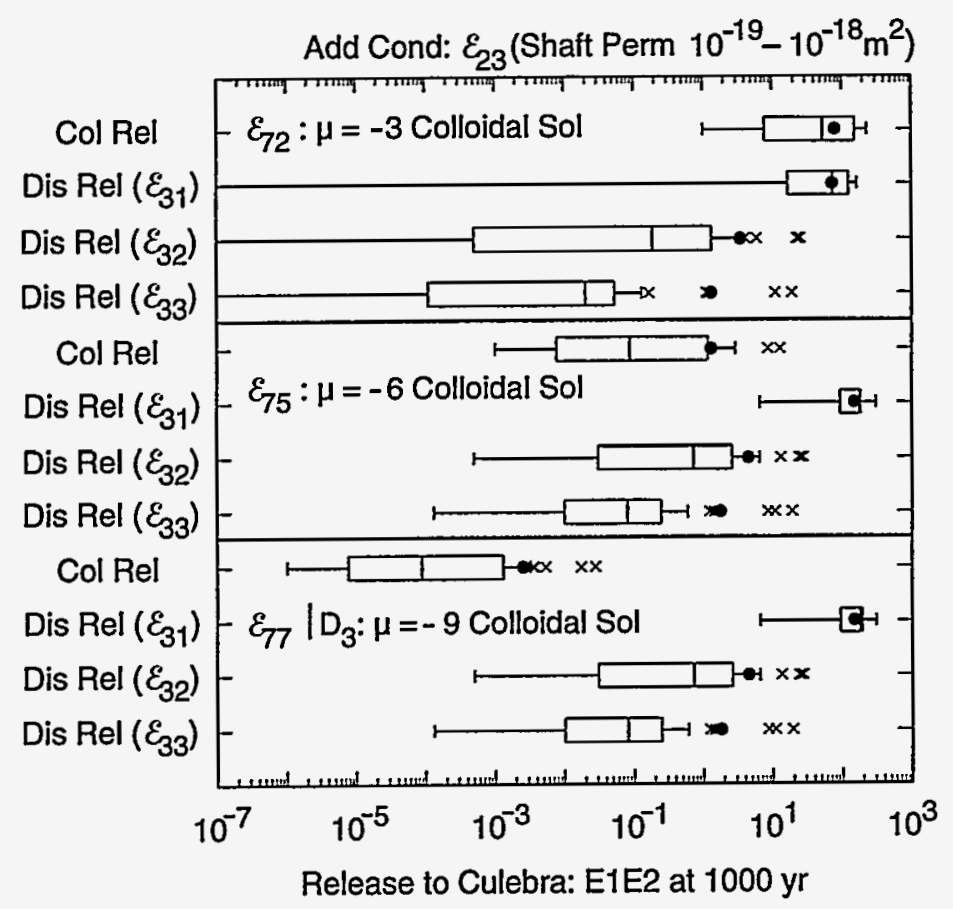

TRl-6342-4611-0

Figure 5.14. Total release of chemically-dissolved radionuclides to the Culebra over 10,000 yr for an E1E2 intrusion at $1000 \mathrm{yr}$, low permeability shaft seals and different assumptions involving colloidal sorption and chemical solubility.

particular, Pu-241 was combined with Am-241; Pu-240 and Pu-242 were combined with Pu-239; and U-234, Pu-238, U-235, U-236 and Np-237 were combined with U-233. This coalescing reduces the number of radionuclide transport calculations required with SECO-TRANSPORT in the Culebra but, as shown by examination of Fig. 5.9, should result in little loss of resolution in estimated groundwater releases to the accessible environment. For perspective, the radionuclide composition of the releases to the Culebra after this coalescing are shown in Figs. 5.15 and 5.16 for colloidally-sorbed and chemically-dissolved radionuclides, respectively. In order of importance, the dominant radionuclides for the colloidally-sorbed release are Pu-239, U-233 and Am-241, with the U-233 releases tending to be only slightly larger than the Am-241 releases. The dominant radionuclides for the chemicallydissolved release are Pu-239, Am-241 and U-233 in the presence of infinite chemical solubility (i.e., $\varepsilon_{31}$ ) and $\mathrm{Am}-241, \mathrm{U}-233$ and $\mathrm{Pu}-239$ in the presence of intermediate (i.e., $\varepsilon_{32}$ ) and low (i.e., $\varepsilon_{33}$ ) chemical solubility As comparison of the bottom four box plots in Fig. 5.15 and the top four box plots in Fig. 5.16 shows, changing from an assumption of low chemical solubility (i.e., $\varepsilon_{33}$ ) to an assumption of infinite chemical solubility (i.e., $\varepsilon_{31}$ ) has little effect on the colloidally-sorbed release. As shown by Fig. 5.9, the importance of Am-241 decreases rapidly with time due to radioactive decay. 


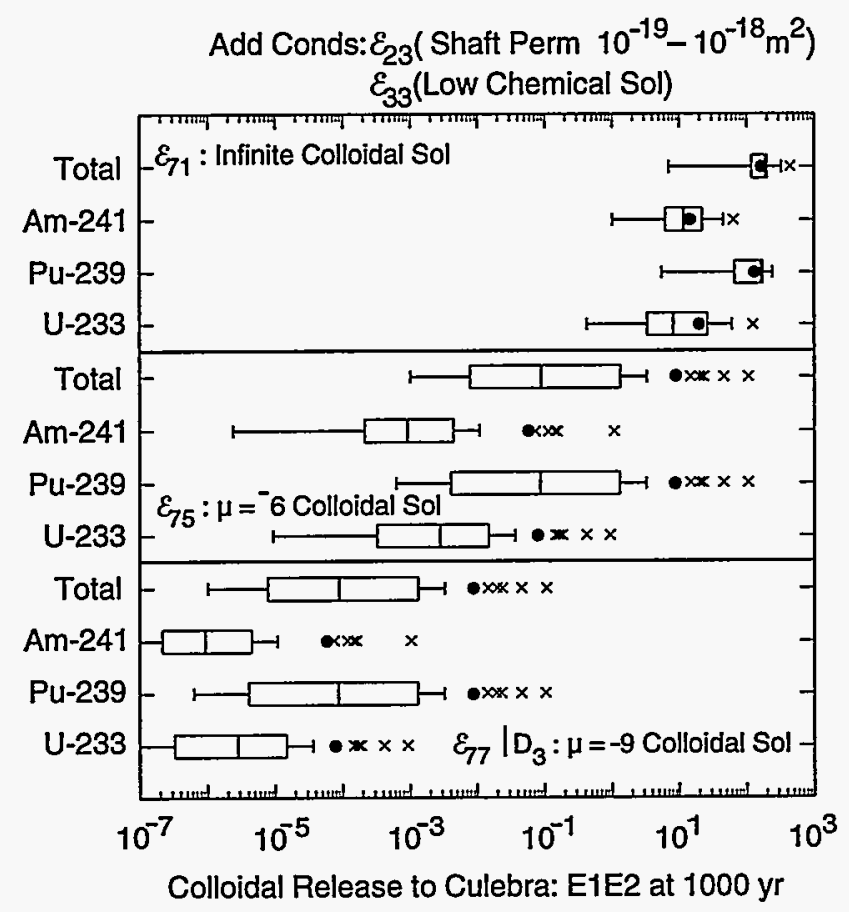

TRI-6342-4612-0

Figure 5.15. Release of individual colloidally-sorbed radionuclides to the Culebra over 10,000 yr for an E1E2 intrusion at $1000 \mathrm{yr}$, low permeability shaft seals and different assumptions involving colloidal sorption.

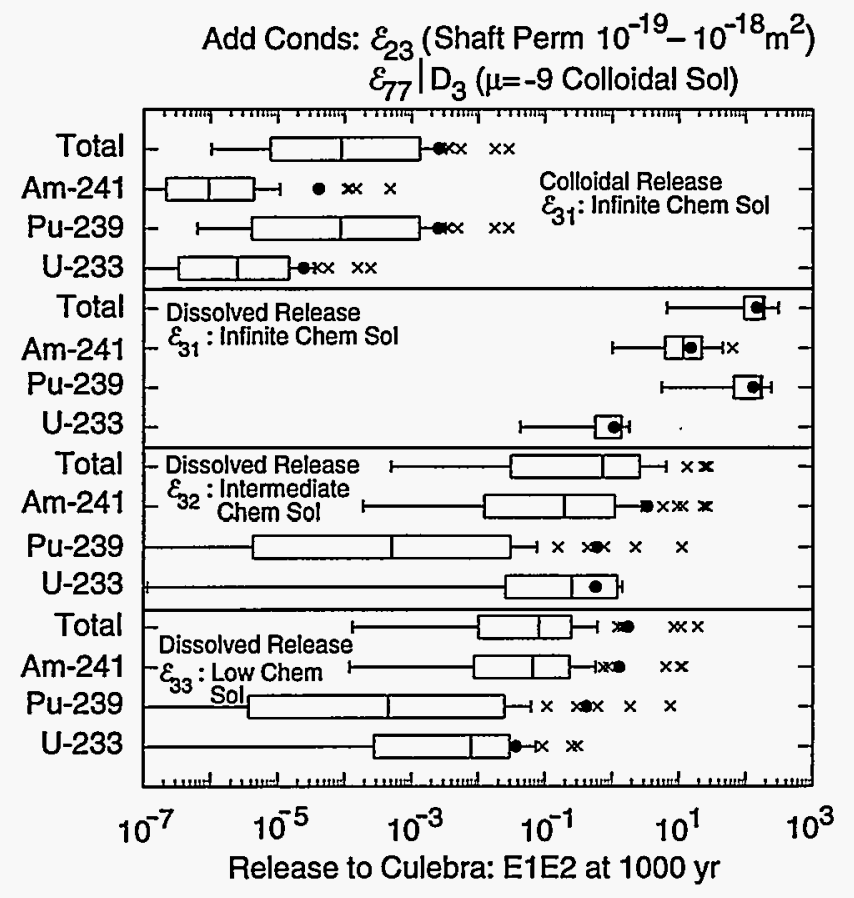

TRI-6342-4614-0

Figure 5.16. Release of individual chemically-dissolved radionuclides to the Culebra over 10,000 yr for an E1E2 intrusion at $1000 \mathrm{yr}$, low permeability shaft seals, $\mu=-9$ for colloidal solubility and different assumptions involving chemical solubility. 
A partial summary of the PANEL results saved for use in later calculations is given in Table 5.6. As previously indicated, the results for $i=1$ and 2 are obtained by scaling the results for $i=3$. The direct release to the surface (i.e., PANOUTS) is always zero in this analysis because BRAGFLO predicted no brine flows up the shaft or up an intruding borehole to the land surface. The integer $l$ designates the time periods over which integrated releases are calculated; most of the release occurs after the drilling intrusion at $1000 \mathrm{yr}$ but there can be some release before 1000 yr due to brine flow up the shaft (see Figs. 5.5 and 5.6). The integer $m$ designates the radionuclides considered for transport in the Culebra with SECO-TRANSPORT (i.e., Am-241, Pu-239, U-233). The colloidally-sorbed radionuclide release to the Culebra (i.e., PANOUTC) is assumed to be transported instantly to the accessible environment. The chemically-dissolved radionuclide release (i.e., PANOUTD) is the input to SECO-TRANSPORT. The actual input to SECO-TRANSPORT is the time-dependent discharge of Am-241, Pu-239 and U-233 to the Culebra as indicated in Fig. 5.17. For the purpose of initiating groundwater transport calculations, the discharges to the Culebra were specified over shorter time intervals (i.e., $50 \mathrm{yr}$ ) than used in conjunction with the results saved in PANOUTD.

Table 5.6. Partial Summary of PANEL Results Calculated for a Drilling Intrusion at $1000 \mathrm{yr}$ and Saved for Use in Later Calculations

PANOUTC $(h, i, j, k, l, m, n)=$ colloidally-sorbed radionuclide release to the Culebra (Ci)

$\operatorname{PANOUTD}(h, i, j, k, l, m, n)=$ chemically-dissolved radionuclide release to the Culebra (Ci)

PANOUTS $(h, i, j, k, l, m, n) \quad=$ direct radionuclide release to surface environment due to brine flow from the repository to the land surface $(\mathrm{Ci})$

where

$h \sim$ shaft permeability $\left(1 \Rightarrow \varepsilon_{21}\right.$, i.e., $10^{-15}-10^{-13} \mathrm{~m}^{2} ; 2 \Rightarrow \varepsilon_{22}$, i.e., $10^{-18}-10^{-16} \mathrm{~m}^{2} ; 3 \Rightarrow \varepsilon_{23}$, i.e., $10^{-19}-10^{-18}$ $\mathrm{m}^{2}$ )

$i \sim$ type of intrusion $(1 \Rightarrow \mathrm{E} 1,2 \Rightarrow \mathrm{E} 2,3 \Rightarrow \mathrm{E} 1 \mathrm{E} 2)$

$j \sim$ outcome of experimental program $E_{3}$ for chemical solubility $\left(1 \Rightarrow \varepsilon_{31}\right.$, i.e., infinite solubility; $2 \Rightarrow \varepsilon_{32}$, i.e., intermediate solubility; $3 \Rightarrow \varepsilon_{33}$, i.e., low solubility)

$k \sim$ outcome of experimental program $E_{7}$ and effect of design modification $D_{3}$ for colloidally-sorbed radionuclides $\left(1 \Rightarrow \varepsilon_{71} \mid D_{3}\right.$ and $\varepsilon_{71}$, i.e., infinite solubility; $2 \Rightarrow \varepsilon_{72}$, i.e., $\mu=-3 ; 3 \Rightarrow \varepsilon_{72} \mid D_{3}$ and $\varepsilon_{73}$, i.e., $\mu$ $=-4 ; 4 \Rightarrow \varepsilon_{73} \mid D_{3}$ and $\varepsilon_{74}$, i.e., $\mu=-5 ; 5 \Rightarrow \varepsilon_{74} \mid D_{3}$ and $\varepsilon_{75}$, i.e., $\mu=-6 ; 6 \Rightarrow \varepsilon_{75} \mid D_{3}$ and $\varepsilon_{76}$, i.e., $\mu=-7 ; 7$ $\Rightarrow \varepsilon_{77} \mid D_{3}$, i.e., $\mu=-8 ; 8 \Rightarrow \varepsilon_{77} \mid D_{3}$ and $\varepsilon_{71}$, i.e., $\mu=-9$ )

$l \sim$ integration period $(1 \Rightarrow 0-2000 \mathrm{yr}, 2 \Rightarrow 0-4000 \mathrm{yr}, 3 \Rightarrow 0-6000 \mathrm{yr}, 4 \Rightarrow 0-8000 \mathrm{yr}, 5 \Rightarrow 0-10,000 \mathrm{yr})$

$m \sim$ radionuclide $(1 \Rightarrow \mathrm{Am}-241,2 \Rightarrow \mathrm{Pu}-239,3 \Rightarrow \mathrm{U}-233)$

$n \sim$ sample element $(n=1,2, \ldots, 25)$ 

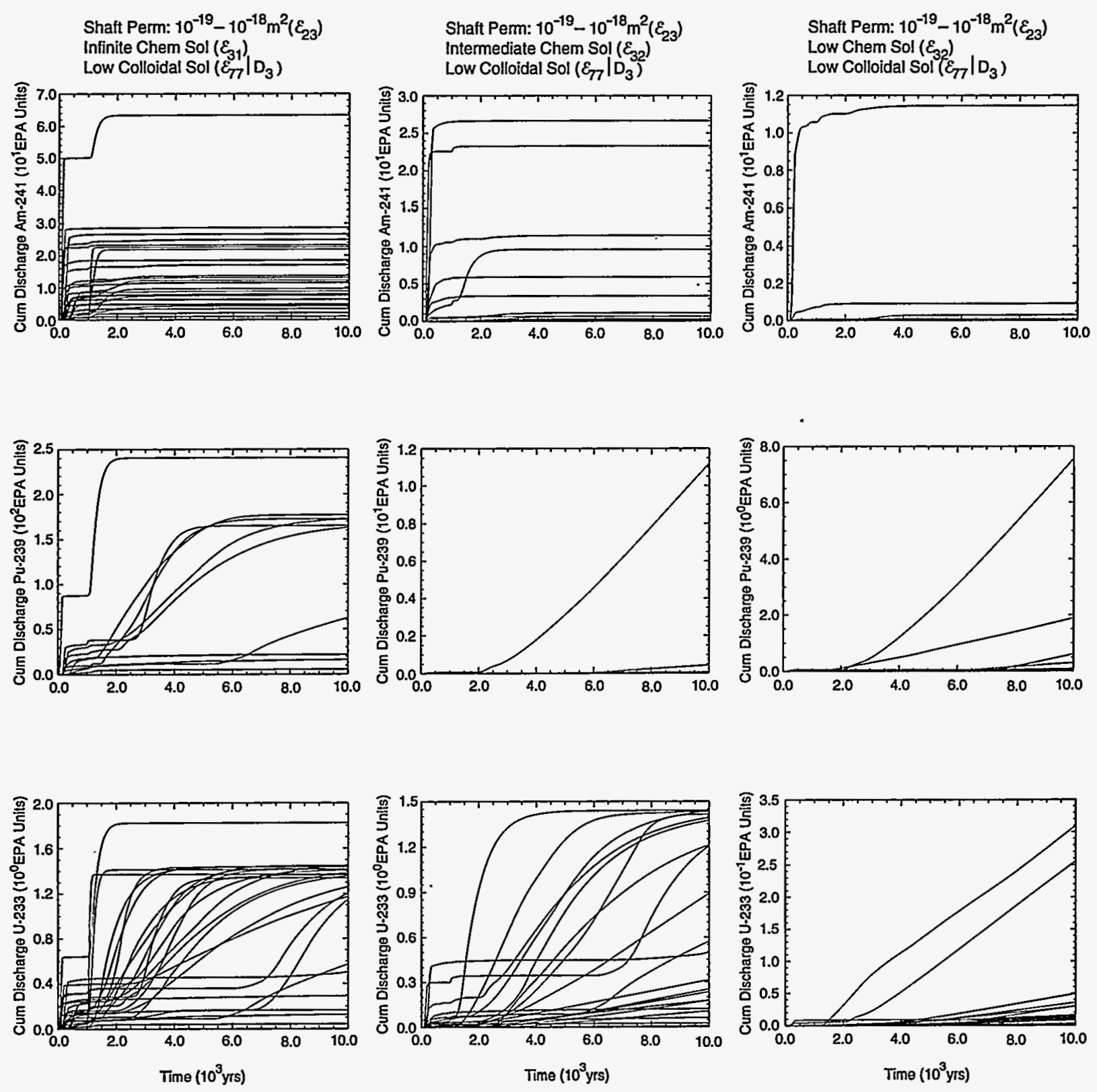

TRl-6342-4615-0

Figure 5.17. Example radionuclide discharges to the Culebra calculated by PANEL and used as input to radionuclide transport calculations performed with SECO-TRANSPORT for an E1E2 intrusion at $1000 \mathrm{yr}$, low permeability shaft seals, $\mu=-9$ for colloidal solubility, and different assumptions involving chemical solubility. Care should be taken in comparing results in different plot frames due to the use of different scales on the ordinate. 


\subsubsection{SECO-FLOW Calculations}

The SECO-FLOW program was used to determine the Culebra flow field for use with the SECO-TRANSPORT

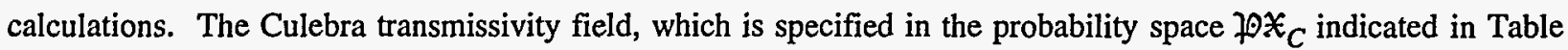
3.21 , is the only uncertain input used in the determination of the Culebra flow field. As a result, the SECO-FLOW calculations were preceded by the generation of an LHS

$$
\mathbf{v}_{m}=\left[v_{m 1}, v_{m 2}, \ldots, v_{m 5}\right], m=1,2, \ldots, n L H S=25,
$$

from the probability space $\not O X_{C}$, where $v_{1}, v_{2}, v_{3}, v_{4}$ and $v_{5}$ correspond to the variables CULCLYF, CULCLYP, CULFRPOR, CULPOR and CULTRFLD associated with $\not O X_{C}$. A SECO-FLOW calculation was then carried out for each sample element $\mathrm{v}_{m}$ in Eq. (5.14) to produce a corresponding flow field for use in conjunction with the SECOTRANSPORT calculations. The SECO-FLOW calculations use only the variable CULTRFLD; the remaining variables associated with $\mathfrak{O X}_{C}$ are used in the radionuclide transport calculations performed with SECOTRANSPORT.

\subsubsection{SECO-TRANSPORT Calculations}

Radionuclide transport calculations within the Culebra are performed with the SECO-TRANSPORT program. In concept, one or more sets of calculations could be required for each path through Fig. 5.11 (i.e., for each set of PANEL calculations). Experimental programs $E_{4}, E_{5}$ and $E_{6}$ affect calculations with SECO-TRANSPORT. As indicated in Fig. 5.18, this could lead to a requirement for 20 sets of SECO-TRANSPORT calculations for each set of PANEL calculations. In addition, results are required for radionuclide releases into the Culebra beginning at a number of different times. If SECO-TRANSPORT calculations were performed for the three shaft seal cases indicated in Fig. 5.4, the three types of intrusions (i.e., E1, E2, E1E2), the 24 cases indicated in Fig. 5.11 for PANEL calculations, the 20 cases indicated in Fig. 5.18 resulting from experimental programs relevant to the Culebra, the four radionuclide decay chains considered in the 1992 WIPP PA (Table 7.3-2, Ref 19) and 5 possible drilling intrusion times (e.g., $1000 \mathrm{yr}, 3000 \mathrm{yr}, 5000 \mathrm{yr}, 7000 \mathrm{yr}, 9000 \mathrm{yr}$ ), a total of 86,400 sets of calculations (i.e., $3 \times 3 \times$ $24 \times 20 \times 4 \times 5$ ) would be required. In addition, the actual number of SECO-TRANSPORT calculations would be larger by a factor of 25 due to the use of an LHS of size 25. As SECO-TRANSPORT does not run quickly enough to allow this number of calculations to be performed, the nature of the PANEL results indicated in Fig. 5.11 and the Culebra conditions indicated in Fig. 5.18 must be examined carefully to determine the situations for which Culebra transport results (1) must be obtained directly from SECO-TRANSPORT calculations performed as part of SPM-1, (2) can be constructed from related SECO-TRANSPORT calculations performed as part of SPM-1, and (3) can be inferred from calculations performed as part of prior analyses (e.g., the 1992 WIPP PA) without the performance of additional SECO-TRANSPORT calculations. Further, as already indicated in Sect. 5.3.3, the transport of 9 


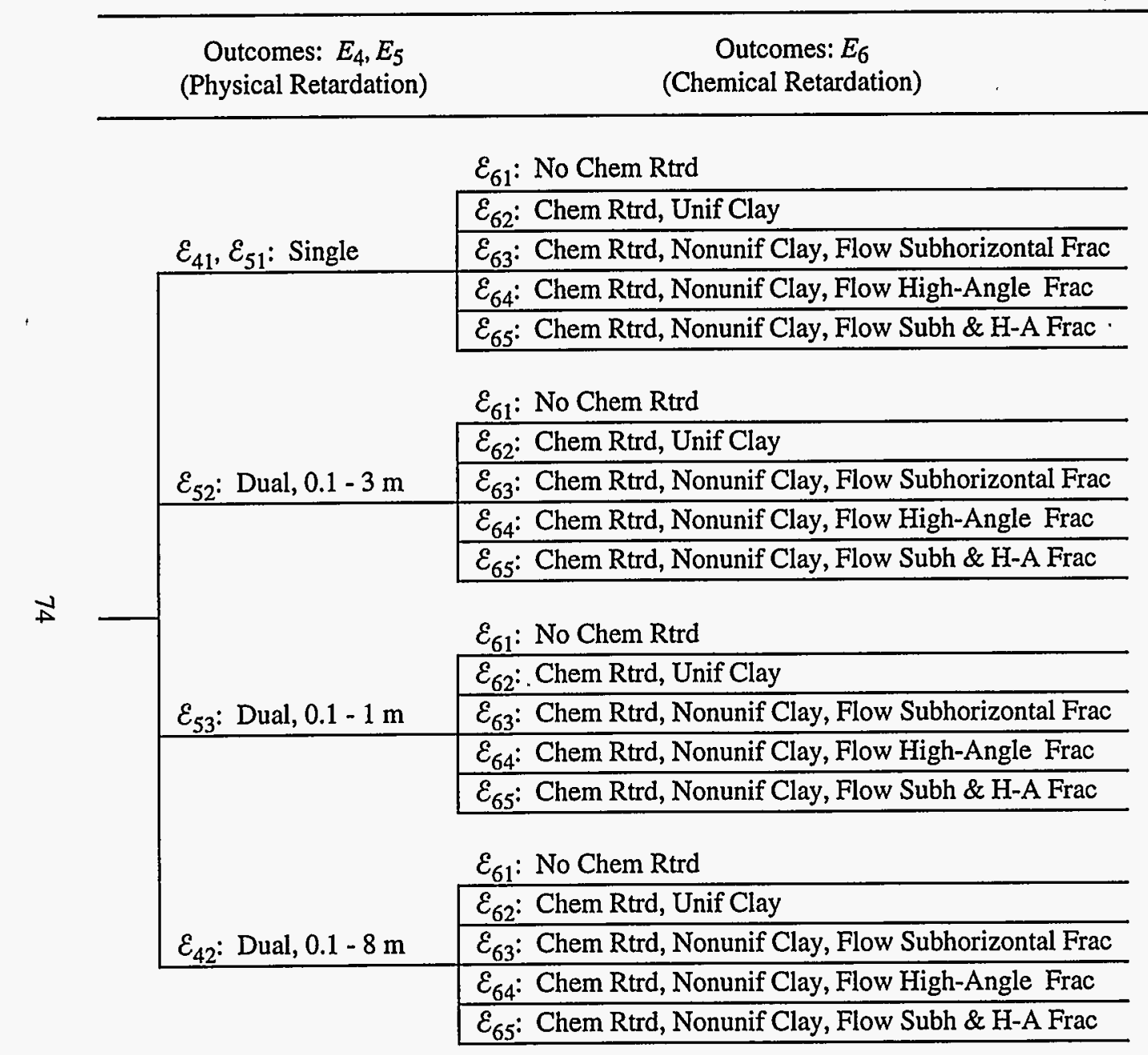

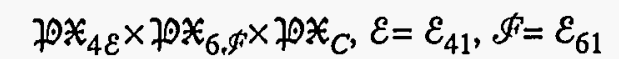

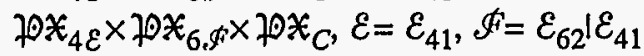

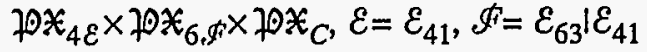

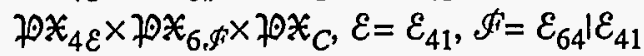

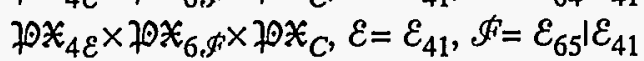

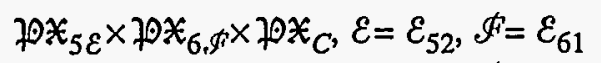

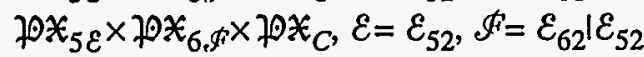

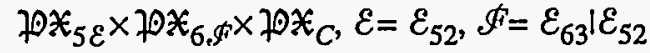

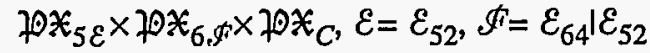

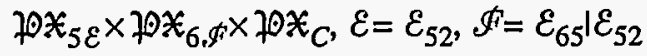

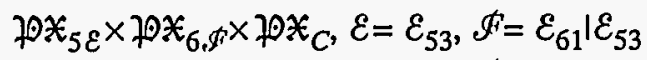

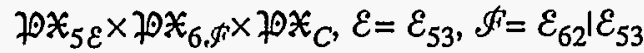

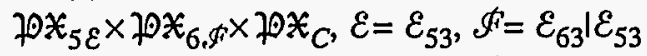

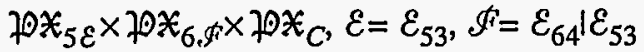

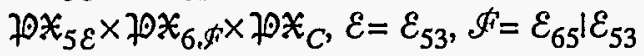

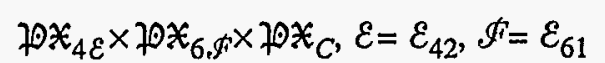
$\not 0 x_{4 \varepsilon} \times 70 X_{6, F} \times 70 X_{C}, \varepsilon=\varepsilon_{42}, \oiint=\varepsilon_{62} \mid \varepsilon_{42}$

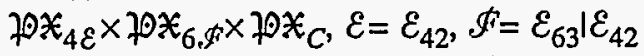

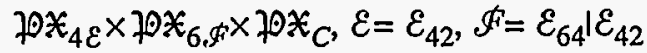

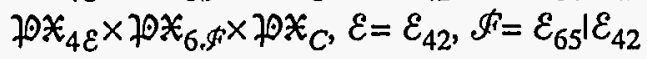

SECO-TRANSPORT

Calculations
No. Use Culebra release Yes

Yes

No. Same as $\varepsilon_{62}$ No. Same as $\varepsilon_{63}$

No. Release $=0$

No. Release $=0$

No. Release $=0$

No. Release $=0$

No. Release $=0$

No. Release $=0$

No. Release $=0$

No. Release $=0$

No. Release $=0$

No. Release $=0$

Yes

No. Release $=0$

No. Release $=0$

No. Release $=0$

No. Release $=0$

Figure 5.18 Logic diagram indicating 20 possible sets of conditions in the Culebra associated with experimental programs $E_{4}, E_{5}$ and $E_{6}$ that could require SECOTRANSPORT calculations. 
radionuclides in 4 decay chains as done in the 1992 WIPP PA can be reduced to the transport of 3 radionuclides (i.e., Am-241, Pu-239, U-233).

The performance of SECO-TRANSPORT calculations for the cases indicated in Fig. 5.18 is considered first. As indicated in Fig. 5.19, the assumptions of single porosity (i.e., fracture only) transport and no chemical retardation results in essentially all radionuclides released to the Culebra reaching the accessible environment. Thus, there is no need to perform SECO-TRANSPORT calculations for this case (i.e., for $\not \cap \mathbb{X}_{4 \varepsilon} \times \not \cap \mathbb{X}_{6 \mathscr{F}} \times \not \cap \mathbb{X}_{C}$ with $\varepsilon=$ $\varepsilon_{41}$ and $\mathscr{F}=\varepsilon_{61}$ in Fig. 5.18); rather, all chemically-dissolved radionuclides released to the Culebra can be assumed to reach the accessible environment. The cases for uniform clay distribution and nonuniform clay distribution with fluid flow primarily in high-angle fractures in conjunction with chemical retardation and single porosity transport

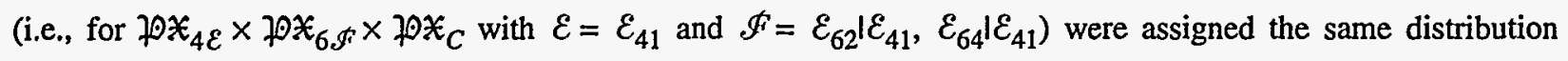
coefficients; thus, one set of SECO-TRANSPORT calculations covers both of these cases. The cases for fluid flow in subhorizontal fractures and for fluid flow in both subhorizontal and high-angle fractures in conjunction with

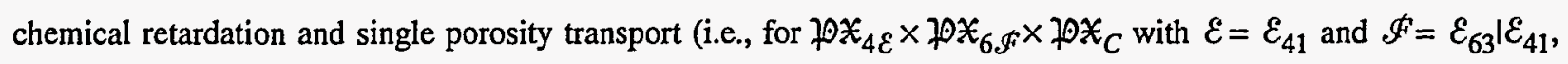
$\varepsilon_{65} \mid \varepsilon_{41}$ ) differ in that flow in subhorizontal fractures (i.e., for $\varepsilon_{63}$ ) results in radionuclides encountering only clay

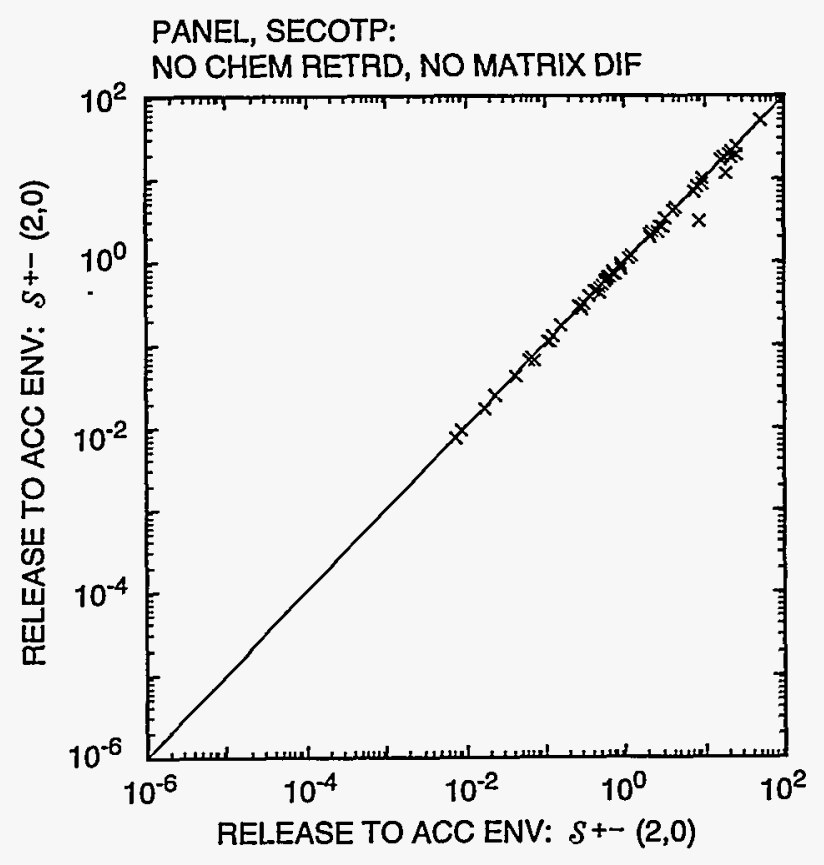

TRI-6342-2139-1

Figure 5.19. Scatterplot for total normalized release to Culebra over 10,000 yr versus total normalized release to the accessible environment due to groundwater transport with no chemical retardation and no matrix diffusion for Scenario $S^{4-}(2,0)$, where $\delta^{+-}(2,0)$ is used to denote an E1E2 intrusion occurring 1000 yr after repository closure (Fig. 8.4-1, Ref. 19). 
materials in the fractures while flow in both subhorizontal and high-angle fractures (i.e., for $\varepsilon_{65}$ ) results in radionuclides being in contact with clay for approximately half their transport distance in fractures and in contact with dolomite for the other half of the transport distance. As the distribution coefficients for clay are considerably higher than the distribution coefficients for dolomite, the results for both cases are dominated by the distribution coefficients for clay. Therefore, a set of SECO-TRANSPORT calculations is performed for the case with $\mathscr{F}=$ $\varepsilon_{63} \mid \varepsilon_{41}$ and the results of these calculations are assumed also to be appropriate for use with the case $\mathscr{F}^{\prime}=\varepsilon_{65} \mid \varepsilon_{41}$.

The cases in Fig. 5.18 associated with dual porosity transport (i.e., for $\mathcal{E}=\varepsilon_{52}, \varepsilon_{53}, \varepsilon_{42}$ ) are now considered. As shown in Fig. 5.20, the presence of both dual porosity transport and chemical retardation effectively cut off radionuclide transport to the accessible environment in the 1992 WIPP PA. In particular, although there was a tendency for the size of the release to increase as the fracture spacing increased, the largest observed release was approximately 0.1 EPA release units. As shown in Fig. 5.21, a similar pattern also occurs for dual porosity transport in the absence of chemical retardation, although the releases tend to be larger. However, all of the releases are less than approximately 0.1 EPA release units for fracture spacings less than $3 \mathrm{~m}$.

Due to the patterns indicated in Fig. 5.20, a set of SECO-TRANSPORT calculations was performed for dual

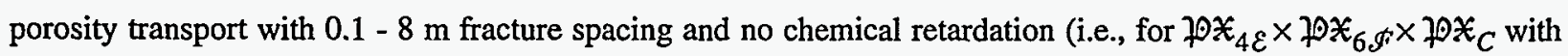
$\mathcal{E}=\varepsilon_{42}, \mathscr{F}^{\prime}=\varepsilon_{61}$ ). However, due to the small releases (i.e., $\leq 0.1 \mathrm{EPA}$ release units) indicated in Fig. 5.21 for fracture spacing less than $3 \mathrm{~m}$, no SECO-TRANSPORT calculations are performed for this case (i.e., for $70 X_{4 \varepsilon} \times$ $\not 0 \mathcal{X}_{6 \varepsilon} \times \not \cap \mathcal{X}_{C}$ with $\mathcal{E}=\varepsilon_{52}, \varepsilon_{53}, \mathscr{F}=\varepsilon_{61}$ ); rather, all radionuclide releases to the accessible environment were assigned a value of 0 . Due to the pattern indicated in Fig. 5.20, all radionuclide releases to the accessible environment for dual porosity transport and chemical retardation were also assigned a value of 0 (i.e., for $\mathfrak{D Q X}_{4 \varepsilon} \times$

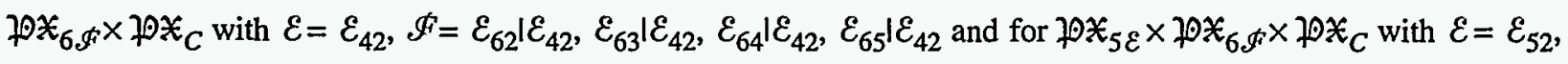
$\varepsilon_{53}, \mathscr{I}^{\prime}=\varepsilon_{62}\left|\varepsilon_{52}, \varepsilon_{63}\right| \varepsilon_{52}, \varepsilon_{64}\left|\varepsilon_{52}, \varepsilon_{65}\right| \varepsilon_{52}$ and also with $\left.\varepsilon=\varepsilon_{53}, \mathscr{F}^{\prime}=\varepsilon_{62}\left|\varepsilon_{53}, \varepsilon_{63}\right| \varepsilon_{53}, \varepsilon_{64}\left|\varepsilon_{53}, \varepsilon_{65}\right| \varepsilon_{53}\right)$, with the result that no SECO-TRANSPORT calculations are required for these cases. The elimination of transport calculations for dual porosity transport, no chemical retardation and fracture spacing in the range 0.1 to $3 \mathrm{~m}$ is fairly aggressive and merits a careful reconsideration in future analyses.

The influence of the case structure indicated in Fig. 5.11 on the required SECO-TRANSPORT calculations is now considered. The SECO-TRANSPORT program is based on the solution of a system of linear partial differential equations. As a result, the radionuclide transport results obtained with SECO-TRANSPORT are a linear function of the radionuclide source rate into the Culebra. That is, if $s(t)$ is the radionuclide source rate $(\mathrm{Ci} / \mathrm{yr})$ into the Culebra and $d(t)$ is the resultant radionuclide discharge rate $(\mathrm{Ci} / \mathrm{yr})$ to the accessible environment calculated with SECOTRANSPORT, then the consideration of a new source rate $k s(t)$, where $k$ is a constant, would result in a discharge rate of $k d(t)$ to the accessible environment. As will be indicated, this linearity can be used to reduce the number of required SECO-TRANSPORT calculations that derive from the cases indicated in Fig. 5.11. 


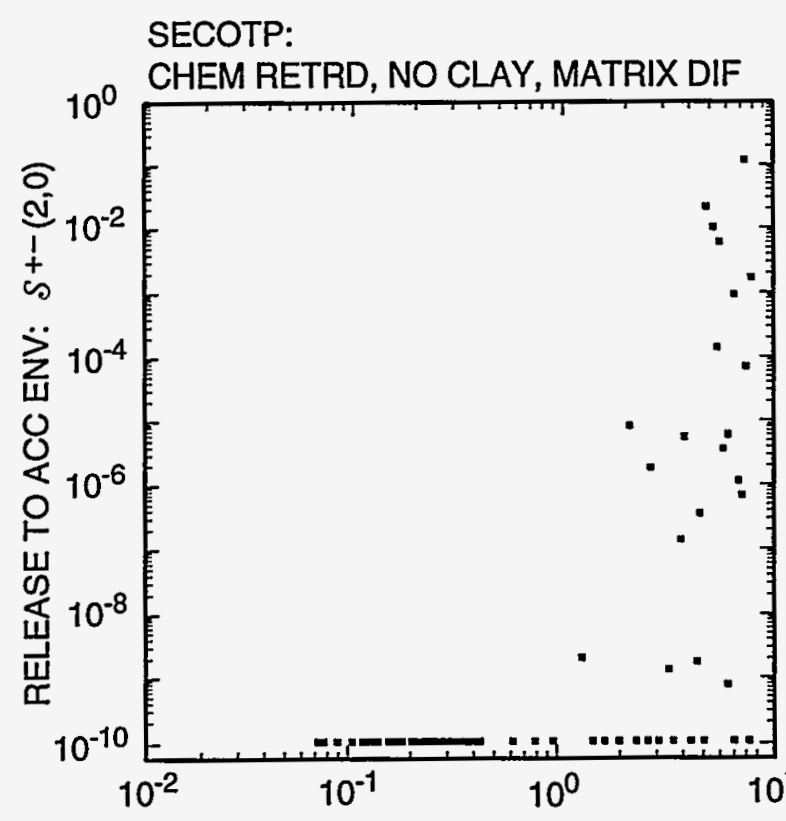

CULEBRA FRACTURE SPACING (CULFRSP)

TRI-6342-2616-1

Figure 5.20. Scatterplot for total normalized release to the accessible environment over 10,000 yr due to groundwater transport with chemical retardation, no clay lining in fractures and matrix diffusion versus CULFRSP (Culebra fracture spacing) for scenario $\delta^{+-}(2,0)$, where $\delta^{4-}(2,0)$ is used to denote an E1E2 intrusion occurring 1000 yr after repository closure (Fig. 8.4-10, Ref. 19).

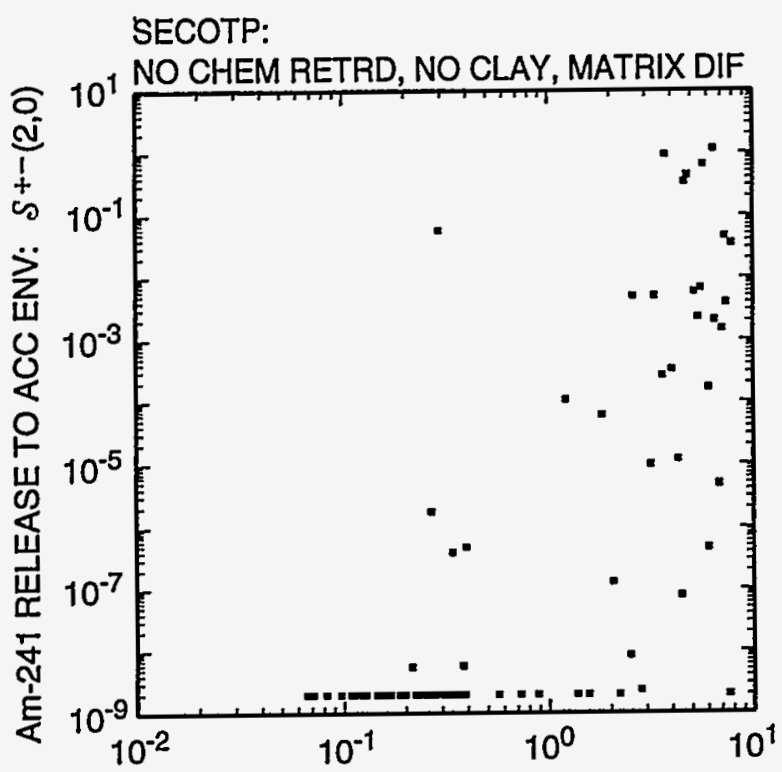

CULEBRA FRACTURE SPACING (CULFRSP)
SECOTP:

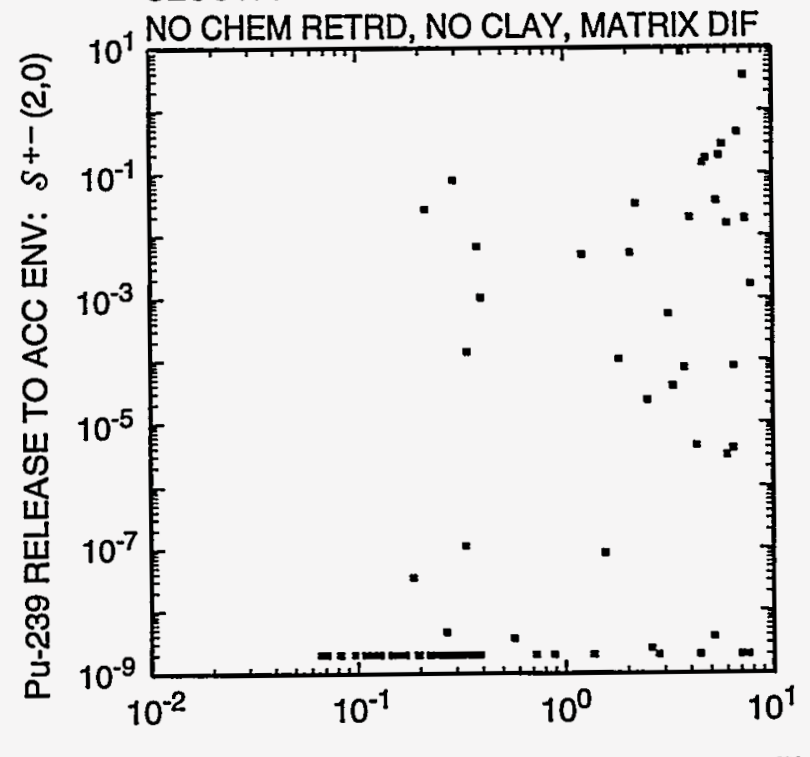

CULEBRA FRACTURE SPACING (CULFRSP)

TRI-6342-2619-1

TRI-6342-2621-1

Figure 5.21. Scatterplots for normalized releases of Am-241 and Pu-239 to the accessible environment over 10,000 yr due to groundwater transport with no chemical retardation, no clay lining in fractures and matrix diffusion versus CULFRSP (Culebra fracture spacing) for scenario $\mathcal{S}^{4-}(2,0)$, where $\mathcal{S}^{4-}(2,0)$ is used to denote an E1E2 intrusion occurring $1000 \mathrm{yr}$ after repository closure (Figs. 8.4-17, 8.4-18, Ref. 19). 
As colloidally-sorbed radionuclides are assumed to undergo instantaneous transport to the accessible environment, SECO-TRANSPORT calculations are only needed for chemically-dissolved radionuclide releases. In SPM-1, radionuclides are assumed initially to attach to colloids to the greatest extent possible, with only the remaining radionuclides being available for chemical dissolution. As a result, the largest releases of chemicallydissolved radionuclides will occur for the analysis case that involves the smallest amount of colloidally-sorbed radionuclides (i.e., for $\mu=-9$ associated with $\varepsilon_{77} \mid D_{3}$ as indicated in Fig. 5.11). As $\mu$ increases from -9 to -3 , the potential importance of the dissolved radionuclide release will decrease because (1) the size of the colloidally-sorbed release is increasing, (2) the size of the chemically-dissolved release is decreasing, and (3) the colloidally-sorbed release is transported instantaneously to the accessible environments while the transport of the chemically-dissolved release is impeded by physical and chemical retardation. As indicated in Fig. 5.13, the dissolved release in SPM-1 turned out to be fairly insensitive to the colloidally-sorbed release.

Because of the preceding considerations, SECO-TRANSPORT calculations were only performed for E1E2 intrusions for the three cases in Fig. 5.11 that have the lowest concentration of colloidally-sorbed radionuclides and

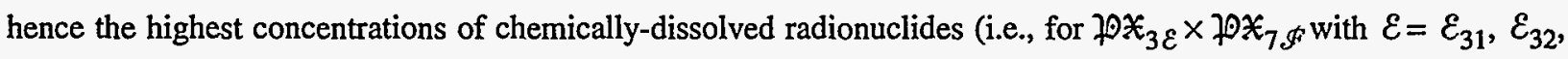

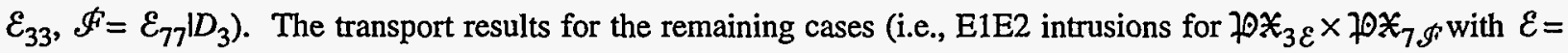
$\varepsilon_{31}, \varepsilon_{32}, \varepsilon_{33}, \&_{F}=\varepsilon_{72}, \varepsilon_{73}, \ldots, \varepsilon_{77}, \varepsilon_{72}\left|D_{3}, \varepsilon_{73}\right| D_{3}, \ldots, \varepsilon_{76} D_{3}$, and E1 and E2 intrusions for $\not 0 \mathcal{F}_{3 E} \times \not Q \mathcal{x}_{7 F}$ with $\left.\varepsilon=\varepsilon_{31}, \varepsilon_{32}, \varepsilon_{33}, \mathscr{F}=\varepsilon_{72}, \varepsilon_{72}, \ldots, \varepsilon_{77}, \varepsilon_{72}\left|D_{3}, \varepsilon_{73}\right| D_{3}, \ldots, \varepsilon_{77} \mid D_{3}\right)$ can be obtained by linearly scaling the releases to the accessible environment obtained in the cases for which SECO-TRANSPORT calculations were performed. The numerical implementation of these scalings is defined at the end of this section in Eq. (5.15) for E1 and $E 2$ intrusions for $\not \partial \mathcal{X}_{3 \varepsilon} \times \not \supset \mathcal{X}_{7 \&}$ with $\mathcal{E}=\varepsilon_{31}, \varepsilon_{32}, \varepsilon_{33}, \mathscr{F}=\varepsilon_{71} D_{3}$ and in Sect. 5.4.2 for the remaining cases. There is no dissolved release in the presence of infinite solubility for colloidally-sorbed radionuclides (i.e., for $\mathscr{F}^{\prime}=$ $\left.\varepsilon_{71} \mid D_{3}, \varepsilon_{71}\right)$ and so no adjustments are required for this case.

As discussed earlier, BRAGFLO and PANEL calculations are performed for drilling intrusions occurring at $1000 \mathrm{yr}$. The SECO-TRANSPORT calculations discussed in this section are also performed for intrusions at 1000 yr. However, the CCDF construction procedure described in Sect. 5.4 requires groundwater releases associated with intrusions occurring at a number of times between 100 and 10,000 yr. To implement the CCDF construction procedure, the previously indicated linear relationship between radionuclide source rate and discharge rate that exists for SECO-TRANSPORT will be used to construct the releases to the accessible environment that would occur for intrusions at times other than 1000 yr. As done with PANEL, integrated discharges to the accessible environment over a number of time intervals will be calculated with SECO-TRANSPORT and saved for an intrusion occurring at $1000 \mathrm{yr}$. With an appropriate correction for radioactive decay as described in Sect. 5.4.2, these discharges can then be used to estimate the releases that would occur for intrusions occurring at times other than $1000 \mathrm{yr}$.

The considerations discussed in this section resulted in SPM-1 requiring 81 sets of SECO-TRANSPORT calculations. In particular, calculations were performed for (1) an E1E2 intrusion occurring at $1000 \mathrm{yr}$ and the 
outcome associated with experimental program $E_{7}$ that results in the lowest concentration of colloidally-sorbed radionuclides (i.e., $\varepsilon_{77} \mid D_{3}$ ), (2) the three outcomes associated with experimental program $E_{2}$ (i.e., $\varepsilon_{21}, \varepsilon_{22}, \varepsilon_{23}$ ), (3) the three outcomes associated with experimental program $E_{3}$ (i.e., $\varepsilon_{31}, \varepsilon_{32}, \varepsilon_{33}$ ), (4) three radionuclides (i.e., Am-241, Pu-239, U-233) and (5) the three cases indicated in Fig. 5.18 that require SECO-TRANSPORT calculations. Example results are presented in Figs. 5.22 and 5.23. As total releases are being presented, each box plot in Fig. 5.22 is based on three sets of SECO-TRANSPORT calculations (i.e., calculations for Am-241, Pu-239 . and U-233). For perspective, example results obtained for individual radionuclides are shown in Fig. 5.23.

A partial summary of the SECO-TRANSPORT results calculated for a drilling intrusion at $1000 \mathrm{yr}$ and saved for use in later calculations is given in Table 5.7. As previously indicated, the results for $i=1$ and 2 (i.e., for $E 1$ and E2 intrusions) are not obtained from separate SECO-TRANSPORT calculations but rather are obtained by scaling the corresponding result for $i=3$ (i.e., for an E1E2 intrusion). In particular,

$$
\begin{aligned}
\operatorname{SECOUT}(h, i, j, k, l, m, n)= & {[\operatorname{PANOUTD}(h, i, j, 8, l, m, n) / \operatorname{PANOUTD}(h, 3, j, 8, l, m, n)] } \\
& \bullet \operatorname{SECOUT}(h, 3, j, k, l, m, n)
\end{aligned}
$$

for $i=1,2$.

\subsection{Monte Carlo Construction of CCDFs}

\subsubsection{Cases Requiring Unique CCDF Constructions}

The purpose of the mechanistic calculations discussed in Sects. 5.3.1 - 5.3.5 is to provide the results needed to approximate the integrals over $\mathscr{I} \not S$ in Eqs. (4.11) and (4.12) with the Monte Carlo procedure indicated in Eqs. (4.18) and (4.19). As a reminder, the integrals over $\mathscr{B} / \mathcal{I} S$ in Eqs. (4.11) and (4.12) yield the exceedance probabilities associated with EPA normalized releases of size 1 and 10, respectively. Thus, evaluation of these integrals is equivalent to the construction of a CCDF. In the implementation of the approximations indicated in Eqs. (4.18) and (4.19), interpolation procedures will be used to obtain normalized releases to the accessible environment due to random sequences of drilling intrusions from the releases calculated at fixed points in time as described in Sects. 5.3.1 - 5.3.5.

The total number of cases requiring CCDF constructions (i.e., approximations to the integrals over $F_{\mathscr{A}} A S$ in Eqs. (4.11) and (4.12)) in SPM-1 is equal to the number of unique values that $20 \ldots 245_{j k}$ can take on in Eqs. (4.5) and (4.6). In turn, this is equal to the total number of paths through the logic diagram that results from combining the first three branches in Fig. 5.4 (i.e., for the outcomes $\varepsilon_{21}, \varepsilon_{22}, \varepsilon_{23}$ associated with experimental program $E_{2}$ ) with the 24 paths through Fig. 5.11 (i.e., for the outcomes associated with experimental programs $E_{3}$ and $E_{7}$ and design modification 


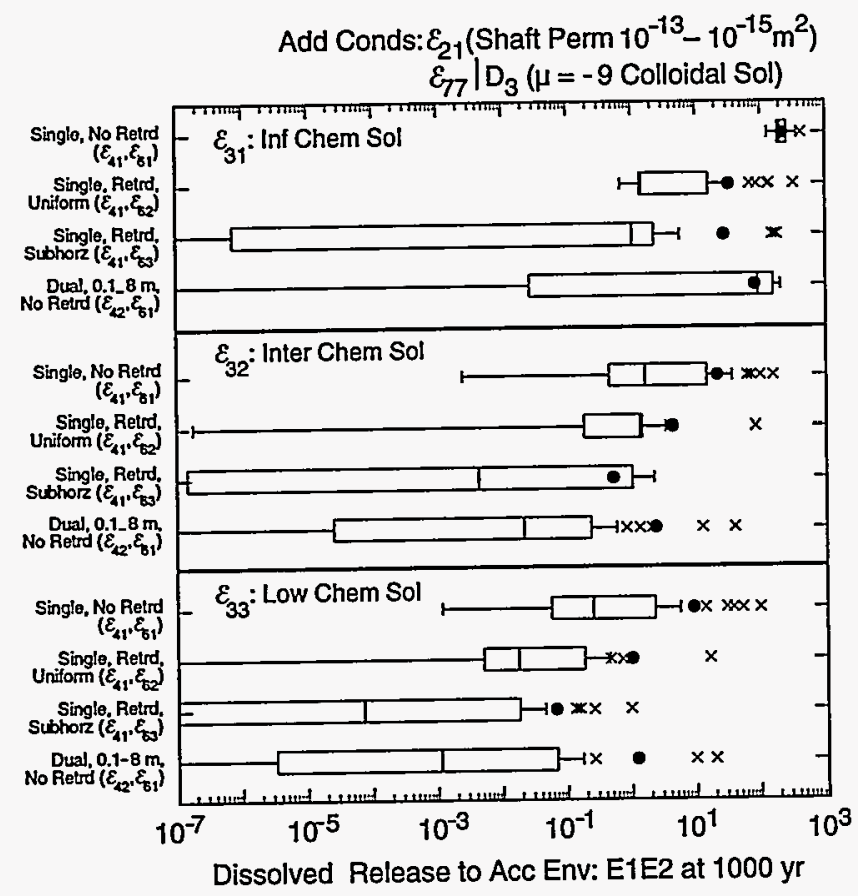

TRl-6342-4617-0
Add Conds: $\varepsilon_{22}$ (Shaft Perm $10^{-18}-10^{-16} \mathrm{~m}^{2}$ ) $\varepsilon_{77} \mid D_{3}(\mu=-9$ Colloidal Sol)

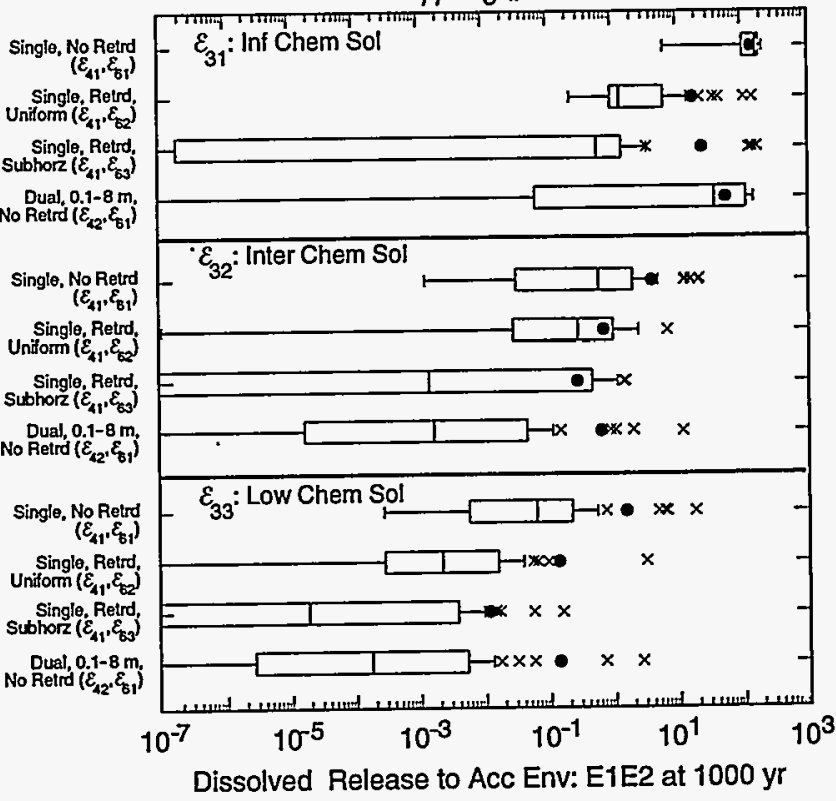

TRI-6342-4618-0

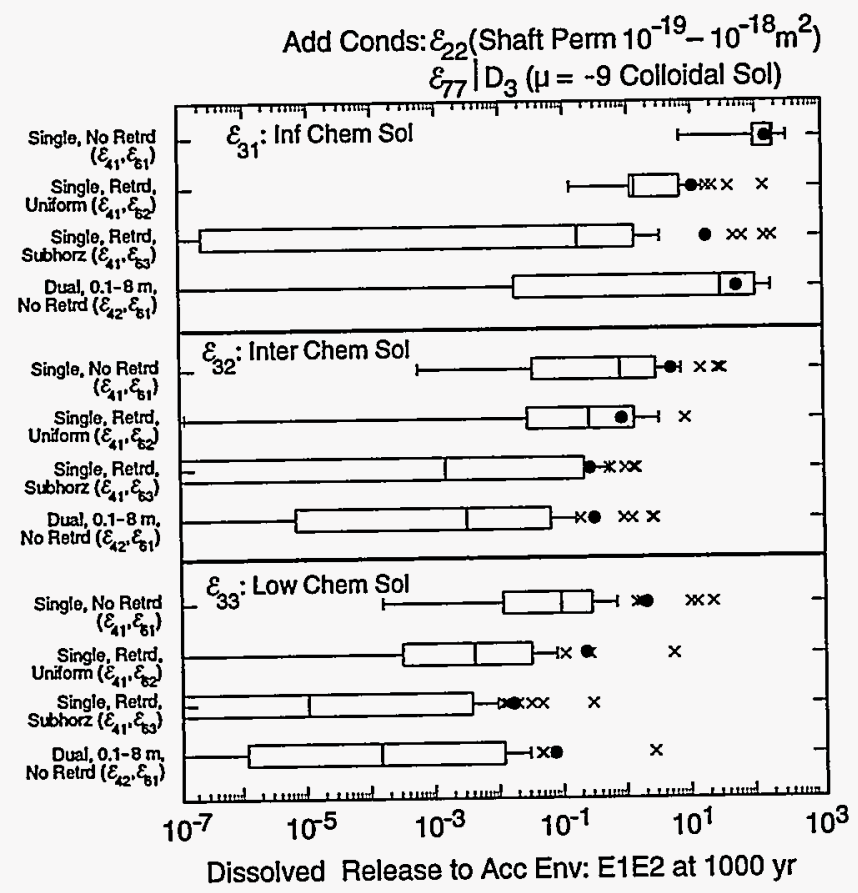

TRI-6342-4616-0

Figure 5.22. Total release of chemically-dissolved radionuclides to the accessible environment over 10,000 yr due to groundwater transport for an E1E2 intrusion at $1000 \mathrm{yr}, \mu=-9$ for colloidal solubility, and different assumptions involving shaft seal permeability, chemical solubility and retardation. 


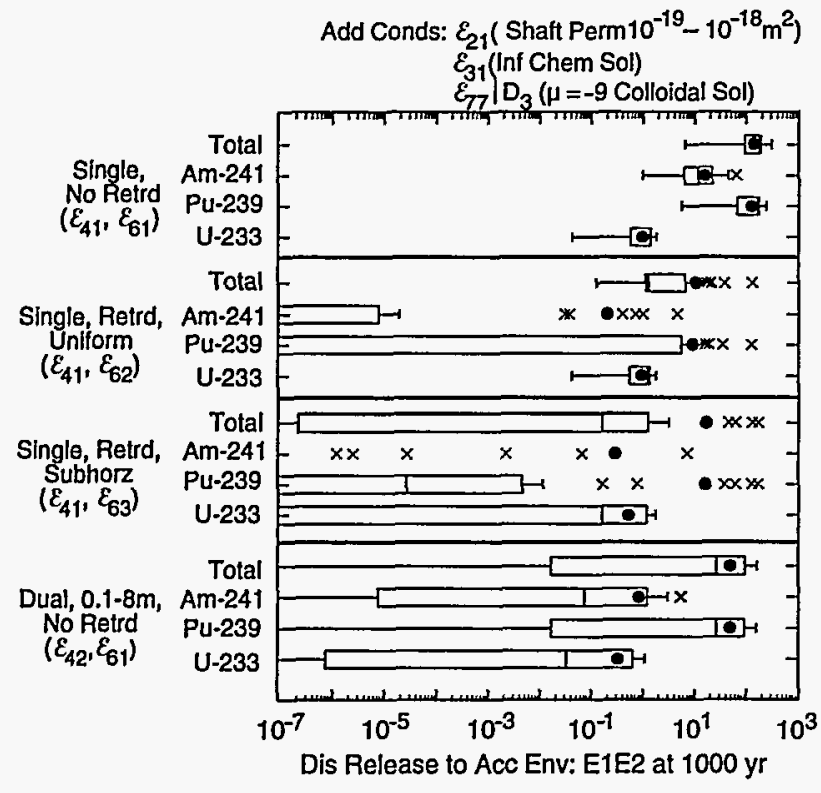

TRI-6342-4621-0

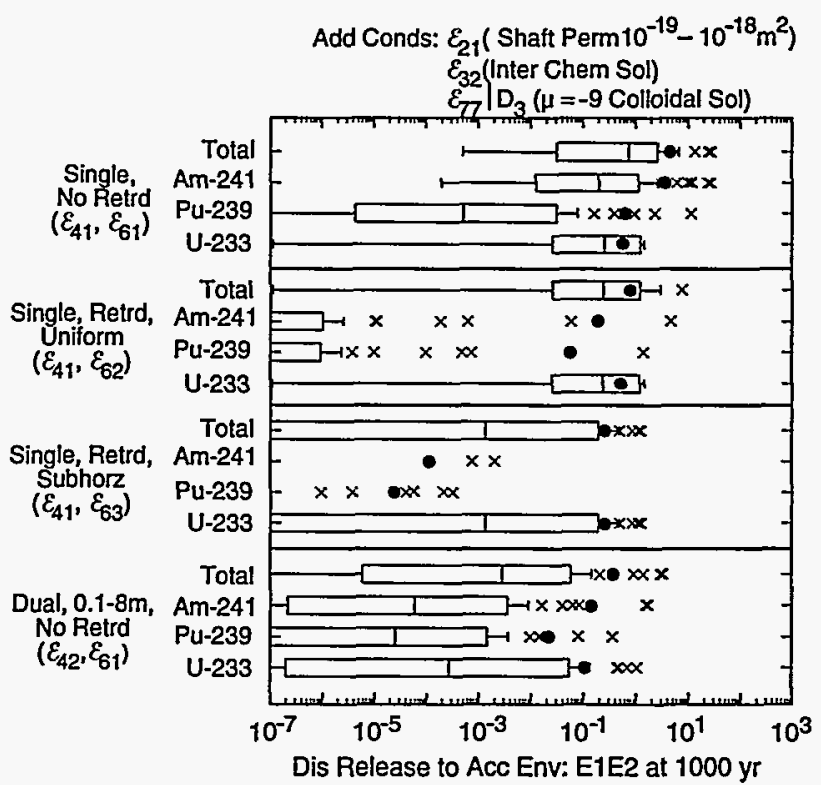

TRI-6342-4620-0

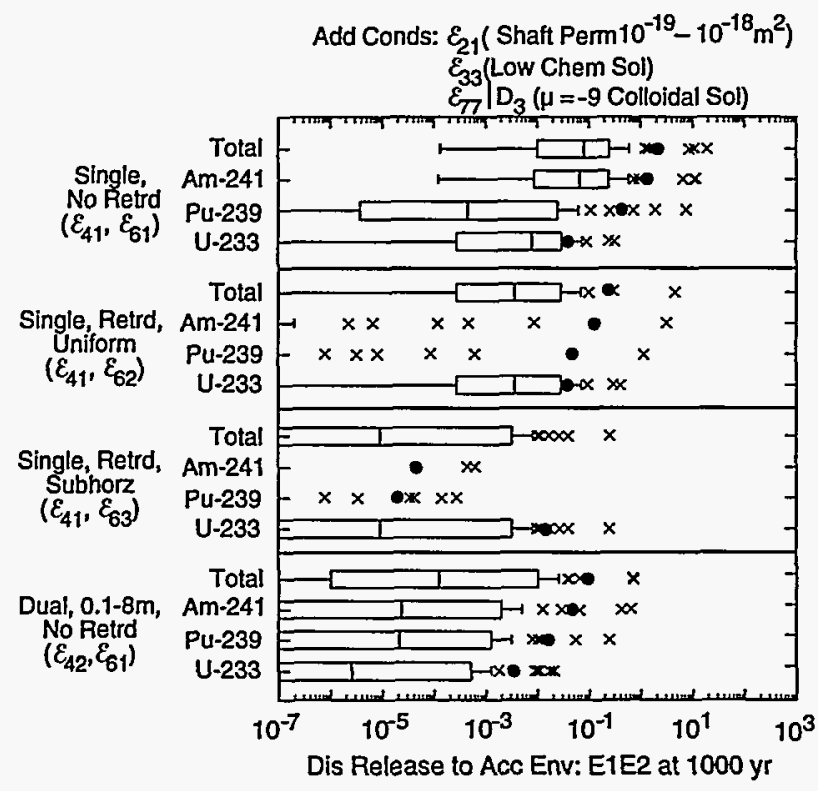

TRI-6342-4619-0

Figure 5.23. Release of individual chemically-dissolved radionuclides to the accessible environment over $10,000 \mathrm{yr}$ due to groundwater transport for an E1E2 intrusion at $1000 \mathrm{yr}$, low permeability shaft seals, $\mu=-9$ for colloidal solubility, and different assumptions involving chemical solubility and retardation. 
Table 5.7. Partial Summary of SECO-TRANSPORT Results Calculated for a Drilling Intrusion at $1000 \mathrm{yr}$ and Saved for Use in Later Calculations

$\operatorname{SECOUT}(h, i, j, k, l, m, n)=$ chemically-dissolved radionuclide release to the accessible environment (Ci)

where

$h \sim$ shaft permeability $\left(1 \Rightarrow \varepsilon_{21}\right.$, i.e., $10^{-15}-10^{-13} \mathrm{~m}^{2} ; 2 \Rightarrow \varepsilon_{22}$, i.e., $10^{-18}-10^{-16} \mathrm{~m}^{2} ; 3 \Rightarrow \varepsilon_{23}$, i.e., $10^{-19}-10^{-}$ $\left.18 \mathrm{~m}^{2}\right)$

$i \sim$ scenario identifier $(1 \Rightarrow \mathrm{E} 1 ; 2 \Rightarrow \mathrm{E} 2 ; 3 \Rightarrow \mathrm{E} 1 \mathrm{E} 2)$

$j \sim$ chemical/colloidal solubility identifier $\left(1 \Rightarrow\right.$ infinite chemical solubility, $10^{-9}$ colloidal solubility, i.e., $\varepsilon_{31}$, $\varepsilon_{77} \mid D_{3} ; 2 \Rightarrow$ intermediate chemical solubility, $10^{-9}$ colloidal solubility, i.e., $\varepsilon_{32}, \varepsilon_{77} \mid D_{3} ; 3 \Rightarrow$ low chemical solubility, $10^{-9}$ colloidal solubility, i.e., $\varepsilon_{33}, \varepsilon_{77} D_{3}$ )

$k \sim$ Culebra transport case $\left(1 \Rightarrow\right.$ single porosity, chemical retardation, subhorizontal fractures, i.e., $\varepsilon_{41}, \varepsilon_{63}, \varepsilon_{65}$; $2 \Rightarrow$ single porosity, chemical retardation, high-angle fractures, i.e., $\varepsilon_{41}, \varepsilon_{62}, \varepsilon_{64} ; 3 \Rightarrow$ dual porosity, no chemical retardation, $0.1-8 \mathrm{~m}$ fracture spacing, i.e., $\mathcal{E}_{42}, \mathcal{E}_{61}$ )

$l \sim$ integration period $(1 \Rightarrow 0-2000 \mathrm{yr} ; 2 \Rightarrow 0-4000 \mathrm{yr} ; 3 \Rightarrow 0-6000 \mathrm{yr} ; 4 \Rightarrow 0-8000 \mathrm{yr} ; 5 \Rightarrow 0-10,000 \mathrm{yr})$

$m \sim$ radionuclide $(1 \Rightarrow \mathrm{Am}-241,2 \Rightarrow \mathrm{Pu}-239,3 \Rightarrow \mathrm{U}-233)$

$n \sim$ sample element $(n=1,2, \ldots, 25)$

$D_{3}$ ), with the 20 paths through Fig. 5.18 (i.e., for the outcomes associated with experimental programs $E_{4}, E_{5}$ and $E_{6}$ ) and finally with the 4 paths through Fig. 5.10 (i.e., for the outcomes associated with experimental program $E_{8}$ ). This produces a total of 5760 cases (i.e., $3 \times 24 \times 20 \times 4$ ) requiring CCDF construction. However, there are only 5 paths through Fig. 5.18 that result in unique CCDFs. Thus, the number of required CCDF constructions is 1440 .

\subsubsection{Organization of Mechanistic Results for Monte Carlo CCDF Construction}

As shown in Fig. 5.24, the outcomes of the calculations described in Sects. 5.3.1 - 5.3.5 were assembled into a standard structure to permit the construction of CCDFs for the 1440 cases indicated in the preceding section. The first row in Fig. 5.24 indicates the particular case and sample element under consideration. In particular,

$I S H A F T \sim \quad$ shaft permeability identifier $\left(1 \Rightarrow 10^{-15}-10^{-13} \mathrm{~m}^{2}\right.$, i.e., $\varepsilon_{21} ; 2 \Rightarrow 10^{-18}-10^{16} \mathrm{~m}^{2}$, i.e., $\varepsilon_{22} ; 3 \Rightarrow$ $10^{-19}-10^{-18} \mathrm{~m}^{2}$, i.e., $\left.\varepsilon_{23}\right)$

ICHMSOL chemical solubility identifier $\left(1 \Rightarrow\right.$ infinite, i.e., $\varepsilon_{31} ; 2 \Rightarrow$ intermediate, i.e., $\varepsilon_{32} ; 3 \Rightarrow$ low, i.e., $\left.\varepsilon_{33}\right)$

JCOLSOL colloidal solubility identifier $\left(1 \Rightarrow\right.$ infinite, i.e., $\varepsilon_{71} \mid D_{3}, \varepsilon_{71} ; 2 \Rightarrow \mu=-3$, i.e., $\varepsilon_{72} ; 3 \Rightarrow \mu=-4$, i.e., $\varepsilon_{72} \mid D_{3}, \varepsilon_{73} ; 4 \Rightarrow \mu=-5$, i.e., $\varepsilon_{73} \mid D_{3}, \varepsilon_{74} ; 5 \Rightarrow \mu=-6$, i.e., $\varepsilon_{74} \mid D_{3}, \varepsilon_{75} ; 6 \Rightarrow \mu=-7$, i.e., $\varepsilon_{75} \mid D_{3}, \varepsilon_{76} ; 7 \Rightarrow \mu=-8$, i.e., $\varepsilon_{76} \mid D_{3}, \varepsilon_{77} ; 8 \Rightarrow \mu=-9$, i.e., $\left.\varepsilon_{77} \mid D_{3}\right)$ 
ISHAFT $=3$, ICHMSOL $=3$, JCOLSOL $=4, \operatorname{KCULTRN~}=2$, LSPAL $=1$, IOBS $=1$ TMCUT $=$

$\begin{array}{lllllll}1.000 E+02 & 1.250 \mathrm{E}+02 & 1.750 \mathrm{E}+02 & 3.500 \mathrm{E}+02 & 1.000 \mathrm{E}+03 \quad 3.000 \mathrm{E}+03 \quad 7.250 \mathrm{E}+03 \quad 1.000 \mathrm{E}+04\end{array}$ $\mathrm{RCUT}=$

$2.317 \mathrm{E}-04 \quad 2.064 \mathrm{E}-04 \quad 1.680 \mathrm{E}-04 \quad 1.060 \mathrm{E}-04 \quad 7.550 \mathrm{E}-05 \quad 6.527 \mathrm{E}-05 \quad 5.613 \mathrm{E}-05 \quad 5.122 \mathrm{E}-05$

$2.317 \mathrm{E}-03 \quad 2.064 \mathrm{E}-03 \quad 1.680 \mathrm{E}-03 \quad 1.061 \mathrm{E}-03 \quad 7.550 \mathrm{E}-04 \quad 6.526 \mathrm{E}-04 \quad 5.612 \mathrm{E}-04 \quad 5.122 \mathrm{E}-04$

2.317E-02 2.064E-02 $1.680 \mathrm{E}-02 \quad 1.061 \mathrm{E}-02 \quad 7.550 \mathrm{E}-03 \quad 6.526 \mathrm{E}-03 \quad 5.612 \mathrm{E}-03 \quad 5.122 \mathrm{E}-03$

$2.317 \mathrm{E}-012.064 \mathrm{E}-01$ 1.680E-01 $1.061 \mathrm{E}-01 \quad 7.550 \mathrm{E}-02 \quad 6.526 \mathrm{E}-02 \quad 5.612 \mathrm{E}-02 \quad 5.122 \mathrm{E}-02$

$\begin{array}{lllllllll}1.185 E-02 & 1.122 E-02 & 1.020 E-02 & 8.019 E-03 & 5.045 E-03 & 3.403 E-03 & 2.837 E-03 & 2.567 E-03\end{array}$

$\begin{array}{lllllllll}5.894 \mathrm{E}-03 & 5.249 \mathrm{E}-03 & 4.273 \mathrm{E}-03 & 2.697 \mathrm{E}-03 & 1.920 \mathrm{E}-03 & 1.660 \mathrm{E}-03 & 1.427 \mathrm{E}-03 & 1.303 \mathrm{E}-03\end{array}$ RSPAL $=$

$0.000 \mathrm{E}+00 \quad 0.000 \mathrm{E}+00 \quad 4.138 \mathrm{E}-02 \quad 5.830 \mathrm{E}-02 \quad 4.287 \mathrm{E}-02 \quad 3.304 \mathrm{E}-02 \quad 2.643 \mathrm{E}-02 \quad 1.947 \mathrm{E}-02$ TMGWTRN =

$1.000 \mathrm{E}+02 \quad 1.000 \mathrm{E}+03 \quad 3.000 \mathrm{E}+03 \quad 5.000 \mathrm{E}+03 \quad 7.000 \mathrm{E}+03 \quad 9.000 \mathrm{E}+03 \quad 1.000 \mathrm{E}+04$

$\operatorname{RE1}=$

1.604E-02 1.604E-02 8.614E-03 1.344E-03 4.727E-04 3.957E-04 0.000E+00

$\mathrm{RE} 2=$

2.601E-02 2.601E-02 1.397E-02 2.180E-03 $7.666 \mathrm{E}-04 \quad 6.417 \mathrm{E}-04 \quad 0.000 \mathrm{E}+00$

$\mathrm{RE} 3 .=$

$2.245 \mathrm{E}-012.245 \mathrm{E}-01 \quad 1.206 \mathrm{E}-01 \quad 1.882 \mathrm{E}-02 \quad 6.618 \mathrm{E}-03 \quad 5.539 \mathrm{E}-03 \quad 0.000 \mathrm{E}+00$

Figure 5.24. Example of Input File Used in Monte Carlo CCDF Construction.

KCULTRN Culebra transport identifier $\left(1 \Rightarrow\right.$ single porosity, no retardation, i.e., $\varepsilon_{61}\left|\varepsilon_{41}, \varepsilon_{61}\right| \varepsilon_{51}$; $2 \Rightarrow$ single porosity, retardation, uniform clay, i.e., $\varepsilon_{62}\left|\varepsilon_{41}, \varepsilon_{64}\right| \varepsilon_{41}, \varepsilon_{62}\left|\varepsilon_{51}, \varepsilon_{64}\right| \varepsilon_{51}$; $3 \Rightarrow$ single porosity, retardation, nonuniform clay, flow in subhorizontal fractures, i.e., $\varepsilon_{63} \mid \varepsilon_{41}$, $\varepsilon_{65}\left|\varepsilon_{41}, \varepsilon_{63}\right| \varepsilon_{51}, \varepsilon_{65} \mid \varepsilon_{51} ; 4 \Rightarrow$ dual porosity, no retardation, $0.1-8 \mathrm{~m}$ fracture spacing, i.e., $\varepsilon_{61} \mid \varepsilon_{42} ; 5 \Rightarrow$ complete retardation of chemically-dissolved release to Culebra, i.e., $\varepsilon_{6 j} \mid \varepsilon_{42}, j=$ $\left.2,3,4,5, \varepsilon_{6 j} \mid \varepsilon_{5 i}, i=2,3, j=1,2,3,4,5\right)$

LSPAL $\quad$ spallings identifier $\left(1 \Rightarrow\right.$ baseline, i.e., $\varepsilon_{81} ; 2 \Rightarrow$ baseline $/ 2$, i.e., $\mathcal{E}_{82} ; 3 \Rightarrow$ baseline $/ 10$, i.e., $\varepsilon_{83}$; $4 \Rightarrow$ baseline $/ 100$, i.e., $\left.\varepsilon_{84}\right)$

IOBS $\quad$ sample element identifier $(I O B S=1,2, \ldots, 25)$.

As indicated earlier, there are 1440 cases (i.e., $3 \times 3 \times 8 \times 5 \times 4$ ) for CCDF construction. Further, 25 CCDFs are constructed for each case. In particular, 1440 is the number of cases for which the integrals in Eqs. (4.5) and (4.6) will be evaluated and 25 is the value for $n L H S$ appearing in Eqs. (4.11) and (4.12).

The row identified by TMCUT contains the times at which cuttings and spallings releases were calculated with CUTTINGS and SPALLINGS. As indicated in Eq. (5.7), these times are 100, 125, 175, 350, 1000, 3000, 7250 and $10,000 \mathrm{yr}$ in SPM-1. 
The rows identified by RCUT contain the cuttings releases calculated at the times associated with TMCUT. In particular, the six rows under RCUT correspond to releases for a borehole encountering waste of activity level 1 , activity level 2 , activity level 3 , activity level 4 , activity level 5 and average activity level, respectively. The indicated releases were calculated with CUTTINGS as described in Sect. 5.3.2.

The rows identified by RSPAL contain the spallings releases calculated at the times associated with TMCUT under the assumption that the first drilling intrusion into the repository occurs at the indicated time. In the Monte Carlo procedure for CCDF construction described in Sect. 5.4.3, only the first drilling intrusion into the repository will result in a spallings release due to the depressurization that results from the high permeability assumed for boreholes. The indicated releases were calculated with SPALLINGS as described in Sect. 5.3.2 and used the corresponding pressure history for ISHAFT and IOBS calculated with BRAGFLO (see Fig. 5.2).

The row identified by TMGWTRN contains the times at which drilling intrusions occur and possibly initiate a radionuclide release from the repository due to groundwater transport, although it is possible that some release may take place prior to the drilling intrusion due to flow up the shaft. In SPM-1, these times are 100, 1000, 3000, 5000, 7000,9000 and 10,000 yr. As a reminder, the preceding times are not the drilling intrusion times actually used in CCDF construction, which are randomly sampled. Rather, the times associated with TMGWTRN are used in calculating releases to the accessible environment that are then used in conjunction with interpolation procedures to determine the releases associated with the random drilling times actually used in CCDF construction.

The next three rows are identified by $R E 1, R E 2$ and $R E 3$ and correspond to the total normalized releases to the accessible environment for $\mathrm{E} 1$ intrusions (RE1), $\mathrm{E} 2$ intrusions (RE2) and E1E2 intrusions (RE3), respectively, at the times associated with TMGWTRN. Each release is the sum of three components: a direct release to the surface (derived from PANOUTS in Table 5.6), which was always zero in SPM-1; a colloidally-sorbed release (derived from PANOUTC in Table 5.6), which is assumed to transport instantaneously to the accessible environment; and the release to the accessible environment that results from the transport of chemically-dissolved radionuclides through the Culebra (derived from PANOUTD in Table 5.6 and SECOUT in Table 5.7). The algorithm used to define RE1, $R E 2$ and $R E 3$ is indicated in Table 5.8. In particular, the counter $i$ associated with $R E L G W$ in Steps 2 and 3 in Table 5.8 corresponds to $R E 1, R E 2$ and $R E 3$ (i.e., $1 \Rightarrow R E 1,2 \Rightarrow R E 2,3 \Rightarrow R E 3$ ) and the counter $j$ corresponds to intrusion time (i.e., $1 \Rightarrow 100 \mathrm{yr}, 2 \Rightarrow 1000 \mathrm{yr}, 3 \Rightarrow 3000 \mathrm{yr}, 4 \Rightarrow 5000 \mathrm{yr}, 5 \Rightarrow 7000 \mathrm{yr}, 6 \Rightarrow 9000 \mathrm{yr}, 7 \Rightarrow 10,000$ yr).

\subsubsection{Numerical Implementation of Monte Carlo CCDF Construction}

The following describes the algorithm used to construct the release to the accessible environment associated with a single $10,000 \mathrm{yr}$ history of random drilling intrusions. This release corresponds to the quantity $F\left(\mathbf{x}_{m}, \mathrm{f}_{n}\right)$ in 
Table 5.8. Summary of Algorithm Used to Define Normalized Releases to the Accessible Environment Due to Groundwater Transport (i.e., RE1, RE2, RE3 in Fig. 5.24)

1. Assign values to CULTRN for transport of dissolved radionuclides to the accessible environment without a correction for radioactive decay:

CULTRN (ISHAFT, $i$, ICHMSOL, JCOLSOL, $j, k$, IOBS)

$=$ PANOUTD(ISHAFT, $i$, ICHMSOL, JCOLSOL, $j, k, I O B S)$ for KCULTRN $=1$

$=[$ PANOUTD (ISHAFT, $i, I C H M S O L, J C O L S O L, j, k, I O B S)$

I PANOUTD(ISHAFT, $i$, ICHMSOL, $8, j, k$, IOBS)]

SECOUT(ISHAFT, $i, I C H M S O L, K C U L T R N-1, j, k, I O B S$ ) for $K C U L T R N=2,3,4$

$=0$ for $K C U L T R N=5$

where $i=1,2,3 \sim$ release type (i.e., $1 \Rightarrow \mathrm{E} 1,2 \Rightarrow \mathrm{E} 2,3 \Rightarrow \mathrm{E} 1 \mathrm{E} 2$ ), $j=1,2,3,4,5 \sim$ integration period (i.e., $1 \Rightarrow 0$ to $2000 \mathrm{yr}, 2 \Rightarrow 0$ to $4000 \mathrm{yr}, 3 \Rightarrow 0$ to $6000 \mathrm{yr}, 4 \Rightarrow 0$ to $8000 \mathrm{yr}, 5 \Rightarrow 0$ to $10,000 \mathrm{yr}$ ), $k=1,2,3 \sim$ radionuclide ( $1 \Rightarrow \mathrm{Am}-241 ; 2 \Rightarrow \mathrm{Pu}-239 ; 3 \Rightarrow \mathrm{U}-233$ ).

2. Assign values to RELGW for normalized release to the accessible environment due to groundwater transport for intrusions at $1000,3000,5000,7000$ and $9000 \mathrm{yr}$ with correction for time of intrusion:

RELGW (i,j, ISHAFT, ICHMSOL, JCOLSOL, IOBS)

$$
\begin{aligned}
=\sum_{k=1}^{3} \quad & {[\text { PANOUTS(ISHAFT, } i, \text { ICHMSOL, JCOLSOL, } 7-j, k, \text { IOBS }) } \\
& + \text { PANOUTC(ISHAFT, } i, \text { ICHMSOL, JCOLSOL, } 7-j, k, I O B S) \\
& + \text { CULTRN }(I S H A F T, i, I C H M S O L, J C O L S O L, 7-j, k, I O B S)] \\
& \cdot[\exp \{[-\ln (2) / H L I F E(k)][j-2][2000 \mathrm{yr}]\}]\left[\left(10^{6} \mathrm{Ci} / C\right) / L_{k}\right]
\end{aligned}
$$

where $i=1,2,3 \sim$ release type (i.e., $1 \Rightarrow \mathrm{E} 1,2 \Rightarrow \mathrm{E} 2,3 \Rightarrow \mathrm{E} 1 \mathrm{E} 2$ ), $j=2,3,4,5,6 \sim$ time at which drilling intrusion occurs (i.e., $2 \Rightarrow 1000 \mathrm{yr}, 3 \Rightarrow 3000 \mathrm{yr}, 4 \Rightarrow 5000 \mathrm{yr}, 5 \Rightarrow 7000 \mathrm{yr}, 9 \Rightarrow 9000 \mathrm{yr}$ ), $k$-radionuclide (i.e., $1 \Rightarrow \mathrm{Am}$ $241,2 \Rightarrow$ Pu-239, $3 \Rightarrow$ U-233), HLIFE (k) halflife for radionuclide $k$ (i.e., $1 \Rightarrow 432 \mathrm{yr}, 2 \Rightarrow 2.41 \times 10^{4} \mathrm{yr}, 3 \Rightarrow 1.59$ $\times 10^{5} \mathrm{yr}$ ), $L_{k} \sim$ release limit for radionuclide $k$ (i.e., $1 \Rightarrow 100 \mathrm{Ci}, 2 \Rightarrow 100 \mathrm{Ci}, 3 \Rightarrow 100 \mathrm{Ci}$ ), $C \sim$ actinide inventory emplaced in repository (i.e., $C=1.27 \times 10^{6} \mathrm{Ci}$ ).

3. Assign values to $R E L G W$ for normalized release to the accessible environment due to groundwater transport for intrusions at 100 and $10,000 \mathrm{yr}$ :

RELGW $(i, 1$, ISHAFT, ICHMSOL, JCOLSOL, IOBS $)=$ RELGW $(i, 2$, ISHAFT, ICHMSOL, JCOL, IOBS)

RELGW $(i, 7$, ISHAFT, ICHMSOL, JCOLSOL, IOBS $)=0$

with notation as in Step 2. 
Eq. (4.17) and will be constructed from an array of results of the form shown in Fig. 5.24. The vector $f_{n}$ designates a single 10,000 yr history of random drilling intrusions (i.e., an element of $\$ \& S$ in Eq. (4.17)) and has the form

$$
\mathbf{f}_{n}=\left[t B H_{1}, l B H_{1}, a B H_{1}, t B H_{2}, l B H_{2}, a B H_{2}, \ldots, t B H_{n B H}, l B H_{n B H}, a B H_{n B H}\right]
$$

in SPM-1, where $t B H_{j}$ is the time (yr) at which the $j^{\text {th }}$ drilling intrusion occurs, $l B H_{j}$ is the location of the $j^{\text {th }}$ drilling intrusion, $a B H_{j}$ is the activity level of waste $\left(\mathrm{Ci} / \mathrm{m}^{2}\right)$ penetrated by the $j^{\text {th }}$ drilling intrusion, $n B H$ is the number of drilling intrusions, and the times $t B H_{j}$ are assumed to be ordered so that $t B H_{j} \leq t B H_{j+1}$ for $j=1,2, \ldots, n B H-1$. The distribution that gives rise to the $f_{n}$ is defined by the probability space $70\left\{245\right.$. Similarly, $\mathbf{x}_{m}$ is an element of $X \not A S_{j \varepsilon}$ and is sampled from a distribution defined by the probability space $70 X \mathcal{A} 5_{j \varepsilon}$. There are 25 values for $x_{m}$ and hence 25 compilations of the form shown in Fig. 5.24 for each of the 1440 cases requiring CCDF construction in SPM-1. For the discussion in this section, $\mathbf{x}_{m}$ is assumed to be fixed (i.e., a particular compilation of the form shown in Fig. 5.24 is under consideration).

With the assumption that drilling intrusions follow a Poisson process with a rate constant $\lambda$, the probability $\operatorname{prob}(n)$ that exactly $n$ drilling intrusions occur in the time interval $\left[t_{0}, t_{\max }\right]$ is given by

$$
\operatorname{prob}(n)=\left[\left(t_{\max }-t_{0}\right)^{n} \lambda^{n} / n !\right] \exp \left[-\left(t_{\max }-t_{0}\right) \lambda\right]
$$

In PAs for the WIPP, $t_{0}=100 \mathrm{yr}$ due to the assumption of $100 \mathrm{yr}$ of administrative control and $t_{\max }=10,000 \mathrm{yr}$ as specified in 191.13(a). The value for $n B H$ in Eq. (5.16) is obtained by randomly sampling a number $r$ from the interval $[0,1]$ and then assigning $n B H$ the smallest integer value for which the inequality

$$
r \leq \sum_{n=0}^{n B H} \operatorname{prob}(n)
$$

is satisfied.

Once a value for $n B H$ is selected, an additional $3 n B H$ random numbers can be used to assign the values for $t B H_{j}, l B H_{j}$ and $a B H_{j}, j=1,2, \ldots, n B H$. Specifically, let $r t B H_{j}, r l B H_{j}$ and $r a B H_{j}, j=1,2, \ldots, n B H$, represent numbers randomly selected from the interval $[0,1]$. Then, the times $t B H_{j}$ are defined by

$$
t B H_{j}=t_{0}+r t B H_{j}\left(t_{\max }-t_{0}\right)
$$

where $t_{0}=100 \mathrm{yr}$ and $t_{\max }=10,000 \mathrm{yr}$ as previously indicated.

The location of drilling intrusions within the repository can be discretized by defining a sequence of locations (points) as indicated in Fig. 5.25. The integers 


$$
i D L=1,2, \ldots, n D L
$$

then designate possible locations of drilling intrusions. Location 1 corresponds to a drilling intrusion in the experimental or operations region. Locations $2,3, \ldots, n D L$ correspond to drilling intrusions within the waste panels. The analysis for SPM-1 used the $n D L=73$ locations indicated in Fig. 5.25.

The random number $r l B H_{j}$ can be used to identify the location (point) in Fig. 5.25 corresponding to the $j^{\text {th }}$ drilling intrusion. For notational convenience, let

$$
\begin{aligned}
& a W D=\text { area }\left(\mathrm{m}^{2}\right) \text { of waste disposal region, } \\
& a O P=\text { area }\left(\mathrm{m}^{2}\right) \text { of operations region, } \\
& a E X=\text { area }\left(\mathrm{m}^{2}\right) \text { of experimental region, } \\
& a T O T=a W D+a O P+a E X
\end{aligned}
$$

Then, the $j^{\text {th }}$ drilling intrusion occurs at Location 1 (i.e., in the experimental or operations region) and is assigned a value of $l B H_{j}=1$ if

$$
0 \leq r l B H \leq(a E X+a O P) / a T O T
$$

If the preceding inequality is not satisfied, then the intrusion occurs in the waste disposal region at Location $l B H_{j}$ given by

$$
l B H_{j}=[r N E W n D L]+2
$$

where $r N E W$ is the random number between 0 and 1 defined by

$$
r N E W=(1-r l B H) \text { aTOT } / a W D
$$

and $[\sim]$ designates the greatest integer function.

Finally, $a B H_{j}$ corresponds to penetration of waste of activity level $m$, where $m$ is the smallest integer such that

$$
r a B H_{j} \leq \sum_{l=1}^{m} p L_{l}
$$

and $p L_{l}$ is the probability that a randomly placed drilling intrusion will penetrate waste of activity level $l$. The values used for $p L_{l}$ in SPM-1 are given in Table 5.2. 


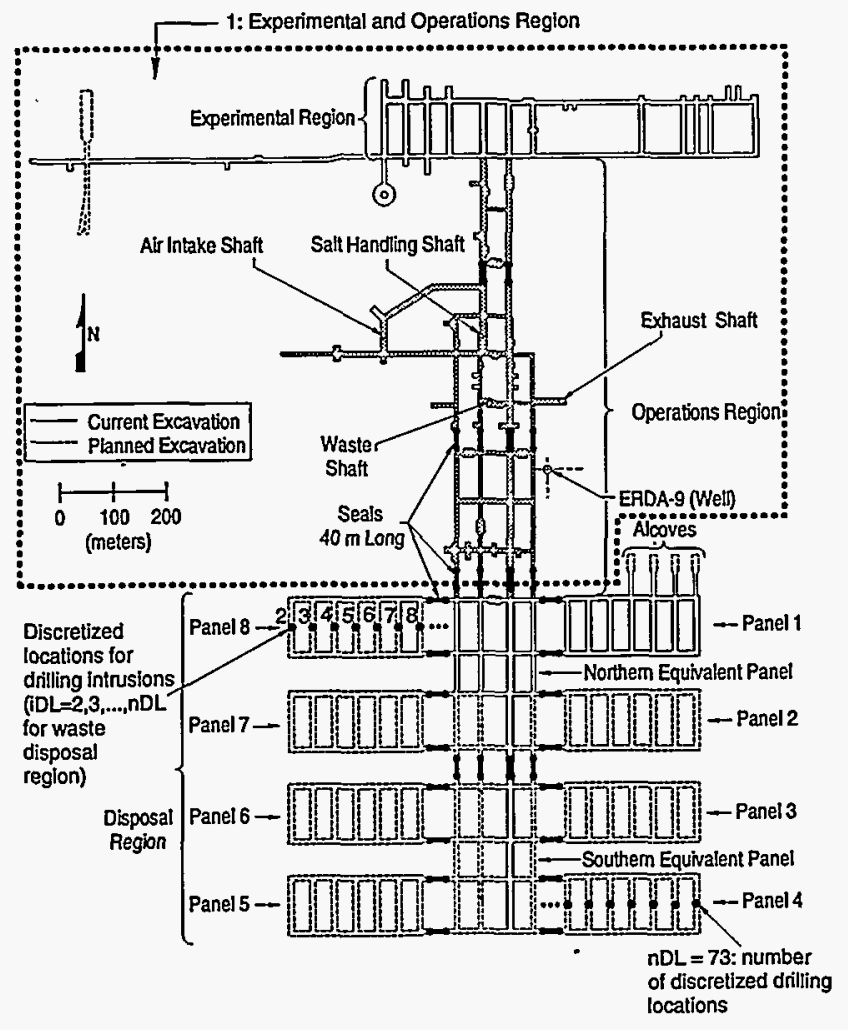

TRI- 6334-206-15

Figure 5.25. Excavated areas and planned seals in the WIPP repository (modified from Refs. 85, 86).

The normalized release to the accessible environment $F\left(\mathbf{x}_{m}, f_{n}\right)$ is given by

$$
F\left(\mathbf{x}_{m}, \mathbf{f}_{n}\right)=n R C\left(\mathrm{f}_{n}\right)+n R G\left(\mathbf{f}_{n}\right)
$$

where $n R C\left(f_{n}\right)$ is the normalized release to the accessible environment due to cuttings and spallings removal, $n R G\left(\mathrm{f}_{n}\right)$ is the normalized release to the accessible environment due to groundwater transport and the dependence on $\mathbf{x}_{m}$ is omitted for notational convenience. The releases $n R C\left(f_{n}\right)$ and $n R G\left(f_{n}\right)$ can be determined separately and then summed to obtain $n R\left(\mathrm{f}_{n}\right)$. The information available for the construction of $n R C\left(\mathrm{f}_{n}\right)$ and $n R G\left(\mathrm{f}_{n}\right)$ is summarized in Table 5.9 and corresponds to the information in Fig. 5.24.

At the same time the vector $f_{n}$ in Eq. (5.16) is generated, the following vectors of summary information can also be produced:

$$
\begin{aligned}
\mathbf{c C}\left(\mathbf{f}_{n}\right) & =[i C(1), i A(1), \ldots, i C(n B H), i A(n B H)], \\
\operatorname{cPB}\left(\mathfrak{f}_{n}\right) & =[t P B(1), i P B(1), l P B(1), \ldots, t P B(n P B), i P B(n P B), l P B(n P B)],
\end{aligned}
$$


Table 5.9 Information Available for Construction of Cuttings Release, $n R C\left(f_{n}\right)$, and Groundwater Release, $n R G\left(\mathrm{f}_{n}\right)$, to the Accessible Environment

\begin{tabular}{|c|c|}
\hline Quantity & Definition \\
\hline$t C_{i}$ & $\begin{array}{l}\text { Time (yr) at which calculated results are available for release to the surface environment due to } \\
\text { cuttings removal and spallings for } i=1,2, \ldots, n C T, t C_{1}=100 \mathrm{yr} \text { and } t C_{n C T}=10,000 \mathrm{yr} \text {. } \\
\text { Corresponds to times associated with TIMCUT in Fig. } 5.24 \text {. }\end{array}$ \\
\hline$r C_{i j}$ & $\begin{array}{l}\text { Normalized release to the accessible environment due to a drilling intrusion at time } t C_{i}, i=1,2 \text {, } \\
\ldots, n C T \text {, that penetrates waste of activity level } j, j=1,2, \ldots, n A L \text {. Corresponds to releases } \\
\text { associated with } R C U T \text { for activity levels } 1,2,3,4,5 \text { in Fig. 5.24. }\end{array}$ \\
\hline$r S_{i}$ & $\begin{array}{l}\text { Normalized release to the accessible environment due to spallings associated with a drilling } \\
\text { intrusion at time } t C_{i}, i=1,2, \ldots, n C T \text {. Corresponds to releases associated with RSPAL in Fig. } \\
\text { 5.24. }\end{array}$ \\
\hline$t G_{i}$ & $\begin{array}{l}\text { Time (yr) at which calculated results are available for release to the accessible environment due } \\
\text { to groundwater transport for } i=1,2, \ldots, n G T, t G_{1}=100 \mathrm{yr} \text { and } t G_{n G T}=10,000 \mathrm{yr} \text {. } \\
\text { Corresponds to times associated with TMGWTRN in Fig. 5.24. }\end{array}$ \\
\hline$r E 1_{i}$ & $\begin{array}{l}\text { Release to accessible environment due to groundwater transport resulting from an } \mathrm{E} 1 \text { intrusion } \\
\text { at time } t G_{i} \text {. Corresponds to releases associated with } R E I \text { in Fig. } 5.24 \text {. }\end{array}$ \\
\hline$r E 2_{i}$ & $\begin{array}{l}\text { Release to accessible environment due to groundwater transport resulting from an E2 intrusion } \\
\text { at time } t G_{i} \text {. Corresponds to releases associated with } R E 2 \text { in Fig. } 5.24 \text {. }\end{array}$ \\
\hline$r E 1 E 2_{i}$ & $\begin{array}{l}\text { Release to accessible environment due to groundwater transport resulting from an E1E2 } \\
\text { intrusion at time } t G_{i} \text { obtained under assumption that entire inventory of a single waste panel can } \\
\text { be accessed. Corresponds to releases associated with } R E 3 \text { in Fig. } 5.24 \text {. }\end{array}$ \\
\hline
\end{tabular}

$$
\operatorname{cNPB}\left(\mathrm{f}_{n}\right)=[t N P B(1), i N P B(1), l N P B(1), \ldots, t N P B(n N P B), i N P B(n N P B), l N P B(n N P B)]
$$

and

$$
\mathrm{CPA}=[t P A(1), i P A(1), \ldots, t P A(n P A), i P A(n P A)],
$$

where $i C(j)$ is the integer such that $t C_{i C(j)} \leq t B H_{j}<t C_{i C(j)+1}, i A(j)$ is the integer designator for the activity level encountered by the $j^{\text {th }}$ drilling intrusion, $t P B(j)$ is the time (yr) at which the $j^{\text {th }}$ drilling intrusion that penetrates pressurized brine occurs, $i P B(j)$ is the integer such that $t G_{i P B(j)} \leq t P B(j)<t G_{i P B(j)+1}, \quad l P B(j)$ is the location of the $j^{\text {th }}$ drilling intrusion that penetrates pressurized brine, $n P B$ is the number of drilling intrusions that penetrate pressurized brine, $t P A(j)$ is the time $(\mathrm{yr})$ at which the initial penetration of the $j^{\text {th }}$ waste panel to experience one or more drilling intrusions occurs with $t P A(j) \leq t P A(j+1)$ for $j=1,2, \ldots, n P A-1, i P A(j)$ is the integer such that the initial penetration of the $j^{\text {th }}$ waste panel to experience one or more drilling intrusions occurs in time interval $\left[t G_{i P A(j)}\right.$, $\left.t G_{i P A(j)+1}\right], n P A$ is the number of waste panels penetrated by one or more drilling intrusions, and $t N P B(j)$, $i N P B(j)$, 
for $i=1,2, \ldots, n D L$. The assumed location of pressurized brine beneath the waste panels in SPM-1 is shown in Fig. 5.26; further, the experimental and operations regions are assumed to be entirely underlain by pressurized brine (Ref. 87).

The determinations of $n R G\left(\mathrm{f}_{n}\right)$ for Cases 2 and 3 are similar and will be presented together. Specifically,

$$
n R G\left(\mathbf{f}_{n}\right)=\sum_{j=1}^{n P A}\left\{r E 1_{i P A(j)}+f T_{j}\left[r E 1_{i P A(j)+1}-r E 1_{i P A(j)}\right]\right\}
$$

for Case 2 (i.e., for $\mathrm{E} 1$ intrusions only) and

$$
n R G\left(\mathbf{f}_{n}\right)=\sum_{j=1}^{n P A}\left\{r E 2_{i P A(j)}+f T_{j}\left[r E 2_{i P A(j)+1}-r E 2_{i P A(j)}\right]\right\}
$$

for Case 3 (i.e., for $\mathrm{E} 2$ intrusions only), where

$$
f T_{j}=\left[t P A(j)-t G_{i P A(j)}\right] /\left[t G_{i P A(j)+1}-t G_{i P A(j)}\right]
$$

is used for interpolation on intrusion time.

The determination of $n R G\left(\mathrm{f}_{n}\right)$ for Case 4 is now considered. As indicated in Eq. (5.39), this case occurs when at least one drilling intrusion penetrates pressurized brine and at least one drilling intrusion does not penetrate pressurized brine. Brine flow is assumed to be channelized in the sense that flow takes place from an E1 intrusion to either the closest E2 intrusion that exists at the time the E1 intrusion occurs or, if no E2 intrusion exists at the time of the first E1 intrusion, to the first E2 intrusion that occurs after the E1 intrusion. Once an E1 intrusion establishes a connection with an E2 intrusion, the resultant preferential flow path is assumed to hold regardless of any subsequent E2 intrusions that may occur (i.e., a new flow path will not be established even if a second E2 intrusion occurs at a later time that is closer to the location of the E1 intrusion than the E2 intrusion used in establishing the initial flow path).

Each E1 intrusion associated with the vector $\mathrm{CPB}\left(\mathrm{f}_{n}\right)$ in Eq. (5.31) generates a flow path to one of the E2 intrusions associated with the vector $\operatorname{CNPB}\left(f_{n}\right)$ in Eq. (5.32). The flow path associated with the $j^{\text {th }}$ drilling intrusion in $\mathrm{CPB}\left(\mathrm{f}_{n}\right)$ will access a fraction $f W_{j}$ of the waste inventory, where (1)

$$
f W_{j}=\min \{f L[l B P(j), l N B P(k)], k=1,2, \ldots, n M, \text { where } n M \text { is the largest integer such that } t N B P(n M) \leq t B P(j)\}
$$

if $t N B P(1) \leq t B P(j)$ (i.e., if the drilling intrusion that penetrates pressurized brine at time $t P B(j)$ is preceded by one or more drilling intrusions that fail to penetrate pressurized brine), (2) 


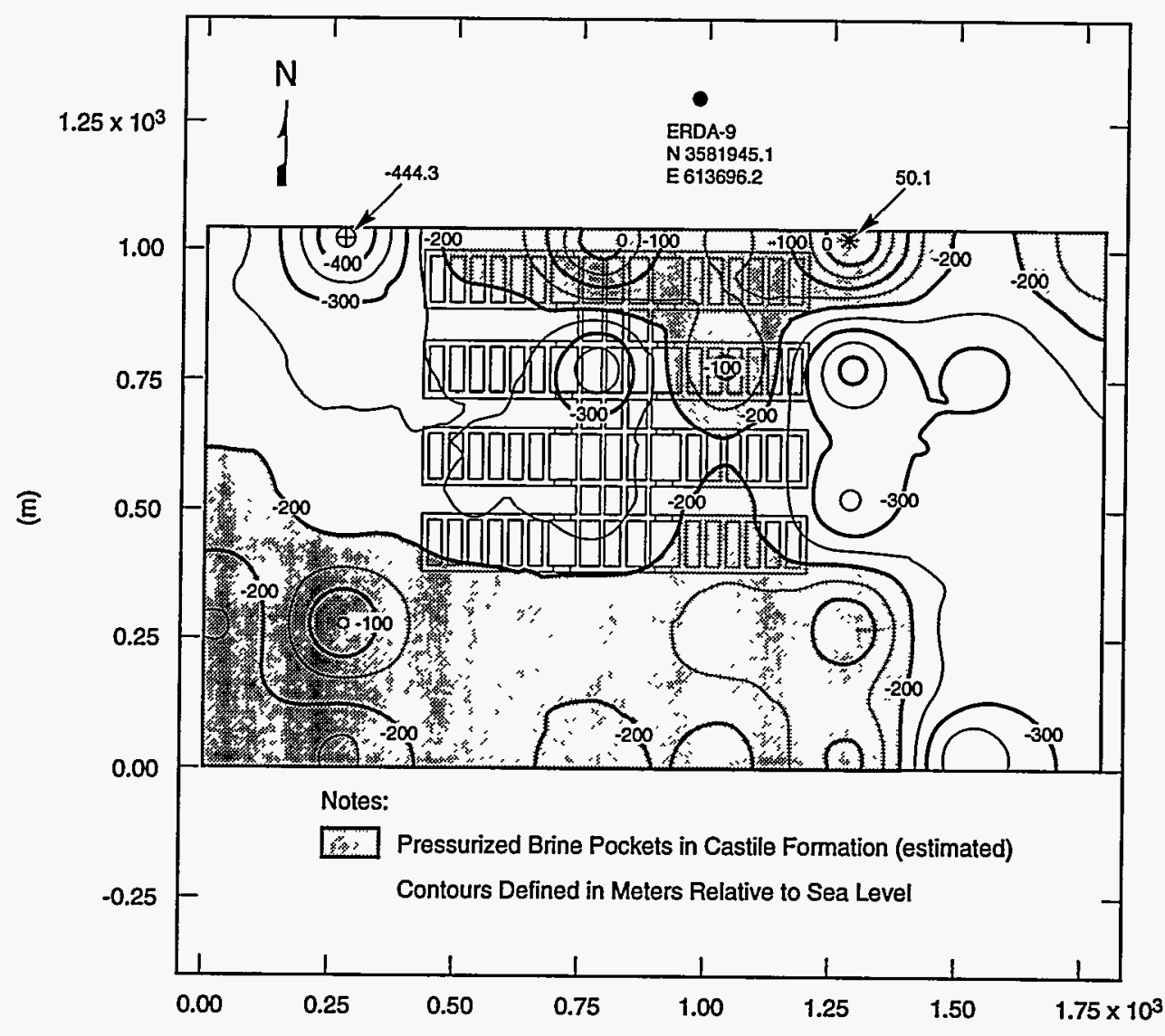

(m)

TRI-6342-1239-2

Figure 5.26. Contour map of elevation to first major conductor below WIPP disposal area (Sect. 5.1.1, Ref. 12; Ref. 88). Stippled areas indicate estimated locations of pressurized brine pockets in the Castile Formation.

$$
f W_{j}=f L[L B P(j), l N B P(1)]
$$

if $t N B P(1)>t B P(j)$ (i.e., if the drilling intrusion that penetrates pressurized brine at the time $t P B(j)$ is not preceded by a drilling intrusion that fails to penetrate pressurized brine), and (3)

$$
f L(i, j)=\text { fraction of waste inventory accessed by flow path connecting Locations } i \text { and } j \text {. }
$$

The fraction $f L(i, j)$ is assumed to be directly proportional to the length of the most direct flow path between locations $i$ and $j$ and is an externally-calculated input supplied to the analysis.

The releases associated with Case 4 are of the form

$$
n R G\left(\mathrm{f}_{n}\right)=n R G_{B}\left(\mathrm{f}_{n}\right)+n R G_{A}\left(\mathrm{f}_{n}\right)
$$


where $n R G_{B}\left(\mathrm{f}_{n}\right)$ is the release that occurs before the initiation of an E1E2-type release and $n R G_{A}\left(\mathrm{f}_{n}\right)$ is the release that occurs after the initiation of an E1E2-type release. The release $n R G_{A}\left(f_{n}\right)$ is given by

$$
n R G_{A}\left(f_{n}\right)=\sum_{j=1}^{n P B} n P f W_{j}\left\{r E 1 E 2_{i P B(j)}+f T_{j}\left[r E 1 E 2_{i P B(j)+1}-r E 1 E 2_{i P B(j)}\right]\right\},
$$

where

$$
f T_{j}=\left[t P B(j)-t G_{i P B(j)}\right] /\left[t G_{i P B(j)+1}-t G_{i P B(j)}\right]
$$

and $n P=10$ is the number of waste panels and enters the calculation because $r E I E 2_{i}$ is calculated with the inventory of a single waste panel being accessed (Table 5.9) and the access fraction $f W_{j}$ is calculated with respect to the entire waste inventory (Eqs. (5.44) and (5.45)).

The form of $n R G_{B}\left(\mathbf{f}_{n}\right)$ depends on whether the initial drilling intrusion (i.e., at $t B H_{1}$ ) does or does not penetrate pressurized brine. If the initial drilling intrusion penetrates pressurized brine (i.e., if $t P B(1)<t N P B(1)$ ), then $n R G_{B}\left(\mathrm{f}_{\mathrm{n}}\right)$ is given by Eq. (5.41) with $n P A$ replaced by $n E 1$, where $n E l$ is the largest integer such that

$$
t P A(j)<t N P B(1)
$$

for $j=1,2, \ldots, n E 1$. If the initial drilling intrusion does not penetrate pressurized brine (i.e., if $t P B(1)>t N P B(1)$ ), then $n R G_{B}\left(\mathrm{f}_{n}\right)$ is given by Eq. (5.42) with $n P A$ replaced by $n E 2$, where $n E 2$ is the largest integer such that

$$
t P A(j)<t P B(1)
$$

for $j=1,2, \ldots, n E 2$.

The construction procedure for $F\left(\mathbf{x}_{m}, \mathbf{f}_{n}\right)$ outlined in this section was used for all 1440 cases indicated in Sect. 5.4.1, with a slight modification for the cases involving a high permeability shaft (i.e., ISHAFT $=1$ as used in conjunction with Fig. 5.24). The presence of the high permeability shaft was introduced into the analysis through two assumptions. First, an E2 intrusion was always assumed to be present at $100 \mathrm{yr}$, with this intrusion corresponding to the presence of the shaft. Second, all E1E2 intrusions were assumed to be connected to the shaft, with this assumption deriving from the high permeability and large cross-sectional area associated with the shaft (e.g., see flows summarized in Fig. 5.5 and Table 5.5).

For a fixed $x_{m}$, repeated sampling of $f_{n}$ and construction of $\mathrm{F}\left(\mathbf{x}_{m}, f_{n}\right)$ results in evaluation of the expression in Eq. (4.17). As indicated in Eq. (4.17), this process is equivalent to the Monte Carlo evaluation of an integral over $\oiint \& I S$ and leads to the CCDF for normalized release to the accessible environment. Specifically, the evaluations for $\delta_{1}$ and $\delta_{10}$ give the exceedance probabilities for normalized releases of size 1 and 10. Further, evaluations with $\delta_{x}$ 
for additional values of $x$ fills out the remainder of the CCDF. As a sample of size 25 is under consideration (i.e., $\mathbf{x}_{1}$, $x_{2}, \ldots, x_{25}$ ), this process is repeated 25 times for each of the 1440 cases required in SPM-1 (see Sect. 4.5.1). The ultimate outcome is 1440 distributions of $25 \mathrm{CCDFs}$ each. A sample of size $n R=1000$ was used in SPM-1. Tests with larger sample sizes indicated that increasing $n R$ above 1000 had little effect on the estimated exceedance probabilities for normalized releases of size 1 and 10 .

As an example, four distributions of CCDFs are shown in Fig. 5.27. For a given distribution, the individual CCDFs result from evaluation of the integral approximation in Eq. (4.17). Associated with each frame are also mean exceedance probabilities for normalized releases of size 1 and 10 (e.g., $1.585 \times 10^{-1}$ and $0.000 \times 10^{0}$ in the upper left frame). These probabilities result from evaluation of the integral approximations in Eqs. (4.18) and (4.19). In the context of Fig. 5.27, the preceding approximations are equivalent to summing the 25 exceedance probabilities associated with normalized releases of size 1 and 10 , respectively, and then dividing by 25 . Also associated with each frame are 90th quantiles for the distributions of exceedance probabilities for normalized releases of size 1 and 10 (e.g., $7.150 \times 10^{-1}$ and $0.000 \times 10^{0}$ in the upper left frame). These quantiles result from solution of the integral equations in Eqs. (2.35) and (2.36) for $p_{1}$ and $p_{2}$ with $q=0.9$. In the context of Fig. 5.27, the indicated solutions were obtained ordering the exceedance probabilities associated with normalized releases of size 1 and 10 from largest to smallest and then using linear interpolation between the 2 nd and $3 \mathrm{rd}$ largest exceedance probabilities to estimate the 90th quantile (i.e., as a sample of size 25 is in use, an estimate of the 90th quantile of the exceedance probabilities associated with a given normalized release is the average of the 2 nd and 3 rd largest exceedance probabilities).

For the cases presented in Fig. 5.27, the dissolved and colloidally-sorbed releases are small, with the result that the CCDFs are dominated by the cuttings and spallings releases. The tendency of the CCDFs to shift to the left as $L S P A L$ increases results from the assumed effects of the possible outcomes of experimental program $E_{8}$ (i.e., $\varepsilon_{81}$, $\varepsilon_{82}, \varepsilon_{83}, \varepsilon_{84}$ ). For $L S P A L=4$ (lower right frame), the spallings releases are considerably smaller than the cuttings releases and so the associated CCDFs are showing the effects of cuttings removal alone. 
ISHAFT $=3, \operatorname{ICHMSOL}=3, \mathrm{JCOLSOL}=8$, KCULTRN $=5$, LSPAL $=1$

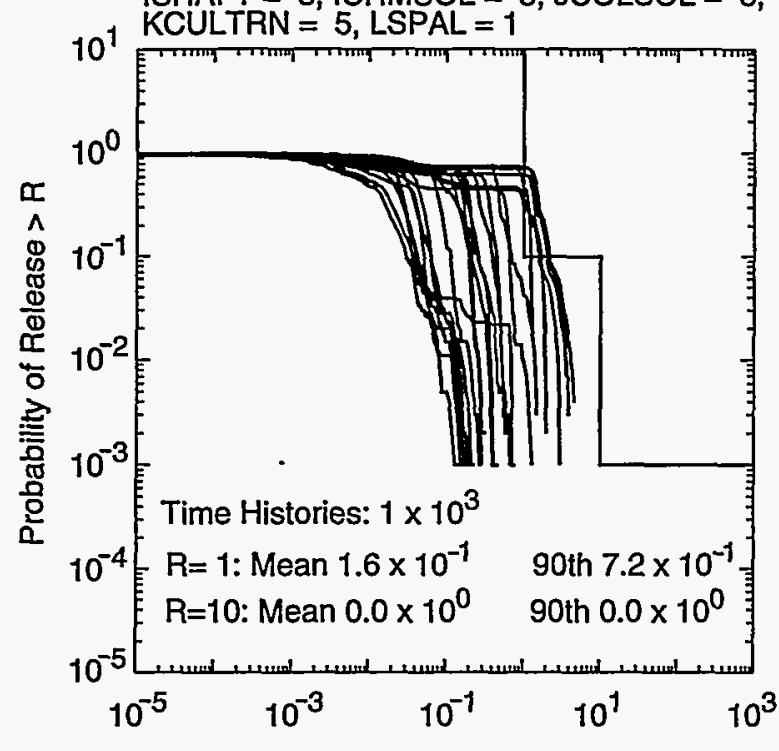

Release to Accessible Environment

TRI-6342-4622-0
ISHAFT $=3, \mathrm{ICHMSOL}=3, \mathrm{JCOLSOL}=8$,

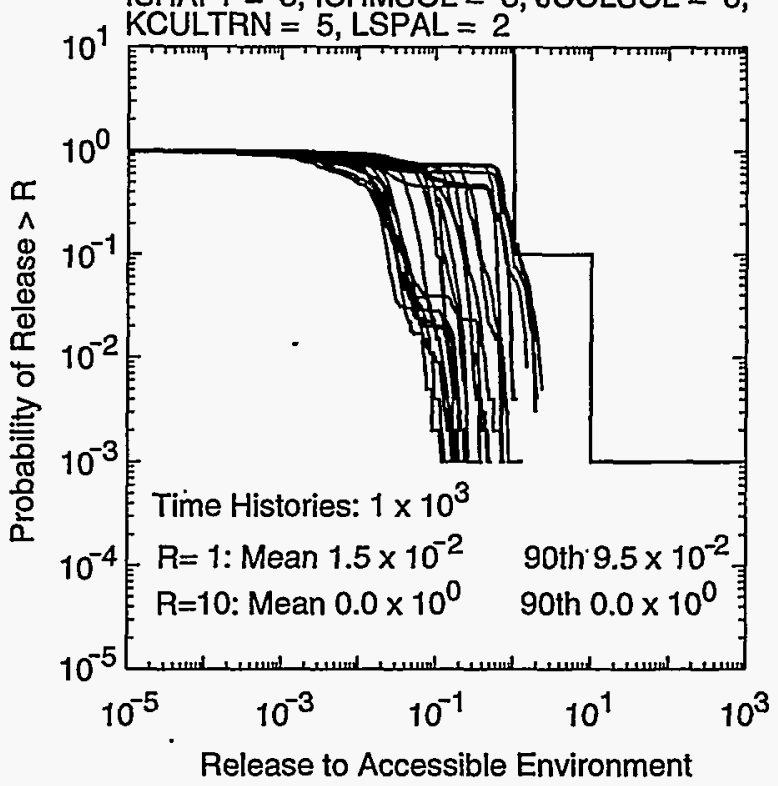

TRI-6342-4623-0

ISHAFT $=3, \mathrm{ICHMSOL}=3, \mathrm{JCOLSOL}=8$

KCULTRN $=5$, LSPAL $=3$

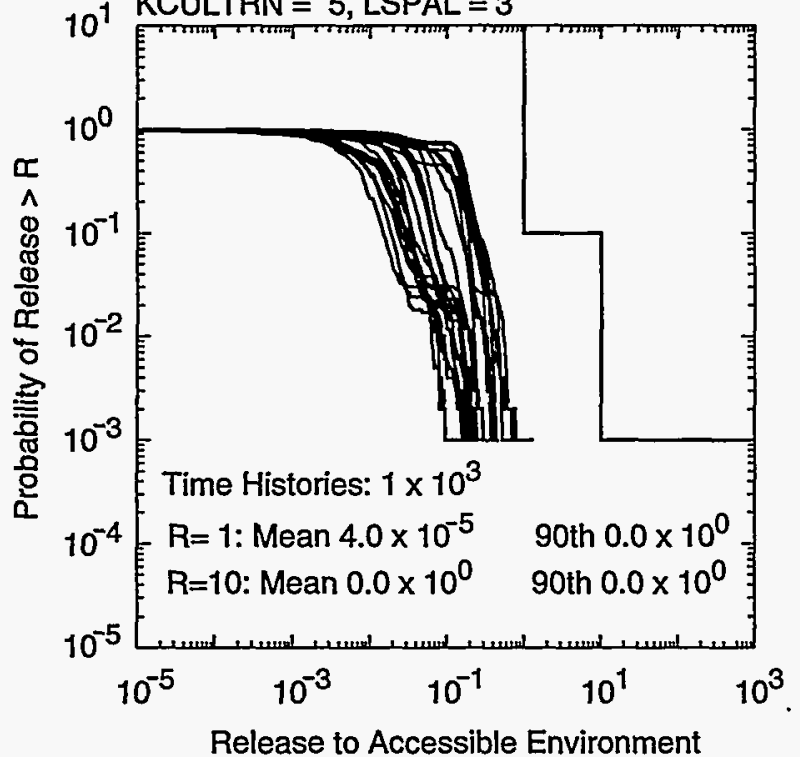

TRI-6342-4624-0
ISHAFT $=3$, ICHMSOL $=3, \mathrm{JCOLSOL}=8$,

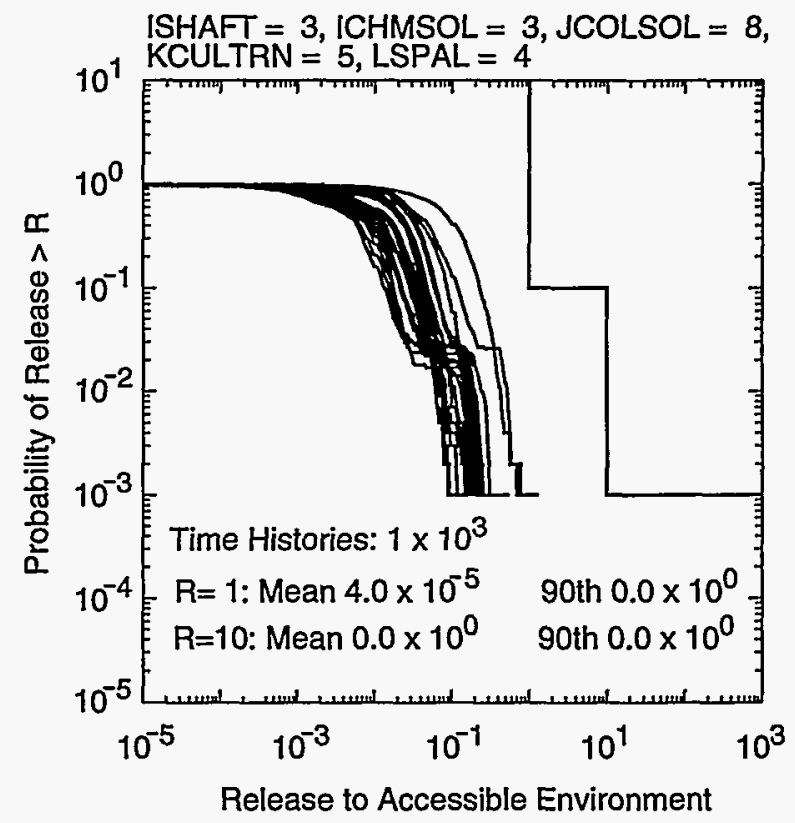

TRI-6342-4356-0

Figure 5.27. Example distributions of CCDFs for normalized release to the accessible environment constructed for low permeability shafts $(I S H A F T=3)$, low chemical solubility $(I C H M S O L=3)$, low colloid solubility $(J C O L S O L=8)$, complete retardation of dissolved release to the Culebra $(K C U L T R N=5)$, and four outcomes for spallings ( $L S P A L=1 \Rightarrow$ baseline, $L S P A L=2 \Rightarrow$ baseline $/ 2, L S P A L=3 \Rightarrow$ baseline $/ 10$, $L S P A L=4 \Rightarrow$ baseline $/ 100)$. 


\section{Systems Prioritization}

As indicated in Eq. (1.1), the SPM is based on consideration of the triples $\left(p A S_{j}, c A S_{j}, t A S_{j}\right), j=1,2, \ldots, n A S$, where $p A S_{j}$ is the probability that performance of activity set $A S_{j}$ will lead to compliance with applicable regulations as defined in Eqs. (2.25) and (2.40), $c A S_{j}$ is the cost (\$) of implementing activity set $A S_{j}$ and $t A S_{j}$ is the time (yr) required to implement activity set $A S_{j}$. Three different ways of determining $p A S_{j}$ are indicated in Sect. 2 (i.e., Eqs. (2.28) - (2.32), Eqs. (2.35) - (2.39), and Eqs. (2.41) - (2.45)). The cost $c A S_{j}$ is the sum of the costs for the individual experimental programs and design modifications associated with activity set $A S_{j}$. The time $t A S_{j}$ is the maximum of the times for the individual experimental programs and design modifications associated with $A S_{j}$.

The three alternative ways to define $p A S_{j}$ lead to the compliance probabilities $p A S M_{j}, p A S Q_{j}$ and $p A S I_{j}$, where

$p A S M_{j}=$ probability that performance of $A S_{j}$ will lead to compliance with applicable regulations on the basis of mean analysis outcomes as indicated in Eqs. (2.28) - (2.32),

$p A S Q_{j}=$ probability that performance of $A S_{j}$ will lead to compliance with applicable regulations on the basis of a specified quantile for analysis outcomes as indicated in Eqs. (2.35) - (2.39),

$p A S I_{j}=$ probability that performance of $A S_{j}$ will lead to compliance with applicable regulations on the basis of individual analysis outcomes as indicated in Eqs. (2.41) - (2.45).

With respect to 40 CFR 191.13, use of $p A S M_{j}$ is equivalent to assessing compliance on the basis of mean CCDFs (e.g., the mean exceedance probabilities in Fig. 5.27) as has been suggested by a number of authors. ${ }^{88-91}$ Use of $p A S Q_{j}$ is equivalent to assessing compliance on the basis of the location of a distribution of CCDFs relative to the boundary line specified in 40 CFR 191.13 (e.g., the 90th quantile values in Fig. 5.27), with this approach to the assessment of the relevance of uncertainty also suggested by a number of authors in various contexts. $36,40,92-97$ Finally, again in the context of $40 \mathrm{CFR} 191.13$, use of $p A S I_{j}$ is equivalent to assessing compliance on the basis of individual CCDFs (e.g., the individual CCDFs in Fig. 5.27) before the incorporation of a probabilistic representation for the subjective uncertainty in the inputs to the PA. In contrast, $p A S M_{j}$ and $p A S Q_{j}$ incorporate the effects of subjective uncertainty in the inputs to the PA before the determination of compliance.

As an example, scatterplots defined by the pairs $\left(c A S_{j}, p A S M_{j}\right),\left(c A S_{j}, p A S Q_{j}\right)$ and $\left(c A S_{j}, p A S I_{j}\right)$ appear in the upper, middle and lower frames, respectively, of Fig. 6.1. As illustrated in Fig. 5.27, the 90th quantile is used in the definition of $p A S Q_{j}$ in this example. Activity sets that result in a zero probability of compliance appear as points on the abscissa corresponding to their cost; activity sets that result in a nonzero probability of compliance appear as points above their cost. If cost was no object, then the activity set giving the highest probability of compliance would be preferred. However, as shown in Fig. 6.1, similar compliance probabilities can result for activity sets with very different costs. Given activity sets with similar probabilities of compliance, the set with the lowest cost is likely 
to be preferred. Thus, activity sets resulting in points in the upper left of the frames in Fig. 6.1 are likely to be preferred over activity sets resulting in points in the upper right.

As comparison of the three frames in Fig. 6.1 shows, $p A S M_{j}, p A S Q_{j}$ and $p A S I_{j}$ are not equal. In this particular example, use of $p A S I_{j}$ produces the largest probabilities of compliance and use of $p A S Q_{j}$ produces the smallest probabilities of compliance. However, as shown in Fig. 6.2, there is a positive correlation between $p A S M_{j}, p A S Q_{j}$ and $p A S I_{j}$.
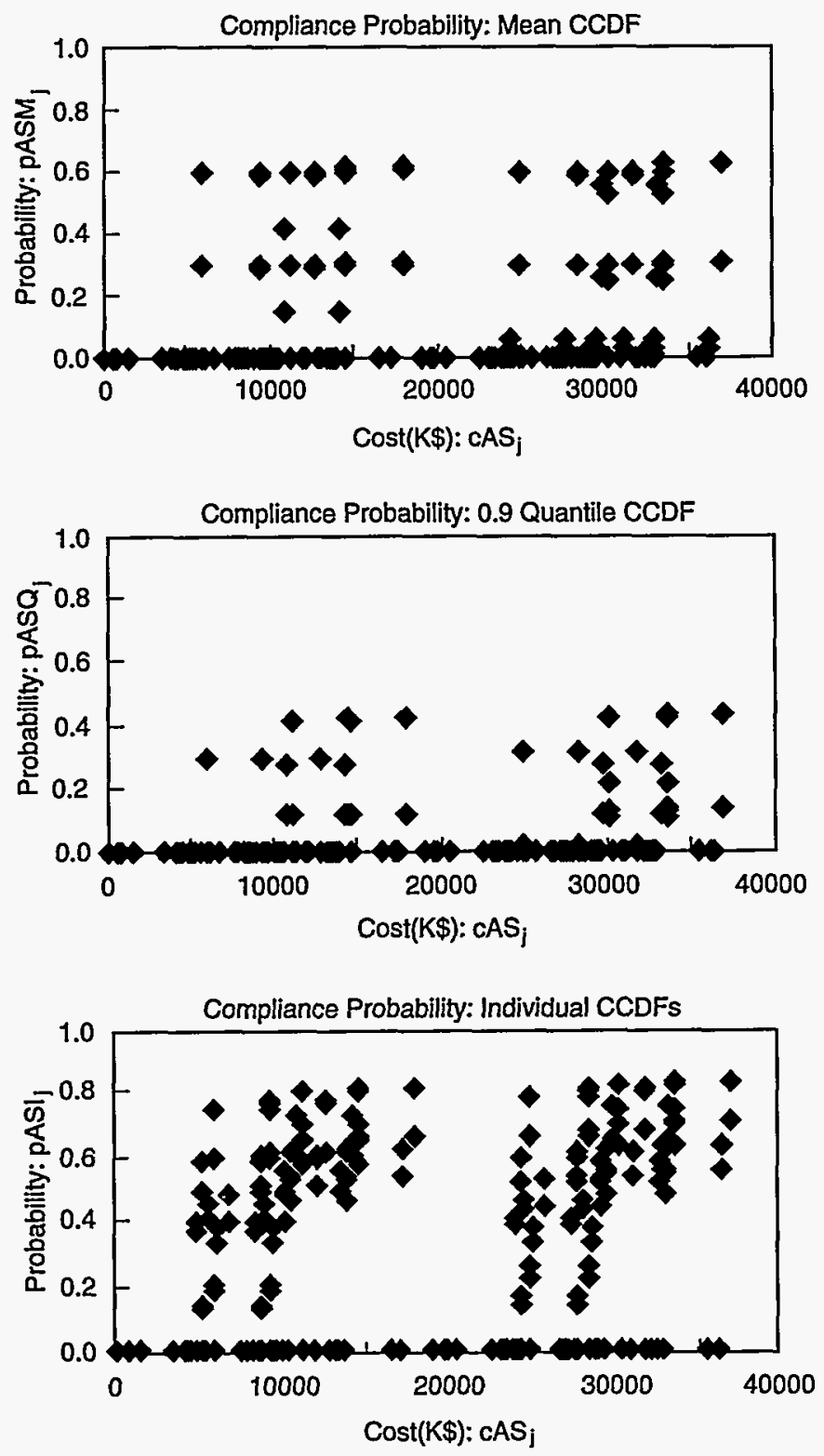

TRI-6342-4378-0

Figure 6.1. Scatterplots of cost $\left(c A S_{j}\right)$ versus compliance probability $\left(p A S_{j}\right)$ for $p A S_{j}=p A S M_{j}$ (upper frame), $p A S_{j}$ $=p A S Q_{j}$ (middle frame) and $p A S_{j}=p A S I_{j}$ (lower frame) for $j=1,2, \ldots, n A S$. 

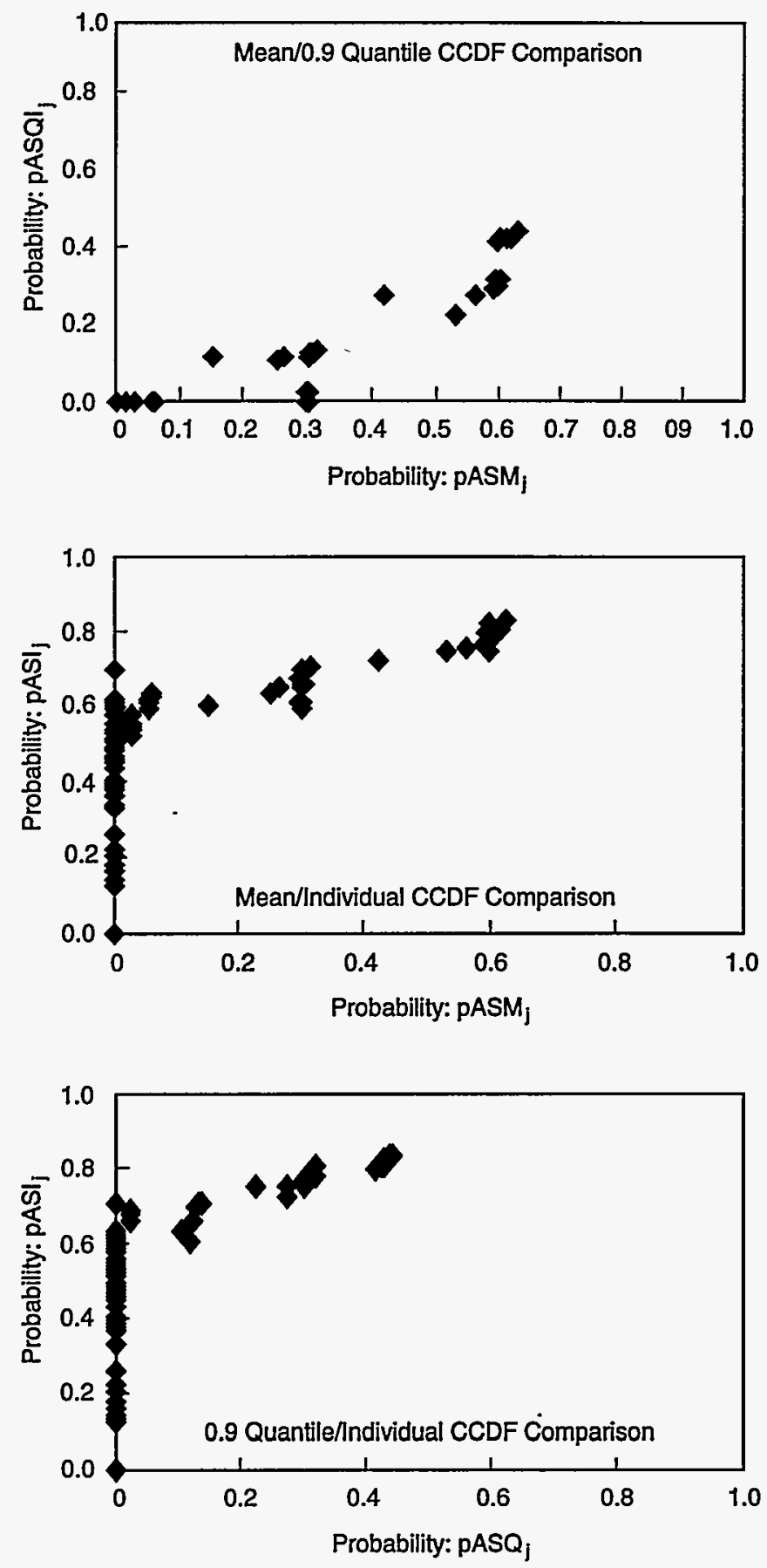

TRI-6342-4379-0

Figure 6.2. Scatterplots for $\left(p A S M_{j}, p A S Q_{j}\right)$ (upper frame), $\left(p A S M_{j}, p A S I_{j}\right)$ (middle frame) and $\left(p A S Q_{j}, p A S I_{j}\right)$ (lower frame) for $j=1,2, \ldots, n A S$. 


\section{Discussion}

The SPM is based on determining the probability that individual activity sets (i.e:, collections of experimental programs and design modifications) will lead to licensing of the WIPP for the disposal of TRU waste. Two types of uncertainty are incorporated into the compliance probabilities calculated in the SPM: stochastic (or aleatory) uncertainty, which arises because many different types of disruptions are possible at the WIPP over the next 10,000 yr and is thus a property of the system under study, and subjective (or epistemic) uncertainty, which arises from a lack of knowledge about the WIPP and is thus a property of the analysts performing the studies that will support the licensing application. ${ }^{36-43,92-96}$ Further, subjective uncertainty is used in two distinct ways within the SPM: first, to characterize the experimentalists' degree of belief as to the possible outcomes of their experimental programs, and second, to characterize the uncertainty in PA inputs given specific experimental outcomes. In a formal description of the SPM, probability spaces are used to carefully define these uses of uncertainty, with the compliance probability for each activity set then resulting from appropriately defined integrals over these probability spaces.

The probability space for stochastic uncertainty gives rise to the CCDF specified in 40 CFR 191.13. In SPM-1, this probability space arises from the assumptions that drilling intrusions constitute the only significant disruptions at the WIPP over the next 10,000 yr and that these intrusions follow a stationary Poisson process (i.e., occur randomly in time and space at a constant rate).

In the SPM, a probability space is defined for each experimental program under consideration. For example, SPM-1 considers 8 experimental programs and so 8 probability spaces are defined, one for each program. The purpose of these probability spaces is to characterize the experimentalists' degree of belief as to what the possible outcomes of each experimental program might be. Each experimental program has infinitely many possible outcomes. As a result, probabilities cannot be usefully assigned to individual outcomes; rather, the individual outcomes must be formed into groups of related outcomes, with probabilities then assigned to these groups. In SPM1 , the number of groupings used for each experimental program varied from 2 to 8 . In general, these groupings should be defined in terms of properties of the experimental programs themselves rather than in terms of their implications for PA. The development of these probability spaces is primarily the responsibility of the relevant experimentalists and should focus on characterizing the possible outcomes of the experiments themselves. However, this is not to imply that the implications for PA should be completely ignored in developing the probability spaces for experimental outcomes; recognition of the potential relationships between experimental results and PA input facilitates the next step in the SPM, which is the definition of probability spaces that characterize the uncertainty in PA input conditional on specific sets of experimental outcomes. In SPM-1, there was a tendency to define sets of experimental outcomes in terms of their implications for parameters used in PA rather than in terms of actual experimental outcomes. To the extent possible, this should be avoided in future applications of the SPM. Rather, the interpretation of the implications of experimental outcomes for PA input should be reserved for the next step in 
the SPM, which is the definition of the probability spaces that characterize the uncertainty in PA input. As indicated earlier, the probability spaces for the individual experimental programs are characterizing subjective uncertainty; specifically, the experimentalists' degree of belief as to what the outcomes of their experiments will be.

Once the probability spaces for the individual experimental programs have been defined, the next step in the SPM is to define the probability spaces that characterize the subjective uncertainty in PA input. In general, definition of these spaces will require a joint involvement of the experimentalists because of their knowledge of the physical processes and experimental outcomes and the PA modelers because of their knowledge of the computational models and the scale on which these models are used. Typically, experimental programs measure quantities on a smaller scale than is actually used in PA calculations. As a result, experimental outcomes are not direct input to PA models. Rather, a considerable amount of insight and manipulation is required to convert from experimental results on one scale to PA input on a possibly quite different scale. Thus, the involvement of both experimentalists and PA modelers is necessary to appropriately define the probability spaces that characterize the uncertainty in PA input. The complexity of this task can vary greatly depending on the characteristics of individual applications of the SPM. The simplest situation arises when the possible values for each PA input are affected by only one experimental program and are unaffected by any of the design modifications. Then, the probability spaces for the individual PA inputs can be constructed for the individual sets of outcomes associated with each experimental program. In particular, there is no need to consider the outcome of more than one experimental program at a time nor is there a need to consider the interplay between experimental outcomes and design modifications. A more complex situation arises when the outcomes of two or more experimental programs may affect the characterization of a single PA input. Effects 'due to design modifications are also a possibility. In such situations, the number of combinations of experimental outcomes and design modifications that require probability spaces for the characterization of uncertainty in PA input can become quite large. In SPM-1, most PA inputs were affected by either one or none of the experimental programs, one group of inputs was affected by two experimental programs; and one input was affected by one experimental program and one design modification.

The development of the probability spaces that characterize the possible outcomes of the experimental programs is part of the SPM and has no role to play in the final analyses that will be performed to support a licensing application for the WIPP. However, the development of probability spaces to characterize the uncertainty in PA input given a particular set of experimental outcomes will remain part of the PA process. In particular, experimental results will be produced by whatever experimental programs are ultimately selected for support, with the resultant need for the interpretation of the implications of these results for PA input.

The definition of a scientifically sound baseline for each experimental program is an important part of the SPM. The baseline for a given experimental program constitutes the least conservative values for the quantities under study in the program that the experimentalists and PA analysts feel can be successfully defended on the basis of presently available information. When an experimental program is not included in an activity set, the baseline values for that 
experimental program are used in the determination of the compliance probability for the activity set. In the definition of the baseline, it is important to avoid overconservatism. As indicated above, the goal is to define the least conservative values that can be successfully defended with presently available information. Definition of the baseline is sometimes complicated by the difficulty of knowing in advance of an analysis for a complex system what constitutes a conservative value for a particular assumption or parameter. For example, high shaft permeabilities are often viewed as a conservative assumption in PAs for the WIPP because of enhanced fluid flow into and out of the repository. However, high shaft permeabilities also reduce fluid pressures in the repository and thus reduce pressuredriven spallings releases in the event of a drilling intrusion; as a result, high shaft permeability is not conservative for this release mode.

In SPM-1, the baseline was always defined as one of the possible outcomes of the particular experimental program under consideration. Conceptually, the baseline can be viewed as characterizing the knowledge that would exist if an experimental program was conducted but failed completely (i.e., generated no new information). However, it is also possible that an experimental program will establish that the baseline does indeed constitute the appropriate set of assumptions for use in PA. The two preceding possibilities lead to the same assumptions for PA but for different reasons. As a result, the probabilities for the baseline in SPM-1 (i.e., $p E_{i}\left(\varepsilon_{i 1}\right)$ ) are a sum of the probabilities that (1) the experimental program fails completely and (2) the experimental program succeeds and implies that the baseline is indeed the appropriate set of assumptions. An explicit distinction between these two possibilities would be informative in future applications of the SPM.

The role of the baseline should be considered carefully in applications of the SPM. In SPM-1, the baseline was intended to be the least conservative values that could be defended with presently available information, with the result that conservative assumptions are incorporated into the analysis for each omitted experimental program. The outcome of this approach is that analyses tend to underestimate the probability of compliance (i.e., provide conservative results), but information on the analysts' degree of confidence in the results is lost. An alternative approach is to define the baseline as a degree of belief distribution as where the appropriate parameter values for use in PA are located in the absence of information from the relevant experimental program. In this case, the SPM provides information on the effectiveness of individual activity sets in reducing the uncertainty in whether or not the WIPP would comply with applicable regulations. The computational procedures associated with both definitions of the baseline are similar. However, the interpretation of the results is not the same as one approach is based on deliberate conservatism and the other is based on best-estimate analyses and measures of the attendant uncertainty.

If a conservative analysis is desired, then conservative assumptions should be used consistently. In particular, both the baseline and the implications of individual experimental outcomes should be the least conservative values that are consistent with all available information. Similarly if a best estimate analysis and estimates of the attendant uncertainties are desired, then degree of belief distributions should be used for both the baseline and the implications of individual experimental outcomes. In retrospect, the SPM-1 analysis tended to mix the two preceding ideas, with 
the baseline intended to be least conservative values and the implications of individual experimental programs taken to be degree of belief distributions. As a result, the final quantities used in assessing compliance with the applicable regulations were a mixture of conservative assumptions and degree of belief distributions. Also, the distributions used to define the baseline do not always seem to be consistent with the concept of conservatism (i.e., if a conservative value is desired, why use a distribution unless there is a nonmonotonic relationship between a parameter and its effect on regulatory outcomes and, as a result, there is uncertainty with respect to where the least conservative value is located). These observations do not detract from the value of SPM-1 as a vehicle to illustrate the SPM. However, they do point out possible problem areas that should be carefully thought through before entering into an SPM analysis that will serve as the basis for important programmatic decisions. The SPM process is too complicated to be embarked on without a clear understanding of what is to be determined and the manner in which the necessary calculations will be carried out.

The SPM involves a complex synthesis of information derived from the detailed modeling of the physical processes taking place at the WIPP under a variety of conditions and several distinct uses of probability to characterize uncertainty in different contexts. To develop the information used in this synthesis and then to organize the necessary calculations requires a clear conceptual model for the overall SPM. This report presents such a model by describing the SPM in terms of probability spaces and integrals over these spaces. Although this description is abstract, the individual components used in the description (i.e., probability spaces, integrals, functions) are real entities within the SPM that must be defined and/or evaluated. The value of the abstract description is that it allows the overall structure of the SPM to be seen. In contrast, excessive description of the many mathematical models and numerical algorithms, dozens of computer programs, and hundreds of thousands of lines of FORTRAN that underlie an analysis such as SPM-1 can obscure the core ideas on which the SPM is based. This is not intended to imply that such descriptions are unimportant and should not be supplied. Rather, some type of road map to such descriptions is needed. A suitable high level abstraction of an analysis provides such a map.

A carefully planned computational strategy is needed to implement the SPM. In turn, the development of this strategy depends on an understanding of both the conceptual basis of the SPM and the characteristics of the individual mathematical models and computer programs in use. As described in this presentation, the SPM involves iterated integrals over three probability spaces. In SPM-1, the integral over the probability space for possible experimental outcomes was implemented by systematically covering all possible combinations of experimental outcomes for each activity set, which was possible due to the relatively small number of outcomes (i.e., 2-8) associated with each experimental program and an efficient procedure to extend mechanistic results to a variety of situations. Even so, this approach required consideration of several thousand combinations of experimental outcomes for some activity sets, with 41,580 being the maximum number considered for any one activity set. The integral over the probability space associated with uncertainty in the PA input parameters was evaluated with a Monte Carlo procedure based on Latin hypercube sampling, and the integral over the probability space associated 
with future occurrences at the WIPP was evaluated with a Monte Carlo procedure based on simple random sampling. Technically, the probability spaces for experimental outcomes and PA input used for each activity set are product spaces that are built up by combining a number of individual probability spaces, with the result that the indicated integrals are really multiple integrals over the individual probability spaces. The function actually being integrated is developed from calculations performed with BRAGFLO, CUTTINGS, PANEL, SECO-FLOW, SECOTRANSPORT and SPALLINGS. Due to the expense of performing these calculations, especially with BRAGFLO, the overall structure of the calculations and their use within the indicated integrals had to be planned very carefully. Considerations in planning these calculations included (1) identification of cases for which different calculations were independent and hence could be performed separately, (2) elimination of calculations for which the results could be reasonably anticipated on the basis of prior analyses, (3) decomposition of calculations so that one computationally-demanding calculation could provide input to a number of less demanding calculations, (4) use of relatively simple models to extend mechanistic results to a number of different situations and (5) use of linearity and/or interpolation to extend mechanistic results to a variety of situations.

As should be apparent from the descriptions contained in Sect. 5, the implementation of the SPM is a large analysis effort that involves many components, many individual analysts and many distinct interfaces. It is important to examine intermediate results throughout the analysis. Due to the complexity of the analysis, it is very difficult to examine a final result (e.g., a probability of compliance for a particular activity set) and determine if it is correct. Rather, individual analysis results (e.g., pressure histories and brine flow rates predicted by BRAGFLO; radionuclide discharge rates to the Culebra predicted by PANEL; flow fields predicted by SECO-FLOW; radionuclide release rates to the accessible environment predicted by SECO-TRANSPORT; cuttings and spallings releases to the accessible environment predicted by CUTTINGS and SPALLINGS) must be examined and understood. Without such examination, it is difficult to have any assurance that the overall analysis is operating correctly. Further, such examination facilitates understanding the implications of the analysis assumptions, contributes to the efficient design of future calculations, and provides an opportunity to learn from the large amount of information that is generated as part of the SPM.

Although not emphasized in this presentation, sensitivity analysis techniques ${ }^{9,73}$ can be used to examine the results obtained in the SPM. In particular, the sampling-based integration procedures in use produce a mapping from analysis input to analysis results. Once this mapping is generated and stored, it can be explored with techniques based on regression analysis, partial correlation analysis and the examination of scatterplots. The preceding techniques provide a powerful way both to identify the uncertainties that are affecting the analysis outcomes and to check for errors in the implementation of the analysis.

A Monte Carlo procedure was used for CCDF construction in SPM-1 (Sect. 5.4). In contrast, the 1991 and 1992 WIPP PAs used a procedure based on importance sampling for CCDF construction. ${ }^{79}$ The Monte Carlo 
procedure worked well and allowed flexibilities that are not possible with the importance sampling procedure (e.g., more detailed treatment of scenarios that involve penetration of pressurized brine pockets). When equivalent analysis problems are considered, both procedures produce similar CCDFs. ${ }^{80}$

The SPM is not tied to one particular measure of compliance. This presentation used three different measures of compliance for illustration: mean CCDFs, percentile curves, and individual CCDFs. Other measures of compliance are possible.

As a concluding caveat, the reader is once again reminded that the example results taken from SPM- 1 are for illustration only. ${ }^{35}$ Although SPM-1 serves well as a vehicle to illustrate the SPM, this analysis was not developed with sufficient care for use in making decisions with respect to the WIPP. 


\section{References}

1. U.S. DOE (Department of Energy). 1980. Final Environmental Impact Statement: Waste Isolation Pilot Plant. DOE/EIS-0026. Washington, DC: U.S. Department of Energy. Vols. 1-2.

2. U.S. DOE (Department of Energy). 1990. Final Supplement Environmental Impact Statement, Waste Isolation Pilot Plant. DOE/EIS-0026-FS. Washington, DC: U.S. Department of Energy, Office of Environmental Restoration and Waste Management. Vols. 1-13.

3. U.S. DOE (Department of Energy). 1991. Strategy for the Waste Isolation Pilot Plant Test Phase. DOE/EM/48063-2. Washington, DC: U.S. Department of Energy, Office of Waste Operations.

4. Bertram-Howery, S.G., M.G. Marietta, D.R. Anderson, K.F. Brinster, L.S. Gomez, R.V. Guzowski, and R.P. Rechard. 1989. Draft Forecast of the Final Report for the Comparison to 40 CFR Part 191, Subpart B, for the Waste Isolation Pilot Plant. SAND88-1452. Albuquerque, NM: Sandia National Laboratories.

5. Marietta, M.G., S.G. Bertram-Howery, D.R. Anderson, K.F. Brinster, R.V. Guzowski, H. Iuzzolino, and R.P. Rechard. 1989. Performance Assessment Methodology Demonstration: Methodology Development for Evaluating Compliance with EPA 40 CFR 191, Subpart B, for the Waste Isolation Pilot Plant. SAND892027. Albuquerque, NM: Sandia National Laboratories.

6. Lappin, A.R., R.L. Hunter, D.P. Garber, P.B. Davies, R.L. Beauheim, D.J. Borns, L.H. Brush, B.M. Butcher, T. Cauffman, M.S.Y. Chu, L.S. Gomez, R.V. Guzowski, H.J. Iuzzolino, V. Kelley, S.J. Lambert, M.G. Marietta, J.W. Mercer, E.J. Nowak, J. Pickens, R.P. Rechard, M. Reeves, K.L. Robinson, and M.D. Siegel. 1989. Systems Analysis, Long-Term Radionuclide Transport, and Dose Assessments, Waste Isolation Pilot Plant (WIPP), Southeastern New Mexico; March 1989. SAND89-0462. Albuquerque, NM: Sandia National Laboratories.

7. Bertram-Howery, S.G., M.G. Marietta, R.P. Rechard, P.N. Swift, D.R. Anderson, B.L. Baker, J.E. Bean, Jr., W. Beyeler, K.F. Brinster, R.V. Guzowski, J.C. Helton, R.D. McCurley, D.K. Rudeen, J.D. Schreiber, and P. Vaughn. 1990. Preliminary Comparison with 40 CFR Part 191, Subpart B for the Waste Isolation Pilot Plant, December 1990. SAND90-2347. Albuquerque, NM: Sandia National Laboratories.

8. Rechard, R.P., H.J. Iuzzolino, and J.S. Sandha. 1990. Data Used in Preliminary Performance Assessment of the Waste Isolation Pilot Plant (1990). SAND89-2408. Albuquerque, NM: Sandia National Laboratories.

9. Helton, J.C., J.W. Garner, R.D. McCurley, and D.K. Rudeen. 1991. Sensitivity Analysis Techniques and Results for Performance Assessment at the Waste Isolation Pilot Plant. SAND90-7103. Albuquerque, NM: Sandia National Laboratories.

10. WIPP PA (Performance Assessment) Division. 1991. Preliminary Comparison with 40 CFR Part 191, Subpart B for the Waste Isolation Pilot Plant, December 1991. Volume 1: Methodology and Results. SAND91-0893/1. Albuquerque, NM: Sandia National Laboratories.

11. WIPP PA (Performance Assessment) Division. 1991. Preliminary Comparison with 40 CFR Part 191, Subpart B for the Waste Isolation Pilot Plant, December 1991. Volume 2: Probability and Consequence Modeling. SAND91-0893/2. Albuquerque, NM: Sandia National Laboratories.

12. WIPP PA (Performance Assessment) Division. 1991. Preliminary Comparison with 40 CFR Part 191, Subpart B for the Waste Isolation Pilot Plant, December 1991. Volume 3: Reference Data. SAND91-0893/3. Albuquerque, NM: Sandia National Laboratories. 
13. Helton, J.C., J.W. Garner, R.P. Rechard, D.K. Rudeen, and P.N. Swift. 1992. Preliminary Comparison with 40 CFR Part 191, Subpart B for the Waste Isolation Pilot Plant, December 1991. Volume 4: Uncertainty and Sensitivity Analysis Results. SAND91-0893/4. Albuquerque, NM: Sandia National Laboratories.

14. WIPP PA (Performance Assessment) Department. 1992. Long-Term Gas and Brine Migration at the Waste Isolation Pilot Plant: Preliminary Sensitivity Analyses for Post-Closure 40 CFR 268 (RCRA), May 1992. SAND92-1933. Albuquerque, NM: Sandia National Laboratories.

15. Helton, J.C., J.E. Bean, B.M. Butcher, J.W. Garner, J.D. Schreiber, P.N. Swift, and P. Vaughn. 1993. Uncertainty and Sensitivity Analyses for Gas and Brine Migration at the Waste Isolation Pilot Plant, May 1992. SAND92-2013. Albuquerque, NM: Sandia National Laboratories.

16. WIPP PA (Performance Assessment) Department. 1992. Preliminary Performance Assessment for the Waste Isolation Pilot Plant, December 1992. Volume 1: Third Comparison with 40 CFR 191, Subpart B. SAND920700/1. Albuquerque, NM: Sandia National Laboratories.

17. WIPP PA (Performance Assessment) Department. 1992. Preliminary Performance Assessment for the Waste Isolation Pilot Plant, December 1992. Volume 2: Technical Basis. SAND92-0700/2. Albuquerque, NM: Sandia National Laboratories.

18. Sandia WIPP Project. 1992. Preliminary Performance Assessment for the Waste Isolation Pilot Plant, December 1992. Volume 3: Model Parameters. SAND92-0700/3. Albuquerque, NM: Sandia National Laboratories.

19. WIPP PA (Performance Assessment) Department. 1993. Preliminary Performance Assessment for the Waste Isolation Pilot Plant, December 1992. Volume 4: Uncertainty and Sensitivity Analyses for 40 CFR 191, Subpart B. SAND92-0700/4. Albuquerque, NM: Sandia National Laboratories.

20. WIPP PA (Performance Assessment) Department. 1993. Preliminary Performance Assessment for the Waste Isolation Pilot Plant, December 1992. Volume 5: Uncertainty and Sensitivity Analyses of Gas and Brine Migration for Undisturbed Performance. SAND92-0700/5. Albuquerque, NM: Sandia National Laboratories.

21: U.S. EPA (Environmental Protection Agency). 1985. "40 CFR Part 191: Environmental Standards for the Management and Disposal of Spent Nuclear Fuel, High-Level and Transuranic Radioactive Wastes; Final Rule," Federal Register. Vol. 50, no. 182, 38066-38089.

22. U.S. EPA (Environmental Protection Agency). 1993. " 40 CFR Part 191: Environmental Radiation Protection Standards for the Management and Disposal of Spent Nuclear Fuel, High-Level and Transuranic Radioactive Wastes; Final Rule," Federal Register. Vol. 58, no. 242, 66398-66416.

23. U.S. EPA (Environmental Protection Agency). 1986. " 40 CFR Part 268: Land Disposal Restrictions," as amended and published in the most recent Code of Federal Regulations. Washington, DC: Office of the Federal Register, National Archives and Records Administration.

24. Public Law 94-580. 1976. Resource Conservation and Recovery Act of 1976.

25. Beyeler, W. 1994. "Initial Thoughts on Quantitative Prioritization." Memo to Paul Davis, February 17, 1994. (Reprinted in Appendix A, pp. 114-140.)

26. Beyeler, W. 1994. "Introduction of the New WIPP System Prioritization Method." Draft, March 17, 1994. (Reprinted in Appendix A, pp. 141-154.) 
27. Tierney, M.S. 1994. "Notes on the WIPP Prioritization Methodology." Draft, April 30, 1994. (Reprinted in Appendix A, pp. 155-167.)

28. Beyeler, W. 1994. "Summary of SP Utility Function Meeting of May 17." Memo, May 19, 1994. (Reprinted in Appendix A, pp. 168-184.)

29. Lincoln, R.C. 1994. "Second Draft Performance Measures for the WIPP System Prioritization." Memo to P.A. Davis, June 27, 1994. (Reprinted in Appendix A, pp. 185-188.)

30. Hora, S.C. 1994. "Computational Strategies for the Systems Prioritization." Draft, June 29, 1994. (Reprinted in Appendix A, pp. 189-199.)

31. Mendenhall, F.T. 1994. "Prototype System Prioritization Baseline." Memo to R.C. Lincoln, July 1, 1994. (Reprinted in Appendix A, pp. 200-207.)

32. Mendenhall, F.T., N.H. Prindle, and W. Beyeler. 1994. "Systems Prioritization Prototype BRAGFLO Baseline and Activity Set Parameter Ranges and Parameter Distributions." Memo to R.C. Lincoln, July 15, 1994. (Reprinted in Appendix A, pp. 208-214.)

33. Hora, S.C. 1994. "Decision Analysis Within the Systems Prioritization Process." July 27, 1994. (Reprinted in Appendix A, pp. 215-225.)

34. Mendenhall, F.T., N.H. Prindle, W. Beyeler, and R.C. Lincoln. 1994. "Prototype Systems Prioritization 1 (SP-1) Activity Set Definition." Memo, August 4, 1994. (Reprinted in Appendix A, pp. 226-235.)

35. "The Waste Isolation Pilot Plant (WIPP) Systems Prioritization Method-Iteration One (SPM-1): A Prototype Decision Analysis, Final." No date or attribution. (Reprinted in Appendix A, pp. 236-276.)

36. Vesely, W.E., and D.M. Rasmuson. 1984. "Uncertainties in Nuclear Probabilistic Risk Analyses," Risk Analysis. Vol. 4, no. 4, 313-322.

37. Paté-Cornell, M.E. 1986. "Probability and Uncertainty in Nuclear Safety Decisions," Nuclear Engineering and Design. Vol. 93, no. 2-3, 319-327.

38. Parry, G.W. 1988. "On the Meaning of Probability in Probabilistic Safety Assessment," Reliability Engineering and System Safety. Vol. 23, no. 4, 309-314.

39. Apostolakis, G.E. 1989. "Uncertainty in Probabilistic Risk Assessment." Nuclear Engineering and Design. Vol. 115, 173-179.

40. International Atomic Energy Agency. 1989. Evaluating the Reliability of Predictions Made Using Environmental Transfer Models. Safety Series Report No. 100. Vienna, Austria: International Atomic Energy Agency.

41. Apostolakis, G. 1990. "The Concept of Probability in Safety Assessments of Technological Systems," Science. Vol. 250, no. 4986, 1359-1364.

42. Helton, J.C. 1993. "Risk, Uncertainty in Risk, and the EPA Release Limits for Radioactive Waste Disposal," Nuclear Technology. Vol. 101, no. 1, 18-39.

43. Helton, J.C. 1994. "Treatment of Uncertainty in Performance Assessments for Complex Systems," Risk Analysis. Vol. 14, no. 4, 483-511. 
44. Feller, W. 1971. An Introduction to Probability Theory and Its Applications. 2nd ed. New York, NY: John Wiley and Sons. Vol. II.

45. Ash, R.B. 1972. Real Analysis and Probability. New York, NY: Academic Press.

46. Breiman, L. 1992. Probability. Philadelphia, PA: Society for Industrial and Applied Mathematics.

47. Helton, J.C., J.W. Garner, M.G. Marietta, R.P. Rechard, D.K. Rudeen and P.N. Swift. 1993. "Uncertainty and Sensitivity Analysis Results Obtained in a Preliminary Performance Assessment for the Waste Isolation Pilot Plant," Nuclear Science and Engineering. Vol. 114, no. 4, 286-331.

48. Brooks, R.H., and A.T. Corey. 1964. Hydraulic Properties of Porous Media. Hydrology Paper No. 3. Fort Collins, CO: Civil Engineering Department, Colorado State University.

49. van Genuchten, R. 1978. Calculating the Unsaturated Hydraulic Conductivity with a New Closed-Form Analytical Model. Research Report 78-WR-08. Princeton, NJ: Princeton University, Department of Civil Engineering.

50. Parker, J.C., R.J. Lenhard, and T. Kuppusamy. 1987. "A Parametric Model for Constitutive Properties Governing Multiphase Flow in Porous Media," Water Resources Research. Vol. 23, no. 4, 618-624.

51. Trauth, K.M., R.P. Rechard, and S.C. Hora. 1991. "Expert Judgment as Input to Waste Isolation Pilot Plant Performance Assessment Calculations: Probability Distributions of Significant System Parameters," Mixed Waste: Proceedings of the First International Symposium, Baltimore, MD, August 26-29, 1991. Eds. A.A. Moghissi and G.A. Benda. SAND91-0625C. Baltimore, MD: Environmental Health and Safety, University of Maryland. 4.3.1 through 4.3.9.

52. Trauth, K.M., S.C. Hora, R.P. Rechard, and D.R. Anderson. 1992. The Use of Expert Judgment to Quantify Uncertainty in Solubility and Sorption Parameters for Waste Isolation Pilot Plant Performance Assessment. SAND92-0479. Albuquerque, NM: Sandia National Laboratories.

53. Hobart, D.E., F.J. Millero, I-Ming Chou, and C.J. Bruton. (Forthcoming.) Estimates of the Solubilities of Waste Element Radionuclides in Waste Isolation Pilot Plant (WIPP) Brines: A Report by the Expert Panel on Source Term Solubilities. Albuquerque, NM: Sandia National Laboratories.

54. Helton, J.C., D.R. Anderson, B.L. Baker, J.E. Bean, J.W. Berglund, W. Beyeler, J.W. Garner, H.J. Iuzzolino, M.G. Marietta, R.P. Rechard, P.J. Roache, D.K. Rudeen, J.D. Schreiber, P.N. Swift, M.S. Tierney, and P. Vaughn. 1994. "Effect of Alternative Conceptual Models in a Preliminary Performance Assessment for the Waste Isolation Pilot Plant," Nuclear Engineering and Design. Vol. 154, no. 3, 251-344.

55. Freeze, R.A., and J.A. Cherry. 1979. Groundwater. Englewood Cliffs, NJ: Prentice-Hall, Inc.

56. Brinster, K. 1990. Appendix A: "Memo 10: Well Data from Electric Logs," Data Used in Preliminary .Performance Assessment of the Waste Isolation Pilot Plant (1990). R.P. Rechard, H. Iuzzolino, and J.S. Sandha. SAND89-2408. Albuquerque, NM: Sandia National Laboratories. A-129 through A-138.

57. Brush, L.H. 1991. "Appendix A: Current Estimates of Gas Production Rates, Gas Production Potentials, and Expected Chemical Conditions Relevant to Radionuclide Chemistry for the Long-Term WIPP Performance Assessment," Preliminary Comparison with 40 CFR 191, Subpart B for the Waste Isolation Pilot Plant, December 1991. Volume 3: Reference Data. WIPP Performance Assessment Division. Eds. R.P. Rechard, A.C. Peterson, J.D. Schreiber, H.J. Iuzzolino, M.S. Tierney, and J.S. Sandha. SAND91-0893/3. Albuquerque, NM: Sandia National Laboratories. A-25 through A-36. 
58. Gorham, E., R. Beauheim, P. Davies, S. Howarth, and S. Webb. 1992. "Appendix A: Recommendations to PA on Salado Formation Intrinsic Permeability and Pore Pressure for 40 CFR 191 Subpart B Calculations," Preliminary Performance Assessment for the Waste Isolation Pilot Plant, December 1992. Volume 3: Model Parameters. Sandia WIPP Project. SAND92-0700/3. Albuquerque, NM: Sandia National Laboratories. A47 through A-67.

59. Howarth, S.M., E.W. Peterson, P.L. Lagus, K-H. Lie, S.J. Finley, and E.J. Nowak. 1991. "Interpretation of InSitu Pressure and Flow Measurements of the Salado Formation at the Waste Isolation Pilot Plant," 1991 Joint Rocky Mountain Regional Meeting, Society of Petroleum Engineers and Low Permeability Reservoirs Symposium, Denver, CO, April 15-17, 1991. SPE-21840; SAND90-2334C. Richardson, TX: Society of Petroleum Engineers. 355-369.

60. Beauheim, R.L., G.J. Saulnier, Jr., and J.D. Avis. 1991. Interpretation of Brine-Permeability Tests of the Salado Formation at the Waste Isolation Pilot Plant Site: First Interim Report. SAND90-0083. Albuquerque, NM: Sandia National Laboratories.

61. Zenz, F.A., and D.F. Othmer. 1960. Fluidization and Fluid Particle Systems. New York, NY: Reinhold.

62. Partheniades, E., and R.E. Paaswell. 1970. "Erodibility of Channels with Cohesive Boundary," Journal of the Hydraulics Division, Proceedings of the American Society of Civil Engineers. Vol. 96, no. HY3, 755-771.

63. Short, J.A. 1982. Drilling and Casing Operations. Tulsa, OK: PennWell Books.

64. Kelley, V.A., and G.J. Saulnier, Jr. 1990. Core Analyses for Selected Samples from the Culebra Dolomite at the Waste Isolation Pilot Plant Site. SAND90-7011. Albuquerque, NM: Sandia National Laboratories.

65. LaVenue, A.M., and B.S. RamaRao. 1992. A Modeling Approach To Address Spatial Variability within the Culebra Dolomite Transmissivity Field. SAND92-7306. Albuquerque, NM: Sandia National Laboratories.

66. Helton, J.C. 1993. "Drilling Intrusion Probabilities for Use in Performance Assessment for Radioactive Waste Disposal," Reliability Engineering and System Safety. Vol. 40, no. 3, 259-275.

67. Cox, D.R., and P.A.W. Lewis. 1966. The Statistical Analysis of Series of Events. London: Chapman and Hall.

68. Haight, F.A. 1967. Handbook of the Poisson Distribution. New York, NY: Wiley.

69. Cox, D.R., and V. Isham. 1980. Point Processes. London: Chapman and Hall.

70. McKay, M.D., W.J. Conover, and R.J. Beckman. 1979. "A Comparison of Three Methods for Selecting Values of Input Variables in the Analysis of Output from a Computer Code." Technometrics 21: 239-245.

71. Iman, R.L., and J.C. Helton. 1988. "An Investigation of Uncertainty and Sensitivity Analysis Techniques for Computer Models," Risk Analysis. Vol. 8, no. 1, 71-90.

72. Iman, R.L., and J.C. Helton. 1991. "The Repeatability of Uncertainty and Sensitivity Analyses for Complex Probabilistic Risk Assessments," Risk Analysis. Vol. 11, no. 4, 591-606.

73. Helton, J.C. 1993. "Uncertainty and Sensitivity Analysis Techniques for Use in Performance Assessment for Radioactive Waste Disposal," Reliability Engineering and System Safety. Vol. 42, no. 2-3, 327-367.

74. Berglund, J.W. 1992. Mechanisms Governing the Direct Removal of Wastes from the Waste Isolation Pilot Plant Repository Caused by Exploratory Drilling. SAND92-7295. Albuquerque, NM: Sandia National Laboratories. 
75. Roache, P.J. 1993. "The SECO Suite of Codes for Site Performance Assessment," High Level Radioactive Waste Management, Proceedings of the Fourth Annual International Conference, Las Vegas, NV, April 26-30, 1993. La Grange Park, IL: American Nuclear Society, Inc. Vol. 2, 1586-1594.

76. Stone, C.M., R.D. Krieg, and Z.E. Beisinger. 1985. SANCHO: A Finite Element Computer Program for the Quasistatic, Large Deformation, Inelastic Response of Two-Dimensional Solids. SAND84-2618. Albuquerque, NM: Sandia National Laboratories.

77. Berglund, J.W. "Memorandum of Record: The Direct Removal of Waste Caused by a Drilling Intrusion into a WIPP Panel-A Position Paper." August 31, 1994. (Reprinted in Appendix D, pp. 302-326.)

78. Bear, J., and A. Veruijt. 1987. Modeling Groundwater Flow and Pollution. Boston, MA: D. Reidel Publishing.

79. Helton, J.C., and H.J. Iuzzolino. 1993. "Construction of Complementary Cumulative Distribution Functions for Comparison with the EPA Release Limits for Radioactive Waste Disposal," Reliability Engineering and System Safety. Vol. 40, no. 3, 277-293.

80. Helton, J.C., and A.W. Shiver. 1994. A Monte Carlo Procedure for the Construction of Complementary Cumulative Distribution Functions for Comparison with the EPA Release Limits for Radioactive Waste Disposal. SAND94-2128. Albuquerque, NM: Sandia National Laboratories.

81. Iman, R.L., and M.J. Shortencarier. 1984. A FORTRAN 77 Program and User's Guide for the Generation of Latin Hypercube and Random Samples for Use with Computer Models. NUREG/CR-3624, SAND83-2365. Albuquerque, NM: Sandia National Laboratories.

82. Iman, R.L., and W.J. Conover. 1982. "A Distribution-Free Approach to Inducing Rank Correlation Among Input Variables," Communications in Statistics: Simulation and Computation. Vol. 11, no. 3, 311-334.

83. Garner, J.W. 1994. "Radionuclides to Transport for System Prioritization (SP) Calculations." Memo, July 13, 1994. (Reprinted in Appendix D, pp. 327-334.)

84. U.S. DOE (Department of Energy). 1994. Waste Isolation Pilot Plant Transuranic Waste Baseline Inventory Report. CAO-94-1005, Revision 0. Carlsbad, NM: Carlsbad Area Office, U.S. DOE. Books 1-2.

85. Bechtel, Inc. 1986. Design Validation Final Report. DOE/WIPP-86-010. Prepared for US Department of Energy. San Francisco, CA: Bechtel National, Inc.

86. Nowak, E.J., J.R. Tillerson, and T.M. Torres. 1990. Initial Reference Seal System Design: Waste Isolation Pilot Plant. SAND90-0355. Albuquerque, NM: Sandia National Laboratories.

87. Earth Technology Corp. 1987. Final Report for Time Domain Electromagnetic (TDEM) Survey at the WIPP Site. SAND87-7144. Albuquerque, NM: Sandia National Laboratories.

88. Bonano, E.J., and K.K. Wahi. 1990. Use of Performance Assessment in Assessing Compliance with the Containment Requirements in 40 CFR Part 191. NUREG/CR-5521, SAND90-0127. Albuquerque, NM: Sandia National Laboratories.

89. Rechard, R.P. 1989. Review and Discussion of Code Linkage and Data Flow in Nuclear Waste Compliance Assessments. SAND87-2833. Albuquerque, NM: Sandia National Laboratories. 
90. Hunter, R.L., R.M. Cranwell, and M.S.Y. Chu. 1986. Assessing Compliance with the EPA High-Level Waste Standard: An Overview. NUREG/CR-4510, SAND86-0121. Albuquerque, NM: Sandia National Laboratories.

91. Cranwell, R.M., J.E. Campbell, J.C. Helton, R.L. Iman, D.E. Longsine, N.R. Ortiz, G.E. Runkle, and M.J. Shortencarier. 1987. Risk Methodology for Geologic Disposal of Radioactive Waste: Final Report. NUREG/CR-2452, SAND81-2573. Albuquerque, NM: Sandia National Laboratories.

92. Kaplan, S., and B.J. Garrick. 1981. "On the Quantitative Definition of Risk," Risk Analysis. Vol. 1, no. 1, 1127.

93. National Research Council. 1993. Issues in Risk Assessment. Washington, DC: National Academy Press.

94. U.S. EPA (Environmental Protection Agency). 1993. An SAB Report: Multi-Media Risk Assessment for Radon, Review of Uncertainty Analysis of Risks Associated with Exposure to Radon. EPA-SAB-RAC-93-014. Washington, DC: Environmental Protection Agency, Science Advisory Board.

95. Hoffman, F.O., and J.S. Hammonds. 1994. "Propagation of Uncertainty in Risk Assessments: The Need to Distinguish Between Uncertainty Due to Lack of Knowledge and Uncertainty Due to Variability," Risk Analysis. Vol. 14, no. 5, 707-7 12.

96. National Research Council. 1994. Science and Judgment in Risk Assessment. Washington, DC: National Academy Press. 

Appendix A

SPM-1 Memos Cited in Report 

25. Beyeler, W. 1994. "Initial Thoughts on Quantitative Prioritization." Memo to Paul Davis, February 17, 1994. 


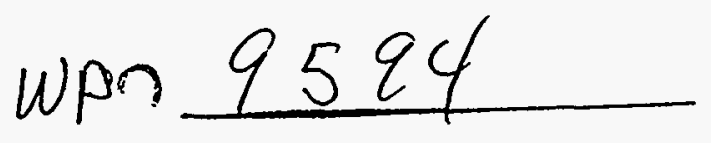

INFORMATION ONLY

Date: March 8, 1994

To: Peter Davies 6115

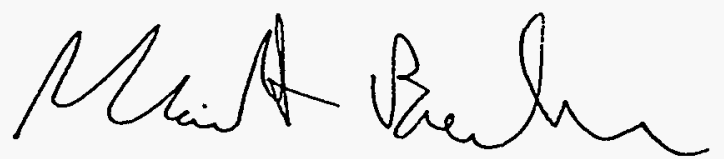

From: Walt Beyeler 6307

Re: Prioritization procedure

I understand that you are interested in the work on prioritization being done in the Technical and Compliance Integration Department. The attached memo to Paul has the most complete discussion of the proposed prioritization procedure. I apologize in advance for the rather rambling and convoluted presentation: at the time it was written I didn't anticipate the interest that it would receive. I am working on a more concise description of the method, but I thought that you might rather have the earlier version now, in spite of its flaws, rather than wait for a revision.

Please let me know your thoughts and comments.

cc: SWCFWBS 1.1.7.2, $P A / C O$ 
Date: February 17, 1994

To: Paul Davis, 6307

From: Walt Beyeler, 6307

Re: Initial thoughts on quantitative prioritization

I recall our discussing the need to keep all concerned parties abreast the developments on the ranking procedure. Whatever the outcome, someone is going to find it disagreeable: everyone should therefore have every opportunity for understanding and criticizing the process used to recommend priorities, prior to having it applied to their work.

One disadvantage of "open" development is that all the missteps, retrenchments, dead ends, unexplored paths and acts of faith are in plain evidence. The history of how a thing was done is always much messier than a bare description of the outcome, but I believe, in this case, it is worth exposing as many people as practical to the development of the ranking procedure to promote the quality and credibility of the result.

The îrst task is to clearly delineate the purpose and scope of the procedure. It is especially important that I understand your requirements, and that the format and limitations of the results conform to the needs of the decision makers. To this end, I've put down some thoughts in these areas. In the spirit of "open" development, I've tried to be complete rather than concise. The result is consequently somewhat rambling and disorganized. I have included digressions on implementation and other issues incidental to definition of purpose and scope, more or less as they occurred to me. Some of the discussion (e.g. on regulatory interpretation) is not included under pretense of insight or novelty, but because there appears to be a strong connection to the problem at hand, which is best illustrated by adopting some specific position and following the consequences. It is important that the formulation of the ranking procedure be consistent with project positions in related-areas. Correction of, or comments on, any incidental material would be helpful in insuring that consistency.

Please let me know your comments, and those of any others you feel might contribute at this early stage, as soon as you can. I propose we submit a finalized purpose and scope, again to as wide an audience as practical and useful, as soon as possible, and will begin to put that together as soon as I have your thoughts. 
A plausible goal of the ranking procedure is to propose priorities for experimental work which leads to the most efficient evaluation of WIPP compliance. This doesn't hold up very well on examination, but is close enough to a more precise and workable purpose to serve as a starting point.

\section{A) Compliance and Evaluation of Compliance}

"Compliance" is understood to mean producing a CCDF for comparison with the 40 CFR191 containment requirements. Although these requirements are not final (and would be sufficient but not necessary conditions if they were final), allowing for the possibility of compliance via unwritten regulations using unspecified procedures introduces too many imponderables. If the CCDF lies to the left of both critical points stipulated in 191, we assume the site complies; if it lies to the right of either one or both, it does not.

Even constrained in this way, interesting questions remain: which CCDF ("mean", "median", and so on) will be used? what will be done about multiple conceptual models? what are we to make of language in 191 that alludes to uncertainty in the CCDF itself? what are the sources of this uncertainty and how is it to be treated? These questions are being addressed, and will presumably be answered before a compliance document is submitted.

An early answer to these questions may be more important for experimental ranking than for compliance assessment per se. As an example, a decision to use the median rather than mean CCDF determines which of two results, simultaneously produced, are emphasized in the compliance document; the mechanism for determining the sensitivity of the median CCDF to new information, however, may be quite different from the mechanism used to determine the sensitivity of the mean CCDF. A conclusive ruling would ideally be made in time to allow development and testing of only the appropriate code.

In addition to these questions, there is a fundamental ambiguity about the CCDF that influences, among other things, our approach to experiment ranking. The regulations stipulate a probabilistic limit on system performance, and mandate consideration of probabilities (e.g. future events) which do not have an unambiguous frequentist interpretation. Probabilities attached to important components of the performance assessment represent a degree of belief in the existence or operation of those components, rather than a frequency of occurrence which can be objectively estimated. Because these probabilities effect the CCDF, the CCDF itself represents the degree of belief in various levels of future repository release. The regulation stipulates what must be believed about the repository, rather than setting out objective criteria to be met.

The immediate question is "Whose beliefs?". The most reasonable answer is EPA. In this view, the decision (e.g.) to present mean rather than median CCDF's would not be based on theoretical considerations at all, but on EPA's answer to the question "which curve is the better representation of your degree of belief about release?". Creating a CCDF would amount to quantifying EPA's degree of belief about various levels of future repository release. This quantification would rely on models which were appropriate in 
the view of the regulators, using parameters which represent their degree of belief about appropriate values. In the extreme, appeals to experimental data would not necessarily be availing: we are seldom in a position to make a direct measurement corresponding to a model parameter which is unambiguously valid for 10,000 years. The EPA is anyway not obliged to be rigorously Baysean in updating their prior beliefs.

The regulatory environment makes this view of the CCDF more than a radical extrapolation of probabilistic esoterica. The regulators, if they deem the repository to comply, will be obliged to defend our work in court. They are not likely to do this without full faith in the analyses we perform. Whether formally or informally, any CCDF which shows compliance must represent the EPA's degree of belief about repository performance.

The relevance of all this to the present work is clear: a quantitative measure of experimental importance will depend on the experiment's expected influence on the CCDF. We obviously can't estimate this influence without a clear definition of the CCDF, but this is not enough. We also need to understand how experimental results will influence calculated release (e.g. through model parameters). This may be straightforward in some cases, but it would be imprudent to proceed without a clear and specific understanding of how experimental results will be regarded by the regulators. As an example, we might consicier spending a great deal of money to measure plutonium solubility to high precision under a wide range of conditions. Whether these results are used by themselves to replace existing estimates, or are used instead to update (in the sense of Baysean updating) a distribution provided by an expert panel may greatly influence the anticipated value of the experiment.

A prior agreement about experimental interpretation is critically important for resolving conceptual model uncertainty. The method used to represent conceptual model uncertainty in the CCDF (whether by selecting the most conservative model, or weighting the models in some way, or selecting the median probability at each release level, or whatever) would seem to be a decision which the regulators must make if we accept that it is their beliefs which the CCDF represents.

Whatever method is used, it will not be possible to evaluate the utility of experiments directed at resolving conceptual model uncertainty without a clear understanding of the connection between the degree of belief in the model and possible measurements. The need for this understanding can be most clearly seen if a single conservative model is selected: without an acknowledged connection between specific observable results and belief in the model, there is no prospect of designing an experiment to test the hypothesis represented by the model, let alone estimating the utility of such an experiment.

I would strongly urge that any proposed conceptual models always be accompanied by a specific a priori description of experiments and experimental outcomes which would constitute disproof. If the regulators are inclined to weight results of conceptual models, the way in which this weighting is affected by data must be understood.

Prioritization of experimental activity on the basis of compliance does not render work which does not feed model parameters irrelevant. If the notion of the CCDF representing the EPA's degree of belief has any validity, such work may be, for 
example, part of establishing credibility with the regulator: an EPA hydrologist may be unwilling to believe anything we say about WIPP hydrology until it is placed in a credible regional context. I mention this here for two reasons: it would seem to be an immediate priority to identify and satisfy all "background" information requirements of the regulator, and it seems important to emphasize within the project our understanding that experimental or analytical work can directly affect compliance without necessarily supplying numbers for a particular code.

Returning at last to the purpose of the experiment ranking, "evaluation of compliance" can be understood as "calculating a CCDF in such a way that, along with supporting background information, the EPA would consider the regulations to be satisfied if the probability of a release value of 1 were less than 0.1 and the probability of a release value of 10 were less than $0.001 "$.

\section{B) Most Efficient Evaluation}

We can be in one of three positions with regard to evaluation of compliance with the containment requirement: unable to evaluate compliance (for example because we know that the EPA would not consider any CCDF we can produce as suitable for comparison with the standard); able to evaluate compliance (because we know the EPA would consider the CCDF suitable); or uncertain about our ability to evaluate compliance (because we don't know what results or evidence the EPA considers suitable). As a separate point, a given CCDF either meets or does not meed the criteria defined in 191. We can represent the possible states in a $3 \times 2$ table:

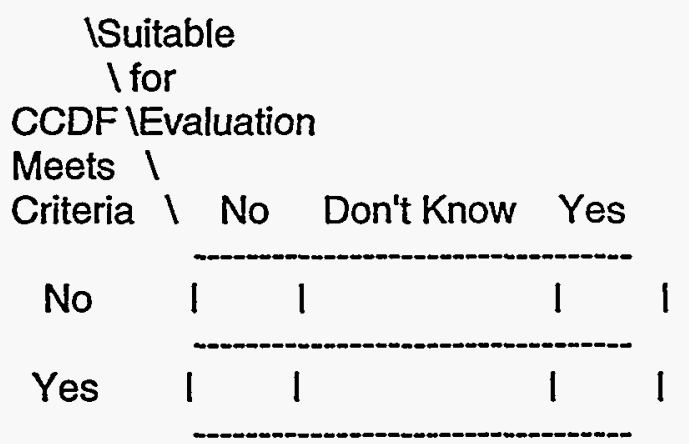

On one construction, Sandia's job is to move from wherever we happen to be to the column on the right, whether or not the CCDF meets the stipulated criteria. The experimental prioritization procedure is intended to recommend an optimal way of doing this.

Postponing for the moment a definition of the cost or utility function to be optimized, lets consider the optimization problem as proposed. First, the minimum cost associated with moving to the 'Yes' column is itself of interest: the DOE might decide that this cost is not justified, whatever the position of the resulting CCDF. Second, and more seriously, we need to define the alternatives for moving from one column to another, across which an optimum is desired, keeping in mind that for the present we are indifferent as to rows.

Presumably, if we are in the 'No' column, we are there because some piece of 
information or documentation is known to be missing, without which the EPA would reject any application as incomplete. Correcting this known deficiency obviously poses an optimization problem only if there are alternative ways of correcting it: e.g. if the inventory was deemed to be too uncertain (again with respect to suitability for evaluating compliance rather than for demonstrating compliance), we might optimize over proposed source term characterization procedures, each of which meet the required accuracy.

We can expect, in general, that while deficiencies in accuracy or completeness might be found, findings of deficiency would refer to the existence of data, not to the data values themselves. We would never hear, for example, that "EPA cannot evaluate your compliance document because Plutonium retardation factors in the Culebra are bëlow 10." An implied interchangeability of data ("We cannot evaluate your application until you either provide 3 additional halite permeability measurements or an Americium solubility") is also never to be expected. It seems much more likely that findings of deficiency with respect to completeness will be rather narrowly focused, and offer a single method of resolution. While it is essential to identify and correct any such deficiencies, correcting them appears unlikely to confront us with an optimization problem.

A variant of the hypothetical Plutonium retardation comment provides an apparent counterexample: "We do not consider your argument that Plutonium retardation is above 10 to be convincing. This assumption requires further support before we can evaluate compliance." This suggests an optimization problem, but a trivial one if the objective is purely to present a document suitable for compliance evaluation, regardless of the ouicome of the evaluation. The least costly option in this and all similar cases is to retract the undersupported assumption in favor of something more conservative.

There are real alternatives if we are in the middle column. We can either prepare a compliance document in the expectation that EPA will consider it complete, or work to insure completeness prior to preparing the compliance document. The more cost effective option will be determined by the EPA's willingness to provide DOE with preliminary indications of suitability. If they are forthcoming, it will obviously be more cost effective to solicit informal guidance rather than prepare a compliance report. If the EPA will give no advance indication as to suitability, we can either collect or compile supporting information which we expect they may require, or submit a possibly incomplete document to get a formal reading.

The optimal choice in this case would depend on our confidence in predictions about the EPA's requirements, the cost of preparing the compliance report, the cost of a finding of incompleteness, the cost of assembling information that we expect will be required (again being concerned with the nature rather than the content of this information).

This decision might be formulated as an optimization problem (given the relevant probabilities and cost estimates), but there is no point. The premise that we are interested in most efficiently supporting a compliance report that is suitable for evaluation, without reference to the finding of compliance, is untenable. If this was the objective, we might assume that the entire repository inventory was instantaneously disgorged by some unspecified mechanism. This estimate of release would be optimal in some sense because it would require no technical justification: 
release could never conceivably exceed this value.

We have assumed that DOE will seek compliance with the containment requirement in 191. Under this assumption, DOE has nothing to gain by preparing a compliance document which contains a CCDF indicating non-compliance. The preparation of a document which adequately defends a finding of non-compliance will have little value for DOE. DOE presumably places great value in finding themselves in the lower right corner of the above table. In recommending experimental priorities to DOE we must take explicit account of the value they place on a finding of compliance.

All this may seem like a long and tortuous path to an obvious conclusion. I include it because I have heard Sandia's role described (roughly) as: supporting the DOE iñ preparation of a compliance report, one way or the other (i.e. whatever the outcome of the compliance evaluation). This seems unworkable if one of the envisioned outcomes is a compliance report which manifestly demonstrates non-compliance: such a report would never be worth preparing. If we accept that any submission to the EPA will illustrate compliance, it seems that a better description would be: finding the best technical support of an argument for compliance. Possible outcomes in this case are: an EPA finding of compliance, an EPA finding of inadequate support, and a decision not to pursue the compliance determination because of cost. The cost function which will be used to recommend steps for moving around the above table must explicitly include the value attached to the CCDF meeting the criteria.

\section{C) Formulation of an Optimization Problem}

The goal of the experimental ranking is to identify the "best" path irom wherever we happen to be to the lower right corner of the above table, to identify the costs associated with the path, and to recommend experimental priorities based on this path. This goal can be formulated as a quantitative optimization problem as long as costs and the notion of "best" can be quantified, and the set of activities from which the optimum path can be built are specified.

The activities that might influence the completeness of the compliance report (i.e which column we're in) have been discussed previously. It seems likely than many of these activities will consist of satisfying requests for specific information (e.g. "where is the derivation for equation 2?" or "please provide copies of the referenced well logs") which do not admit a range of alternative responses. In these cases, there is no role for optimal resource allocation. (If the cost of responding to requests of this kind is thought to be significant, however, these activities might contribute "uncertain fixed costs" which influence the decision to pursue compliance.) Any activities needed to establish credibility with the regulator (e.g. the regional groundwater study discussed above) are in this category.

Other activities influencing completeness will depend on the EPA's willingness to provide informal guidance, as discussed previously. Although questions arising in this regard (e.g. "Should we collect additional information, or submit the existing information to EPA to see what they say?") can be posed as optimization problems, I am suspicious of the value of doing this unless experience allows us to quantify the likelihood of EPA's response with some degree of reliability. 
The third type of completeness-related problem discussed above ("Your support for a Plutonium retardation factor of 10 is inadequate") is potentially amenable a cost/benefit analysis, in that it implies that compliance might be demonstrated if either more data were provided or a different value was used. In this sense, the problem is very similar to making decisions about how to "optimally" influence the CCDF, which are discussed in detail below. The important difference is that the need to make this decision is hard to anticipate a priori (before submission of the compliance document), and is mandatory a posteriori.

The activities which influence the position of the CCDF (which row we are in) include anything that changes the degree of belief (presumably of the regulators) in the value of integrated release over 10,000 years. Moving from the upper to the lower row entails changing the belief of the regulators about release. With respect to compliance, the ultimate aim of experimentation is to affect the belief of the regulators, rather than to understand the natural behavior of the system. The latter goal is relevant only because it is instrumental to the former. This "unscientific" situation arises because the CCDF is not an inherent property of the repository system, in the sense that the repository location and size, or even the integrated release over the next 10,000 years, are inherent properties of the system. The repository has no "true" CCDF which can be uncovered by experimentation: it is instead a function of the information we have about the system.

Let's begin considering the "optimal" path to an argument supporting compliance by imagining that all completeness requirements have been satisfied (i.e. we are in the rightmost column), and that we can calculate a CCDF which the EPA accepts. If the CCDF satisfies the containment requirements, then we are done. If it doesn't, then the DOE may either abandon the site or seek to modify the CCDF so that the containment requirements are satisfied. To make this decision, our job is to identify the "best" method for modifying the CCDF (by modifying the regulators beliefs), and the costs associated with this method.

The regulators beliefs about repository release will be derived from the site description, available information about the site, and predictive models which they consider applicable. The types of activities which affect the CCDF therefore include:

1) Supplementing data available about the repository;

2) Modifying the way this data is used to predict future repository states;

3) Modifying the description (design) of the repository.

Each of these categories represents a degree of freedom in finding an "optimal" path to compliance, whatever definition we adopt for "optimal". In principle there is no advantage to artificially constraining the optimal path to consist of activities from only one or two of these categories. The lowest cost alternative identified using all three types of activity can never be greater than the lowest cost using (only) one or two types, and is very likely to be less. To insure DOE that the "best" path is identified, optimization of activities should include all mechanisms for influencing the CCDF unless there are insurmountable practical problems associated with including activities of a particular type. 
To define the optimization problem, we need to describe costs and benefits associated with each activity.

\section{1) Benefit Measures}

Given the constraints adopted above (consideration of only the containment requirement, current completeness of the compliance application), the benefit of each activity or set of activities is related to its effect on the CCDF. Whether the CCDF shows compliance after some activity is performed is of particular interest. One possible measure of benefit is based only on this consideration: if an activity or set of activities results in a CCDF which shows compliance, it has a benefit value of 1 ; if not, its benefit value is 0 .

This measure may not capture some desirable consequence of the activity. Suppose alternatives $A$ and $B$ have identical costs, but different expected effects on the CCDF:

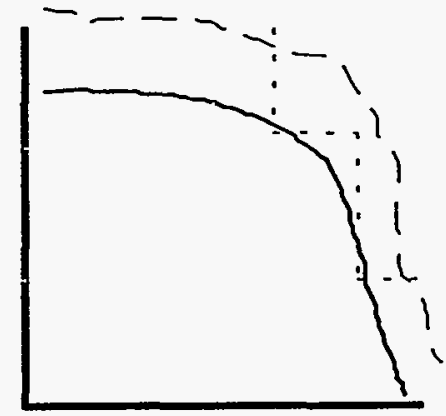

A

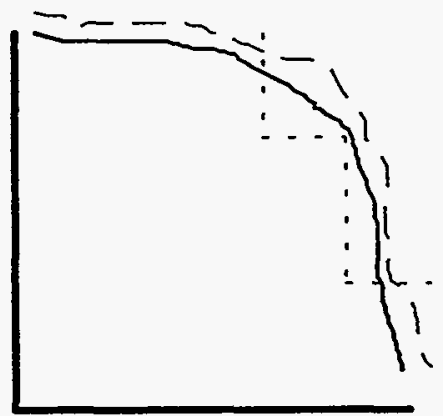

B

Neither CCDF complies, but the larger effect of activity A may make it the better choice if combined with other activities which also reduce expected release. Similarly, if the effect of alternatives $C$ and $D$ are:

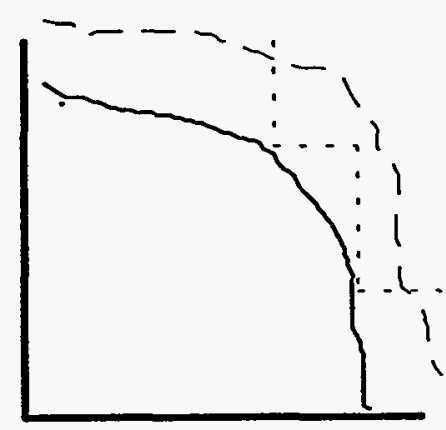

C

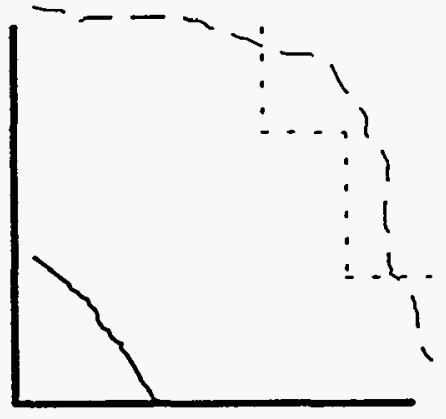

D 
we are led to prefer $D$ if all costs are equal. The CCDF resulting from $D$ does not comply "more" than the CCDF from $\mathrm{C}$, but appears to provide a larger margin of safety from the standpoint of demonstrating compliance. We can influence beliefs about release by collecting new information or by demonstrating a better use for existing information. Similarly, new information or requirements to consider different interpretations may cause predicted release to increase. The CCDF resulting from $D$ appears to provide a larger "buffer" for future changes in predicted release arising from unexpected information.

These hypothetical situations suggest a benefit measure that includes some notion of degree of compliance or distance from compliance, even though this has no regulatory meaning.

The benefit measure should also include the uncertainty in the prediction of the effect of an activity. If we consider the range of outcomes for activities $E$ and $F$ :

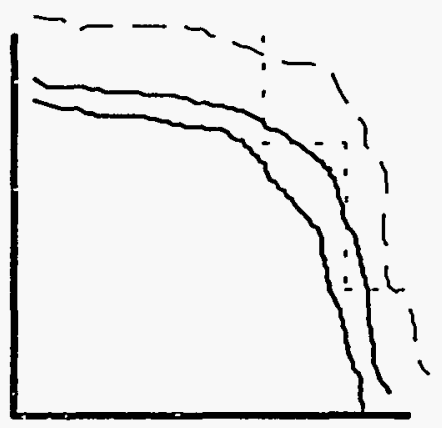

$E$

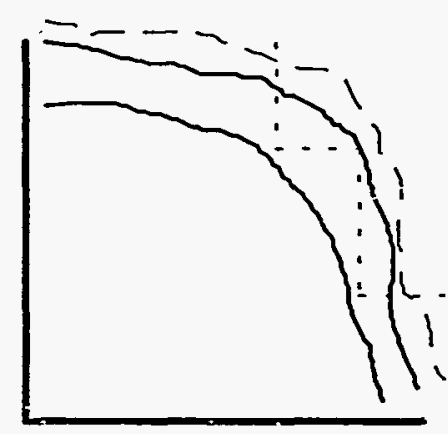

$\mathbf{F}$

With equal costs, $E$ is preferred because it is more likely to lead to a complying CCDF. In the figure below, G might be preferred for the same reason:

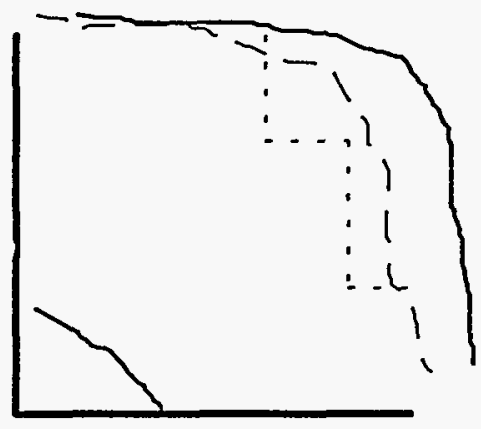

G

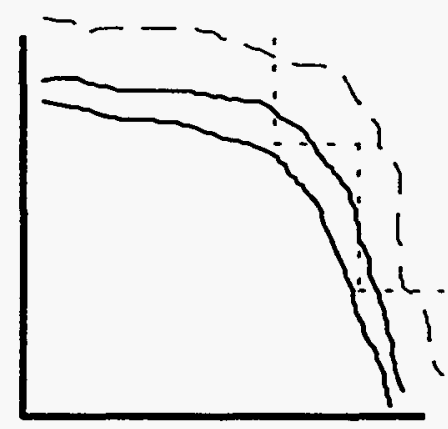

H

although the possibility of producing a CCDF which is very "far" from compliance may argue against it. This is only important, however, if other activities following determination of the effect of $\mathrm{G}$ are contemplated.

The goal proposed for the optimization is to identify the path which maximizes the probability of producing a CCDF that illustrates compliance within given cost constraints. 
If the cost constraints are reached without producing a complying CCDF, no further action will be considered. This goal seems a sensible basis for making resource recommendations, and resolves most of the dilemmas presented by the hypothetical situations described above:

If the identical costs of $A$ and $B$ both exhaust the cost constraints, there is no reason to prefer $A$ over $B$. If either activity can be part of a larger set of activities, any advantage represented by $A$ will be reflected in a larger probability of compliance for the sets which contain A rather than $B$.

No such distinction is made between $C$ and $D$. Neglecting uncertainty in predicting the effects of $C$ and $D$, the activities are indistinguishable from the standpoint of compliance. As discussed, D may offer an advantage against unanticipated changes in the information or models used to predict performance. We have already assumed, however, that we are in the rightmost column of the compliance table, i.e. producing a CCDF which complies with the containment requirements will result in compliance. From this position $C$ and $D$ are indistinguishable. If we are not in the rightmost column, $D$ may offer a real advantage over $\mathrm{C}$. As discussed before, determining an optimal course of action becomes a much different and much more complicated problem in this case.

If either activity $E$ or $F$ would exhaust the cost constraints, $E$ would be preferred. If either one could be part of a larger set of activities, any advantage offered by the "predictability" of $E$ would be reflected in the final maximum probability of compliance.

Again, if $\mathrm{G}$ or $\mathrm{H}$ would consume all costs, $\mathrm{G}$ would be preferred. As alternative parts of a set of activities, the possible disadvantage of $G$ (potential to increase predicted release) will be properly reflected by the presence of mitigating measures in paths including high-release outcomes for $\mathrm{G}$.

The product of the optimization would be the set or sequence of activities which maximize the probability of producing a CCDF showing compliance, given constraints on cost. We would naturally be interested in the effects of different cost constraints on the maximum compliance probability. Results would be presented as an illustration of the tradeoffs between limits on the various components of cost and the maximum probability of compliance, along with the sequence or set of activities corresponding to the optimal path for each limit considered.

\section{2) More Comprehensive Benefit Measures}

It is tempting to try to identify some function that subsumes the various components of cost, and the cost of failure to produce a CCDF showing compliance, into a single utility function. I strongly suggest that we resist this temptation. Establishing such a function would be a complicated and potentially very inaccurate process.

We might develop or collect cost estimates for alternative waste disposal options if WIPP is not used, which would include costs for closing WIPP. The costs and likelihood of seeking compliance by some other means would be important, but difficult to estimate. There would also naturally be many intangible costs to abandoning WIPP, 
which might be determining, but would elude quantification.

If we assumed that money and time are the only components of cost, establishing the perceived tradeoff between them would also be a challenge. Is it worth spending $\$ 100$ million to submit a compliance report in 1997 vs. 1998? Would it be worth waiting until 1999 if $\$ 500$ million could be saved? What if this delay also raised the probability of compliance from 0.8 to 0.95 ?

A practical way of developing a global utility function would be to present the decision makers with a large set of alternative pairs of numbers for cost and compliance probability, and ask them to order them. How seriously would this be taken? Would the same person make the same decision when confronted with actual tradeoffs? Would the same people even be involved? How would priorities change with time? Although this sort of elicitation seems the only way of identifying the client's utility function, the process, even if it yielded reliable results, is quite silly: we would confront the decision maker with countless pairs of hypothetical alternatives in order to avoid presenting them with a comprehensible set of actual alternatives.

I strongly feel that the final answer we provide should reduce the dimensionality of the decision-making problem through technical considerations, while allowing latitude for the final decision in terms that are relevant for programmatic direction and public policy. Presenting the results as the maximum probability of compliance corresponding to different constraints on cost creates an effective interface between two separate decision processes. Technical issues are effectively compressed into something that is relevant for programmatic decision making (probability of success), while the technical activities appropriate urider established cost limits are identified through a scientific understanding of system behavior.

\section{3) Cost Measures}

For discussion, I propose we consider time and money to be the only components of cost. This assumes, for instance, that capital can be converted to personnel and vice versa. That this is not strictly the case will at least influence experimental interactions, in which a key individual can't be scheduled to do two things at once.

This simple view of cost will probably have to be enlarged. If new components of cost have externally established limits (i.e. they show up as another dimension in the "cost space" of the result), there is no formal difference in the optimization problem. If the new components do not have separately established limits, I assume that we can establish an exchange rate between the new components and other contributors to fixed constraints. This tradeoff would be included in the solution of the optimization problem.

As discussed before, it is important to include all possible mechanisms for influencing the CCDF in order to find the truly optimal solution. The three types of activities (data collection, design modifications, model alteration) have the same type of result: modification of the CCDF. They are also assumed to have the same types of cost. I suspect that, if this is not true, it would complicate the discussion but not preclude a solution which considers all three activity types. In any case it is important to be sure of this, and to flesh out the definition of cost in the near future. 


\section{4) Overview of the Optimization Problem}

Again, we assume that we have satisfied all requirements for completeness, in that if we calculated a CCDF which met the containment requirements then the compliance application would be accepted. The CCDF we calculate, however, does not meet these requirements, and we have number of specific activities available for modifying the CCDF. We also assume that if we perform any of these activities, or any combination of them, the completeness requirements would still be met.

Our job is to identify the activity or set or sequence of activities which maximize the probability of producing a CCDF which complies with the containment requirements, given specified cost constraints. The analysis will be repeated over a range of values for each constraint.

For convenience the optimization problem may be dēscribed as a decision tree, although this is not necessarily the best model for solving it. Beginning with a "noncompliant" CCDF, we consider each combination of activities which remain within the cost constraints. The possible outcomes of each combination are considered: the probability of compliance following those activities is then determined. For each noncompliant outcome, the combinations of available activities are again considered and screened against cost limitations. This need not be the same set of activities as was initially considered: activities available at any stage may be influenced by activities performed at previous stages. The possible effects of performing these activities are again estimated, and the process is repeated. The expansion terminates when all activity paths exhaust the cost constraints or lead to compliance.

Beginning with the last expansion, the activity path is selected which maximizes the probability of compliance. This probability then becomes the probability of compliance given the outcome which led to the alternatives considered. The conditional probabilities given all outcomes of the prior activity are used to calculate the probability of compliance given that activity, and so on.

This description is somewhat misleading, in that there will in general be a continuum of outcomes which do not comply, rather than a finite number. How this is resolved is another issue: the purpose here is to convey a picture of the problem.

\section{D) Activity Models}

A remaining problem is to decide how the activities are to be modeled in such a way that the models make sense for the types of activities considered, are compatible with the structure of the optimization problem, and have parameters which can be reliably estimated.

\section{1) Components of Activity Models}

On the simplest view, the activities we consider convert cost into some effect on release prediction:

$$
\text { Cost } \rightarrow \text { Activity } \rightarrow \Delta \text { (Release) }
$$


The link to release is always indirect: the activity produces some result or condition that, in turn, influences the release prediction.

$$
\text { Cost } \rightarrow \text { Activity } \rightarrow \text { Result } \rightarrow \text { Inference } \rightarrow \Delta \text { (Release) }
$$

In general the effect of the activity on release (at any probability level) is uncertain because the result is uncertain.

We assume that the connection between a given result and the change in release (at all probability levels) is not uncertain, because the inference method has been adequately supported as part of establishing the completeness of the compliance application. Note that this does not preclude, for example, updating a parameter distribution using experimental results in such a way that the parameter value is uncertain after the experiment: we instead assume that the way in which the parameter is updated on the basis of experimental results has been agreed to.

The details of the connection between result and predicted release depend on the type of activity (experimental, design modification, model modification), and are discussed in later sections. For the time being, we assume that each activity has an associated result, which is uncertain, and that this result has some effect on predicted release (the result may have other effects as well).

It will prove helpful to consider time as a separate component of cost, so that the model of activity i looks like:

\section{$\Delta$ Release}

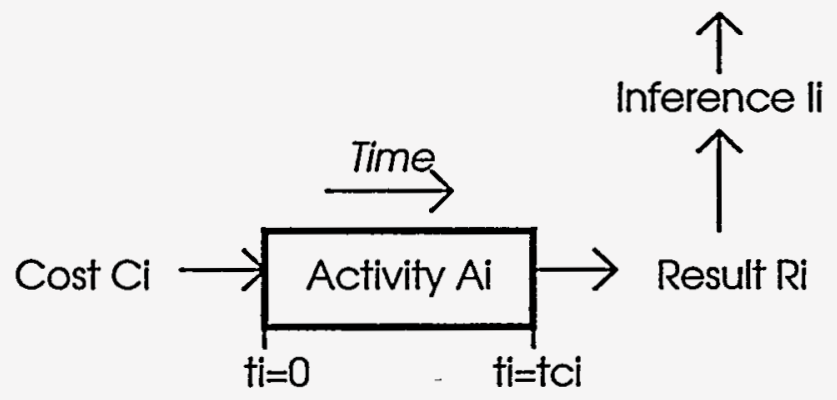

This model, along with the associated inference procedure, is all that is needed to characterize each activity for the purpose of optimization. Does it make sense to require each considered activity to be described by a known cost, a fixed duration, and a distribution of outcomes?

Some activities may fit this description; most will not. First, a result may not be obtained at all, or the outcome may be such that the regulators will not allow an inference to be drawn. The possibility of a null result should be included. The experiment may produce a number of types of result, rather than the single result suggested, and the result values and possibilities of null values may be correlated across result types. In addition, 
the result will be obtained with some accuracy, which should be included.

The cost and duration parameters may be oversimplified as well. Exact values may not be known in advance. Often this is due to contingencies built into the activity plan. Other results are observed which, while not affecting the predicted release per se, determine subsequent events. A more accurate model can be built.using elaborations of the simple model proposed above:

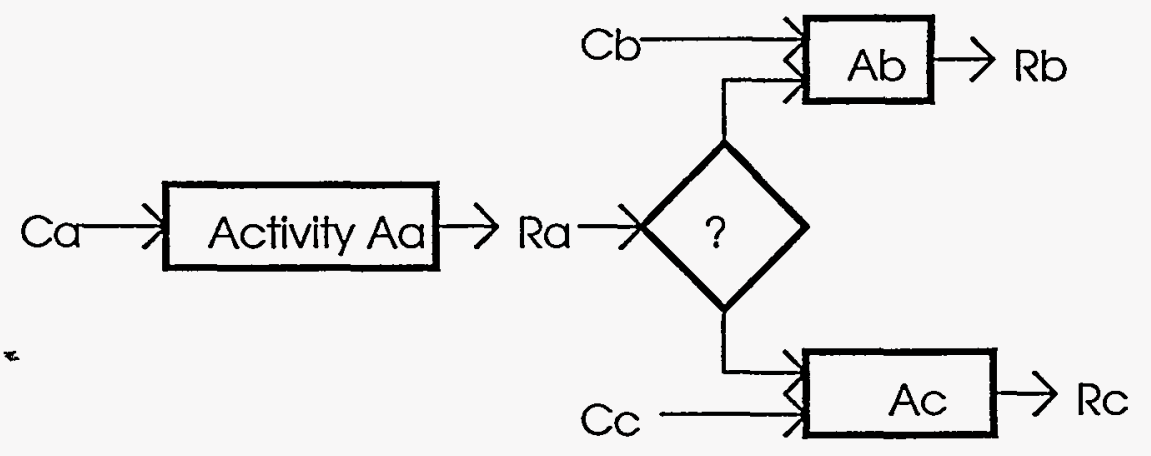

The decision elements in the model allow for costs to be conditionally incurred and results to be conditionally available. The individual sub-activities are still assumed to have fixed costs and durations. If the more elaborate model is "wrapped" as a single activity, it appears, from the standpoint of the optimization problem, identical to the original model, except that the overall cost, duration, and result availability are uncertain, and are appropriately interrelated. If the assumptions of fixed cost and known duration are not appropriate for the component activities of the more elaborate model, these components could presumably be expanded in terms of simpler, more predictable activities which satisfied these conditions, along with the appropriate_decision elements.

A remaining limitation is the assumption that the decision rules can be specified in advance. If these rules are allowed to refer to the value or existence of results from other activities this does not seem too restrictive.

The decision elements within activities create additional branching in the "decision tree" structure of the overall optimization problem within the activity branches. Each outcome of each possible decision needs to be represented. It is not necessary, but it may sometimes be desirable, to have activity decision elements which coincide with release distribution updates.

In these cases, it may be worthwhile to divide an activity into phases, junctions of which correspond to revision of predicted release. In constructing possible activity sets, a later phase would be available only if the preceding phase had been completed. As an example, suppose some experimental variable can be determined with increasing precision with increasing time. The experimental model might look like: 
Phase 1

Phase 2

Phase 3

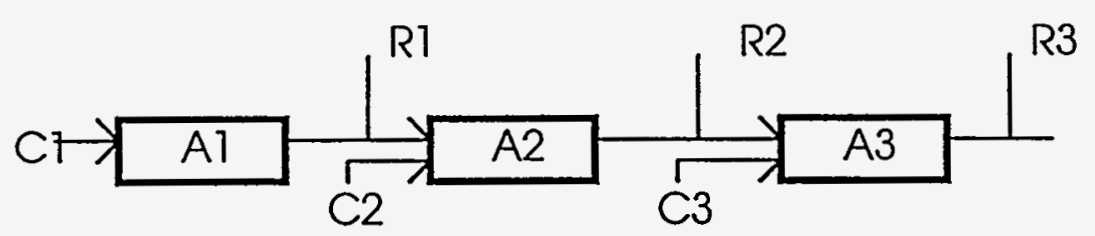

In which the first phase contains start-up overhead costs, and successive phases provide updated estimates of the variable value with increasing precision. The benefit of proceeding to successive phases would be determined by the inability to-demonstrate compliance given the result and precision of the preceding phase.

The elaborated activity description seems sufficiently flexible to accommodate most anticipated activities, but it is essential to confirm this by discussing the model with researchers, operations management experts, and by trying to interpret existing plans in light of the model. Interesting complications arise around the interactions and sequences of activities. In the above example, for instance, would the costs remain the same if the program start date were delayed by a year? Is it possible to delay the start of Phase 2, or must it follow Phase 1 immediately if at all? If it can be delayed, are there additional restart costs not included in the original description?

Our job is not to answer these questions, but to be able to represent the answers if they are relevant. In building a sequence of activities to find an optimum path, it is essential that each path represented make sense by itself. Determination of global reasonableness (e.g. are two incompatible activities scheduled to occur in the same place at the same time) is an important aspect, but is not a requirement for the reasonableness of the activity model per se.

Activity models for experiments, engineered alternatives, and system model modifications differ in the types of results expected and the types of inferences used to predict changes in release. Some interesting general points about the proposed activity model can be seen before considering models for particular activity types.

In each case we need to identify the type of result expected, the techniques available for making prior estimates of result values, and the inferential methods connecting results to release predictions. Result types and the methods used to estimate distributions are particular to the activity type. Like the CCDF itself, the result distribution represents a degree of belief about various outcomes, however there is no regulatory significance to this distribution: rather than representing the regulators degree of belief, it should reflect the knowledge of the DOE, or Sandia, or knowledgeable experimentalists or design engineers. This point is discussed in more detail in the context of the experimental model.

The connection between the activity result and the change in predicted release can be divided into two parts: the effect of the result on the modelling system, and the effect of the modified modeling system on predicted release. Again, because the activity being considered should not degrade the completeness of the compliance application, the way in which the results would be interpreted (i.e. the connection between the results and changes in the modeling system) must be approved by the regulators, and therefore 
must be agreed to before the results are available.

Ideally, the effect on release given some change to the modelling system would come from 'turning the crank' using the regulator-approved implementation of the modified modeling system. As discussed later, I don't think this will be possible in practice. The important point here is, again, the optimization problem has no regulatory significance: we are free to use whatever method seems appropriate to implement the modified modeling system: we need to approximate the approved implementation only because the approved implementation would be used to update release estimates using actual experimental results.

The same reasoning is valid in principle for the first inferential'stage (coninecting rèsults to model changes), but is not worth pursuing. A given approved method for changing the modeling system in response to results is not likely to be so inefficient as to make approximation worthwhile, even if some standard for approximation was available. On the other hand, if the way in which results will be interpreted by the regulators is unknown, a meaningful assessment of the value of the experiment is impossible.

The following sections discuss the result types, result distribution estimates, and firststage inferential procedures associated with the three activity types.

\section{2) Experiment Models}

For many experimental activities, the result will be a measurement that closely corresponds to a model parameter, such as plutonium solubility, gas gerieration rate, or transmissivity. In the simplest case, the experimental resuit (with the associated experimental error) would be identified with a particular model parameter. In this case, the prior (present) distribution for the parameter could be used as the distribution of the experimental outcome. As discussed above, this is not necessary: if the present distribution includes values which the experimentalist considers unrealistic (and, again, the regulator has agreed to replace the current distribution with the result of the proposed experiment), the experimentalist's prior distribution would be more appropriate.

In a more realistic situation, the experimental result would be more loosely connected with the model parameter, and would be used to update the parameter distribution in some way (e.g. because of concern over extrapolation over time, or estimation of volume averages from point measurements, or weight given to prior estimates). In principle this presents no problem as long as the updating procedure is specified. Prior estimates of experimental results might come from experimentalists, or might be derived from existing data. For example, if the experiment was meant to measure far-field fluid pressures in the Salado, Martin Tierney's regression model, fitted to existing data, could be used to predict the distribution of outcomes of "point" measurements at new distances; these possible experimental outcomes could in turn be used to derive estimates of volume-averaged pressure distributions. As another example, a prior distribution for Culebra transmissivity could be obtained by kriging existing values.

In the above cases, the experimental result influences release because it is directly or indirectly related to parameters of the system model. Two other possibilities come to mind: the result changes the system model (i.e. changes the way the inference of 
release is made), or the result has no direct influence on release, but is important for some subsequent activity. In the proposed framework, the first situation is considered under 'Analysis' activities, along with other possible changes to the inferential mechanism. Although the underlying activity is experimental, the effect on estimated release is similar to (e.g.) a change in the differencing scheme used to solve the transport equation. The second situation is handled by making subsequent activities contingent on the experimental outcome through explicit reference to the result in decision elements.

\section{3) Design Models}

Like experimental results, design results may directly correspond to system modël parameter changes, or may indirectly influence model parameters, or may change the system models themselves. (It seems unlikely that we would consider design modifications with no direct connection to release estimates.) The 'result' of the design modification may include a index of the expected repository state, which might appear in the decision elements of subsequent activities.

From the standpoint of the optimization problem, design changes appear to be indistinguishable from data collection: a separate activity class is useful because they are different types of activities from any other viewpoint, and because important structural differences may appear under more careful examination.

Many interesting questions surrounding design modifications are expressible and answerable in the proposed framework, for example, "Is it worth making preparations for waste treatment now, even though later information may show that this is not needed?" Other questions, such as the tradeoffs between waste processing options based on operational risks, are beyond the proposed scope of this procedure and of Sandia's role in WIPP. The interdependence of operational risk, total cost, and long-term performance should somehow be addressed, perhaps by adding another dimension to the fixed cost constraints considered in prioritization.

\section{4) Analysis Models}

Any activity that changes the inferential procedure (i.e. the structure of the system models) is considered in this category. Examples include changing numerical procedures in computational models, changing the resolution of discrete approximations, changing the dimensionality of models, modifying the governing equations, modifying the boundary conditions or initial conditions, or modifying the design.

Some of these cases are not relevant for the optimization problem. We can assume, for instance, that in order to satisfy completeness requirements, current numerical procedures would have been verified, in that the results are shown to be 'close' to analytical solutions. Alternative procedures will either produce similar results or, if not, will either be wrong or refute the verification of the current procedure.

We might be in a position of deciding between two acceptable discretizations or dimensionalities which produce substantially different results if we use a simpler representation for computational efficiency and have demonstrated that the 
simplification always gives conservative results. The 'cost' of using the more complex procedure comes from the additional time and computer resources required to calculate the CCDF compared to some current simplification. Unlike the experimental activities, it seems that we must incur this cost in order to estimate the benefit of incurring it! For the time being, this problem is excluded from the scope of the prioritization procedure.

The inferential procedure may be changed by changing the design or conceptual model. If the influence of design changes on the current model structure is accepted in advance, the effect of the new structure can (in principle) be easily explored by incorporating it in the inferential structure of all descendant paths which incorporate the design modification. The viability of the design modification, as represented by the change in system model structure, can be made appropriately contingent on the outcome of experiments. If the influence of the design change on model structure is uncertain, we essentially have a 'conceptual model' problem overlaid on the design modification problem.

The 'conceptual model' problem arises if there is more than one tenable inferential procedure which connects available information to release estimates. The inferential procedure which meets the 'completeness' criteria can be thought of as a composite of all tenable models in which the results of each are weighted. The weights may be fractional, or may be 1 for the 'most conservative' and 0 for the remaining models, or any other scheme which satisfies the completeness criteria. As mentioned earlier, conceptual model uncertainty can be sensibly incorporated into the prioritization problem (or sensibly dealt with at all) only if the connection between observations and model weights is specified. The situation is analogous to experimental activities: we cannot evaluate the significance of an experiment without an a priori understanding of how the results affect the system model.

If we have, as I think we should require, an explicit description of the dependence of model weights on observables, the problem becomes tractable. We can design experiments to make the relevant measurements, and use the prior distributions of experimental outcomes (measurements)-to-assess the-selectivity of the-experiment with respect to models.

A possibly interesting aside: the above procedure is applicable whether the weights assigned to the models are fractional (i.e. represent a model 'probability') or give exclusive weight to one model in preference to all others. We only require that the model weights be connected to observables in some specified way. This doesn't seem too demanding if the model is meant as a scientific hypothesis about the real world. Once the connection between observables and weights is established, however, we can derive the distribution of weights from the prior distribution of quantitative observations. While this may be difficult in practice, the prior distributions of quantitative experimental outcomes are at least meaningful in principle. Because the model weights are defined in terms of the experimental outcomes, their distributions also have meaningful probabilistic interpretations. The expected values of these weights therefore represent the prior probabilities of the models under consideration.

5) Interactions

To find the set or sequence of activities which leads to the maximum probability of 
compliance for a given cost, we should consider, at each decision point, all possible combinations of activities. Not every combination or sequence of activities, however, is sensible: key personnel or resource requirements may preclude two activities from occurring simultaneously, or one activity may be contingent on the successful completion of another. There may be positive interactions as well, such as cross-hole logging between two holes drilled for different experiments. The goal is not to design a procedure for identifying important interactions, but to allow the important types of interactions to be appropriately represented. With the possible exception of resource allocation, the specific interactions to be considered must be anticipated by the PI's.

The decision elements are suitable for most of the examples that come to mind, provided references are allowed to the results of other activities: a hole drilled to obtain a core sample might show up as a contingent drawdown observation in a long-term

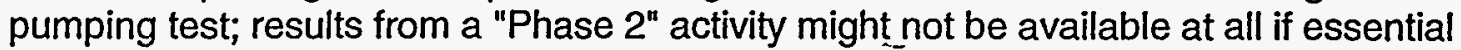
"Phase 1 " results were not satisfactory. In practice, this latter type of contingency should be imposed "externally" (i.e. in constructing the activity sets at the decision points) to prevent needless branching of the decision tree.

There may be situations where it appears reasonable to impose external constraints, but these constraints are either unneeded or lead to a non-optimal solution. Suppose we are considering a design modification which requires confirming experiments to demonstrate its (beneficial) effect. We might represent this as one activity, consisting of the experiment followed by the modification, contingent on the success of the experiment. We have more flexibility if we describe it instead as three activities: an experiment, a design modification, and an analysis modification contingent on the successful outcome of the experiment and design modification. The optimization will filter out silly combinations (design modification without demonstration of its utility, successful experimental outcome without the design modification), but unanticipated utility may be identified: because of time constraints, it may be desirable to begin the design modifications at the same time as the experiment, incurring the risk of misspent start-up costs. If efficiency is not a consideration, external constraints should only be imposed when logically necessary.

"Analytical" activities, which modify the system model structure, have a particular interaction problems. If the model structure is changed, the effect of the results of all subsequent experiments on release should be inferred using the revised model. This may be trivial in some cases. In other cases, the experimental result may be meaningless in light of the new model. In any case, we should consider in advance how each experiment would be interpreted within the framework of each model, and also anticipate experiments which might be useful in supplying parameters for models which are not currently used, but which may be adopted later.

Aggregations of system models need to be fully specified as well, for example "how do we represent design modifications $A$ and $M$ under conceptual model X?" This activity is further complicated by the great desirability for the concurrence of the regulators on the acceptable representation of each permutation.

Right now, a number of important interactions seem to be representable within the activity model, while others may require a global screening of activities prior to expansion of the "decision tree". There is no point in being more specific about the 
mechanisms for representing interactions until the activity model has been finalized and 'validated' against current proposals, and the specific interactions between all current activities have been identified and classified.

\section{E) Tentative Description of the Prioritization Procedure}

The following 'straw man' prioritization procedure summarizes conclusions reached from the preceding considerations:

If all 'completeness' requirements for the compliance application have been satisfied, and the quantitative regulatory performance measures do not satisfy the regulations, we want to identify the subset of activities (from a specified set) which are most likely-to lead to compliance given various cost constraints.

Cost constraints will include (at least) the present value of expenditures and the time until a compliance decision is required, but may include other components such as operational risk.

Activities available for modifying release predictions are described by their costs and the type of result they produce. All costs are certain, but may be functions of absolute starting time, or starting time relative to other activities. The result can be of any type, but must be characterized by a prior distribution. The distribution can include the probability of a 'null' or ambiguous outcome. Result distributions can be conditional on the-results of other activities.

Activities influence predicted release through their results. Results can directly influence predicted release by modifying the parameters of the system model or by changing the structure of the system model. In either case, the procedure for updating the system model given the result must be specified, and must not effect the completeness of the compliance application.

More complex activities can be assembled using decision elements. Internal decision elements can refer to prior results for the activity or results from other activities. External decision elements identify natural opportunities for programmatic redirection (increase, decrease, or termination of funding) based on compliance status.

Groups of exclusive activities can constructed, e.g. where each member corresponds to a different 'level' of effort. Sequences of activity groups, connected by external decision elements, can be defined, where each group in the sequence corresponds to a different 'phase' of the overall activity. The most general description of activities, which is used in describing the optimization problem, looks like: 


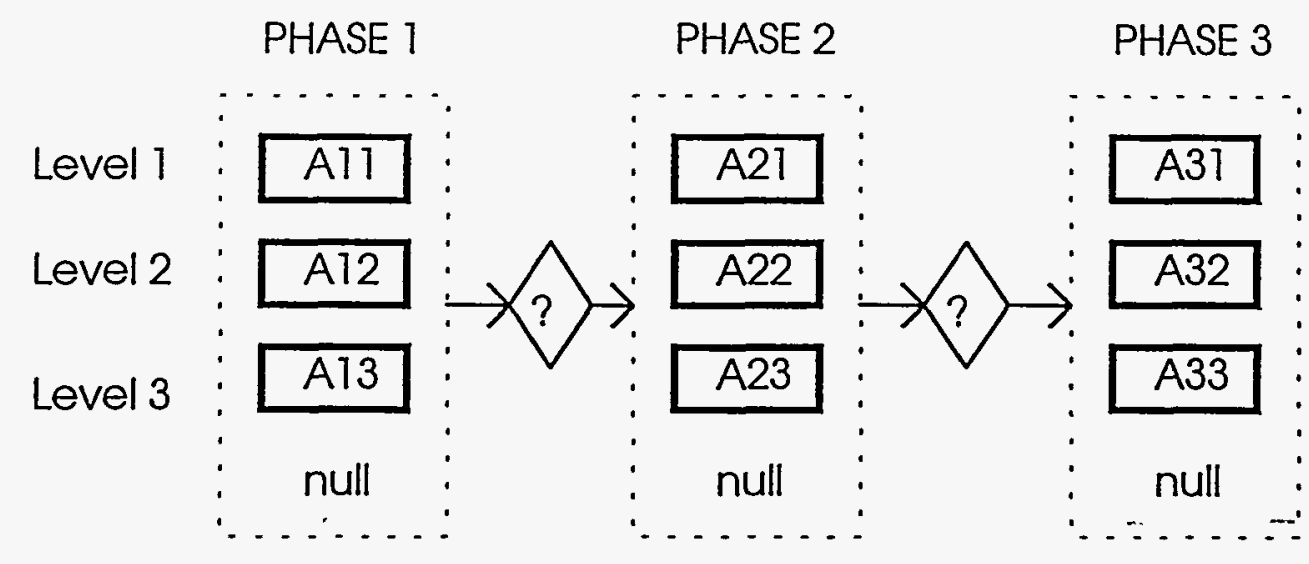

Where the 'null' activity, meaning termination, has been added for completeness. Each level in each group may be a complex activity, containing several sub-activities and internal decision elements.

Anticipated interactions between activities are described either through internal decision elements, or by explicitly disallowing or mandating specific combinations or sequences of activities.

External decision elements are required to occur only at regular intervals which make sense programmatically (e.g. 6 months or 1 year). The continuity of long-term activities across external decision elements can be insured using the 'interaction' restrictions described above.

Once all proposed activities have been specified, along with their interactions, and the way in which results influence the system models, the 'brute force' approach to the optimization problem is straightforward in principle:

1) We start at a decision point with releases which do not.satisfy regulatory requirements, and with stipulated cost constraints.

2) Identify all possible combination of activity 'levels' (including null) for all activities which satisfy interaction restrictions. Each combination defines an activity 'set' which can be followed from the current state.

3) Discard any activity set which violates any cost constraint (because of internal decision elements the cost of a set is uncertain in general. Expected cost or maximum cost might be used).

4) For each activity set, identify the distribution of results available at the earliest external decision element in the set.

5) Calculate the probability of compliance given this result distribution.

6) For each non-compliant outcome, repeat 2 through 5.

7) Once all paths terminate due to compliance or cost constraints, back up the probability of compliance associated with each activity set as described earlier. 
Identify the initial activity set which leads to the maximum probability of compliance.

\section{F) Implementation Considerations}

For the purpose of this memo, implementational details of the straw man described above are not appropriate. Some general considerations, however, may be relevant for the final formulation.

The process described is possible in principle, but creates what I think are impractical computation demands. Evaluation of release following each possible outcome of each possible sequence of each possible activity set would require a ridiculous number of PA calculations. Presumably there are techniques (e.g. within the framework of decision trees) for managing the combinatorial explosion, and there may be other efficiencies which turn up after a more thorough look at our particular problem. I don't think we can practically expect to do more than a small number (say 10) sets of PA calculations per year using the current PA models, or any models that are similarly complex: I don't expect than any reformulation of the optimization problem would allow us the scope envisioned here within these limits. Two options come to mind: the scope can be narrowed, and the release estimation procedure (vastly) simplified.

The degree of branching in the decision tree is controlled by the number of external decision points allowed between now and the final compliance evaluation. If we do not incorporate programmatic redirection between now and then, we can identify the optimal 'big step.' We might also retain the higher resolution activity descriptions, but identify the optimal 'small-step' based on compliance probabilities following the first step only. This seems less useful, as activities which do not influence release estimates after the first decision interval would have no apparent value. We can, in any case, control the degree of branching by reducing the number of external decision points considered at the expense of the 'optimality' of the result.

As emphasized above, estimating the value of activities with respect to compliance requires using the same procedures to connect results to release as would be used in a 'complete' PA. Because there is no regulatory significance to this optimization procedure, we are free to do otherwise, but risk misleading ourselves. I believe the distinction I tried to make earlier is worthwhile: it is important to accurately reflect how (if at all) the regulators will modify their beliefs about the system model based on results (because this procedure may be very uncertain and difficult to approximate); it is desirable to use the same calculational model as would be used in a 'complete' PA, but this part of the connection between result and release is much more amenable to approximation. We can tolerate more detail in the decision tree if we use simplified computational models to estimate release. There are also auxiliary benefits for confidence-building in developing simplified computational models which parallel the more elaborate codes used in PA.

The purpose of the optimization procedure is to see that project costs result in the maximum return of value. The same type of thinking should (informally) be applied to the optimization procedure itself. It is very easy to underestimate the difficulty of any 
activity involving computers: in preparing a program plan, I think it is important to insure that, if the capricious gods that control software development conspire against us, we have contingency plans in place, and interim results that are useful to the project. As an example, it seems that describing experimental and design modification activities in terms of costs, specific result types, connections between results and release estimates, and potential interactions has value beyond the use of the description in an optimization procedure. Although much of this has undoubtedly been done, consolidating and distributing this information in a common concise format may help Pl's and design engineers improve their designs by taking advantage of concurrent activities.

I suspect that one of the two options suggested for making the optimization problem manageable will be required, or both to some degree. Whatever approximations or simplifications are contemplated, we must be certain that the resulting prioritization is not changed as a result. In case the optimization problem remains intractable under this restriction, I think we should be prepared to abandon the quantitative prioritization rather than pursue it using simplifications which have an unknown effect on the quality of the result.

G) Miscellaneous

After a little thinking about the prioritization problem, some important questions remain:

What about regulations other than 40 CFR 191.13? It seems that the problem is not fundamentally changed if we consider 'compliance' to mean simultaneously satisfying all quantitative long-term performance regulations. Although, for nonprobabalistic regulations, we are no longer formally in the business of identifying the regulator's beliefs about the system, the practical situation is the same: the regulators must concur with the way the analysis was done, and the need to use 'approved' methods of updating and weighting models remains.

Is the probability of compliance a complete measure of benefit? New information can change release predictions. Although there is no real degree of compliance, a release prediction that is far below the regulatory limits is better than a release prediction that is just below, because the anticipated finding of compliance in the former case is less sensitive to the effects of unexpected discoveries. The proposed benefit measure does not represent this value.

Is the optimal solution meaningful if the compliance application is incomplete? Strictly, no. There is little point in deciding how to 'best' modify release estimates if the regulators reject the way such estimates are made. Practically, the optimization can be performed, but the results may have limited usefulness. Obviously, any activities which are known to be required for completeness trump recommendations based on the assumption of completeness. The optimization procedure may help resolve specific defensibility challenges (e.g. "either defend the retardation values used or reduce them").

Can the process of assuring completeness be usefully formalized and optimized? I don't think so, for the reasons described.

Is there any need for feedback to Pl's which will help experimental design? In 
general, questions like "How well do you need to know solubility?" cannot be answered in isolation. If this type of feedback would be useful, one approach would be for PI's to propose several specific 'levels' of experimental activity, which differ in cost and accuracy. The optimal 'level' could then be identified in the context of all other activities.

How can we efficiently estimate the effect of abandoning computationally efficient conservative models in favor of less conservative but more complex models?

How do we get 'unbiased' prior distributions of activity results?

\section{H) Expected Sequence of Events}

The following is a guess about how best to proceed. Again, I think it is important to involve as many people as practical in the design process. To this end, all evident opportunities for review and comment are taken.

1) Agree on purpose (specific content of the result) and scope (what activities and contingencies will be includied)

2) Distribute a concise description of the purpose, scope, and tentative problem formulation to all concerned parties (PI's, PA, systems analysts, WID, DOE,...) to determine whether the results will be useful and the problem is tractable in that the required information is either available or can be readily acquired and the optimization problem can be solved.

3) Design an activity model which is appropriate for activities within the scope of the problem.

4) Validate against available instances of activities (e.g. EPP)

5) Distribute the description of the proposed activity model and validation to Pl's and systems analysts, and resolve important problems or conflicts.

6) Decide on a framework for solving the optimization problem considering the structure of the activity model (e.g. decision tree, influence diagram, ....)

7) Develop a schedule for implementing the optimization problem based on software development time.

8) Distribute a description of the proposed implementation and schedule, and of the final activity model to all concerned parties, along with a request for the PI's, design engineers, etc. to describe their activities in terms of the final model.

9) Develop a 'test case' which includes a variety of activities within the scope of the ultimate application to WIPP. The purpose of the test case is both to verify the operation of the optimization procedure (particularly elements developed specifically for this application), and to illustrate the operation of the optimization 
procedure. The test case could, and perhaps should, be generic rather than 'WIPP-like'.

10) Distribute a description of the test case to concerned parties ( PI's, systems analysts, $\mathrm{PA}, \ldots$ ) and incorporate comments where possible.

11) Assemble and distribute the description of all proposed activities in terms of the activity model, with obvious interactions identified, to Pl's, design engineers, etc. Request that contributors identify interactions between their activities and all other activities.

12) Apply optimization technique to the test case. Distribute the results and an interpretation of any interesting or surprising findings.

13) Incorporate interactions (and revisions) in the 'activity catalog' and distribute

14) Apply the optimization procedure to proposed WIPP activities; report and interpret results.

15) Duck

This sequence depends heavily on the final purpose and scope being very close to what has been described. It doesn't seem appropriate to be more specific until the purpose and scope have been finalized. Only the first and last items are certain. 
26. Beyeler, W. 1994. "Introduction of the New WIPP System Prioritization Method." Draft, March 17, 1994. 


\section{Summary}

The Department of Energy (DOE) has committed to reaching a disposal decision for the Waste Isolation Pilot Plant (WIPP) in January 1998. The disposal decision will be based on satisfying regulatory requirements by that date. This report summarizes a method for establishing priorities for current and proposed technical programs on the basis of regulatory compliance.

The goal of the procedure is to identify the trade-offs between schedule constraints, budget constraints, and the likelihood of demonstrating compliance within these constraints. An understanding of these trade-offs is important for establishing the program budget and schedule, and for the disposal decision. The relationship between, schedule, and the likelihood of compliance may also influence broader public policy issues.

A path to a disposal decision which maximizes the likelihood of demonstrating regulatory compliance can be identified, as long as evaluation of regulatory compliance is limited to comparing quantitative performance measures to regulatory limits. Confidence of the technical and regulatory communities in the procedures used to derive quantitative performance measures is essential. As a necessary preliminary step to quantitative prioritization, the first section of this report outlines procedures designed to help establish that confidence.

The second section of the report describes a decision tree approach as the proposed mechanism for finding the optimal path to a disposal decision. Decision trees provide a very general and flexible framework for formulating and solving certain classes of optimization problems. As applied programmatically to WIPP, a decision tree approach will be used to identify the mix of all activities affecting quantitative performance measures (data collection, repository design changes, waste treatments, backfill amendments) that maximizes the likelihood of meeting those performance measures. A detailed presentation of the decision tree approach will be made at the thursday meeting.

\section{Evaluation of Compliance}

Regulations goveming long-term disposal at WIPP establish specific quantitative limits for potential release. Calculations can be performed for comparison with these limits given information on the repository design, waste characteristics, the geological setting, and assumptions about events and processes which may occur in the regulatory time frame. These calculations are only relevant for demonstrating compliance when they provide a reasonable degree of assurance that quantitative limits will be met. Reasonable assurance comes from the reliability of the calculations themselves, the appropriateness of the assumptions and parameters used, and the range of events and processes considered.

To support a compliance decision, the regulator and stakeholders must have confidence in the way quantitative performance measures are calculated. There are two approaches to creating this confidence: defending a single set of assumptions, 
Introduction of the New WIPP System Prioritization Method

parameters, and methods; or screening all plausible altemative sets of assumptions, parameters, and methods. The two approaches are suited to different problems, but the WIPP program feels that there is an important role for second approach in providing reasonable assurance to the regulator.

Identifying and defending a single set of events or processes as appropriate for longterm performance assessment is extremely difficult because of the long time frames considered in regulations pertaining to WIPP. The WIPP program will seek to provide reasonable assurance to the regulator and stakeholders about the scenarios and conceptual models used in performance assessment by actively identifying and incorporating their concems into performance assessment calculations as altemative scenarios and conceptual models.

A conceptual model elicitation and tracking procedure is being developed to provide a mechanism for including the concems of the regulator and other stakeholders in the performance assessment. Performance assessment will evaluate the consequences of all conceptual models which are plausible, are consistent with existing information, have not been adequately addressed in past studies, and affect the regulatory performance of WIPP. The purpose of conceptual model elicitation is to provide the regulator with reasonable assurance of the adequacy or completeness of the processes and events used in performance assessment. The screening process will be designed to produce a manageable number of alternatives without compromising this assurance.

A proposed elicitation and tracking procedure will be described in a forthcoming document, but the results of this procedure are critical inputs to the quantitative prioritization described in this report:

A set of conceptual models that provide reasonable assurance to the regulator that the important processes and events which influence performance measures are included;

A defensible mechanism for calculating performance measures for each model and scenario;

A description of data or analyses that would test the plausibility of each conceptual model;

The approach to providing reasonable assurance that calculated performance measures adequately capture concems over the long term safety of WIPP can be briefly summarized: the WIPP program will endeavor to assess performance in such a way that, using existing information, if the quantitative performance measures are met, the regulator would consider the compliance demonstration complete. This approach includes identifying and using the conceptual models which encompass the regulator's concerns, but also extends to the use of existing data, interpretation of proposed experiments, and demonstration of the effectiveness of possible engineering modifications. 
This approach requires a new dialogue between the WIPP program, the regulator, and other stakeholders. Formal review comments are helpful in identifying concems about data completeness or interpretation, given the current assumptions used in performance assessment. More conservative assumptions, which the current data support, can be inferred in some cases. To find the most timely and cost-effective way to address stakeholder concerns over long-term safety, a more direct mechanism for identifying assumptions supported by existing data would be helpful.

\section{Compliance-Based Prioritization}

As described above, the WIPP project approach is to first that the method of calculating performance measures is defensible and would be acceptable to the regulator given existing data. If any performance measure exceeds the regulatory limit, all activities which influence that performance measure will be considered to identify the most timely and cost-effective means for addressing the safety-related issues reflected in the performance measure. The activities considered might include conducting an experiment to test the plausibility of the conceptual model leading to exceedance, conducting experiments to measure parameters which are currently assigned conservative values, or modifying the waste form to reduce the performance measure given the existing model and parameters.

The remainder of this report describes a method for establishing project priorities for experimental programs, engineering altematives, waste acceptance criteria, and other activities based on their influence on quantitative performance measures. The terminology is first described, followed by a summary of the approach and information requirements. The last two sections describe the structure of the inference models and activity models used in the optimization problem.

\section{A) Terminology}

Several terms are used frequently in describing the optimization procedure. In this context each term has a narrow and specific meaning, which is described in this section.

Throughout, the term calculated perfomance measure should be understood to mean performance measure calculated in such a way that, if the value satisfied the regulatory limits, the regulator would consider the regulation to be satisfied. As long as the safetyrelated concems of the regulator and stakeholders are incorporated in the method of calculating the performance measure, complying with a given regulation corresponds to meeting the quantitative regulatory limits using this method.

In this description, compliance is assumed to be demonstrated when all calculated performance measures satisfy the quantitative limits of all relevant regulations.

Activity refers to any action which has an effect on the performance measure, such as data collection, repository design modifications, waste form modifications, or changes in waste acceptance criteria. 
Introduction of the New WIPP System Prioritization Method

The outcome of an activity refers to the effect of the activity on information about the disposal system. Outcomes include experimental measurements, changes to repository geometry, changes to the waste form, or limits on waste acceptance. In general, and particularly for experimental activities, the outcome is uncertain. The way the outcome influences performance measures, however, is assumed to be known (for example, the way a solubility measured in a particular experimental program could be used in a defensible performance assessment is assumed to be known, even though the value that would be measured is not known).

Activity set refers to a viable combination of activities.

B) Goal of Prioritization

Given that one or more performance measures do not meet the regulatory limits, the WIPP program will identify the most timely and cost effective activities for demonstrating compliance. Whether these activities are in fact pursued will depend on the estimated time, cost, and probability of success. Because the outcome of any given activity is uncertain, the ability to demonstrate compliance following the activity is also uncertain.

The objective of the prioritization process is to identify, for given schedule and budget constraints, the activity set which leads to the maximum probability of compliance. The analysis will be repeated considering different values for budget and schedule constraints. The result will illustrate the tradeoffs between costs, schedule, and the probability of demonsirating regulatory compliance, in terms that are relevant both for programmatic direction and public policy decisions.

[Note: the following description of the optimization procedure has been adapted from a draft intemal Sandia letter report. A revised draft is in progress. The text is included here in its present form as the best available description of the prioritization method.]

C) Overview of the Optimization Problem - to be presented 
Beginning with calculated performance measures that do not satisfy regulatory limits, we consider each combination of activities which remain within the cost constraints. The possible outcomes of each combination of activities are identified. For each outcome that does not lead to compliance, the combinations of available additional activities are considered and screened against cost limits. This need not be the same set of activities considered at first: activities available at any stage may be influenced by activities performed at previous stages. The possible effects of performing additional activities are again identified, and the process is repeated. The expansion terminates when all activity paths either exhaust the cost constraints or lead to compliance.

For the last set of activities along each path. the probability of compliance can be estimated from the range of possible outcomes. Beginning with the last decision point, the activity path is selected which maximizes the probability of compliance. This probability then becomes the probability of compliance given the outcome which led to the altematives considered. The conditional probabilities given all outcomes of the previous activity are then used to calculate the probability of compliance given that activity, and so on.

D) Activity Models

In practice, this approach requires a description of all activities which influence performance measures, and a description of the possible outcomes of the activities as prior probability distributions. An activity model describes technical programs or procedures in such a way that the models make. sense for the types of activities considered, are compatible with the structure of the optimization problem, and have parameters which can be reliably estimated.

1) Components of Activity Models

On the simplest view, the activities we consider convert cost into some effect on performance measure:

Cost Activity $\quad$ \$(Performance Measure)

The link to the performance measure is always indirect: the activity produces some result or condition that, in tum, influences the performance measure.

\section{Cost Activity Result Inference * $\quad$ (Performance Measure)}

In general the effect of the activity on the performance measure is uncertain because the result is uncertain. 
Introduction of the New WIPP System Prioritization Method

We assume that the connection between a given result and the performance measure is not uncertain, because the inference method is defensible in the judgment of the regulator. Note that this does not preclude, for example, updating a parameter distribution using experimental results in such a way that the parameter value is uncertain after the experiment: we instead assume that the way in which the parameter is updated on the basis of experimental results has been agreed to.

The details of the connection between result and the performance measures depend on the type of activity (experimental, design modification, etc.), and are discussed in later sections. For the time being, we assume that each activity has an associated result, which is uncertain, and that this result has some effect on some performance measure (the result may have other effects as well).

Considering time as a separate component of cost, the model of activity i looks like:

- Release

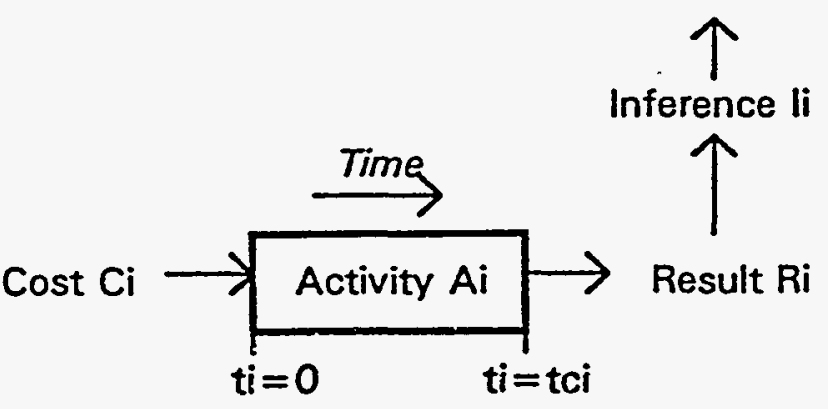

This model, along with the associated inference procedure, is all that is needed to characterize each activity for the purpose of optimization.

Some activities may fit this simple description; most will not. First, a result may not be obtained at all, or the outcome may be such that the regulator will not allow an inference to be drawn. The possibility of a "null" result should be considered. The experiment may produce a number of types of result, rather than a single result type, and the result values and possibilities of null values may.be correlated across result types. In addition, the result will be obtained with some accuracy, which should be included.

For many activities, exact values of cost and duration may not be known in advance. Often this is due to contingencies built into the activity plan. Other results are observed which, while not affecting the performance measure per se, determine subsequent events. A more flexible model can be built using elaborations of the simple model proposed above: 


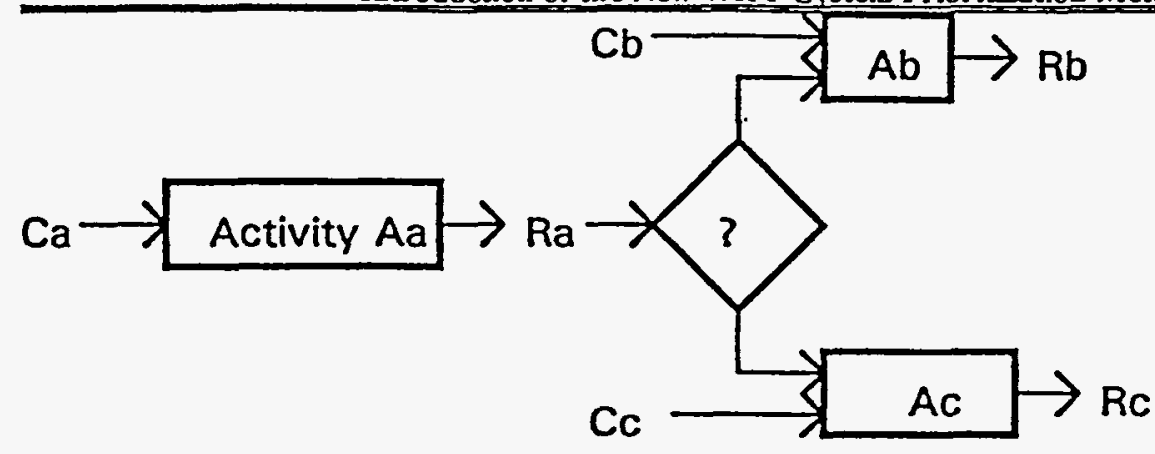

The decision elements in the model allow for costs to be conditionally incurred and results to be conditionally available. The individual sub-activities are still assumed to have fixed costs and durations. If the more elaborate model is considered as a single activity, it appears, from the standpoint of the optimization problem, identical to the original model, except that the overall cost, duration, and result availability are uncertain, and are appropriately interrelated. If the assumptions of fixed cost and known duration are not appropriate for the component activities of the more elaborate model, these components could be expanded in terms of simpler, more predictable activities which satisfied these conditions, along with the appropriate decision elements.

A remaining limitation is the assumption that the decision rules can be specified in advance. If these rules are allowed to refer to the value or existence of results from other activities this does not seem too restrictive.

Activities may be divided into phases. Compliance could be evaluated at junctions between phases. If the actual outcomes support a' finding of compliance, no further phase would be required. In constructing possible activity sets, a later phase would be available only if the preceding phase had been completed. As an example, consider some experimental measurement that can be made with increasing precision with increasing time. The experimental model might look like:

Phase 1 Phase $2 \quad$ Phase 3

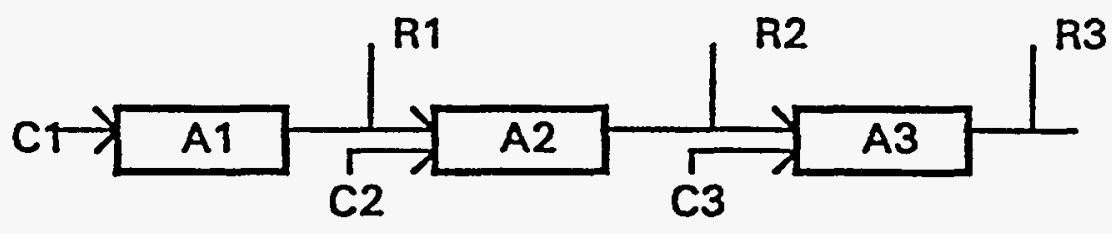

In which the first phase contains start-up overhead costs, and successive phases provide updated estimates of the variable.value with increasing precision. The benefit of proceeding to successive phases would be determined by the inability to demonstrate compliance given the result and precision of the preceding phase. 
Interesting complications arise around the interactions and sequences of activities. In the above example, for instance, would the costs remain the same if the program start date were delayed by a year? is it possible to delay the start of Phase 2 , or must it follow Phase 1 immediately if at all? If it can be delayed, are there additional restart costs not included in the original description? in building a sequence of activities to find an optimum path, it is essential that each path represented make sense by itself.

Activity models for experiments, engineered altematives, and system model modifications differ in the types of results expected and the types of connection to the performance measure. Some general points about the proposed activity model can be made before considering models for particular activity types.

In each case we need to identify the type of result expected, the techniques available for making prior estimates of result values, and the inferential methods connecting results to performance measures. Result types and the methods used to estimate distributions are particular to the activity type. The result distribution represents a degree of belief about various outcomes, however there is no regulatory significance to this distribution: it need not represent the regulator's degree of belief, but it should reflect the knowledge of the DOE, or Sandia, or knowiedgeable experimentalists or desigri engineers. This point is discussed in more detail in the context of the experimental model.

The connection between the activity result and the change in performance measure can be divided into two parts: the effect of the result on the modelling system, and the effect of the modified modeling system on the performance measure. Again, because the activity being considered should not impair the regulator's confidence in the calculated performance measure, the way in which the results would be interpreted (i.e. the connection between the results and changes in the modeling system) must be acceptable to the regulator.

Ideally, the effect on performance measures given some change to the modelling system would come from using the same regulator-approved implementation of the changed modelling system as would be used in a formal peiformance assessment. This may not be possible in practice. The important point is that the optimization problem has no regulatory significance: we are free to use whatever method seems appropriate to implement the modified modeling system: we need to approximate the formal, defensible implementätion because the formal implementation would be used to update performance measures using actual experimental results.

The following sections discuss the result types, result distribution estimates, and firststage inferential procedures associated with the three activity types.

\section{2) Experiment Models}

For many experimental activities, the result will be a measurement that closely corresponds to a model parameter, such as plutonium solubility, gas generation rate, or 
Introduction of the New WIPP System Prioritization Method

transmissivity. In the simplest case, the experimental result (with the associated experimental error) would be identified with a particular model parameter. In this case, the prior (present) distribution for the parameter could be used as the distribution of the experimental outcome. As discussed above, this is not necessary: if the present distribution includes values which the experimentalist considers unrealistic (and, again, the regulator has agreed to replace the current distribution with the result of the proposed experiment), the experimentalist's prior distribution might be more appropriate.

In a more realistic situation, the experimental result would be more loosely connected with the model parameter. and would be used to update the parameter distribution in some way (e.g. because of concem over extrapolation over time, or estimation of volume averages from point measurements, or weight given to prior estimates). In principle this presents no problem as long as the updating procedure is specified. Prior estimates of experimental results might come from experimentalists, or might be derived from existing data. For example, if the experiment was meant to measure farfield fluid pressures in the Salado, a regression model, fitted to existing data, could be used to predict the distribution of outcomes of "point" measurements at new distances; these possible experimental outcomes could in tum be used to derive estimates of volume-averaged pressure distributions. As another example, a prior distribution for Culebra transmissivity could be obtained by kriging existing values.

In the above cases, the experimental result influences performance measures because it is directly or indirectly related to parameters of the system model. Other possibilities exist: the result might change the system model (i.e. change the way the performance measure is calculated), or the result may have no direct influence on performance measures, but be important for some subsequent activity. In the proposed framework, the first situation is considered under 'Analysis' activities, along with other possible changes to the inferential mechanism. Although the underlying activity is experimental, the effect on calculated performance measures is similar to (e.g.) a change in the differencing scheme used to solve the transport equation. The second situation is handled by connecting subsequent activities to the experimental outcome through decision elements.

\section{3) Design Models}

Like experimental results, design results may directly correspond to system model parameter changes, or may indirectly influence model parameters, or may change the system models themselves. The 'result' of the design modification may include $a$ index of the expected repository state, which might be used in the decision elements of subsequent activities.

Many questions surrounding design modifications are expressible and answerable in the proposed framework, for example, "Is it worth making preparations for waste treatment now, even though later information may show that this is not needed?"

4) Analysis Models

Watt Beyeler, 6307 draft 
Any activity that changes the inferential procedure (i.e. the way performance measures are calculated) is considered in this category. Examples include changing numerical procedures in computational models, changing the resolution of discrete approximations, changing the dimensionality of models, modifying the governing equations, modifying the boundary conditions or initial conditions, or modifying the repository design.

Some of these cases are not relevant for the optimization problem. We can assume, for instance, that as part of establishing reasonable assurance, current numerical procedures would have been verified, in that the results are shown to be 'close' to analytical solutions. Altemative procedures will either produce similar results or, if not, will either be wrong or refute the verification of the current procedure.

We mighit be in a position of deciding between two acceptable discretizations or dimensionalities which produce substantially different results if we use a simpler representation for computational efficiency and have demonstrated that the simplification always gives conservative results. The 'cost' of using the more complex procedure comes from the additional time and computer resources required to calculate the performance measure compared to some current simplification. Unlike the experimental activities, we must incur this cost in order to estimate the benefit of incurring it. This problem is excluded from the current scope of the prioritization procedure.

The inferential procedure may be changed by changing the design or conceptual model. If the influence of design changes on the current model structure is acceptable to the regulator, the effect of the new structure can (in principle) be easily explored by incorporating it in the inferential structure of all descendant paths which incorporate the design modification. The viability of the design modification, as represented by the change in system model structure, can be made appropriately contingent on the outcome of experiments. If the influence of the design change on model structure is uncertain, we essentially have a 'conceptual model' problem overlaid on the design modification problem.

The 'conceptual model' problem arises if there is more than one tenable inferential procedure which connects available information to performance measures. The inferential procedure which provides reasonable assurance to the regulator can be thought of as a composite of all tenable models in which the results of each are weighted. The weights may be fractional, or may be 1 for the 'most conservative' and 0 for the remaining models, or any other scheme which provides reasonable assurance. As mentioned earlier, conceptual model uncertainty can be sensibly incorporated into the prioritization problem (or sensibly dealt with at all) only if the connection between observations and model weights is specified. The situation is analogous to experimental activities: we cannot evaluate the significance of an experiment without an a priori understanding of how the results affect a defensible system model.

If we have an explicit description of the dependence of model weights on observables, the problem becomes tractable. We can design experiments to make the relevant 
Introduction of the New WIPP System Prioritization Method

measurements, and use the prior distributions of experimental outcomes

(measurements) to assess the selectivity of the experiment with respect to models.

5) Interactions

To find the set or sequence of activities which leads to the maximum probability of compliance for a given cost, all possible combinations of activities should be considered, at each decision point. Not every combination or sequence of activities, however, is sensible: key personnel or resource requirements may preclude two activities from occurring simultaneously, or one activity may be contingent on the successful completion of another. There may be positive interactions as well, such as cross-hole logging between two holes drilled for different experiments. The goal is not to design a procedure for identifying important interactions, but to allow the important types of interactions to be appropriately represented. With the possible exception of resource allocation, the specific interactions to be considered must be anticipated by the project personnel.

The decision elements are suitable for most interactions, provided references are allowed to the results of other activities: a hole drilled to obtain a core sample might allow an additional drawdown observation in a long-term pumping test; results from a "Phase 2 " activity might not be available at all if essential "Phase 1 " results were not satisfactory.

"Analytical" activities, which modify. the way performance measures are calculated. have particular interaction problems. If the model structure is changed, the effect of the results of all subsequent experiments on the performance measures should be inferred using the revised model. This may be trivial in some cases. In other cases, the experimental result may be meaningless in light of the new model. It is important to consider in advance how each experiment would be interpreted within the framework of each model, and also anticipate expeniments which might be useful in supplying parameters for models which are not currently used, but which may be adopted later. Aggregations of system models need to be fully specified as well, for example "how do we represent design modifications $A$ and $M$ under conceptual model X?" The answer to this question must also be acceptable to the regulator.

\section{E) Summary Description of the Prioritization Procedure}

If performance assessment calculations have been designed to provide reasonable assurance to the regulator, and the quantitative regulatory performance measures do not satisfy the regulatory limits, the goal is to identify the subset of activities (from a specified set) which are most likely to lead to compliance given various cost constraints.

Cost constraints will include (at least) the present value of expenditures and the time until a compliance decision is required, but may include other components.

Activities which affect the performance measure are described by their costs and the type of result they produce. All costs are certain, but may be functions of absolute 
Introduction of the New WIPP System Prioritization Method starting time, or starting time relative to other activities. The result can be of any type, but must be characterized by a prior distribution. The distribution can include the probability of a 'null' or ambiguous outcome. Result distributions can be conditional on the results of other activities.

Activities influence performance measures through their results. Results can directly influence performance measures by modifying the parameters of the system model or by changing the structure of the system model. In either case, the procedure for updating the system model given the result must be specified, and must not degrade the defensibility of the performance assessment.

More complex activities can be assembled using decision elements. Internal decision elements can refer to prior results for the activity, or to results from other activities. Extemal decision elements identify natural opportunities for programmatic redirection (increase, decrease, or termination of funding) based on compliance status.

Groups of exclusive activities can constructed, e.g. where each member corresponds to a different 'level' of effort. Sequences of activity groups, connected by external decision elements, can be defined, where each group in the sequence corresponds to a different 'phase' of the overall activity. The most.general description of activities, which is used in describing the optimization problem, can be illustrated as follows:

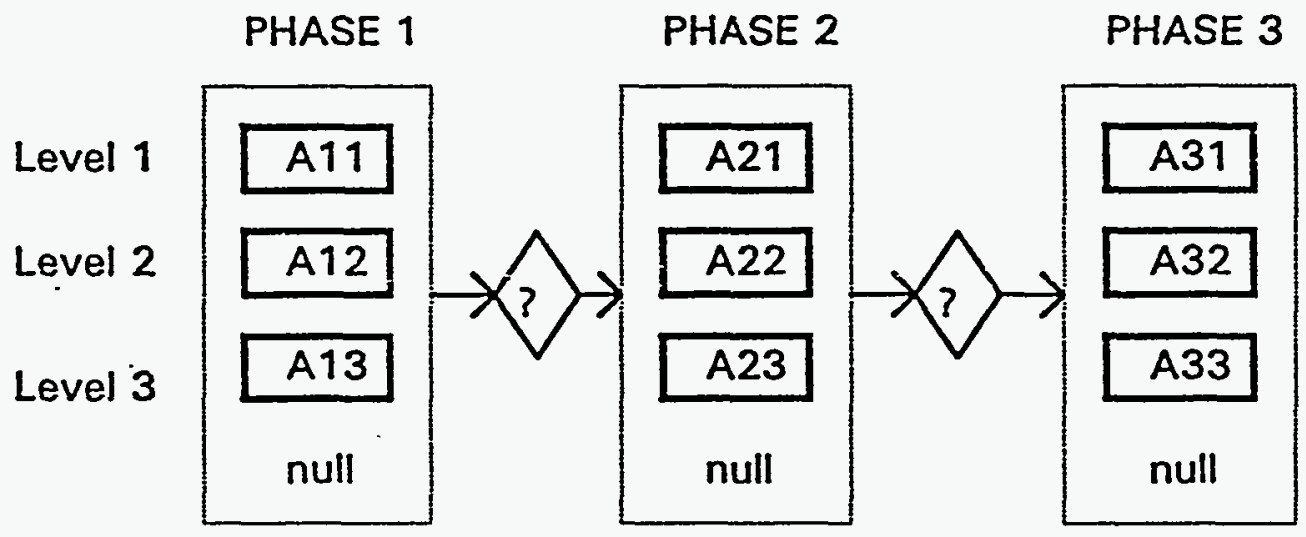

where the 'null' activity, meaning termination, has been added for completeness. Each level in each group may be a complex activity, containing several sub-activities and internal decision elements.

Anticipated interactions between activities are described either through intemal decision elements, or by explicitly disallowing or mandating specific combinations or sequences of activities.

Extemal decision elements are required to occur only at regular intervals which make sense programmatically (e.g. 6 months or 1 year). The continuity of long-term activities 
across external decision elements can be insured using the 'interaction' restrictions described above.

Once all proposed activities have been specified, along with their interactions, and the way in which results influence the system models, the decision tree approach to the optimization problem is straightforward in principle:

1) We start at a decision point with performance measures which do not satisfy regulatory requirements, and with stipulated cost constraints.

2) Identify all possible combination of activity 'levels' (including null) for all activities which satisfy interaction restrictions. Each combination defines an activity 'set' which can be followed from the current state.

3) Discard any activity set which violates any cost constraint (because of internal decision elements the cost of a set is uncertain in general. Expected cost or maximum cost may be used).

4) For each activity set, identify the distribution of results available at the eariiest external decision element in the set.

5) For each outcome which does not result in compliance, repeat 2 through 4.

6) Once all paths terminate due to compliance or cost constraints, back up the probability of compliance associated with each activity set as previously described. Identify the initial activity set which leads to the maximum probability of compliance. 
27. Tierney, M.S. 1994. "Notes on WIPP System Prioritization Methodology." Draft, April 30, 1994. 
Dreft 4/30/94

Qutine

in \& $T$

"Votes an the wiPP Priaritipation Medioldopy

Stuterserst of the problem

Sonce Oefinition

Matherseticid Stotennent of the problener

Complutational Conciderations

Pafernamec Wesures

Whenuses for 40 CFR Part 191, Sulpart $B$

Baskeranase on Caloulatini CCDF 1

Peforanarec Hencuras far the RCRA

Utility Fenstomes

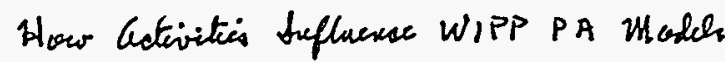

Chaice of Tharlel Canfiguration Dectors

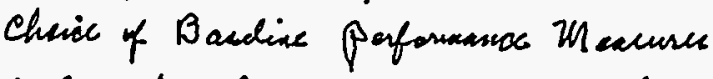

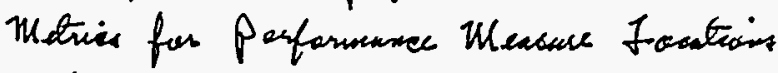

calculetion of Utitity of an Aetirity. Set

chaies of objestive utitity Fruaction

Sonce Cederestager of This Cupposech

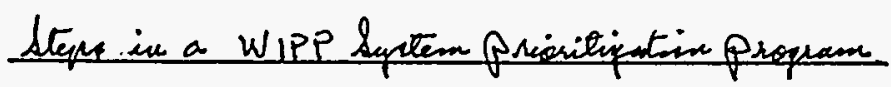

Date Sathesing Phase:

- Compilation of Cuctivitas Data Buce

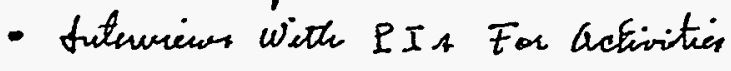

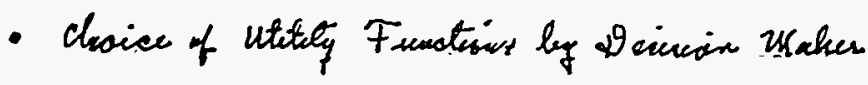

Smalytige pluce:

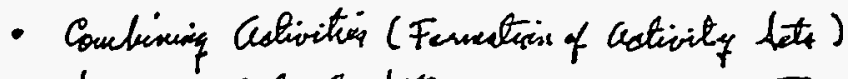

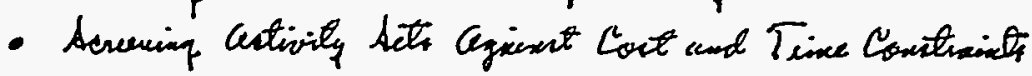

Calculationed pteres:

- Celculating Whetricas of Socenced actioity Sts

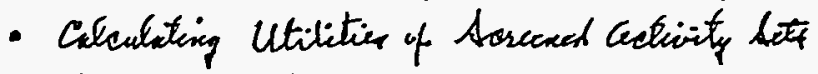

- Ractring actioity Loto by Their Mlilitici

Referemeres

157 
1 op

Natet on WIPP Syptem Prisictigatione Methadalagft

Martion \&. Teeracey

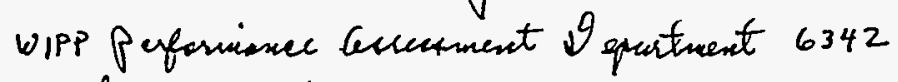
A curdia Xlational I alvosationies

Altonquesque, Hew Hesiss

(Orpt sated $4130 / 1994)$

Statement of the prodem

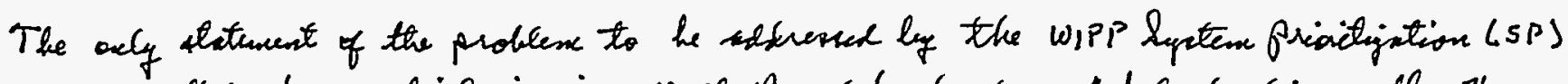

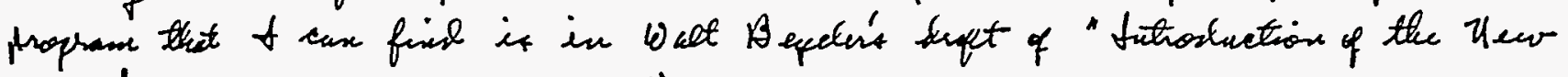

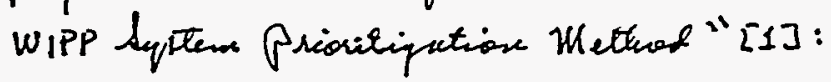

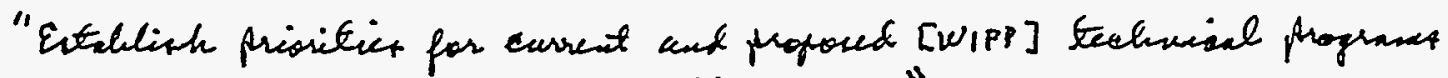
on the baier of repulatory complinince. ... " $[1 ; p g .1]$

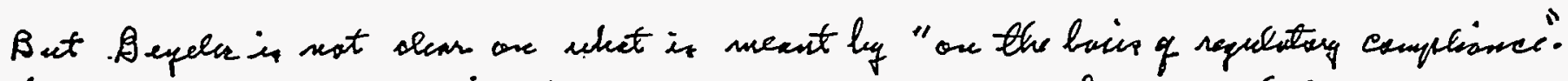

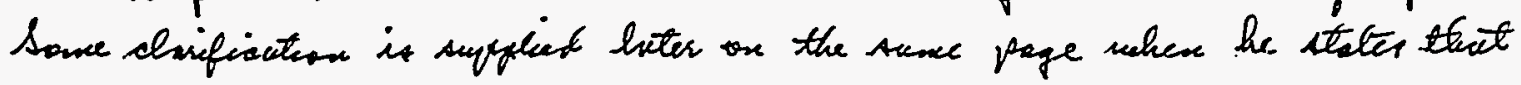

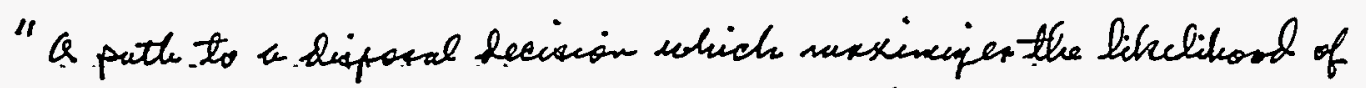
demontrating regulwery complinsece sur be idertifies [provides that]

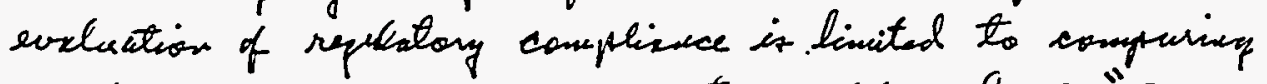

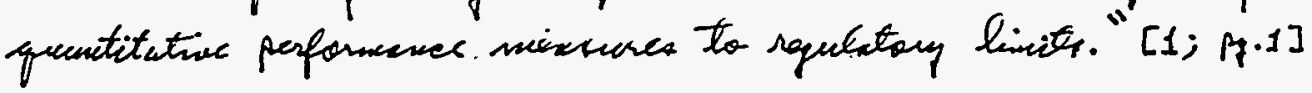

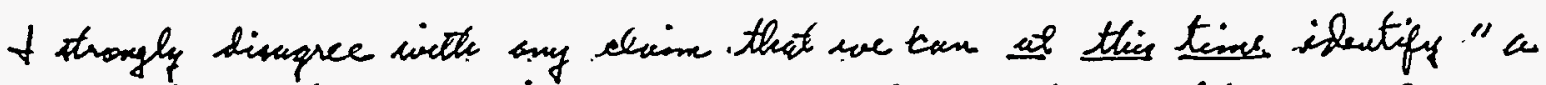

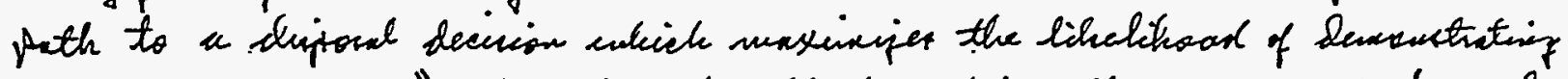

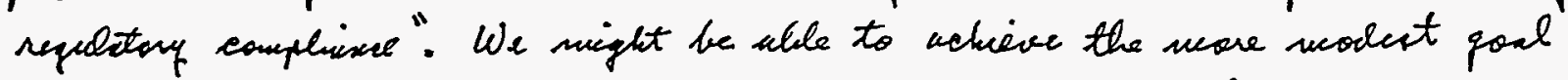

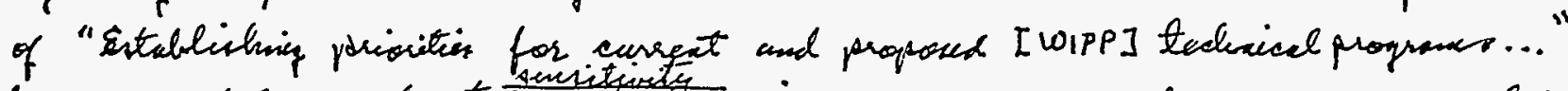

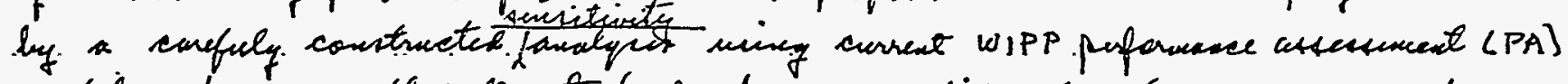

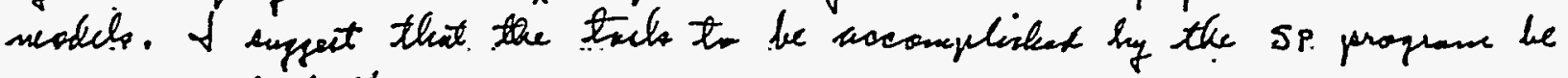
Squied in the folhwing way:

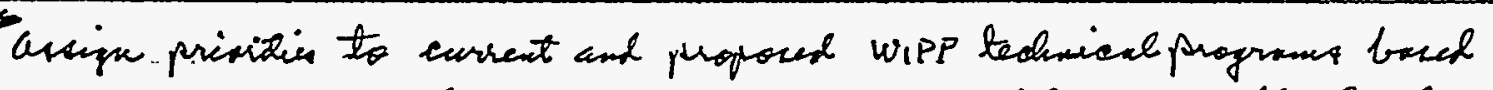
on the relative infrestanse of exch propren for determining the locetion

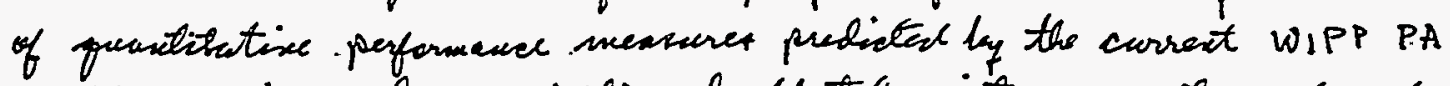

Box

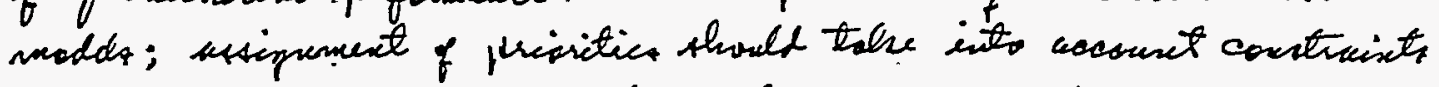
the are cont und time thit conth be inporet on the wipp projest.

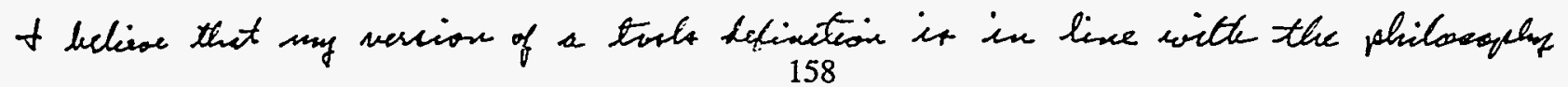


$($ Draft $4 / 30 / 84)$
2 of

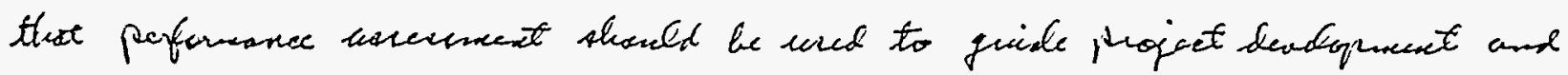
to prionitige activitien with the progeat.

Sone Pefinition:

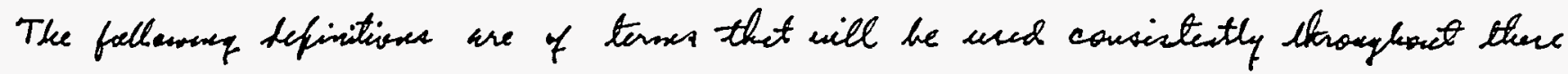
notes.

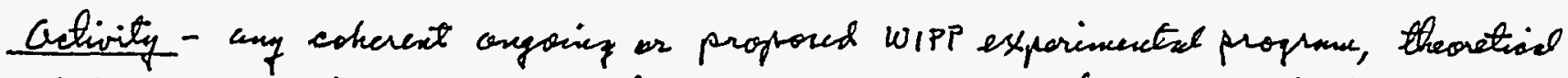

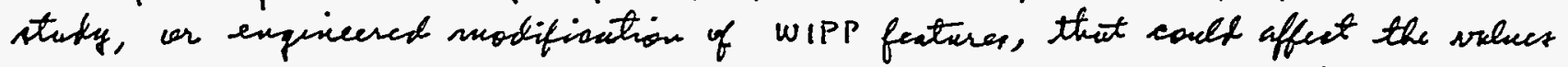
of the parameters of the PA model spetere. Porrible examplas: see the 90 itemes densibied in Lation 5.1 throng 5.4 of "Espreriocented proquan Plase for the Wute fralation pilat plant " [2].

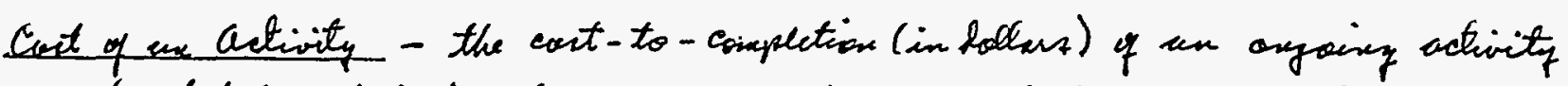

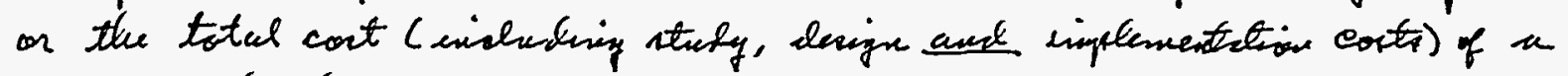
puprosed astivity.

Time of an (hetivity - the time-to-congletion ( ine year) of are ongsing activity

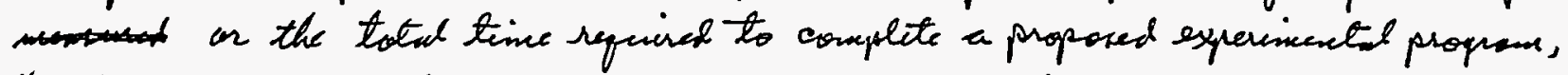

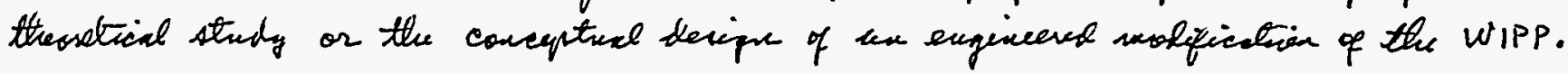

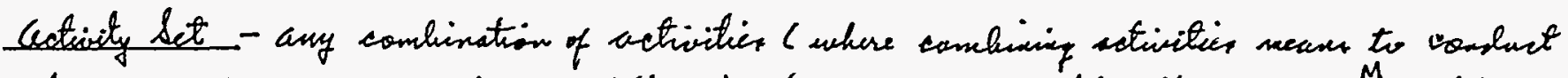

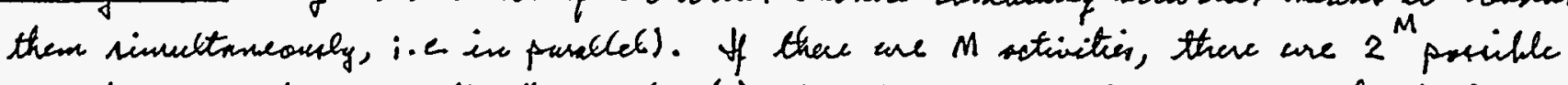

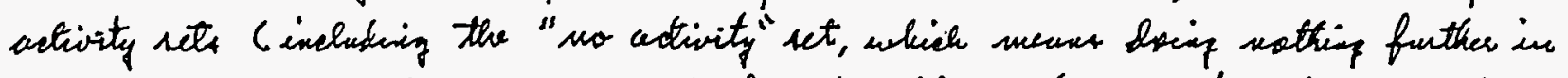

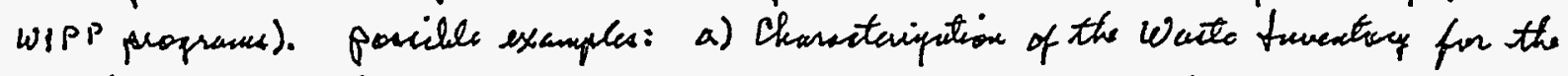

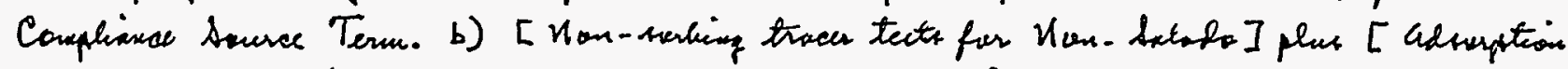

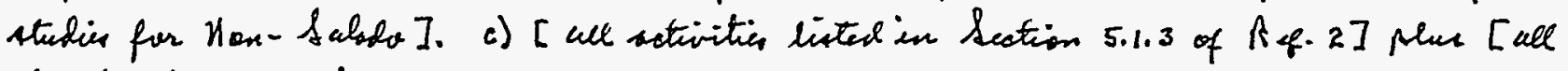
wetivities lister in Section 5.1 .2 of Ref. 2 ].

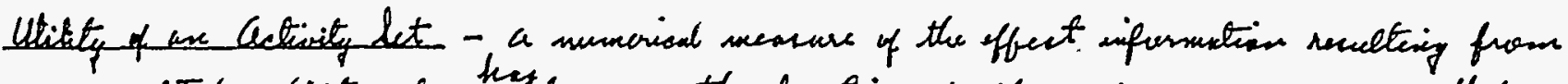

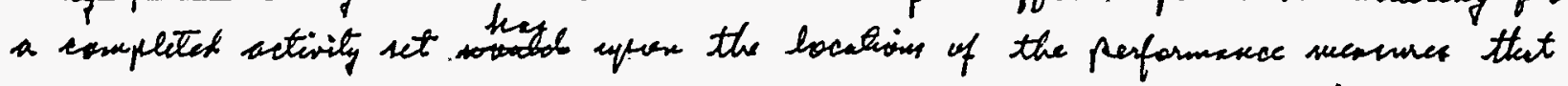

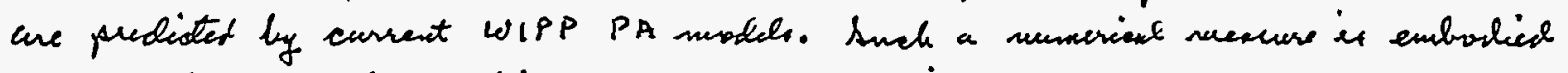

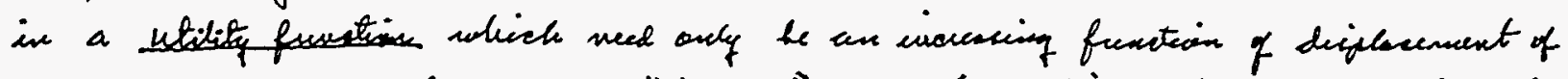

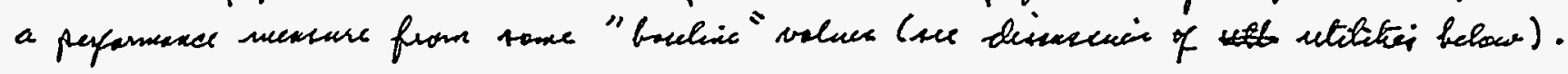

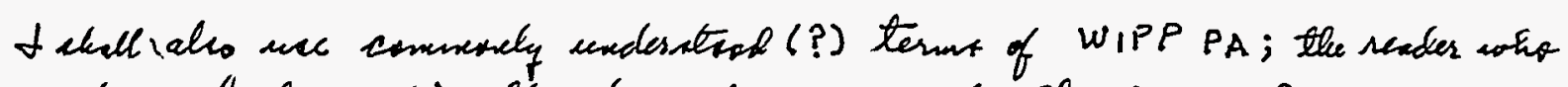

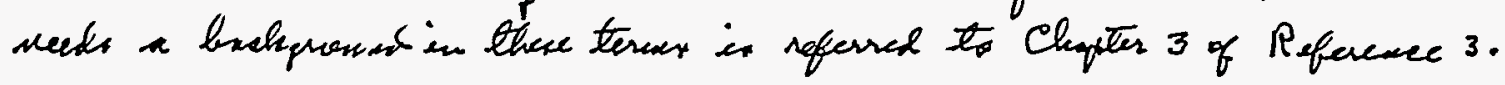

159 


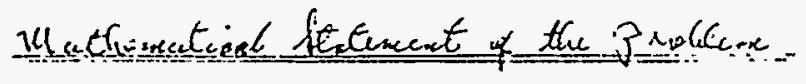

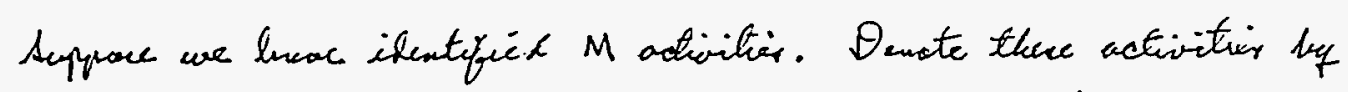

$A_{2 m}, \quad m=1,2,3, \cdots, M$

and let $c_{m}$ and $t_{m}$ be requectivicly their coste cund teiner.

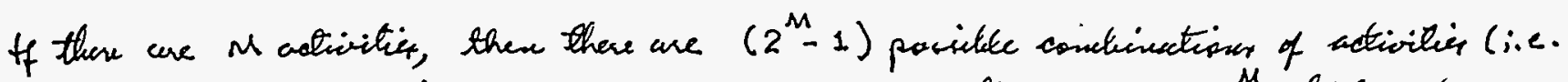
wetivity sets). If we incheste the "us activity" set, there are $N=2^{M}$ actirity sets reluick we lakel as

$$
S_{n}, n=0,1,2,3, \cdots,(5-1) \text {. }
$$

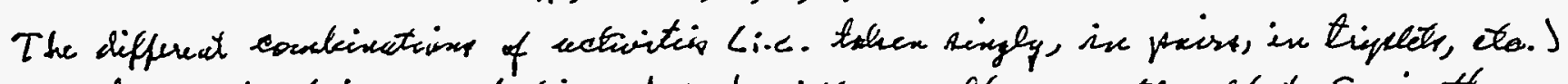

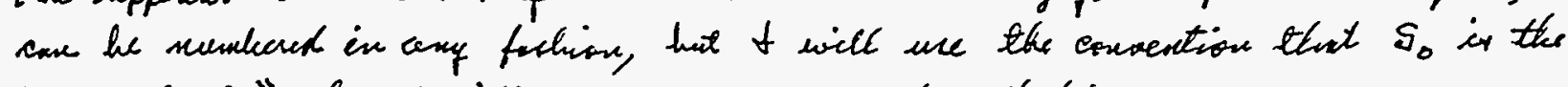

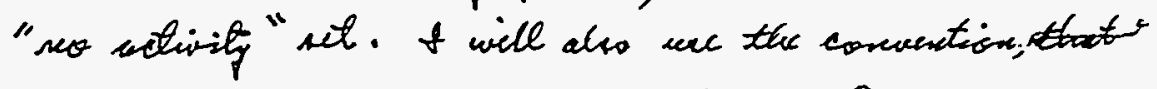

$$
A_{m} \in S_{n} \text {, }
$$

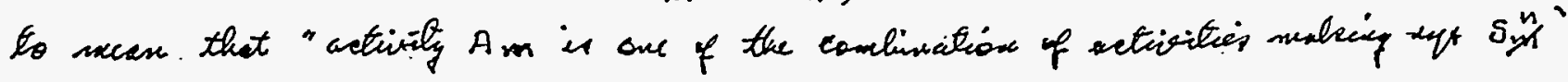

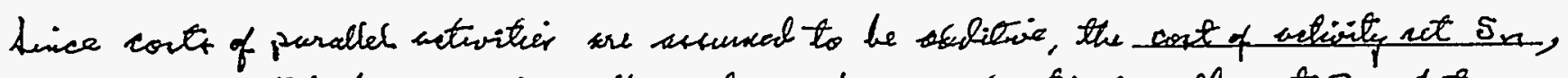

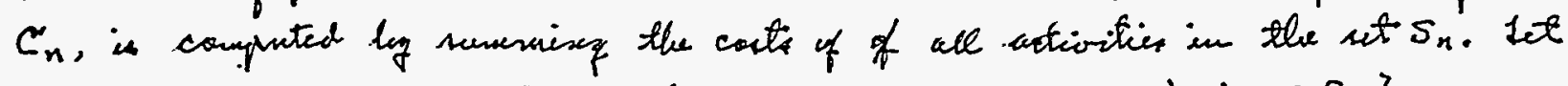

$$
I(n)=\left\{m: k=1,2,3, \cdots, n \text {, ak.d } A_{m} \in S_{n}\right\}
$$

Theen

$$
C_{n}^{v}=\sum_{k \in \in I(x)} c_{n}
$$

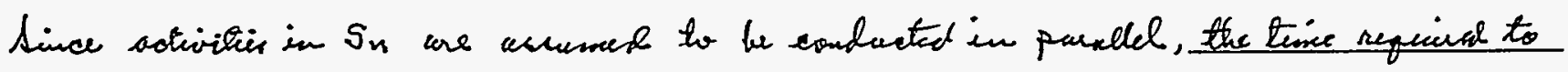
renglete actionty at $S_{n}, T_{n}$, is compinted by

$$
T_{n}=\max _{n \in I(n)}\left\{t_{n}\right\} \quad \text { Equ. } 2
$$

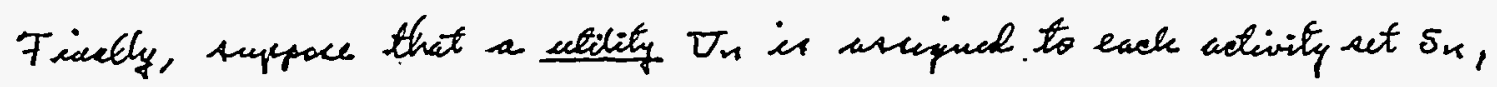

$$
U_{n}, n=0,1,2,3, \cdots,(N-1) \text {, }
$$

geveing the triplets of rumckers, ase triplet for embe $S_{n}$,

$$
\left\{C_{n}, T_{n}, \sigma_{n} ; n=0,1,2,3, \ldots,(N-1)\right\} \ldots \quad E_{2 n} .3 .
$$

The trigiet in Eqe. (3) is all the infernation we need to soluc the probleme,

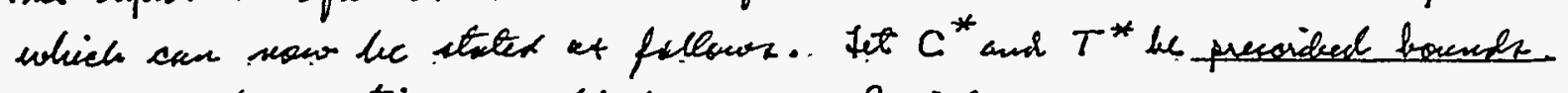

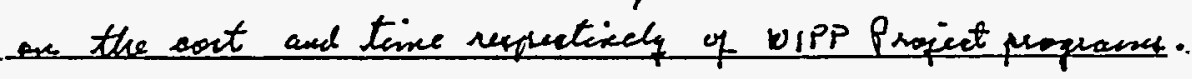

arrign prianitien to all activity ecte $\left\{s_{n}\right\}$ by ordering thene asonding to thai wilitien $U_{n}$, sulject only to the constraint that ond thore membere of $\left\{S_{n}\right\}$ that sutify

$$
C_{n} \leq C^{*} \text { and } T_{n} \leq T^{*}
$$

be cocented in the ordanis of the ett. 160 
Simapt 4136184
4 of

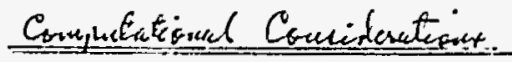

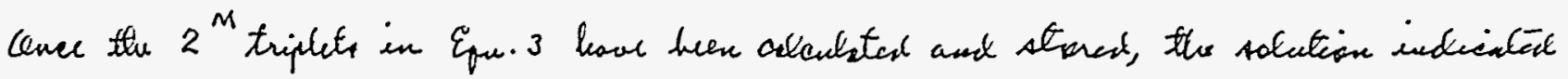
in $\beta_{0} 1$ should he eacy to cirsy cout uning a moderse conplater. The only

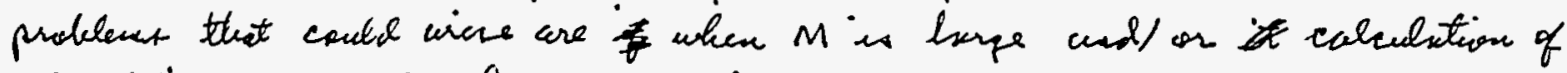
the utitidie, $\left\{U_{n}\right\}$, is time consusing.

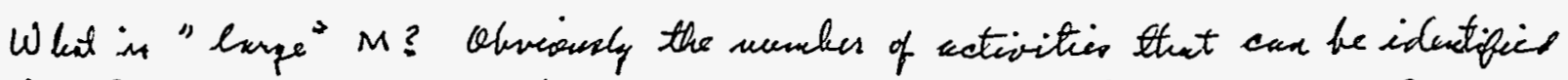

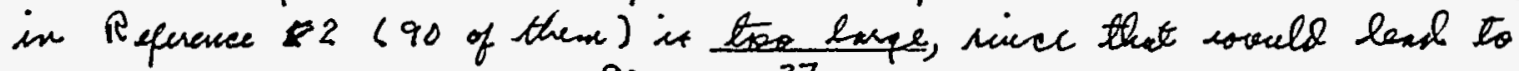
$2^{90} \approx 10^{27}$ attivity $\mu$ t.

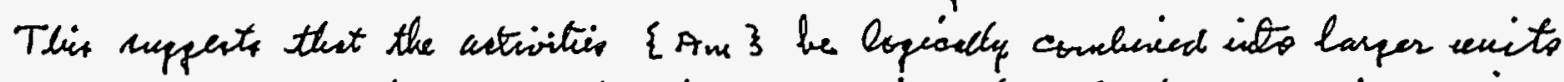
in some way; the ponibiliter for sombinang the activitios are deicursed in

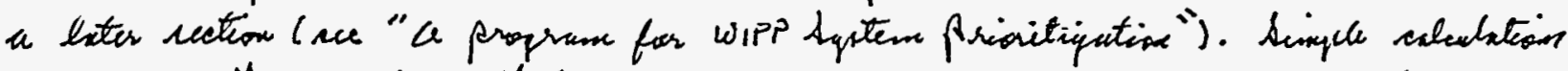
with $N=2^{M}$ will sluow thit $M=30$ is probaley tor laye for current starape epracitice.

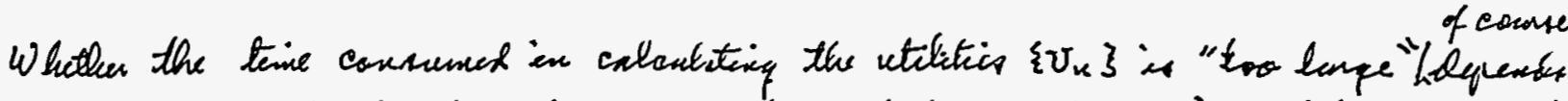

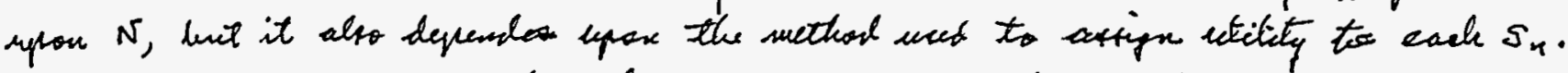

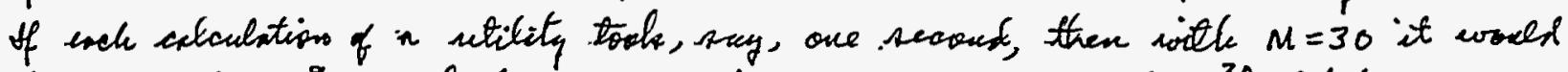

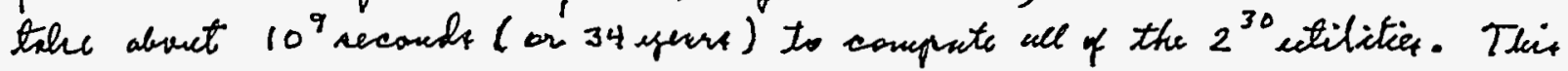

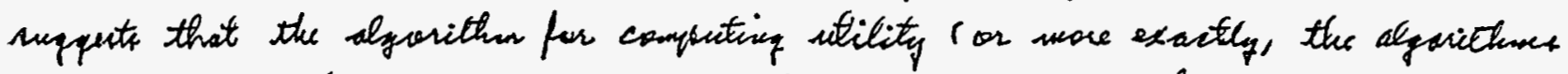

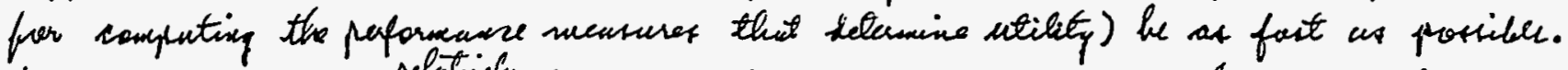

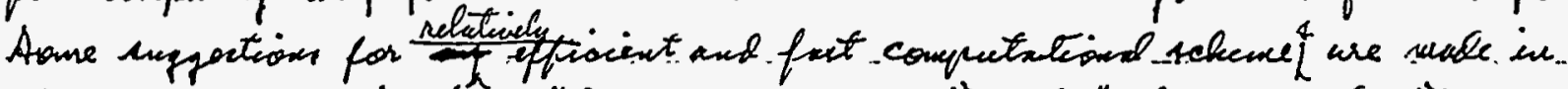

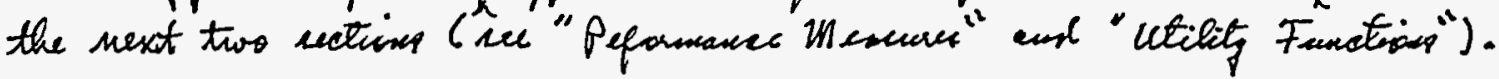

Pesforsearce Wesures

The Acientifie pant of ituadards; the EPA'A 40 CFR Part 191, hulytart B, cuse the RCRA, 40 CFR Pait $26 P$.

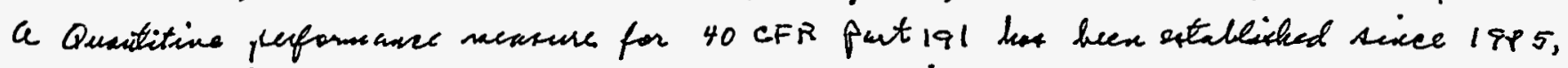
lut there has at yet been wo peroved peformince menume for the $R C R A$.

Merures for 40 CFR Pant 191, hulpat B

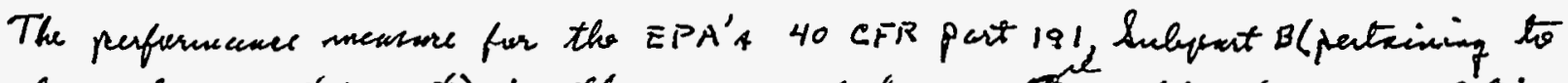

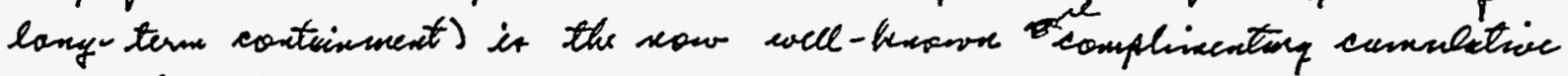

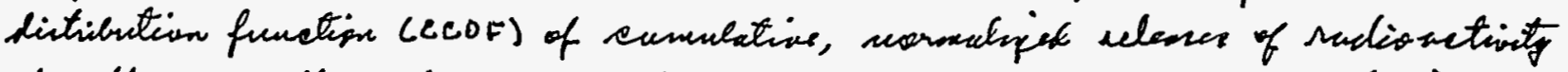
to the ancerible envirarment. The regulatory CDDF $C_{i}$.e. the cisuc thet is

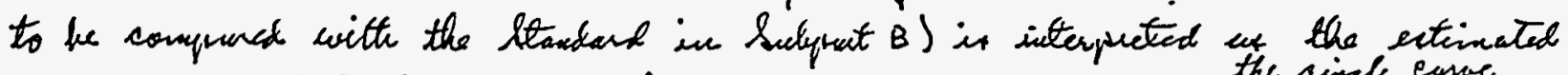

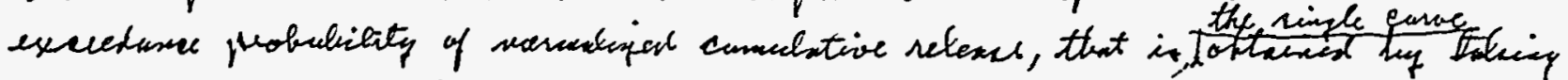

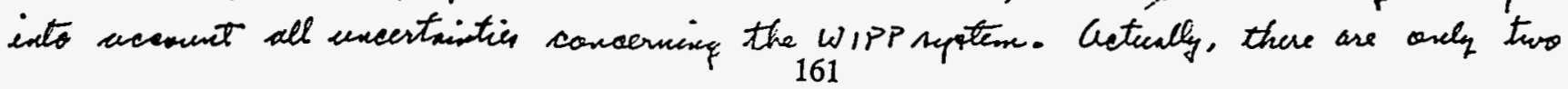




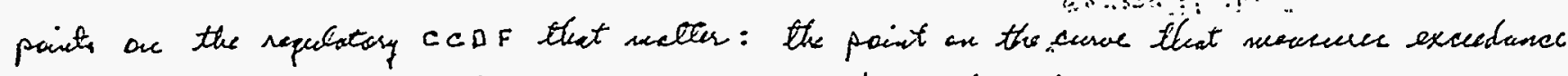

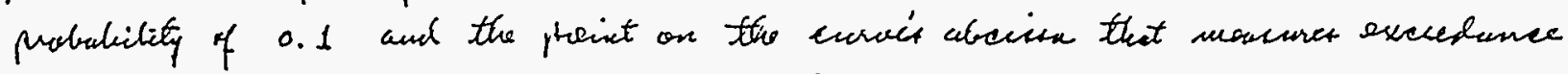

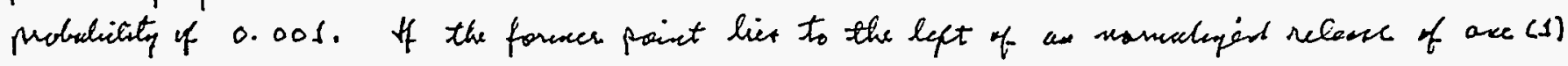

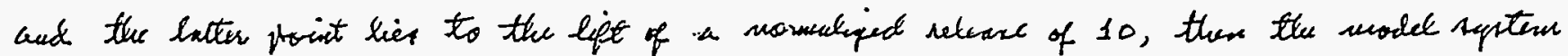

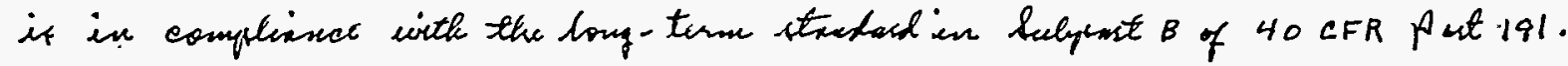
(Sec Fejure 1).

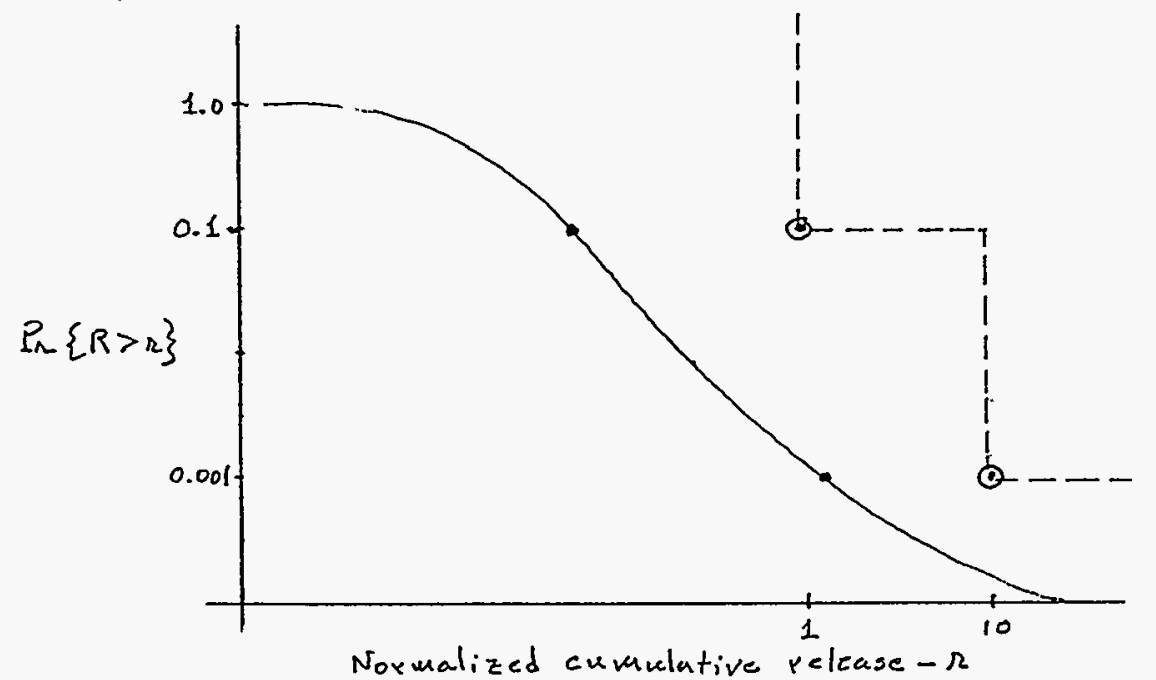

Figuxe 1. A complementaxy cumulative distribution function (CCDF). Curve shown is in compliance with long-texin standard in Sulpoat $B$ of 40 CFR Paxt 191.

\section{Bodensend Calculation a CDPF}

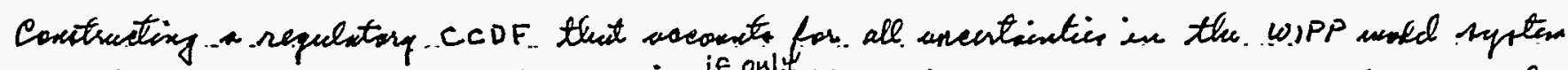

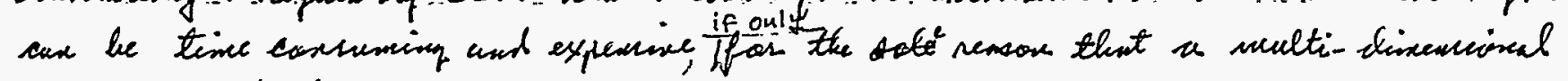
integral of the form

$$
\operatorname{Ir}_{r}\{R>r\}=\int_{D} u[R(\underline{x})-r] d F(\underline{x})
$$

Equ. 4

must be calculated [3; Chequter 3]. In Equ. 4,

$$
\underline{x}=\left(x_{1}, x_{2}, x_{3}, \cdots, x_{L}\right)
$$

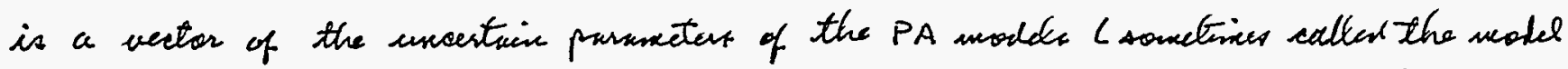

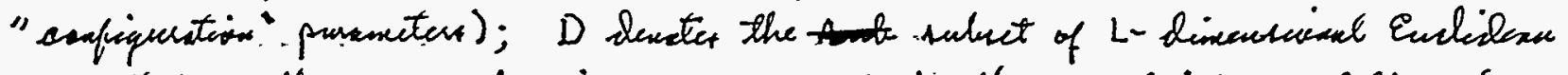

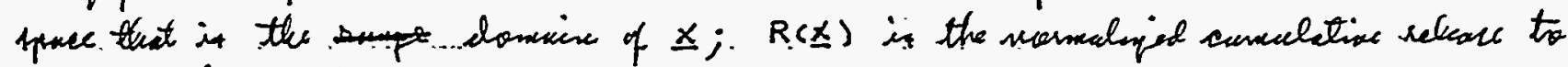

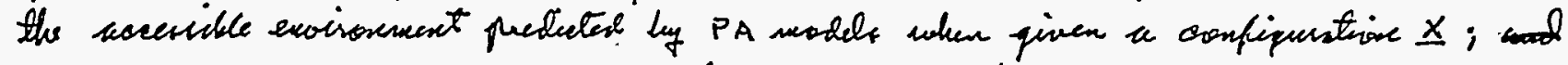

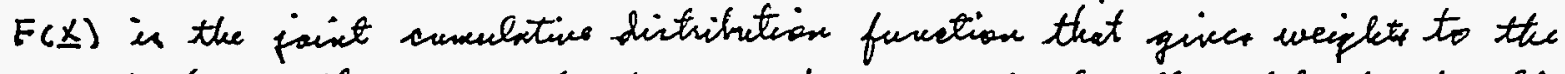

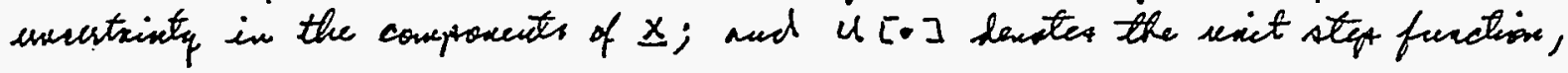

$$
\begin{aligned}
u(y) & =1 \quad \text { if } y>0 \\
& =0 \quad \text { if } y \leq 0 .
\end{aligned}
$$

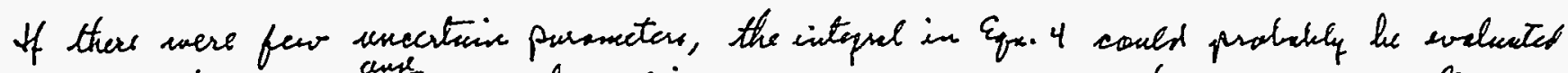

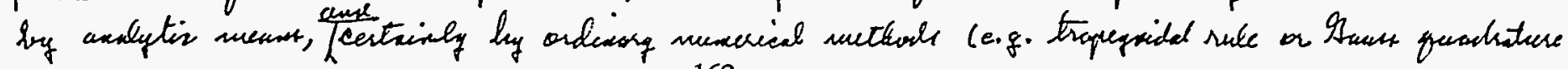




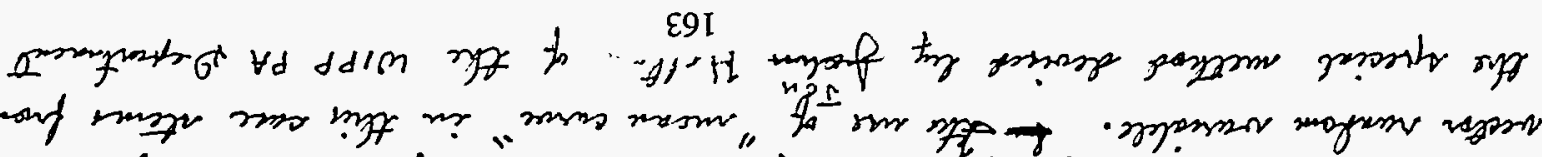

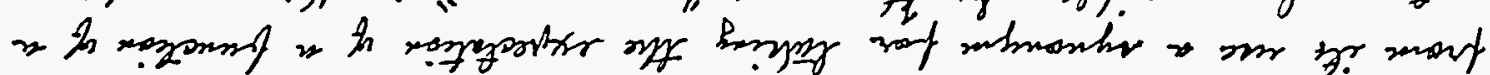

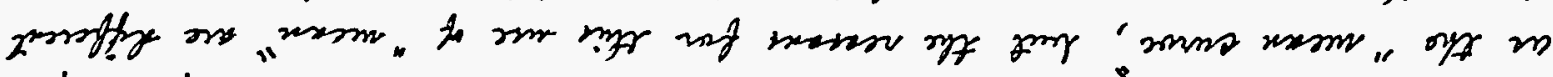

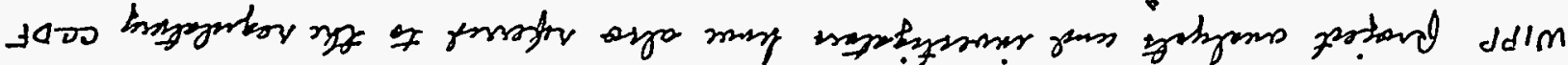

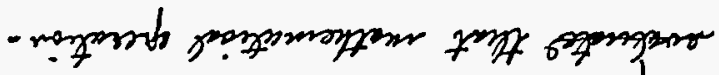

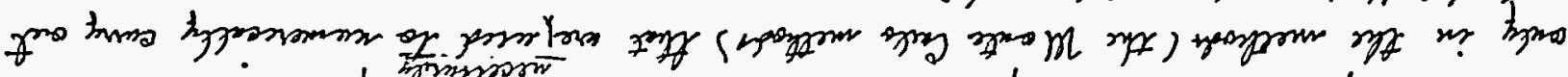

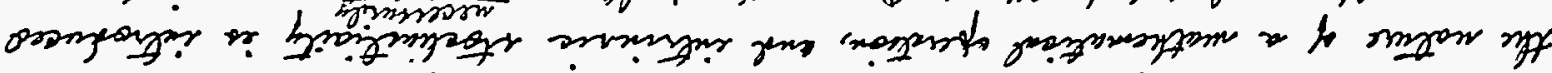
no

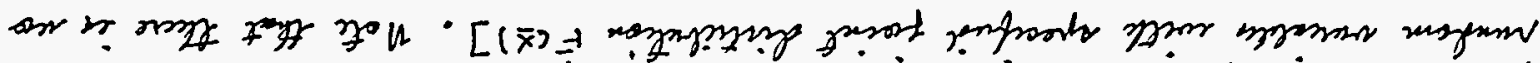

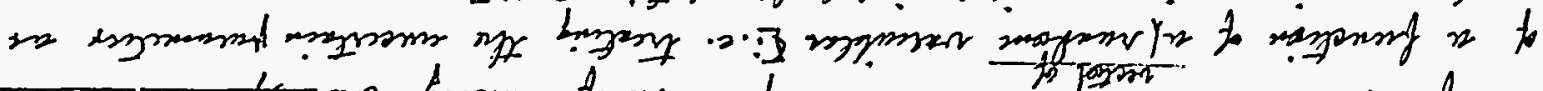

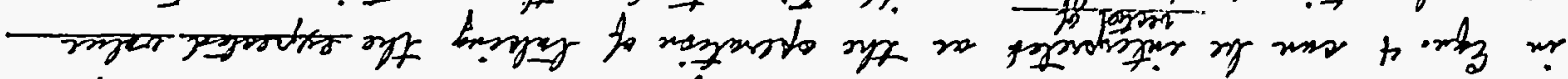

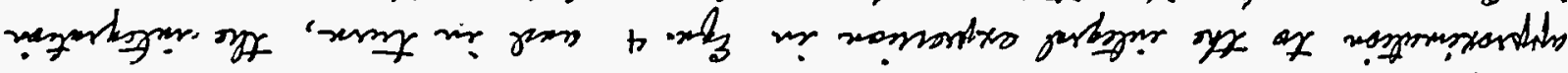

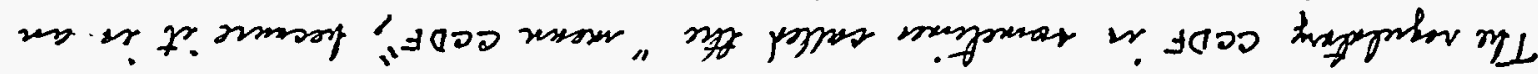

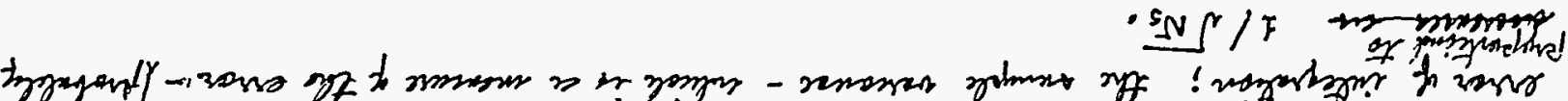

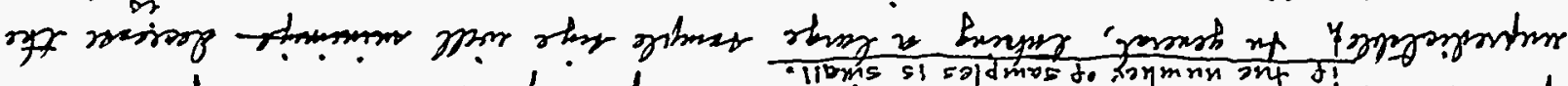

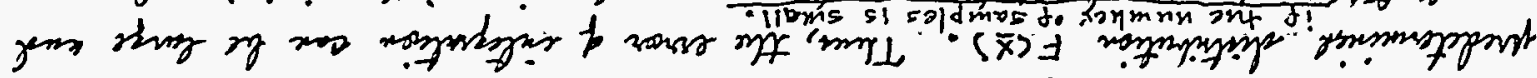

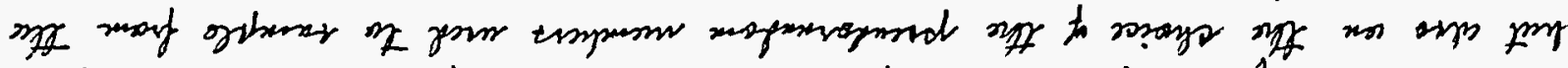

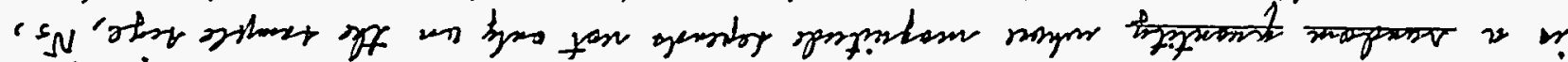

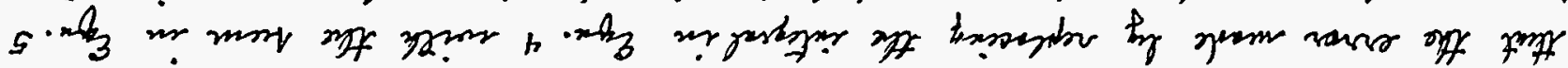

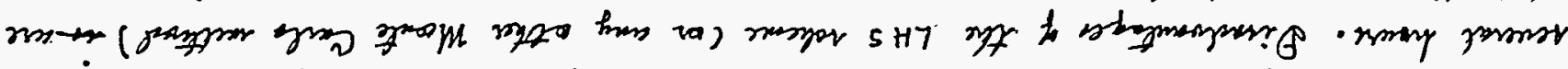

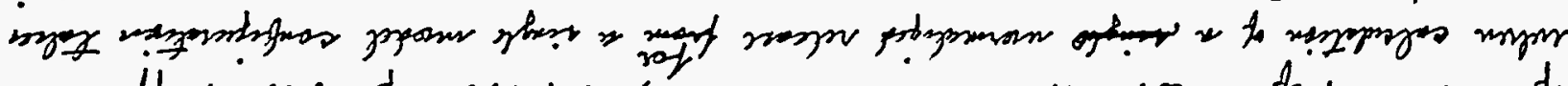

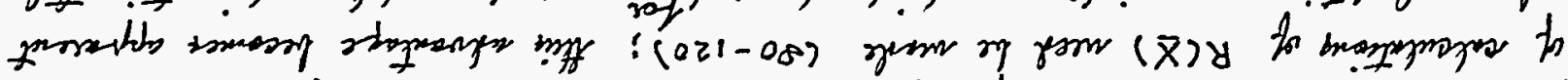

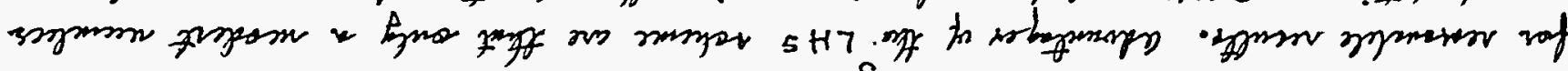
$7 \frac{\varepsilon}{h}<{ }^{s} N$

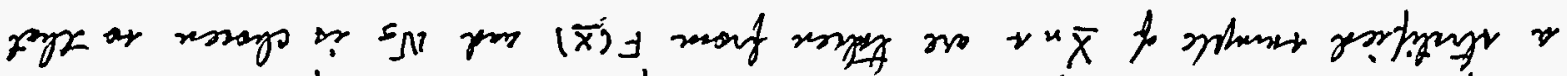

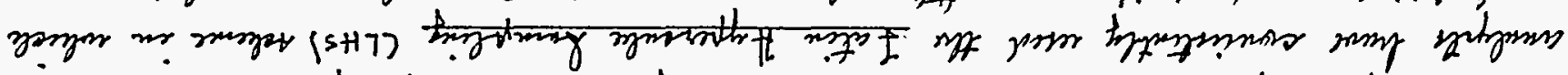

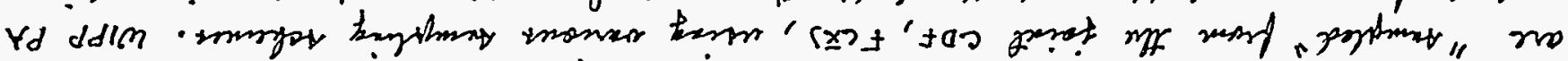
si $\bar{x} \cdot \ldots \cdot \varepsilon \bar{x}{ }^{r z} \bar{x}{ }^{\prime} \cdot \bar{x}$

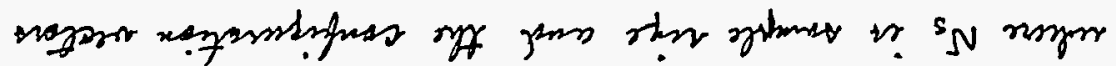

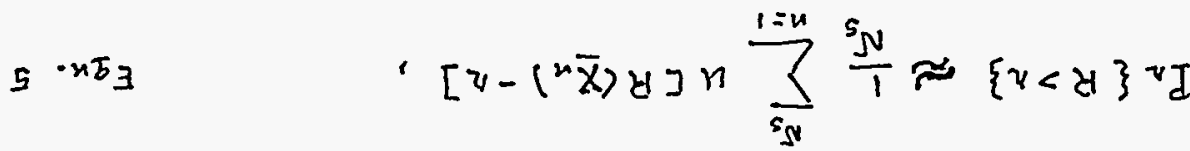

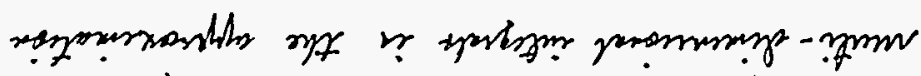

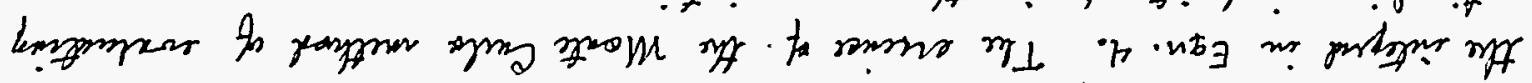

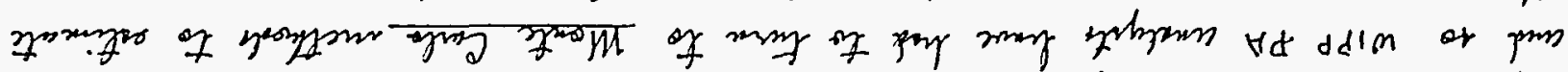

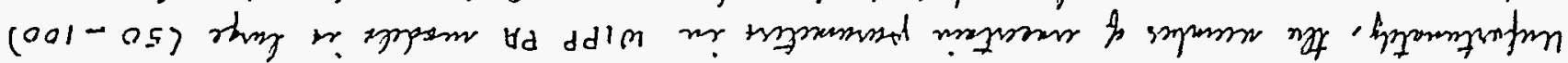




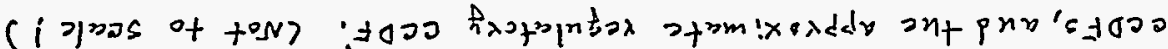

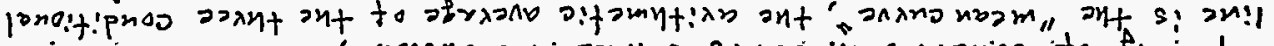

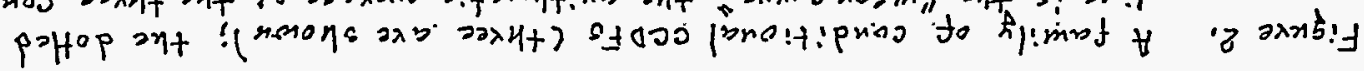

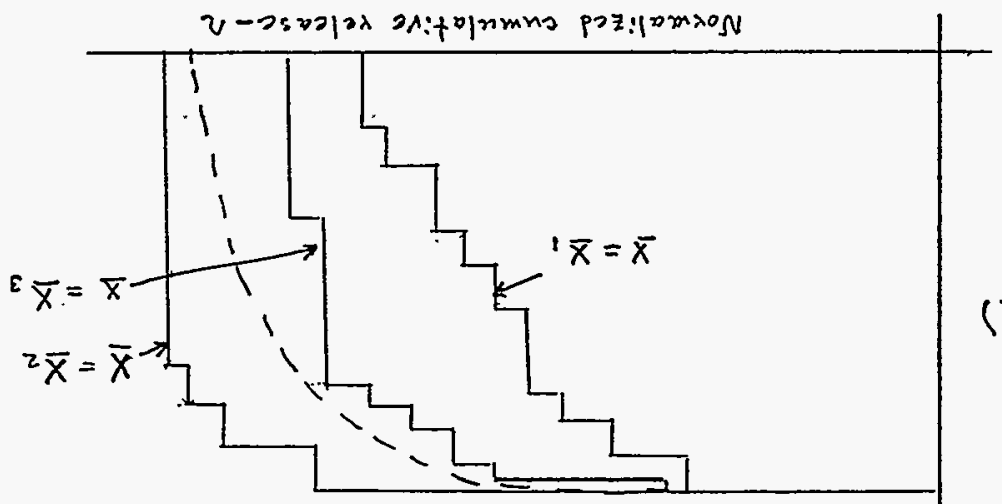

$(\bar{x} \mid v) \exists$

$\because 4020$ morse,

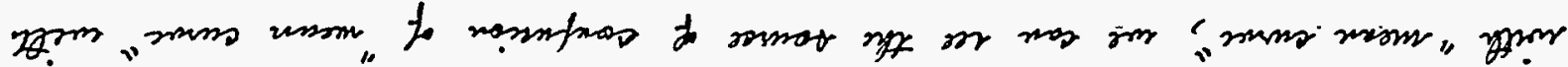

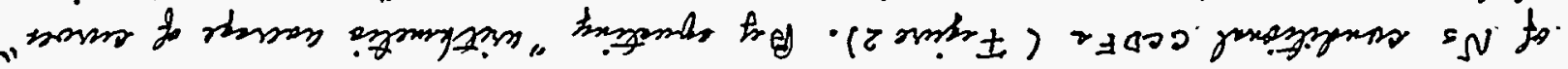

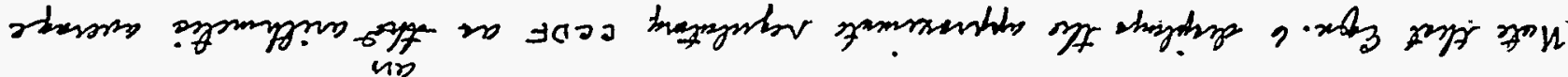

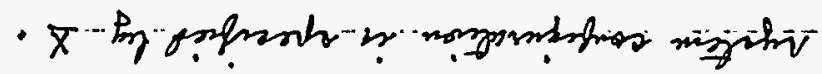

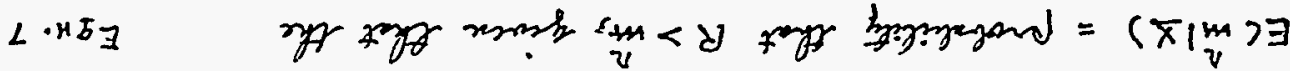

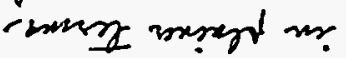

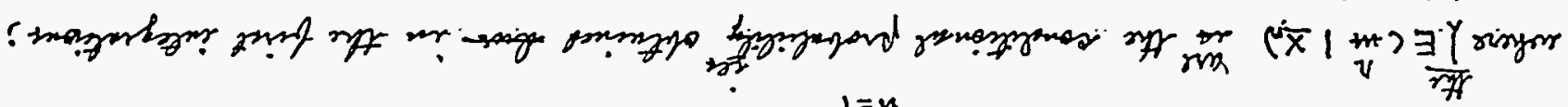
$2-x+2$

$$
(n \bar{X} \mid \underset{v}{m}) \underset{\frac{s_{N}}{1=n}}{\frac{s_{N}}{1}} \approx\{v<\gamma\} v_{-J}
$$

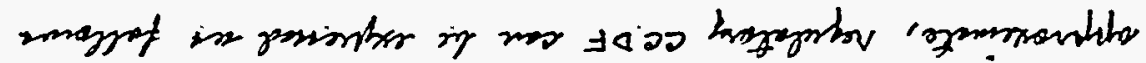

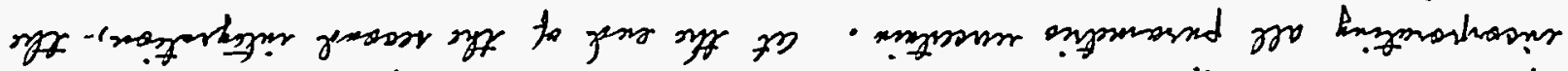

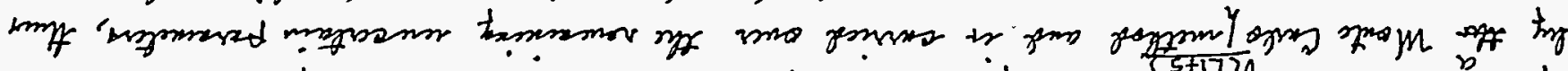

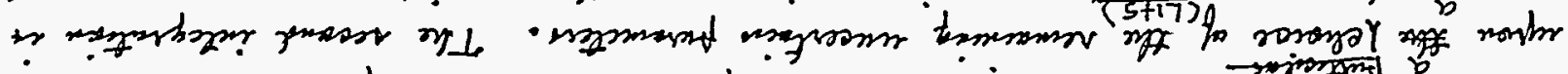

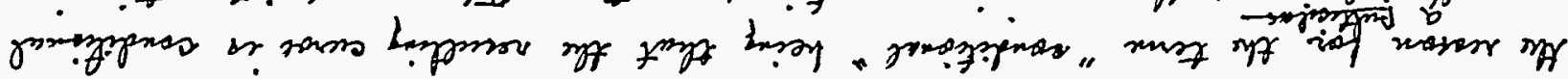

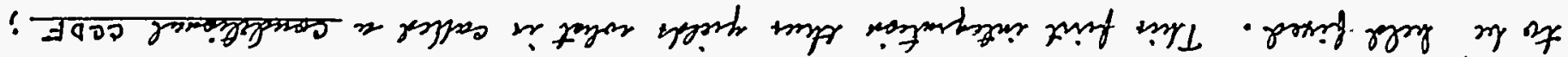

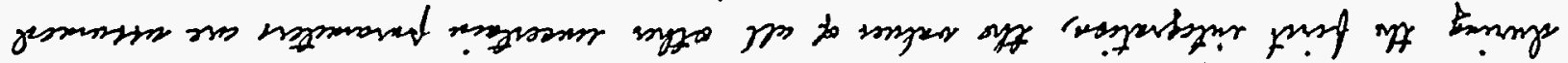

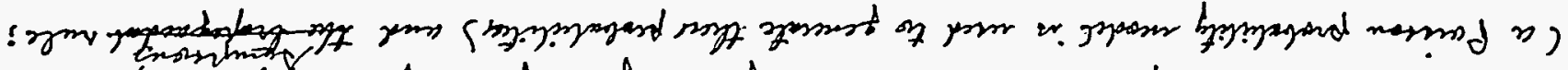

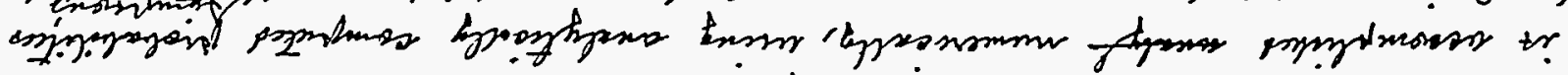

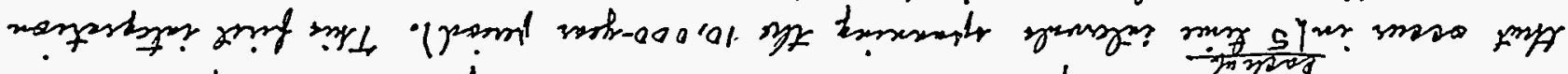

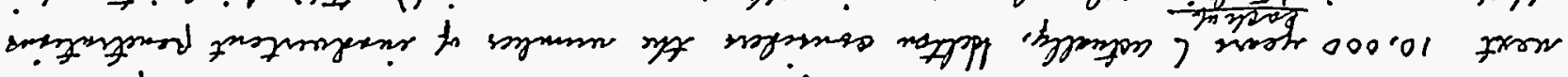

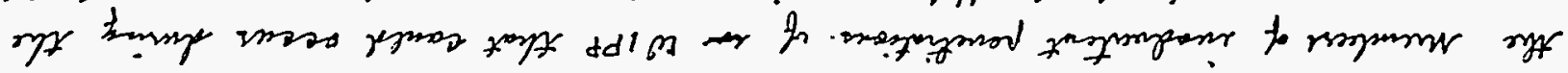

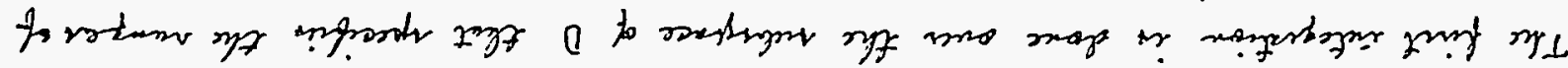

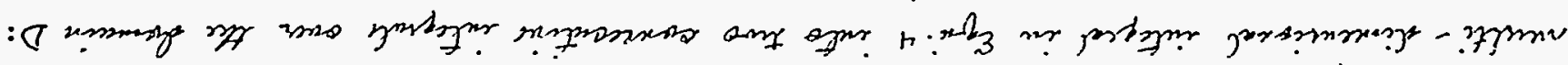

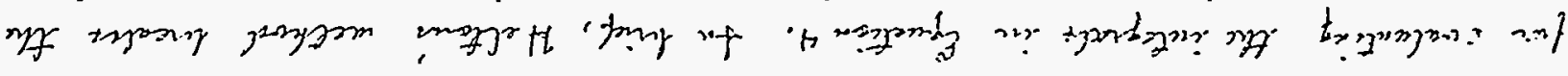




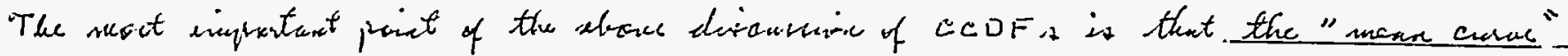

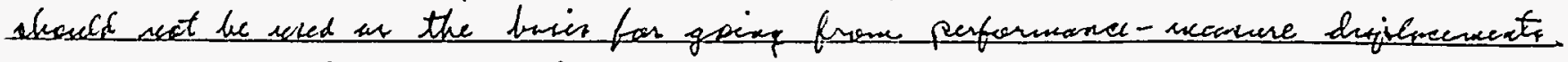
to calculation of ulititic for activity Act in the wIPP SP proprem. (Thir actertion

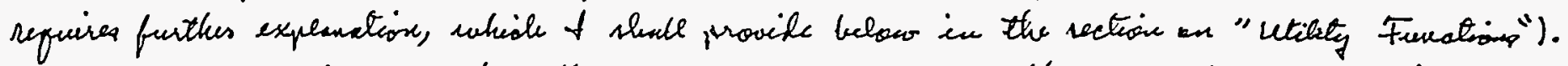

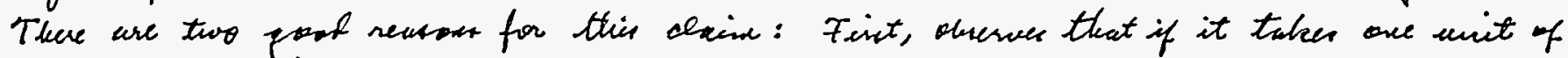

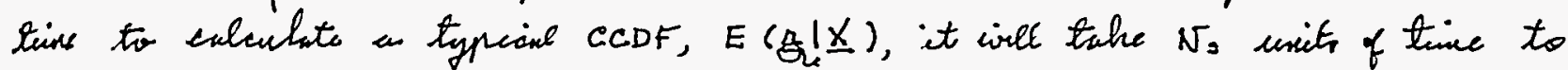

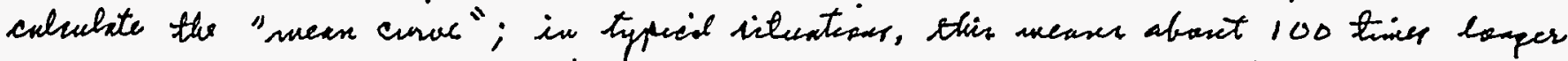

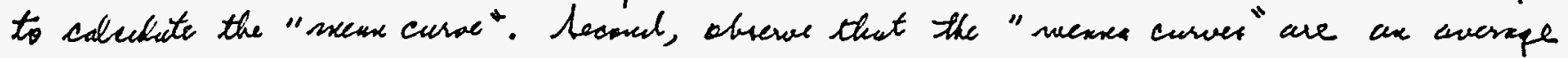

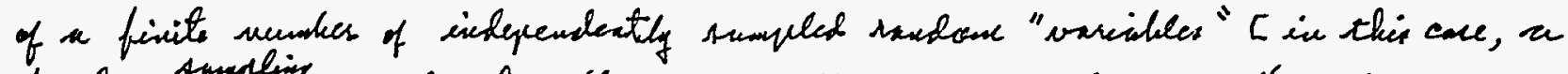

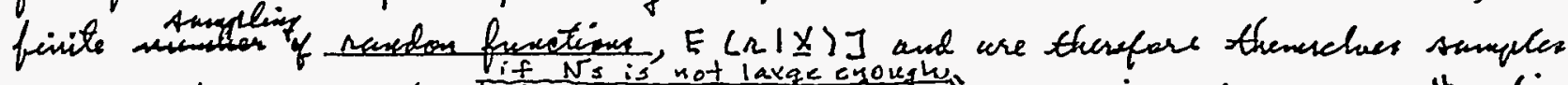

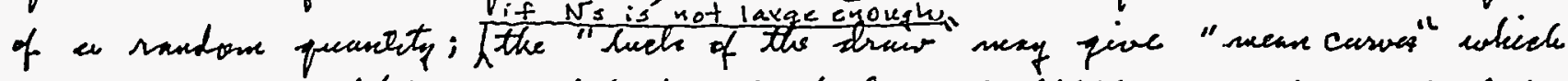

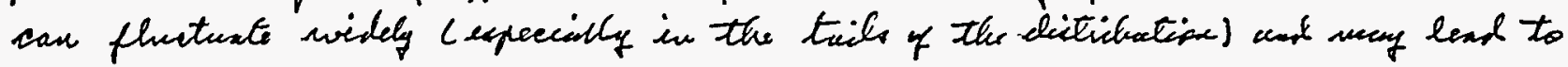

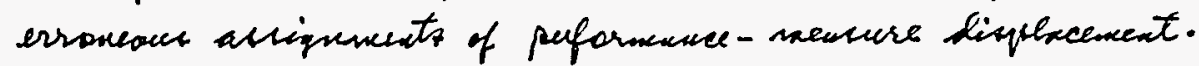

Fur these rearax, f proproce the une of a single conditionel CCDF, E. (rl X),

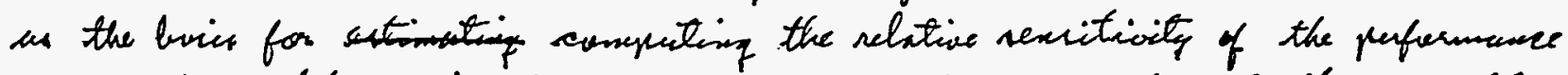

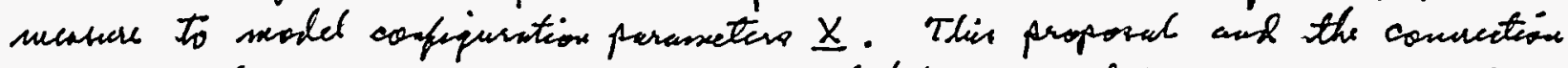

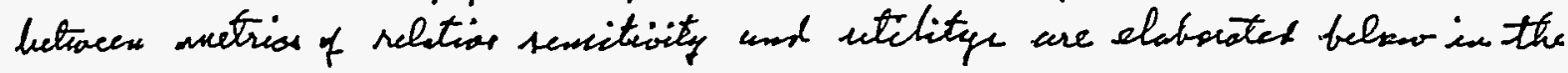
rection "Utility Function".

\section{Pestomenese Mesures for RCRA}

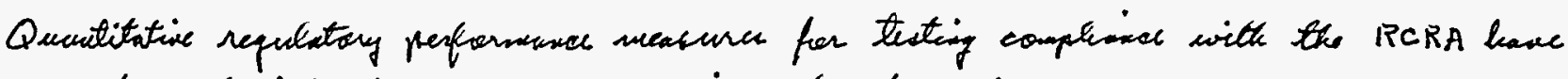

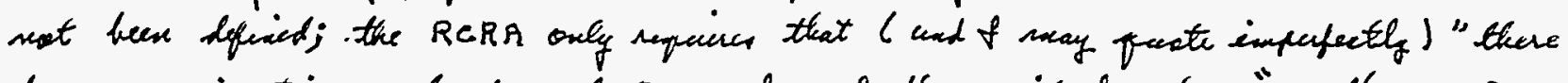
be no mignation of toxic substanaes begons the unit bounkary" of the WipP.

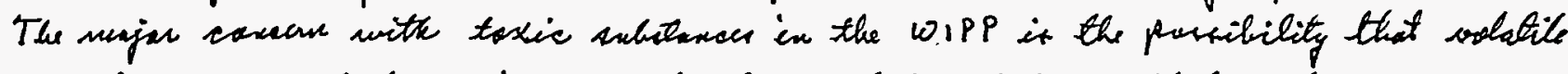

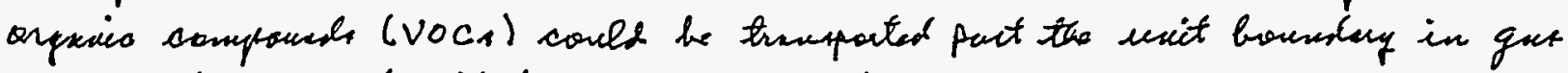

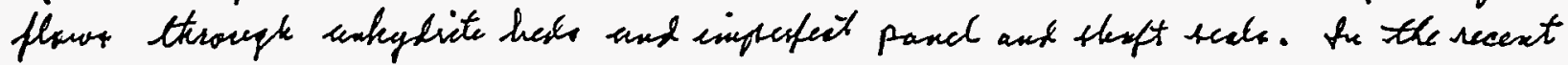

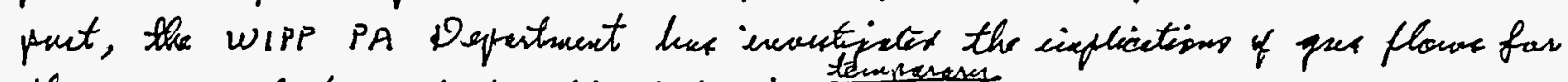

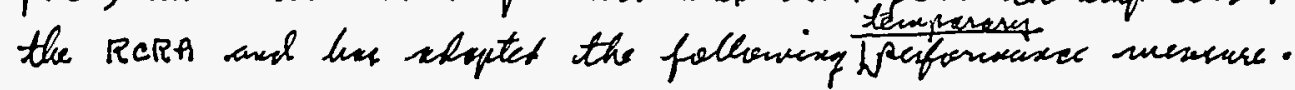

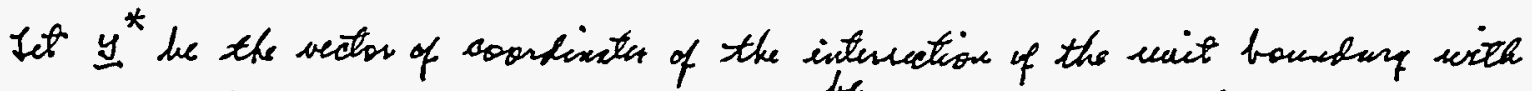

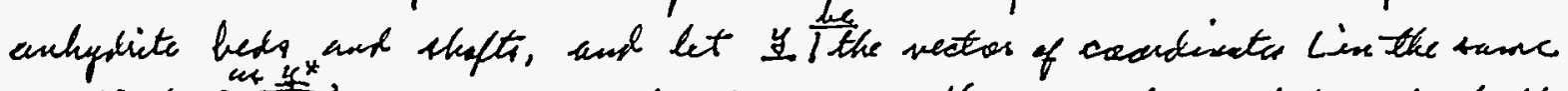

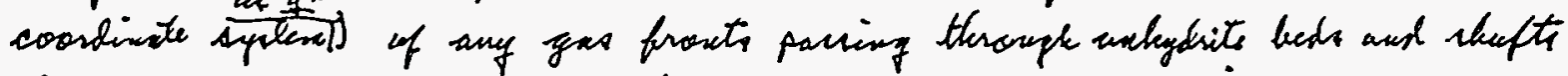

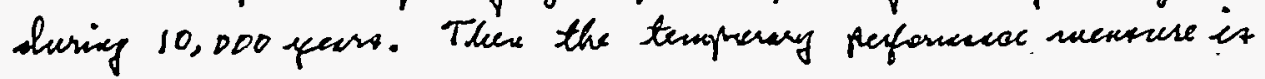

$$
\begin{aligned}
& R(\underline{x})=1 \text { if at leat one oomponeat of }\left(\underline{\underline{y}}-\underline{y}^{*}\right) \text { is poitio } \\
& =0 \quad \text { cetheravie }
\end{aligned}
$$




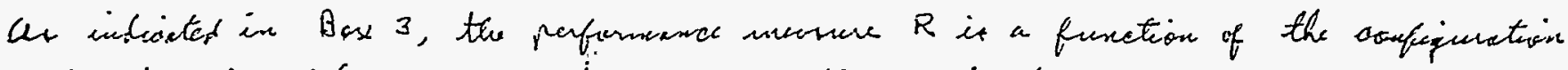

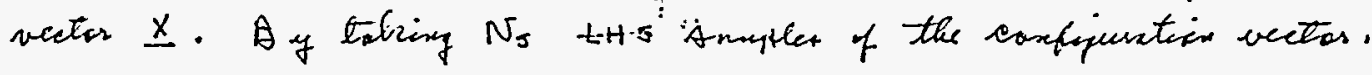

$$
\underline{x}_{1}, \underline{x}_{2}, \underline{x}_{3}, \cdots, \underline{x}_{N_{3}} \text {, }
$$

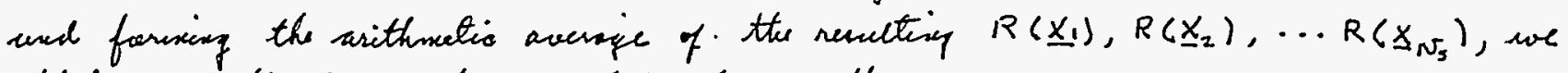

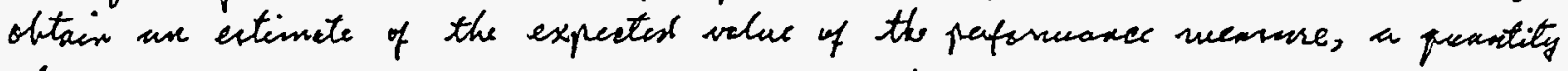

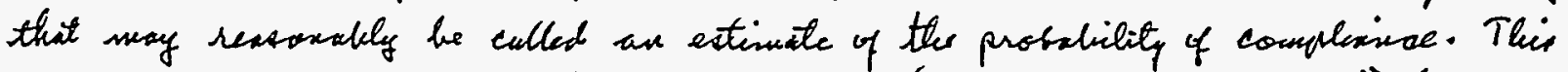

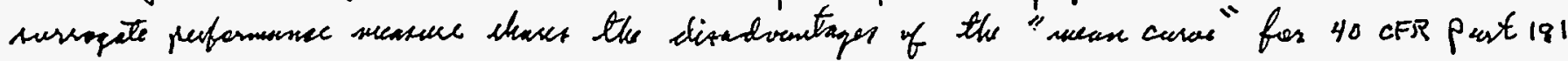

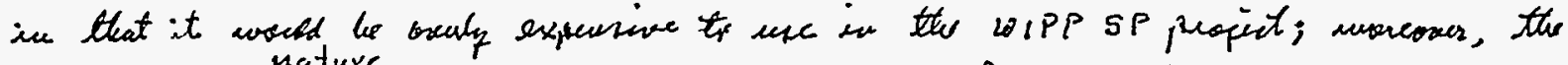

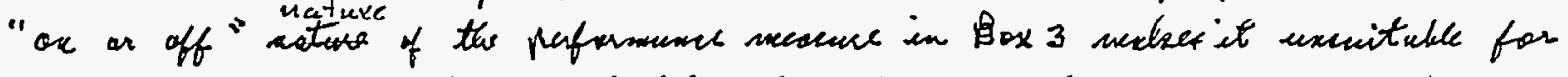

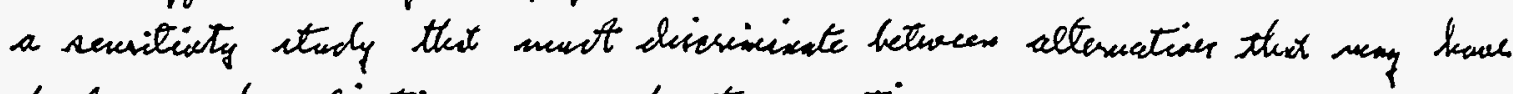

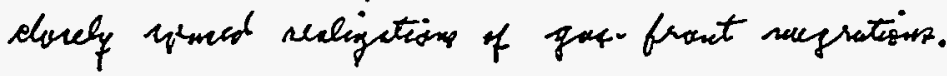

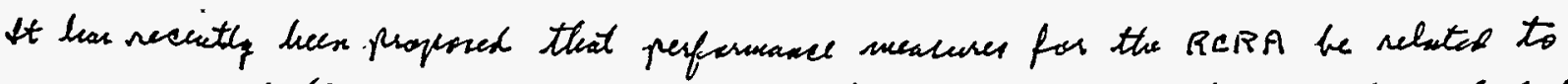

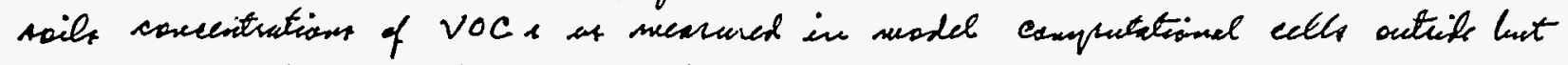
adjicent to tho exict boundary. It

$$
V_{R S}=\text { mensisumen sail concentration of the } k \text { theses of VOC is }
$$

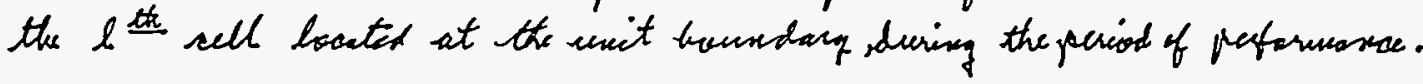

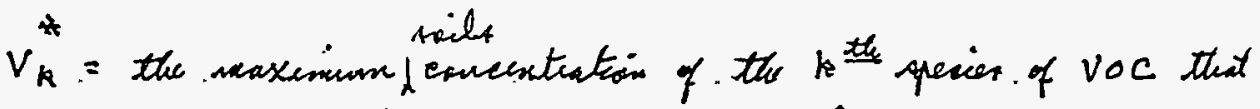

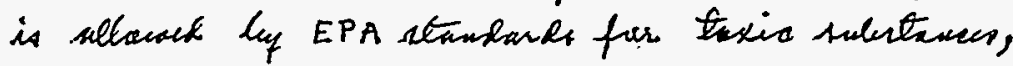

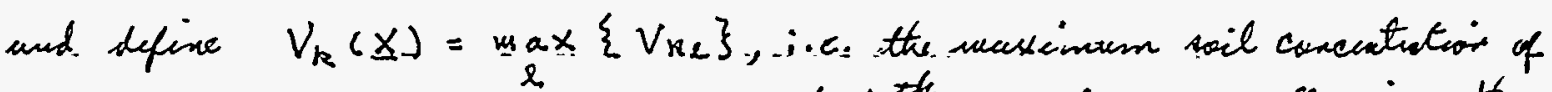
the 15 vOC ix any ecll, givere the suald oonfequintion vector $X$.

and $\quad \rho_{k}(\underline{x})=\frac{\underline{Y}_{k}(\underline{x})}{! V_{k}^{*}}$

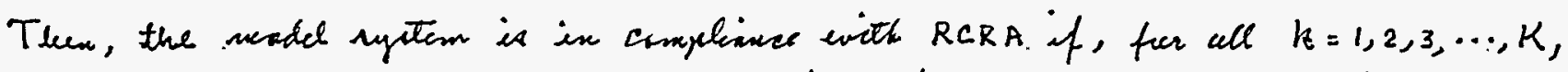
$P_{k}(X) \leq 1$; and if, for cot lant ore vecher of $k, P_{n}(\underline{X})>1$, then the mestel

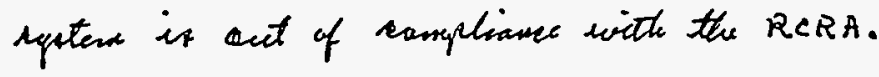

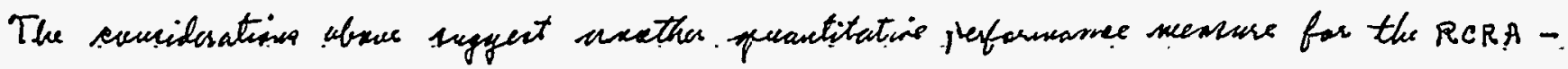

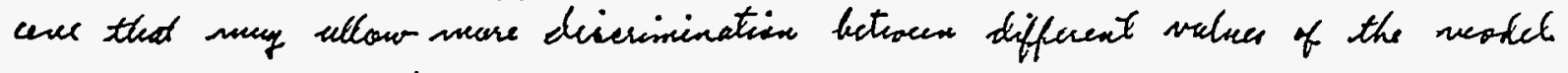
confegiueration uever $x$ :

$$
\rho(\underline{x})=\max _{k}\left\{\rho_{h}(\underline{x})\right\}
$$

Equ. 8

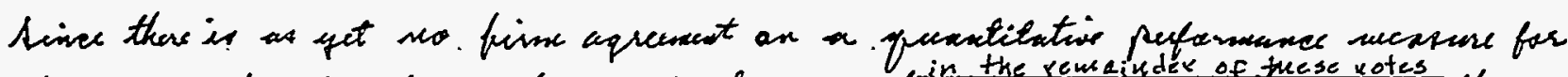

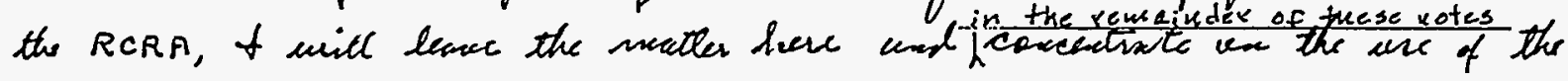

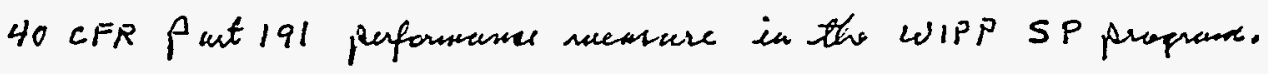




\section{Utilitig Frenterios}

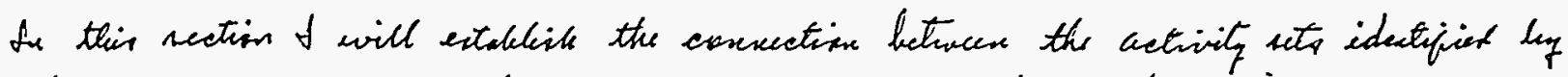
the WIPP SP proquam and ax oljectioie meacure of the etility of exch activity ret. An outline of the line of rearosing presented in this section is stersors in Box 4 .

$$
\text { Gativity Seti, } S_{n}
$$

$\underline{x}_{n}$, the moded configurative pector for astrivify ect $S_{n}$ $\downarrow$

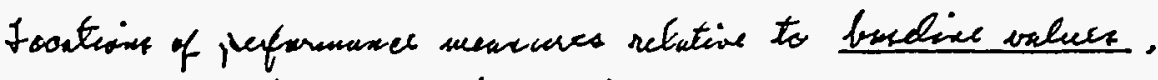

$$
\begin{gathered}
d_{1}\left(\underline{x}_{n}\right), d_{2}\left(\underline{x}_{n}\right) \text { for } 40 \text { CFR Pact.191 } \\
d_{3}\left(\underline{x}_{n}\right) \text { for the RCRA } \\
\downarrow
\end{gathered}
$$$$
\text { axiqument of utitity to actioity set } S_{n}
$$$$
v_{n}=\Phi\left[d_{1}\left(\underline{x}_{n}\right), d_{2}\left(\underline{x}_{x}\right), d_{3}\left(\underline{x}_{n}\right)\right]
$$

Box 4

\section{How Gativitic Let tufloenes WIPP PA Madels}

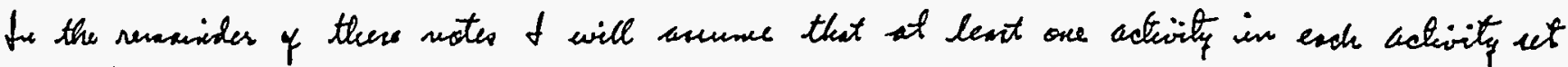

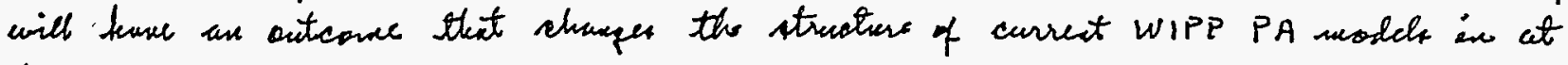
lewit one of three ways:

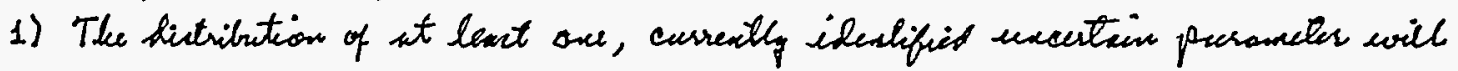
chenge; i.c. the range of the parameter changes or its central values lescan, metion or seote) change.

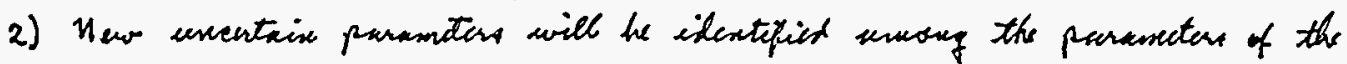
curreat coreplex of WIPP PA reodek.

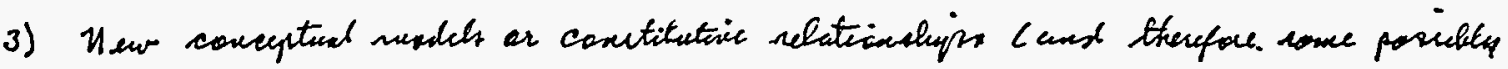

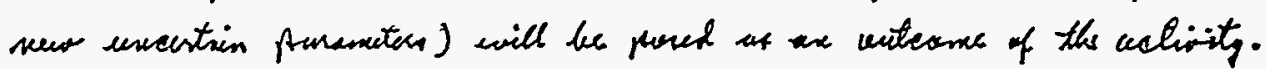

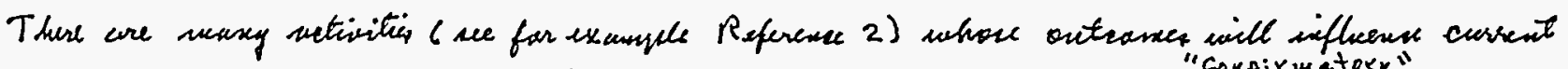

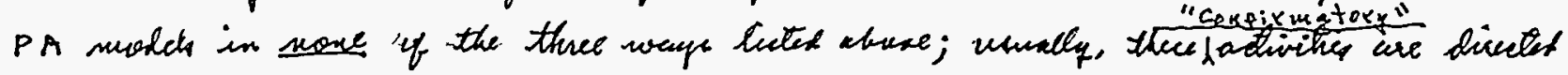

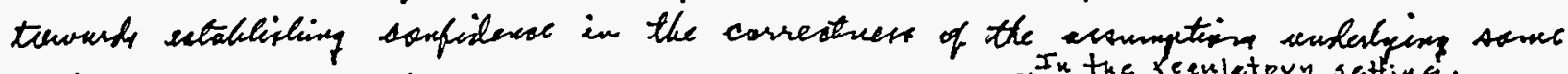

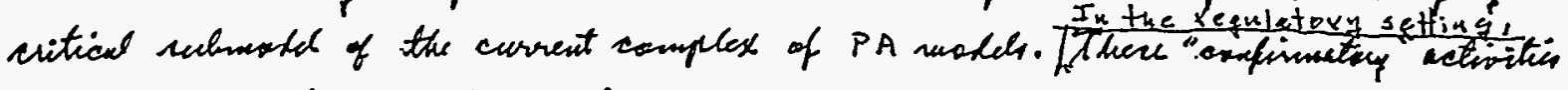

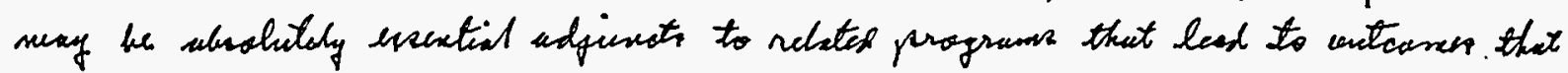

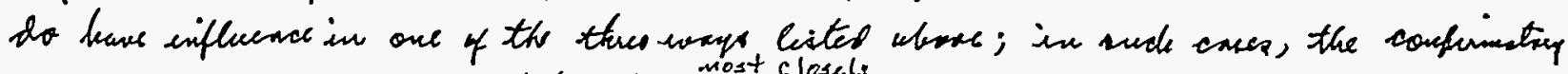

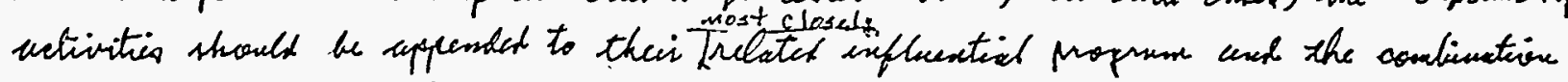

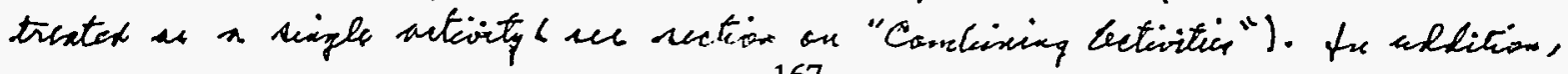




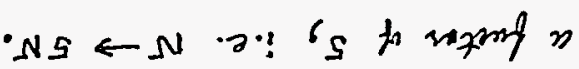

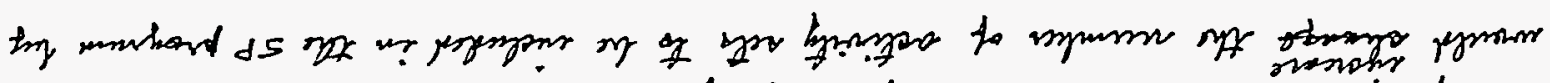

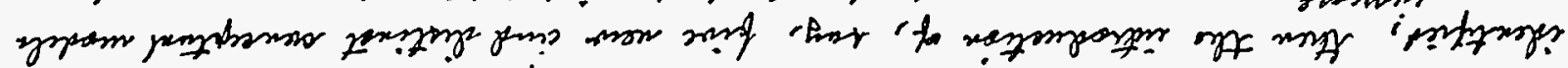

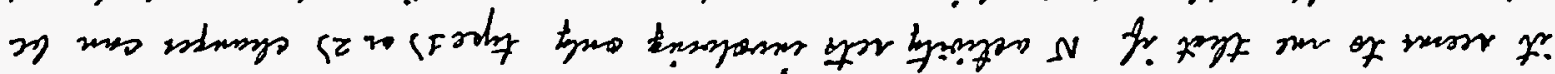

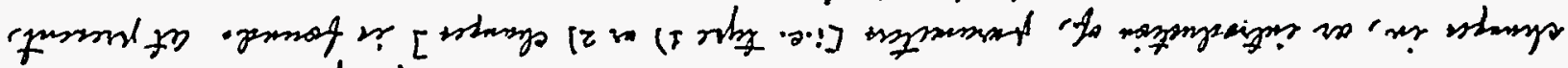

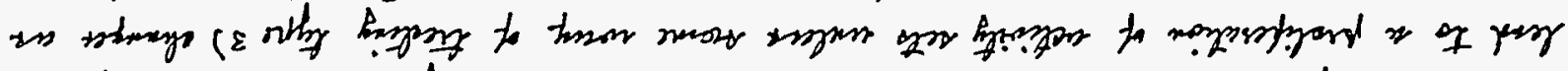

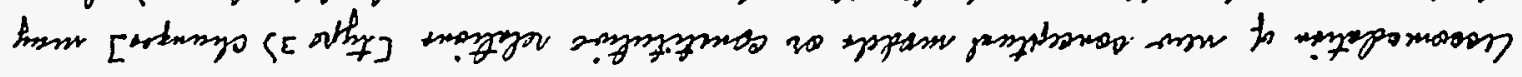

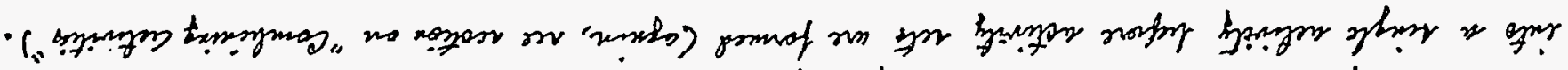

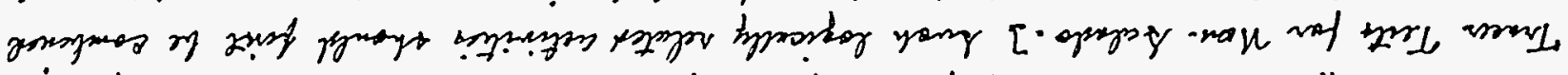

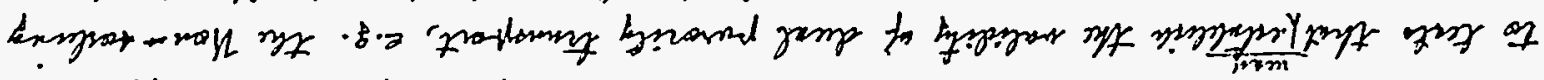

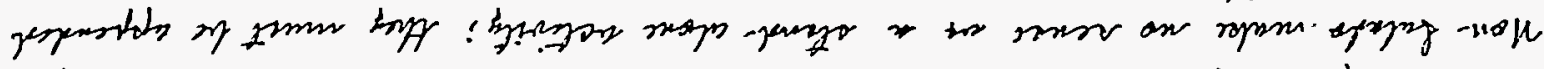

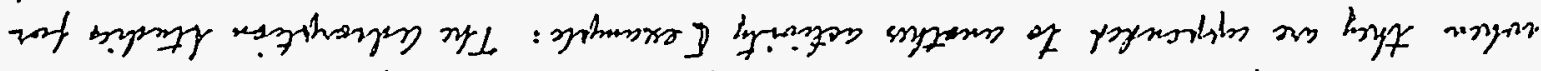

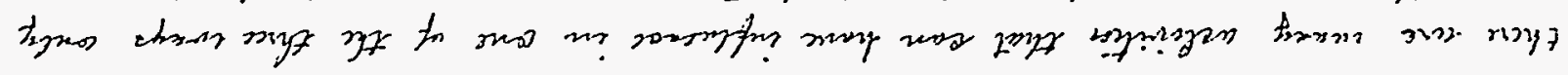


28. Beyeler, W. 1994. "Summary of SP Utility Function Meeting of May 17." Memo, May 19, 1994. 
Summary of SP Utility Function Meeting of May 17, 1994

with Corrections and Additional Comments Provided by Participants

\author{
prepared by \\ Walt Beyeler \\ Dept. 6307/SAIC \\ June 2, 1994
}

\title{
List of Participants:
}

$\begin{array}{ll}\text { D.R. Anderson } & 6342 \\ \text { R. Cox } & 6403 \\ \text { J. Helton } & 6342 \\ \text { S. Hora } & 6342 \\ \text { M. Marietta } & 6342 \\ \text { D. Rudeen } & 6307 \\ \text { P. Swift } & 6307 \\ \text { M. Tierney } & 6342 \\ \text { W. Beyeler } & 6307\end{array}$




\section{Description of Utility Functions}

\section{A) Common Features}

The utility functions discussed do not try to represent the way that the decision makers preferences change with the cost and duration of available options. The narrower purpose of the proposed utility functions is to identify the "best" option among a set of options, each of which satisfies constraints on cost and duration. For any of the proposed utilities, the "best" option would be identified under various possible constraints on cost and duration.

\section{B) "Probability of Compliance" (PC)}

Steve Hora illustrated this utility function using a single activity (a tracer test) as an example:

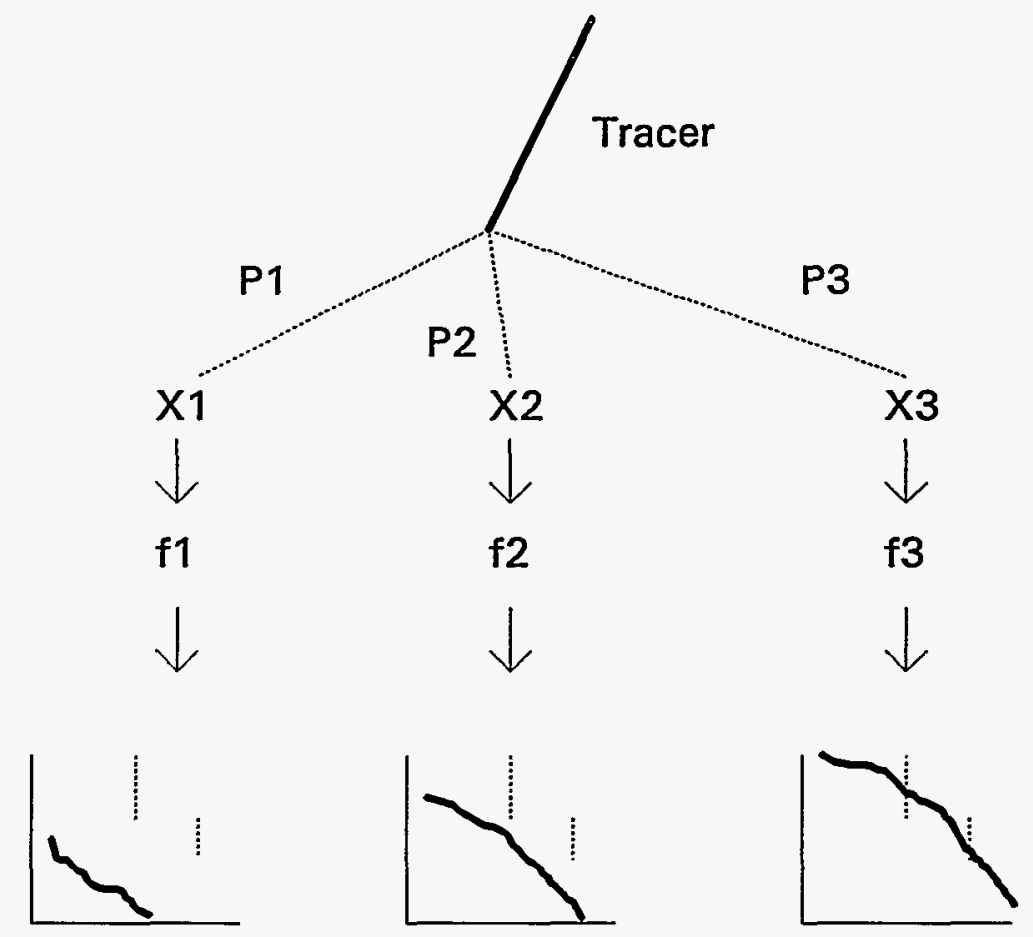

In this example, the possible outcomes of the tracer test have been binned into three classes. $\mathrm{P} 1, \mathrm{P} 2$, and $\mathrm{P} 3$ are probabilities that represent the experimentalist's (or other informed party's ) degree of belief that the outcome of the tracer test will fall in the corresponding class. $X 1, X 2$, and $X 3$ are representative outcomes characterizing each class, and $\mathrm{f} 1, \mathrm{f2}$, and $\mathrm{f} 3$ are the modified distributions of the model parameters that would be produced as a result of observing $X 1, X 2$, and $X 3$, respectively. Each of these distributions, along with the distributions of all other model parameters, would lead to different PA results, including a different mean CCDF. The probability that the mean CCDF would satisfy the regulatory requirements, following completion of the tracer test, is then the sum of the probabilities of observing outcomes that lead to a CCDF that satisfies regulatory requirements ( $P 1+P 2$ in the example). 
The concept can be generalized to multiple concurrent activities, and to multiple regulatory performance measures.

\section{C) "Displacement-based Utility" (DU)}

Martin Tierney described a utility function based on displacement of performance measures. Performance assessment calculations would be performed using a single set of parameter values ("one-shot" calculation) which characterize the central tendency of the parameter distribution (the mean value). Each one-shot calculation would lead to a CCDF of integrated normalized release, which reflects stochastic uncertainty but not the full range of subjective uncertainty. Each parameter set would also produce a performance measure related to RCRA, such as concentrations of hazardous constituents at the RCRA compliance boundary.

Each set of activities considered, and each outcome of a given set of activities, would lead to modified distributions for model parameters, as in the tracer test example described above. The central value of parameters affected by the activities could therefore be changed, leading to a modified one-shot performance calculation:

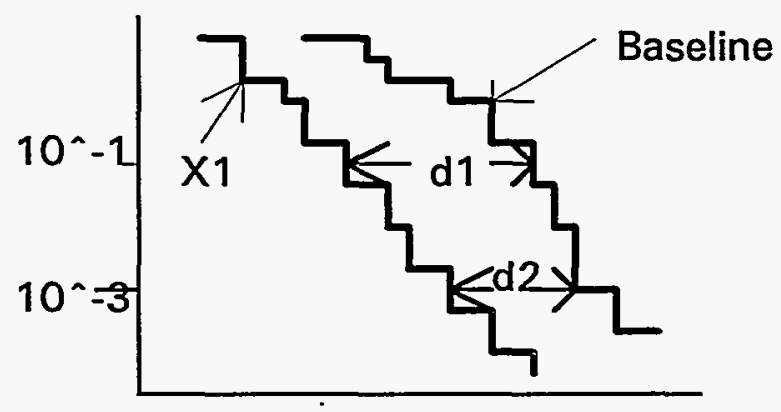

For integrated normalized release, two metrics are defined: the displacement of release at the $10^{-3}$ and $10^{-1}$ percentile levels. The baseline from which the displacements are measured is arbitrary: either the regulatory CCDF or a CCDF produced by current distributions might be used.

A third metric, d3, would be defined for RCRA-related performance calculations. The maximum, over all regulated constituents, of the ratio of calculated concentrations to regulatory concentration limits, is a possible summary measure for RCRA. The metric $\mathrm{d} 3$ would then be calculated as the difference between the summary measure resulting from a given mean/median parameter set, and an arbitrary baseline value (usually one ) for this measure.

An overall utility would be defined for the three displacement metrics:

$$
\phi(\vec{d})=\phi_{1}\left(d_{1}\right)+\phi_{2}\left(d_{2}\right)+\phi_{3}\left(d_{3}\right)
$$

where the individual utility functions describe the value that the decision-maker places on values of the different displacement metrics. 
Possible functional forms for the individual utility functions were discussed, although there is no restriction on the functional form that may be used, other than

$\phi_{i}(0)=0, \quad i=1,2,3$. Functions that are symmetrical about displacement values of zero represent indifference of the decision-maker to the direction of change of the performance measure. Asymmetrical functions can be used to encode preference for positive or negative displacements, or indifference to displacements beyond specific threshold values. The appropriate functional form of the utility functions, and the parameters of the utility functions, would be developed through elicitation of decisionmakers.

\section{D) "Large-Scale" Sensitivity Analysis}

The possibility of using a sensitivity analysis, analogous to sensitivity analyses done for previous PA's, was raised, but was not discussed in detail. This would involve sampling on all parameters which are influenced by any activity under consideration. The utility of a given activity would have to be related in some way to the sensitivity of performance measures to parameters influenced by that activity.

\section{Issues Discussed in Connection with Proposed Utility Functions}

The issues and concerns raised in the discussion of the proposed utility functions have been organized into five categories: A) Probability of compliance as a measure of utility, B) Displacement-based utility, C) One-shot CCDF as an estimate of the mean CCDF, D) Identification of a defensible baseline, E) Utility-independent implementation concerns. Responses addressed to those concerns are described in section III.

\section{A) Probability of compliance as a measure of utility}

1. An extremely large number of samples is required to reliably estimate the mean CCDF (i.e. one that accounts for the full range of subjective uncertainty) for a single outcome of a single activity set. It is practically impossible to calculate a single mean CCDF before September, let alone the number required by the decision problem.

2. A binary distinction on compliance/non-compliance does not distinguish between performance measures that are very near to regulatory limits and performance measures that are very far from regulatory limits.

3. By focusing on quantitative performance measures only, the need to provide "reasonable expectation" that the quantitative limits are satisfied (40 CFR 191c) is not addressed.

4. Activities that have the potential to shift performance measures "to the right" (further from compliance) could be improperly discounted.

5. Association with an effort to "maximize the probability of compliance" makes Sandia, either actually or in the view of stakeholders, an advocate for WIPP, and is not compatible with Sandia's role as scientific advisor. 


\section{Additional /ssues}

6. How this "utility function" is to be propagated through the decision tree is something that needs further exploration.

7. A mean CCDF provides an insufficient measure of system performance for purposes of optimizing the allocation of resources for several reasons:

a) The impact of uncertainties will not be adequately incorporated into the analysis

b) Political considerations are not incorporated into the analysis (e.g. the EEG may be very antagonistic if certain types of experiments are not conducted).

c) [It] does not take into account the quality or robustness of analyses to show the suitability of the WIPP as a waste disposal site (e.g. does not give any merit to the use of multiple barriers).

d) Does not take into account regulations other than 40 CFR 191.13

\section{B) Displacement-based utility}

1. The utility that a decision-maker assigns to a particular displacement will depend in part on the quality of supporting documentation.

2. Why are horizontal (release) displacements preferable to vertical (probability) displacements?

3. In order for a DOE decision-maker to assign a utility to a displacement value, they will require an understanding of the connection between the one-shot CCDF and the regulatory CCDF, or will respond as though they are identical.

\section{Additional /ssues}

4. It is unlikely that DOE decision makers will be able to define or understand appropriate utilities. It is likely that individuals at DOE will have little or no understanding of what is done in an analysis of this type.

5. Provided a utility function can be developed that captures the decision maker's preferences for displacements when costs and durations are equal, it is unclear how a decision maker might decide whether it is worth changing the schedule or budget in order to gain some incremental utility.

\section{C) One-shot CCDF as an estimate of the mean CCDF}

1. The one-shot CCDF requires a single execution of the PA codes, and does not require random sampling of parameter values. It is therefore far easier to compute than the mean CCDF, and is not subject to sampling variability.

2. Because the PA models are non-linear, the CCDF resulting from mean parameter values is not the same as the mean of the CCDFs resulting from sampled parameter 
values. $A$ one-shot mean value $C C D F$ is only the first term an expansion of the mean CCDF.

3. By recognizing that the model parameters are generally averages over large volumes, while the current parameter distributions often describe small-scale variability, the ranges of many parameter values should be reduced from current ranges. A oneshot mean value $C C D F$ converges to the mean CCDF as parameter variance decreases: a one-shot mean CCDF may therefore be a better approximation of the mean CCDF than is apparent from current PA calculations, provided scaling issues are resolved.

\section{Additional Issues}

4. By emphasizing means, important considerations with respect to the quality of the argument that can be put forth on the suitability of the WIPP for waste disposal are lost.

\section{D) Identification of a defensible baseline}

1. Because the baseline will be used to evaluate projects and therefore influence funding, there is a strong incentive to bias assumptions in the direction of larger performance measures. This bias will distort the evaluation, and the resulting assumptions may misrepresent the value of past project work to outside groups.

2. Because many processes (e.g. gas generation) influence different performance measures in different directions, or have contrary influences on different release mechanisms considered in the same performance measure, the definition of the baseline in terms of defensible parameters and assumptions will probably be nonunique or impractically difficult.

3. We cannot claim that a baseline can be identified such that all future performance measures will consistently move towards lower release. Unanticipated information, reconsideration of assumptions, or interactions that are overlooked in the original baseline definition may all cause future performance measures to be larger than a current baseline. This would result in a perception of inconsistency, or a loss of credibility of the process.

4. Why are "defensible" parameter distributions expected to differ from parameter distributions that have been provided in the past, in cases where no new information has become available?

5. Non-uniqueness in the baseline definition (e.g. no gas generation for 40 CFR 191 and high gas generation for RCRA) will produce glaring inconsistencies in the system description, and might provoke.criticism of the program from the regulator and the public.

\section{Additional Issues}

6. The "state of knowlege" quantification problem is more complex that that addressed in past PA's for the WIPP. This has been a major problem in these PA's and is yet to 
be resolved to everyone's satisfaction. I am not optimistic about carrying a considerably more complex quantification problem to completion in less time and with less resources than available to past PA's.

7. It isn't clear what "defensible" means. Anything to remove subjectivity will help.

8. [We] should not confuse defensible with conservative: defensible and conservative may be self-exclusive.

9. [The baseline is intended to be] conservative with respect to what?

10. [ldentifying the basline will be] hard in non-linear coupled systems.

\section{E) Utility-independent implementation concerns}

1. Identifying the connection between activity outcomes and parameter distributions (i.e. between $\mathrm{X} 1$ and $\mathrm{f} 1$ in the above figure) is vital to the process, and is not straightforward. Past efforts to define connections between data and PA model parameters have been difficult and controversial.

2. Upscaling of data from the measurement scale to the model scale has only been systematically investigated for the Culebra flow field. The results of an exploratory study of solubility upscaling suggest that discharges to the accessible environment can be significantly influenced by recognizing the influence of spatial variability.

3. Will the elicitation of possible activity consequences focus on outcomes or parameters? For example, will Rick Beauheim be asked to predict pressures, flow rates, and heads, or interpreted transmissivities at individual wells, or entire transmissivity fields?

4. The tracer test example in the above figure includes a binning of outcomes into three classes. For the tracer test, and in general, the outcome will be continuous. How will the binning decision be made?

\section{Additional /ssues}

5. I am very concerned that the scale of the analysis, the quality of information that can be obtained to go into the analysis, the resolution with which modeling can be performed, and the computing resources required are not being adequately considered. None of the performance measures discussed are adequate to capture all of the important considerations in the selection of an optimum development plan for WIPP.

6. Values of one parameter will effect the sensitivity of parameters in other areas that are coupled.

III. Responses to Issues

A) Probability of compliance as a measure of utility 
1. Because it is not practically possible to calculate convergent mean CCDFs (i.e. CCDFs that incorporate the full range of subjective uncertainty) for each future state, we should identify and evaluate procedures for estimating the location of the mean CCDF.

\section{No response offered}

\section{No response offered}

4a. If the baseline is properly defined, then potential experimental outcomes that might lead to large releases should establish the set of defensible assumptions. All anticipated outcomes should therefore produce performance measures that are smaller than performance measures calculated with baseline assumptions.

4b. Definition of such a baseline is problematic (see II-D-3)

5a. Looking for a technical program that maximizes the probability of compliance does not in any way require that Sandia advocate WIPP or adopt the DOE's desire to operate WIPP, but merely takes cognizance of the DOE's objectives.

The situation is analogous to other engineering design problems. If the DOD were interested in producing a bomb with a certain yield, they might propose a specific design, and request an analysis from Sandia. Sandia's job would then be to objectively estimate the yield, which the DOD would compare against the desired yield. If the requirements were not met, the DOD might modify the design in some way, for which Sandia would provide another objective scientific analysis. DOD's underlying question ("Is it possible to produce a bomb with a minimum yield of $X$ subject to the following constraints.....?") would be answered more efficiently if asked directly. In searching for a design that fell within the stipulated constraints and satisfied the stated criterion, the analysis of each design would still be conducted in an objective, scientifically defensible way. Sandia would in no way be required to compromise the integrity of the analysis, or share in DOD's desire that the bomb be produced, in order to answer the technical question of whether DOD's objective could be met within the stated constraints.

No response was offered to the perception of advocacy.

$5 b$. Adopting a utility function which represents indifference to the direction of displacement of performance measures avoids the perception of advocacy.

B) Displacement-based utility

1. No response offered

2. No response offered

3. No response offered

C) One-shot CCDF as an estimate of the mean CCDF 


\section{No response offered}

2. It should be possible to estimate the second term in the expansion of the mean CCDF by perturbing the mean parameter values. This would provide an improved estimate of the mean CCDF.

3. Volume averaging may not be an appropriate upscaling method for some parameters.

D) Identification of a defensible baseline

1. No response offered.

2. There is no requirement for uniqueness.

No response offered to practical problems of non-monotonic influence.

3. New information can always change the baseline. Any analysis will be dependent on the information available and the assumptions made.

4. With reference to the tracer test example in the above figure, the defensible distribution would be $\mathrm{f} 3$, which corresponds to the least favorable experimental outcome, while the experimentalists prior distribution would be built from all conditional distributions ( $\mathrm{f} 1, \mathrm{f} 2$, and $\mathrm{f} 3$ ) and the corresponding outcome probabilities.

5. Consistency is important of we want a single "best" description of the system, independent of regulatory performance. If, instead, we want to know what can defensibly be claimed about the performance of the system with respect to specific performance measures, then the description may be different for different regulations. The description used to address one regulation may not be, and does not need to be, consistent with the description used for a different regulation.

E) Utility-independent implementation concerns

1a. It might be useful to include definition of outcome/parameter connections as an activity in the decision process.

1b. Defining the connection between outcomes and parameters is a neccessary preliminary step to evaluating the potential impacts of other activities.

\section{No response offered}

3. The elicitation might in principle be focused on anything between gage measurements and PA model parameters. The level of abstraction may need to be tailored to each activity. Rick Beauheim should not be asked to anticipate transducer readings or to sketch regional transmissivity fields.

4. The elicitation will be based on a few ( 4 or 5 ) intervals for continuous parameters. Coverage of the model parameter range is more important than coverage of the outcome range. 


\section{Further Responses}

\section{A) Probability of compliance as a measure of utility}

2a. Not a valid issue for this scheme, which would weight the $[0,1]$ outcomes with probabilities.

$2 \mathrm{~b}$. The utility function does not measure "degrees" of compliance because the regulation does not acknowlege "degrees" of compliance. By setting a limit on continuous quantities, the regulations will always create a situation where arbitrarily similar systems can be different in terms of compliance, and vastly different systems can be insitinguishable. This is an issue to raise with the EPA if this consequence of setting specific limits is objectionable. The ability of new information to change the status with respect to compliance creates a technical reason for preferring a performance measure that is "far" from the boundary to one that is "close". To the extent that the possible range of new information is anticipated, and will be provided by an activity under consideration (the tracer test in the example discussed), this preference is represented in the probability of compliance: if the expected mean CCDF is "close" to the regulatory limits, the probability of compliance will be near $1 / 2$; if it is "far" from the regulatory limits, the probability of compliance will instead be near either 0 or 1.

3a. Reasonable expectation does not imply a quantitative measure such as a degree of confidence or probability. The EPA standard (40 CFR 191) is, itself, probabilistic. There is no need to add probabilities upon probabilities. Reasonable expectation is best interpretaed as meaning the underlying process for constructing the CCDF is sound -- without substantial flaws.

3b. "Reasonable expectation" is not clearly defined, and can plausibly be established by the way in which the quantitative performance measure is calculated and the arguments that support the calculation. It would indeed be pointless to predicate resource allocation on the basis of a calculation that the EPA clearly would not accept as satisfying the "reasonable expectation" requirement. This is the primary purpose of insisting on a "technically defensible" baseline: the performance measures are assumed, as noted, to be calculated in such a way that, if the quantitative limits are satisfied, then the regulator would agree that the site complied with respect to those aspects of the regulations. The SP process seeks to establish reasonable expectation by soliciting and incorporating the public's concerns about release scenarios and processes (i.e. "conceptual models") in the performance measure calculations.

4a. While this is true for maximizing the probability of compliance, an alternative was suggested several years ago that involves the maximization of the probability of resolution of the issue, whether good or bad.

Let CCDF $(r)$ the probability that the standardized release exceeds $r$ and let $S(r)$ the probability of a release $r$ or greater limited by the standard. 
The criterion $P\left\{\min _{r}[S(r)-C C D F(r)]>0\right\}$ has been suggest for use by the SP effort. This criterion, however, is only one of many candidate functions. The decision regarding the appropriate choice is a policy decision. The choice $\mathrm{P}\left\{\min _{\mathrm{r}}[S(r)-\operatorname{CDF}(r)]>0\right\}$ has both advantages and disadvantages. One advantage is that the expected value of the criterion has a directly interpretable meaning -- it is the probability that CCDF( $r)$ is below $S(r)$ and thus the performance assessment shows that the repository meets the standard. This criterion is useful in evaluating experiments only if, a priori, it is believed that the repository satisfies the standard. If the performance assessment indicates that the repository does not meet its goal, experimental information, on average, will drive the expected criterion lower. This behavior suggests that a criterion such as

$$
\left.\max _{\{}\left\{\min _{r}[S(r)-\operatorname{CCDF}(r)]>0\right\}, P\left\{\min _{r}[\operatorname{CCDF}(r)-S(r)]>0\right\}\right\}
$$

which is more neutral in a policy sense because the criterion can be maximized by showing the repository is either satisfactory or not satisfactory. An apparent drawback to such a criterion is that it does not extend to desgin modifications. For example, making the waste as soluble as possible might also help maximize the probability of noncompliance.

4b. This perception overlooks the significance of the definition of the technically defensible baseline. Again, the objective is to calculate performance measures in such a way that, if the quantitative requirements are satisfied, then the regulator would agree that the regulation is satisfied in this regard (see IV.A.3 above). The proposed utility function does not depend on the location of the baseline CCDF, but rather on the range of locations of CCDFs that are expected after an activity set is performed: whether this range lies to the right or left of the baseline CCDF is immaterial. I believe that the undelying concern can be represented using a hypothetical example:

Suppose we are comparing two activity sets, $A$ and $B$. The mean CCDFs for the possible outcomes of $A$ and $B$ each form a family of mean CCDFs, all of which lie within some limiting curves:

$A$, with a probability of compliance of 1 , will be selected over $B$, with a probability of compliance less than 1. This is a sensible decision only if all of the calculated CCDFs, for both $A$ and $B$, would be technically defensible to the regulator given their respective outcomes. If $B$ contains an experiment that $A$ does not, it may be (mistakenly) alleged that set $A$ is selected because of the potential for the experiment in $B$ to provide unwelcome information. This allegation is unsupportable because, as part of the definition of the baseline, each experimentalist has been asked to describe the assumptions that they would defend to the regulator given existing information. These assumptions would be defined in consideration of the possible performance-related 
concerns that cannot be disqualified with existing data. Each calculation in A will have been done in accordance with the assumptions that the experimentalist identified as supportable using exising information, in effect assuming the least favorable outcome for the experiment excluded from $A$. The reason that $A$ has a higher probability of compliance is that some mitigating measure has been adopted (an engineering modification or an experiment in a different area) that compensates for our ignorance about the experimental outcome, not because the potentially negative outcomes of the experiment have been conveniently ignored.

There are additional criticisms that might be made following a decision to select $A$ over B:

"I have changed my mind. I no longer consider the assumptions under which $A$ was evaluated to be defensible, but instead insist that some other set of assumptions be used"

This challenge has the same effect as the introduction of a new conceptual model or scenario. All bets are off, and the evaluation would have to be repeated. I don't believe that the possibility that this argument might be proposed is a special weakness of this process: if any evaluation procedure results in the suspension of any program we can expect the assumptions under which the evaluation was conducted to be challenged. In this case it is the investigator's own assumptions that are questioned. The merit of this approach, unlike the Systems Evaluation for example, is that the investigator has been consulted to define the technical consequences of suspending their program.

"The potential benifits of the mitigating or compensating programs adopted in A are overstated. An honest assessment of these programs would reveal the need for my experiment."

Bias may be introduced through the subjective probabilities of activity outcomes, or through the claims made about the possible utility of outcomes. The formal elicitation is intended to control the first source. The second source is controlled by requiring the connection between the possible outcomes and PA to be technically defensible in the view of the regulator, i.e. subject to review and comment by stakeholders. Depending on the way the decision analysis is implemented, it may also be very easy to examine the sensitivity of the decision (the optimal activity set) to subjective priors.

"Conducting the experiment might disclose unanticipated phenomena that were not considered in establishing the baseline assumptions, and that have a large effect on performance measures."

If a specific phenomenon or process is envisioned, this is essentially the first argument. If the point is rather that if we look we may find something we did not expect, this will always be true of any experiment. We cannot design or conduct an experimental program to comprehensively search for things that are unknown, unanticipated, and inarticulable. 
"The assumptions are fine, but the limitations of the method used to estimate the location of the performance measures, or the discretization of outcomes, makes $A$ appear better than $B$, when in fact it is worse."

It is important to understand the limitations of any approximations required to estimate the values of performance measures, and to estimate the probability of compliance. Ideally we should acknowlege these limitations by identifying all activity sets which satisfy the given cost and schedule constraints and are indistinguishable from the optimal set within the limitations of the analysis (e.g. sampling error). Note that we risk missing the optimal set of activities by committing this error, but do not risk compromising the defensibility of the compliance application if the selected (sub-optimal) activities lead to acceptable performance measures.

5a. The argument in the engineering design analogy (IIl.A.5a) is the reason why the displacement-based utility was proposed.

$5 b$. The perception of advocacy may, in some cases, be effectively corrected by arguments that illustrate the fallacy of this perception (see III.A.5a). Sandia's association with the corrolary effort to actively solicit and incorporate stakeholder's concerns may also mitigate negative perceptions.

6. The utility function would be propagated upward only if future decisions were included in the analysis. In the tracer test example, if outcome $X 3$ were observed, and if budget and schedule constraints allowed, the effect of additional activities following the tracer tests would be estimated, conditional on the tracer test outcome X3. As in the example, the probability of compliance for the initial decision to conduct the tracer test is:

$$
P(C)=P(C \mid X 1)^{\star} P 1+P(C \mid X 2)^{*} P 2+P(C \mid X 3)^{*} P 3
$$

$P(C \mid X 1)$ and $P(C \mid X 2)$ are both 1 , as before, while $P(C \mid X 3)$ would (presumably) be greater than zero.

7. The mean CCDF is sufficient to the extent that the compliance decision will be based on the mean CCDF. If the use of the mean CCDF as the basis for comparison against the standard (which is the anticipated guidance in 40 CFR 194) is not considered appropriate, this concern is properly addressed to the EPA. Uncertainties may either be represented in the CCDF (as contributing to the probability of integrated release exceeding certain values) or may be about the CCDF (How accurate is the estimate of the mean? Which of several alternatives is appropriate?). As in previous PA analyses, both stochastic and subjective uncertainty are represented in the mean CCDF. By actively soliciting and incorporating alternative conceptual models in calculating the mean $C C D F$, and by predicating resource allocation decisions on the least optimistic of the resulting mean CCDFs, the (arguably) most significant contribution to uncertainty about the mean CCDF is explicitly incorporated. (See also IV.A.3a and $b$ ). 
The objective function, as proposed, is intended to identify those activities that are most cleary required for a technical defense of the compliance application given existing regulations. (Although the example focuses on 40 CFR 191, the probability of compliance with all long-term performance requirements would be used in practice). The DOE is not bound to act strictly in accordance with the results of this analysis. They may impose (or allow others to impose) additional requirements which would mandate work not justifiable on the basis of compliance with the existing regulatory definition of safety. If they can be formalized, these requirements might be used to extend the proposed objective function. If not, the DOE might choose to supplement the set of activities identified as "optimal" for meeting the quantitative performance requirements with activities that fill political needs (e.g.. EEG recomendations, multiple barrier characterization). The risk is that the supplemented activity set might not be optimal for satisfying both technical and political requirements.

A robust argument is sought through public solicitation and consideration of alternative modelling assumptions, rather than through evaluating performance with respect to other safety concepts (e.g. redundant barriers).

\section{B) Displacement-based utility}

2. See II.A.2, IV.A.2

\section{C) One-shot CCDF as an estimate of the mean CCDF}

2. Estimating the second term in the expansion of the CCDF would be computationally expensive.

3a. Permeability is an example of a parameter for which volume averaging is inapproporate.

$3 b$. The point is not what average to use but that some change in distribution due to upscaling needs to be added.

\section{See IV.A.7}

\section{D) Identification of a defensible baseline}

1. The formal elicitation process by which the baseline is defined will help to control this tendency. The proper measure of the value of past work is not the current baseline, but rather the difference between the current baseline and a baseline that would have been defined in the past.

2. See III.D.5 for comments on consistency of the system model between regulations. The practical problem created by gas generation is that low rates tend to increase liquid-borne releases considered in 191, while high rates may tend to increase spallings releases which are also considered in 191. The total integrated release, across the range of gas generation rates consistent with our current understanding, is probably not monotinic. We could in principle find the gas generation rate which is consistent with existing information and leads to the least optimistic integrated release using a 
separate modeling study. This approach is probably impractical, and the answer might vary with other system parameters, such as Salado permeability. One solution would be to calculate spalling releases using large gas generation rates, and liquid-borne releases using small gas generation rates. This solution creates an inconsistency in the system models that is perhaps more distasteful, but not essentially different, from any inconsistencies between the assumptions used to address different regulations.

We can subsequently estimate the value of removing this inconsistency through the modeling activity described above: identifying the unique "defensible" gas generation rate would lead to an integrated release that is more than the integrated release due to either mechanism (spalling or liquid flow) considered seperately and "inconsistently", but is less than the sum of the two integrated release values. We might (optimistically) approximate the effect of the modeling study by selecting the maximum release due to the separate mechanisms, or, perhaps more fairly, as an integrated release midway between the maximum and the sum. However this approximation is made, it appears that defining a consistent gas generation rate has value only if spallings releases and liquid release have similar values: in all other situations the difference between the summed integrated release and the "consistent" integrated release will be bounded by the integrated release due to the less significant mechanism. To the extent that either direct removal or liquid-borne releases dominate integrated discharge for a particular vector and scenario, there is no practical value in searching for a consistent gas generation rate. Because this has been the case in past PA calculations, the proposed inconsistent approximation appears to be quite acceptable, even if counter-intuitive.

Note that the above argument does not pertain to the potential value of an experimental program designed to reduce uncertainty about gas generation, but only to the value of a consistent representation of existing uncertainty.

\section{See IV.A.4b}

4a. Why aren't all outcomes $(X 1, \times 2, X 3)$ defensible?

4b. All outcomes are defensible, in the sense that they are not disqualified by existing data. The performance measure calculations are made defensible by adopting distribution f3.

6. Although the time available for the "state of knowlege" quantification is quite short, other resources need not be severely limited. The difficulties inherent in similar past efforts may have been exacerbated by a perception among upper management of secondary importance, in view of other project priorities. In order for this effort to succeed, the importance of arriving at an internal concensus regarding the defensible baseline must be emphasized strongly and consistently by project management.

Note that a "state of knowlege" quantification would be required by any rational evaluation process, and is not a special burden imposed by the SP which might be avoided by adopting another process. If the DOE contemplates the suspension of any information-gathering program, regardless of how they arrive at the decision to suspend that program, they must consider the technical ramifications of that decision, i.e. what may be defensibly claimed about the process under investigation using existing 
information. If, for some program, we cannot identify a defensible position using existing information, we are in effect stating that completion of that program is essential for evaluation of compliance, regardless of outcome. Any such assertion should be strongly supported by a thorough, documented effort to understand the possible uses and limitations of existing information, but cannot be supported by a failure, due to internal conflicts, to confront the prospect of suspending the program.

\section{E) Utility-independent implementation concerns}

1. It is not possible to evaluate the usefulness of an experiment by any method unless the way the experimental results will be used is understood. It would also be neccessary to confront this problem if the experiment were conducted, whether or not it was conducted as a consequence of some evaluation process. The fact that we are required to define in advance the possible representations of the system corresponding to different experimental outcomes, even though this is a difficult process, is a strong argument in favor of SP, and the result has value independent of the formal evaluation process.

2. Threshold pressure is an example of a parameter whose large-scale value depends on the model of spatial variation, and cannot be treated by volume averaging. 
29. Lincoln, R.C. 1994. "Second Draft Performance Measures for the WIPP System Prioritization." Memo to P.A. Davis, June 27, 1994. 


\section{Sandia National Laboratories}

date: June 27,1994

Albuquerque, New Mexico 87185

to:

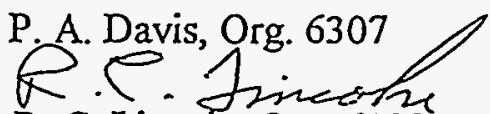

from: R. C. Lincoln, Org. 6308

subject: Second Draft Performance Measures for the WIPP System Prioritization

S. Bertram implemented a 6307 review of the draft performance measures defined in my June 20,1994, memo. After that review she asked that I modify the proposed performance measures. This memo formally transmits the second draft of the WIPP System Prioritization Performance Measures. The major differences are that 1) these performance measures are digital in nature and only indicate compliance or non-compliance, and 2) the post-closure RCRA performance measure averages hazardous constituent concentrations over a smaller region of space and includes both gas and liquid phase transport of hazardous constituents.

Attached to this memo is a draft of Performance Measures for the WIPP System Prioritization. These two performance measures are designed to indicate either compliance or non-compliance for both radionuclide and hazardous constituent post-closure regulatory compliance. The definition of these performance measures specifically excludes any consideration of operational time scale regulatory compliance requirements as a part of the system prioritization effort and also excludes from this effort undisturbed performance related to 40 CFR 191 (specifically the dose requirements of 191.15, Subpart B, and the Environmental Standards for Ground-Water Protection of Subpart C).

Please review the draft performance measures and arrange for review by $\mathrm{CAO}$ and WID. We will continue developing the system prioritization analysis using these draft performance measures. Comments received prior to July 9,1994 , will have the potential to impact the September 30,1994, analysis. It will be very difficult, if not impossible, to change the performance measures after July 9 and meet the September milestone. If the performance measures do not represent the regulatory requirement the system prioritization will result in a flawed decision matrix being the basis for potential CAO decisions. Therefore, it is critical that $\mathrm{CAO}, \mathrm{WDO}$ and EPA review the performance measures in a timely manner.

Note that implementing the post-closure RCRA performance measure may require a health based level (for soil) for any hazardous constituent identified in the WTPP inventory. This may require implementing the EPA procedure for hazardous constituents not included in 55 FR 30865 through 55 FR 30868. We need to identify what organization (other than 6308) is responsible for implementing this procedure consistent with the system prioritization schedule. 
Distribution w/1 attachment:

6300 D. E. Ellis

6303 W. D. Weart

6305 S. A. Goldstein

6306 A. L. Stevens

6305 N. H. Prindle

6307 F. T. Mendenhall

6307 S. G. Bertram

6308 W. Beyeler

6308 D. Rudeen

6141 R. C. Lincoln

6342 D. R. Anderson

6347 D. R. Schafer

6348 J. T. Holmes

6351 R. E. Thompson

6352 S. E. Sharpton

6115 P. B. Davies

6117 W. R. Wawersik

6119 E. J. Nowak

6121 J.R. Tillerson

6700 P.Brewer

6743 V. Harper-Slaboszewicz

SWCF CO/SP WBS 1.1.7.2 
Implementation of the WIPP system prioritization requires the definition of a performance measure for each regulatory requirement that will be addressed by the analysis. These definitions of performance measures assume that the regulatory requirements to be addressed by the WIPP system prioritization will be the radionuclide containment requirement of 40 CFR 191, Subpart B, section 191.13(a), and the hazardous constituent concentration requirement of 40CFR268.6 with health based levels for soil as defined in 55 FR 30865 . through 55 FR 30868.

The scope of the system prioritization effort has been defined as post-closure regulatory compliance. Based on this scope definition operational time scale RCRA requirements and 40 CFR 191, Subpart A, requirements will not be included in the analysis. The focusing of system prioritization on the post-closure radionuclide containment portion of 40 CFR 191 is based on the results of prior modeling of undisturbed performance. This modeling has indicated that radionuclide releases without human intrusion are very small. Based on these prior modeling results the system prioritization analysis will not address 40 CFR 191, Subpart B, section 191.15 (Individual protection requirements) and 40 CFR 191, Subpart C (Environmental Standards for Ground-Water Protection).

\section{RADIONUCLDE CONTAINMENT PERFORMANCE MEASURE}

Radionuclide containment regulatory compliance will be assumed when the mean CCDF meets the requirements of 40 CFR 191, Subpart B, section 191.13 (a).

\section{HAZARDOUS CONSTITUENT CONCENTRATION PERFORMANCE MEASURE}

Post-closure hazardous constituent concentration regulatory compliance will be assumed when the hazardous constituent concentration calculated at the unit boundary is less than the health based level for soil (as defined in 55 FR 30865 through 30868) for each hazardous constituent. Hazardous constituents transported to the unit boundary by gas will be compared with the health based levels. The anhydrite interbed pathway will use from the top of marker bed 138 to the bottom of marker bed 139 and gas available porosity for concentration calculations. The shaft pathway will use the model shaft diameter and the gas available porosity for concentration calculations. The focusing of system prioritization on hazardous constituent transport by the gas phase is based on the results of prior modeling of undisturbed performance. This modeling has indicated that contaminated brine does not reach the unit boundary. The hazardous constituent concentrations will be calculated from the larger of the mean or median of the multiple deterministic concentration calculations to include the effect of parameter uncertainty. 
30. Hora, S.C. 1994. "Computational Strategies for the Systems Prioritization." Draft, June 29, 1994. 
Draft -- June 29, 1994

\section{COMPUTATIONAL STRATEGIES FOR THE SYSTEMS PRIORITIZATION}

Prepared by stephen C. Hora

The system Prioritization (SP) process requires that the performance measures be associated with various potential outcomes of the activity sets. The paper discusses some issues concerning how the calculations can be performed in order to establish these relations. It will be assumed, in this discussion, that the performance measure reflects compliance with both $40 \mathrm{CFR} 191$ and RCRA. Using Richard Lincoln's notation (memorandum from R. Lincoln to P. Davis, June 20, 1994), the outcomes of the activity sets map into values $\mathrm{PM}_{\mathrm{R}}$ and $\mathrm{PM}_{\mathrm{HC}}$ and the performance indicator, $\mathrm{PI}$, would be

$$
\begin{aligned}
\mathrm{PI} & =1 \text { if } \mathrm{PM}_{\mathrm{R}}>0 \text { and } \mathrm{PM}_{\mathrm{HC}}>0 \\
& =0 \text { otherwise. }
\end{aligned}
$$

The first issue discussed here is the choice of activity outcomes to be evaluated. The notation used is as follows:

$$
\begin{aligned}
& \text { Let } A_{i} \text { be activities and } A=\left\{A_{i}, \ldots, A_{k}\right\} \text { an } \\
& \text { activity set. }
\end{aligned}
$$

Let $\theta_{i j}, j=1, \ldots, m_{i}$ be the potential outcomes associated with the activity $i$ and let $p_{i j}$ be outcomes: $\theta=\left(\theta_{i j}, \ldots, \theta_{i 1 j}\right)$ is a vector of potential outcomes of an activity set. Denote by $P(\theta)$ the probability distribution on $\theta$.

$\mathrm{PI}(\theta)$ is the performance indicator evaluated given the outcome vector $\theta$.

\section{SELECTING THE SET OF ACTIVITY OUTCOMES FOR EVALUATION}

This section presents several options for selecting the outcome vectors for evaluation.

Complete Enumeration

The first option is to evaluate every possible outcome vector of a given activity set. With $n$ activities, the number or vectors is $I_{i=1, n} m_{i}$. For example, a set of twenty activities, each having five out'comes would require the evaluation of $5^{20^{\prime}} \approx 10^{14}$ vectors. Supposing that each vector requires one second for evaluation, this would require several million years. Even at 1000 evaluations per second, several thousand years would be required. Complete enumeration is not feasible except for activity sets with very few activities. 
Sampling Methods

It is not necessary to evaluate all possible outcomes of an activity set. The outcomes $\theta$, their probabilities $P(\theta)$, and the performance measure PI $(\theta)$, give rise to a population of performance measures, each element of the population being a 0 or $a 1$. The probability of compliance is simply the mean of this population. The problem them becomes one of sampling from a population of zeroes and ones in such a way that one accounts for the differences in probabilities of the various elements.

Monte Carlo sampling:

To implement Monte carlo sampling, it is first necessary to construct a cumulative probability representation for the outcomes, $\theta$. There are two methods for constructing the Monte Carlo sample. The first method is completely general while the second method assumes independence among the outcomes.

Let $\theta_{\ell}, \ell=1, \ldots$, I be the outcome vectors placed in some arbitrary order, and let $Q_{i}=\Sigma_{\ell=1, i} P\left(\theta_{\ell}\right)$. An element in the Monte Carlo sample is.chosen by generating a uniform $[0,1]$ random deviate, say $U$, finding the smallest $\ell$ such that $U<Q_{\ell}$, and returning $P I\left(\theta_{\ell}\right)$ as the sampled value. The process is done repeatedly by drawing successive values of $U$. The probability of compliance is estimated by the mean of the sampled values.

If the outcomes are probabilistically independent, then each activity's may be sampled separately, avoiding the need to construct a table of all possible outcomes. Thus, if there are $k$ activities, $k$ uniform random variables must be drawn and the sampled outcome for each activity determined from its respective random variable using the table look-up method.

With a large number of activities, the listing the potential vectors required by the first method may be too large a task and the time required for table look-ups may be excessive without indexing schemes. Thus, the second method is to be preferred when there is independence among the outcomes or even among subsets of the outcomes.

Comments on Monte Carlo sampling:

The variance of the estimate is $V_{M C}=E[P I(\theta)]\{1-E[P I(\theta)]\} / K$ where $K$ is the size of the Monte Carlo sample.

The addition of more experiments to the activity set(s) drives $E[P I(\theta)]$ close to unity which will, counter intuitively, decrease the number of sampled vectors required to obtain a given degree of accuracy. 
The same outcome vector may be sampled more than once. Keeping track of the evaluations may help reduce the number of evaluations of PI and thus reduce the computational effort.

Latin hypercube sample (LHS):

LHS is a version of stratified sampling where the stratification is usually on cumulative probabilities. As with the case of Monte Carlo sampling, the sampling can be done on vectors (general case) or by outcomes when the outcomes are independent. If the sampling is done on vectors, then it is advantageous to order the vectors in a meaningful way so as to reduce the variance of the estimate of compliance. Ordering the vectors by their impact on releases would accomplish this goal but it would require that somehow one is able to impute a release to each vector. But this is what we want to avoid doing.

When the outcomes are independent or the dependencies can be represented through normal-score rank correlations, it is advantageous to sample the variables independently and assemble the LHS sample from the individual samples. It will be assumed that this is the case for the discussion of LHS sampling.

The stratification can be done on cumulative probabilities of the outcome or by the outcome categories themselves. The second alternative results in sampling cells of unequal probabilities. There may be some ádvantages to such an approach if some outcomes have probabilities so small that there is some probability that they will not be sampled.

When sampling is stratified on the distribution of the outcomes, the distribution is divided into $N$ categories with equal probability of $1 / \mathrm{N}$. The estimate of compliance is then

$$
\operatorname{E}[\operatorname{PI}(\theta)]=\Sigma_{i=1, H}(1 / N) \operatorname{PI}\left(\theta_{i}\right)
$$

where the $\theta_{i}$ are the vectors sampled. In contrast, when stratification is done on the outcomes, the various sampled cells in the outcome space have unequal probabilities, say $p_{i}$. The estimate of compliance becomes

$$
E[\operatorname{PI}(\theta)]=\Sigma_{i=1, N} N^{k-1} p_{i} \operatorname{PI}\left(\theta_{i}\right)
$$

where $k$ is the number of activities.

Comments of LHS sampling:

LHS sampling usually produces more efficient estimates (less variance) than Monte carlo sampling. 
The variance of an LHS is often difficult or impossible to compute. However, replicated LHS samples are independent and thus an estimate of the variance of the estimator can be obtained. For example, rather than using a sample of 100 LHs vectors, one could generate five samples of twenty vectors. The estimate of the probability of compliance would be computed as the mean of the five estimates. Errors bounds can be approximated using student's $t$ distribution.

When stratifying on discrete outcomes, it maybe desirable to sample each outcome several times knowing that each sampling is apt to result in different pairings with outer variables.

\section{EVALUATION OF PERFORMANCE MEASURES}

Each sampled outcome vector translates into a set of parameter input distributions to the performance assessment (PA) system. There are some alternative strategies for the repeated evaluation of PA model. Since evaluations are costly, there is a motivation to use efficient methods.

Evaluation using means:

Martin Tierney has proposed the evaluation of the PA model be done by passing through the mean vector of parameters conditional on the sampled outcomes. While the performance measure evaluated at the mean of the parameter distributions is not the mean of the performance measure, it does provide a first order approximation. Each sampled outcome vector would then translate into a set of parameter means and one deterministic evaluation of the PA model would be done for each outcome vector.

Evaluation using LHS reweighted samples

Suppose that the PA system has been evaluated using LHS and the defensible baseline distributions or some other set of distributions. Each sampled cell in the evaluation has a probability $p_{i}$ in the initial evaluation. Using the new input distributions associated with an outcome vector $\theta$, the cell probabilities are recalculated. Denote the recalculated cell probabilities by $q_{i}$. Then the LHS estimate of compliance is calculated as

$$
E[P I(\theta)]=\Sigma_{i=1, N} q_{i} \operatorname{PI}\left(\theta_{i}\right) /\left(\Sigma_{j=1, N} q_{j}\right) \text {. }
$$

This process avoids the new evaluations of the PA model. 
Comments on reweighted LHS samples:

This process does not accommodate resampling from distributions with dependencies represented through correlations. Deterministic dependencies (complete dependence) can be represented, however,

It is not clear how the reweighting is done with discrete input distributions and stratification on the distribution function.

The more equally the probability is spread across cells, the smaller the variance of the estimate of compliance is thought to be. Reweighting, however, can lead to very different probabilities across cells and thus reduce the efficiency of the estimate. It will most likely occur that some vectors are given zero weight in the reweighting process. This is not a problem as long as there are sufficient remaining vectors to estimate release probabilities. But one cannot be assured that this is the case. with a large number of variables and some experimental outcomes that produce relatively sharp parameter distributions, a large number of vectors may be trimmed.

Evaluation using nearest neighbors

Again suppose that the PA system has been evaluated using LHS or some other sampling method. For a given outcome vector, a sample of input parameters is developed. Rather than passing these input parameters through"the PA model, the sample is associated with the evaluated sample that it most closely resembles. The output from the evaluated sample is then associated with the new vector of input parameters as though the model had been evaluated. Because the dimensionality of the input variable space is large (forty-nine dimensions in the $1992 \mathrm{PA}$ ), a large library of vectors would be needed to insure results that are sufficiently close.

The nearest neighbor approach requires a metric to measure the distance between vectors. Such a measure should be invariant to scale changes for obvious reasons.

\section{RECOMMENDATIONS}

\section{Experiment outcomes}

The experiment outcomes should be sampled using LHS. The sampling scheme should be partially stratified on outcomes to ensure that each outcome is sample at least once or $m$ times and partially stratified on probabilities to retain as much efficiency as possible by sampling the most relevant part of the outcome space. Several LHS samples should be constructed so that an error estimate for the probability of demonstrating compliance can be developed. 
PA Model Evaluation

Reweighting LHS parameter vectors provides an computationally efficient method of evaluating the distribution of releases given a particular set of experiments and outcomes. Consideration should be given to constructing an initial set of vectors such that the parameter space is well covered. It is suggested that the predictive distribution of each parameter be sampled. The predictive distribution is constructed from the outcomes $\theta_{1}, \ldots, \theta_{m}$, the probability distribution of the outcomes $p\left(\theta_{i}\right)$, and the conditional parameter distributions $F\left(\tau \mid \theta_{i}\right)$ and is given by:

$$
F(\tau)=\Sigma_{i=1, m} F\left(\tau \mid \theta_{i}\right) p\left(\theta_{i}\right)
$$

These distributions should be sampled be stratifying the distribution function into equal probability intervals.

As in the case of sampling the experiment outcomes, the parameter sample should be replicated. The variance of the estimate of $\mu=E(P I)$, the probability of demonstrating compliance can be calculated using the decomposition of the variance into the expectation of the conditional variance and the variance of the conditional expectation. Denoting the parameter samples by $S$ and the outcome samples by $\theta, \operatorname{Var}(\mu)=\mathrm{E}_{\theta} \operatorname{Var}_{\mathrm{s} \mid \theta}(\mu)+\operatorname{Var}_{\theta} \mathrm{E}_{\mathrm{s} \mid \theta}(\mu)$.

If it occurs that the reweighting scheme becomes infeasible because the number of vectors assigned zero probability is very large, then the nearest neighbor approach should be implemented. It is important that we determine, as soon as possible, whether the reweighting scheme will work.

\section{AN EXAMPLE WITH LHS SAMPLING OF OUTCOMES AND A REWEIGHTED IHS SAMPLE OF PARAMETERS}

Consider a very elementary $P A$ model where releases, $R$, are related to three variables, say solubility $\left(x_{1}\right)$, permeability $\left(x_{2}\right)$, and source term $\left(x_{3}\right)$. The PA model is $R=x_{1} x_{2} x_{3}$. Compliance is shown only when $P(R<1)>.9$. For simplicity, the baseline distributions of the variables are assumed to be lognormal all with parameters $\mu=-.4$ and $\sigma=1.0$.

Experiments are available for $\mathrm{x}_{1}, \mathrm{x}_{2}$, and $\mathrm{x}_{3}$. The characteristics of the experiments are shown in the following table: 
Draft -- June 29, 1994

TABLE 1

Experiment outcomes and Parameter Distributions

\begin{tabular}{||l|l|l|l||}
\hline Variable & Outcome & Probability & $\begin{array}{l}\text { Parameter } \\
\text { Distribution }\end{array}$ \\
\hline \hline$x_{1}$ & A & $1 / 2$ & Lognormal $(-.4, .5)$ \\
\hline & B & $1 / 3$ & Lognormal $(-.6, .5)$ \\
\hline \hline$x_{2}$ & C & $1 / 6$ & Lognormal $(-.8, .5)$ \\
\hline & D & $1 / 3$ & Lognormal $(-.4, .5)$ \\
\hline & E & $1 / 3$ & Lognormal $(-.8, .5)$ \\
\hline \hline$x_{3}$ & F & $1 / 3$ & Lognormal $(-1.0, .5)$ \\
\hline & D & $1 / 4$ & Lognormal $(-.4, .5)$ \\
\hline & E & $1 / 2$ & Lognormal $(-1.0, .5)$ \\
\hline & F & $1 / 4$ & Lognormal $(-1.5, .5)$ \\
\hline
\end{tabular}

Using the baseline distributions and a lHs sample size of ten with equal probability intervals, the following input vectors are obtained:

Table 2

LHS Vectors and Releases

Baseline Distributions

\begin{tabular}{||l|l|l|l|l||}
\hline Vector & $x_{1}$ & $x_{2}$ & $x_{3}$ & $R$ \\
\hline$I$ & $.163(1)$ & $1.222(8)$ & $.588(5)$ & .117 \\
\hline 2 & $.283(2)$ & $.715(6)$ & $.195(2)$ & .039 \\
\hline 3 & $.349(3)$ & $.099(1)$ & $.467(4)$ & .016 \\
\hline 4 & $.435(4)$ & $2.068(9)$ & $.139(1)$ & .125 \\
\hline 5 & $.660(5)$ & $1.044(7)$ & $.714(6)$ & .492 \\
\hline 6 & $.686(6)$ & $3.142(10)$ & $1.649(9)$ & 3.552 \\
\hline 7 & $1.035(7)$ & $.202(2)$ & $2.661(10)$ & .556 \\
\hline 8 & $1.132(8)$ & $.383(3)$ & $.376(3)$ & .163 \\
\hline 9 & $2.387(9)$ & $.467(4)$ & $1.038(7)$ & 1.158 \\
\hline 10 & $9.508(10)$ & $.562(5)$ & $1.291(8)$ & 6.899 \\
\hline
\end{tabular}

The numbers shown in parentheses in Table 2 are the LHS intervals from which the associated value was drawn. The ten intervals are 
$[0, .186),[.186, .289),[.289, .397),[.397, .520),[.520, .670)$, $[.670, .864),[.864,1.132),[1.132,1.555),[1.555,2.415),[2.415, \infty)$. These interval limits are needed in the reweighting of vectors conditional on the experimental outcomes.

Table 2 also shows the release, $R$, associated with each vector. Seven of the ten vectors show $R<1$. Since the vectors are initially equally likely, the estimate of the probability of $R<1$ is $7 / 10$. The analytically derived probability of demonstrating compliance is .756.

Next, a LHs sample of experiment outcomes is drawn. In this demonstration we have chosen to stratify on the outcomes, selecting each of the outcomes three times so that a total of nine LHS vectors are drawn. The IHS sample of outcomes and their probabilities normalized to sum to one are given in Table 2 .

TABLE 3

IHS Sample of Outcomes and Normalized Probabilities

\begin{tabular}{||l|l|l|l|l||}
\hline $\begin{array}{l}\text { Outcome } \\
\text { Vector }\end{array}$ & $\mathrm{x}_{1}$ outcome & $\mathrm{x}_{2}$ outcome & $\mathrm{x}_{3}$ outcome & Probability \\
\hline 1 & $\mathrm{~A}$ & $\mathrm{D}$ & $\mathrm{G}$ & .12 \\
\hline 2 & $\mathrm{~A}$ & $\mathrm{~F}$ & $\mathrm{I}$ & .12 \\
\hline 3 & $\mathrm{~A}$ & $\mathrm{E}$ & $\mathrm{H}$ & .24 \\
\hline 4 & $\mathrm{~B}$ & $\mathrm{E}$ & $\mathrm{H}$ & .16 \\
\hline 5 & $\mathrm{~B}$ & $\mathrm{D}$ & $\mathrm{H}$ & .16 \\
\hline 6 & $\mathrm{~B}$ & $\mathrm{~F}$ & $\mathrm{I}$ & .08 \\
\hline 7 & $\mathrm{C}$ & $\mathrm{F}$ & $\mathrm{G}$ & .04 \\
\hline 8 & $\mathrm{C}$ & $\mathrm{E}$ & $\mathrm{G}$ & .04 \\
\hline 9 & $\mathrm{C}$ & $\mathrm{D}$ & $\mathrm{I}$ & .04 \\
\hline
\end{tabular}

Vector 5 ( $B, D, H)$ has been chosen to demonstrate the reweighting process. The first step in the reweighting process is to use the parameter distributions for $x_{1}, x_{2}$ and $x_{3}$ conditional on the experiment outcomes $B, D$, and $H$, to find the marginal probabilities of the LHS sampling intervals. These probabilities are shown in Table 4 . 
Draft -- June 29, 1994

Table 4

Reweighted Interval probabilities

\begin{tabular}{||l|l|l|l||}
\hline Interval & $\begin{array}{l}x_{1} \text { Probability } \\
\text { After B }\end{array}$ & $\begin{array}{l}x_{2} \text { Probability } \\
\text { After D }\end{array}$ & $\begin{array}{l}\text { x Probability } \\
\text { After H }\end{array}$ \\
\hline 1 & .015 & .005 & .086 \\
\hline 2 & .085 & .041 & .229 \\
\hline 3 & .158 & .101 & .245 \\
\hline 4 & .199 & .159 & .196 \\
\hline 5 & .198 & .194 & .129 \\
\hline 6 & .163 & .194 & .071 \\
\hline 7 & .108 & .159 & .038 \\
\hline 8 & .055 & .101 & .004 \\
\hline 9 & .018 & .041 & .002 \\
\hline 10 & .001 & .005 & .000 \\
\hline
\end{tabular}

Once the intervals are reweighted, the new probabilities are associated with the correct vectors. For example, the first vector was created by drawing $x_{1}$ from the first interval, $x_{2}$ from the eighth interval, and $x_{3}$ from the fifth interval as shown in Table 2. The correct interval probabilities are then multiplied and normalized to obtain the reweighted vector probabilities shown in the last column of Table 5 . 
Table 5

Reweighted Vector Probabilities

\begin{tabular}{||l|l|l|l|l|l||}
\hline \multirow{2}{*}{ Vector } & \multicolumn{2}{l}{ Interval } & \multicolumn{2}{l|}{ Probabilities } & \multicolumn{2}{l|}{ Vector Probabilities } \\
\cline { 2 - 6 } & $\mathrm{x}_{1}$ & $\mathrm{x}_{2}$ & $\mathrm{x}_{3}$ & Unnormalized & Normalized \\
\hline \hline 1 & .015 & .101 & .129 & .000195 & .023 \\
\hline 2 & .085 & .194 & .229 & .003776 & .442 \\
\hline 3 & .158 & .005 & .196 & .000155 & .018 \\
\hline 4 & .199 & .041 & .086 & .000702 & .082 \\
\hline 5 & .198 & .159 & .071 & .002235 & .262 \\
\hline 6 & .163 & .005 & .002 & .000002 & .000 \\
\hline 7 & .108 & .041 & .000 & .000000 & .000 \\
\hline 8 & .055 & .101 & .245 & .001361 & .159 \\
\hline 9 & .018 & .159 & .038 & .000109 & .013 \\
\hline 10 & .001 & .004 & .004 & .000001 & .000 \\
\hline
\end{tabular}

Remembering that all vectors except $6,9,10$ result in releases less than unity, one can calculate $P(R<1)=.987$ posterior to observing the outcomes $B, D, H$. (The analytic probability is .989.) Thus, this set of experiment outcomes results in compliance being shown and thus $P I(B, D, H)=1$. The process is repeated for each vector of experiment outcomes, determining whether or not compliance will be shown. The probability of demonstrating compliance is then estimated by taking the expectation of PI with respect to the probabilities of the experiment outcomes. 
31. Mendenhall, F.T. 1994. "Prototype System Prioritization Baseline." Memo to R.C. Lincoln, July 1, 1994. 


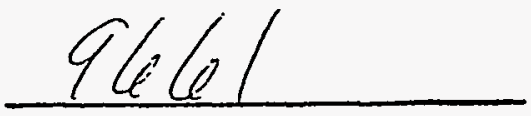

date: July 1, 1994

to: R.C. Lincoln, acting 6341

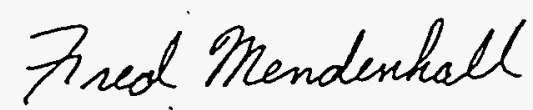

from: F.T. Mendenhall, 6308

subject: Prototype System Prioritization Baseline
INFORMATION ONLY

Sandia National Laboratories

Albuquerque, New Mexico $87185 \cdot 1341$

\section{Prototype SP Baseline}

This document defines, at a conceptual level, the models for the first iteration of the 191B and RCRA systems prioritization baseline calculations. Systems Prioritization (SP) is-concerned with identifying activities that will lead the WIPP project to a timely and cost-effective regulatory compliance application. Because the output of SP is used to define program direction, and is not part of the compliance application, its computer programs and data sets are not required to be at the same level of quality as the compliance application. SP does not deal with any operational period concerns that may be required by the regulator to demonstrate compliance. However, the modeling used may need to take into account physical and chemical conditions and processes active during the operational phase in order to predict long term performance measures. Issues or models not specifically discussed in this document are assumed to be the same as used in the $12 / 92$ PA effort. We believe that this document and the associated performance measures [Lincoln 1994] are sufficient to initiate the calculation of the SP baseline performance analysis and to request that the analysis be completed expeditiously. Any deviation from the baseline defined here needs to be documented and brought to our attention as soon as the deviation is recognized.

While we believe this baseline to be reasonable and technically defensible, it:

1) Did not include sufficient SNL staff, WID, DOE, regulator or stakeholder involvement to represent a full range of concerns.

2) Assumes appropriate personnel are available to do both baseline calculations and the identified side calculations as well as meet other important compliance commitments such as moving the codes to QA level A.

3) Contains buried assumptions that presuppose the completion of certain activities, e.g. the assumption of Darcian flow in the Salado may require the completion of some subset of Salado experiments in order to defend the Darcy assumption.

While these buried assumptions are important and will be identified in the project white papers 
used as input for the stakeholder interactions, the need to identify and discuss these assumptions in this document is secondary to the need to initiate the SP process.

\section{Culebra}

The SP baseline will use the flow model used in the 12/92 PA with no sorption and no dual porosity transport in the Culebra. Specifically, there will be no porosity in the dolomite matrix and no clay fracture linings. (Both of these assumptions are the focus of activities and experimental work that may lead to a different set of SP models for the activity sets.) The 100 calibrated Tfields, as prepared for the 12/94 PA calculation, will be used, as required, for the regional flow. In addition two specific assumptions remain as they were in $12 / 92$ :

1) The Culebra will be modeled as a $2 \mathrm{D}$ confined aquifer (no leakage) both because results from the $3 \mathrm{D}$ regional flow transport model will not be available in time for the prototype $S P$ and because we believe that the $2 \mathrm{D}$ confined approach is conservative regarding releases to the site boundary. The $3 \mathrm{D}$ model is expected to confirm or refute this assumption as one of its objectives.

2) Colloids are assumed to be transported at the same rate as dissolved mobilized actinides, i.e., they simply travel with the flow field. This assumption could be made absolutely conservative for the baseline by assuming that colloids would preferentially travel in the middle of the flow stream and hence travel at a rate of 1.2 time the mean flow velocity, however, the uncertainty in the flow velocities tend to be so large as to make this multiplication meaningless. Also, the source term model for the baseline, described below, does not resolve the colloidal forms from the dissolved forms of actinides.

\section{Stakeholder Comments:}

Do not model sorption or dual porosity until you can show that they are appropriate. Clearly we are addressing this concern with the baseline model.

Regional flow and transport may be affected by climate change and you should assume things are going to be wetter than what you are modeling. To deal with this issue we will raise the heads in the "recharge strip" to the land surface and keep them there for the duration of the analysis. This should bound any effects of climate change within the confined model.

\section{Salado}

Flow in the Salado will be modeled as planned for the intended 1994 PA calculation. This includes the 12/92 PA Salado model with the additions of a pressure-sensitive altered anhydrite 
model to estimate fracture behavior. However, if at any time the pressures within the repository reach lithostatic or above in the simulation, it will be assumed that the gas phase in the repository is directly connected to the markerbeds at the regulatory boundary, i.e. that the gas concentrations and species present in the repository will be the same gas concentrations and species filling the void space in the anhydrite just outside the regulatory boundary. This assumption bounds the expected behavior caused by a very thin crack that might be created by the over-pressurization of the repository and readily allow gas transport to the boundary. Liquid transport, however, is not expected to reach the boundary because it is able to migrate into the halite. Therefore, the twophase flow of the brine out of the room, if it occurs, is expected to be adequately modeled by BRAGFLO.

\section{Stakeholders Comments:}

Consider gas solubility in brine. For the case where gas dissolves in brine, gas transport is by far the faster mechanism and hence not allowing gas to dissolve in brine is conservative. If the concern is gases like nitrogen coming out of the brine into the room, then as the room pressure rises and approaches hydrostatic, most if not all of those gases will be redissolved in the brine. Hence for the purposes of the SP analysis we will assume that gas simply remains gas and does not go into solution with the brine. We believe this assumption to be consenvative.

What about vertical fracturing in the Salado due to gas over pressurization? Currently we do not expect vertical fractures to exist in the Salado. An investigation has been started to see if internal pressurization can create fractures that will leave the interbeds and climb toward the surface and or turn vertical. We hope to have early results from this study to present at stakeholder meetings and to inchde in subsequent SP iterations if deemed appropriate. However, we also note that because of the way we propose to model transport in the Salado, it does not matter if the gas reaches the regulatory boundary at the side or at the top of the regulated unit; once lithostatic pressure is reached we are assuming that disposal room gas is transporled outside of the regulated whit, therefore, we feel vertical fracturing is no worse than horizon fracturing for the baseline.

\section{Inventory}

The baseline inventory report (BIR) is due out 6/30/94 and is expected to have the required information to update the $12 / 92$ PA waste inventory parameters. This report is also to contain error estimates on the inventory. This report should be used to define the waste data set used in the prototype baseline SP analysis. The waste and backfill should be parameterized the same way they were in 12/92 PA, i.e., homogenous, same parameters, etc..

Pige i 


\section{Stakeholder Comments:}

What is the VOC source term? For the SP process we will use the best inventory information available to CAO (June 1997 BIR). Also we would note that for the baseline, by not taking credit for any VOC retardation or degradation, we believe we are being very conservative.

You should assume bounding waste distribution. DOE is currently initiating an effort to define a bounding waste envelope and a Performance Based Waste Acceptance Criteria (PB-WAC). However, for the purpose of the prototype SP the information from the June 1994 BIR will be used to define the waste characteristics.

\section{Repository Near Field}

For the baseline, room closure occurs according to the 1992 porosity surface until gas pressure reaches lithostatic. Thereafter, the room porosity and permeability remains'constant. We would note that the constraint of the room porosity and permeability remaining constant after lithostatic pressure is achieved should automatically result if anhydrite fracturing occurs at lithostatic pressure.

We feel that the reaction path gas generation model is the defendable gas generation model, however, it is not ready to be included in the SP calculations. Therefore, the baseline will use the gas generation model as was defined by Brush for 94 PA. Specifically this is the 92PA gas generation model that has been updated with rate information documented in the June 18, 1993 memorandum of record from L.H. Brush to M.S. Tierney titled, " Likely Gas-Generation Reactions and Current Estimates of Gas-Generation Rates for the Long-Term Performance Assessment." In addition, the baseline will assume that all plastic and rubber material are available for degradation reactions. Furthermore, we will do a side calculation comparing the gas generation results of this SP baseline model against the reaction path model to gain insight on how the reaction path model may impact the performance measures.

The issue of repository and/or anhydrite dip has been raised. For the purpose of the prototype SP, dip will be modeled in a side calculation for both a dipped repository and dipped anhydrite to estimate its impact on performance measures both for human intrusion and for Salado flow. If these side calculations show important results, the methodology will be available to incorporate in the next $S P$ iteration.

The human intrusion model will include cuttings, cavings and for the first time a simplified spalling model as will be defined by Berglund and Butcher for use in SP. Note that for flow calculations it is acceptable to assume that the waste permeability remains constant at $10^{-12} \mathrm{~m}^{2}$, however, for the cavings, cuttings, and spalling calculations the waste permeability should be sampled as a uniform distribution over the range of $10^{-12}$ to $10^{-17} \mathrm{~m}^{2}$.

Page 4 
The DRZ will be modeled as it was in the 12/92 PA. Specifically the halite regions of the DRZ will be treated as having far-field halite properties for porosity but having an enhanced permeability, i.e., $k=10^{-15} \mathrm{~m}^{2}$, so that it does not act as a significant barrier to fluid flow.

The concrete elements for all repository and panel seals will be assumed to have a permeability of from $10^{-12}$ to $10^{-14} \mathrm{~m}^{2}$ for the duration of the analysis. This value is indicative of silty sand and is the best that can be defended until it is demonstrated that large WIPP concrete sealing elements can be manufactured without fracturing the concrete. Once fractured, it is not expected that the fractures will heal. The repository seals contain 120 feet of concrete to be modeled either as a continuous column or as 3 discrete elements according to the seal design. The salt columns are to be modeled having a permeability of two orders of magnitude less than the concrete elements for the first hundred years. Then the salt columns begin to transition from their initial permeability to a permeability range from $10^{-17}$ to $10^{-19} \mathrm{~m}^{2}$ in a column of salt 100 meters long by the end of their second hundred years. If a transition is difficult to model it is acceptable to have a step transition at the end of two hundred years. It should be noted that the panel seals do not have halite elements. However, backfill is emplaced near the panel seals that may consolidate and act as a sealing member. This backfill will not be modeled separately in the baseline SP.

For actinide source term and colloid transport, we will assume that the total actinide inventory will be turned either into dissolved or colloid-mobilized forms. [ Note the implication for colloid $\mathrm{K}_{d} \mathrm{~s}$ for Culebra transport in activity sets.] These assumptions are based on: a) the complete lack of information concerning the crystallinity of actinide-bearing materials in the WIPP waste; 2) The anticipated local variability of Eh and $\mathrm{pH}$ conditions within the waste/backfill; and 3) the lack of agreement concerning whether the data pertaining to actinide solubilities in fresh water are applicable and/or bounding .

For the baseline the initial brine saturation of the waste has been defined by Butcher and Vaughn as Min.=0.04 Median=0.44 and Max=5.2 percent.

\section{Stakeholder Comments:}

What is the VOC source term? For the SP process we will use the best inventory information available to CAO (.June 199٪ BIR).

Use a VOC degradation model. Unil defensible data are available SP intends to assume no degradation of the VOCs as a bounding case. However, VOC degradation will be modeled as an activity set to evaluate its potential impact on the performance measures.

You should model the impacts of Nuclear Criticality. This is an inportant issue that needs to be studied, but it is best studied as a side issue not as part of the SP modeling at this time.

You should assume early repository flooding due to poor seal performance, i.e., the concrete plugs crack and do not perform as effective initial barriers to Culebra flow. The range of seal

\section{Page 5}


parameters selected for the SP baseline calculation reflects our concern in defending highperformance seals until they are demonstrated. The amount of Culebra inflow to the repository will be calculated.

\section{Human Intrusion}

\section{Stakeholder Comments:}

Assume a brine slurry for room contents. We do not see how the total room contents can turn into a brine slurry. We would like the opportunity to discuss this with stakeholders before putting it into systems prioritization. Therefore it will not be in the prototype analysis.

Calculate releases during the drilling operation prior toplug emplacement as well as after plug emplacement. We intend to do this either as part of the prototype baseline calculations or as a separate side calculation.

Allow human intrusion borehole plugs to degrade with time. For the prototype baseline, we expect to treat the plugs with subjective uncertainty and sample to create a time-independent value for the plugs. The sample range will be the same as used for degraded plug properties in the I2/92 PA. Again a side calculation will be done to evaluate if this assumption is conservative over the transient response of a time-degrading plug.

Calculate effects of drill holes near but not through the repository. We believe that this was done for the 1991 PA calculations in response to an EEG comment. At that time we believe we showed using single-phase flow models that a near miss had very little effect on the CCDF. We wish to discuss the need to due further calculations with stakeholders before including it in the SP baseline.

Model the impacts of subsidence due to potash mining. We intend to use the $3 D$ modeling program to understand this issue well enough to decide how to incorporate it into the SP baseline, therefore, it will not be part of the first SP.

Use a borehole intrusion rate of 30 boreholes $/ \mathrm{km}^{2}$ for 10,000 years. For the purposes of the SP baseline we will assume an intrusion rate of 30 boreholes $/ \mathrm{km}$ for 10,000 years. Furthermore, for the SP baseline we will assume that the sampling will be random in time and that the first human intrusion can occur as early as 101 years after closure. Note that this does not mean 30 boreholes will penetrate the WIPP in 10.000 years because the projected area of the WIPP is less than a square kilometer.

Don't assume markers and institutional controls work during and after the first 100 years? For the purposes of the SP baseline we will assume no credit for institutional controls or markers after the first 100 years. The EPA clearly allows credit for institutional controls for the first 
100 years and the baseline SP will follow this regulatory approach. However it may be appropriate to define an activity set that elicits guidance from the EPA regarding the appropriate level of credit that may be taken for markers

Reference:

R. C. Lincoln, Memorandum of Record to P.A. Davis, June 27. 1994. " Second Draft Performance Measures for the WIPP Systems Prioritization."

Distribution:

6307 Staff

6308 Staff

6341 Staff

6342 Staff

6303 W.D. Weart

6305 S.A. Goldstein

SWCF CO/SP WBS 1.1.7.2 
32. Mendenhall, F.T., N.H. Prindle, and W. Beyeler. 1994. "Systems Prioritization Prototype BRAGFLO Baseline and Activity Set Parameter Ranges and Parameter Distributions." Memo to R.C. Lincoln, July 15, 1994. 


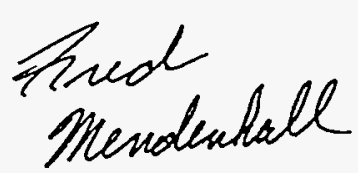

from: F.T. Mendenhall, 6308/MS1341

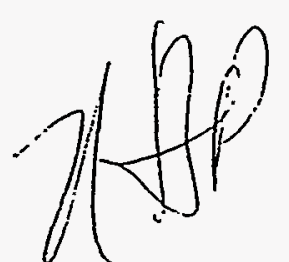

N.H.Prindle, 6308/MS1341

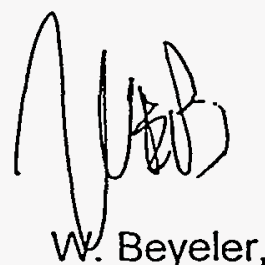

6308/MS1341

subject: Systems Prioritization Prototype Bragflo-Baseline and Activity Set Parameter Ranges and Parameter Distributions.

In order to facilitate the initiation of the Bragflo calculations for the Systems Prioritization prototype calculations, referred to as SP-1, we have given priority to identifying the activities, parameters, parameter ranges and distributions, that are required to perform the SP-1 Bragflo analysis. Following is a list of these parameters, ranges and distributions for the SP-1 Baseline. In addition, we have identified code capabilities and parameter ranges we expect to be required from the activity sets that may impact the Bragflo analysis. While we have primarily examined the seals and Salado activities, we also expect that Engineering Enhancements (EE) may require additional Bragflo capabilities not identified in this document. The issue of $E E$ is presently being worked. We request that you do not wait for further definition of $E E$ activities to begin modeling and calculating the SP-1 baseline. It is most important to begin the SP-1 baseline calculations now.

The process used in determining these listings was :

Step 1. List the parameters essential for Performance Assessment modelling (with respect to the Bragflo runs) of the Activity Sets and SP Baseline for both disturbed and undisturbed performance measures.

Step 2. Do a first-pass screen of listed parameters based on the ' 91 and '92 sensitivity studies for identifying the most influential parameters: Based on the assumptions made for the SP Baseline and the Activity Sets, evaluate whether the parameter is likely to continue to have the same degree of influence. The parameter is chosen for sampling by SP if it is judged likely, under SP Baseline and Activity Set assumptions, to continue to be of first or second order influence on the PA results. Note that this is a relatively subjective call made by the authors under a tight time constraint.

Step 3. Add all new parameters that are likely to influence PA from here-to-fore unconsidered scenarios or conceptual models that are now being considered by SP. 


\section{Baseline Variables}

A) Culebra

No Parameters are sampled.

B) Salado

\begin{tabular}{|c|c|c|c|c|}
\hline Parameter & \multicolumn{2}{|c|}{$\begin{array}{l}\text { Level of } \quad \text { Description } \\
\text { Importance }\end{array}$} & Why Included & $\begin{array}{l}\text { Range \& } \\
\text { Distribution }\end{array}$ \\
\hline MBPERM & 1 & Anhydrite Perm & Identified 12/92 & Use $12 / 92$ \\
\hline BCFLG & 2 & $\begin{array}{l}\text { Switch between Brooks Corey } \\
\text { and Parker Van Genuchten }\end{array}$ & Identified 12/92 & $50 \%$ each \\
\hline MBPOR & 2 & Anhydrite Porosity & Identified 12/92 & $\begin{array}{l}\text { Fix at bottom } \\
\text { of } 12 / 92 \text { Dist. }\end{array}$ \\
\hline SALPERM & 2 & Halite Perm & Identified 12/92 & use $12 / 92$ \\
\hline C) Inventory & & & & \\
\hline Parameter & $\begin{array}{l}\text { Level } \\
\text { Impo }\end{array}$ & $\begin{array}{l}\text { of } \\
\text { tance }\end{array}$ & Why Included & $\begin{array}{l}\text { Range \& } \\
\text { Distribution }\end{array}$ \\
\hline VMETAL & 2 & $\begin{array}{l}\text { Initial Vol Fraction of Metals } \\
\text { Glass in Waste }\end{array}$ & Identified 12/92 & use BIR \\
\hline WWOOD & 2 & $\begin{array}{l}\text { Initial Vol Fraction of } \\
\text { combustibles in Waste }\end{array}$ & Identified 12/92 & use BIR \\
\hline VPLAS & TBD & $\begin{array}{l}\text { New - Plastic and Rubber } \\
\text { in Waste }\end{array}$ & $\begin{array}{l}\text { SP Baseline allows } \\
\text { All Plastic and } \\
\text { Rubber to degrade }\end{array}$ & use BIR \\
\hline
\end{tabular}

[NOTE: We will need a RCRA Source term, Defined by the BIR as well for these Calculations] 
D) Near Field

\begin{tabular}{|c|c|c|c|c|}
\hline Parameter & $\begin{array}{l}\text { Leve } \\
\text { Impo }\end{array}$ & Description & Why Included & $\begin{array}{l}\text { Range \& } \\
\text { Distribution }\end{array}$ \\
\hline $\begin{array}{l}\text { SEALPERM } \\
\text { SEALPERM } 1\end{array}$ & $\begin{array}{ll}2 & 1 \\
1 & \end{array}$ & $\begin{array}{l}\text { Main Shaft Seal Permeability } \\
0-200 \& 200-10000 \text { Yrs }\end{array}$ & Identified 12/92 & $\begin{array}{l}\text { Use Baseline } \\
\text { Memo. }\end{array}$ \\
\hline BRSAT & 1 & $\begin{array}{l}\text { Initial Brine Contents } \\
\text { of Room }\end{array}$ & Identified 12/92 & $\begin{array}{l}\text { Use Baseline } \\
\text { Memo. }\end{array}$ \\
\hline ??? & TBD & Waste Permeability & $\begin{array}{l}\text { New Spalling } \\
\text { Model }\end{array}$ & $\begin{array}{l}\text { Use Baseline } \\
\text { Memo. }\end{array}$ \\
\hline STOICMIC & 1 & Bio Degrade Coef & Identified 12/92 & $\begin{array}{l}\text { Fix at the } \\
\text { Expected } \\
12 / 92 \text { value }\end{array}$ \\
\hline GRCORI & 1 & Corrosion GG Rate Inundated & Identified 12/92 & $\begin{array}{l}\text { Use Brush } \\
6 / 93 \text { Memo }\end{array}$ \\
\hline GRMICl & 1 & Bio Degrade GG Rate Inundated & Identified $12 / 92$ & $\begin{array}{l}\text { Use Brush } \\
6 / 93 \text { Memo }\end{array}$ \\
\hline GRCORHF & 2 & Corrosion GG Rates Humid & Identified 12/92 & $\begin{array}{l}\text { Use Brush } \\
6 / 93 \text { Memo }\end{array}$ \\
\hline GRMICHF & 2 & Bio Degrade GG Rates Humid & Identified 12/92 & $\begin{array}{l}\text { Use Brush } \\
6 / 93 \text { Memo }\end{array}$ \\
\hline STOICCOR & 2 & Corrosion Coef & Identified 12/92 & $\begin{array}{l}\text { Fix at the } \\
\text { expected } \\
12 / 92 \text { value }\end{array}$ \\
\hline TZPORF & 2 & $\begin{array}{l}\text { Transition Zone Disturbed } \\
\text { Porosity }\end{array}$ & $\begin{array}{l}\text { Bound in } \\
\text { Baseline Memo }\end{array}$ & $\begin{array}{l}\text { Do } r \\
\text { sample }\end{array}$ \\
\hline BHPERM & 1 & Borehole Permeability & Identified 12/91 & Use $12 / 91$ \\
\hline DBDIAM & 1 & Drill Bit Diameter & Identified 12/91 & Use $12 / 91$ \\
\hline BPPRES & 2 & Brine Pocket Pressure & Identified 12/91 & $\begin{array}{l}\text { Fix at } \\
\text { Lithostatic }\end{array}$ \\
\hline
\end{tabular}


In addition to above parameters, we recommend that the following values be used for the specified code variables for the baseline analysis:

$\begin{array}{ccccc}\text { Parameter } & 1991 & 1992 & \text { Description } & \text { Bounding Values } \\ \text { Name } & \text { Vol } 3 & \text { Vol } 3 & & \\ & \text { Ref. } & \text { Ref. } & & \end{array}$

\begin{tabular}{lc}
\hline \hline BKFLPOR & $3.14(?)$ Backfill, drift, lower shaft poresity Use 95\% Salado Density \\
DSEALPRM & 3.14 Panel and drift seal permeability Defined in Baseline
\end{tabular}

2.352 .41 Porosity of undisturbed halite 0.001 from 1992 Vol3, pp3-41,2

2.37 2.43 Porosity of disturbed halite Defined as DRZ in Baseline

2.602 .61 Permeability of disturbed $\quad 1 E-15 \mathrm{~m}^{2}, 1992 \mathrm{Vol} 3, \mathrm{p} 2-61$ anhydrite

2.642 .67 Porosity of disturbed anhydrite Use the Value for Undisturbed Halite

3.22 Consolidation thickness in lower Defined in Baseline shaft

3.26a 3.14 Initial permeability of drift backfill

Same as initial Salt Elements for Seals- Defined in Baseline

$3.26 \mathrm{~b} \quad 3.14$ Final permeability of drift backfill $10^{-14} \mathrm{~m}^{2}$

3.28a 3.14 Permeability of intact concrete Defined in Baseline

3.120 3.57 Threshold pressure of waste Per '92 PA

3.121a 3.57 Residual brine saturation of waste Per '92 PA

$3.121 \mathrm{~b} \quad 3.57$ Residual gas saturation of waste Per'92 PA $\begin{array}{lll}3.122 & 3.57 & \begin{array}{l}\text { Brooks-Corey pore-size } \\ \text { distribution parameter for waste }\end{array}\end{array}$ Per '92 PA 

$3.126 \quad 3.57$ Absolute roughness of waste
Per'92 PA
3.128
3.57 Effective shear strength for erosion of unmodified waste
Per'92 PA
3.149
3.57(?) Effective shear strength for erosion of salt-packed waste
Per'92 PA
$3.150 \mathrm{~b}$
3.57(?) Porosity of consolidated
Defined in Baseline room

\section{Other Capabilities:}

In reviewing the activity sets from the Seals, Salado, Culebra, Solubility, Spalling, EE, and PB-WAC activities, we have identified the following additional code capabilities that would be advantageous to have in the Bragflo models. Please understand that we are not at this time defining how these activities need to be sampled for SP, the process for sampling on activity sets will be defined shortly. In the meantime, the definition of the baseline and additional code abilities should be sufficient to initiate the calculations for the baseline with confidence that the modeling design will have sufficient capability for the vast majority of SP-1 Bragflo runs.

The main repository seals need to be modeled over the range of permeabilities from $10^{-12}$ to $10^{-19} \mathrm{~m}^{2}$ and seal length of 300 to 500 feet.

For the Salado we expect to need to be able to:

1) Model complete analysis using either Brooks Corey or Parker Van Genuchten two phase flow relationships.

2) The ability to sample on the following parameters and ranges.

$\begin{array}{llrr}\text { Parameter } & \text { Description } & \text { Why Included } & \begin{array}{c}\text { Range \& } \\ \text { Distribution }\end{array} \\ \text { PHIMAX } & \begin{array}{l}\text { Altered Anhydrite } \\ \text { Porosity }\end{array} & \text { Fracture Model } & \text { fix at } 0.1\end{array}$




\begin{tabular}{|c|c|c|c|}
\hline POROSITY & $\begin{array}{l}\text { Far field Anhydrite } \\
\text { Porosity }\end{array}$ & Fracture Model & fix at 0.014 \\
\hline KMAX_LOG & $\begin{array}{l}\text { Altered Anhydrite } \\
\text { Permeability }\end{array}$ & Fracture Model & $\begin{array}{l}-12 \text { to }-9 \\
\text { Uniform }\end{array}$ \\
\hline PRMX_LOG & $\begin{array}{l}\text { Far Field Anhydrite } \\
\text { Permeability }\end{array}$ & Fracture Model & $\begin{array}{l}-21 \text { to }-18 \\
\text { Uniform }\end{array}$ \\
\hline MBTHPRES & $\begin{array}{l}\text { Threshold displacement } \\
\text { pressure in MB } 139\end{array}$ & Identified 12/91 & Use $12 / 91$ \\
\hline MBPOR & Anhydrite Porosity & Identified $12 / 92$ & Use $12 / 92$ \\
\hline
\end{tabular}

Distribution:

All WIPP Managers

6307 Staff

6308 Staff

6341 Staff

6342 Staff

6115/MS1324 S. Howarth

SWCF CO/SP WBS 1.1.7.2 
33. Hora, S.C. 1994. "Decision Analysis Within the Systems Prioritization Process." July 27, 1994. 
July 27, 1994

\title{
DECISION ANALYSIS WITHIN THE SYSTEMS PRIORITIZATION PROCESS
}

\author{
prepared by \\ stephen C. Hora \\ University of Hawaii at Hilo
}

\section{Iritroduction}

This report has been prepared to provide an overview of the decision analysis component the WIPP SP process. Included in the report are a description of the decision analysis process and its information requirements, a explanation of how the findings of the process may be used, and a discussion of the limitations of the method.

System Prioritization (SP) provides a rational approach to evaluating proposals for activities and developing alternative strategies related to the licensing of the WIPP. These activities can include information gathering activities, refinement of performance assessment models, repository system modifications, and policy issues such as waste form modification and waste acceptance criteria. The goal of the SP is to provide information about how these various activities, when viewed singly or in combination, can contribute to the successful licensing of the WIPP. This objective is explicit and arives the SP. The output from the SP, therefore, is the likelihood of demonstrating compliance given that certain activities are undertaken, and the costs in time and dollars necessary to undertake these activities. Decision analysis provides a logical approach to developing this information and presenting it in a form that aids decision making.

A key to understanding how the prioritization process works is in the relation between the output of the performance assessment model and the regulatory requirements, and how decision analysis can be used to exercise the performance assessment model to judge the likelihood that various activities will result in the demonstration of compliance. It is also necessary to understand that expert input about proposed activities and how they may change our view of the WIPPs performance is critical.

The systems performance model produces performance measures that can be compared to regulatory requirements. One such measure is a complementary cumulative distribution function (CCDF) that represents the probability distribution of releases to the accessible environment. In Figure 1 such a CCDF is shown along another stair step CCDF which is the regulatory requirement given in CFR 191. The WIPP is said to be in compliance with CFR 191 if the performance assessment CCDF lies below and to the left of the regulatory CCDF.

While the regulatory CCDF is fixed by statute, the performance 


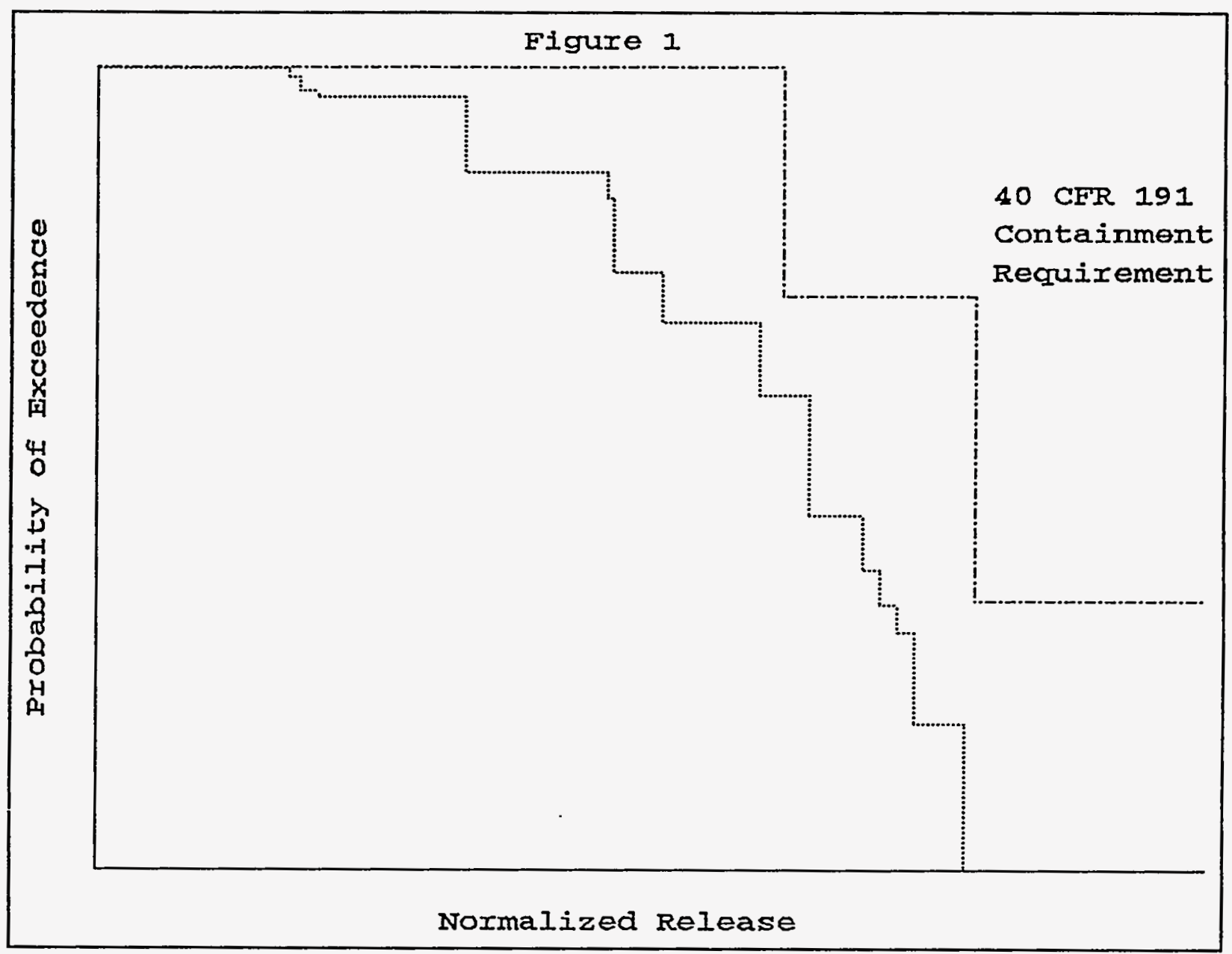

assessment CCDF's position is determined by our knowledge of the WIPP processes and characteristics. Changing our state of knowledge through data acquisition or changing the design of the WIPP or the waste acceptance criteria can move the CCDF either left or right.

Similarly, RCRA provides another regulatory requirement that the WIPP must meet. This standard is terms of a hazardous constituent concentration.

Our current state of knowledge about WIPP features and processes characterizes both what we know and what we know we do not know. This knowledge is usually best expressed through a probability distribution.: For example, while something is known about the solubility of plutonium in WIPP brines, no one can say definitively what the solubility will be. Our state of knowledge about such a parameter is based on many types of knowledge including experimental data (both from WIPP and from other sources), theory, 
and models. Not all of these sources, however, may be defensible in a court of law. Thus, we define a "defensible" probability distribution for a parameter to be the one that an investigator would be willing to defend to the EPA. The defensible CCDF is simply the CCDF generated by evaluating the performance assessment model using the collection of defensible distributions.

\section{Evaluating an Activity through Decision Analysis}

Suppose that an activity is to be evaluated. For concreteness, let this activity be an experiment to determine the solubility of plutonium in brine. Before conducting the experiment, one might reflect on what the experiment will determine, that is, what might be the experimental outcomes. For simplicity, suppose that the experimental outcomes can be classified into five ranges, from lowest to highest solubility. Denote the event that the experimental outcome is the first range by $x_{1}$, in the second range by $\mathrm{x}_{2}$, etc.

After the experiment is complete, our knowledge of solubility changes to reflect the new information. It is highly unlikely, however, that all uncertainty is resolved by the experiment. Unknown room conditions such as the presence of carbonates, make it impossible to know with certainty what the solubility will be. Thus, after the experiment is completed, there is still residual uncertainty about the solubility which can, again, be expressed through a probability distribution. since there are multiple potential outcomes to an experiment there are multiple potential states of knowledge that can result.

Again consider the idea of a defensible distribution. After one of the potential experimental outcomes is observed, one constructs a distribution for the solubility of plutonium that one would be able to defend in light of the hypothetical new evidence. We denote the five possible defensible probability distributions corresponding to the five ranges of experimental outcomes by $f_{1}, f_{2}$, eta.

Now, suppose that we specify $f_{1}, f_{2}$, etc. prior to conducting the experiment and use these distributions in the performance assessment model to estimate the corresponding CCDFs, CCDF ${ }_{1}, \mathrm{CCDF}_{2}$, etc. Five such CCDFs are shown in Figure 2 . In addition to providing the $f_{i}$, we also evaluate the relative likelihoods or probabilities of various outcomes, $x_{i}$, denoted by $p_{i}$.

From Figure 2 ; one can see that outcomes $x_{1}, x_{2}, x_{3}$, and $x_{4}$ result in a state of knowledge such that compliance can be demonstrated. In contrast, $x_{5}$ results in a state of knowledge where compliance cannot be demonstrated. The probability of showing compliance, viewed prior to conducting the experiment, is then $p_{1}+p_{2}+p_{3}+p_{4}$. 


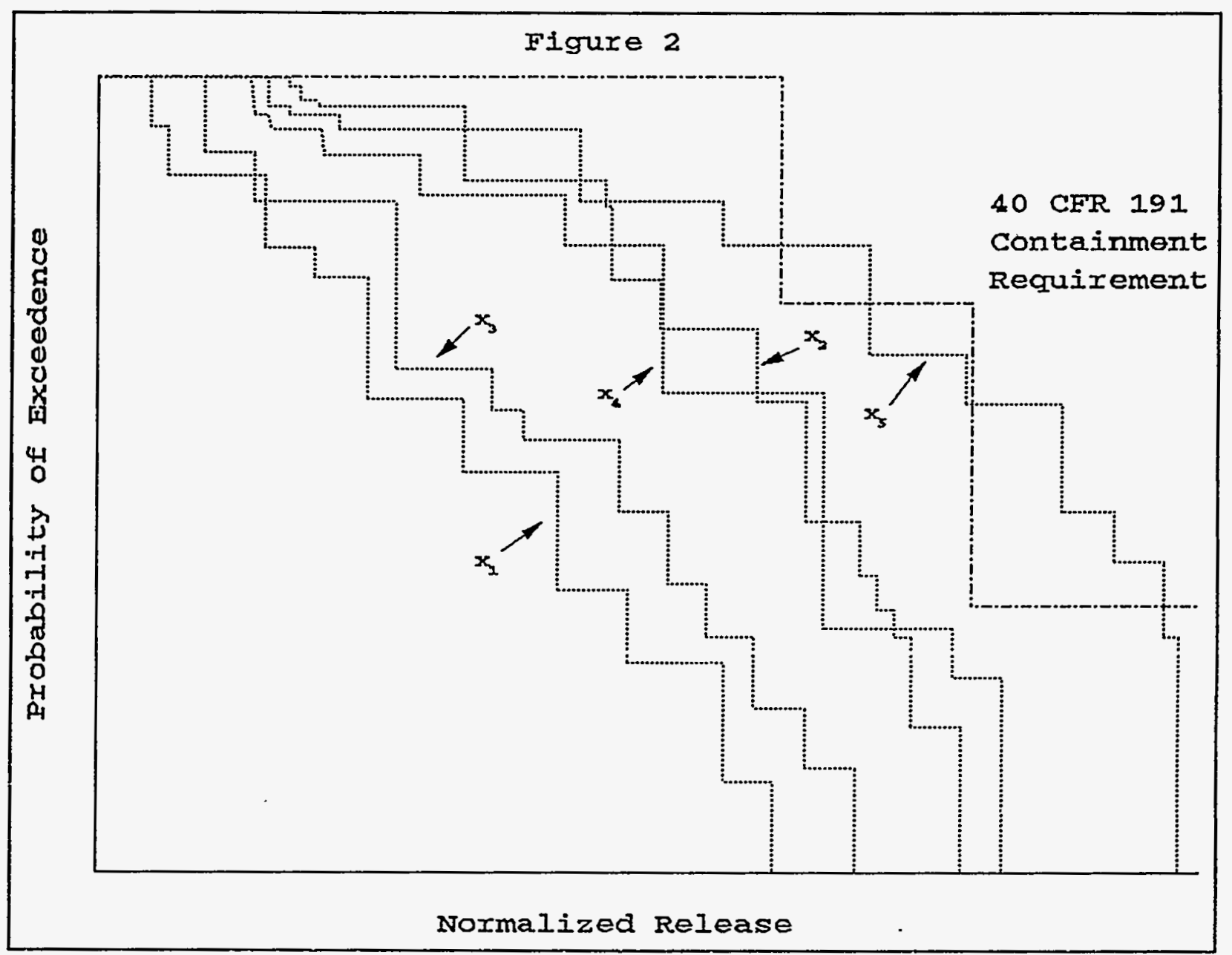

This is the basis for valuing experiments. The technique extends to other activities such repository design modification, waste form treatment, and acceptance criteria. With these activities, the evaluation may be even simpler because the effect of the activity may be known with relative certainty. Thus, only two. CCDFs may be relevant -- the CCDF prior to modification and the CCDF posterior to modification.

Thus far, the evaluation of a single activity has been discussed. SP must be able to consider sets of activities as well as individual activities. The effect of implementing several activities cannot be judged from the individual effects alone. Instead, a joint evaluation must be conducted. Suppose, for example, that a change in waste acceptance criteria is proposed along with two experimental programs. The effect of the waste acceptance criteria may be deterministic but the effects of the two experimental programs are uncertain since the outcomes of the experiments are uncertain. Suppose that the effect of the waste 
modification is to change the distribution of a source term parameter and that the resulting density of this parameter is $g_{1}$. The first experiment has potential outcomes $x_{1}, \ldots, x_{5}$ with probabilities $p_{1}, \ldots, p_{5}$ and the corresponding parameter input distribution $f_{1}, \ldots, f_{5}$, while the second experiment has outcomes $y_{1}, \ldots, y_{3}$ with probabilities $q_{1}, \ldots, q_{3}$ and corresponding parameter input distributions $h_{1}, \ldots, h_{3}$. There are then 15 CCDFs generated corresponding to the various combinations of experimental outcomes. If these outcomes are independent, the joint probabilities can be computed by taking the product of the probabilities of the individual outcomes. Figure 3 depicts 15 such CCDFs. Here only two of the CCDFs, those corresponding to the outcome pairs $\left(x_{2}, y_{3}\right)$ and $\left(x_{4}, y_{3}\right)$ result in not being able to show compliance. Therefore, this activity set has a $1-\left(p_{2} q_{3}+p_{4} q_{3}\right)$ probability of a state of knowledge and repository/waste configuration such that compliance can be shown.

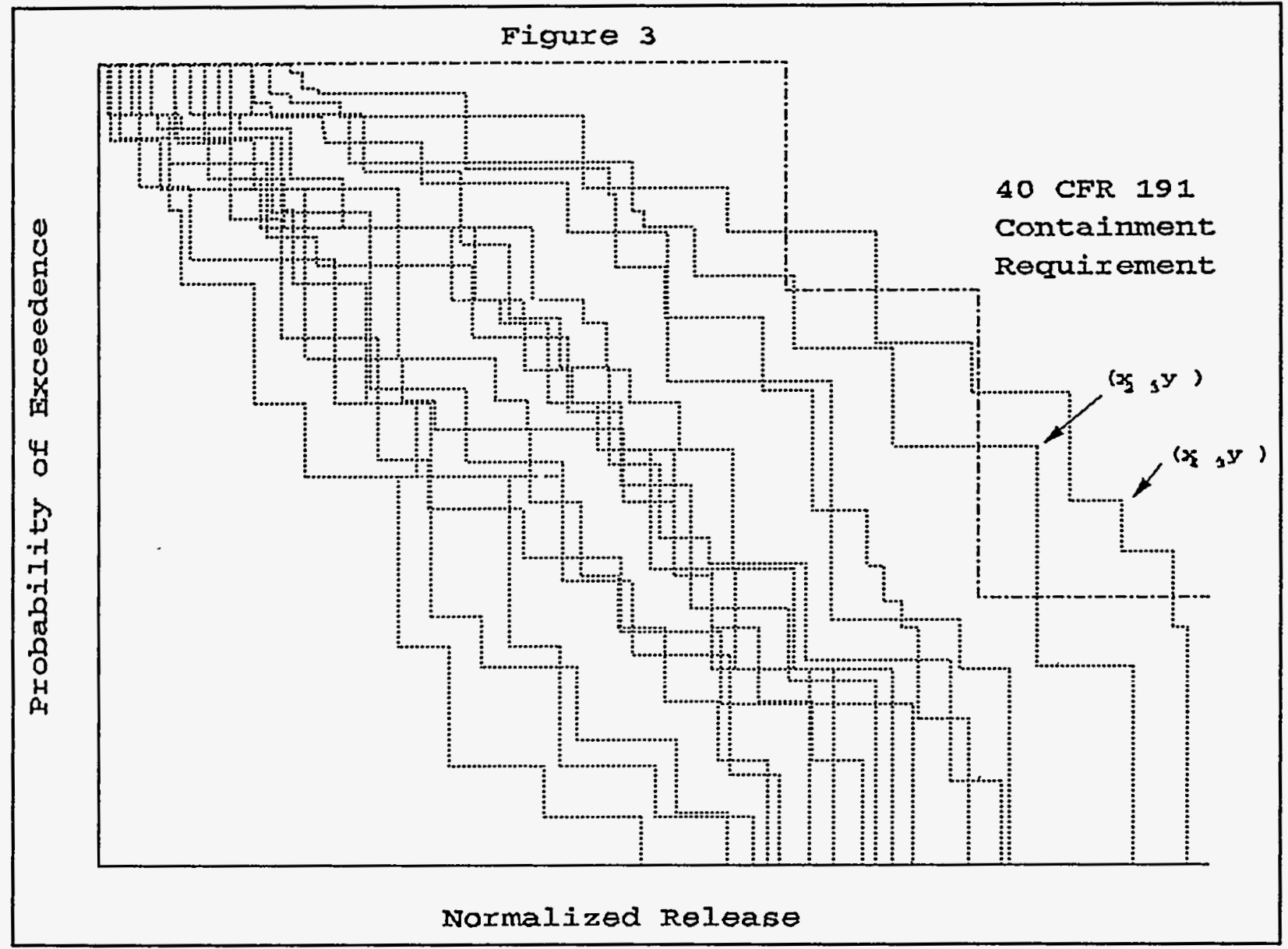

Although this discussion has been restricted to compliance with 40 CFR 191, WIPP must also comply with requirements in RCRA. To integrate both criteria into the analysis is a simple matter. The 
criterion for success is that both the CCDF lie below and to the left of the regulatory CCDF and that the RCRA soil concentration requirements are met. The performance measures given any of the hypothetical states of knowledge are computed from the PA model and a 1 assigned to the outcome sets for which compliance can be shown for both regulations and a 0 assigned to other outcome sets. The probability of compliance with both criteria is just the expectation of this 0-1 indicator variable.

\section{The Decision Output}

The decision analysis produces information about the probability of demonstrating compliance. This information, along with information about costs and schedules, provides input for decision making. one such display of information is a decision matrix such as that shown in Figure 4 .

Figure 4

Probabilities of Demonstrating Compliance

Budget Level

Schedule

3 Years

4 Years

\begin{tabular}{|c|c|c|c|}
\hline A & B & C & $D$ \\
\hline $\begin{array}{l}10 \\
\text { Activity } \\
\text { Set } 11 \\
\end{array}$ & $\begin{array}{c}20 \\
\text { Activity } \\
\text { Set } 64 \\
-\end{array}$ & \begin{tabular}{l}
40 \\
Activity \\
Set 18 \\
\hdashline-10
\end{tabular} & $\begin{array}{c}.50 \\
\text { Activity } \\
\text { Set } 46 \\
-\end{array}$ \\
\hline $\begin{array}{l}30 \\
\text { Activity } \\
\text { set } 77 \\
-\end{array}$ & \begin{tabular}{c}
50 \\
Activity \\
Set 52 \\
\hdashline$-10-$
\end{tabular} & $\begin{array}{c}\text {.70 } \\
\text { Activity } \\
\text { Set } 68 \\
-0\end{array}$ & \begin{tabular}{c}
.80 \\
Activity \\
Set 34 \\
\hdashline---
\end{tabular} \\
\hline $\begin{array}{c}.40 \\
\text { Activity } \\
\text { Set } 13 \\
0\end{array}$ & $\begin{array}{c}.60 \\
\text { Activity } \\
\text { Set } 22 \\
\end{array}$ & \begin{tabular}{c}
.80 \\
Activity \\
Set 42 \\
\hdashline-0
\end{tabular} & $\begin{array}{c}90 \\
\text { Activity } \\
\text { Set } 8 \\
-10\end{array}$ \\
\hline
\end{tabular}

Each cell in the decision matrix contains the maximum probability of demonstrating compliance over all activity sets meeting the cost and time goals shown on the column and row headings respectively. The method of construction ensures that the probabilities of demonstrating"compliance are nondecreasing as one move from the upper left of the table to the lower right of the table. Not shown in the table are combinations of activity sets that, while meeting the appropriate cost and time goals, have lower probabilities of 
demonstrating compliance. These combinations of activity sets are said to be dominated by those having higher probabilities.

An alternative display is shown in Figure 5. Here the vertical axis is the probability of demonstrating compliance and the horizontal axis is cost. The various combinations of activity sets are coded by symbols showing the schedule requirements. Within each group of activity sets having the same schedule requirement, a dotted line is drawn connecting those combinations of activity sets that dominate within the particular schedule limitation.

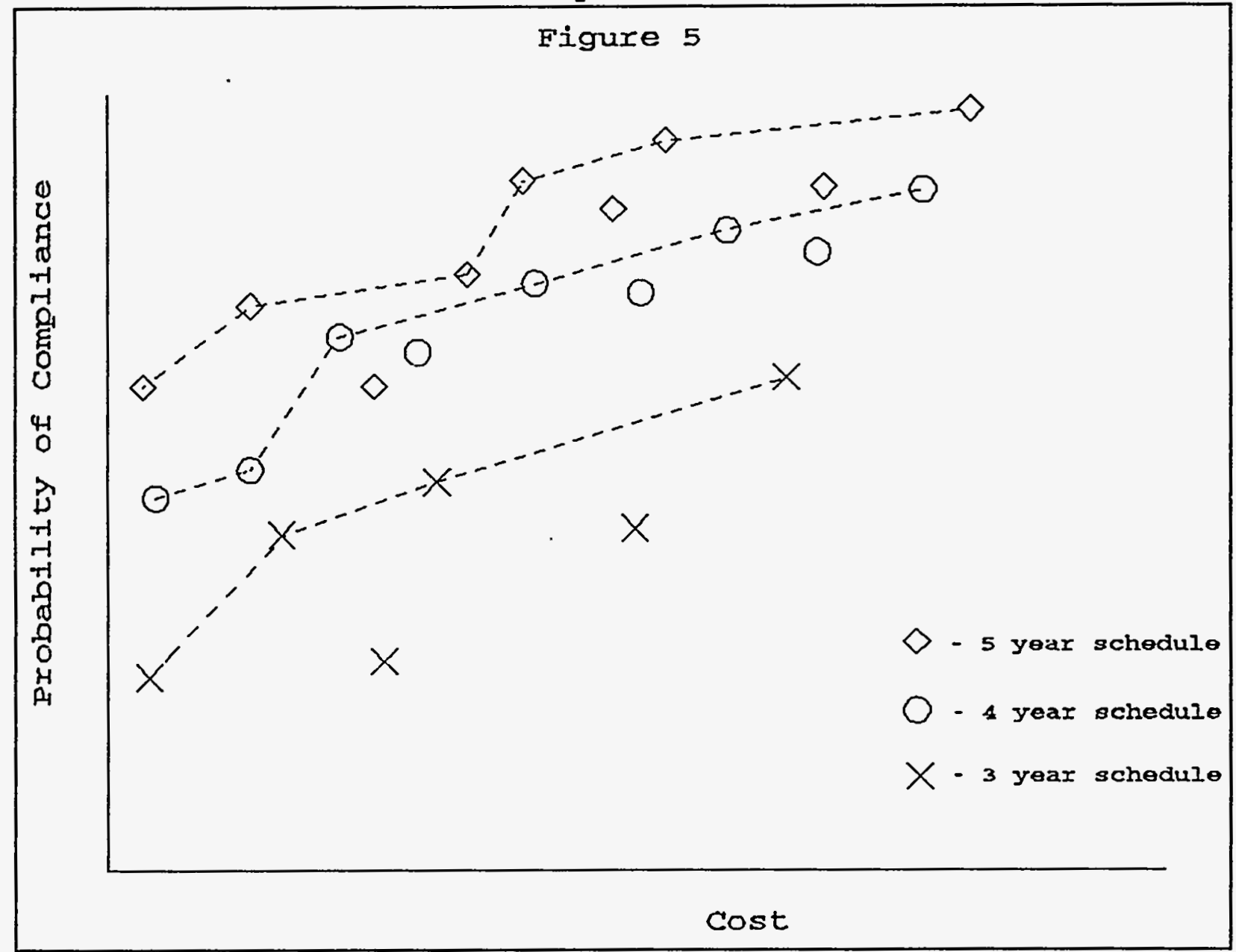

The presentations shown in Figures 4 and 5 represent the type of information that will be produced during the decision analysis phase of the SP. Although decision analysis is often carried to the point of recommending a particular action, the analysis presented here stops short of this point, showing instead those actions from which a best action may be chosen. stopping at this point avoids the problem of defining a utility function over 
probability of demonstrating compliance, cost, and schedule.

\section{Information Requirements}

The usefulness of a decision analysis depends upon the quality of the information used in the analysis. While the activity sets, their schedules and costs, and the defensible baseline are considered to be external to the decision analysis portion of the $S P$, there are extensive data requirements concerning the experimental outcomes, their probabilities, and the state of knowledge about system parameters and conceptual models that would follow particular experimental results. Similar data requirements exist for design and waste modifications and, to lesser extent, for changes in acceptance criteria.

The evaluation of experimental programs relies on the ability to specify the possible outcomes of an experimental program, to provide probabilities for these outcomes, and to identify changes that these outcomes will produce in the performance assessment. The last step, identifying the changes to the performance assessment is most critical and perhaps most difficult.

Several sources may contribute to the needed information concerning the relation of experimental findings and parameter distributions. First, the scientist engaged in the research should have insights into what types of information their experiments will produce and how likely are the various outcomes. The relative likelihoods of these outcomes must be encoded as probability distributions. Each experimental program must also be associated with parameter sets and conceptual models used in the performance assessment. Understanding the relation between the experimental outcomes and the performance assessment requires knowledge both of the experimental environment and the performance assessment environment. It is therefore plausible that both experimental and $P A$ staff members will be involved in specifying these relationships. As the $\mathrm{SP}$ evolves and more external review is sought, stakeholders should be given the opportunity to review, criticize, and perhaps endorse these relationships.

Nonexperimental activities such as design modification, etc. may also produce uncertain outcomes. The information requirements would then be much the same as for the experimental programs: the potential outcomes, the probabilities of these outcomes, and the relation of the outcomes to the $\mathrm{PA}$.

What Is Not Included in the Decision Analysis

A decision analysis is necessarily an abstraction from a real problem. Of course, this abstraction will be successful if the 
essence of the real problem is represented. In making the abstraction from the real problem assumptions must be made. These assumptions involve both what is assumed to be prerequisite to the analysis and a termination point for consideration of future actions. This concept is illustrated in the pyramid depicted in Figure 6. The apex of the pyramid contains those things that are assumed as a basis for the decision analysis. Here, one key element is the performance assessment model itself. Because the decision analysis employs the PA model as part of its structure, the PA model is taken as a given. Iikewise the sets of activities to be evaluated are imposed from outside the decision analysis. Also taken as given are the regulations even though it is

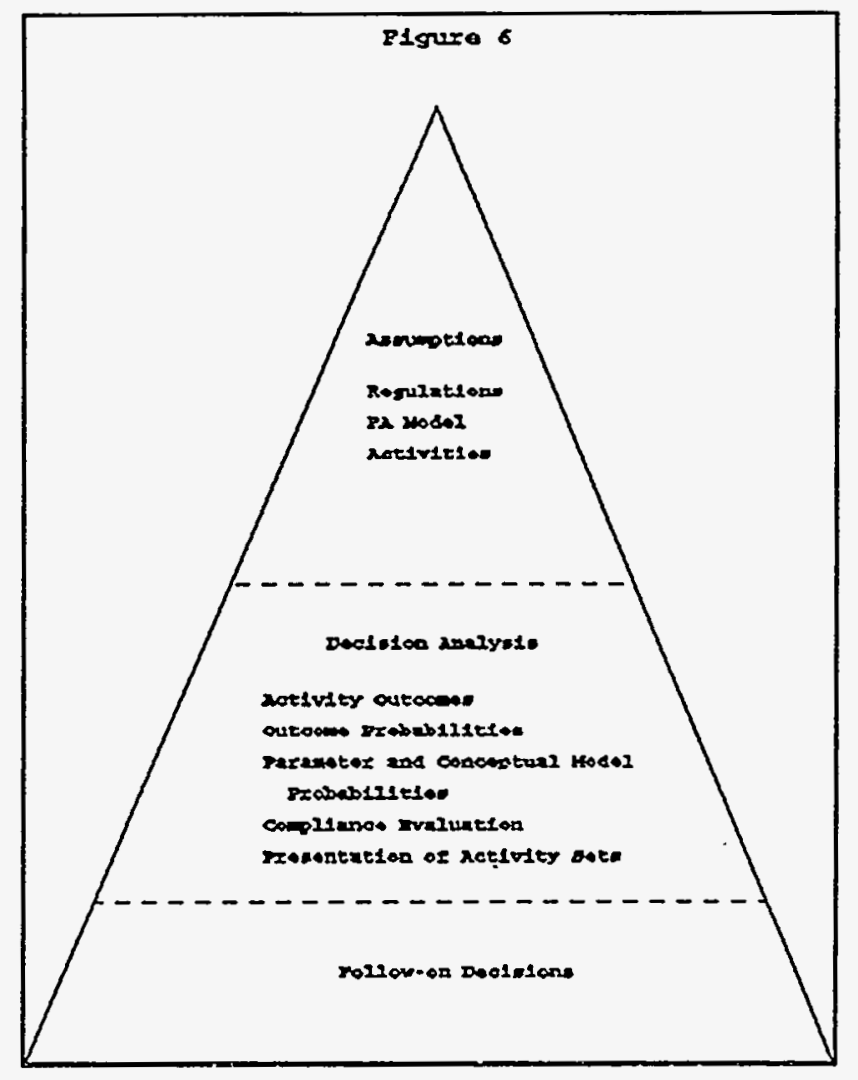
possible that in the next several years these regulations could be modified. Such considerations are simply placed outside the scope of the decision analysis in order to keep the analysis tractable.

The bottom tiex of the pyramid represents those decisions that may ensue after the decisions about funding activities are made. In may be, for example, that after the experiments and modifications have been conducted, new concerns arise or there is a need to verify certain results or new opportunities for design modifications are found. These possibilities are also excluded from the decision analysis. This avoids the problem of an infinite time horizon and the complexity of a long sequence of decisions.

This leaves the center slice of the pyramid -- those aspects of the problem included in the decision analysis. It is important here that these aspects define an abstraction that is sufficiently close to the real problem facing the decision maker so that the analysis provides meaningful and useful information.

\section{Iimitations}

The decision analysis described in this paper provides a general approach to evaluating activities that can enhance the prospect of 
licensing the WIPP. The analysis cannot, however, accommodate all potential activities because of some practical limitations. Perhaps the most important limitation is the inability of the performance assessment model to accommodate some activities. For example, if an activity produces information that cannot be represented in the structure of the $P A$ model or through the input distributions, the PA model cannot assist in evaluating this information. Among such types of information is information about the PA model itself. Examples include gas generation work, fracture work, seal work, etc. (from memo of P. Swift May 20, 1994). However, if the effects of such information on the output measures or internal values within the $\mathrm{PA}$ model can be predicted, it is possible to evaluate such information through the SP process by emulating the effect the information would have on the PA results.

There are also computational limitations to the decision analysis. Suppose, for example, that seventy activities are to be considered. the number of portfolios of these activities is one less than $2^{70}$. Clearly, not all combinations of activities can be evaluated. But this is where common sense and an understanding of repository system can be used to create reasonable sets of activities for evaluation. For example, portfolios of activity sets that greatly exceed reasonable budget allocations should not evaluated nor should portfolios with contradictory activities such as measuring the solubility of current waste forms along with a proposal for waste form modification. 
34. Mendenhall, F.T., N.H. Prindle, W. Beyeler, and R.C. Lincoln. 1994. "Prototype Systems Prioritization 1 (SP-1) Activity Set Definition." Memo, August 4, 1994. 


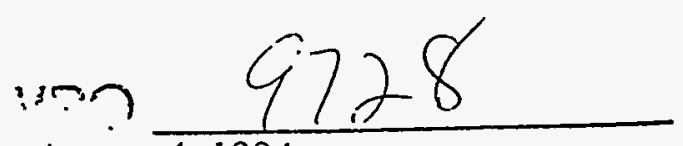

date: August 4, 1994
INFORMATION ONLY

Sandia National Laboratories

Albuquerque. New Mexico $87185-1345$

to: Distribution

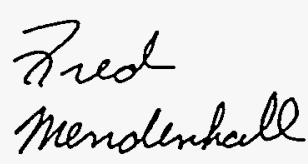

from: F.T. Mendenhall, 6308/MS1341

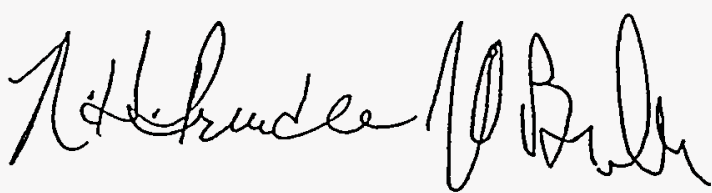

N.H.Prindle, 6308/MS1341
W. Beyeler,

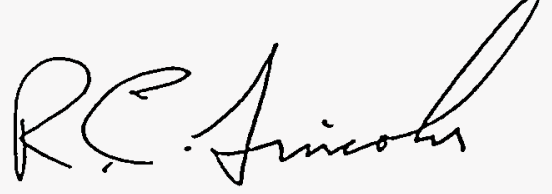

R.C. Lincoln

6308/MS1341

subject: Prototype Systems Prioritization 1 (SP-1) Activity Set Definition

This is the last of four memorandum that define the performance measures, input models, input parameter ranges and parameter distributions needed for the Systems Prioritization Methodology - 1 (SPM-1) analysis. The other three memorandum are: 1) "Second Draft Performance Measure for the WIPP Systems Prioritization," from R.C. Lincoln to P.A. Davis, June 27, 1994.

2)"Prototype Systems Prioritization Baseline, " from F.T. Mendenhall to R.C. Lincoln, July 1, 1994. and 3) "Systems Prioritization Prototype Bragflo Baseiine and Activity Set Parameter Ranges and Parameter Distributions," from F.T. Mendenhall, et.al., to R.C. Lincoln, July 15,1994.

This memorandum is intended for the SNL SP- 1 analytic team in order to allow them to initiate the SP-1 calculations. The authors recognize the need to document the rationale behind these parameter values, however the logic is expected to be part of later documentation.

The SP-1 Baseline was defined in the July 1, 1994 Memorandum "Prototype Systems Prioritization Baseline." In that memorandum the conceptual models were defined for the SP-1 baseline calculations. In this memorandum, we define the changes to the baseline as a result of groups of WIPP related activities, referred to as SP activity sets, and provide the cumulative distribution functions for these possible future states. For the purpose of the SP-1 activity set calculations, issues and models not specifically discussed in this document are assumed to be the same as used in the SP-1 baseline calculation. Furthermore, for this prototype effort the same caveats that were expressed in the baseline memorandum are still operable. Specifically while we believe the baseline to be reasonable and technically defensible, it:

1) Did not include sufficient SNL staff, WID, DOE, regulator or stakeholder involvement to represent a full range of concerns.

2) Assumes appropriate personnel are available to do SP baseline and activity set calculations and the identified side calculations as well as meet other important compliance commitments such as moving the codes to QA level A.

3) Contains buried assumptions that presuppose the completion of certain activities, e.g. 
the assumption of Darcian flow in the Salado may require the completion of some subset of Salado experiments in order to defend the Darcy assumption. While these buried assumptions are important and will be identified in the project white papers used as input for the stakeholder interactions, the need to identify and discuss these assumptions in this document is secondary to the need to initiate the SP process.

In addition for the activity set calculations we would add that:

4) The required computational complexity for the activity set analysis is at least two orders of magnitude larger than the most ambitious WIPP PA calculation ever conducted. While we are proceeding with these calculations with the expectation of success, there is a possibility that computational difficulties may arise that may limit the suite of activity sets that can be analyzed for our September deliverable to DOE-CAO.

5) The future states and associated activity sets are not at this point in the SP process linked to the resource commitments. Specifically, we have selected these activities on the basis of technical enhancements to repository performance. We have not yet identified budget or schedule needed for these activities.

The activities have been grouped into 9 major categories, specifically: Salado, Seals, Source Term, Culebra Physical Retardation, Culebra Chemical Retardation, Colloids, VOC Degradation, Spalling, and the last category is a combination of Performance Based Waste Acceptance Criteria (PB-WAC) and Engineering Enhancements activities.

\section{Salado}

Much of the Salado activity is aimed at supporting a buried assumption in the present SP-1 baseline, namely the assumption of Darcian two phase fluid flow in the Salado halite and anhydrite regions. This effort is aimed at both supporting the two-phase flow conceptual model assumption and at defining the appropriate parameter set needed to perform a two-phase flow calculation. In order to achieve a end of September deliverable it was decided that SP-1 would use the codes in the 12/92 PA analysis, which are two phase flow codes. Hence the baseline assumes two-phase flow, and we believe that this will be defendable. However, because of the buried nature of these assumption in the baseline, SP-1 will not be able to completely identify the data worth, i.e., to estimate the value of the data relative to compliance, of the Salado activities; some of the data worth will be essentially hidden from the analysis. SP-2 will explore ways to compensate for buried assumptions.

For the purposes of SP-1 Salado activities, we expect future states of knowledge to change in two areas. We expect to be able to better define the two-phase flow relationships in Salado flow. We also expect to better define the nature of preferential fluid flow in the Salado

The Brooks-Corey and Parker/Van Genuchten two-phase flow relationships produce 
fundamentally different fluid flow behaviors and they may generally bound the expected fluid flow behaviors in the Salado. They may or may not however, capture the extremes in flow behaviors. The WIPP project currently has a two-phase flow parameter measurement program underway that should define the range of behaviors in FY95. However, for the purposes of SP-1 we will assume that there is a $50 \%$ probability that fluid flow in the Salado will be controlled by two-phase flow characteristics that are represented by the Brooks-Corey relationship. Furthermore, we will assume for SP-1 that if Salado flow is not controlled by Brooks-Corey then it will be controlled by two-phase flow characteristics that are represented by the Parker/Van Genuchten relationship. Thus for activities calculation we would expect to construct results using only Brooks-Corey relations for one outcome and results using only Parker/Van Genuchten relations for another outcome.

We expect as a result of Salado activities to be able to defend calculated gas flow and VOC transport in the Salado instead of using the baseline conceptual model of instantaneous gas transport to the regulatory boundary when the pressure reaches lithostatic. This assumes that we will be able to account for preferential flow behavior either directly in the flow modeling or through the use of simplified modifications to symmetric flow modeling. For SP-1 the approach will be to use the altered anhydrite flow model with parameter ranges and distributions as defined in the July 15, 1994 memorandum from F.T. Mendenhall, et.al., to R. C. Lincoln, acting 6342, titled "Systems Prioritization Prototype Bragflo Baseline and Activity Set Parameter Ranges"and Parameter Distributions." In addition we will multiply the gas transport distance by a factor that is our current best estimate of the effects of preferential flow process, i.e. anhydrite dip, heterogeneity and fingering. [These factors are base on preliminary calculation and estimates that have not passed QA processes at this time.] This factor will be used to determine the earliest time that gas might cross the regulatory boundary. Once it is determined that gas has crossed the boundary, VOC concentrations will be determined by the VOC source term and the dilution caused by the net gas generation at that time.

The expected future state of knowledge is:

Gas Flow Conceptual

Models Outcomes

Baseline Gas Flow Model

Altered Anhydrite Gas Flow Model
CDF Probability

0.90

[All outcome probabilities are presented as cumulative distributions. For example, the above table states that the probability of using the baseline gas flow model following the completion of Salado activities is 0.10 , while the probability of using the altered anhydrite model for gas flow is 0.90 ] 
Preferential Gas Flow Factor

Outcomes

10

6

4

2

1 ( Axisymmetric Flow)
CDF Probability

1.0

0.8

0.5

0.2

0.1

\section{Seals}

The seal effort contains many issues, such as concrete elements, salt elements, and DRZ grouting to name a few.

For the SP1 Activity sets there are no new variables for seals, however the seals activities can be expected to result in the following future states:

Seal Permeablities

Outcomes

CDF Probability

Baseline Values

1.0

$10^{-16}-10^{-18} \log$ Uniform

$10^{-18}-10^{-19} \log$ Uniform

0.90

Note that the seal program not only improves the permeability of the seal, it gives a better performing seal much earlier in the repository life. Therefore, the above permeability ranges are good from emplacement throughout the 10,000 year regulatory period of interest.

For SP-1, we will assume the length of the seal, as a result of seal program activities, will be the same as the baseline length, i.e., 100 meters.

\section{Source Term}

For SP-1 we will assume that the Source term activities may result in the following future states:

Outcomes

Inventory Limited (Baseline Values)

CDF Probability

$10^{-14}-10^{1}$ (Expert Judgement Values)

$10^{-14}-10^{-4}$ (Possible Reduced Range)
1.00

0.99

0.50

Note that the distributions for the baseline are defined in the baseline document and the other distributions are the same as used for the 12/92 PA analysis. In the case of the reduced range, these distributions will be truncated at the specified upper endpoint of the range. 


\section{Culcbra Physical Retardation}

For the SP-I Culebra Physical Retardation activities we will assume the following future states: Culebra Fracture Spacing CDF Probability Outcomes

Baseline values

Based on single well testing

0.1 to $8 \mathrm{~m}$ (Uniform)

Based on single and multiwell testing

$\begin{array}{ll}0.1 \text { to } 3 \mathrm{~m} \text { (Uniform) } & 0.95 \\ 0.1 \text { to } 1 \mathrm{~m} \text { (Uniform) } & 0.57\end{array}$

\section{Culebra Chemical Retardation}

The probability of potential results of the Cuiebra chemical retardation activities are conditional upon results for single versus dual porosity flow representation in the Culebra. The dual porosity flow model has a $95 \%$ probability of being shown by Activity 4 , so joint pdfs will be constructed using the $\mathrm{K}_{\mathrm{y}}$ 's below in the SP1 calculations. The following potential outcomes are in terms of the conceptual models and associated parameter distributions for $\mathrm{K}_{\mathrm{d}}$ 's.

Outcome 1: Conceptual Model -- no retardation

Outcome 2: Conceptual Model -- retardation with uniform distribution of $5 \mathrm{wt} \%$ clay minerals throughout the unit. All sorption is modeled as matrix sorption using a bulk rock $\mathrm{K}_{\mathrm{d}}$. Outcome 3: Conceptual Model -- retardation; non-uniform distribution of clay minerals in unit (i.e., the clay minerals are concentrated adjacent to sub horizontal fractures but not adjacent to high-angle fractures) The experiments will distinguish between the following three cases for flow with corresponding interpretations of $\mathrm{K}_{d}$ 's to use for describing the retardation:

3a) advective flow = sub-horizontal fractures only

3b) advective flow $=$ high-angle fractures only

3c) advective flow $=$ sub-horizontal + high-angle fractures

Probability Distribution Function for Outcomes:

Probability of Outcome 1 double porosity $=.01$

Probability of Outcome 2/double porosity $=.95$

Probability of Outcome $3 /$ double porosity $=.04$

Probability of outcome 3a|double porosity $=.04 / 3=.0133$

Probability of outcome 3 b/double porosity $=.04 / 3=.0133$ 
Probability of outcome $3 \mathrm{c}$ double porosity $=.04 / 3=.0133$

Probability of Outcome 1/single porosity $=.01$

Probability of Outcome 2/single porosity $=0.0$

Probability of Outcome 3/single porosity $=.99$

Probability of Outcome 3 alsingle porosity $=.33$

Probability of Outcome 3 b/single porosity $=.33$

Probability of Outcome $3 \mathrm{c} \mid$ single porosity $=.33$

Parameter Means and Distributions for Outcomes:

Outcome 1 -- $K_{d}=0$

Outcome 2 -- Double Porosity

Means for bulk rock $K_{d}$ 's are derived by taking a correspondingly weighted average of the '92 PA mean values for $\mathrm{K}_{\mathrm{d}}$ 's in the clay and the dolomite. The shape of the probability distribution function is that of the matrix $\mathrm{K}_{\mathrm{d}}$ used for the ' $92 \mathrm{PA}$. The following means are obtained:

\section{Table 1. Adjusted Mean Values Bulk Rock $\mathrm{K}_{4}(\mathrm{ml} / \mathrm{g})$ :}

$A m=1142 \quad \mathrm{~Np}=51 \quad \mathrm{Pu}=1626 \quad \mathrm{Th}=20 \quad \mathrm{U}=28$

Outcome 3 -- Double Porosity

3a) $\mathrm{K}_{\mathrm{d}}$ 's for clay and matrix as used in '92 PA and corresponding model for double porosity transport through matrix and clay "linings".

3b) $K_{d}$ 's same as for outcome 2 above.

3c) $50 \%$ of the time the mean $\mathrm{K}_{d}$ and distribution will be identical to $3 \mathrm{a} ; 50 \%$ of the time the mean $K_{d}$ and distribution will be identical to $3 b$.

Outcome 3 -- Single Porosity

3a) $K_{d} s=$ '92 PA clay mineral $K_{d} ' s$

3b) $K_{d}$ 's are those given by Table 1 above

Question: Can the current $P A$ models simulate sorption with $K d^{\prime}$ 's in the current formulation of this condition?

3c) $K_{d}$ 's = equally likely sampling between a) and b)

\section{Colloids}

For the purposes of SP-1 future states, colloid ranges and distributions are defined below. Colloids will be preferentially mobilized over solute species and once mobilized they will be transported with no retardation in the Culebra, i.e., no chemical and no physical retardation.

The impact of outcomes for colloids from the experiments are likely to be straightforward. However, the application of the results will depend on the scenarios posed by other activities (such as source 
term), policy decisions (such as PBWAC), and/or modelling assumptions. The two cases below account for-a potential effect of humic acids on the concentration of actinides in mobile colloids.

Case I) Present and past waste inventories having no significant amounts of high molecular weight organic materials such as may be found in soils.

Outcome: The total concentration of actinides (moles/liter) in mobile colloids can be represented by the following probability distribution table on the outcome categories:

Table 2. Probability of Outcomes for Colloid Experiments

Outcome

Category $\leq 10^{-9} \quad 10^{-8}$

$10^{-7}$

$10^{-6}$

$10^{-5}$

$10^{-4}$

Probability $\quad 1 / 3 \quad 1 / 3$

$16 / 87$

$8 / 87$

$4 / 87$

$1 / 87$

Within each outcome category, the pdf's for the total concentration of actinides is given by the following table:

Table 3

Conditional Probability Distributions for

Performance Assessment Total Actinide

Concentration in Colloids

\begin{tabular}{|l|l|l|l|l|l|l|}
\hline $\begin{array}{l}\text { Experimental } \\
\text { Outcome }\end{array}$ & $10^{-9}$ & $10^{-8}$ & $10^{-7}$ & $10^{-6}$ & $10^{-5}$ & $10^{-4}$ \\
\hline $\begin{array}{l}\text { Lognormal } \\
\text { Parameters }\end{array}$ & $\begin{array}{l}\mu=-9 \\
\sigma=1.03\end{array}$ & $\begin{array}{l}\mu=-8 \\
\sigma=1.03\end{array}$ & $\begin{array}{l}\mu=-7 \\
\sigma=1.03\end{array}$ & $\begin{array}{l}\mu=-6 \\
\sigma=1.03\end{array}$ & $\begin{array}{l}\mu=-5 \\
\sigma=1.03\end{array}$ & $\begin{array}{l}\mu=-4 \\
\sigma=1.03\end{array}$ \\
\hline
\end{tabular}

The concentrations to be used in the SP-1 activity sets code evaluations are given by:

$$
f(x ; \mu, \sigma)=(2 \pi \sigma x)^{-1} \exp \left[\frac{-.5(x-\mu)^{2}}{\sigma^{2}}\right]
$$

\section{Case 2)}

For wastes containing significant amounts of high molecular weight organic materials such as may be found in soils (i.e., ER and/or D\&D wastes), the outcomes in Table 2 and Table 3 are multiplied by 10 and otherwise the tables remain unchanged. 


\section{VOC Degradation}

WID has agreed to identify VOC half lives for the WIPP-relevant VOCs in the literature. For the purposes of SP-1 future states we will assume:

VOC's half lives

Infinite (Baseline)

WID Supplied
Probability

1.00

TBD when the literature references are reviewed

\section{$\underline{\text { 8. Spalling }}$}

For the SP-1 Spalling activities we will assume the following future states:

Based on the ability to control the waste permeability or on the ability develop a spalling model that includes a waste strength parameter and defending a non-zero waste strength:

Spalling Release- Divide baseline (zero strength)

release by a factor of:
1 (baseline)
1.00
10
0.95
100
0.75
0.40

Probability

2

\section{PB-WAC \& Hypothetical Engineering Enhancement}

A) Limit or exclude soils.

The impact of limiting or excluding soils is shown in section 6 of this report entitled Colloids.

B) Limit VOCs.

If the hazardous constituent concentration performance measure shows non-compliance due to the transport of a single or small number of VOCs, a solution may be found by simply applying a PB-WAC limitation on the inventory of specific VOCs.

C) Limit the influence of gas generation.

Numerous engineering enhancements can be thought of that in some fashion are aimed at limiting the impacts of gas-generation from waste degradation processes. For the purposes of SP-I 
these will be lumped into a single category that limit the total gas potential by a factor of 10 from the baseline. Note that the rate of gas generation, both ranges and distribution, are to be identical to those used in the baseline calculation.
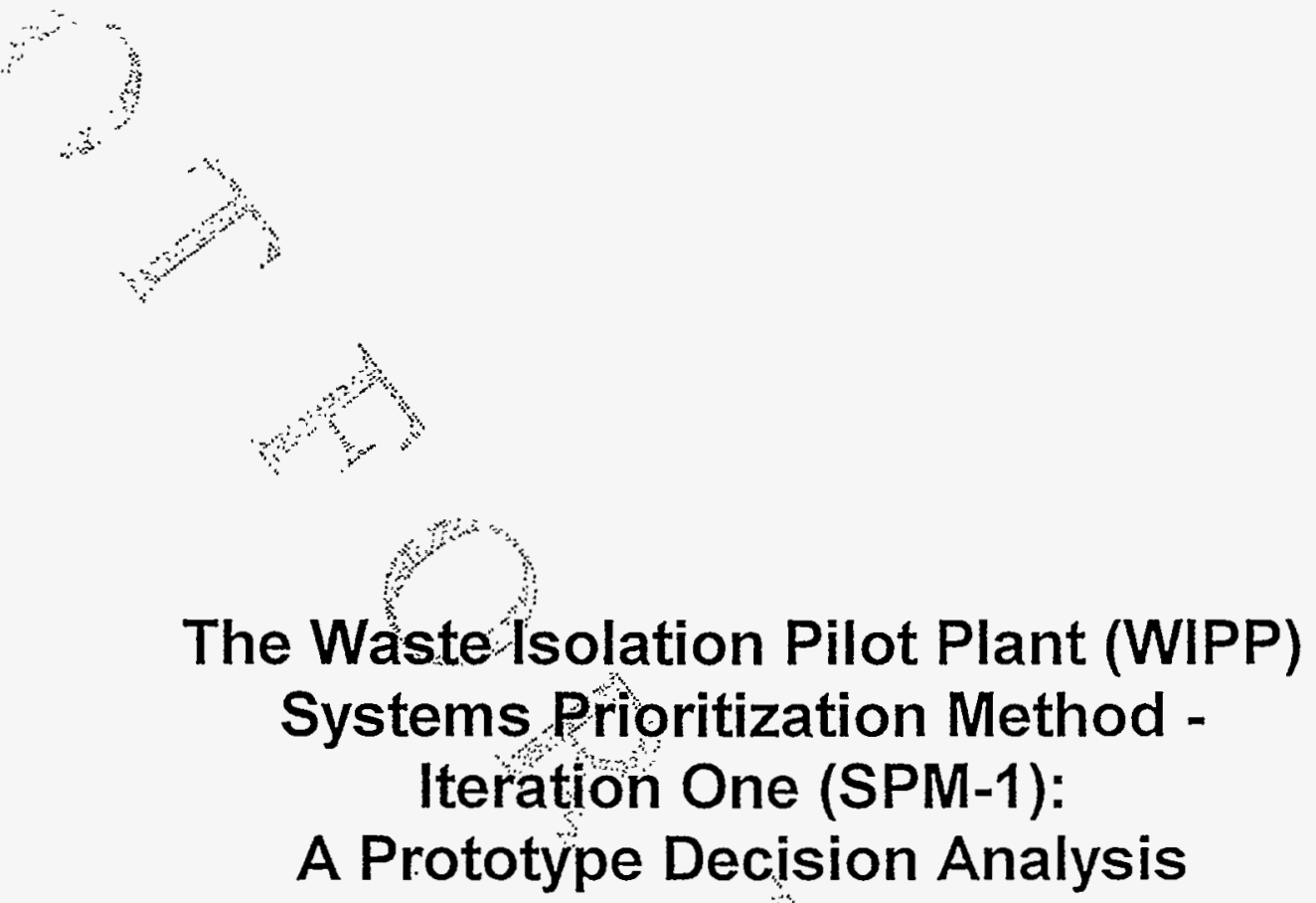

Final 


\section{EXECUTIVE SUMMARY}

The System Prioritization Method (SPM) is a decision analysis tool developed to provide an analytical basis for programmatic decisions regarding activities undertaken in support of a compliance application for the Waste Isolation Pilot Plant (WIPP) to meet selected portions of applicable Environmental Protection Agency (EPA) long-term performance regulations. SPM will calculate the probabilities of certain sets of activities demonstrating compliance with portions of 40 CFR 191 Subpart B and 40 CFR 268.6. SPM will provide this information in the form of a decision matrix to identify low risk and/or cost-effective paths for demonstrating this compliance.

SPM has eleven key steps. They are: the specification of the SPM compliance indicator (CI), a binary measure of whether the WIPP disposal system is predicted to succeed or fail in meeting selected performance requirements in 40 CFR 191 and 40 CFR 286.6 (step 1); the development of a baseline consisting of models and data necessary to evaluate the CI (step 2); the evaluation of the baseline CI for the WIPP disposal system using models and data developed in step 2 (step 3); the identification of activities available to the WIPP project that, if implemented, have the potential to impact the system's CI (step 4); the elicitation of information from the project about what might evolve if specific activities are implemented (potential outcomes) (step 5); the evaluation of the performance of the disposal system using the potential outcomes of the activities and combinations of activities (activity sets) (step 6); the performance of a decision analysis and the creation of a decision matrix that includes the probability of demonstrating compliance, cost, and duration for the activity sets (step 7); the selection by DOE of activities to implement (step 8); the implementation of selected activities (step 9); the reiteration of steps 2 through 8 as necessary (step 10); and, when the baseline calculations indicate compliance, the execution of PA calculations with the Quality Assurance (QA) requirements necessary to prepare a compliance application (step 11).

The SPM uses existing WIPP Performance Assessment (PA) methods and computational tools, or modifications of these tools, to estimate disposal system performance. "Unlike previous WIPP PAs, which used models based on best estimates of the natural processes or properties, SPM assumptions and parameters are based on existing information that is believed to be defensible in a regulatory environment.

A prototype of the WIPP SPM was successfully completed on a tight schedule. The time constraints imposed on the SPM-1, and the fact that it was a prototype, required modifying and condensing the SPM steps in several ways: 1) Models and data were based on the 1992 PA, stakeholder concerns received in writing prior to May 1994 that could be easily implemented as simple code modifications, and some new PA models developed in 1993.2 2) System 
performance was estimated using the baseline prescribed by the SPM team. The baseline for subsequent iterations of SPM will be documented in the WIPP Project Technical Baseline (PTB) report. 3) Potential outcomes of activity sets and their probabilities were prescribed by the SPM team (as .opposed to formal elicitations on a project-wide reference basis).

To implement SPM-1, it was necessary to make several assumptions and decisions, and to use several processes that limit the prototype's applicability. As a result, the reader of this report is cautioned that the results of the SPM-1 are unsuitable for making programmatic decisions. The principal caveats are listed below.

Caveat 1. The SPM-1 baseline is an estimate of the future Project Technical Baseline (PTB) used for the purposes of prototyping the SPM and reflects only the beliefs of members of the SPM team at Sandia National Laboratories (SNL). It does not include sufficient SNL, Westinghouse Waste Isolation Pivision (WD), DOE-CAO, regulator, or stakeholder involvement to represent a full range of concerns and therefore is not useful for drawing programmatic conclusions.

Caveat 2. Only conceptual models that could readily be incorporated into the modeling structures used in the 1992 PA analysis were considered for the SPM-1 baseline and the activity set outcomes.

Caveat 3. Possible outcomes of the SPM-1 activities were defined in part using information obtained in limited and informal elicitation of the Principal Investigators (PD). These activity outcomes are estimates for the purposes of prototyping the SPM. The interpretation of these outcomes and their use in SPM-1 are the sole responsibility of the SNL SPM team.

Caveat 4. The tie between activity sets and cost and schedule for SPM-1 is an estimate for the purposes of prototyping the SPM.

Caveat 5. While in general the SNL SPM team believes that the decision matrix information correctly reflects the results of the computer modeling, there may be isolated errors in results because of insufficient time to check the output and behavior of each run of each code in detail, due to the large amount of information handled:

Caveat 6. SPM-1 analysis is limited to evaluating the ability of the WIPP disposal system to meet selected post-closure regulatory requirements. Any other requirements from the regulator are not covered in this analysis.

Based on prior PA results, SPM-1 does not address 40 CFR 191.15 (individual protection requirements), and $40 \mathrm{CFR}$ 191, Subpart C (environmental standards for groundwater protection).

The SPM-1 team implemented steps 1-7 of the SPM. The results of the evaluation of the CI for the SPM-1 baseline indicated that radionuclide containment and hazardous consituent concentration requirements were not both met. The results of modeling the potential outcomes 
of identified activities were compiled into a threc dimensional plot representing a "decision matrix" showing the probability of demonstrating compliance of the activity set that leads to the highest probability of demonstrating compliance, against each associated cost and schedule category.

SPM-1 has successfully shown that the SPM is computationally feasible and that the type of information desired can be generated given sufficient resources. The results of the SPM prototype will serve both as a benchmark and as a test bed for developing the tools needed for the anticipated requirements of the second iteration of SPM (SPM-2). The SPM-1 effort has demonstrated: 1) The ability to reconfigure the original 1992 PA codes in a timely fashion to contain or approximate altered conceptual models for both a baseline calculation and a suite of activity sets. 2) The development of databases and the execution of decision analysis software to handle large files of information. 3) The ability to handle a large number of input vectors to the PA systems models, computations, and PA output files. 4) The ability to reduce a very large amount of information into a deçision matrix.

A number of lessons were learned during SPM-1. One was that the SPM-1 decision matrix for compliance probability as a function of program duration and total costs resulted in no schedule discrimination because the SNL five-year plan had been modified to make all currently planned activities consistent with the WIPP Disposal Decision Plan (DDP). SPM-2 will analyze multiple potential outcomes corresponding to different total costs and durations for selected activities to allow duration to impact compliance probability. Another lesson learned was that SPM can measure only the "regulatory compliance worth" of activity sets that will change the baseline information. This means that the regulatory compliance worth of activity sets with potential outcomes that are the same as the baseline will be calculated to be zero.

The SPM-1 prototype has demonstrated the ability to supply a decision maker with important information to aid in directing a program as complex as WIPP. The tool, however, is only as useful as the quality of the input data including cost and schedule information. Although the SNL SPM team has just started to fully analyze the results from the SPM-1 prototype, it is clear (given activity cost, schedule, and potential outcomes) that SPM is a viable tool for identifying:

1) activity sets necessary for a given probability of demonstrating compliance (Caveat 6),

2) activity sets that give the maximum probability of demonstrating compliance (Caveat 6),

3) activities that have minimal impact on probability of demonstrating compliance (Caveat 6), and

4) the potential worth, with respect to demonstrating compliance (Caveat 6), of new activities. 


\section{CONTENTS}

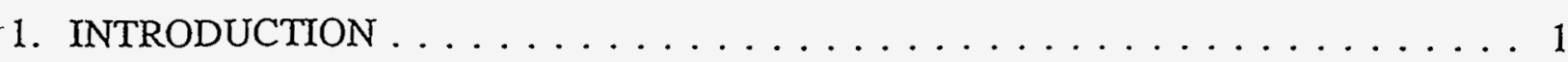

2. SPM OVERVIEW $\ldots \ldots \ldots \ldots \ldots \ldots \ldots \ldots \ldots \ldots \ldots \ldots \ldots \ldots$

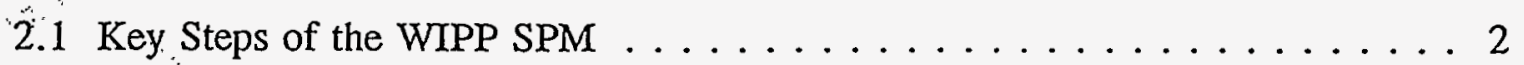

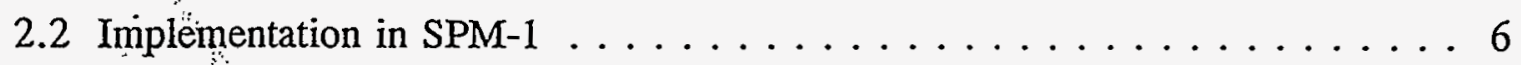

2.3 SPM-1 and 1992 PA Modeling Differences . . . . . . . . . . . 7

3. CAVEATS

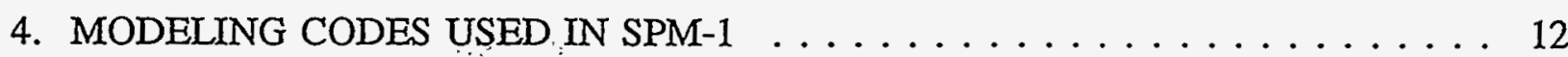

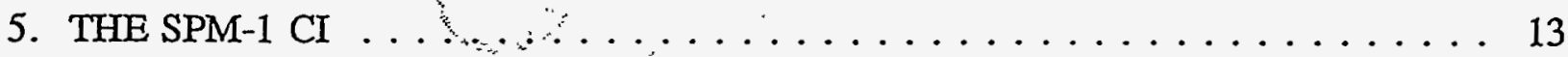

5.1 Radionuclide Containment $\ldots \ldots \ldots \ldots \ldots \ldots \ldots$

5.2 Hazardous Constituent Concentration $\ldots \ldots \ldots \ldots \ldots$

5.3 SPM-1 WIPP Disposal System CI . . . . . . . . . . . . 14

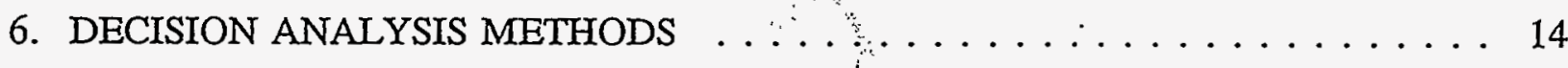

6.1 Computational Approaches . . . . . . . . . . . . . . 14

6.2 Construction of Decision Matrix . . . . . . . . . . . . . 16

6.2.1 Definition of Measure of Value and Discriminators . . . . . . 16

6.2.2 Probability of Demonstrating Compliance and the CI . . . . . . 16

6.2.3 Constructing the Decision Matrix . . . . . . . . . 17

6.2 .4 Analysis of the Decision Matrix . . . . . . . . . . . . . 18

7. SPM-1 RESULTS AND CONCLUSIONS $\ldots \ldots \ldots \ldots \ldots \ldots \ldots$

7.1 SPM-1 Results . . . . . . . . . . . . . . . . . . 18

7.2 Lessons Learned from SPM-1 . . . . . . . . . . . . . . 22

7.2.1 Budget and Schedule Resolution . . . . . . . . . 22

7.2 .2 Embedded Assumptions . . . . . . . . . ....... 22

7.2 .3 Elicitation Process . . . . . . . . . . . . . . . . . 24

7.2 .4 SPM-2 Computational Needs . . . . . . . . . . . . 25

7.2.5 Automated Code Operations and Alternate Computational Platforms

7.2 .6 Post-Closure Design . . . . . . . . . . . . . . . 25 
7.2.7 Configuration Control and Information Flow Requirements . . . . 26

7.2 .8 Decision Analysis Post-Processing .............. 26

$7.3 \mathrm{SPM}$ as a Decision Tool . . . . . . . . . . . . . 26

APPENDIX A: SPM-1 BASELINE $\ldots \ldots \ldots \ldots \ldots \ldots \ldots \ldots \ldots \ldots \ldots$

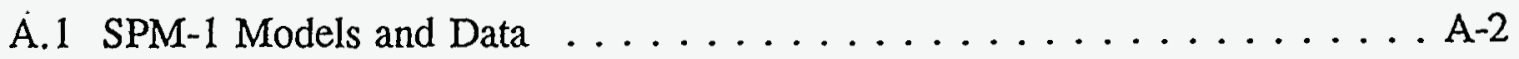

A.1.1 SPM-1 Baseline Assumptions about Flow and Transport in the Culebra

Dolomite Member of the Rustler Formation . . . . . . . . . . A-2

A.1.2 SPM-1 Baseline Assumptions about Flow and Transport in the Salado

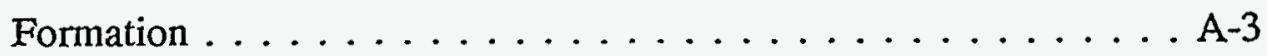

A.1.3 SPM-1 Baseline Assumptions About the Inventory . . . . . . . . . A-6

A.1.4 SPM-1 Baseline Assumptions About the Repository and Near Field

A.1.5 SPM-1 Assumptions about Human Intrusion . . . . . . . . A-8

APPENDIX B: SPM-1 ACTIVTTIES . . . . . . . . . . . . . B-1

B.1 Salado Flow Activities . . . . . . . . . . . . . B $\ldots \ldots$

B.3 Actinide Solubilities . . . . . . . . . . . . B-6

B.4 Physical Retardation in the Culebra ... . . . . . . . . . B 6

B.5 Chemical Retardation in the Culebra $\ldots \ldots \ldots \ldots \ldots \ldots$. . . . . . . . . . . . . . . . .

B.6 Actinide Colloids . . . . . . . . . . . . . . . . . B-9

B.6.1 Case $1 \ldots \ldots \ldots \ldots \ldots \ldots \ldots$ B $\ldots \ldots \ldots$

B.6.2 Case $2 \ldots \ldots \ldots \ldots \ldots \ldots \ldots$ B $\ldots \ldots \ldots \ldots$

B.7 Cuttings/Spallings . . . . . . . . . . . . . . . . B $\ldots \ldots$

B.8 PB-WAC and Engineering Enhancements . . . . . . . . . B

B.8.1 PB-WAC Excluding Soils . . . . . . . . . . . . B-13

B.8.2 PB-WAC Excluding RCRA VOCs .............. . . . . . . . . . . . . . . . . . . .

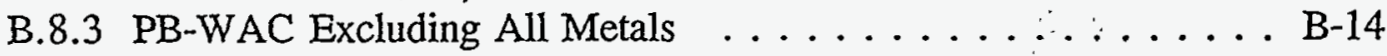

APPENDIX C: COMPLETE LIST OF SPM-1 CAVEATS AND ASSUMPTIONS . . . C-1

APPENDIX D: GLOSSARY AND ACRONYMS $\ldots \ldots \ldots \ldots \ldots \ldots \ldots$ 


\section{INTRODUCTION}

The System Prioritization Method (SPM) is a decision analysis tool developed at Sandia National Laboratories (SNL) to provide an analytical basis for programmatic decisions regarding activities undertaken in support of a compliance application for the Waste Isolation Pilot Plant (WIPP) to meet selected portions of the applicable Environmental Protection Agency (EPA) long-term performänce regulations. SPM is designed to calculate the probabilities of certain sets of activities demonstrating compliance with portions of 40 CFR 191 Subpart B and 40 CFR 286.6. SPM provides this information in the form of a decision matrix for identifying low risk and/or cost-effective paths for demonstrating this compliance.

SPM involves an eleven-step process that uses existing WIPP Performance Assessment (PA) methods and computation tools, or modifications of these tools, to estimate the WIPP disposal system performance. The first iteration of the WIPP SPM (SPM-1) was successfully completed at the close of September 1994. The results of this prototype will serve both as a benchmark and as a test bed for developing the tools needed for the anticipated requirements of the second iteration of SPM (SPM-2).

Section 2 of this report provides an overview of the SPM and describes the key steps of the WIPP SPM, the modifications that were made for SPM-1, and the differences in modeling between SPM- 1 and the $199^{\circ}$ PA. Section 3 discusses the caveats associated with the SPM-1. The modeling codes used in SPM-1 and the SPM-1 Compliance Indicator (CD) are described in Sections 4 and 5, respectively. Section 6 reviews the SPM decision analysis methods, including the computation approaches, the method of calculating the probability of demonstrating compliance using the $\mathrm{CI}$, and the construction and analysis of the decision matrix. The results and conclusions of SPM-1 are presented in Section 7 with an overview of the SPM-1 lessons learned.

\section{SPM OVERVIEW}

The SPM is a decision analysis tool developed to provide an analytical basis for programmatic decisions regarding activities undertaken in support of a compliance application for the WIPP to meet applicable EPA long-term performance regulations. SPM will calculate the probabilities of certain sets of activities leading to a demonstration of compliance with portions of 40 CFR 191 and 40 CFR 268.6 (see Section 3, Caveat 6, and Section 5). . The SPM calculations will provide information for identifying low risk and/or cost-effective paths for demonstrating this compliance. 
As currently defined, the activities considered are those managed by the Department of Energy (DOE) that have the potential to affect selected quantitative aspects of regulatory compliance, such as experimental and other information-gathering programs, engineered alternatives to current repository design or waste form, and possible modifications to the waste acceptance criteria. Non-quantitative aspects of regulatory compliance will require consideration of other activity sets. SPM-1 includes activities from experimental programs at SNL and selected potential performance-based waste acceptance criteria (PB-WAC).

The SPM uses existing WIPP Performance Assessment (PA) methods and computational tools, or modifications of these tools, to estimate the WIPP disposal system performance, but it uses a fundamentally different approach to iterative analyses than those used in past PAs. In previous WIPP PAs, Principal Investigators (PIs) were asked what they believed about the current state of knowledge and data in their area of expertise. PIs typically provided the PA analysts with models or data distributions that were their informal best estimates of the natural processes or properties. There was no explicit constraint that assumptions and parameters used in the PA be based on existing information believed to be defensible in a regulatory environment. Each iteration of these PAs used increasingly refined models and data as they became available, but the responsibility of establishing the suitability of the models and data with respect to existing information was essentially outside the PA process. In contrast to these previous PA analyses, the PA calculations of the baseline in future SPM iterations will use only information that can withstand significant scrutiny. As such, the SPM PA is more conservative than the 1992 PA.

The SPM requirement to use only defensible information as a starting point is derived from the following premise: information that is used in a compliance application must ultimately be acceptable to the regulator for the application to be successful. This premise drives the key steps of the SPM process illustrated in Figure 1 and described below.

\subsection{Key Steps of the WIPP SPM}

\section{Specify the SPM(CD).}

The CI provides a binary measure of whether the WIPP disposal system is predicted to succeed or fail in meeting the specific post-closure performance requirements in $40 \mathrm{CFR}$ 191.13(a) and 40 CFR 286.6. Compliance with the requirements in Section 5 is indicated when $\mathrm{CI}$ equals the value 1 (see Caveat 6 ). If the $\mathrm{CI}$ equals 0 , compliance is not indicated. The first : step in SPM is to specify how the CI will be evaluated. 


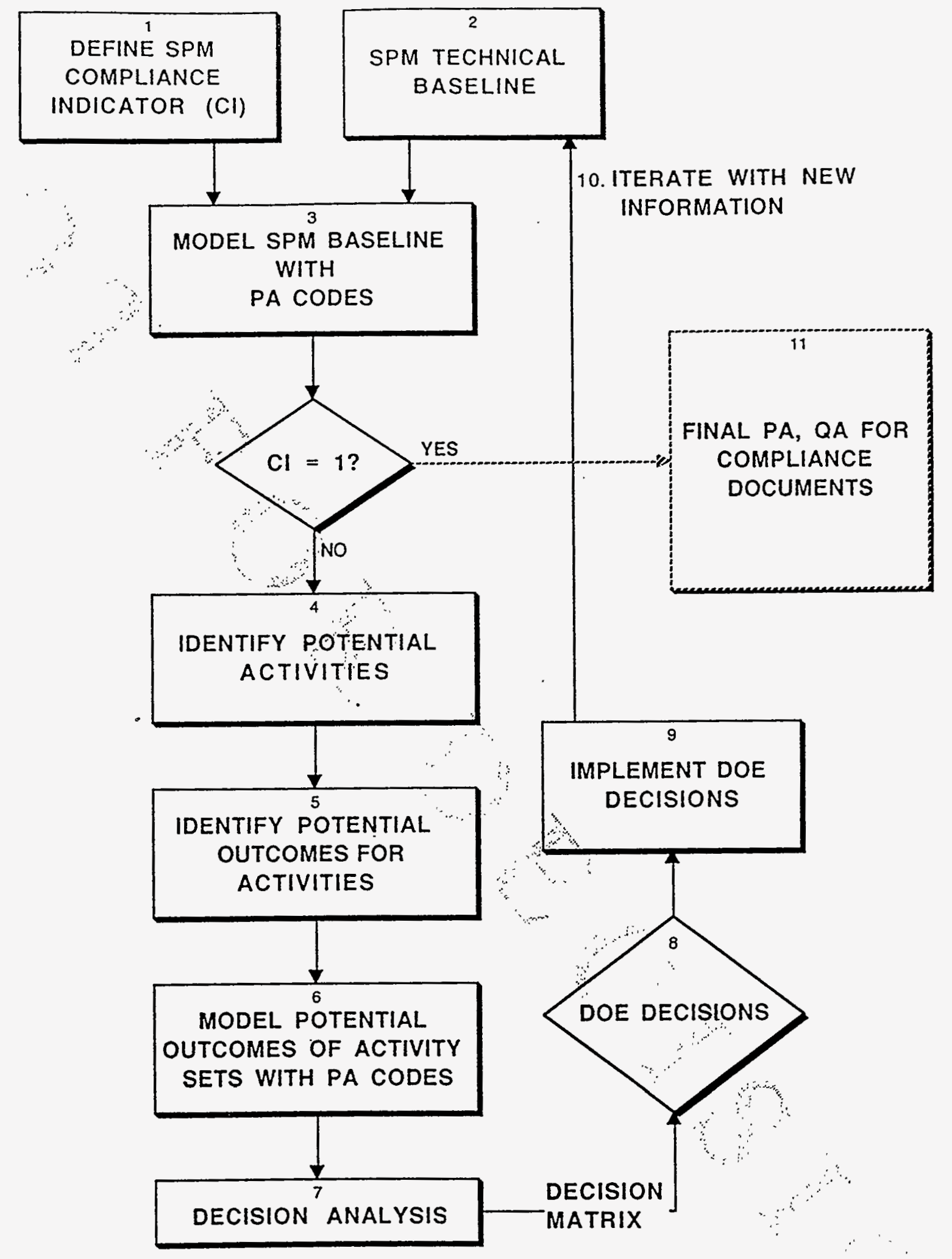

Figure 1. Overview of the SPM. Steps 1-7 were implemented in SPM-1; steps 1-10 will be implemented in all subsequent iterations. 
2. Identify the models and data that are necessary for evaluating the baseline $\mathrm{Cr}$, and that are defensible based on existing information.

Models and data identified in this step are used to analyze the SPM baseline. For SPM-1, the baseline was developed by the SNL SPM team. The baseline for subsequent iterations of SPM will be documented in the WIPP Project Technical Baseline (PTB) report.

3. Evaluate the CI for the WIPP disposal system using models and data from the baseline. If the baseline $\mathrm{CI}$ shows compliance with quantitative standards $(\mathrm{CI}=1)$, prepare a compliance application. This involves implementing a full PA with appropriate Quality Assurance (QA) (step 11). If the $\mathrm{CI}$ does not indicate potential compliance $(C I=0)$, proceed to SPM step 4.

4. Identify activities available to the WIPP project that, if implemented, have the potential to impact the system's CI.

The activities may include experimental program elements or design changes to the facility, such as changes to the waste acceptance criteria.

5. Elicit from the project participants their beliefs about what new information, or changes to existing information, might evolve if specific activities are implemented.

These "potential outcomes" should be as realistic and unbiased as possible, but all parties must recognize that they are essentially informed estimates that are provided before the activity has been implemented.

The process for eliciting information from the PIs and other project participants includes answering four basic questions:

1) What is the purpose of the activity with respect to demonstrating compliance with quantitative standards $(\mathrm{CI}=1)$ ?

2) What do you expect to be the potential outcomes (or outcome categories) of your activity with respect to $\mathrm{PA}$ models and/or parameters that are important for assessing compliance with quantitative standards $(\mathrm{CI}=1)$ ?

3) In your best judgment, what do you think are the probabilities of these possible outcomes?

4) What are the appropriate PA parameters and/or models for each of these outcomes?

6. Model potential outcomes of activities using PA codes. 
PA results are calculated assuming that the specific activities are implemented and the results of the activities match their potential outcomes. For SPM-1, this means calculating Complementary Cumulative Distribution Functions (CCDFs) for radionuclide releases and concentrations of Volatile Organic Compounds (VOCs) in soil. Other iterations of SPM could address additional quantitative regulatory standards.

7. Create a decision matrix that includes the probability of demonstrating compliance (see Caveat 0 ; cost, and duration for all activity sets.

The probability: of demonstrating compliance is calculated from the PA results in step 6 for combinations of activity outcomes and the probabilities of those potential outcomes. These are compiled into probabilities of demonstrating compliance for activity sets with the associated cost and schedule. These analyses of thousands of combinations of activity outcomes are condensed into a decision matrix displaying those activity sets leading to the highest probability of compliance within given cost and schedule categories.

8. DOE CAO decides which activities to implement.

The decision matrix will provide information for selecting activities to implement.

\section{Implement the selected activities.}

Update the PTB after the activities have been completed. Note that the results of the selected activities may or may not match their potential outcomes.

10. Repeat step 2, and iterate as necessary until the baseline CI equals 1 .

11. When the baseline calculations indicate that the $\mathrm{CI}$ equals one, remun a full suite of PA calculations for a compliance application with the corresponding $Q \mathrm{~A}$ requirements.

The calculations performed in step 11 will follow a different process than those in other SPM steps, and will adhere to all applicable regulatory QA requirements. Some work getting the PA codes and models used in SPM up to QA Level A is expected; especially where new conceptual and numeric models have been added to the SPM baseline.

There are two important concepts integrated into the SPM: first, the QA level required for SPM is different than the level required for a compliance application; and second; DOE is the customer for SPM, and ultimately the sole decision maker of which activities to implement. 
Note that calculations using the PA computational system are performed three times in Figure 1. PA calculations occur first in step 3, which consists of an analysis using the current baseline. PA calculations occur a second time in step 5, where multiple analyses are performed using the activity sets' potential outcomes. The PA calculations occur a third time in step 11, for the compliance application. The calculations in steps 3 and 5 are for DOE's internal use for making programmatic budget decisions, and cannot be used in a compliance application. Neither the calculations for making programmatic budget decisions nor the data used in these calculations are at the QA level necessary for a compliance demonstration. The data used in step 3 is based on the current state of knowledge as contained in the PTB. It will be of whatever QA level the PTB has available. The data used in step 5 is, by definition, hypothetical. Only the data and calculations used in step 11, which take place outside of the SPM loop (steps 2 through 10) (see Figure 1), will be at the QA level appropriate for a compliance demonstration.

Also note that the decision about which activities to perform, in step 7 , is a decision made by DOE. There is always some risk that the activities will not actually yield the anticipated outcomes. If the actual outcomes do not support an acceptable compliance application, DOE may need to implement additional activities and iterate the SPM as necessary.

\subsection{Implementation in SPM-1}

The SPM overview above describes the ideal process. The time constraints imposed on SPM-1, and the fact that it is a prototype process, required modifying and condensing the SPM steps in the following ways:

1) For the most part, models and data were based on the 1992 PA. Only those stakeholder concerns received in writing prior to May 1994 and that could be easily implemented as simple code modifications were addressed. Some new PA models developed in 1993 were implemented.

2) System performance was estimated using the baseline prescribed by the SPM team (as opposed to the PTB), as described in Appendix A.

3) Potential outcomes of activity sets and the probabilities of these outcomes were prescribed by the SPM team (as opposed to formal elicitations on a project-wide basis), as described in Appendix B.

4) The performance of the system was estimated using a "Selective Brute Force" approach described in Section 6.1 . 


\subsection{SPM-1 and 1992 PA Modeling Differences}

Table 1 summarizes the differences between the models for the 1992 PA, SPM-1 baseline, and SPM- 1 activity sets. Table 2 summarizes the regulatory requirements addressed in the 1992 PA, SPM- 1 baseline, and SPM-1 activity sets.

\section{CAVEATS}

SPM- 1 is the prototype of a complex set of conceptual and numeric models and computer calculations performed on a tight schedule. As such, it was necessary to make several assumptions and decisions, and use several processes, that limited the scope of SPM-1, in order to complete the analysis within the desired time frame. As a result, the reader is cautioned that the results of SPM-1 are not suitable for making programmatic decisions. To understand the SPM-1 results and their range of applicability, it is important to understand the caveats associated with this prototype. The high-level caveats are listed below. A complete list of SPM-1 caveats appears in Appendix $\mathrm{C}$ :

Caveat 1. The SPM-1 baseline is an estimate of the future PTB used for the purposes of prototyping the SPM and reflects only the beliefs of members of the SPM team at SNL. It does not include sufficient SNL; Westinghouse Waste Isolation Division (WD), DOE-CAO, regulator, or stakeholder involvement to represent a full range of concerns and therefore is not useful for drawing programmatic conclusions.

To measure the potential value of the data from the WIPP Experimental Program, the SPM must use a "technical baseline" as a starting point. The technical baseline is a compendium of scenarios, conceptual models, numerical models, experimental data, parameter ranges and distributions, and computer codes that the WIPP project is willing to defend to the regulator as appropriate for demonstrating the performance of the repository. The technical baseline includes both the repository design and a baseline inventory. Defining the SPM-2 technical baseline is an ongoing process that entails the consideration of input from the stakeholder meetings and the production of the PTB document.

Caveat 2. Only conceptual models that could readily be incorporated into the modeling structures used in the 1992 PA analysis were considered for the SPM-1 baseline and the activity set outcomes.

This results from a need to rapidly explore the feasibility of SPM technology. 
Table 1. Model Differences Between the 1992 PA, SPM-1 Baseline, and SPM-1 Activity Sets

\begin{tabular}{|c|c|c|c|}
\hline \multirow[b]{2}{*}{$\begin{array}{c}\text { Processes and } \\
\text { Parameters } \\
\end{array}$} & \multicolumn{3}{|c|}{ Model and Parameter Values } \\
\hline & $1992 \mathrm{PA}$ & SPM-1 Baseline & SPM-1 Activity Sets \\
\hline $\begin{array}{l}\text { Salado - Anhydrite } \\
\text { Relative } \\
\text { Permability Curve }\end{array}$ & $\begin{array}{l}\text { Brooks Corey used } 66 \% \text { of the } \\
\text { time/Parker Van Genuchten } \\
\text { used } 33 \% \text { of the time }\end{array}$ & $\begin{array}{l}\text { Brooks Corey used } 50 \% \text { of the } \\
\text { time/Parker Van Genuchten } \\
\text { used } 50 \% \text { of the time }\end{array}$ & $\begin{array}{l}\text { Either Brooks Corey or Parker } \\
\text { Van Genuchten depending on } \\
\text { Salado activity outcome (see } \\
\text { Appendix B.1) }\end{array}$ \\
\hline $\begin{array}{l}\text { Salado - Anhydrite } \\
\text { Gas Flow Model }\end{array}$ & $\begin{array}{l}\text { Two-phase flow with constant } \\
\text { permeability and porosity }\end{array}$ & $\begin{array}{l}\text { Instant gas transport to boundary } \\
\text { when repository pressure } \\
\text { reaches } 12.5 \mathrm{MPa}\end{array}$ & $\begin{array}{l}\text { Either baseline model or pressure- } \\
\text { dependent permea-bility with } \\
\text { preferential gas flow model } \\
\text { depending on Salado activity } \\
\text { outcome (see Table B-1) }\end{array}$ \\
\hline $\begin{array}{l}\text { Shaft Seals } \\
\text { Permeability }\end{array}$ & Sample range: $10^{-20}$ to $10^{-21} \mathrm{~m}^{2}$ & $\begin{array}{l}0-100 \text { years, sample range: } 10^{-12} \\
\text { to } 10^{-14} \mathrm{~m}^{2} \\
100-10,000 \text { years, sample range: } \\
10^{-13} \text { to } 10^{-15} \mathrm{~m}^{2}\end{array}$ & $\begin{array}{l}\text { Sample range depends on Seals } \\
\text { activity outcome (see Table B-2) }\end{array}$ \\
\hline $\begin{array}{l}\text { Panel Seals } \\
\text { Permeability }\end{array}$ & Zero & $10^{-12}$ to $10^{-14} \mathrm{~m}^{2}$ & $10^{-12}$ to $10^{-14} \mathrm{~m}^{2}$ \\
\hline $\begin{array}{l}\text { Initial Room } \\
\text { Moisture }\end{array}$ & $\begin{array}{l}\text { Sample range: } 0.0 \text {, to } 14.0 \% \\
\text { Mean: } 7.0 \%\end{array}$ & $\begin{array}{l}\text { Sample Range: } 0.04 \text { to } 5.2 \% \\
\text { Mean: } 0.44 \%\end{array}$ & $\begin{array}{l}\text { Sample Range: } 0.04 \text { to } 5.2 \% \\
\text { Mean: } 0.44 \%\end{array}$ \\
\hline Waste Inventory & $\begin{array}{l}\text { Used the } 1990 / 91 \text { Integrated } \\
\text { Database }\end{array}$ & $\begin{array}{l}\text { Used the } 1994 \text { BIR w/limits on } \\
\text { U }_{235}\end{array}$ & Used 1994 BIR w/limits on $U^{235}$ \\
\hline $\begin{array}{l}\text { Plastic and Rubber } \\
\text { Degradation }\end{array}$ & $50 \%$ available for gas production & $\begin{array}{l}100 \% \text { available for gas } \\
\text { production }\end{array}$ & $100 \%$ available for gas production \\
\hline $\begin{array}{l}\text { Actinide Solute } \\
\text { Concentrations }\end{array}$ & Limited by actinide solubility & Actinide inventory limited & $\begin{array}{l}\text { Solubility sample range depends on } \\
\text { Actinide Source Term activity } \\
\text { outcome (see Table B-3) }\end{array}$ \\
\hline
\end{tabular}


Table 1. Model Differences Between the 1992 PA, SPM-1 Baseline, and SPM-1 Activity Sets (Continued)

\begin{tabular}{|c|c|c|c|}
\hline \multirow[b]{2}{*}{$\begin{array}{l}\text { Processes and } \\
\text { Parameters }\end{array}$} & \multicolumn{3}{|c|}{ Model and Parameter Values } \\
\hline & $1992 \mathrm{PA}$ & SPM-1 Baseline & SPM-1 Activity Sets \\
\hline $\begin{array}{l}\text { Colloid } \\
\text { Concentration }\end{array}$ & Not modeled & Actinide inventory limited & $\begin{array}{l}\text { Solubility sample range depends on } \\
\text { Actinide Colloid activity outcome } \\
\text { (see Table } B-7 \text { ) }\end{array}$ \\
\hline Colloid Transport & Not modeled & $\begin{array}{l}\text { No chemical or physical } \\
\text { retardation }\end{array}$ & No chemical or physical retardation \\
\hline $\begin{array}{l}\text { Culebra Physical } \\
\text { Retardation of } \\
\text { Solute }\end{array}$ & $\begin{array}{l}\text { Both dual and single porosity } \\
\text { cases }\end{array}$ & Not modeled & $\begin{array}{l}\text { Fracture spacing sample range } \\
\text { depends on Culebra Physical } \\
\text { Retardation activity outcome (see } \\
\text { Table B-4) }\end{array}$ \\
\hline $\begin{array}{l}\text { Culebra Chemical } \\
\text { Retardation of } \\
\text { Solutes }\end{array}$ & Modeled (see Appendix B.5) & $\begin{array}{l}\text { Not modeled } \\
\ldots\end{array}$ & $\begin{array}{l}\text { Retardation sample range depends } \\
\text { on Culebra Chemical Retardation } \\
\text { activity outcome (see Table B-5) }\end{array}$ \\
\hline $\begin{array}{l}\text { Groundwater Level } \\
\text { in Recahrge Area }\end{array}$ & $\begin{array}{l}\text { Sample range: Present depth to } \\
\text { land surface }\end{array}$ & $\begin{array}{l}\text { Fixed at land surface for } 10,000 \\
\text { years }\end{array}$ & $\begin{array}{l}\text { Fixed at land surface for } 10,000 \\
\text { years }\end{array}$ \\
\hline $\begin{array}{l}\text { Human Intrusion - } \\
\text { Spallings }\end{array}$ & Not modeled & Modeled & $\begin{array}{l}\text { Sample range depends on Spalling } \\
\text { activity outcome (see Table B-9) }\end{array}$ \\
\hline $\begin{array}{l}\text { Human Intrusion - } \\
\text { Borehole Intrusion } \\
\text { Rate }\end{array}$ & $\begin{array}{c}\text { Sampled as Poisson process with } \\
\text { sampled rate: } 030 / \mathrm{km}^{2} \\
\vdots\end{array}$ & Fixed at $30 / \mathrm{km}^{2}$ & Fixed at $30 / \mathrm{km}^{2}$ \\
\hline $\begin{array}{l}\text { Human Intrusion - } \\
\text { Earliest Intrusion } \\
\text { Time }\end{array}$ & $\begin{array}{c}1000 \text { years } \\
\ddots\end{array}$ & 101 years & 101 years \\
\hline Borehole Plugs & All flow diverted into Culebra & $\begin{array}{l}\text { Degraded (approximates the } \\
\text { permeability of silty sand) }\end{array}$ & $\begin{array}{l}\text { Degraded (approximates the } \\
\text { permeability of silty sand) }\end{array}$ \\
\hline PB-WACs & Nót considered & Not considered & Considered (see Section B.8) \\
\hline
\end{tabular}


Table 2. Regulatory Requirements Addressed in the 1992 PA, SPM-1 Baseline, and SPM-1 Activity Sets

\begin{tabular}{|c|c|c|c|}
\hline Regulatory Requirements & 1992 PA & $\begin{array}{l}\text { SPM-1 } \\
\text { Baseline }\end{array}$ & $\begin{array}{c}\text { SPM-1 } \\
\text { Activity Sets }\end{array}$ \\
\hline $\begin{array}{l}40 \text { CFR 191.13(a) } \\
\text { Radionuclide Containment }\end{array}$ & Yes & Yes & Yes \\
\hline $\begin{array}{l}40 \text { CFR 268.6 Hazardous } \\
\text { Constituents }\end{array}$ & No & Yes & Yes \\
\hline $\begin{array}{l}40 \text { CFR } 191.15 \text { Individual } \\
\text { Protection }\end{array}$ & No & No & No \\
\hline $\begin{array}{l}40 \text { CFR } 191 \text { Subpart C } \\
\text { Groundwater Protection }\end{array}$ & No & No & No \\
\hline
\end{tabular}

Caveat 3. Possible outcomes of the SPM-1 activities were defined in part using information obtained in limited and informal elicitation of the PIs. These activity outcomes are estimates for the purposes of prototyping the SPM. The interpretation of these outcomes and their use in SPM-1 are the sole responsibility of the SNL SPM team.

In addition to the technical baseline, the SPM process requires the elicitation of possible future states of knowledge based on possible activities. "Activity" here has a broad definition, and could include things as diverse as field experiments, lab experiments, novel analyses, changes in the engineering design, changes in the waste acceptance criteria, discussions with the regulator, and literature searches of existing information bases. The limited and informal elicitation of the PIs, as in the case of the conceptual models, resulted from the need to rapidly explore the feasibility of SPM technology.

Caveat 4. The tie between activity sets and cost and schedule for SPM-1 is an estimate for the purposes of prototyping the SPM.

Information required for the SPM analysis also includes the cost:and duration necessary to accomplish the activity sets. For the purposes of SPM-1, the cost and duration figures were obtained from the SNL portion of the budget only, and are therefore incomplete. Also note that the definition of the potential outcomes of the activity sets occurred before the recent budget validation exercise, which may lead to mismatches between the potential future states of knowledge and the associated budget and schedule. 
Caveat 5. While in general the SNL SPM team believes that the decision matrix information correctly reflects the results of the computer modeling, there may be isolated errors in results because of the insufficient time to check the output and behavior of each run of each code in detail, due to the large amount of information handled.

SPM-1 required hundreds of runs of complex codes such as BRAGFLO (BRine And Gas FLOw), NUTS (NUclide Transport System), and SECO/TP (Sandia ECOdynamics TransPort). Normally the output of each of these codes is carefully examined to ensure that the code behaved appropriately. In addition, a number of new codes were developed to process PA results for decision analysis. While in general the SNL SPM team has confidence in the codes and their execution, the large amount, of information generated in a short time frame did not allow the PA analysts to check each run of each code for normal behavior. This leads to the need, as discussed in Section 6.2 .5$, to automate the analysis of the output of some codes, allowing reviews of key indicators to quickly determine if the code ran as expected.

Caveat 6. SPM-1 analysis is limited to evaluating the ability of the WIPP disposal system to meet specific regulatory requirements as defined in Section 5. Any other requirements from the regulator are not covered in this analysis.

The SPM-1 concerns itself only with post-closure regulatory compliance. As such, regulations related to the operational period of: WIPP are not considered. Furthermore, based on prior modeling results, the SPM-1 does not address 40 CFR 191, Subpart B, 191.15 (individual protection requirements), and $40 \mathrm{CFR} 191$, Sübpart $\mathrm{C}$ (environmental standards for groundwater protection). Compliance with the radionuclioge containment regulation requirements is assumed when the mean CCDF meets the requirements of 40 CFR Part 191, Subpart B, 191.13 (a), i.e., below the values specified in the regulation:

Compliance with the post-closure hazardous constituents concentration regulatory requirement is assumed in SPM-1 when the hazardous constituent concentration calculated at the Resource Conservation and Recovery Act (RCRA) unit boundary is less than the health-based level for soils for each hazardous constituent. For SPM-1, the anhydrite interbed pathway uses from the top of marker bed 138 to the bottom of marker bed 139, the shaft pathway uses the model shaft diameter, and both pathways use gas-available porosity for concentration calculations. The hazardous constituent concentrations are calculated from the mean of the multiple deterministic concentrations calculations to include the effect of parameter uncertainty. For SPM-1, only five VOCs were looked at, and values for the VOC source term and for the health-based level for soils were assumed. 
SPM- 1 was run to demonstrate that the SPM is computationally feasible and that the type of information desired could be generated. None of these caveats should thus cause any concern, as long as the SPM-1 results are not interpreted for the purpose of making programmatic decisions.

\section{MODELING CODES USED IN SPM-1}

The PA process for the SPM-1 prototype required the modeling of the repository shaft system for the undisturbed scenario, and either one or two boreholes for the E1, E2, and E1E2 human intrusion scenarios (see Appendix $D$ for definitions of the three scenarios). BRAGFLO was used to model two-phase flow in the repository shaft system, boreholes, and in the Salado Formation. The PANEL code used the borehole flow to mobilize radionuclides and transport them to the Culebra. SECO2D and SECO/TP were used to model radionuclide flow and transport in the Culebra.

The VAST code used the BRAGFLO flow fields to model transport of VOCs in the gas phase for the RCRA CI determination. The NUTS code was used to model transport of a nonreactive tracer in brine to allow the determination of the potential for contaminated brine flow. The CUTTINGS code was used to model three separate physical processes causing direct releases to the surface, including cuttings, cavings, and spallings.

SPM-1 required the development of new software for assembling and post-processing PA results, storing activities and activity set data (cost, duration, connection to PA results), performing the decision analysis, and displaying and exploring the decision analysis results. The new codes developed for SPM-1 are briefly summarized below:

MIXMASTER: combines and re-scales output from PANEL, SECO/TP, and Cuttings for each CCDF construction case.

CONREL: uses MCXMASTER output to calculate CCDFs for histories of random drilling intrusions.

CI191B: compares CONREL mean CCDF for each calculational case to the 40 CFR 191.13(a) performance requirements.

CIRCRA: compares VOC concentrations calculated by VAST to evaluate compliance with RCRA health-based soil concentrations.

CISET: uses the results of CI191B and CIRCRA to update the activities database. 
CPROB: calculates probability of demonstrating compliance using the activities, activity sets, outcomes, and compliance results in the activities database.

Database queries and macros were also developed for:

1) generating activity sets

2) calculating costs and durations for activity sets

3) exploring and displaying decision analysis results

\section{THE SPM-1 CI}

The scope of the system prioritization effort has been defined as post-closure regulatory compliance. Activities that are to take place during the operational period of the WIPP are not considered. Results of prior modeling of undisturbed performance have indicated that radionuclide releases without human intrusion are very small. Based on these prior modeling results, the system prioritization analysis does not address 40 CFR Part 191, Subpart B, 191.15 (individual protection requirements), and 40 CFR Part 191, Subpart C (environmental standards for groundwater protection).

The CI addressed by the system prioritization effort is based on the radionuclide containment requirements of 40 CFR Part 191, Subpart B, 191.13(a), and the hazardous constituent concentration requirements of 40 CFR 286.6 with health-based levels for soil.

\subsection{Radionuclide Containment}

Compliance with the radionuclide containment regulatory requirements is assumed when the mean CCDF meets the requirements of 40 CFR Part 191, Subpart B, 191.13(a), i.e., below the values specified in the regulations.

\subsection{Hazardous Constituent Concentration}

Compliance with post-closure hazardous constituent concentration regulatory: requirements is assumed when the hazardous constituent concentration calculated at the unit boundary is less than the health-based level for soil for each hazardous constituent.

Hazardous constituents transported to the RCRA unit boundary by gas are compared with the health-based levels. This focus on transport by the gas phase is based on the results of prior 
modeling of undisturbed performance, which indicated that contaminated brine does not reach the unit boundary. The anhydrite interbed pathway model uses from the top of marker bed 138 to the bottom of marker bed 139; the shaft pathway uses the model shaft diameter; and both pathways use gas-available porosity, for concentration calculations. The hazardous constituent concentrations are calculated from the mean of the multiple deterministic concentration calculations to include the effect of parameter uncertainty.

\subsection{SPM-1 WIPP Disposal System CI}

The SPM-1 WIPP disposal system CI is equal to 1 if, and only if, the assumptions on radionuclide containment and hazardous constituent concentration compliance (as defined in Sections 5.1 and 5.2) are met; otherwise, the CI is equal to zero.

\section{DECISION ANALYSIS METHODS}

\subsection{Computational Approaches}

The following computational approaches were considered as potential ways to implement the SPM-1 analysis: Straight Brute Force, Selective Brute Force, Median/Median Calculations, and Interpolation Approaches.

Straight Brute Force: This potential computational approach involves the direct calculation of CCDFs for radionuclide releases and soil concentrations for hazardous constituents for every projected outcome of each combination of activities. Previous PA analyses have used this Straight Brute Force method, treating all input parameters and scenarios probabalistically, and generating about 70 different vectors for CCDFs to compare with the applicable 40 CFR 191 requirements. However, for the SPM-1 analysis, the Straight Brute Force would have required the calculation of many thousands of CCDFs and VOC concentrations to calculate the CI for each activity set combination.

Selective Brute Force: This computational approach involves the direct calculation of CCDFs for radionuclide releases and/or post-closure VOC soil concentrations for only the potential outcomes that are expected, on the basis of prior calculations or an understanding of the isolation system, to significantly impact the CI. For example, once compliance is indicated for chemical retardation above a certain threshold, there is no need for additional calculations of related activities with higher chemical retardation. 
Median/Median Calculations: This computational approach involves the direct calculation of CCDFs for radionuclide releases and post-closure VOC concentrations using the single valued median of each parameter distribution instead of current PA methodology (i.e., Latin Hypercube sampling of each parameter distribution with multiple Monte Carlo calculations).

Interpolation Approaches: These potential computational approaches (there are as many of these approaches as there are interpolation algorithms) involve approximating the results for CCDFs and VOC concentrations associated with a given set of conceptual models and parameters by interpolating between previously calculated results created with the Straight Brute Force method.

Straight Brute Force was not used for the SPM prototype because of the large computational resources needed to implement this alternative. Median/median calculations were not used for the SPM prototype because of the unknown nature of potential systematic errors due to nonlinear models resulting in the median of the input distributions not resulting in the median of the output distribution. Interpolation approaches were not used for the SPM prototype because of the unknown extent of potential random errors associated with these alternatives prior to benchmarking.

The Selective Brute Force computational approach was chosen for the SPM prototype. As discussed in the definition above, once compliance is indicated for chemical retardation above a certain threshold, there is no need for additional calculations of related activities with a higher chemical retardation. Therefore, for SPM-1, the SNL SPM team elected not to overtly calculate the CCDF for cases above the threshold. We did, however, account for the probability that these cases will occur and will lead to a favorable demonstration of compliance in the decision matrix. In preparation for SPM-2, we plan to use the results from the SPM-1 PA calculations as a library for rerunning SPM-1 with alternative interpolation computational approaches to benchmark the interpolation approaches. The benchmarking information, along with the results of rerunning SPM-1 with the median/median technique, will be used to select a computational approach for SPM-2. 


\subsection{Construction of Decision Matrix}

\subsubsection{Definition of Measure of Value and Discriminators}

The SPM involves the evaluation of whether the CI that was calculated using the baseline indicates compliance of the WIPP disposal system (see Caveat 6). If the answer is no, the goal of the SPM decision analysis is to predict the value to DOE-CAO of implementing different activities or activity sets on the basis of increasing the probability of demonstrating compliance.

The probability of demonstrating compliance, as indicated by the $\mathrm{CI}$, is the sole measure of value used by SPM-1.' In the terminology of decision analysis, the probability of demonstrating compliance is the utility function for the analysis.

For the different activity sets, the SPM decision analysis derives the functional relations among the three discriminators: the probability of demonstrating compliance, cost, and duration. The SPM decision matrix is an array of numbers defining the functional relationship among these discriminators.

\subsubsection{Probability of Demonstrating Compliance and the $\mathrm{Cl}$}

The results of the PA calculations for the assumed activities and their potential outcomes are CCDFs for radionuclide releases and soil concentrations of VOCs. The $\mathrm{CI}$ is determined by comparing these results to the applicable quantitative regulatory standard.

The computational approach used for the SPM decision analysis allows for separate analysis of the value of the activity sets with respect to RCRA and 40 CFR 191. This can be particularly useful when considering PB-WAC and engineered design alternatives to implement for purposes of increasing the probability of demonstrating compliance with one or the other regulation.

The probabilities of demonstrating compliance for individual activities and activity sets are calculated. The probabilities for the potential outcomes for activities in the set(s) are combined with the calculated results for the CIs to calculate the probability of demonstrating compliance (see Caveat 6) for each activity set combination. Figure 2 shows Activity Set K,

\footnotetext{
${ }^{1}$ Note that decision analysis allows for additional measures of value to be used if desired.
} 
an example of an SPM activity set composed of activities 1 and 3 . The potential outcomes for each activity and the estimated probability of occurring are listed.

\section{ACTIVITY SET K}

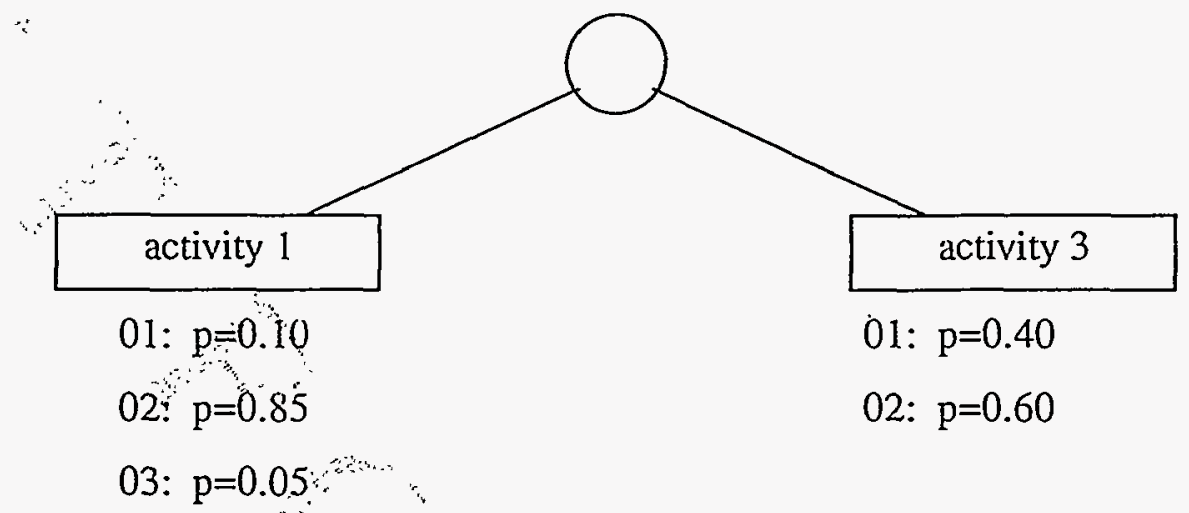

Figure 2. Activity Set $\mathrm{K}$

In this example, assume the PA analyses show that all outcomes, with the exception of Outcome 3 for Activity 1 in combination with Outcome 2 for Activity 3, which results in demonstrating compliance. Then the probability that Activity Set $\mathrm{K}$ will demonstrate compliance is the sum of the probabilities of the outcome combinations that demonstrate compliance, or

$(0.1)[(0.4)+(0.6)]+(0.85)[(0.4)+(0.6)]+(0.05)(0.4)-0.97$

The probability that Activity Set $K$ will result in outcomes that, when analyzed by PA, will not demonstrate compliance, is

$$
(0.05)(0.60)=0.03
$$

\subsubsection{Constructing the Decision Matrix}

The SNL SPM team constructed the decision matrix by associating the calculated probability of demonstrating compliance with cost and schedule information for all activity sets considered. The cost and schedule data used in SPM-1 are from the pre-validated, rebaselined SNL five-year plan, and are broken down according to the current WIPP Work Breakdown Structure (WBS) elements. The resulting decision matrix contains, for each activity set 
combination, the relationship between the discriminators of cost, schedule, and probability of demonstrating compliance.

\subsubsection{Analysis of the Decision Matrix}

It is possible to display in a 3-D plot the probability of demonstrating compliance of the activity set that leads to the highest probability of demonstrating compliance (see Caveat 6) within each cost and schedule category (see Section 7.1, Figure 3). In SPM-1, the SNL SPM team also found other analyses useful to understand the results. The SPM-1 results and corresponding database will be used to design corresponding analysis algorithms for SPM-2.

It is important to note that the goal of SPM is to provide DOE-CAO with a decision matrix that shows the activity sets leading to the highest probability of demonstrating compliance with the selected regulatory standards. Correspondingly, the utility function for the SPM decision analysis is the probability of demonstrating compliance with the selected regulatory standards. However, one can also analyze the information produced from the PA analyses of potential outcomes of activities using a different utility function, such as one based on demonstrating the probability of compliance with other portions of 40 CFR 191.

\section{SPM-1 RESULTS AND CONCLUSIONS<smiles>[AlH2]</smiles>

This section discusses the results and conclusions of the SPM-1 effort. It is divided into three subsections: 1) results of the SPM-1, 2) lessons learned from the implementation of SPM1, and 3) a discussion of SPM as a decision tool.

\subsection{SPM-1 Results}

The major purpose of the SPM-1 effort was to conduct a prototype of the SPM in order to determine the feasibility and usefulness of this technology. This effort has demonstrated:

1) The ability to reconfigure the original $1992 \mathrm{PA}$ codes in a timely fashion to contain or approximate altered conceptual models for both a baseline calculation and a suite of activity sets. Examples of reconfigurations include: the addition of a spallings model (see Appendix B.7), the inclusion of actinide colloids (see Appendix B.6), an accounting for preferential (nonaxisymetric) flow in the activity sets (see Appendix B.1), the adjustment of the recharge heads: 
- to bound climatic effects (see Appendix A.1.1), and the elimination of effective panel seals (see Appendix C.2).

2) The development of databases and the execution of decision analysis software to handle large files of information regarding budget and schedule, and the CI resulting from the PA models.

3) The ability to handle a large number of: input vectors to the PA models $(\sim 37,000$ input vectors); computations (500 Cray central processing unit (CPU) hours, 340 Alpha CPU hours, and 600 Paragon nade hours); and PA output files ( $\sim$ 1,500 mean CCDFs for 40 CFR 191 and 300 RCRA concentration calculations for each of five VOCs).

4) The ability to reduce a very large amount of information, including results from $\sim 1500$ separate activity sêts, into a decision matrix.

The SPM-1 team implemented steps 1-7 of the SPM. The team specified how the SPM-1 CI would be evaluated (step 1) (see Section 5), identified the models and data believed necessary for evaluating the baseline CI (step 2) (see Appendix A), and then evaluated the CI for the WIPP disposal system using models and data from the basseline (step 3) (see Section 4 and 5). The results of the baseline evaluation for RCRA and 40 CFR 191.13(a) are presented in Tables 3 and 4 , respectively. Table 3 shows that the calculated baseline mean soil concentrations are less than the assumed health-based levels. Table 4 shows that the SPM-1 baseline calculated probabilities for the normalized releases exceed the probability criteria in 40 CFR 191.13(a). For the CI to equal 1 , both assumptions on radionuclide containment and hazardous constituent concentration (see Section 5 and Caveat 6 in Section 3) must be met. Since both assumptions were not met in SPM-1, the SPM-1 baseline CI equaled 0.

Activities available to the WIPP project that, if implemented, would have the potential to impact the system's CI, were identified (see Appendix B) (step 4). The SPM-1 team prescribed what new information, or changes to existing information, might evolve if specific activities were implemented (step 5). They also identified potential outcomes and appropriate PA parameters and/or models for each of these outcomes (see Appendix B; see also Table 1). The potential outcomes of the identified activities were then modeled using PA codes (step 6), and the probability of demonstrating compliance for combinations of activity outcomes, and the probabilities of those potential outcomes, were calculated. These were compiled into a three dimensional (3-D) plot of the probability of demonstrating compliance of the activity sets that 


\begin{tabular}{|c|c|c|c|}
\hline $\begin{array}{c}\text { Hazardous Constituent } \\
\end{array}$ & $\begin{array}{l}\text { Assumed VOC } \\
\text { Headspace } \\
\text { Concentration } \\
\text { (ppmv) }\end{array}$ & $\begin{array}{c}\text { Calculated } \\
\text { SPM-1 Baseline } \\
\text { Mean Soil } \\
\text { Concentrations } \\
(\mathrm{mg} / \mathrm{kg})\end{array}$ & $\begin{array}{l}\text { Assumed } \\
\text { Health- } \\
\text { Based Level } \\
\text { for Soil } \\
(\mathrm{mg} / \mathrm{kg})\end{array}$ \\
\hline Carbon Tetrachloride & 441 & 0.01 & 5.38 \\
\hline Methylene Chloride & 177 & 0.001 & 93.33 \\
\hline 1,1,1-Trichloroethane & 391 & 0.01 & 7,200 \\
\hline Trichloroethylene & 14 & 0.001 & 63.63 \\
\hline $\begin{array}{l}\text { 1,1,2-Trichloro- } \\
\text { 1,2,2-Trifluoroethane }\end{array}$ & 41 & 0.001 & $2,400,000$ \\
\hline
\end{tabular}

Table 4. Bàseline 40 CFR 191.13(a) Results

\begin{tabular}{|c|c|c|}
\hline $\begin{array}{c}\text { Normalized Release } \\
\text { (40 CFR 191, Appendix } \\
\text { A, Table 1) }\end{array}$ & $\begin{array}{l}40 \text { CFR 191.13(a) } \\
\text { Probability Criteria }\end{array}$ & $\begin{array}{c}\text { SPM-1 Baseline } \\
\text { Calculated Mean } \\
\text { Probability of } \\
\text { Exceedence }\end{array}$ \\
\hline 1 & $<0.1$ & 0.93 \\
\hline 10 & $<0.001$ & 0.85 \\
\hline
\end{tabular}

leads to the highest probability of demonstrating compliance against each associated cost and schedule category (step 7). A representation of the SPM-1 "decision matrix" is shown in Figure 3. Note that all of these SPM-1 numeric results are subject to the caveats in Section 3 and are not to be used for decision purposes.

This decision matrix has only one duration category, with all activities being completed between one to two years. This lack of discrimination in the duration variable is a result of the process used to define the SPM-1 budget and schedule input and is discussed in Section 7.2.1. 


\section{әวuе!|duoว}

\section{Бu!nexpsuoməd to Kł!!!qeqoıd}

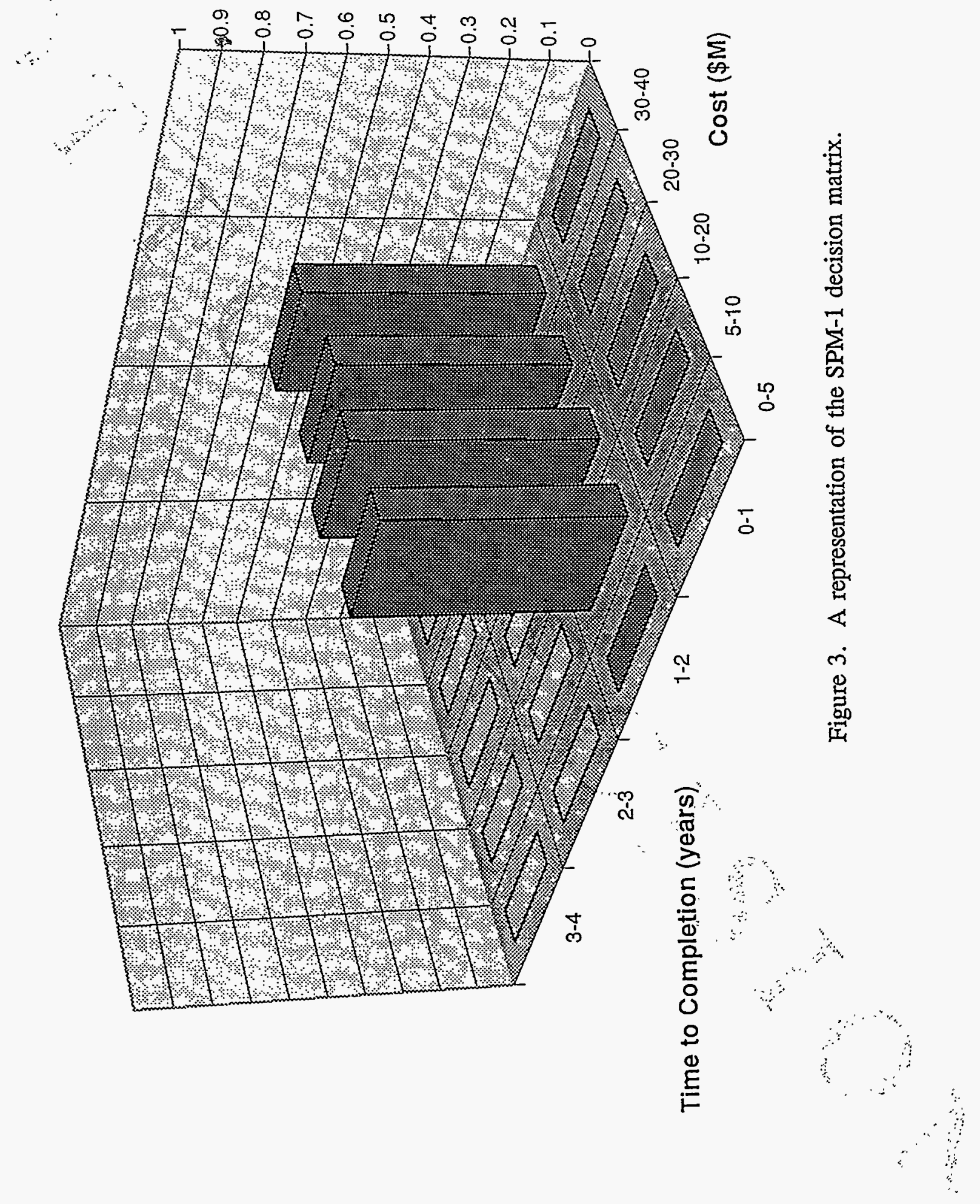


Figure 4 is a representation of a hypothetical fully 3-D form of the decision matrix expected from the SPM-2 budget and schedule process, which will include activity durations that are not constrained by the Disposal Decision Plan (DDP).

A major conclusion of the SPM-1 effort is that SPM analysis is possible given sufficient resources. Future iterations of SPM will need to handle an even larger number of activities and activity sets. This prototype effort has identified methods that are expected to address this need. Furthermore, the results of the SPM prototype will serve both as a benchmark and as a test bed for developing the tools needed for the anticipated requirements of SPM-2.

\subsection{Lessons Learned from SPM-1}

In addition to the high-level process results discussed in Subsection 7.1, additional information was obtained in the prototype that may impact future iterations of the WIPP SPM process. In this section, we document these "lessons learned."

\subsubsection{Budget and Schedule Resolution}

The SPM-1 decision matrix resulted in no discrimination in the schedule variable. This is because the SPM team simply imported the SNL portion of the WIPP schedule, which is already in alignment with the DDP, into the analysis. The current plan for SPM-2 includes budget information relating to the Experimental Program Plan (EPA) costs, the total WIPP project, and the National Transuranic (TRU) Program. The plan also includes defining activities with various durations and corresponding outcomes that are not constrained by the DDP. To take full advantage of the SPM as a decision-making tool, solid cost and schedule information of the right scope is needed, just as solid conceptual and computer models are needed to calculate the CI.

\subsubsection{Embedded Assumptions}

A lesson learned during implementation of the SPM-1 is that the SPM cannot measure the "regulatory compliance worth" of information in the baseline. The process is designed to measure the regulatory compliance worth of activity sets that will change the baseline. The worth is calculated from the potential change to the baseline resulting from implementing the activity sets. The regulatory compliance worth of activity sets with potential outcomes that are the same 


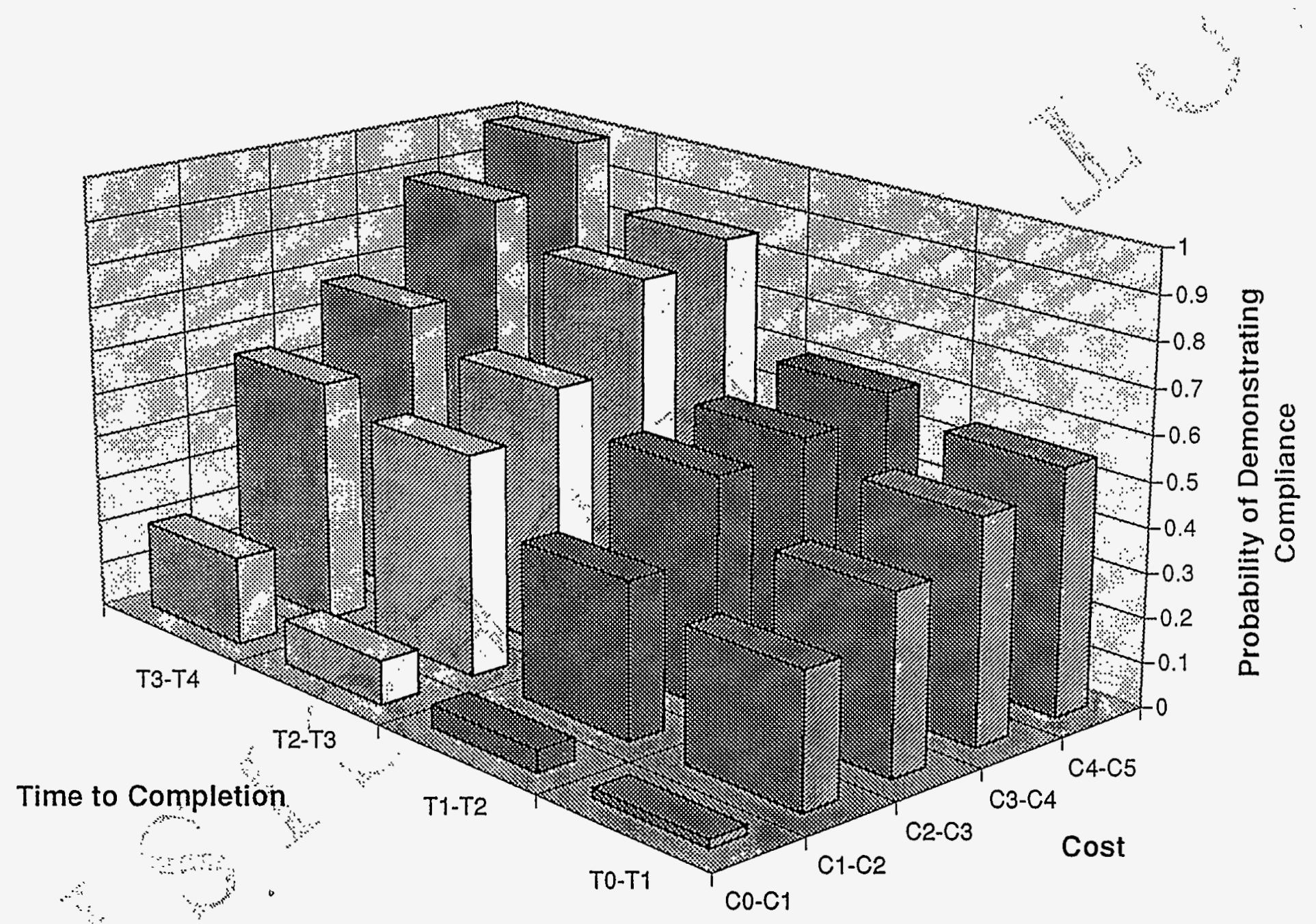

Figure 4. A representation of a hypothetical fully 3-D form of the decision matrix expected from the SPM-2 budget and schedule process. 
as the baseline will be calculated to be zero. In some cases, the SPM-1 baseline contains modeling parameters that are unknown. If all possible combinations of these parameters can be used and the set that corresponds to the most significant regulatory impact is identified, that set may be used in the baseline, and the regulatory compliance worth of an activity set designed to measure the actual value of one or more of these parameters can be calculated using the SPM.

This phenomenon of embedded assumptions represents a limit to the applicability of SPM. The primary impact of it in SPM-1 appears to be in the area of the Salado modeling. Whether it will have an impact on SPM-2 depends on the nature of the SPM-2 baseline currently being defined in the PTB process. The principle risk associated with the embedded assumption phenomenon will arise when the decision to implement the activity sets is made. It appears that the risk will be at a maximum if the embedded assumption has not been identified. The decision risk can be minimized by the identification of these embedded assumptions.

\subsubsection{Elicitation Process}

There were three important lessons learned about the elicitation process.

The first lesson learned is that the credibility of the SPM depends largely on the credibility of the elicitation process. For SPM-1, the elicitation process involved informal interviews with PIs, when available, performed by SPM team members. For consistency, credibility, and improved quality of information, SPM-2 elicitation needs to be performed by consultants who are familiar with the WIPP project and who specialize in expert elicitations. The questions and documentation of the SPM-2 elicitations need to follow a standardized process.

The second lesson learned is that the elicitation process should allow for more time and for interactions among PIs and between PIs and PA analysts. The information provided by PIs about individual activities, when aggregated into information about potential outcomes of activity sets, can have dependent relationships between activity outcomes that must be verified. Additionally, there must be agreement between the PIs and PA modelers on the representation of the potential experimental outcomes in the PA computational approach for SPM-2. In some cases, potential outcomes for SPM-1 did not have adequate PA representation in the current models. An example is the full effect of colloid retardation, which was not phenomenologically modeled by the SPM-1 PA codes. 
The third lesson learned is that more time needs to be allotted for the elicitation process than originally provided for SPM-1. The goal of SPM-2 will be to maximize the information in the position papers and information acquired from stakeholder interactions in order to obtain the necessary input to SPM-2 baseline for PA parameter input and models. We can only do this, however, if the position papers prepared for input to the PTB contain the baseline information required for SPM-2.

\subsubsection{SPM-2 Computational Needs}

The computational needs of SPM-2 are expected to be significantly larger than those required for SPM-1 Seetion 6.1 described the computational approaches considered for SPM-1. The results of SPM-1 will be used to develop and evaluate these approaches for use in SPM-2.

\subsubsection{Automated Code Operations and Alternate Computational Platforms}

One observation made while implementing the SPM-1 was that significant effort by individual analysts was needed to initiate the computer runs of various preprocessor codes, analysis codes, and post-processor codes. The SPM analysis would be faster if the execution of the PA codes were automated, and more analyst time would be available to review calculations, design analyses, or modify codes. The analytical codes that dominate the computer time required for an analysis have been modified for execution on a variety of computer platforms. Calculations in support of the prototype SPM were performed on Alphas using the Open-VMS operating system and Crays, HP work stations, and the Paragon massively paralleled processor using various UNIX-based operating systems. An additional observation was that a UNIX-based computational platform is more readily available thăn Open-VMS platforms to support the large scale intense computational efforts of SPM.

\subsubsection{Post-Closure Design}

An observation made during the definition of the prototype SPM analysis is that a formal definition of the post-closure facility conditions is needed to define the initial conditions of the post-closure analysis. 


\subsubsection{Configuration Control and Information Flow Requirements}

The amount of information generated by SPM-1 was large, and the amount of information generated by SPM-2 will be at least an order of magnitude greater. This will lead to significant information management requirements and complicate a meaningful analysis of the results. The results contained in the decision matrix should be traceable back to the original proposed potential outcomes and their probabilities, through the conceptual model used to represent the outcome, the computer platform used to analyze the outcome, and the postprocessing of results. This requires an automated process for information flow and a controlled configuration to process the information.

\subsubsection{Decision Analysis Post-Processing}

The decision matrix distills a large amount of information about the behavior of the WIPP disposal system. Once activity sets that maximize the probability of demonstrating compliance for a given cost and duration had been identified, the SPM team recognized the need to answer auxiliary questions in order to understand the results:

1) How sensitive is the result to the outcome probability?

2) Which activities have the greatest influence on compliance probability?

3) What is the effect of removing activities from, or adding activities to, selected sets?

Additional post-processing of the decision analysis results is needed to help answer these and related questions. Software should be developed to support SPM-2.

\subsection{SPM as a Decision Tool}

SPM-1 has demonstrated the ability to supply a decision maker with important information to aid in directing a program as complex as the WIPP program. The tool, however, is only as useful as the quality of the input data, including cost and schedule information. Although the SNL SPM team has just started to fully analyze the results from the SPM-1 prototype, it is clear (given activity cost, schedule, and potential outcomes) that SPM is a viable tool for identifying:

1) activity sets necessary for a given probability of demonstrating compliance (Section 3, Caveat 6), 
2) activity sets that give the maximum probability of demonstrating compliance 3. (Section 3, Caveat 6),

3) activities that have minimal impact on probability of demonstrating compliance (Section 3, Caveat 6), and

4) the potential worth, with respect to demonstrating compliance (Section 3, Caveat O, of new activities.
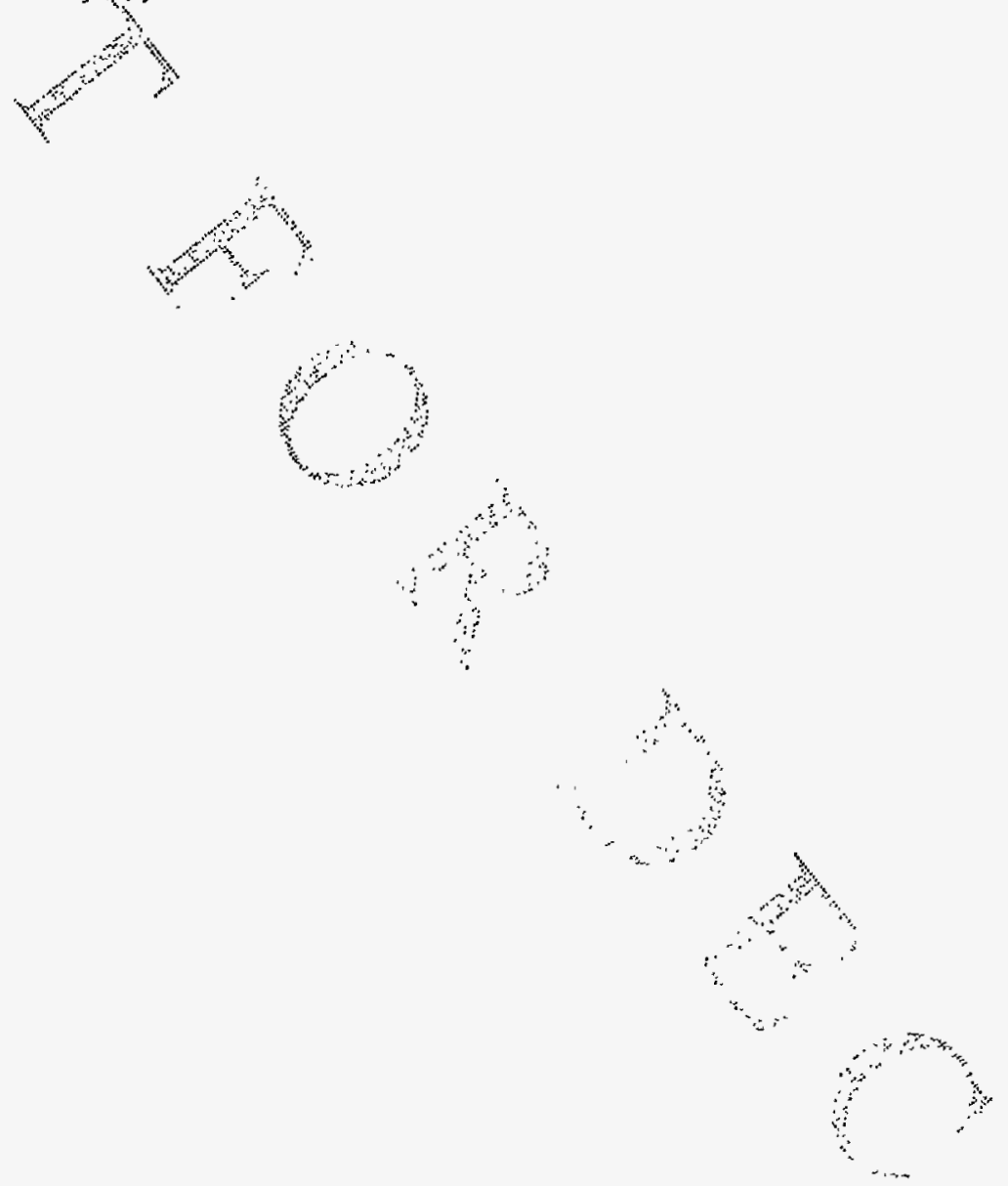

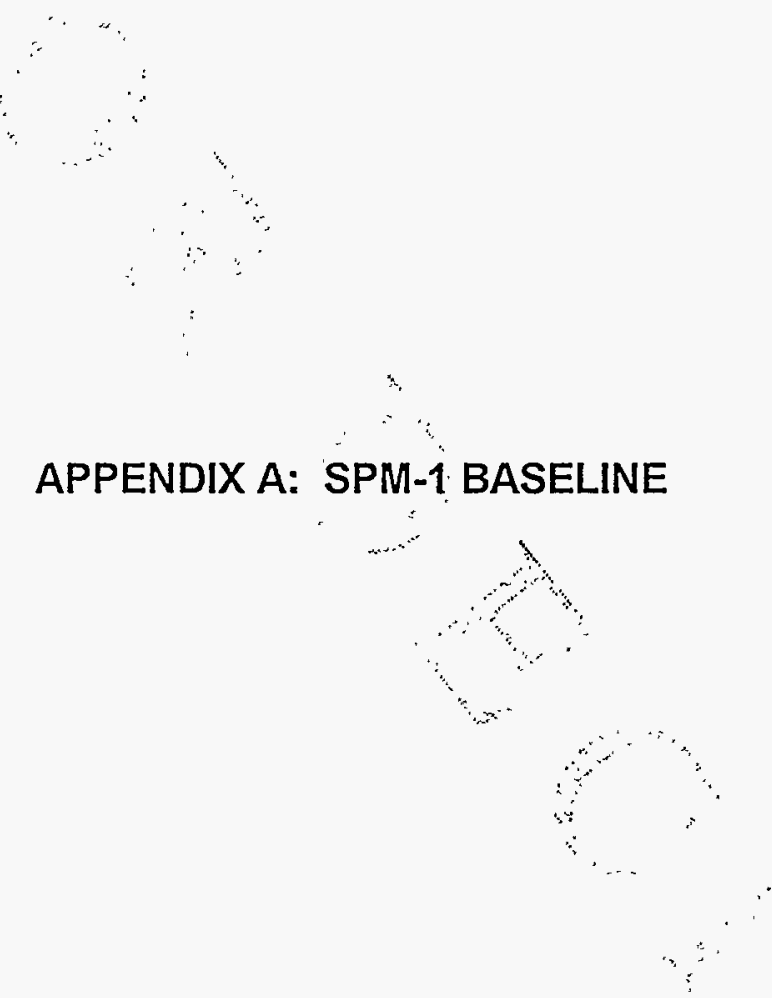
The SPM-1 baseline estimates the current state of knowledge with which the proposed activities and project design can be evaluated in terms of the regulatory requirements. The SPM-1 baseline is an estimate of the PTB made by the SPM team for the purposes of SPM-1 calculations only.

The following caveats apply specifically to the SPM-1 baseline:

Caveat A-1. The baseline does not include sufficient SNL staff, Westinghouse Waste Isolation Division (WD), DOE-CAO, regulator, or stakeholder involvement to represent a full range of concerns and therefore is not useful for drawing final programmatic conclusions.

Caveat A-2. Baseline model assumptions and parameters used in SPM-1 could not be defended in a compliance application because of Caveat A-1. The SPM-1 results cannot be used to draw conclusions about compliance.

Caveat A-3. The baseline contains embedded assumptions that presuppose the completion of certain activities; e.g., the assumption that fluid flow in the Salado Formation behaves according to Darcy's Law may require the completion of some subset of Salado experiments in order to be defensible.

These embedded assumptions are discussed further in Subsection 7.2.2.

\section{A.1 SPM-1 Models and Data}

Conceptual models, computational models, and data not described specifically in this section are the same as those used in the 1992 PA for the WIPP.

\section{A.1.1 SPM-1 Baseline Assumptions about Flow and Transport in the Culebra} Dolomite Member of the Rustler Formation

Regional groundwater flow is modeled using the 70 calibrated transmissivity fields used in the 1992 PA from which 20 sample fields were randomly drawn. Other assumptions, models, and data used in the 1992 PA remain unchanged. Specifically, the Culebra is modeled as a twodimensional (2D) confined aquifer (no leakage) because the SPM team believes that confining transport through the Culebra is conservative with respect to subsurface releases at the site boundary, since the Culebra is the most permeable unit of the Rustler Formation. The regional 
three-dimensional (3D) modeling activity is intended to confirm or refute this assumption as one of its objectives. In response to stakeholder concerns that the treatment of climate change is not defensible, SPM-1 uses the approach used in the 1992 PA of varying boundary heads in a northern "recharge strip," except that heads remain at their elevated position for the entire 10,000 years. This results in the maximum possible head gradient across the site, and provides an upper bound on the effect of climate change within the context of the $2 \mathrm{D}$ flow model.

The baseline model for radionuclide transport in the Culebra is one of the conceptual models analyzed in the 1992 PA with no chemical sorption and no physical retardation transport (i.e., single-porosity, fracture-only transport). Specifically, there is no porosity in the dolomite matrix and no clay fracture linings. These assumptions are made in keeping with the interpretation offered by the Environmental Evaluation Group (EEG) of the terms of the Agreement for Cooperation and Consultation between DOE and the State of New Mexico. Colloids are assumed to be transported at the same rate as dissolved mobilized actinides (i.e., they simply travel with the flow field). Uncertainty about the rate at which colloids are transported is believed to be insignificant compared to the uncertainty in the flow field already incorporated in the analysis. Furthermore, the source term model for the baseline, described below, does not distinguish between colloidal and dissolved forms of actinides.

\section{A.1.2 SPM-1 Baseline Assumptions about Flow and Transport in the Salado Formation}

Several changes have been made since the 1992 PA in the modeling of gas and brine flow and contaminant transport in the Salado Formation. These conceptual changes are included in the SPM-1 baseline and activity set evaluations. The BRAGFLO model now approximates pressure-dependent fracturing of anhydrite interbeds by varying porosity and permeability as a function of pressure when gas generation creates pressures that approach or exceed lithostatic. Additional stratigraphic layers have been added above the Salado Formation to model possible flow into units in addition to the Culebra and to the ground surface. The regions above the Culebra consist of the Santa Rosa, just below the ground surface, and Dewey Lake, 15.76 meters and 149.3 meters thick, respectively, with the water table 24.6 meters above the lower boundary of Dewey Lake.

Above the water table, the brine saturation was taken to be 0.20 and pressure was 1 atm. Below the water table, initial pressures were calculated assuming hydrostatic gradient with fluid (brine) density of $1230 \mathrm{Kg} / \mathrm{m}^{3}$ and $\mathrm{g}=9.79 \mathrm{~m} / \mathrm{sec}^{2}$. Initial excavated regions had pressure equal to $1 \mathrm{~atm}$. Additional properties are shown in Table A-1. 


\begin{tabular}{|c|c|c|c|c|}
\hline $\begin{array}{l}\text { Stratigraphic } \\
\text { Layers }\end{array}$ & Porosity & $\begin{array}{l}\text { Permeability } \\
\qquad\left(\mathrm{m}^{2}\right)\end{array}$ & $\begin{array}{l}\text { Residual } \\
\text { Brine } \\
\text { Saturation }\end{array}$ & $\begin{array}{c}\text { Residual } \\
\text { Gas } \\
\text { Saturation }\end{array}$ \\
\hline Santât Rosa & 0.175 & $10^{-10}$ & 0.20 & 0.20 \\
\hline Dewey Lake & 0.20 & $10^{-15}$ & 0.20 & 0.20 \\
\hline
\end{tabular}

An unsaturated zone was added in the uppermost layer. Transport codes were incorporated for both $\mathrm{VCS}$ and radionuclides, allowing the tracking of contaminants within the repository and the surrounding strata.

Uncertainty remains high about the model used to approximate the effects of fracturing and the validity of the assumption of radial homogeneous property flow used in BRAGFLO (i.e., possible preferential flow effects of channeling, fingering, and non-uniform fracturing were not simulated). The SPM-1 baseline thus contains the following assumption: if at any time pressure within the repository approaches or exceeds $12.5 \mathrm{MPa}$ in the simulation, the gas phase in the repository is assumed to be directly connected to the regulatory boundary through the anhydrite interbeds. Gas concentrations and species filling the void space in the anhydrite just outside the regulatory boundary are the same as those calculated in the repository. No estimate is made of possible VOC degradation or sorption during transport. These assumptions bound the consequences of gas migration with respect to $40 \mathrm{CFR} 268.6$.

A comparable bounding assumption is not made for liquid-phase flow and transport in the Salado. Brine migration distances are much less than those for gas, and effects of preferential flow in the anhydrite are greatly diminished because capillary effects are assumed to allow brine into brine-saturated halite porosity while gas remains within the anhydrite pores. The system CI related to liquid-phase transport of contaminants in the Salado is estimated using the current BRAGFLO and NUTS models.

The dissolution of gas in brine is not addressed in the SPM-1 baseline Gas transport is faster than brine transport; not allowing gas to dissolve in brine is therefore conservative.:

Vertical fracturing was not considered in SPM-1 because vertical fractures are not: expected in the Salado. The modeling of gas-phase transport in the Salado leaves no distinction: between gas at the side boundary and at the top boundary of the regulated unit. Once $12.5 \mathrm{MPa}$ pressure is reached in the disposal room, we assume that all disposal room gas is instantaneously transported outside of the regulated unit. For evaluating compliance with 40 CFR 268.6, we 
therefore considered vertical fracturing to be no worse than horizontal fracturing. Note that vertical flow up the shaft is a separate issue, and calculated independently of the fracture issue.

In the shaft, initial brine saturation was taken to be 0.25 except in the zones above the water table, where the value 0.20 was used. Residual brine and gas saturations were 0.20 in all shaft regions, panel seals, backfill, and experimental rooms. Initial saturation in panel seals, experimental, and backfill was 0.25 . Capillary pressures were assumed to be 0 in shaft regions above the top of the Culebra, the Dewey Lake, and Santa Rosa as well as in the MB139, $\mathrm{MB138}$, and Anhydrite A and B.

\section{A.1.3 SPM-1 Baseline Assumptions About the Inventory}

Physical properties of waste and backfill were treated in the same manner as in the 1992 PA; for repository and Salado modeling, waste and backfill is a homogeneous material characterized by the same parameters used in 1992. Parameter values in some cases have been updated to reflect new information.

The radionuclide inventory was based on the 1994 Baseline Inventory Report (BIR) with the exception that values for remote handled $(\mathrm{RH}) \mathrm{U}^{235}$ were reduced to transportation limits.

The VOC inventory was based on the best information available to DOE-CAO at this time.

Alternative inventories were not included in the SPM- 1 baseline. However, DOE-CAO is currently initiating an effort to define a bounding waste envelope based on the performance of the waste in the disposal system. This envelope will be the basis for the PB-WAC that may be considered in future iterations of the SPM.

\section{A.1.4 SPM-1 Baseline Assumptions About the Repository and Near Field}

Room closure occurs, according to the 1992 porosity surface, until gas pressure reaches lithostatic. Thereafter, the room porosity and permeability remain constant. We note that the assumption of constant room porosity and permeability after lithostatic pressure is achieved is consistent with the assumption that anhydrite fracturing occurs at lithostatic pressure.

The gas-generation model used in SPM-1 was that used in the 1992 PA, updated with rate information documented in the June 18, 1993, memorandum of record from L.H. Brush to M.S. Tierney titled, "Likely Gas-Generation Reactions and Current Estimates of Gas-Generation Rates for the Long-Term Performance Assessment." In response to stakeholder concerns about 
the treatment of the plastic and rubber inventory in past analyses, all plastic and rubber material are available for degradation reactions.

For SPM-1, the repository and surrounding strata are assumed to be horizontal.

The human intrusion model estimated cuttings and cavings in the same manner as in the 1992 PA. A simplified spalling model was included as defined by Berglund and Butcher. For two-phase flow calculations, waste permeability remained constant at $10^{-12} \mathrm{~m}^{2}$. However, for the cavings, cuttings, and spalling calculations, waste permeability values were sampled from a loguniform probability distribution of $10^{-12}$ to $10^{-17} \mathrm{~m}^{2}$.

The Disturbed Rock Zone (DRZ) was modeled with the halite regions having far-field halite properties for porosity, but enhanced permeability (i.e., $\mathrm{k}=10^{-15} \mathrm{~m}^{2}$ ), under the assumption that they do not act as a significant barrier to fluid flow.

In response to concerns from stakeholders that previous PA modeling of seals has taken unwarranted credit for the ability of concrete seals to prevent groundwater from the Culebra from reaching the repository, the concrete elements for all repository and panel seals were assumed to have a permeability range from $10^{-12}$ to $10^{-14} \mathrm{~m}^{2}$ for the full 10,000 years. This range is comparable to that of silty sand, and is the best that can be defended until it can be demonstrated that large WIPP concrete sealing elements can be manufactured without fracturing the concrete. The repository seals contain 120 feet of concrete modeled either as a continuous column or as three discrete elements according to the seal design. If fractures occur in these concrete sections, they are not expected to heal. The crushed salt portions of the seals were modeled as having permeability two orders of magnitude less than the concrete elements for the first 200 years. From then on, the lowest $100 \mathrm{~m}$ of crushed salt was assigned a permeability value from the range $10^{-13}$ to $10^{-15} \mathrm{~m}^{2}$. As modeled in SPM-1, the panel seals do not have halite elements, and have the properties of concrete for the full 10,000 years. Possible consolidation of backfill in the drifts was not modeled.

For the actinide source term, it was assumed that the total actinide inventory was available for liquid-phase transport as either dissolved or colloid-mobilized forms. This assumption was based on: 1) the lack of information concerning the solubility of actinidebearing materials in the WIPP waste; 2) the anticipated local variability of Eh and pH conditions within the waste/backfill; and 3) the lack of agreement concerning whether the data pertaining to actinide solubilities in fresh water are applicable and/or bounding.

The initial water content of the waste has been estimated as ranging from $0.04 \%$ to $5.2 \%$, with a median value of $0.44 \%$. This paramcter describes all water initially present in the 
waste, including that remaining as residual moisture in the pore volume of well-drained material. The parameter value is expressed as a percent of the total porosity that is filled with water.

\section{A.1.5 SPM-1 Assumptions about Human Intrusion}

Consistent with the current EPA guidelines, the intrusion rate for SPM-1 was modeled as 30 boreholes $/ \mathrm{km}^{2}$ for 10,000 years for all realizations. Intrusions were random in time, and the first intrusion was assumed to occur as early as 101 years after closure.

The arbitrary plugs used in the 1992 and previous PAs to divert flow into the Culebra were removed. All SPM- 1 borehole plugs were assigned permeability values from the range used in the 1992 PA, approximating that of silty sand. 


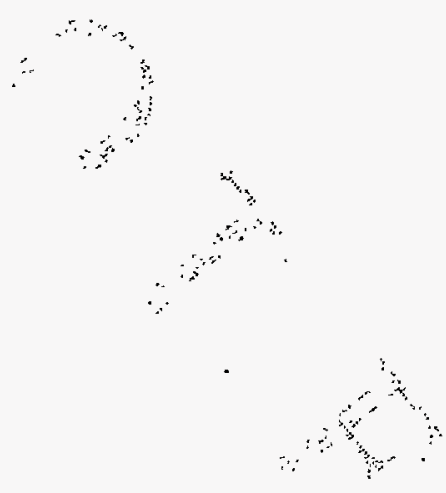

APPENDIX B: SPM-1 ACTIVITIES

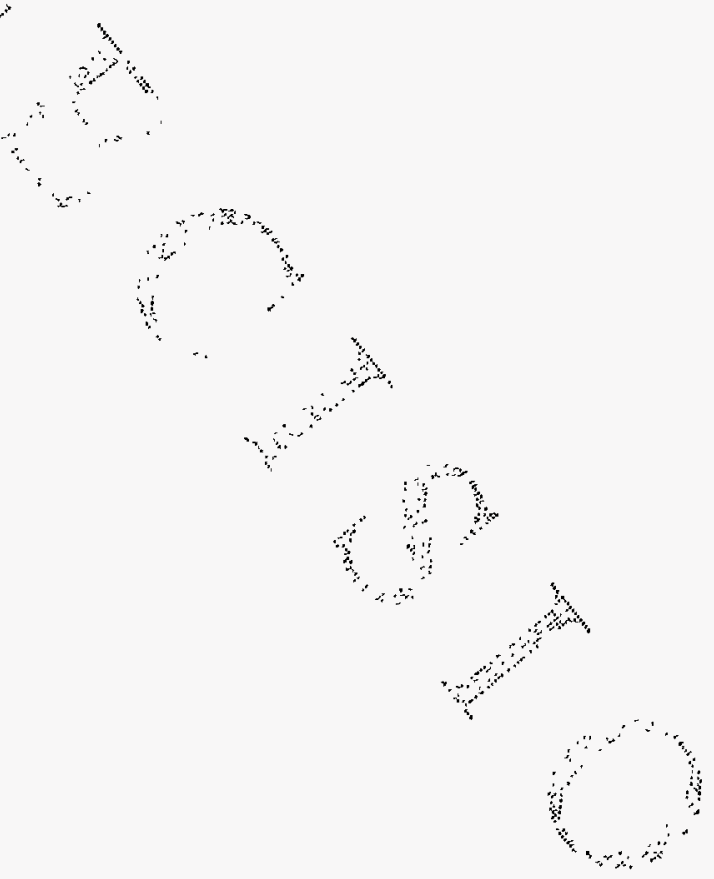




\section{APPENDIX B. SPM-1 ACTIVITIES}

The primary goal of the SPM is to calculate the probability for each activity set identified of demonstrating compliance with portions of $40 \mathrm{CFR} 191$ Subparts B and 40 CFR 268.6. The activities to be considered in a comprehensive application of the SPM will include activities managed by DOE that have the potential to affect quantitative aspects of regulatory compliance; for example, experimental programs at SNL, engineered alternatives to current repository design or waste form, and possible modifications to the waste acceptance criteria.

For SPM-1, a limited group of activities were selected by the SPM team at SNL based on the following criteria:

- The activities should cover a broad range of program options to provide a useful demonstration of the modeling system and the decision analysis methodology.

- Definition of the activities and their potential outcomes should be based on real WIPP examples. As stated in the following caveats, these outcomes are hypothetical and not for use in programmatic decision-making. However, the usefulness of the demonstration depends on analyzing problems similar to those that will be faced in future iterations.

- The total number of activity sets should be small enough that analyses can be completed within the time available.

- All models and data not explicitly discussed in this appendix remained the same as in the baseline.

Results based on these activities should not be used for programmatic decisions for the following reasons:

Caveat B-1. The choice of activities and the characterization of their potential outcomes do not have project-level input, and reflect only the beliefs of members of the SPM team at SNL.

Caveat B-2. The activities considered in SPM-1 do not cover the full range of options available to DOE-CAO. Some activities have not been considered at all (e.g., various engineered alternatives), and others may have been simplified in inappropriate ways.

Eleven activities were considered in the SPM-1. These activities are: Salado Flow, Shaft Seals, Actinide Solubilities, Culebra Physical Retardation, Culebra Chemical Retardation, Actinide Colloids, VOC Degradation, Cuttings/Spallings, and a combined category, PB-WAC and Engineering Enhancements activities. 


\section{B.1 Salado Flow Activities}

Many of the ongoing activities related to the Salado Formation are aimed at supporting the assumption that two-phase fluid flow in halite and anhydrite behaves according to Darcy's Law. This effort is aimed at both supporting the two-phase flow conceptual model and defining the appropriate parameter set needed to perform a two-phase flow calculation. Because of the inherent Darcy flow assumption in the present modeling system, SPM-1 cannot completely identify the data worth (i.e., estimate the value of the data relative to compliance), of all Salado activities. Some of the data worth will be essentially hidden from the analysis.

For the purposes of SPM-1 Salado activities, we expect future states of knowledge to change in two areas. We expect to be able to better define the two-phase flow characteristics of Salado halite, and we expect to better define the nature of preferential fluid flow in the Salado.

\section{The Brooks-Corey and Park}

The Brooks-Corey and Parker-Van Genuchten two-phase flow relationships produce fundamentally different fluid flow behaviors, and they may generally bound the expected fluid flow behaviors in the Salado. They may or may not, however, capture the extremes in flow behaviors. The WIPP project currently has a two-phase flow parameter measurement program underway that should define the range of behaviors in FY95. However, for the purposes of SPM-1, we assume that there is a $50 \%$ probability that fluid flow in the Salado will be controlled by the two-phase flow characteristics represented by the Brooks-Corey relationship. Furthermore, we assume that if Salado flow is not controlled by the Brooks-Corey relationship, it will be controlled by two-phase flow characteristics represented by the Parker-Van Genuchten relationship. Thus, for activity-set analysis, we would construct results using only Brooks-Corey relations for one outcome and results using only Parker/Van Genuchten relations for another outcome.

As a result of Salado activities, we expect to be able to defend calculated gas flow and VOC transport in the Salado instead of using the baseline conceptual model of instantaneous gas transport to the regulatory boundary when the pressure reaches $12.5 \mathrm{MPa}$. This assumes that we will be able to account for preferential flow behavior either directly in the low modeling or through the use of simplified modifications to symmetric flow modeling. SPM-1 uses a model for pressure-dependent fracturing of anhydrite where permeability and porosity of the anhydrite are increased as a function of pressure when pressure exceeds the undisturbed pore pressure of the unit. This model is referred to as the "altered anhydrite model." In addition, we multiply: the gas transport distance by a factor that is our current best estimate of the effects of . preferential flow process (i.e., anhydrite dip, heterogeneity, and fingering). This factor is used to determine the earliest time that gas might cross the regulatory boundary. Once it is 
determined that gas has crossed the boundary, VOC concentrations are determined based on the VOC source term and the dilution caused by the net gas generation at that time.

Probabilities assigned to these models, based on the assumption that all Salado activities are implemented, are as follows:

Table B-1. Salado Outcomes.

\begin{tabular}{|c|c|}
\hline Gas Flow Conceptual Models & Probability \\
\hline Baseline Gas Flow Model & 0.10 \\
\hline Altered Anhydrite Gas Flow Model & 0.90 \\
\hline \multicolumn{2}{|l|}{ Preferential Gas Flow Factor } \\
\hline 10 & 0.2 \\
\hline 6 & 0.3 \\
\hline 4 & 0.3 \\
\hline 2 & 0.1 \\
\hline 1 (Axisymmetric radial flow) & 0.1 \\
\hline
\end{tabular}

Activities related to sealing address many issues, such as performance of concrete elements, consolidation of salt elements, and DRZ grouting.

Table B-2. Seal Outcomes.

\begin{tabular}{|c|c|c|}
\hline \multicolumn{2}{|c|}{ Seal Permeability } & Probability \\
\hline \multicolumn{2}{|c|}{ Baseline Values } & 0.01 \\
\hline $10^{-16}-10^{-18}$ & Loguniform & 0.09 \\
\hline $10^{-18}-10^{-19}$ & Loguniform & 0.90 \\
\hline
\end{tabular}

Note that the seal program not only improves the permeability of the seal, it gives a better performing seal much earlier in the repository life. Therefore, the above permeability: ranges apply, beginning with emplacement, throughout the 10,000-year regulatory period. 
For SPM-1, we assume the length of the seal, as a result of seal program activities, will be the same as the baseline length; that is, 100 meters.

\section{B.3 Actinide Solubilities}

The following probabilities are assigned for ranges of actinide concentrations, assuming completion of the source-term related activities.

Table B-3. Actinide Solubilities Outcomes.

\begin{tabular}{ll}
\hline & Probability \\
\hline Inventory Limited (Baseline Values) & 0.01 \\
$10^{-14}-10^{1} M$ (Expert Judgment Values) & 0.49 \\
$10^{-14}-10^{-4} \mathrm{M}$ (Possible Reduced Range) & 0.50 \\
\hline
\end{tabular}

Note that the distributions for the baseline are defined in Appendix A.1.4 and the other distributions are the same as used for the 1992 PA analysis. In the case of reduced range, these distributions will be truncated at the specified upper endpoint of the range.

\section{B.4 Physical Retardation in the Culebra}

Sensitivity analyses conducted as part of the 1992 PA identified fracture spacing within the Culebra as the single parameter in the PA model that had the largest effect on physical retardation when using the dual porosity conceptual model. Fracture spacing in the PA transport model controls the amount of available surface area on fracture walls where contaminants can diffuse into the matrix.

The probabilities assigned for possible value ranges of Culebra fracture spacing, assuming implementation of activities related to physical retardation are shown in Table B-4.

\section{B.5 Chemical Retardation in the Culebra}

The chemical retardation activity determines effective values for the partitioning coefficients $\left(\mathrm{K}_{\mathrm{d}} \mathrm{s}\right)$ and chemical retardation models used in PA, based on the type of the : groundwater flow, on a laboratory scale, in Culebra core (i.e., whether it occurs in sub-: horizontal fractures and/or high-angle fractures, or whether it can be discriminated at all). The type of groundwater flow affects the selection of $\mathrm{K}_{\mathrm{d}} \mathrm{s}$ and the modeling of retardation, because 
Table B-4. Probability of Outcomes for

Physical Retardation Experiments.

\begin{tabular}{lcc}
\hline \multicolumn{1}{c}{ Culebra Fracture Spacing } & Probability \\
Baseline values & & 0.05 \\
Based on single well testing & 0.95 \\
0.1 to $8 \mathrm{~m}$ (Uniform) & \\
Based on single and multiwell testing & \\
0.1 to $3 \mathrm{~m}$ (Uniform) & 0.38 \\
0.1 to $1 \mathrm{~m}$ (Uniform) & 0.57 \\
\hline
\end{tabular}

clay deposits are located along the sub-horizontal fractures and in horizontal clay lenses. The flow in horizontal fractures will have much more contact with clay deposits than will the flow in high-angle fractures, which only intersect the thin clay layers. Probabilities for the five selected potential outcomes of the chemical retardation activity are shown in Table B-5.

For computational expediency. in SPM-1, Cases 2 and $3 \mathrm{~b}$ were both modeled assuming bulk rock $\mathrm{K}_{d} \mathrm{~s}$ based on a mixture of $95 \mathrm{wt} \%$ dolomite and $5 \mathrm{wt} \%$ clay. In the SPM-1 analysis, the $\mathrm{K}_{\mathrm{d}} \mathrm{s}$ were calculated based on $1992 \mathrm{PA}$ distributions for dolomite and clay. For each vector, a matrix $K_{d}$ and a clay $K_{d}$ were sampled then weighted. Cases $3 a$ and $3 c$ were both modeled assuming a clay lining and a fracture retardation factor calculated from 1992 clay $\mathrm{K}_{\mathrm{d}} \mathrm{s}$. Therefore, the 20 possible permutations of physical and chemical retardation $(4 \times 5)$ can be reduced to the five distinct cases shown in Table B- 5 .

Single Porosity:

1. Fracture flow where clay lining and fracture concentration equilibrium is assumed; fracture retardation is calculated from $\mathrm{K}_{\mathrm{d}} \mathrm{s}$ based on a $5 \mathrm{wt} \%$ clay mixture (Cases 2 and $3 b)$.

2. Fracture flow retardation where clay and fracture concentration equilibrium is assumed; fracture retardation is a function of clay $\mathrm{K}_{d} \mathrm{~s}$ from the 1992 PA (Cases $3 \mathrm{a}$ and 3c).

Dual Porosity:

3. Fracture flow; no clay; matrix $K_{d}=0.0$; use fracture spacing physical retardation outcome 2 (see Table B-5).

4. Fracture flow; no clay; matrix $K_{d}=0.0$; use fracture spacing physical retardation outcome 3 (see Table B-5).

5. Fracture flow; no clay; matrix $K_{d}=0.0$; use fracture spacing physical retardation outcome 4 (see Table B-5). 
Table B-5. Probabilities of Potential Outcomes

for the Chemical Retardation Activity

\begin{tabular}{|c|c|}
\hline Outcome & Probability \\
\hline $\begin{array}{l}\text { Case } 1 \text { - No retardation } \\
\text { (baseline) }\end{array}$ & 0.01 \\
\hline $\begin{array}{l}\text { Case } 2 \text { - No distinction } \\
\text { between flow in sub- } \\
\text { horizontal and high-angle } \\
\text { fractures }\end{array}$ & 0.95 \\
\hline $\begin{array}{l}\text { Case } 3 a-\text { Flow in sub- } \\
\text { horizontal fractures only }\end{array}$ & 0.0133 \\
\hline $\begin{array}{l}\text { Case } 3 b-\text { Flow in high- } \\
\text { angle fractures only }\end{array}$ & 0.0133 \\
\hline $\begin{array}{l}\text { Case } 3 c-\text { Flow in both } \\
\text { sub-horizontal and high- } \\
\text { angle fractures w/ significant } \\
\text { distinguishing differences }\end{array}$ & 0.0133 \\
\hline
\end{tabular}

Note 1: In the case of single porosity and no retardation, all radionuclides released in the Culebra were assumed to instantaneously travel to the regulatory boundary. Release to the accessible environment was assumed equal to release to the Culebra as calculated by the PANEL code. Past PAs have shown that the conceptual model.consisting of single porosity fracture flow with no retardation leads to relatively fast travel times, much less than 10,000 years.

Note 2: In the case of dual porosity and chemical retardation (cases $2,3 \mathrm{a}, 3 \mathrm{~b}$, and $3 \mathrm{c}$ ), past PAs have demonstrated that integrated release at the regulatory boundary is very small. Therefore, it was not necessary to recalculate these cases.

\section{B.6 Actinide Colloids}

Colloids are solid particles small enough to remain suspended in liquids. Rádionuclidebearing colloids may form in the repository environment or during transport within the Culebra. Colloids have the potential to affect radionuclide transport because they will have different physical and chemical properties than dissolved species. Specifically, physical retardation (diffusion) may not occur if colloid diameters are sufficiently large, relative to fracture. apertures, that most transport occurs in central portion of the flow path. Furthermore, chemical retardation by sorption may not occur for colloids. 
Colloids are assumed to be preferentially mobilized over dissolved species and, once mobilized, they are assumed to be transported with no chemical or physical retardation in the Culebra.

Table B-7 shows the probabilities of the possible outcomes for the colloid investigation activity expressed as total actinide concentrations in mobile colloids (moles/liter).

Table B-7. Probabilities of Outcomes for Colloid Experiments

\begin{tabular}{ccc}
\hline $\begin{array}{c}\text { Outcome Category Total } \\
\text { Concentration of Actinides } \\
\text { in Moles/Liter }\end{array}$ & & Probability \\
\cline { 1 - 1 } $10^{-9}$ & & 0.33 \\
$10^{-8}$ & & 0.33 \\
$10^{-7}$ & & 0.18 \\
$10^{-6}$ & & 0.09 \\
$10^{-5}$ & & 0.05 \\
$10^{-4}$ & & 0.01 \\
Inventory Limited & & 0.01 \\
\hline
\end{tabular}

Interpretation of experimental outcomes from colloid-related activities are likely to be straightforward. However, application of the results will depend on the outcomes of other activities (such as source term), and policy decisions (such as PB-WAC). In particular, the formation of colloids in the repository environment is expected to be sensitive to the presence of humic acids contained in soil-rich waste forms. The two cases below account for a potential effect of humic acids on the concentration of actinides in mobile colloids.

\section{B.6.1 Case 1}

If the waste inventory has no significant amounts of high molecular weight organic materials, such as those found in soils, the parameters for the corresponding lognormal probability distribution for the total concentration of actinides to be used in the PA model are those given in Table B-8. 
Table B-8. Conditional Probability Distributions for

PA Total Actinide Colloid Concentration

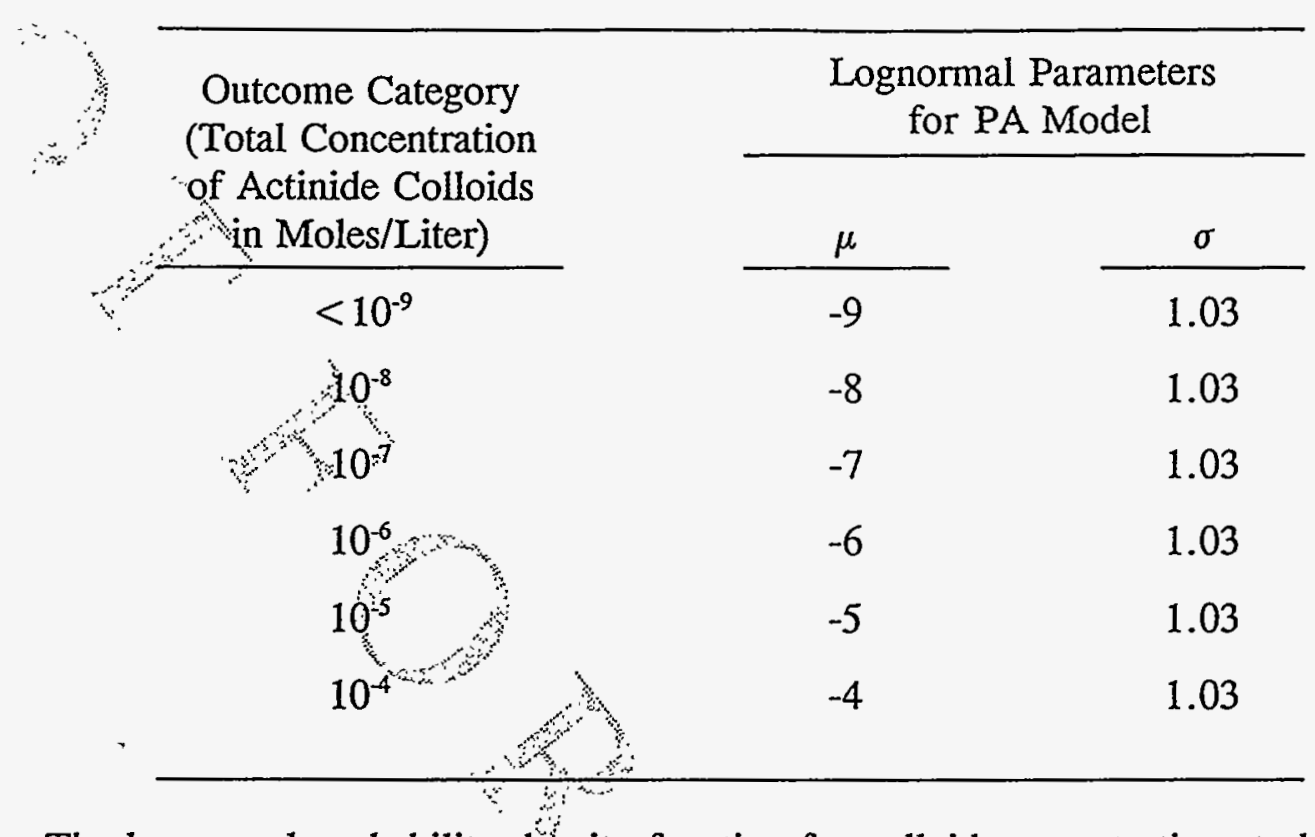

The lognormal probability density function for colloid concentrations to be used in the SPM- 1 activity sets code evaluations is given by:

$$
f(x ; \mu, \sigma)=(\sigma x)^{-1}(2 \pi)^{-1 / 2} \exp \left[\frac{-(\ln x-\mu)^{2}}{\sigma^{2}}\right]
$$

where $\mu$ is the mean and $\sigma$ is the standard deviation:

\section{B.6.2 Case 2}

For wastes containing significant amounts of high molecular weight organic materials such as those found in soils (for example, wastes related to decommissioning and environmental restoration), the concentrations used in the PA model (see Table B-8) are multiplied by 10. Otherwise the information in Tables B-7 and B-8 remain unchanged.

\section{B.7 Cuttings/Spallings}

Spalling occurs when solid waste material is transported into an intruding borehole as the repository depressurizes. The amount of waste released by spalling will depend on the strength: and permeability of the waste, and the pressure within the repository, among other factors. 'The' ongoing spalling activities are designed to provide better understanding of the waste's response to rapid clepressurization. 
Based on the assumption that either a decision will be made to control the waste permeability, or that completion of spalling-related activities will result in a spalling model that includes a waste strength parameter and a non-zero value for waste strength, the following probabilities are assigned to a factor used to scale the baseline spalling release. Calculated baseline spalling releases are multiplied by the scaling factor.

Table B-9. Spalling Outcomes

\begin{tabular}{ccc} 
Spalling Scaling Factor & & Probability \\
$\begin{array}{c}\text { 1 (baseline release, } \\
\text { zero waste strength) }\end{array}$ & & 0.05 \\
0.5 & & 0.20 \\
$\ddots \quad 0.1$ & 0.35 \\
0.01 & 0.40 \\
\hline
\end{tabular}

\section{B.8 PB-WAC and Engineering Enhancements}

The idea behind PB-WAC is to control the type, form, or quantity of waste accepted by the WIPP in a fashion that improves the performance of the system with respect to the applicable regulations. For the purposes of SPM-1, only three conditions were investigated. These are PBWAC Excluding Soils, PB-WAC Excluding RCRA VOCs, and PB-WAC Excluding All Metals.

\section{B.8.1 PB-WAC Excluding Soils}

Modifying the waste acceptance criteria to limit or exclude soil from the waste could reduce humic acids in the waste, and thereby limit colloid formation. The treatment of this hypothetical change is discussed in Appendix B.6.

\section{B.8.2 PB-WAC Excluding RCRA VOCs}

If the hazardous constituent concentration CI shows non-compliance due to the transport of a single or small number of VOCs, a solution may be found by simply applying a PB-WAC limitation on the inventory of specific VOCs.

Possible benefits of this hypothetical PB-WAC are considered in SPM by assuming that it is possible to exclude VOCs from the inventory. 


\section{B.8.3 PB-WAC Excluding All Metals}

Numerous engineering enhancements can be thought of as limiting the impacts of gasgeneration from waste degradation processes. For the purposes of SPM-1, these are combined into a single category that limits the total gas potential by reducing the amount of corrodible metal in the repository to zero. Note that the cellulosic and plastic content are the same as defined in the June 1994 BIR, and are therefore the same as those used in the SPM-1 baseline.
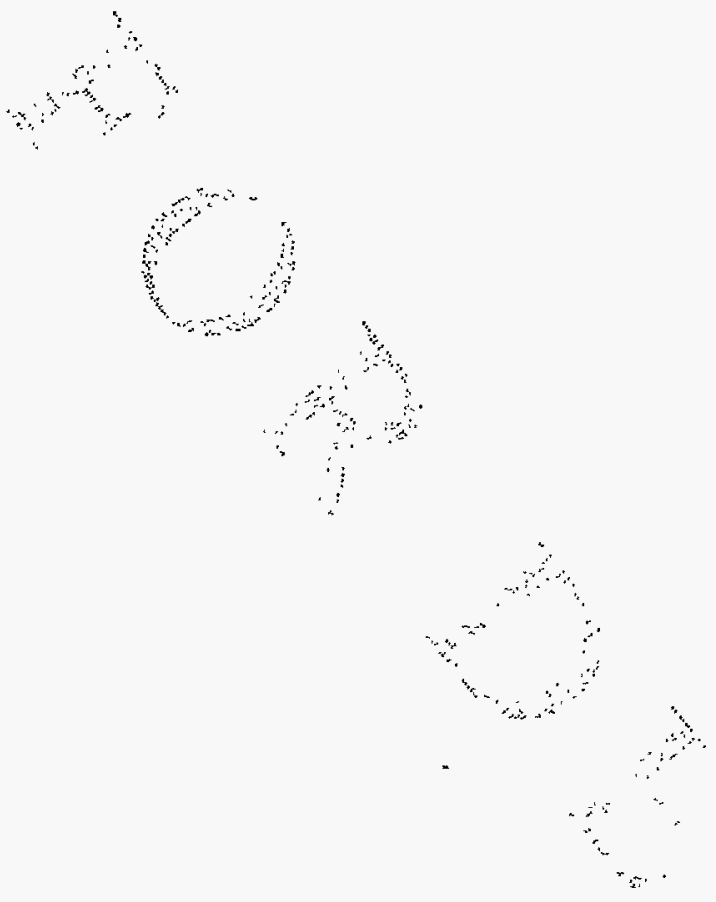
APPENDIX C: COMPLETE LIST OF SPM-1 CAVEATS AND ASSUMPTIONS 
1. The SPM-1 baseline is an estimate of the future PTB used for the purposes of prototyping the SPM and reflects only the beliefs of members of the SPM team at SNL. It does not include sufficient SNL, Westinghouse Waste Isolation Division (WID), DOE-CAO, regulator, or stakeholder involvement to represent a full range of concerns and therefore is not useful for drawing programmatic conclusions.

2. Only conceptual models that could readily be incorporated into the modeling structures used in the 1992 PA analysis were considered for the SPM-1 baseline and the activity set outcomes.

3. Possible outcomes of the SPM-1 activities were defined in part using information obtained in limited and informal elicitation of the PIs and are estimates for the purposes of prototyping the SPM. These activity outcomes are estimates for the purposes of prototyping the SPM. The interpretation of these outcomes and their use in SPM-1 are the sole responsibility of the SPM team at SNL.

4. The tie between activity sets and cost and schedule for SPM-1 is an estimate for the purposes of prototyping the SPM.

5. While in general we believe that the decision matrix information correctly reflects the results of the computer modeling, there may be isolated errors in results because of insufficient time to check the output and behavior of each run of each code in detail, due to the large amount of information handled.

6. The SPM-1 analysis is limited to evaluating the ability of the WIPP disposal system to meet the post-closure regulatory requirements as defined in Section 5. Any other requirements from the regulator are not considered in this analysis.

7. Baseline model assumptions and parameters used in SPM-1 could not be defended in a compliance application because of Caveat 1. The SPM-1 results cannot be used to draw conclusions about compliance.

8. The baseline contains embedded assumptions that presuppose the completion of certain activities; e.g., the assumption that fluid flow in the Salado Formation behaves according to: Darcy's Law may require the completion of some subset of Salado experiments in order to be: defensible. 
9. The choice of activities and the characterization of their potential outcomes do not have project-level input, and reflect only the beliefs of members of the SPM team at SNL.

10. The activities considered in SPM-1 do not cover the full range of options available to DOECAO. Some activities have not been considered at all (e.g., various engineered alternatives), and others may have been simplified in inappropriate ways.

11. For SPM-1, only those stakeholder concerns received in writing prior to May 1994 and that could be easily implemented as simple code modifications were addressed.

12. For SPM-1, system performance was estimated using the baseline prescribed by the SPM management team (as opposed to the PTB), as described in Appendix A.

13. For SPM-1, potential outcomes of activity sets and the probabilities of these outcomes were prescribed by the SPM management team (as opposed to formal elicitations or a project-wide reference base), as described in Appendix B.

14. For SPM-1, assumptions based on experience from past PAs were used to screen unnecessary calculations.

15. The extent of the information derived from the SPM analysis, coupled with the short time available to study the resulting decision matrix, makes it virtually certain that more conclusions and insights will be obtained in the future.

16. For SPM-1, activities that are to take place during the operational period of the WIPP are not considered.

17. SPM-1 does not address 40 CFR Part 191, Subpart B, 191.15 (individual protection requirements), and 40 CFR Part 191, Subpart C (environmental standards for groundwater protection).

18. For SPM-1, compliance with the radionuclide containment regulatory requirements is assumed when the mean CCDF meets the requirements of 40 CFR Part 191, Subpart B, 191.13(a), i.e., below the values specified in the regulations.

19. For SPM-1, compliance with post-closure hazardous constituent concentration regulatory requirements is assumed when the hazardous constituent concentration calculated at the unit boundary is less than the health-based level for soil for each hazardous constituent. 
20. For SPM-1, the anhyclrite interbed pathway uses from the top of marker bed 138 to the bottom of marker bed 139, the model shaft diameter, and gas available porosity, for concentration calculations.

21. The hazardous constituent concentrations are calculated from the mean of the multiple deterministic concentration calculations to include the effect of parameter uncertainty.

22. For the baseline, regional groundwater flow is modeled using the 70 calibrated transmissivity fields used in the 1992 PA from which 20 sample fields were randomly drawn.

23. For the baseline; the Culebra is modeled as a two-dimensional (2D) confined aquifer (no leakage).

24. In response to stakeholder concerns that the treatment of climate change is not defensible, SPM-1 uses the same approach used in the 1992 PA of varying boundary heads in a northern "recharge strip," except that heads remain-at their elevated position for the entire 10,000 years. This results in the maximum possible head gradient across the site, and provides an upper bound on the affect of climate change within the context of the $2 \mathrm{D}$ flow model.

25. The baseline model for radionuclide transport in the Culebra is one of the conceptual models analyzed in the 1992 PA with no chemical sorption and no physical retardation transport, i.e., single-porosity fracture-only transport.

26. For the baseline, colloids are assumed to be transported at the same rate as dissolved mobilized actinides; i.e., they simply travel with the flow freld.

27. Uncertainty about the rate at which colloids are transported is believed to be insignificant compared to the uncertainty in the flow field that is already incorporated in the analysis.

28. The BRAGFLO model now approximates pressure-dependent fracturing of anhydrite interbeds by varying porosity and permeability as a function of pressure when gas generation creates pressures that approach or exceed lithostatic.

29. Above the water table, the brine saturation was taken to be 0.20 and pressure was 1 atm. Below the water table, pressures were calculated (initial conditions) assuming hydrostatic gradient with fluid (brine) density of $1230 \mathrm{Kg} / \mathrm{m}^{3}$ and $\mathrm{g}=9.79 \mathrm{~m} / \mathrm{sec}^{2}$.

30. Initial excavated regions had pressure equal to $1 \mathrm{~atm}$. 
31. For the baseline, if at any time pressure within the repository approaches or exceeds 12.5 $\mathrm{MPa}$ pressure in the simulation, the gas phase in the repository is assumed for the purposes of estimating a CI to be directly connected to the regulatory boundary through the anhydrite interbeds.

32. For the baseline, no estimate is made of possible VOC degradation or sorption during transport.

33. For the baseline, Caveats 31 and 32 bound the consequences of gas migration with respect to 40 CFR 268.6.

34. A comparable bounding assumption (Caveats 31 and 32) is not made for liquid-phase flow and transport in the Salado.

35. The dissolution of gas in brine is not addressed in the SPM-1 baseline. Gas transport is faster than brine transport; not allowing gas to dissolve in brine is therefore a conservative assumption.

36. Vertical fracturing was not considered in SPM-1 because vertical fractures are not expected in the Salado.

37. In the shaft, initial brine saturation was taken to be 0.25 except in the zones above the water table, where the value 0.20 was used.

38. Residual brine and gas saturations were 0.20 in all shaft regions, panel seals, backfill, and experimental rooms.

39. Initial saturation in panel seals, experimental, and backfill was 0.25 .

40. Capillary pressures were assumed to be 0 in shaft regions above the top of the Culebra, the Dewey Lake, and Santa Rosa as well as the MB139, MB138, and Anhydrite A and B.

41. Physical properties of waste and backfill were treated in the same manner as in the 1992 PA.

42. The radionuclide inventory was based on the 1994 Baseline Inventory Report (BIR) with the exception that remote handled $(\mathrm{RH}) \mathrm{U}^{235}$ were reduced to transportation limits.

43. The VOC inventory was based on the best information available to DOE-CAO at this time. 
44. Room closure occurs, according to the 1992 porosity surface, until gas pressure reaches lithọstatic. Thereafter, the room porosity and permeability remain constant.

45. The gas-generation model used in SPM- 1 was that used in the 1992 PA, updated with rate information documented in the June 18, 1993, memorandum of record from L.H. Brush to M.S. Tierney titled, "Likely Gas-Generation Reactions and Current Estimates of Gas-Generation Rates for the Long-Term Performance Assessment."

46. In response to stakeholder concerns about the treatment of the plastic and rubber inventory in past analyses, all plastic and rubber material are available for degradation reactions.

47. For the purpose of SPM-1, the repository and surrounding strata are assumed to be horizontal.

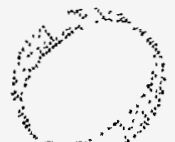

48. The human intrusion model estimated cuttings and cavings in the same manner as in the 1992 PA.

49. For two-phase flow calculations, waste permeability remained constant at $10^{-12} \mathrm{~m}^{2}$. However, for the cavings, cuttings, and spalling calculations, waste permeability values were sampled from the uniform probability distribution of $10^{-12}$ to $10^{-17} \mathrm{~m}^{2}$.

50. The Disturbed Rock Zone (DRZ) was modeled with the halite regions having far-field halite properties for porosity, but an enhanced permeability, $\mathrm{i}_{\mathrm{i}} \mathrm{e}, \mathrm{k}=10^{-15} \mathrm{~m}^{2}$, under the assumption that they do not act as a significant barrier to fluid flow.

51. The concrete elements for all repository and panel seals were be assumed to have permeability values from $10^{-12}$ to $10^{-14} \mathrm{~m}^{2}$ for the full 10,000 years.

52. For the baseline, the crushed salt portions of the seals were modeled as having permeability two orders of magnitude less than the concrete elements for the first 200 years. From then on, the lowest $100 \mathrm{~m}$ of crushed salt was assigned a permeability value from the range $10^{-13}$ to $10^{-15} \mathrm{~m}^{2}$.

53. Possible consolidation of backfill in the drifts was not modeled.

54. It was assumed that the total actinide inventory was available for liquid-phase transport as . either dissolved or colloid-mobilized forms. 
55. The initial water content of the waste has been estimated in a June 4, 1993, memorandum by B.M. Butcher and Lincoln as ranging from $0.04 \%$ to $5.2 \%$, with a median value of $0.44 \%$.

56. The SPM-1 baseline did not include the effects of nuclear criticality.

57. The intrusion rate for SPM-1 was modeled as 30 boreholes $/ \mathrm{km}^{2}$ for 10,000 years for all realizations.

58. Intrusions were random in time, and the first intrusion was assumed to occur as early as 101 years after closure.

59. All SPM-1 borehole plugs were assigned permeability values from the range used in the 1992 PA, approximating that of silty sand.

60. Brine releases to the ground surface during drilling was not examined in SPM-1.

61. Because of the inherent Darcy flow assumption in the present modeling system, SPM-1 can not completely identify the data worth, i.e., estimate the value of the data relative to compliance, of all Salado activities.

62. For the purposes of SPM-1 Salado activities, we expect future states of knowledge to change in two areas. We expect to be able to better define the two-phase flow characteristics of Salado halite, and we expect to better define the nature of preferential fluid flow in the Salado.

63. For the purposes of SPM-1, we assume that there is a $50 \%$ probability that fluid flow in the Salado will be controlled by the two-phase flow characteristics represented by the BrooksCorey relationship.

64. We assume for SPM-1 that if Salado flow is not controlled by the Brooks-Corey relationship, it will be controlled by two-phase flow characteristics represented by the ParkerVan Genuchten relationship.

65. For SPM-1, we expect as a result of Salado activities to be able to defend calculated gas: flow and VOC transport in the Salado. 
66. For SPM-1, we assume that we will be able to account for preferential flow behavior either directly in the flow modeling or through the use of simplified modifications to symmetric flow modeling.

67. For SPM-1, we assumed the information in Table B-1.

68. For SPM-1, we assumed the information in Table B-2.

69. For SPM-1, we assumed the length of the seal, as a result of seal program activities, will be the same as the baseline length; that is, 100 meters.

70. For SPM-1, we assumed the information in Table B-3.

71. Sensitivity analyses conducted as part of the 1992 PA identified fracture spacing within the Culebra as the single parameter in the PA model that had the largest effect on physical retardation when using the dual porosity conceptual model.

72. For SPM-1, we assumed the information in Table B-4.

73. For SPM-1, we assumed the information in Table B-5.

74. For computational expediency in SPM-1, Cases 2 and $3 \mathrm{~b}$ were both modeled assuming bulk rock $\mathrm{K}_{\mathrm{d}} \mathrm{s}$ based on a mixture of $95 \mathrm{wt} \%$ dolomite and 5 wt \% clay.

75. Cases $3 a$ and $3 c$ were both modeled assuming a clay lining and a fracture retardation factor calculated from 1992 clay $\mathrm{K}_{\mathrm{d}} \mathrm{s}$.

76. The 20 possible permutations of physical and chemical retardation $(4 \times 5)$ can be reduced to the following five distinct cases.

Single Porosity:

1. Fracture flow where clay lining and fracture concentration equilibrium is assumed; fracture retardation is calculated from $\mathrm{K}_{\mathrm{d}} \mathrm{s}$ based on a $5 \mathrm{wt} \%$ clay mixture (Cases 2 and $3 \mathrm{~b}$ ).

2. Fracture flow retardation where clay and fracture concentration equilibrium is assumed; fracture retardation is a function of clay $\mathrm{K}_{d}$ s from the 1992 PA (Cases 3a and 3c).

Dual Porosity:

3. Fracture flow; no clay; matrix $\mathrm{K}_{\mathrm{d}}=0.0$; use fracture spacing physical retardation outcome 2 (see Table B-5). 
4. Fracture flow; no clay; matrix $\mathrm{K}_{\mathfrak{d}}=0.0$; use fracture spacing physical retardation outcome 3 (see Table B-5).

5. Fracture flow; no clay; matrix $\mathrm{K}_{\mathrm{d}}=0.0$; use fracture spacing physical retardation outcome 4 (see Table B-5).

77. For.SPM-1, in the case of single porosity and no retardation, all radionuclides released in the Culebra were assumed to instantaneously travel to the regulatory boundary.

78. In the case of dual porosity and chemical retardation (cases 2, 3a, 3b, and 3c), past PAs have demonstrated that integrated release at the regulatory boundary is very small. Therefore, it was not necessary to recalculate these cases.

79. For SPM-1, colloids are assumed to be preferentially mobilized over dissolved species.

80. For SPM-1, once mobilized, colloids are assumed to be transported with no chemical or physical retardation in the Culebra.

81. For SPM-1, we assumed the information in Table B-7.

82. For SPM-1, we assumed the information in Table B-8.

83. For SPM-1, wastes containing significant amounts of high molecular weight organic materials such as those found in soils (for example, wastes related to decommissioning and environmental restoration), the colloids concentrations used in the PA model (as given in Table B-8) are multiplied by 10 .

84. For SPM-1, we assumed the information in Table B-9. 

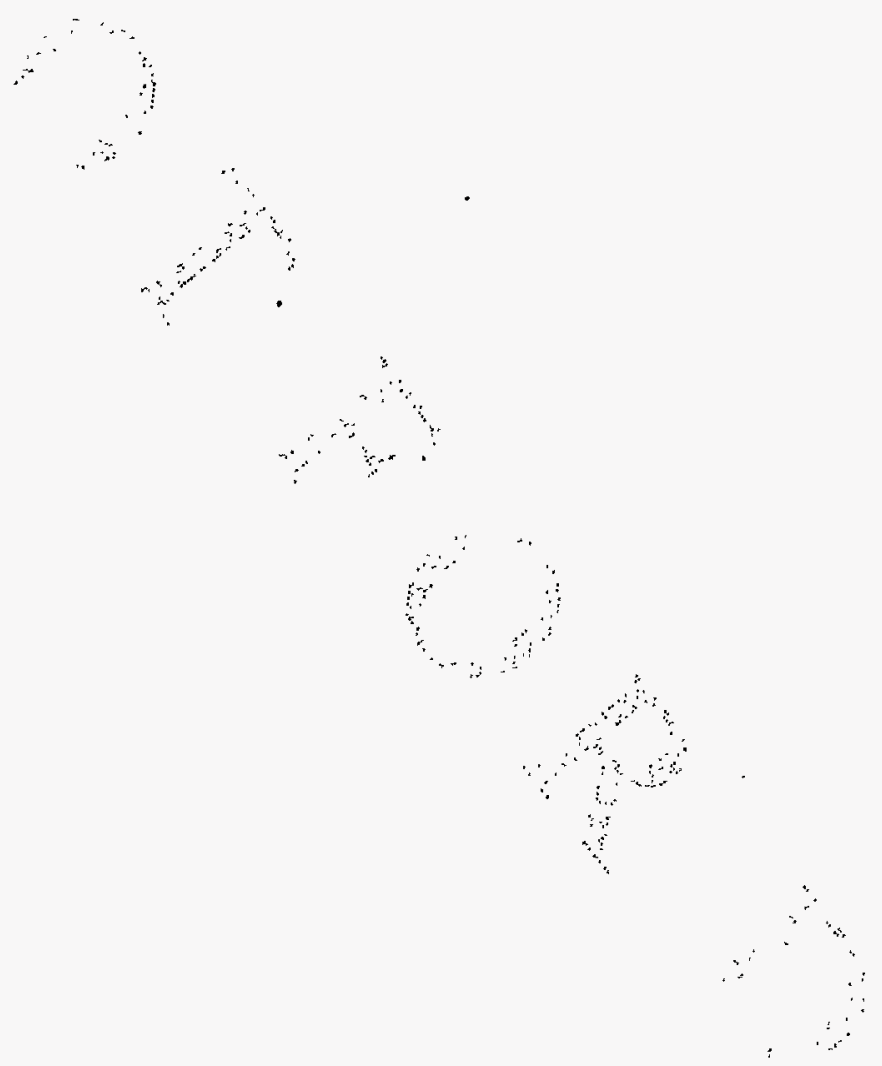
APPENDIX D: GLOSSARY AND ACRONYMS 


\section{APPENDIX D: GLOSSARY AND ACRONYMS}

\section{Glossary}

Activity - A field experiment, lab experiment, novel analysis, change in the engineering design, change in the waste acceptance criteria, discussion with the regulator, literature search of existing information bases or other option available to the WIPP Project, that has the potential to influence regulatory Cls:

Activity Set - A collection of activities that constitutes a coherent program option. SPM associates a probability of demonstrating compliance with each activity set.

Compliance Indicator (CI) The binary measure that indicates whether the WIPP disposal system is predicted to succeed or fail with respect to meeting the specific post-closure performance requirements in 40 CFR 191.13(a) and 40 CFR 286.6.

Dual Permeability - A conceptualization of flow and transport through a fractured porous media where flow, advective transport and mechanical dispersion take place through both the fractures and pore space simultaneously. Exchange of fluid and solute between the two continua are also modeled.

Dual Porosity - An idealized conceptualization of contaminant transport through a fractured, porous media where advective transport is assumed to take place only in the fracture and there is a local exchange of solute between the fracture and matrix controlled by one dimensional diffusion. No transport is assumed to take place in the matrix. The dual-porosity model results in physical retardation of solute. There is also an equivalent dual-porosity conceptualization for fluid flow that is not modeled in the SPM-1 PA.

E1 Intrusion Scenario - A characterization of an alternative future state of the WipP disposal system that models an inadvertent exploratory borehole intersecting the repository and a hypothetical pressurized brine reservoir in the underlying Castile formation.

E2 Intrusion Scenario - A characterization of an alternative future state of the WIPP disposal system that models an inadvertent exploratory borehole intersecting the repository but missing the hypothetical brine reservoir.

E1E2 Intrusion Scenario - A characterization of an alternative future state of the WIPP disposal. system that modes two inadvertent exploratory boreholes intersecting the repository, only one of which hits an underlying brine reservoir. With robust pancl seals both boreholes must intersect a 
panel: panels are isolated. With degraded or tailed seals, any two such boreholes must intersect the repository

Probability of Demonstrating Compliance - The probability that, if a particular activity set is implemented, a defensible argument for compliance could be made based on the outcome of the activity set.

\section{Acronyms}

BIR - Baseline Inventory Report

CCDF - Complementary Cumulative Distribution Function

CI - Compliance Indicator

DDP - Disposal Decision Plan

DOE - Department of Energy

DOE-CAO - Department of Energy Carlsbad Area Office

DRZ - Disturbed Rock Zone

EPA - Environmental Protection Agency

IDB - Integrated Data Base

PA - Performance Assessment

PI - Principal Investigator

PTB - Project Technical Baseline

RCRA - Resource Conservation and Recovery Act

SNL -Sandia National Laboratories

SPM - Systems Prioritization Method 
SPM-1 - The first iteration of SPM

SPM-2 - The second iteration of SPM

PB-WAC - Performance Based Waste Acceptance Criteria

WBS - Work Breâkdown Structure

WID - Westinghouse Waste Isolation Division
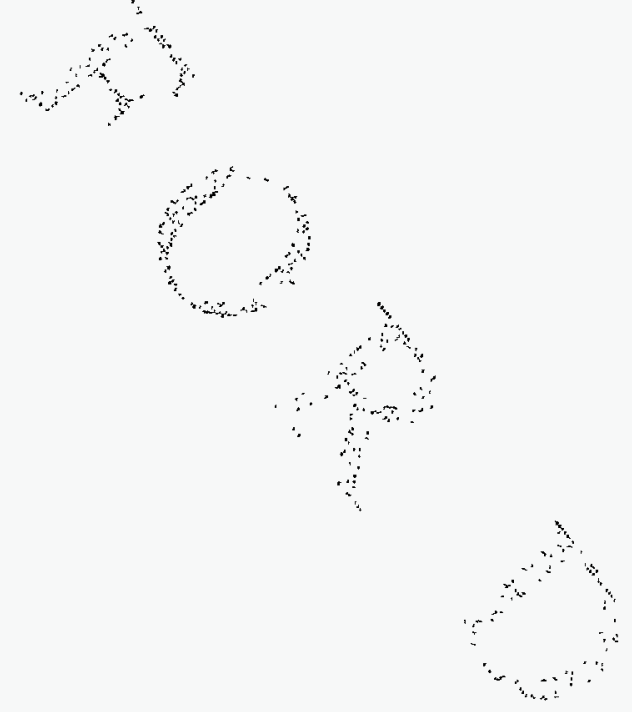
77. Berglund, J.W. "Memorandum of Record: The Direct Removal of Waste Caused by a Drilling Intrusion into a WIPP Panel-A Position Paper." August 31, 1994. 


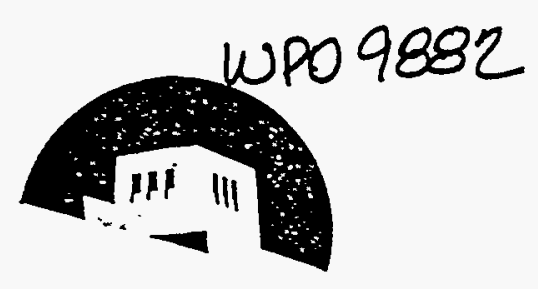

NFORMATION ONLY

The University of New Mexico

New Mexico Engineering Research Institute

Albuquerque, NM 87131-1376

USA

Date: 31 August 1994

From: J. W. Berglund Org 6342 (505 272-7228)

Subject: The Direct Removal of Waste Caused by a Drilling Intrusion Into a WIPP Panel --.A Position Paper

\section{Release Processes}

The 1992 Preliminary Performance Assessment of WIPP discusses three separate physical processes that can influence the quantity of waste brought to the ground surface as the result of the inadvertent penetration of the repository by an exploratory drill bit (WIPP PA, 1992a, 1992b, 1992c). These are:

*cuttings - waste contained in the cylindrical volume created by the cutting action of the drill bit passing through the waste.

*cavings - waste that erodes from the borehole in response to the upward-flowing drilling fluid within the annulus, and

*spallings - waste introduced into the drilling fluid caused by the release of wastegenerated gas escaping to the lower-pressure borehole.

For the first two processes (cuttings and cavings) a quantitative model was developed and forms the basis for the code CUTTINGS (Rath, 1994) currently being used in performance assessment (PA) of the WIPP and forms a part of the CAMCON (Rechard,1990) system of PA codes. The CUTTINGS code as currently configured does not address the third process, namely direct waste removal during a drill intrusion that may arise from the presence of high pressure waste decomposition gas in the waste (spallings). A model for spall that approximates an upper bound for releases has been developed for systems prioritization (SP) but has not been used in prior PA. computations. The model assumes a waste form that has no cohesive strength and includes additional assumptions concerning drilling practices and transport rates up the borehole.

The direct removal of waste to the surface caused by a drilling intrusion is limited by environmental standards for disposal of transuranic waste as defined in 40 CFR 191 Subpart B (US EPA, 1985). The direct removal of waste to the surface does not fall under regulations governed by the Resource Conservation and Recovery Act (RCRA) for the management of hazardous wastes as described in 40 CFR 268 (US EPA, 1986). 
Two additional processes that have not been included in previous PA's that may contribute to surface releases are:

*brine spall - waste introduced into the drilling fluid caused by the flow of pressurized brine through the waste into the borehole. This process may occur due to a dipped repository. The brine introduced into the borehole and then to the surface also must be considered a release since it contains dissolved radionuclides.

*brine slurry - somewhat related to brine spall where the waste, drilling fluid and repository brine form a slurry that is carried to the surface.

In the following sections each of these conditions will be addressed and conceptual models for waste release discussed.

\section{Conceptual/Mechanistic Models and Assumptions}

Appendix C of 40 CFR 191 (US EPA, 1985) presents guidance for the implementation of subpart $B$ which suggests that the most severe intrusion scenario into WIPP can be assumed to be that caused by intermittent exploratory drilling for resources. For the 1992 performance assessment of WIPP it has been assumed that such exploratory drilling is limited to the drilling for hydrocarbon resources and that the procedures used in drilling are comparable to the practices in use today for drilling in the Delaware basin.

\section{Drilling in the Delaware Basin Near WIPP}

In considering the problem of the direct releases caused by drilling it is important to understand the procedures currently utilized in drilling a gas or oil well near the WIPP site. Typically, independent drilling companies respond to requests for fixed price bids from oil companies to provide drilled and cased holes of a specified diameter to a specified depth at a location leased by the oil company. The bids are competitive and generally the low bidder is selected. As a result of this bidding process, profit margins for the winning driller are governed by the effectiveness of the driller in maintaining efficient drilling parameters and by solving unforeseen difficulties that may arise quickly and safely. The principal goal of the driller is to "make hole" and meet contract requirements. If this is accomplished the drilling operation will be profitable (Jensen, 1993).

Within the Delaware Basin near the WIPP site, gas and oil wells are started by clearing the site, and drilling a shallow hole $\left(-40^{\prime}\right)$ to house a conductor pipe. The conductor pipe is set in cement and serves to prevent surface sands from sloughing into the wellbore during later drilling. Below the conductor, drilling is continued to $300-600$ feet (to top of salt section) using a large diameter (17-26") drill bit and another steel casing is set. This "surface" casing is cement grouted into the hole and a blowout preventer tree is attached to the casing at the surface. Drilling below the surface casing (in the salt section) uses a drilling mud that consists of a saturated brine solution to prevent dissolution of the salt. Drilling through the salt section proceeds at rates from 50 to 100 feet/hour depending on bit diameter. If higher rates are attempted by increasing the weight on bit (WOB) there is the danger of producing a crooked hole. While in the salt section, drilling mud (brine) is supplied from a large plastic lined reserve pit dug in the ground with a surface area of approximately $4000 \mathrm{ft}^{2}$. Drilling mud is pumped from the reserve pit down through the drillpipe and drillbit and up the annulus formed by the drillstring and drilled hole. The drilling mud and drill cuttings are returned directly to the reserve pit where the cuttings settle out. While drilling in the salt section no formal attempt is made to monitor the character of the cuttings or the fluid volume of the reserve pit. A gas analyzer is not attached to the retums until the hole is much deeper than the depth of the WIPP repository. The drillstring generally consists of a drillbit attached to approximately 20,30 foot long drill collars followed by multiple sections of 30 foot long $41 / 2$ " diameter drillpipe. One to four drillbit changes may be necessary when drilling 
through the salt section. This process requires the removal of the entire drillstring (tripping). Once the drill has passed the salt section $(-4500 \mathrm{ft})$ the hole is again cased and set with cement. At this point, if the repository had been penetrated no further contact would occur between the drilling mud and the exposed WIPP waste (Jensen, 1993).

For the conceptual models described herein the releases to the surface generally occur over a time scale from a few minutes to tens of hours. In the guidance given in Appendix C of 40 CFR 191 (US EPA, 1985) the assumption is made that because of passive institutional controls or the "intruders' own exploratory procedures" the intruders will "soon detect, or be warned of, the incompatibility of the area with their activities." The interpretation of "soon" is ill defined and it has been assumed in the 1992 PA and other analyses to date that sufficient time is available for the release mechanisms described to be fully implemented prior to the driller becoming aware of the incompatibility of the area with drilling activities. Furthermore, as explained above, the financial incentive to continue drilling is strong and only very unusual circumstances will tikely cause a drilling operation to be significantly delayed beyond the normal time it takes to resolve drilling problems. In the conceptual models to be discussed it will be assumed that actions taken by the driller in normal drilling operations and in problem resolution consist of standard drilling practices. Therefore, in determining the quantity of waste that is released to the surface it will be assumed that the driller either does not become aware of the nature of the waste that is brought to the surface or that its nature is ignored and drilling is continued.

\section{Cuttings}

For a gauge borehole, the volume of cuttings removed and transported to the surface is equal to the drill bit area and the drill depth. Thus, to estimate the total volume of waste removed due to the cutting action of the drill bit $(\mathrm{V})$, it is only necessary to know the compacted repository height (h) and the drill bit area (A).

$$
V=A h
$$

The cuttings volume calculated in this manner is a lower bound to the total quantity of waste removed by drilling. In the CUTTINGS code the waste is assumed to be uniformly distributed throughout the disposal region. Thus the actual computation for release requires only the drill bit area and the waste curie content per unit area.

\section{Cavings}

The cavings component of direct surface release consists of that quantity of waste material that is eroded from the borehole wall by the action of the flowing drilling fluid after a waste disposal room is penetrated. The erosion process is assumed to be driven solely by the shearing action of the drilling fluid (mud) on the waste as it moves up the borehole annulus. Although a number of factors may influence borehole erosion (e.g., eccentricity of the drill pipe in the borehole, impact of solid particles in the mud on the walls, and time of contact between the mud and walls [Broc, 1982]), industry opinion singles out fluid shear stress as the most important factor driving erosion (Darley, 1969; Walker and Holman, 1971). For cavings it is further assumed that the repository is not pressurized by either brine or gas, i.e. the repository pressure is less than the hydrostatic pressure caused by a brine column to the surface. Pressurization effects are included in other release processes and will be discussed in turn.

In the annulus formed by the collars or drill pipe and the borehole wall, the flow of the drilling fluid has both a vertical and rotational component. Within this helical flow pattern, shear stresses are generated by the relative motion of adjacent fluid regions and by the action of the fluid on the borehole wall. In this model, it is assumed that if the fluid shear stress at the wall exceeds the effective shear resistance to erosion of the wall material (filter cake or compacted repository 
wastes), erosion of the wall material will occur, increasing the diameter of the bored hole. The eroded material will then be passed to the surface in the flowing drilling fluid.

Flow in the annulus between the drillpipe and borehole wall is usually laminar (Darley and Gray, 1988). Adjacent to the collars, however, the flow may be either laminar or turbulent as a consequence of the larger collar diameter and resulting higher mud velocities (Pace's letter on pages A-159 through A-164 of (Rechard et al., 1990)). For laminar flow, the analysis lends itself to classical solution methods. Turbulent flow, where the flow is assumed to be axial with no rotational component, requires a more approximate approach. A discussion of these two cases follows.

Below Reynolds numbers of about 2100 for Newtonian fluids and 2400 for some nonNewtonian fluids (Walker, 1976), experiments have shown that the flow of a fluid'in a circular pipe or annulus is well behaved and can be described using a well-defined relationship between the velocity field and the fluid shear stress. This type of flow is called laminar.

Some of the early work on laminar helical flow of a non-Newtonian fluid in an annulus was performed by Coleman and Noll (1959), and Fredrickson (1960). The laminar helical flow solution procedure outlined below is, for the most part, an adaptation of methods described in a paper by Savins and Wallick (1966).

One of the principal difficulties in solving for the shear stresses within a helically flowing drilling fluid is the shear rate dependence of the fluid viscosity. This non-Newtonian fluid behavior necessitates choosing a functional form for the variation of viscosity with shear rate for the fluid. There are several functional forms for the viscosity of drilling fluids that can be assumed. For example, in the oil and gas industry the Bingham and power law models are often used to approximate the shear rate dependence of the fluid viscosity. A less common function is a form chosen by Oldroyd (1958) and used in the analysis by Savins and Wallick (1966). Oldroyd asslimed that the viscosity varied according to the functional relation

$$
\eta=\eta_{o}\left[\frac{1+\sigma_{2} \Gamma^{2}}{1+\sigma_{1} \Gamma^{2}}\right],
$$

where $\sigma_{1}$ and $\sigma_{2}$ are constants, $\eta_{o}$ is the limiting viscosity at zero rate of shear, $\eta_{\infty}$ (defined as $\left.\eta_{o}\left(\sigma_{2} / \sigma_{1}\right)\right)$ is the limiting viscosity at infinite rate of shear, and $\Gamma$ is the shear rate. The viscous shear stress is described by $\tau=\eta \Gamma$.

Using the Oldroyd viscosity, Equation (2), the viscous shear stress can be illustrated graphically as in Figure 1. 


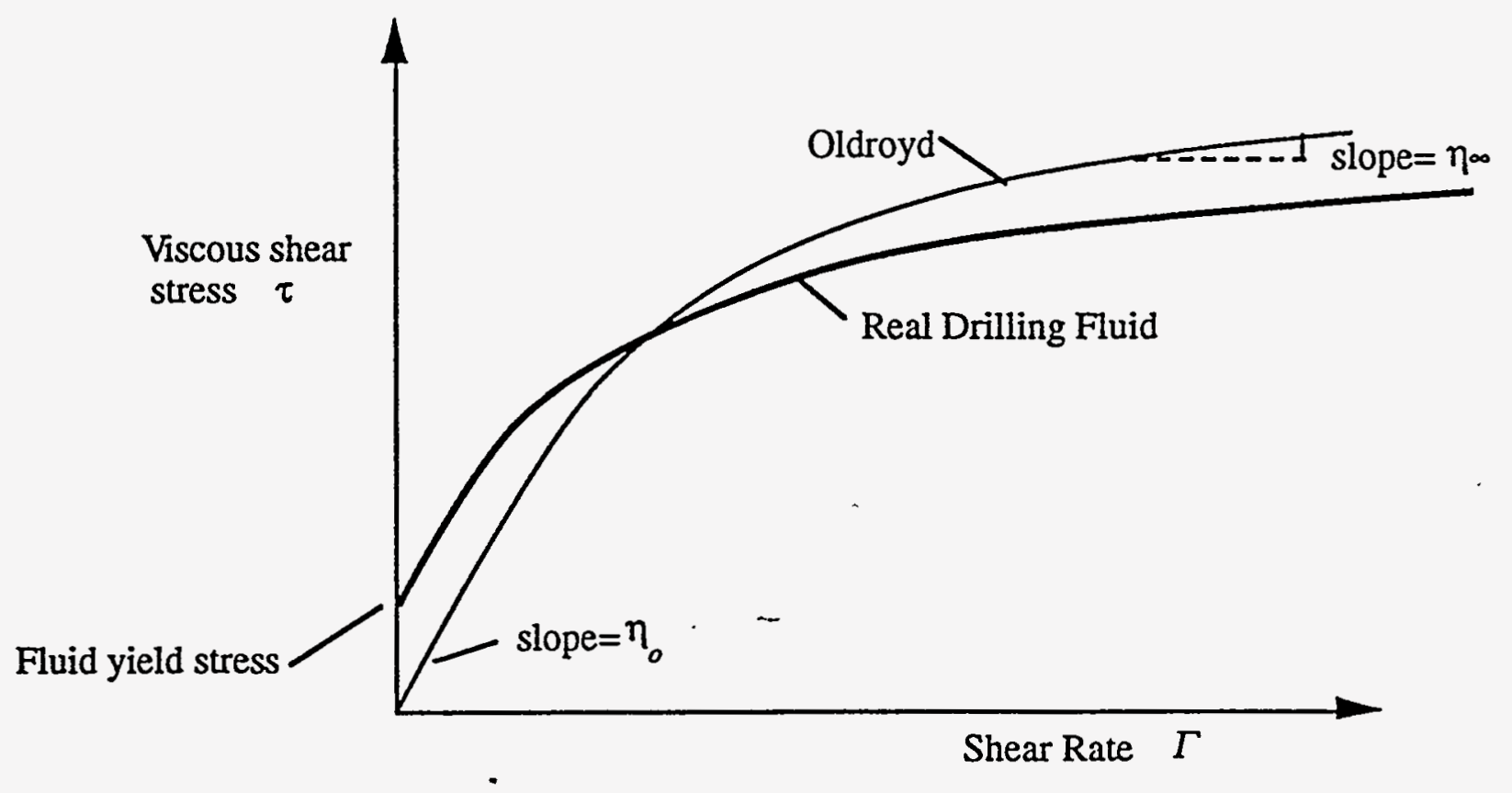

Figure 1. Oldroyd Drilling Fluid

This is a rate softening (pseudoplastic) model that has an initial slope of $\eta_{0}$ and a limiting slope of $\eta_{\infty}$ for large shear rates.

The Oldroyd model cannot account for drilling fluids that exhibit a yield stress. However, above a shear rate of zero, parameters can be chosen so that the model can be made to approximate the pseudoplastic rate response of many drilling fluids (see Figure 1).

Savins and Wallick (1966), expanding on the work of Coleman and Noll (1959) and Fredrickson (1960), showed that the solution for laminar helical flow of a non-Newtonian fluid in an annulus could be written in terms of three nonlinear integral equations.

$$
\begin{aligned}
& F_{1}=\int_{\alpha}^{1}\left(\frac{\rho^{2}-\lambda^{2}}{\rho}\right) \frac{d \rho}{\eta}=0 \\
& F_{2}=c \int_{\alpha}^{1} \frac{d \rho}{\rho^{3} \eta}-\Delta \Omega=0 \\
& F_{3}=\frac{4 Q}{\pi R^{3}}+4\left(\frac{R J}{2}\right) \int_{\alpha}^{1}\left(\frac{\alpha^{2}-\rho^{2}}{\eta}\right)\left(\frac{\rho^{2}-\lambda^{2}}{\rho}\right) d \rho=0,
\end{aligned}
$$

where $\alpha$ is the ratio of the collar radius over the cutting radius $\left(R_{i} / R\right.$ ) (Figure 2), $\Delta \Omega$ is the drill string angular velocity, $Q$ is the drilling fluid (mud) flow rate, $r$ is the radial coordinate, and $\rho$ is the non-dimensional radial coordinate representing the ratio $r / R$.

The unknown parameters $\lambda^{2}, R J / 2$, and $C$ are related to the fluid shear stresses through the relations 


$$
\begin{gathered}
\tau_{r \theta}=\frac{C}{\rho^{2}} \\
\tau_{r z}=\frac{R J}{2}\left(\frac{\rho^{2}-\lambda^{2}}{\rho}\right) \\
\tau^{2}=\tau_{r \theta}^{2}+\tau_{r r}^{2},
\end{gathered}
$$

where $r, \theta$, and $z$ represent radial, tangential, and vertical coordinates associated with the cylindrical geometry of Figure 2.
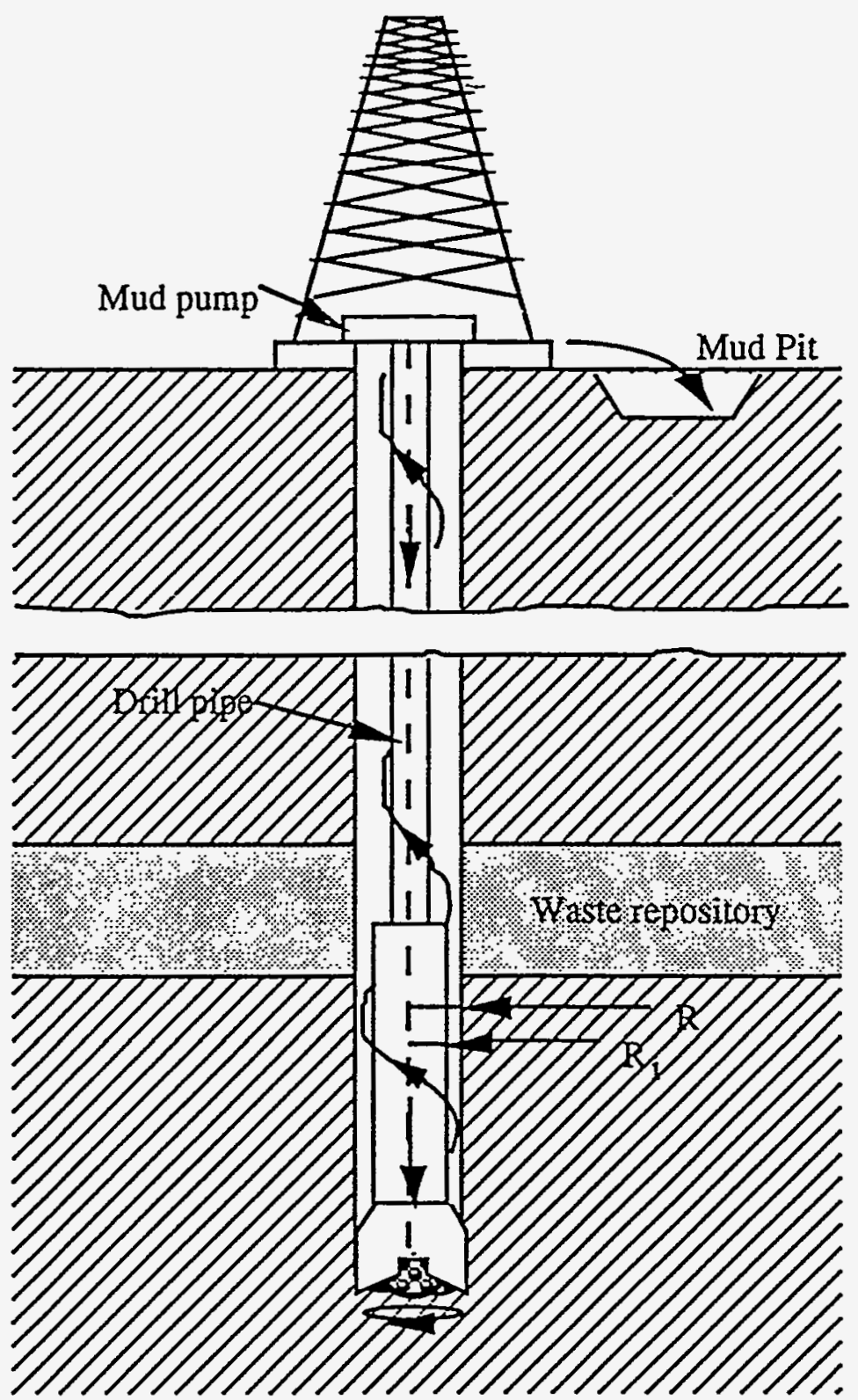

Figure 2. Detail of rotary drill string adjacent to drill bit 
The three nonlinear integral equations represented by Equations (3) generally must be solved numerically, a process which is described in detail in Berglund (1992). In that report, the final eroded diameter is determined through an iterative process that equates the fluid shear stress adjacent to the waste to a measure of the erosion resistance of the waste. The erosion resistance is governed by the effective shear resistance to erosion, a parameter not directly related to the shear strength as normally determined for granular materials using conventional soil tests.

For Newtonian fluids with Reynolds numbers greater than about 2100 , flow in a circular pipe or annulus starts to become more or less random in character, which makes orderly mathematical analysis of the flow difficult, if not impossible. With increasing Reynolds numbers, this random behavior increases until, at a Reynolds number of about 3000, the flow becomes fully turbulent. In fully turbulent flow, momentum effects dominate and the fluid viscösity is nö longer important in characterizing pressure losses.

The Reynolds number $\left(R_{e}\right)$ is defined as

$$
R_{e}=\frac{\bar{\rho} \bar{V} D_{e}}{\bar{\eta}},
$$

where $D_{e}$ is the equivalent hydraulic diameter, $\bar{\rho}$ is the drill fluid density, $\bar{V}$ is the average fluid velocity, and $\bar{\eta}$ is the average fluid viscosity.

For Newtonian fluids, the value to use for the viscosity is clear since the viscosity is constant for all rates of shear. Non-Newtonian fluids exhibit a changing viscosity with shear rate and present a special problem in calculating $R_{e}$. For fluids that exhibit a limiting viscosity at high rates of shear (such as the Bingham model and in our case the Oldroyd model), it has been suggested (Broc, 1982) that the limiting viscosity $\left(\bar{\eta}=\eta_{\infty}\right)$ be used in calculating the Reynolds number.

The Reynolds number for an Oldroyd fluid in an annulus can then be written as (Broc, 1982)

$$
R_{e}=\frac{0.8165 D \bar{V} \bar{p}}{\bar{\eta}},
$$

where the hydraulic diameter is expressed as $D=2\left(R-R_{i}\right)$ (see Figure 2).

The most important influence viscosity has on the calculation of pressure losses in fully turbulent flow of non-Newtonian fluids appears to be in the calculation of the Reynolds number. A far more important parameter is the surface roughness past which the fluid must flow. The Reynolds number, however, does have a role in determining the onset of turbulence. For Newtonian fluids this number is about 2100. For non-Newtonian, rate-thinning fluids, the critical value of $R_{e}$ tends to be greater than 2100 but less than 2400 (Walker, 1976). For our purposes, a value of 2100 will be used to represent $R_{e c}$ (the critical Reynolds number) for the Oldroyd fluid model. Since turbulent flow is more effective in generating fluid shear stresses at the borehole wall, this assumption is conservative.

There is a transition region beyond $R_{e_{c}}$ before the development of fully turbulent flow. In this regime the flow has the character of both laminar and turbulent flow. However, since pressure losses increase rapidly in turbulent flow and affect borehole shear stresses more severely, it will be assumed that beyond $R_{e_{c}}$ the flow is fully turbulent.

Turbulent flow is very complex and, thus, to characterize the turbulent flow regime, the great bulk of analysis has concentrated on empirical procedures. For axial flow in an annulus, the pressure loss under turbulent conditions can be approximated by (Broc, 1982)

$$
\Delta P=\frac{2 f L \bar{\rho} \bar{V}^{2}}{(0.8165) D}
$$


where $f$ is the coefficient of pressure head loss (Fanning friction factor) and $L$ is the borehole length.

If the shear stress due to the flowing fluid is assumed to be uniformly distributed on the inner and outer surfaces of the annulus, it can be easily shown using Equation (7) that the shear stress is related to the average fluid velocity through the relation

$$
\tau=\frac{f \bar{\rho} \bar{V}^{2}}{2(0.8165)},
$$

The Fanning friction factor is empirically related to the Reynolds number and relative roughness by the equation (Whittaker, 1985)

$$
\frac{1}{\sqrt{f}}=-4 \log _{10}\left[\frac{\varepsilon}{3.72 D}+\frac{1.255}{R_{e} \sqrt{f}}\right],
$$

where $\varepsilon / D$ is the relative roughness. For circular pipes, $D$ in this equation represents the inside diameter and $\varepsilon$ is the absolute roughness or the average depth of pipe wall irregularities. In the absence of a similar equation for flow in an annulus, it is assumed that this equation also applies here, where $D$ is the hydraulic diameter as defined earlier and $\varepsilon$ is the absolute roughness of the waste-borehole interface.

For laminar flow within the annulus both axial and rotational flow are modeled. Some of the availabie literature (Khader and Rao, 1974 ; Bilgen E., Boulos, R., and Akgungor A.C., 1973) indicate the importance of also accounting for drillstring rotation when the drilling mud flow within the annulus is turbulent. Consequently,to account for rotational flow in the turbulent regime, an axial flow velocity correction factor is also introduced into the above formulation that maintains eroded diameter compatibility across the laminar- turbulent flow transition.

Using a relative roughness and a calculated Reynolds number, a Fanning friction factor can be determined by iteratively solving Equation (9). The value of the shear stress acting on the borehole wall can then be determined from Equation (8). Using an iterative procedure similar to that for the laminar flow problem, the fluid shear stress can be forced to equal the repository shear resistance to erosion $\left(\tau_{\text {fail }}\right)$ to obtain the final eroded borehole radius (Berglund, 1992).

The equations governing erosion (cavings) based on laminar and turbulent flow were combined into a single Fortran computer code called CUTTINGS (Rath, 1994). Using appropriately selected input based on the physical properties of the waste and other drilling parameters, this code calculates the final eroded diameter of the borehole that passes through the waste. The drilling parameters chosen must reflect data typical of that valid near the WIPP repository.

For drilling operations through salt in the Delaware basin , the drilling mud most likely to be used is a brine (Pace's letter on pages A-159 through A-164 of (Rechard et al., 1990)), with the density cut somewhat with an emulsified oil. The mud density and viscosity parameters required in the laminar and turbulent regime erosion calculations can be estimated based on the assumption of the use of such a brine-based drilling mud. For the 1992 PA the drilling mud properties used were (WIPP PA, 1992b, Table 4.4-1):

\begin{tabular}{llllll} 
Parameter & Median & \multicolumn{2}{c}{ Range } & Units & Distribution Type \\
Density & 1211 & $1139-$ & 1378 & $\mathrm{~kg} / \mathrm{m}^{3}$ & Constructed \\
Viscosity & 0.00917 & $0.005-$ & 0.03 & $\mathrm{~Pa} \cdot \mathrm{s}$ & Constructed \\
Yield Stress & 4 & $2.4-$ & 19.2 & $\mathrm{~Pa}$ & Constructed
\end{tabular}


For drilling through salt, the drilling speeds can vary from 40 to $220 \mathrm{rpm}$ (Austin, 1983 and Pace's letter on pages A-159 through A-164 of (Rechard et al., 1990)). The most probable speed is about $70 \mathrm{rpm}$ (Pace's letter on pages A-159 through A-164 of (Rechard et al., 1990)).

Mud flow rates are usually selected to be from 30 to 50 gallons/minute per inch of drill bit diameter (Austin, 1983) and usually result in flow velocities in the annulus between the drill collars and the borehole wall at or near the critical flow state (laminar-turbulent transition) (Pace's letter on pages A-159 through A-164 of (Rechard et al., 1990)).

The drill bit diameter is related to the total planned depth of the hole to be drilled. For gas wells in the 4000- to 10,000-foot range, it is likely that the drill bit used that passes through a waste room would have a diameter of 10.5 to 17.5 inches. The collar diameter is assumed to be less than the drill bit diameter by 2 inches. For the 1992 PA these parameters are presented in table 4.2-1 (WIPP PA, 1992b)

\begin{tabular}{lllllll} 
Parameter & Median & \multicolumn{2}{c}{ Range } & Units & Distribution Type \\
$\begin{array}{l}\text { Drill bit } \\
\text { diameter }\end{array}$ & 0.355 & 0.267 & -0.444 & $\mathrm{~m}$ & Uniform \\
$\begin{array}{l}\text { Drill string } \\
\text { angular velocity }\end{array}$ & 4.2 & -23 & $\mathrm{rad} / \mathrm{s}$ & Constructed \\
$\begin{array}{l}\text { Drilling mud } \\
\text { flowrate }\end{array}$ & 0.09935 & 0.0745 & -0.124 & $\mathrm{~m}^{3} /(\mathrm{s} \cdot \mathrm{m})$ & Uniform
\end{tabular}

For turbulent flow, the shear stress acting at the borehole at the repository is dependent on the absolute surface roughness. The value chosen for the cailculations exceeds that of very rough concrete or riveted steel piping (Streeter, 1958).

The amount of material eroded from the borehole wall is dependent upon the magnitude of the fluid-generated shear stress acting on the wall and the effective shear resistance to erosion of the compacted, decomposed waste. In the absence of experimental data, the effective shear resistance to erosion of the repository material is assumed to be similar to that of a montmorillonite clay, on the order of several $\mathrm{Pa}$ (Sargunam et al., 1973).

For the 1992 PA, the roughness and shear strength parameters were given in table 3.4-1 (WIPP PA, 1992b).

Of the eight parameters required to compute caving (erosion) adjacent to the repository arising from a drill bit intrusion the value for erosion shear resistance is the most speculative. A model for the erosion properties of decomposed transuranic (TRU) has been described by Butcher, 1994. Butcher assumes that for erosion the properties of various states of decomposed and corroded TRU waste can be no worse than those of a mixture of sand and clay. Thus, a clay/sand mixture represents the most erodable state possible and the erosion of actual waste 
would be much less. This conclusion is based on the observation that "the fabric existing in waste (caused by unreacted materials such as pieces of metal, plastic, etc.) constitutes an internal reinforcement that makes the waste less prone to erosion than would be observed in an equivalent clay/hard particle mixture."

Experimental data for the erosion characteristics of sand/clay waste surrogates have not been generated but an experimental device to acquire such data has been designed (Lenke, 1994). Another test device, to acquire data on gas induced spall in an axisymmetric geometry, can also be used to generate data on erosion. This latter device has yet to be fully designed but should be able to address the effect of pressurized brine or entrained gas on the erosion properties of waste surrogates.

\section{Spallings}

Berglund 1992, describes three mechanisms that could contribute to the direct removal of waste from WIPP as the result of a drilling intrusion. Computational models for two of the mechanisms (cuttings and cavings) are included in the code CUTTINGS (Rath, 1994). The CUTTINGS code does not address the third mechanism, spallings, that may occur as the result of high pressure waste decomposition gas.

The failure, sloughing, or "spalling" of borehole walls is a common occurrence in oil and gas drilling and can be caused by number of different mechanisms, including an encounter with a geopressurized formation. Short (1982) describes in general terms the spalling or "popping off" of shale fragments into the borehole caused by formation pore pressures greater than the borehole pressure. He states that the condition occurs primarily in shales but also indicates that there is field evidence that this behavior may also occur in very-fine-grained, well-cemented sands or in other formations with very low permeability. Cheatham (1984) reviewed the causes of wellbore instability and indicated that one mechanism for collapse was the result of low borehole pressure adjacent to a geopressurized formation.

Fluid flow from a formation to a borehole is necessary for wells to maintain gas or oil production. High differential pressures between the formation pores and the borehole enhance fluid production but tend to cause sloughing of the borehole wall. In poorly consolidated sand formations, the sloughing of sand into the borehole (sand production) is an important problem and has been studied by numerous authors. The problem of sand production is related to the ability of sand to form a stable arch. Hall and Harrisberger (1970), through experiment, concluded that stability of a sand arch requires dilatancy and cohesiveness or some other constraint on the surface of the grains. Bratli and Risnes (1981) studied the failure of spherical sand arches both experimentally and theoretically using an elastic-plastic (Mohr-Coulomb) approach. They concluded that collapse of the inner Coulomb zone of the spherical arch will occur if the pore pressure exceeds the radial stress and the difference becomes equal to the uniaxial tensile strength of the sand. Similar conclusions of stability were reached for sand in a cylindrical geometry by Risnes et al. (1982). In this work, borehole stability in an uncased hole was related to the permeability variation with radius and to the cohesive strength of the sand. In both of the latter works the pore fluid was assumed to be incompressible and that steady-state conditions prevailed. Vaziri (1989) extended the work of Risnes et al. (1982) by considering the time-dependent changes in the stress and fluid fields by using finite-element procedures.

The effects of gas escaping from a geopressurized formation to a borehole are also related to the phenomenon of coal outbursts. An outburst is the violent and potentially dangerous failure of a freshly exposed coal surface that occasionally occurs in underground mining. An extensive review of outbursts and some of the theories hypothesized to explain them was given by Shepherd et al. (1981). In this work, the role of geologic structures, coal stresses, and matrix gases is discussed. A definitive model for coal outbursts was presented by Paterson (1986). The model is based on the pressure gradients generated by the flow of gas from the micropores within 
the coal to the lower-pressure mine surface. These gradients generate body forces within the coal and a tensile stress state that can exceed the tensile capacity of the coal. The outburst occurs when the coal near the mined surface fails at a naturally weakened plane.

Numerous methane-gas-induced outbursts have also been observed in sandstone formations located near coal seams. Sato and Itakura (1989) describe in detail one such event that occurred in the Horonai coal mine in 1978 . While mining advanced progressively along a $17.45-\mathrm{m}^{2}$ crosscut in a sandstone formation using explosives, the volume of fractured sandstone following a blast abruptly increased by more that a factor of twenty and a large volume of methane ( 1800 $\left.\mathrm{m}^{3}\right)$ was released. Sato and Itakura (1989) postulated that the tensile strength of the sandstone in this case was too high for the outburst to occur spontaneously from the entrained gas alone and was initiated by unloading stress waves from the blast.

More pertinent to the possibility of spall caused by a borehole penetrating a gas-saturated waste repository is the phenomenon of outbursts that occur when drilling through deep coal seams. Willis (1978) relates occurrences where "deeply buried coal seams are drilled and the coal literally 'explodes' into the wellbore. ... Cases have been cited where only $1 \mathrm{ft}$ of coal has been drilled and the hole is tight for 30 to $60 \mathrm{ft}$ as the driller pulls out of it." In such a case, although only a small length of coal was drilled, the "explosive" response of the penetrated coal causes quantities of fractured coal to be projected up to $60 \mathrm{ft}$ into the drill annulus, thus restricting mud flow and potentially jamming the bit. Several reasons for this phenomenon are suggested, including the release to the wellbore of entrapped, high-pressure gas in the coal.

Some experiments have investigated the outbursting phenomenon. Ujihira et al. (1985) demonstrated the importance of tensile strength and gas pressure within the porous material on failure. Their experiments illustrated the one-dimensional, progressive, catastrophic failure of a porous coal simulant when a sudden pressure drop was generated at one end. High-speed photography revealed that failure started at the specimen face exposed to the pressure drop and rapidly (within $0.025 \mathrm{~s}$ ) propagated through the specimen. Mini outbursts were also generated in circular cylindrical briquettes of coal pressurized with $\mathrm{CO}_{2}$ and $\mathrm{N}_{2}$ by Bodziony et al. (1989). In the latter, when the pressure surrounding the briquette was suddenly reduced, the ensuing outburst caused the briquette to become divided into layers much like a sliced salami.

The calculations performed by Berglund(1992) and preliminary results from ID experiments conducted in late 1993 and early 1994 (Berglund and Lenke 1994) on gas pressurized porous granular media have indicated that if a drillbit inadvertently penetrates a gas pressurized WIPP disposal room, failure (spall) of the waste adjacent to the drillbit can occur followed by the compression of the waste against the drillstring surface. The compressed waste is likely to cause either sticking of the drillstring assembly or to exacerbate erosion adjacent to the drillpipe and consequently cause a quantity of waste to reach the ground surface. Gas induced spallation of the waste will occur only if certain conditions are met at the time of intrusion. The principal variables governing such spall behavior are the waste permeability, gas pressure and some measure of the strength of the compacted and partially decomposed waste. Experimental data on waste strength is currently unavailable and data on the phenomenology of waste response is limited. In order to assess the potential importance of the effect of gas on direct releases most analyses to date have assumed the decomposed waste has no cohesive strength (Berglund, 1994).

Three possible spall conditions can occur if the drillbit penetrates the repository pressurized with gas at a pressure above hydrostatic: blowout, stuck pipe, and gas flow assisted erosion (as the result of gas induced spall). In the following sections each of these conditions will be described and computational models for waste release discussed. Much of the following sections on blowout, stuck pipe, and gas spall were taken directly from Berglund, 1994. 


\section{Blowout}

For compacted, partially decomposed waste with a high permeability it is possible that a large volume of decomposition gas will be introduced into the drilling mud creating a blowout condition within the mud fluid column when the drillbit penetrates the pressurized repository. For large waste permeabilities (e.g. $\mathrm{k}=1 \times 10^{-13} \mathrm{~m}^{2}$ ) and high gas pore pressure (14.8 $\mathrm{MPa}$ ), gas will flow into the hole at approximately $0.1 \mathrm{~m}^{3} /\left(\mathrm{m}^{2}\right.$ of exposed waste area)/s (Berglund, 1992). This volume increases by a factor of 86 as it rises in the annulus and the rate of gas inflow into the drilling mud at the waste elevation is further exacerbated by the drop of hydrostatic pressure as the mud is displaced out of the hole and the length of the fluid column decreases. This is a classic blowout condition.

There have been several recent instances of shallow salt section high pressure air pockets being penetrated near WIPP (Dyer,1993). Typically during these events, all of the drilling mud is blown out of the borehole. The events occur suddenly, are of short duration (1 or 2 minutes), blowout preventers are not engaged, and drilling is centinued after refilling the hole with drilling fluid. The typical depths where these air pockets occur are between 800-2600 feet (Dyer,1994a). During blowout, drilling mud, rocks, and drill cuttings that may have settled into borehole washouts are ejected at the surface and occasionally surface equipment is damaged. Generally, the drillstring does not get stuck and the hole is for the most part cleared all the way to the surface by the flowing air.

For a future penetration at the shallow depth of the WIPP repository, a similar blowout event is likely to occur if the permeability and gas pressure is high causing drilling fluid to be blown out of the hole and allowing some quantity of WIPP waste to be propelled into the borehole annulus. The large volume of decomposition gas available in a waste panel, if unrestricted, would continue to flow for many hours even at large waste permeabilities (Berglund, 1992). Blowout preventers may eventually be closed and the gas allowed to flow out in a controlled manner or the mud weighted up to eliminate gas flow into the well. After the well mud and repository gas pressures are equalized, drilling can continue with no additional waste removed except by simple erosion (cavings).

Estimates of the quantity of waste propelled into the wellbore which will eventually reach the surface as the result of blowout from a disposal room at WIPP are speculative and are dependent on the characteristics of the decomposed waste and rate of gas flow. Air pocket penetrations that have occurred near the WIPP site suggest that very little material other than residual drill cuttings that have settled into washouts are blown out of the borehole. Indeed, in the events that have been recorded, the borehole remains quite clear and drilling can be continued after refilling the hole with drilling mud. Stuck pipe has not been observed. This suggests that for air pockets in salt, only small amounts of material from the pressurized region are projected into the borehole during blowout. However, decomposed WIPP waste is likely to behave quite differently from the intact salt surrounding the high pressure air pockets encountered to date. The decomposed WIPP waste may have the granular character of a soil - like material with little or no cohesive strength and consequently may be carried into the gas stream and up the borehole annulus.

The entrainment of solid particles by the flow of gas is a well known phenomenon and is in fact a process used to move large quantities of bulk materials through fluidization (Stoess,1983). For smooth, disperse-phase fluidization, moving gas can entrain and move as much as ten percent solids (Zenz,1960).

If blowout has occurred a simple model for the rate of transport of unconsolidated waste can be based on the limits for disperse-phase fluidization, namely a sampling of entrainment rates between $0-10 \%$ of the gas flow rate. In addition to the entrainment percent, total release to the surface requires the gas flow rate and the duration of flow. The rate of gas flow (single phase 
flow is assumed) can be computed for various waste permeabilities and repository gas pressures using the equation

$$
\frac{k}{2 \mu} \nabla^{2} p^{2}=\varphi_{o} \frac{\partial p}{\partial t}
$$

where $k$ is the permeability, $\mu$ is the gas viscosity, $\varphi_{0}$ is the gas porosity, $\nabla^{2}=\frac{\partial^{2}}{\partial r^{2}}+\frac{I}{r} \frac{\partial}{\partial r}$, and $r$ is the radial coordinate (Berglund, 1993). This equation can also be used to determine the range of gas pressures and permeabilities likely to cause a blowout event.

As discussed earlier, when air pocket blowouts occur in salt they are allowed to drain completely permitting the drilling to continue after the borehole is replenished with drilling mud. For an encounter with WIPP gas (primarily hydrogen) the large capacity of a WIPP panel would allow the blowout to continue for hours or longer depending on waste permeability. Under these conditions the driller would likely close the blowout preventers within 5 minutes and proceed to kill the well by weighting up the mud (Dyer, 1994b). The 5 minute maximum permits the total volume of gas release to be computed and additionally provides a means of estimating the volume of waste released to the surface because of a blowout. For high permeability waste (above $\mathrm{k}=10^{-13} \mathrm{~m}^{2}$ ) the gas flows at such a high rate into the borehole annulus that gas compressibility effects become important. Compressible, isothermal flow in a channel with friction is governed by the equation (Binder,1958)

$$
\frac{f l}{D}=\frac{1}{K M_{1}^{2}}\left[1-\frac{p_{2}^{2}}{p_{1}^{2}}\right]-2 \ln \frac{p_{1}}{p_{2}}
$$

where $\mathrm{f}=$ friction factor, $\mathrm{D}=$ pipe diameter, $l=$ pipe length, $\mathrm{K}=$ gas constant, $\mathrm{p}_{1}$ and $\mathrm{p}_{2}=$ inlet and outlet gas pressures, and $M_{1}=$ inlet Mach number.

Table 1 presents the gas flowrate into a blownout borehole from the WIPP repository as calculated by equations (10) and (11). The gas flowrate is given in units of $\mathrm{m}^{3} / \mathrm{s} /\left(\mathrm{m}^{2}\right.$ of exposed borehole surface). The flowrate is greatest immediately after penetration and achieves a quasistatic rate within a short period of time. Table 1 presents the gas (hydrogen) flowrate adjacent to the repository at $100 \mathrm{~s}$ after a sudden penetration for a 15.75 in borehole. In order to compute these values it was assumed that $\mu=9.32 \times 10^{-6} \mathrm{~Pa} \cdot \mathrm{s}, \varphi_{0}=0.19, \mathrm{~K}=1.4, \frac{f l}{\mathrm{D}}=166$, and $\mathrm{p}_{2}=0.0896 \mathrm{MPa}$ (atmospheric pressure).

Table 1

\begin{tabular}{|c|c|c|c|c|c|c|c|c|}
\hline $\begin{array}{l}\text { Repository Gas } \\
\text { Pressure } \mathrm{MPa}\end{array}$ & 7.8 & 8.8 & 9.8 & 10.8 & 11.8 & 12.8 & 13.8 & 14.8 \\
\hline \multicolumn{9}{|l|}{$\begin{array}{l}\text { Waste } \\
\text { Permeability } \mathrm{m}^{2}\end{array}$} \\
\hline $10^{-12}$ & 6.46 & 6.46 & 6.46 & 6.46 & 6.46 & 6.46 & 6.46 & 6.46 \\
\hline $10^{-13}$ & 3.82 & 4.42 & 4.82 & 5.23 & 5.53 & 5.73 & 5.88 & 6.03 \\
\hline $10^{-14}$ & 0.640 & 0.797 & 0.969 & 1.157 & 1.36 & 1.58 & 1.81 & 2.06 \\
\hline $10^{-15}$ & 0.100 & 0.124 & 0.150 & 0.178 & 0.208 & 0.241 & 0.275 & 0.312 \\
\hline $10^{-16}$ & 0.019 & 0.0232 & 0.028 & 0.033 & 0.038 & 0.043 & 0.0495 & 0.0557 \\
\hline
\end{tabular}

Gas Flowrate to Borehole at Repository During Blowout 
The volume of compacted waste propelled into the wellbore and to the surface as the result of a blowout can be estimated by sampling over the entrainment percentage $(0-10 \%)$ and by assuming that the well would be shut in within 5 minutes. The maximum compacted waste volume released during blowout (based on 10\% entrainment and the gas flows of table 1) is shown in figure 3. These values are calculated assuming that waste gas flows out of exposed borehole surface area of $1 \mathrm{~m}^{2}$. The minimum volume that could be released during blowout is zero and corresponds to no solids entrainment in the flowing gas.

For compacted waste with very low permeability the gas flowrate into a borehole after an intrusion is too small to cause blowout. Generally a 2 pound "kick" ( $-20 \%$ gas in $10 \mathrm{lb} / \mathrm{gallon}$ brine drilling mud) is an indication of impeding blowout conditions (Austin, 1983). Waste permeability above $10^{-16} \mathrm{~m}^{2}$ exceeds this blowout criterion. For high gas pressures blowout will occur even at a lower waste permeability but for model simplicity a $10^{-16} \mathrm{~m}^{2}$ limit was chosen. This selection is conservative since releases below this value are greater.

At lower permeabilities gas flow to the borehole continues to occur and may either go undetected by the driller or be allowed to trickle slowly along the drillstring and be released at the surface (Jensen, 1993). The gas flow may initiate waste failure and force the waste against the drill collars exacerbating erosion, or if the waste is jammed against the drillcollar sufficiently hard, cause the drillpipe to stick.

\section{Stuck Pipe}

If the waste has a low permeability when penetrated, the gas flow into the drilling mud may cause waste failure adjacent to the borehole (Berglund, 1993) and jam the drillbit preventing further drilling. Prior to becoming completely stuck the driller will notice an increase in torque on the drillstring and a decrease in the rotational speed. When sticking occurs the driller will usually initiate a cleanout procedure wherein the drill bit is raised and lowered repeatedly into the sticking formation to clear the obstruction. This process can be continued for as much as 12-24 hours if it is shown to be effective. After this time the problem must be solved by weighting up the mud, spot sealing with cement or setting casing (Short, 1982).

During the cleanout procedure waste will be transported to the surface with each thrust of the drillbit into the obstruction. The quantity of waste removed is related to the maximum carrying capacity of the drilling mud and can be estimated based on the observation that for drill cutting loadings above 5\% in the drilling mud (Darley \& Gray, 1988) tight hole conditions or stuck drillpipe may occur when circulation is stopped for any reason. Thus the maximum waste removal rate would consist of $5 \%$ of the drilling mud flowrate. The total quantity of waste transported to the surface can thus be computed as the waste removal rate multiplied by the cleanout time. By sampling on the cleanout time and the drillbit diameter (which drives the mud flowrate) variations of waste removal would occur for inclusion in performance assessments or systems prioritization. The range of releases possible is based on variations in drillbit diameter (10.5 to 17.5 inches), duration of the cleanout procedure (12 to 24 hours) and the drilling mud flowrate (30 to 50 gallons/minute/inch of drill diameter). The releases are based on equation (12)

$$
\mathrm{V}_{S}=0.05 \mathrm{QT}
$$

where

$$
\begin{aligned}
& \mathrm{V}_{S}=\text { compacted waste volume brought to ground surface } \\
& \mathrm{Q}=\text { drilling mud flow rate } \\
& \mathrm{T}=\text { cleanout duration }
\end{aligned}
$$

and varies between 43 to $238 \mathrm{~m}^{3}$ of compacted waste as shown in figure 3 . 
The lower limit of repository gas pressure at which sticking would occur (10MPa) is based on a drillstring power of $800 \mathrm{hp}$ and a coefficient of friction between the waste and drillcollars of 0.3 . For these conditions the drill string angular velocity would decrease by more than $50 \%$ from the normal operating range alerting the driller to sticking conditions.

\section{Gas Spall (Erosion)}

This again occurs when the waste has a low permeability and the gas flow to the borehole is very low and either is not detected by the driller or is allowed to trickle slowly along the drillstring and be released at the surface. The waste fails adjacent to the borehole perhaps causing some pipe sticking but the driller is able to continue and does not detect the unusual nature of the drill cuttings being brought to the surface. The flow of gas from the waste to the borehole generates a stress state in the waste adjacent to the borehole that depending on waste strength and the magnitude of the pressure gradient impresses the failed waste against the drillstring causing a continuous process of gas assisted erosion (Berglund, 1992). As waste erodes, more waste may continue to move toward the drillstring in response to the gas pressure gradient. The process ceases when sufficient gas has been released from the panel to preclude waste failure or until casing is set. Because the driller is either not aware of, or ignores the nature of the drill cuttings being removed in this case, the final volume of waste removed can be substantial.

For compacted waste with little or no strength, waste failure will generally occur for all repository gas pressures that exceed the hydrostatic stress of the drilling mud. The failed waste is then transported to the accessible environment in the drilling mud. As with the sticking mode described above, the volume of waste removed can be computed based on the observation that above drill cutting loadings above 5\% in the drilling mud (Darley \& Gray, 1988) tight hole conditions or stuck drillpipe may occur when circulation is stopped for any reason. Thus under these conditions the driller is not likely to remove waste to the surface at a rate faster than continuous drilling at the 5 percent limit.

The 5 percent cuttings loading will consist of both cuttings (from the hole bottom) and gas spallings. For a fixed mud flowrate the cuttings percentage will vary with the penetration rate. The penetration rate varies between $50-100 \mathrm{ft} / \mathrm{hr}$. For high penetration rates the cuttings percentage will be high leaving only a small amount for spallings to add up to the assumed 5 percent limit. For low penetration rates the cuttings percentage will be small and the spallings percentage correspondingly greater. The quantity of waste removed to the surface will be equal to (spall percentage) X (mud flowrate) X (drilling time). The drilling time is governed by the time required to drill from the elevation of the repository to the elevation at which casing is set which is below the Castile formation at $4500 \mathrm{ft}$.

The volume of waste brought to the surface can be readily computed based on available drilling parameters utilizing the following equation.

where

$$
V_{S}=\left[0.05 Q-\pi\left(D^{2} / 4 .\right) R_{p}\right] \Delta / R_{p}
$$

$\mathrm{V}_{S}=$ Compacted waste volume brought to ground surface

$Q=$ Drilling mud flow rate

$\mathrm{D}=$ Drill bit diameter

$\mathrm{R}_{\mathrm{p}}=$ Penetration rate

$\Delta=$ Differential Drilling Depth (distance from repository depth to depth where casing is set) 
The independent variables must be sampled over their expected ranges. Mud flow rates (Q) are usually selected to be from 30 to 50 gallons/minute per inch of drill diameter. Drillbit diameter (D) varies from 10.5 to 17.5 inches depending upon the planned depth of the drilled hole (Berglund, 1992). The expected penetration rate $\left(R_{p}\right)$ is from 50 to 100 feet /hour (Jensen, 1993). Based on a differential drilling depth of $2350 \mathrm{ft}$, and placing the remaining variables at their extreme values the range of volumes of compacted waste released to the surface varies from 44 to $356 \mathrm{~m}^{3}$. These quantities are shown in figure 3. For comparison, a typical volume of compacted waste brought to the surface based on erosion (cavings) alone (CUTTINGS code) is also shown in figure 3 .

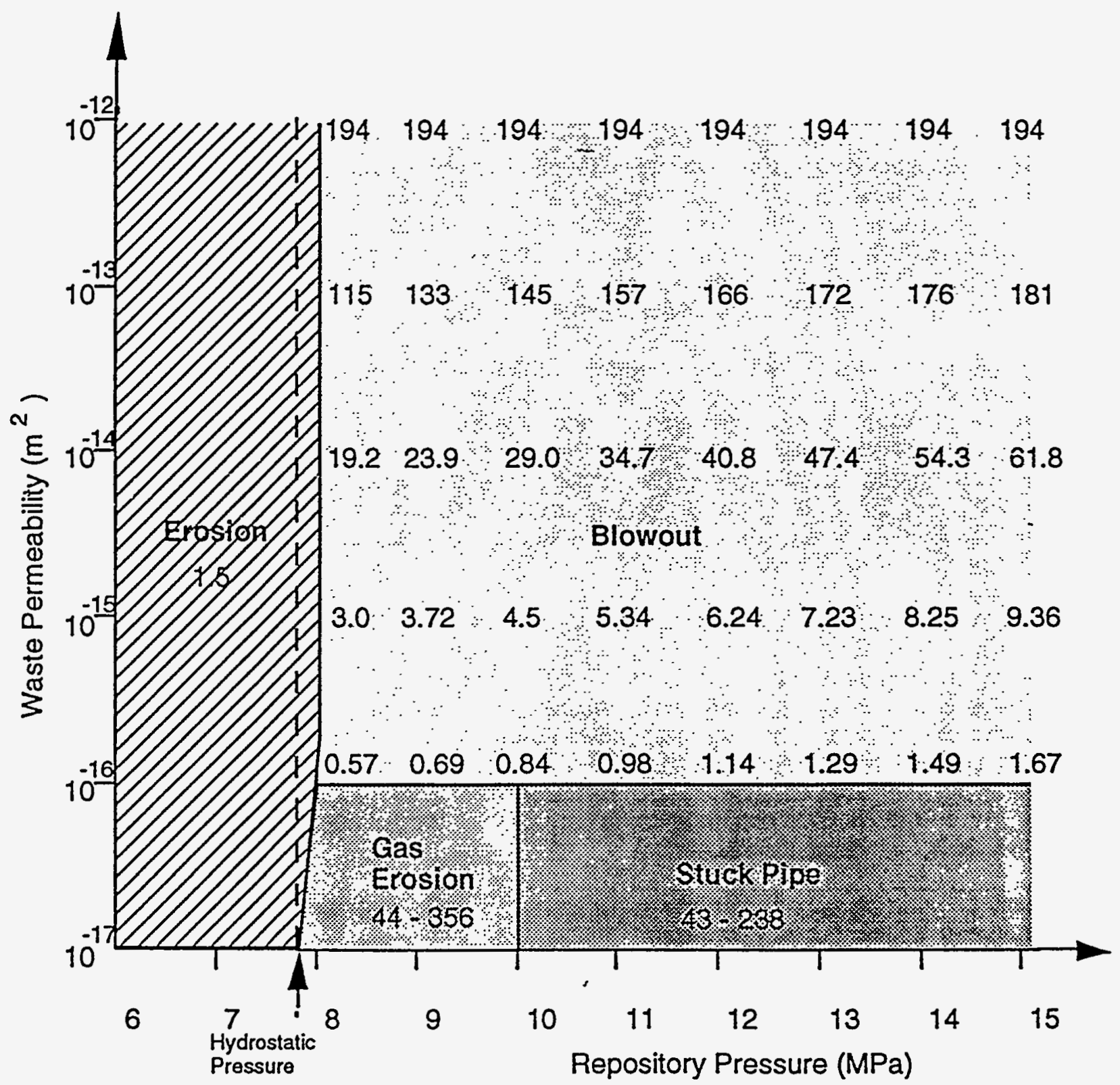

Figure 3 Volume $\left(\mathrm{m}^{3}\right)$ of Compacted Waste Released to the Accessible Environment (Upper bound for blowout, lower and upper bounds for Gas Erosion and Stuck Pipe) 
Figure 3 clearly indicates the importance of gas in the repository in determining the volume of waste that can reach the ground surface if the waste is assumed to have no strength. For repository gas pressures below hydrostatic the volume of waste removed is from pure erosion only and is generally a small fraction of that released when the repository gas pressure is high. At high gas pressures and permeabilities, blowout waste volumes are high but diminish rapidly with decreasing waste permeability. For a waste permeability below $10^{-16} \mathrm{~m}^{2}$ blowout no longer occurs and waste transport becomes a function of either gas assisted erosion or stuck pipe. For these latter modes, volumes of compacted waste 30 to 237 times greater than that for pure erosion could reach the ground surface.

Of the three modes of release caused by high pressure waste gas, stuck pipe and gas erosion would be most affected by increases in waste strength. This occurs because the drilling mud remains in the borehole and the pressure difference between the waste gas and borehole pressure is less than $7 \mathrm{Mpa}$. With blowout the borehole pressure is considerably reduced (near atmospheric) and thus pressure differentials can be much greater (approaching $14 \mathrm{Mpa}$ ).

Aside from severely reducing gas generation in the waste, methods to reduce surface releases include reducing waste permeability and increasing waste strength. Restricting the compacted waste permeability to a narrow range above $10^{-16} \mathrm{~m}^{2}$ (ensuring a low volume blowout) would also reduce the effects of high pressure gas on waste transport to the surface.

In considering the effect of direct releases on PA for the purpose of SP several new variables require sampling and the waste gas pressure at the time of intrusion must be available from the BRAGFLO code. The new sampled variables are:

$$
\begin{aligned}
& T=\text { Cleanout duration for stuck pipe (12-24 hours) } \\
& E_{p}=\text { Solids entrainment percent for blowout }(0-10 \%) \\
& R_{p}=\text { Drillbit penetration rate }(50-100 \mathrm{ft} / \mathrm{hr})
\end{aligned}
$$

There is some indication that $R_{p}$ is inversely related to drillbit diameter and, as with drilling mud flowrate, can be directly calculated based on the sampled value of borehole diameter. This leaves only $\mathrm{T}$ and Ep as the new variables to be sampled.

For spallings, the decomposed waste gas pressure, permeability, gas porosity, and waste gas viscosity play important roles in determining the rate of gas flow from a disposal room breached by a drill bit. In PA analysis the gas pressure in the waste is computed as a function of time in the two phase flow code BRAGFLO (WIPP PA $1992 \mathrm{~b}$ p1-21). Prior to the 1992 PA the effects of disposal room closure on porosity and permeability resulting from salt creep and gas generation were largely ignored (WIPP PA Division, 1991a, Volume 3, Sections 3.4.7 and 3.4.8). In 1992 the effects of room deformation and gas generation were indirectly included in BRAGFLO computations using a porosity surface generated by the finite element code SANCHO (WIPP PA 1992 , p 1-43). Ideally, the value of these parameters (gas pressure, porosity, and permeability) at the time of drill bit intrusion can be made available for spall calculations. The role of spatially variable brine saturation and multiphase flow on spalling has not been investigated.

An experimental program was initiated in 1993 to demonstrate the effect of gas pressure release on waste surrogate response in a simple geometry. Experimental data for the spall behavior of a clay/sand waste surrogate in one dimension were generated to assess the validity of the computational models, and to determine the relationship between spall response in the test chamber to the tensile strength of the material (Berglund and Lenke, 1994). The 1D tests measured the dynamic response of a gas pressurized circular cylindrical clay/sand test sample to the sudden release of pressure on one end. The sudden release of pressure is comparable to the pressure drop that occurs adjacent to the drill bit when the bit suddenly penetrates a gas 
pressurized repository. At low gas pressures the pressure decay within the $15 \%$ kaolin clay/silica sand test sample and the locations of cracks verified the calculated response of the material and the results correlated with tests of the tensile strength of the sand/clay mixture. The principal conclusions of the ID tests were:

- Clay/sand test samples will spall in response to the pressure gradient generated by the 1D flow of gas from the sample to a lower pressure region.

- For gas pressure drops several times greater than the tensile strength of the test sample the spall response forces the sample material against a reaction plate and the time varying stress state between the test sample and the reaction plate is related to the instantaneous gas pressure profile. is observed.

-For gas pressure drops less than the tensile strength of the clay/sand test sample no spall

-For gas pressure drops on the order of the sample tensile strength, cracks form in the test sample that correlate with computational models and test data on sample tensile strength.

A test series to investigate spalling phenomena (blowout, stuck pipe, gas spall) in an axisymmetric geometry is currently being planned. The test device will permit the study of the radial flow of gas toward a borehole and will allow the generation of multiaxial stress states to be created in the test sample. In addition to spall response of a test sample near a borehole, the device will also be able to generate data for the effective shear resistance to erosion.

\section{Brine spall}

Brine spall consists of waste introduced into the drilling fluid caused by the flow of pressurized brine through the waste into the borehole. In a dipped repository it is possible that the gas generated by the decomposition of the waste will be segregated to the high end because of buoyancy effects. The low end of the repository could then be filled with pressurized brine. A drill bit penetration of the repository when it is saturated with pressurized brine may introduce waste into the drilling fluid in a process similar to that caused by pressurized gas. The brine introduced into the borehole and then to the surface also must be considered a release since it contains dissolved radionuclides. Other processes could also cause the repository to be pressurized with brine, such as a penetration through the repository into a Castile brine pocket followed by the partial failure of the borehole plug.

Scoping calculations are required to determine whether brine will accumulate in a low region of a repository as the result of waste decomposition and brine influx. If it is shown that a brine pressurized repository is likely, models will be developed for brine spall. To date no calculations have been performed to assess the importance of brine spall on releases to the surface. Brine spall can also be studied experimentally in the device designed for gas spalling effects.

\section{Brine slumy}

In a 1987 PA memorandum (PA, 1987) and subsequently in comments by the Environmental Evaluation Group (EEG) (WIPP PA, 1991b, Appendix B; WIPP PA,1992d, Appendix B) the issue of the formation of a brine slurry within a disposal room and its potential impact on direct releases were discussed. As defined in (PA, 1987) a brine slurry corresponds to a state where the waste and backfill are fully saturated with brine. If a fully saturated state were to occur prior to 
the creep consolidation of the repository it was conjectured that the waste would not have sufficient strength to resist the erosive effects of an inadvertent drill bit penetration and some quantity of waste would reach the surface. As discussed in (WIPP PA,1992d) present modeling does not indicate that the volume of brine in a panel will be sufficient to form a slurry. However, for extreme cases of seal permeability (e.g. seal failure), panels may be fully flooded with fluid that flows down from the Culebra.

Shortly after the cessation of the 100 year period of active institutional controls, and before the completion of creep consolidation, the waste containers will be, for the most part, intact (with minimal corrosion) and not tightly embedded within the backfill materials. Assuming a fully flooded condition, a drill bit penetration at this time will intersect one or more waste canisters directly in the drill path and carry the contents of these canisters and some backfill material to the surface in the drilling fluid. Intact canisters not directly adjacent to the drill bit will maintain their ability to contain the waste even though they are not embedded in a matrix. For Tater penetrations of the repository, after creep consolidation and additional canister decomposition, the effective stresses in the waste will serve to bind the waste and backfill and waste removal will occur from cuttings and cavings. Thus the most serious consequence of an early time penetration into a brine saturated room results in effects comparable to the cutting and cavings mechanisms discussed earlier. However, if the brine pressure adjacent to the penetrating drill bit exceeds hydrostatic pressure, additional forces may be generated to drive waste towards the borehole and up the annulus. As discussed above, this brine spall phenomenon needs further study.

\section{Summary}

Four direct release processes (cuttings, cavings, spallings, brine spall) have been identified as potentially important to meeting the long term compliance requirements of 40 CFR 191. A fifth process (brine slurry) has been screened out as a separate entity by concluding that the formation of a brine slurry is unlikely based on calculations performed to date and the consequences are not likely to be worse than those already generated by cuttings and cavings. A brine slumy at brine pressures above hydrostatic is included in the mechanism of brine spall.

The computational model for cuttings and cavings is complete and except for values of the effective shear resistance to erosion the data are adequate. No experimental data for waste shear resistance are available and consequently values typical for sea bed erosion have been used for performance assessment computations. These values are probably conservative and generate releases greater than for partially decomposed unmodified waste. Experiments to obtain data for shear resistance to erosion of clay/sand waste surrogates have been planned.

Spalling results from drill bit intrusions into waste which contains decomposition gas at pressures above hydrostatic. Blowout, stuckpipe or gas spall are the three possible outcomes from such an intrusion and the surface releases for cohesionless waste can be substantial. Of the four direct release processes, spalling has the potential of being the most important contributor to surface releases. The physical description of processes for spalling are based on computer models, oil and coal field experience, data from experiments conducted on coal outbursting, and 1D experiments on clay/sand waste surrogates. Worst case computational models that quantify releases have been developed but more data are necessary to assess the importance of waste strength on spall. Since spall is so potentially important to direct surface releases, small scale experiments are being designed to validate processes and quantify waste response in an axisymmetric geometry. These experiments will also help determine whether changes in waste properties such as waste strength or permeability through engineered enhancements would be beneficial.

Brine spall is closely related to gas induced spall discussed above. Little work has been done to characterize the effect of pressurized brine in the repository on the response of waste adjacent to a borehole wall. Oil industry data and analysis indicates that waste can spall off the wall in a manner similar to the process that occurs when the waste is pressurized with gas. The possibility 
of encountering pressurized brine saturated waste has yet to be demonstrated. Scoping calculations with BRAGFLO on a dipped repository are required.

\section{References}

Austin, E.H., 1983, Drilling Engineering Handbook, International Human Resources Development Corporation, Boston, p78.

Berglund, J.W., 1992, Mechanisms Governing the Direct Removal of Wastes from the WIPP Repository Caused by Exploratory Drilling, SAND-92-7295, Sandia National Laboratories, Albuquerque, NM.

Berglund, J.W., 1994, "An Upper Bound Model for the Direct Removal of Waste Caused by a Drilling Intrusion Into a WIPP Panel --For Use in Systems Prioritization Studies", Memorandum to R. Lincoln, New Mexico Engineering Research Institute, University of New Mexico, $18 \mathrm{July}$ 1994.

Berglund, J.W. and Lenke L., 1994, One Dimensional Experiments of Gas Induced Spall, To be published.

Bilgen E., Boulos, R., and Akgungor A.C., 1973, "Leakage and Frictional Characteristics of Turbulent Helical Flow In Fine Clearance, "Journal of Fluids Engineering, Volume 95, pp 493497.

Binder, R.C., 1958, Advanced Fluid Mechanics, Prentice-Hall , p63.

Bodziony, J., A. Nelicki, and J. Topolnicki. 1989. "Results of Laboratory Investigations of Gas and Coal Outbursts," Archiwum Gornictwa. Vol. 34, no. 3, 581-591.

Bratli, R. K., and R. Risnes. 1981. "Stability and Failure of Sand Arches," Society of Petroleum Engineers Journal (SPEJ). Vol. 21, no. 2, 236-248

Broc, R., ed. 1982. Drilling Mud and Cement Slurry Rheology Manual., Houston, TX: Gulf Publishing Company.

Butcher, B.M., 1994, "A Model for Cuttings Release Waste Properties", Memorandum to Distibution, Sandia National Laboratories, Albuquerque, NM, January 6, 1994.

Cheatham, J. B., Jr. 1984. "Wellbore Stability," Journal of Petroleum Technology. Vol. 36, no. $7,889-896$.

Coleman, B.D., and W. Noll. 1959. "Helical Flow of General Fluids," Journal of Applied Physics. Vol. 30, no. 10, 1508-1515. 
Darley, H.C.H. 1969. “A Laboratory Investigation of Borehole Stability," Journal of Petroleum Technology. Vol, 21, no. 7, 883-892.

Darley, H.C.H., and G.R. Gray. 1988. Composition and Properties of Drilling and Completion Fluids. Houston, TX: Gulf Publishing Company. 243.

Dyer, J., 1993, Personal Communication, Drilling Supervisor for McVay Drilling, Hobbs, NM., April 9, 1993

Dyer, J., 1994a, Personal Communication, Drilling Supervisor for McVay Drilling, Hobbs, NM., February 3,9, 1994.

Dyer, J., 1994b, Personal Communication, Drilling Supervisor for McVay Drilling, Hobbs, NM., April 7, 1994.

Fredrickson, A.G. 1960. "Helical Flow of an Annular Mass of Visco-Elastic Fluid," Chemical Engineering Science. Vol. 11, no. 3, 252-259.

Hall, C. D., Jr., and W.H. Harrisberger. 1970. "Stability of Sand Arches: A Key to Sand Control," Journal of Petroleum Technology. Vol. 22, July 1970, 821-829.

Jensen, W.,1993, Personal Communication, Driller for Ziadril, Hobbs, NM.

Lenke, L.R., Wang, J., Berglund, J.W., "Design of Soil Erosion Device", Memorandum to B. Butcher, New Mexico Engineering Research Institute, University of New Mexico, 1 March 1994.

Oldroyd, J.G. 1958. "Non-Newtonian Effects in Steady Motion of Some Idealized ElasticoViscous Liquids," Proceedings of the Royai Society of London. Series A, Vol. 245, no. 1241, 278-297.

Paterson, L. 1986. "Technical Note: A Model for Outbursts in Coal," International Journal of Rock Mechanics and Mining Sciences and Geomechanics Abstracts. Vol. 23, no. 4, 327-332.

Performance Assessment Group, 1987, Early PA Scoping Calculations -- To Help Refocus the Research, Development, and Engineering Parts of the WIPP Project, If Necessary, Draft Memorandum to Distribution, April 7, 1987, Albuquerque, NM: Sandia National Laboratories.

Rath, J.S., 1994, User's Reference Manual for CUTTINGS: A Code for Computing the Quantity of Wastes Brought to the Ground Surface When a Waste Disposal Room of a Radioactive Waste Repository is Inadvertently Penetrated by an Exploratory Borehole. SAND94-XXXX.

Albuquerque NM: Sandia National Laboratories.

Rechard, R.P., H. Iuzzolino, and J.S. Sandha. 1990. Data Used in Preliminary Performance Assessment of the Waste Isolation Pilot Plant (1990). SAND89-2408. Albuquerque, NM: Sandia National Laboratories.

Rechard, R.P., H.J. Iuzzolino, J.S. Rath, A.P. Gilkey, R.D. McCurley, and D.K. Rudeen, 1990,Users Manual for CAMCON: Compliance Assessment Methodology Controller. SAND881486. Albuquerque NM: Sandia National Laboratories.

Risnes, R., R.K. Bratli, and P. Horsrud. 1982. "Sand Stresses Around a Wellbore," SPEJ, Society of Petroleum Engineers Journal. Vol. 22, no. 6, 883-898.

Sato, K., and K. Itakura. 1989. "The Occurrence and Mechanism of Outbursts in Sandstone," Proceedings, Rock at Great Depth, International Symposium of Rock Mechanics, Pau, France, 
August 28-31, 1989. Eds. V. Maury and D. Fourmaintraux. Boston, MA: A.A. Balkema. Vol. 2, 635-641.

Savins, J.G., and G.C. Wallick. 1966. "Viscosity Profiles, Discharge Rates, Pressures, and Torques for a Rheologically Complex Fluid in a Helical Flow," A.I.Ch.E. Journal. Vol. 12, no. $2,357-363$.

Shepherd, J., L.K. Rixon, and L. Griffiths. 1981. "Rock Mechanics Review - Outbursts and Geological Structures in Coal Mines: A Review," International Journal of Rock Mechanics and Mining Sciences and Geomechanics Abstracts. Vol. 18, no. 4, 267-283.

Short, J.A. 1982. Drilling and Casing Operations. Tulsa, OK: PennWell Books, 183-184.

Stoess Jr., H. A., 1983, Pneumatic Conveying, John Wiley and Sons, New York.

Ujihira, M., K. Higuchi, and H. Nabeya. 1985. "Seale Model Studies and Theoretical Considerations on the Mechanism of Coal and Gas Outbursts," 21st International Conference of Safety in Mines, Sydney, Australia, October 21, 1985. Boston, MA: A.A. Balkema. 121-128.

US EPA (Environmental Protection Agency). 1986 "40 CFR 268: Land Disposal Restrictions.", as amended and published in the most recent Code of Federal Regulations. Washington, DC: Office of the Federal Register, National Archives and Records Administration.

US EPA (Environmental Protection Agency). 1985 " 40 CFR 191: Environmental Standards for the Management and Disposal of Spent Nuclear Fuel, High Level Transuranic Radioactive Waste, Final Rule." Federal Register. Vol 50, No. 182, 38066-38089.

Vaziri, H.H. 1989. "A Procedure for Analysing Seepage-ĩnduced Collapse of Weakly Cemented Formations," Proceedings, Rock at Great Depth, International Symposium of Rock Mechanics, Pau, France, August 28-31, 1989. Eds. V. Maury and D. Fourmaintraux. Boston, MA: A. A. Balkema. Vol. 2, 799-807.

Walker, R.E. 1976. "Hydraulic Limits are Set by Flow Restrictions," Oil and Gas Journal. Vol. 74 , no. $40,86-90$.

Walker, R.E., and W.E. Holman. 1971. "Computer Program Predicting Drilling-Fluid Performance," Oil and Gas Journal. Vol. 69, no. 13, 80-90.

Willis, C. 1978. "Rocky Mountain Coal Seams Call for Special Drilling Techniques," The Oil and Gas Journal. Vol. 76, no. 28, 143-152.

WIPP PA (Performance Assessment) Division. 1992a, Preliminary Performance Assessment for the Waste Isolation Pilot Plant, December 1992, Volume 2: Technical Basis, Sandia Report SAND92-0700/2, Sandia National Laboratories, Albuquerque NM, December 1992, pp. 7-23 to 7-29.

WIPP PA (Performance Assessment) Division. 1992b, Preliminary Performance Assessment for the Waste Isolation Pilot Plant, December 1992, Volume 3: Model Parameters, Sandia Report SAND92-0700/3, Sandia National Laboratories, Albuquerque NM, December 1992, pp. 1-30 to $1-34$.

WIPP PA (Performance Assessment) Division. 1992c, Preliminary Performance Assessment for the Waste Isolation Pilot Plant, December 1992, Volume 4: Uncertainty and Sensitivity Analysis for 40 CFR Part 191, Subpart B, Sandia Report SAND92-0700/4, Sandia National Laboratories, Albuquerque NM, December 1992, pp.

7-1 to 7-11. 
WIPP PA (Performance Assessment) Division. 1992d, Preliminary Performance Assessment for the Waste Isolation Pilot Plant, December 1992, Volume I: Third Comparison with 40 CFR Part 191, Subpart B, Sandia Report SAND92-0700/1, Sandia National Laboratories, Albuquerque NM, December 1992.

WIPP PA (Performance Assessment) Division. 1991a, Preliminary Comparison with 40 CFR Part 191, Subpart B, for the Waste Isolation Pilot Plant, December 1991, Volume 3: Reference Data, Sandia Report SAND91-0893/3, Sandia National Laboratories, Albuquerque NM, December 1991.

WIPP PA (Performance Assessment) Division. 1991b, Preliminary Comparison with 40 CFR Part 191, Subpart B, for the Waste Isolation Pilot Plant, December 1991, Volume 1: Methodology and Results, Sandia Report SAND91-0893/1, Sandia National Laboratories, Albuquerque NM, December 1991.

Zenz, F. A. and Othmer, D.F., 1960, Fluidizatioñ and Fluid-Particle Systems, Reinhold Publishing Corporation, New York, p330. 


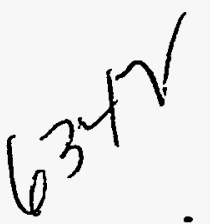

Copy to:

D.R. Anderson

MS-1328 (Org 6342)

MS-1341 (Org 6308)

R. Lincoln

MS-1341 (Org 6348)

J. Holmes

MS-1345 (Org 6307)

P. Davis

MS-1341 (Org 6347)

D. Schafer

M. Marietta

MS-1328 (Org 6342)

B. Butcher

MS-1341 (Org 6348)

F. Mendenhall

MS-1341 (Org 6308)

W. Beyeler

Geo Cen (Org 6308)

D. Rudeen

Geo Cen (Org 6308)

P. Swift

MS-1345 (Org 6307)

L. Lenke

R. Cole

(NMERI)

SWCF PA WBS 1.1.6.3.1 
83. Garner, J.W. 1994. "Radionuclides to Transport for System Prioritization (SP) Calculations." Memo, July 13, 1994. 
Date 13 July, 1994

To Distribution

From J W Garner

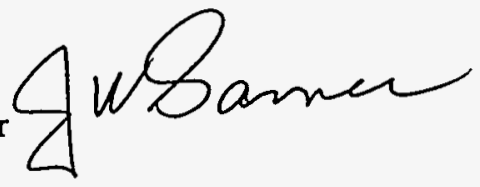

Subject Radionuclides to Transport for System Prioritization (SP) calculations

For a normal performance assessment(PA) calculation, we would transport 10 radionuclides $\left({ }^{241} \mathrm{Am}\right.$, ${ }^{240} \mathrm{Pu},{ }^{239} \mathrm{Pu},{ }^{238} \mathrm{Pu},{ }^{237} \mathrm{~Np},{ }^{234} \mathrm{U},{ }^{233} \mathrm{U},{ }^{230} \mathrm{Th},{ }^{229} \mathrm{Th}$, and ${ }^{226} \mathrm{Ra}$ )

If it becomes necessary to reduce calculation time in the Culebra transport codes, we could implement the following:

1) Transport only ${ }^{241} \mathrm{Am},{ }^{239} \mathrm{Pu}$, and ${ }^{233} \mathrm{U}$

Define $\mathrm{M}^{\# \# \#} \mathrm{Nn}$ as the mass to transport and $\mathrm{P}^{\# \#} \mathrm{Nn}$ as the mass from PANEL of radioonuclide \#n

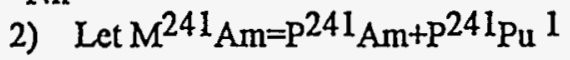

3) Also, let $\mathrm{M}^{239} \mathrm{Pu}=\mathrm{P}^{239} \mathrm{Pu}+\mathrm{P}^{240} \mathrm{Pu} * 3.7 \mathrm{E} 0+\mathrm{P}^{242} \mathrm{Pu} * 6.4 \mathrm{E}-2{ }^{2}$

4) Also,let $\mathrm{M}^{233} \mathrm{U}=\mathrm{P}^{233} \mathrm{U}+\mathrm{P}^{234} \mathrm{U} * 6.5 \mathrm{E}-1+\mathrm{P}^{238} \mathrm{Pu} * 6.5 \mathrm{E}-1+\mathrm{P}^{235} \mathrm{U} * 2.3 \mathrm{e}-4+\mathrm{P}^{238} \mathrm{U} * 3.5 \mathrm{E}-5$ $+\mathrm{P}^{236} \mathrm{U} * 6.8 \mathrm{E}-3+\mathrm{P}^{237} \mathrm{~Np} * 7.4 \mathrm{E}-2 \mathrm{2}, 3$

5) Assume that each radionuclide is a single chain

6) Assume that the half life of ${ }^{233} \mathrm{U}$ is infinite ${ }^{4}$

This process should take place at the SECO-TP/PANEL interface, probably in PRESECO-TP

This is based on the contact-handled and remote-handled inventory published by DOE, $1994^{5}$, and could change if there is significant changes in the radionuclide inventory for the SP calculations.

1241 Pu decays to ${ }^{241}$ Am with a 14.4 year half life.

2 The multiplication factors in 3) and 4) are to maintain the number of Curies since Curies are the units of the $40 \mathrm{CFR} 191$ release limits. The output of transport done using this weighted accumulation should not be used in mass units, but only in Curies.

${ }^{3238} \mathrm{Pu}$ decays to ${ }^{234} \mathrm{U}$ with a half life of 87.7 years. ${ }^{237} \mathrm{~Np}$ decays to ${ }^{233} \mathrm{U}$, but the half life is $2.1 \mathrm{E} 6$ years. This substitution is equivalent to saying $\mathrm{Np}$ transports the same as $\mathrm{U}$.

${ }^{4}$ This will in some manner account for the daughters of $U$ by assuming they transport the same as $U$.

${ }^{5}$ DOE (1994) Waste Isolation Pilot Plant Transuranic Waste Baseline Inventory Report. CAO-94-1005, Carlsbad Area Office, DOE, Carlsbad, NM 
DOE 1994 INVENTORY

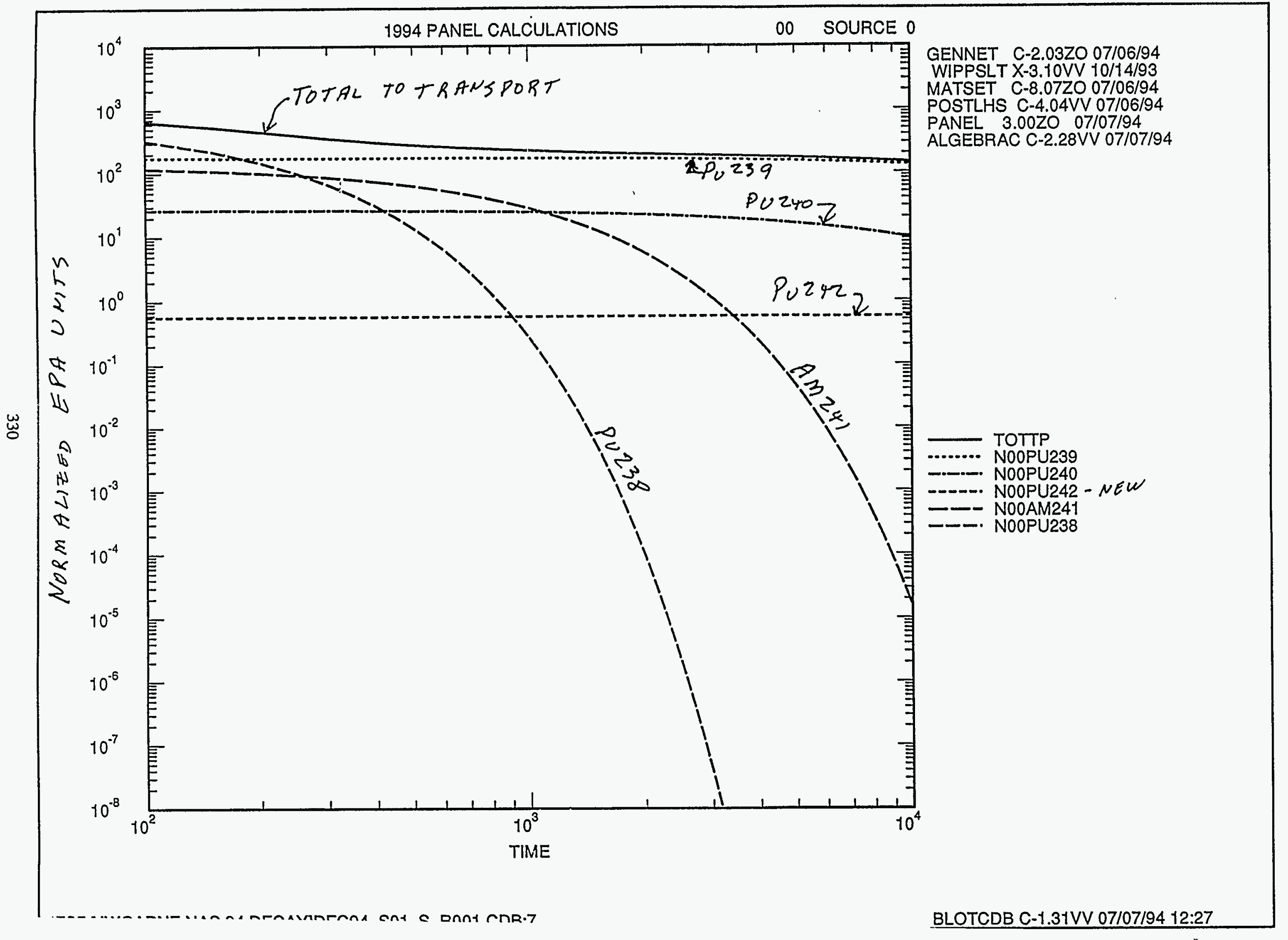


עu以,

$-$

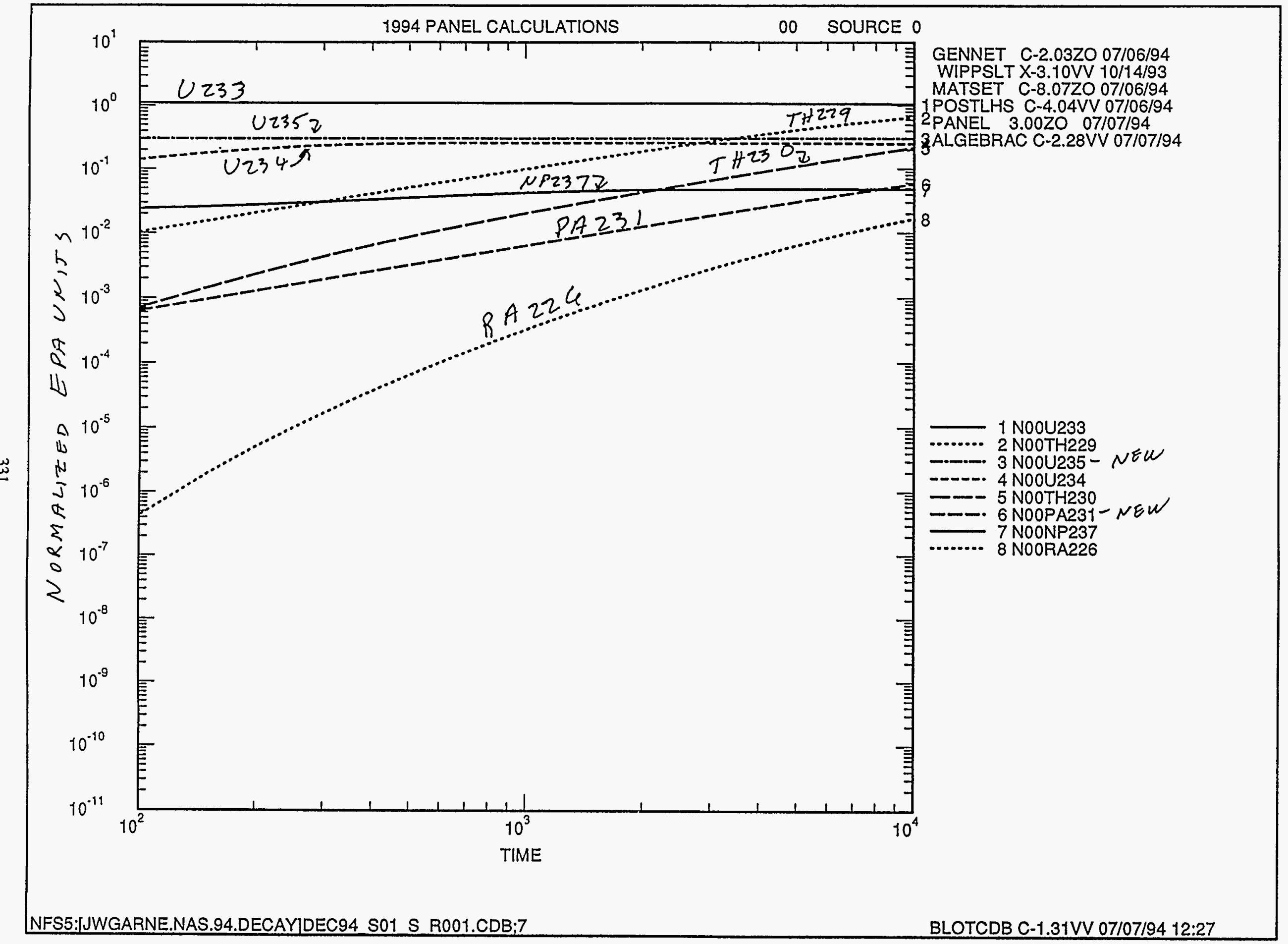


DOE, 1994 INVENTORY

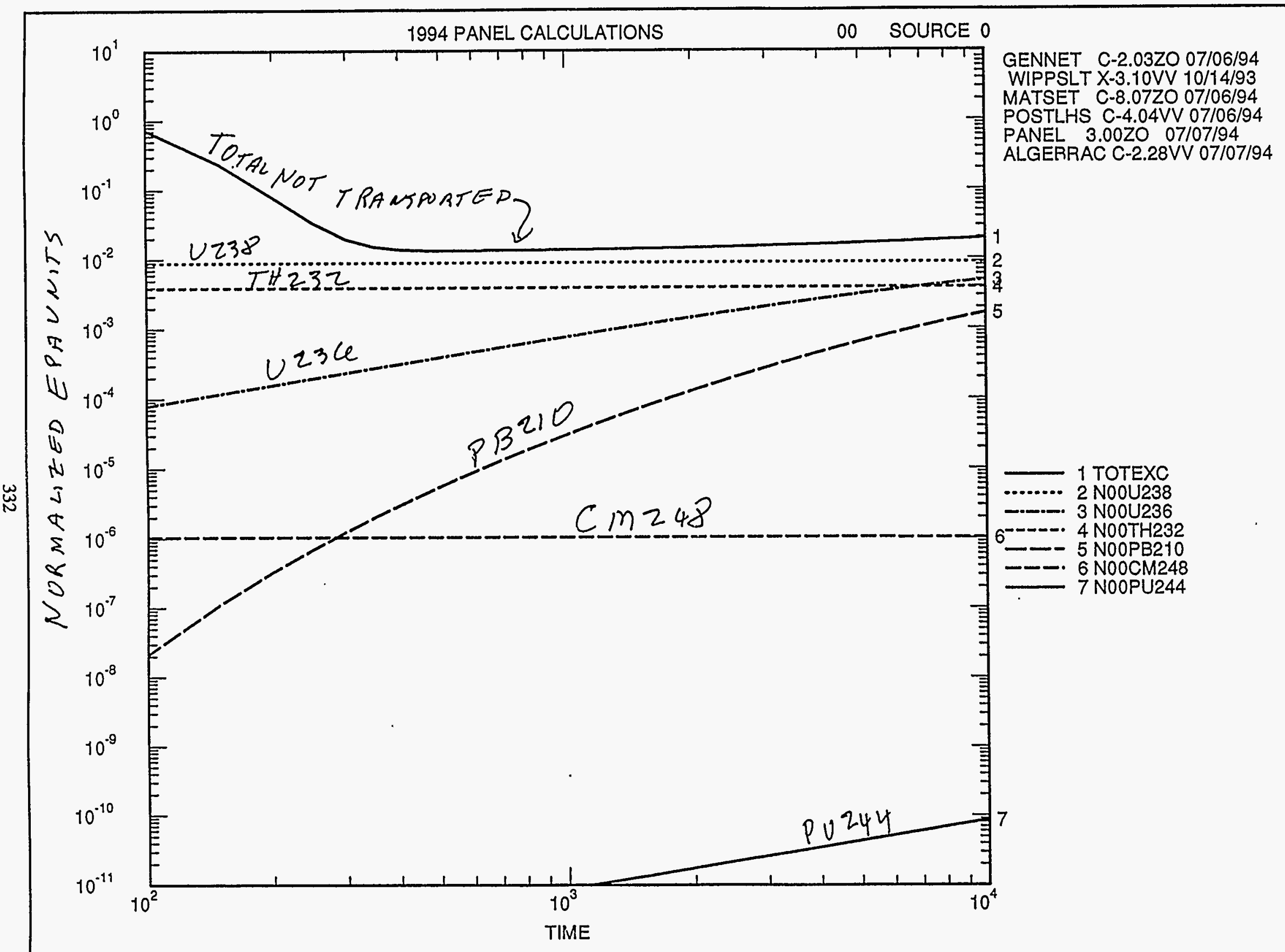


VUV, அサ

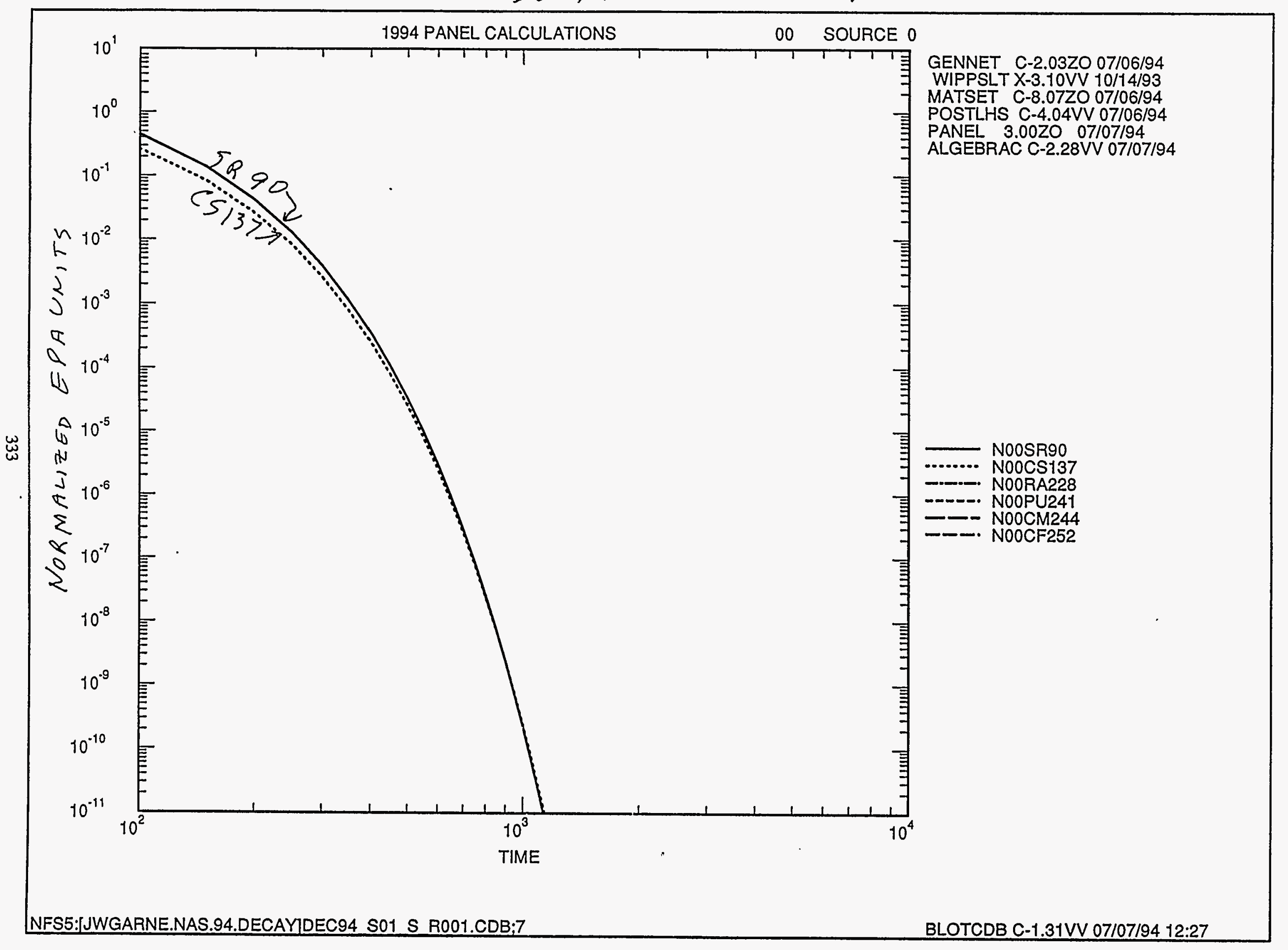


1. SR-90

2 CS -137

$3 \mathrm{~PB}-210$

$4 \mathrm{RA}-226$

$5 \mathrm{RA}-228$

$6 \mathrm{TH}-229$

$7 \mathrm{TH}-230$

8 TH -232

$9 \mathrm{PA}-231$

i0 U -233

$\begin{array}{lll}11 & \mathrm{U} & -234\end{array}$

12 U -235

$13 \mathrm{U}-236$

$14 U-238$

$15 \quad$ NP -237

16 PU-238

$17 \mathrm{PU}-239$

18 PU -240

19 PU-241

20 PU-242

21 PU-244

$32 \mathrm{AM}-241$

$? 3 \mathrm{CM}-244$

$34 \mathrm{CM}-248$

$\pm 5 C F-252$

$36 \mathrm{PM}-147$

$37 \quad S M-147$
RHL

2. $9120 \mathrm{E}+01$

3. $0000 \mathrm{E}+01$

$2.2300 \mathrm{E}+01$

1. $6000 \mathrm{E}+03$

$5.7500 \mathrm{E}+00$

$7.3400 \mathrm{E}+03$

$7.7000 \mathrm{E}+04$

1. $4050 \mathrm{E}+10$

3. $2760 \mathrm{E}+04$

1. $5850 \mathrm{E}+05$

$2.4450 \mathrm{E}+05$

$7.0380 \mathrm{E}+08$

2. $3420 \mathrm{E}+07$

4. $4680 \mathrm{E}+09$

2. $1400 \mathrm{E}+06$

8.7740E+01

2. $4070 \mathrm{E}+04$

$6.5370 \mathrm{E}+03$

1. $4400 \mathrm{E}+01$

3. $7630 \mathrm{E}+05$

$8.2600 \mathrm{E}+07$

$4.3220 \mathrm{E}+02$

1. $8110 \mathrm{E}+01$

$3.3900 \mathrm{E}+05$

$2.6380 \mathrm{E}+00$

$2.6234 \mathrm{E}+00$

$1.0600 \mathrm{E}+06$
IDAUG

$\mathrm{PB}-210$

$R A-226$

$R A-228$

TH -229

TH -230

$\mathrm{PA}-231$

$\mathrm{TH}-232$

$\mathrm{U}-234$

U -233

U -234

U -235

U -236

$A M-241$

U -238

PU-240

NP -237

PU -240

PU-244

$\mathrm{CM}-248$

SM-147

\section{CUPMO}

1. $2277 \mathrm{E}+04$

1. $1917 \mathrm{E}+04$

1. $6031 \mathrm{E}+04$

2. $2344 E+02$

$6.2173 E+04$

$4.8705 \mathrm{E}+01$

$4.6428 \mathrm{E}+00$

2. 5445E-05

1. $0913 \mathrm{E}+01$

2. $2555 E+00$

1. $4622 \mathrm{E}+00$

$5.0795 \mathrm{E}-04$

1.5265E-02

8.0013E-05

1. $6705 \mathrm{E}-01$

4. $0745 \mathrm{E}+03$

1. $4852 \mathrm{E}+01$

$5.4688 \mathrm{E}+01$

$2.4826 E+04$

9.5003E-01

4. 3280E-03

$8.2716 \mathrm{E}+02$

1.9740E+04

$1.0546 \mathrm{E}+00$

1. $3552 E+05$

1. $3627 \mathrm{E}+05$

3. $3726 \mathrm{E}-01$
CUPKG

1. $3641 \mathrm{E}+05$

8. $6982 \mathrm{E}+04$

$7.6339 \mathrm{E}+04$

$9.8865 \mathrm{E}+02$

$2.7269 E+05$

$2.1269 \mathrm{E}+02$

$2.0186 \mathrm{E}+01$

$1.0968 \mathrm{E}-04$

4.7241E+01

$9.6803 \mathrm{E}+00$

$6.2485 E+00$

$2.1615 \mathrm{E}-03$

$6.4680 \mathrm{E}-02$

3. $3619 \mathrm{E}-04$

7.0487E-01

$1.7120 \mathrm{E}+04$

$6.2144 \mathrm{E}+01$

2. $2787 \mathrm{E}+02$

1. $0301 \mathrm{E}+05$

$3.9257 \mathrm{E}+00$

1. $7738 \mathrm{E}-02$

$3.4322 \mathrm{E}+03$

8.0903E+04

$4.2523 \mathrm{E}+00$

$5.3777 \mathrm{E}+05$

$9.2702 \mathrm{E}+05$

$2.2943 E+00$
WIPP CURIES

$5.9520 \mathrm{E}+04$

3. $2170 \mathrm{E}+04$

$0.0000 \mathrm{E}+00$

$0.0000 \mathrm{E}+00$

$0.0000 \mathrm{E}+00$

$0.0000 \mathrm{E}+00$

$0.0000 \mathrm{E}+00$

4.7400E-01

$0.0000 \mathrm{E}+00$

1. $3400 \mathrm{E}+0.3$

$0.0000 \mathrm{E}+00$

3. $6839 \mathrm{E}+02$

$0.0000 \mathrm{E}+00$

1. $0810 \mathrm{E}+01$

$2.4266 \mathrm{E}+01$

$8.7370 \mathrm{E}+05$

$2.1280 \overline{\mathrm{E}}+05$

3. $2780 \mathrm{E}+04$

$6.3200 E+05$

$6.8995 \mathrm{E}+02$

$0.0000 \mathrm{E}+00$

1. $4760 \mathrm{E}+05$

$0.0000 \mathrm{E}+00$

$0.0000 E+00$

1. $6400 \mathrm{E}+02$

1. $8620 \mathrm{E}+03$

$0.0000 E+00$
WIPP GRAMS

$4.3634 \mathrm{E}+02$

$3.6985 \mathrm{E}+02$

$0.0000 \mathrm{E}+00$

$0.0000 E+00$

$0.0000 \mathrm{E}+00$

$0.0000 \mathrm{E}+00$

$0.0000 \mathrm{E}+00$

4.3219E+06x

$0.0000 \mathrm{E}+00$

1. $3843 \mathrm{E}+05$

$0.0000 E+00$

1.7043E+08

$0.0000 \mathrm{E}+00$

3.2155E+07

3. $4426 \mathrm{E}+04$

$5.1035 \mathrm{E}+04$

3. $4243 E+06$

1. $4386 \mathrm{E}+05$

$6.1351 \mathrm{E}+03$

1.7575E+05

$0.0000 \mathrm{E}+00$

4. 3005E+04

$0.0000 \mathrm{E}+00$

$0.0000 \mathrm{E}+00$

3. $0496 \mathrm{E}-01$

2. $0086 \mathrm{E}+00$

$0.0000 \mathrm{E}+00$

$$
\text { DOE, } 1994 \text { INVENTORY }
$$

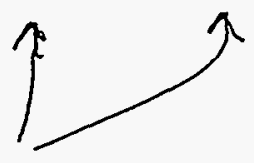

* radionuclides with large mass,
small number of curies 
Distribution (MS)

1341 R. C. Lincoln

1341 W. E. Beyclor

1341 F. T. Mendenhall

1341 D. K. Rudeen

1328 D. R. Anderson

1328 M. G. Marietta

1328 J. C. Helton

1328 L. C. Sanchez

1328 C.T. Stockman $1.1 .7 ; c 0 / 5 \phi$

P. Roache,EcoDymanics 
. 


\section{WIPP \\ UC721 - DISTRIBUTION LIST}

\section{Federal Agencies}

US Department of Energy (6)

Office of Civilian Radioactive Waste Mgmt.

Attn: Deputy Director, RW-2

Associate Director, RW-10/50

Office of Prog. \& Resources Mgmt. Office of Contract Business Mgmt.

Director, RW-22

Analysis \& Verification Division

Associate Director, RW-30

Office of Systems \& Compliance

Associate Director, RW-40

Office of Storage \& Transportation

Director, RW-4/5

Office of Strategic Planning and

International Programs

Office of External Relations

Forrestal Building

Washington, DC 20585

US Department of Energy

Albuquerque Operations Office

Attn: National Atomic Museum Library

P.O. Box 5400

Albuquerque, NM 87185-5400

US Department of Energy

Research \& Waste Management Division

Attn: Director

P.O. Box E

Oak Ridge, TN 37831

US Department of Energy (5)

Carlsbad Area Office

Attn: G. Dials

D. Galbraith

M. McFadden

R. Lark

J. A. Mewhinney

P.O. Box 3090

Carlsbad, NM 88221-3090

US Department of Energy

Office of Environmental Restoration and

Waste Management

Attn: J. Lytle, EM-30

Forrestal Building

Washington, DC 20585-0002
US Department of Energy (3)

Office of Environmental Restoration and Waste Management

Attn: M. Frei, EM-34, Trevion II

Washington, DC 20585-0002

US Department of Energy

Office of Environmental Restoration and Waste Management

Attn: S. Schneider, EM-342, Trevion II Washington, DC 20585-0002

US Department of Energy (2)

Office of Environment, Safety \& Health

Attn: C. Borgstrom, EH-25

R. Pelletier, EH-231

Washington, DC 20585

US Department of Energy (2)

Idaho Operations Office

Fuel Processing \& Waste Mgmt. Division

785 DOE Place

Idaho Falls, ID 83402

US Environmental Protection Agency (2)

Radiation Protection Programs

Attn: M. Oge

ANR-460

Washington, DC 20460

Tomothy M. Barry

Office of Policy, Planning \& Evaluation

PM 223X U. S. EPA

Washington, DC 20460

US Department of Energy

Rocky Flats Area Office

Attn: T. Lukow

P. O. Box 928

Golden, CO 80402-0928

US Nuclear Regulatory Commission (3)

Division of High Level Waste Management

Attn: Seth Coplan

R. Codell

N. Eisenberg

Mail Stop 4-H-3

Washington, DC 20555 


\section{Boards}

Defense Nuclear Facilities Safety Board Attn: D. Winters

625 Indiana Ave. NW, Suite 700

Washington, DC 20004

Nuclear Waste Technical Review Board (2)

Attn: Chairman

S. J. S. Parry

1100 Wilson Blvd., Suite 910

Arlington, VA 22209-2297

\section{State Agencies}

Attorney General of New Mexico

P.O. Drawer 1508

Santa Fe, NM 87504-1508

Environmental Evaluation Group (3)

Attn: Library

7007 Wyoming NE

Suite F-2

Albuquerque, NM 87109

NM Energy, Minerals, and Natural

Resources Department

Attn: Library

2040 S. Pacheco

Santa Fe, NM 87505

NM Environment Department (3)

Secretary of the Environment

Attn: Mark Weidler

1190 St. Francis Drive

Santa Fe, NM 87503-0968

NM Bureau of Mines \& Mineral Resources

Socorro, NM 87801

NM Environment Department

WIPP Project Site

Attn: P. McCasland

P.O. Box 3090

Carlsbad, NM 88221

\section{Laboratories/Corporations}

Battelle Pacific Northwest Laboratories

Attn: R. E. Westerman, MSIN P8-44

Battelle Blvd.

Richland, WA 99352
INTERA, Inc.

Attn: G. A. Freeze

1650 University Blvd. NE, Suite 300

Albuquerque, NM 87102

INTERA, Inc.

Attn: J. F. Pickens

6850 Austin Center Blvd., Suite 300

Austin, TX 78731

INTERA, Inc.

Attn: W. Stensrud

P.O. Box 2123

Carlsbad, NM 88221

Los Alamos National Laboratory

Attn: B. Erdal, INC-12

P.O. Box 1663

Los Alamos, NM 87544

$\mathrm{RE} / \mathrm{SPEC}$, Inc

Attn: Angus Robb

4775 Indian School NE, Suite 300

Albuquerque, NM 87110-3927

RE/SPEC, Inc

Attn: J. L. Ratigan

P.O. Box 725

Rapid City, SD 57709

Tech Reps, Inc. (3)

Attn: J. Chapman (1) Loretta Robledo (2)

5000 Marble NE, Suite 222

Albuquerque, NM 87110

Westinghouse Electric Corporation (5)

Attn: Library

J. Epstein

J. Lee

B. A. Howard

R. Kehrman

P.O. Box 2078

Carlsbad, NM 88221

S. Cohen \& Associates

Attn: Bill Thurber

1355 Beverly Road

McLean, VA 22101

Battelle Northwest

Attn: Pamela Doctor

P. O. Box 999

Richland, WA 99352 
Los Alamos National Laboratory

Analysis and Assessment Division

A-1, MS F600

Attn: M. D. McKay

P. O. Box 1663

Los Alamos, NM 87544

Kenneth T. Bogen

Environmental Sciences Division

Lawrence Livermore National Laboratory

Livermore, CA 94550

David Burmaster

Alceon Corporation

P. O. Box 2669

Harvard Square Station

Cambridge, MA 02239

Idaho National Laboratory

Westinghouse-Idaho Nuclear Co.

Attn: R. Klingler

Mail Stop 3422

P. O. Box 4000

Idaho Falls, ID 83415-3422

Beta Corporation Int.

Attn: E. Bonano

6613 Esther NE

Albuquerque, NM 87109

Ecodynamics

Attn: P. Roache

P. O. Box 9229

Albuquerque, NM 87119-9229

A. E. Van Luik

INTERA/M\&O

The Valley Bank Center

101 Convention Center Drive

Las Vegas, NV 89109

Center for Nuclear Waste Regulatory Analysis (CNWRA)

Southwest Research Institute

Attn: B. Sagar

P. O. Drawer 28510

6220 Culebra Road

San Antonio, TX 78284

Dr. F. Owen Hoffman

SENES Oak Ridge

102 Donner Drive

Oak Ridge, TN 37830
Dr. Alison Cullen

2125 North 90 th Street

Seattle, WA 98103

\section{National Academy of Sciences, WIPP Panel}

Howard Adler

Oxyrase, Incorporated

7327 Oak Ridge Highway

Knoxville, TN 37931

Bob Andrews

Board of Radioactive Waste Management

GF456

2101 Constitution Ave.

Washington, DC 20418

Rodney C. Ewing

Department of Geology

University of New Mexico

Albuquerque, NM 87131

Charles Fairhurst

Department of Civil and Mineral Engineering

University of Minnesota

500 Pillsbury Dr. SE

Minneapolis, MN 55455-0220

B. John Garrick

PLG Incorporated

4590 MacArthur Blvd., Suite 400

Newport Beach, CA 92660-2027

Leonard F. Konikow

US Geological Survey

431 National Center

Reston, VA 22092

Carl A. Anderson, Director

Board of Radioactive Waste Management

National Research Council

HA 456

2101 Constitution Ave. NW

Washington, DC 20418

Christopher G. Whipple

ICF Kaiser Engineers

1800 Harrison St., 7th Floor

Oakland, CA 94612-3430 
John O. Blomeke

720 Clubhouse Way

Knoxville, TN 37909

Sue B. Clark

University of Georgia

Savannah River Ecology Lab

P.O. Drawer E

Aiken, SC 29802

Konrad B. Krauskopf

Department of Geology

Stanford University

Stanford, CA 94305-2115

Della Roy

Pennsylvania State University

217 Materials Research Lab

Hastings Road

University Park, PA 16802

David A. Waite

$\mathrm{CH}_{2} \mathrm{M}$ Hill

P.O. Box 91500

Bellevue, WA 98009-2050

Thomas A. Zordon

Zordan Associates, Inc. 3807 Edinburg Drive

Murrysville, PA 15668

\section{Universities}

University of New Mexico

Geology Department

Attn: Library

141 Northrop Hall

Albuquerque, NM 87131

University of Washington

College of Ocean \& Fishery Sciences

Attn: G. R. Heath

583 Henderson Hall, $\mathrm{HN}-15$

Seattle, WA 98195

Terry Reilly

Decision Sciences Department

University of Oregon

Eugene, OR 97403-1208

John Evans

Harvard School of Public Health

665 Huntington Avenue

Boston, MA 02115
F. E. Haskin

Dept. of Chemical and Nuclear Engr.

University of New Mexico

Albuquerque, NM 87131

M. Granger Morgan

Dept. of Engineering and Public Policy

Carnegie-Mellon University

Pittsburgh, PA 15213

Yacov Y. Haimes

Center for Risk Management of Engineering

D111 Thornton Hall

University of Virginia

Charlottsville, VA 22901

Elisabeth Pate-Cornell

Industrial Engineering \& Engineering Mgmt.

Stanford University

Stanford, CA 94305

University of Hawaii at Hilo

Business Administration

Attn: S. Hora

Hilo, HI 96720-4091

\section{Alan Gutjahr}

Department of Mathematics

New Mexico Institute of Mining \& Tech.

Socorro, NM 87801

Professor George Apostolakis

Room 24-221

Massachusetts Institute of Technology

Cambridge, MA 02139-4307

Professor H. Christopher Frey

North Carolina State University

Dept. of Civil Engineering

Box 7908

Raleigh, NC 27695

Professor Dale Hattis

Center for Technology, Environment \& Dev.

Clark University

950 Main Street

Worcester, Massachusetts 01610

Thomas E. McKone

School of Public Health

University of California

Berkeley, CA 94720-7360 


\section{Libraries}

Thomas Brannigan Library

Attn: D. Dresp

106 W. Hadley St.

Las Cruces, NM 88001

Government Publications Department

Zimmerman Library

University of New Mexico

Albuquerque, NM 87131

New Mexico Junior College

Pannell Library

Attn: R. Hill

Lovington Highway

Hobbs, NM 88240

New Mexico State Library

Attn: N. McCallan

325 Don Gaspar

Santa Fe, NM 87503

New Mexico Tech

Martin Speere Memorial Library

Campus Street

Socorro, NM 87810

WIPP Public Reading Room

Carlsbad Public Library

$101 \mathrm{~S}$. Halagueno St.

Carlsbad, NM 88220

\section{Foreign Addresses}

Studiecentrum Voor Kernenergie

Centre d'Energie Nucleaire

Attn: A. Bonne

SCK/CEN Boeretang 200

B-2400 Mol, BELGIUM

Atomic Energy of Canada, Ltd.

Whiteshell Laboratories

Attn: B. Goodwin

Pinawa, Manitoba, CANADA R0E IL0

Francois Chenevier (2)

ANDRA

Route de Panorama Robert Schumann

B. P. 38

92266 Fontenay-aux-Roses, Cedex

FRANCE
Claude Sombret

Centre d'Etudes Nucleaires de la Vallee Rhone

CEN/VALRHO

S.D.H.A. B.P. 171

30205 Bagnols-Sur-Ceze, FRANCE

Commissariat a L'Energie Atomique

Attn: D. Alexandre

Centre d'Etudes de Cadarache

13108 Saint Paul Lez Durance Cedex

FRANCE

Bundesanstalt fur Geowissenschaften und

Rohstoffe

Attn: M. Langer

Postfach 510153

D-30631 Hannover, GERMANY

Bundesministerium fur Forschung und

Technologie

Postfach 200706

5300 Bonn 2, GERMANY

Institut fur Tieflagerung

Attn: K. Kuhn

Theodor-Heuss-Strasse 4

D-3300 Braunschweig, GERMANY

Gesellschaft fur Anlagen und Reaktorsicherheit (GRS)

Attn: B. Baltes

Schwertnergasse 1

D-50667 Cologne, GERMANY

Shingo Tashiro

Japan Atomic Energy Research Institute

Tokai-Mura, Ibaraki-Ken, 319-11

JAPAN

Netherlands Energy Research Foundation ECN

Attn: J. Prij

3 Westerduinweg

P.O. Box 1

1755 ZG Petten

THE NETHERLANDS

Svensk Karnbransleforsorjning AB

Attn: F. Karlsson

Project KBS (Karnbranslesakerhet)

Box 5864

S-102 48 Stockholm

SWEDEN 
Nationale Genossenschaft fur die Lagerung

Radioaktiver Abfalle (2)

Attn: S. Vomvoris

P. Zuidema

Hardstrasse 73

CH-5430 Wettingen

SWITZERLAND

AEA Technology

Attn: J. H. Rees

D5W/29 Culham Laboratory

Abington, Oxfordshire OX14 3DB

UNITED KINGDOM

AEA Technology

Attn: W. R. Rodwell

044/A31 Winfrith Technical Centre

Dorchester, Dorset DT2 8DH

UNITED KINGDOM

AEA Technology

Attn: J. E. Tinson

B4244 Harwell Laboratory

Didcot, Oxfordshire OX11 ORA

UNITED KINGDOM

Terry Andres

AECL Research

Whiteshell Laboratories

Pinawa, Manitoba ROE 1LO

CANADA

Russell Cheng

University of Kent at Canterbury

Cornwallis Building

Canterbury, Kent, CT2 7NF

UNITED KINGDOM

Christian Ekberg

Chalmers University of Technology

Dept. of Nuclear Chemistry

S-41296 Goteborg

SWEDEN

Sven Erik Magnusson

Lund University

P.O. Box 118

22100 Lund

SWEDEN
Delft University of Technology

Dept. of Mathematics

Attn: Roger Cooke

Julianalaan 132

Delft

THE NETHERLANDS

J. Marivoet

Centre d'Etudes de l'Energie

Nucleaire (CEN/SCK)

Boeretang 200

B-2400 Mol, BELGIUM

Timo Vieno

Technical Research Centre of Finland

(VTT)

Nuclear Energy Laboratory

P. O. Box 208

SF-02151 Espoo, FINLAND

Claudio Pescatore

Division of Radiation Protection \& Waste Mgmt

OECD Nuclear Energy Agency

38, Boulevard Suchet

F-75016 Paris

FRANCE

Tonis Papp

Swedish Nuclear Fuel \& Waste Mgmt. Co.

Box 5864

S 10248 Stockholm

SWEDEN

Alan J. Hooper

UK Nirex Ltd.

Curie Avenue

Harwell, Didcot

Oxfordshire, OX11 ORH

UNITED KINGDOM

Eduard Hofer

Gesellschaft fur Reaktorsicherheit

(GRS) MBH

Forschungsgelande

D-8046 Garching, GERMANY

Andrea Saltelli

Commission of the European Communities

Joint Research Centre of Ispra

I-21020 Ispra (Varese)

ITALY 
Daniel A. Galson

Galson Sciences Ltd.

35, Market Place

Oakham

Leicestershire LE15 6DT

UNITED KINGDOM

Ghislain de Marsily (GXG Chairman)

University Pierre et Marie Curie

Laboratorie de Geologie Applique

4, Place Jussieu

T.26 - 5e etage

75252 Paris Cedex 05

FRANCE

Atomic Energy of Canada, Ltd.

Whiteshell Research Establishment

Attn: D. Wushke

Pinewa, Manitoba ROE ILO, CANADA

Johan Andersson

Swedish Nuclear Power Inspectorate

Statens Karnkraftinspektion (SKI)

Box 27106

S-102 52 Stockholm

SWEDEN

Professor Jack P. C. Kleijnen

Department of Information Systems

Tillburg University

5000 LE Tilburg

THE NETHERLANDS

Dr. Marion Scott

Department of Statistics

Mathematics Building

University of Glasgow

Glasgow, G12 8QW

SCOTLAND

Performance Assessment Peer Review Panel

G. Ross Heath

College of Ocean \& Fishery Sciences

University of Washington

583 Henderson Hall, HN-15

Seattle, WA 98195

Thomas H. Pigford

Department of Nuclear Engineering

4159 Etcheverry Hall

University of California

Berkeley, CA 94720
Thomas A Cotton

JK Research Associates, Inc.

4429 Butterworth Place NW

Washington, DC 20016

Robert J. Budnitz

President, Future Resources Assoc., Inc.

2000 Center Street, Suite 418

Berkeley, CA 94704

C. John Mann

Department of Geology

245 Natural History Bldg.

1301 West Green Street

University of Illinois

Urbana, IL 61801

Frank W. Schwartz

Dept. of Geology and Mineralogy

Ohio State University

Scott Hall

1090 Carmack Rd.

Columbus, $\mathrm{OH} 43210$

\begin{tabular}{lll} 
MS & Org. & \multicolumn{1}{l}{ Internal } \\
\hline 0415 & 5411 & N. H. Prindle \\
0425 & 5415 & R. C. Lincoln \\
1324 & 6115 & P. B. Davies \\
1320 & 6719 & E. J. Nowak \\
1322 & 6121 & J. R. Tillerson \\
1328 & 6749 & D. R. Anderson \\
1328 & 6749 & M. E. Fewell \\
1328 & 6749 & G. K. Froehlich \\
1328 & 6749 & B. L. Baker \\
1328 & 6749 & J. W. Berglund \\
1328 & 6749 & R. Blaine \\
1328 & 6749 & J. W. Garner \\
1328 & 6749 & D. Rudeen \\
1328 & 6749 & J. D. Schreiber \\
1328 & 6749 & P. Vaugh \\
1328 & 6749 & D. M. Boak \\
1328 & 6741 & H. N. Jow \\
1328 & 6741 & J. C. Helton (10) \\
1328 & 6741 & M. S. Tierney \\
1328 & 6741 & K. E. Economy \\
1328 & 6741 & J. E. Bean \\
1328 & 6741 & A. W. Shiver \\
1328 & 6741 & L. N. Smith \\
1335 & 6705 & M. Chu \\
1341 & 6416 & W. Beyeler \\
1341 & 6811 & A. L. Stevens \\
1341 & 6747 & D. R. Schafer \\
1341 & 6748 & J. T. Holmes
\end{tabular}




$\begin{array}{lll}1341 & 6821 & \text { P. N. Swift } \\ 1345 & 6751 & \text { K. W. Larson } \\ 1395 & 6700 & \text { P. Brewer } \\ 1395 & 6800 & \text { L. Shephard } \\ 1395 & 6707 & \text { M. Marietta } \\ 1395 & 6841 & \text { V. H. Slaboszewicz } \\ 9133 & 8220 & \text { F. T. Mendenhall } \\ & & \\ 1330 & 6752 & \text { C. B. Michaels (2) } \\ 1330 & 6752 & \text { NWM Library (20) } \\ 9018 & 8523-2 & \text { Central Technical Files } \\ 0899 & 4414 & \text { Technical Library (5) } \\ 0619 & 12615 & \text { Print Media } \\ 0100 & 7613-2 & \text { Document Processing (2) } \\ & & \text { for DOE/OSTI }\end{array}$



